\title{
Residual Radioactive Contamination From Decommissioning
}

Technical Basis for Translating

Contamination Levels to Annual Dose

Draft Report for Comment

Manuscript Completed: January 1990

Date Published: January 1990

Prepared by

W. E. Kennedy, Jr., R. A. Peloquin

Pacific Northwest Laboratory

Richland, WA 99352

\section{Prepared for}

Division of Regulatory Applications

Office of Nuclear Regulatory Research

U.S. Nuclear Regulatory Commission

Washington, DC 20555

NRC FIN B2908

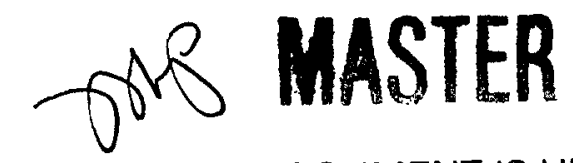


$$
\text { . }
$$ 


\section{DISCLAIMER}

This report was prepared as an account of work sponsored by an agency of the United States Government. Neither the United States Government nor any agency thereof, nor any of their employees, makes any wartanty, express or implied, or assumes any legal liability or responsibility for the accuracy, completeness, or usefulness of any information, apparatus, product, or process disclosed, or represents that its use would not infringe privately owned rights. Reference herein to any specific commercial product, process, or service by trade name, trademark, manufacturer, or otherwise does not necessarily constitute or imply its endorsement, recommendation, or favoring by the United States Government or any agency thereof. The views and opinions of authors expressed herein do not necessarily state or reflect those of the United States Government or any agency thereof. 


\section{DISCLAMMRR}

Portions of this docoment myy be illegible in electronic irogige products. Images are produced from the best available original doevonem. 


\section{ABSTRACT}

This document describes the generic modeling of the total effective dose equivalent (TEDE) to an individual in a population from a unit concentration of residual radioactive contamination. Radioactive contamination inside buildings and soil contamination are considered. Unit concentration TEDE factors by radionuclide, exposure pathway, and exposure scenario are calculated. Reference radiation exposure scenarios are used to derive unit concentration TEDE factors for about 200 individual radionuclides and parentdaughter mixtures. For buildings, these unit concentration factors list the annual TEDE for volume and surface contamination situations. For soil, annual TEDE factors are presented for unit concentrations of radionuclides in soil during residential use of contaminated land and the TEDE per unit total inventory for potential use of drinking water from a ground-water source. Because of the generic treatment of potentially complex ground-water systems, the annual TEDE factors for drinking water for a given inventory may only indicate when additional site data or modeling sophistication are warranted. Descriptions are provided of the models, exposure pathways, exposure scenarios, parameter values, and assumptions used. An analys is of the potential annual TEDE resulting from reference mixtures of residual radionuclides is provided to demonstrate application of the TEDE factors. 



\section{FOREWORD}

The following report was prepared at the request of the U.S. Nuclear Regulatory Commission (NRC) to provide a technical basis for calculating the residual radioactive contamination limits allowable for the unrestricted release of decommissioned facilities. The conversion factors and annual doses contained in the report are not intended for uses beyond the scope of this report, and accordingly, should not be applied to other circumstances. Even within the proper scope of decommissioning, it is emphasized that external dose rates should be determined from actual survey measurements, wherever practical, rather than calculating the dose rates from the models used in this report.

This report is now published in draft to afford the benefit of comments from a broad spectrum of readers. After responding to the comments, the report will be re-issued as a final report. In the interim, the NRC staff will use the report as a technical basis document. Publication of this report will be followed by a companion document for use in implementation of the Commission Policy Statement on Exemptions from Regulatory Control. The NRC staff expects that the radionuclide-specific values that correspond $+n$ the individual dose criterion of the policy statement will serve as the bas: : for future decommissioning decisions. As such, these values would replace the values in Option 1 of the NRC Office of Nuclear Material Safety and Safeguards, Branch Technical Position (Federal Register, Vol. 46, No. 205, October 23, 1981, pp. 52061-3) and the numerical values contained in Regulatory Guide 1.86 . Thus, this technical basis report serves as the foundation for the revised decommissioning criteria.

Comments on this report will be accepted for 90 days after publication and should be directed to:

Dr. Robert A. Meck

Office of Nuclear ReguTatory Research

U.S. Nuclear Regulatory Commission

Washington, D.C. 20555 


\section{EXECUTIVE SUMMARY}

This document describes a generic method for evaluating conditions of unrestricted release of slightly radioactive material in buildings and soil following decommissioning of licensed facilities. The generic evaluation utilizes a radiation exposure scenario analysis that addresses the major exposure pathways of direct exposure to penetrating radiation, inhalation, and ingestion. The result of the evaluation is the total effective dose equivalent (TEDE) to an average individual in a population group exposed to a unit concentration of residual radioactive material after decommissioning. Unit concentration TEDE factors are provided for each exposure pathway and scenario for about 200 individual radionuclides, including key parentdaughter chains. For long-lived parents with short-lived daughters, and for decay chains in equilibrium, the factors are presented in terms of activity of the total parent-daughter mixture. Additional values for the parents and daughters are listed separately so that the annual TEDE can be estimated for non-equilibrium cases.

The annual TEDE resulting from a specific concentration of a radionuclide or mixture of radionuclides can be estimated using the unit concentration TEDE factors provided in this document. The input parameters for each exposure pathway and scenario were selected to provide a reasonable (not worst case) estimate of the likely radiation dose to an average individual in a limited population group exposed to residual radioactive contamination. However, the TEDE factors are still thought to produce overestimates of the potential dose an average individual in the general public (not a limited population group) may receive. Whenever practical, external dose rates determined from actual survey measurements, instead of the results from generic modeling, should be used when estimating the external radiation doses resulting from the conditions found at specific sites.

For unrestricted release of buildings, two sets of generically derived unit concentration annual TEDE factors are provided. They include a set of factors for building renovation (accounting for volume sources), relating the TEDE mrem per $\mathrm{pCi} / \mathrm{g}$ (or $\mu \mathrm{Sv}$ per $\mathrm{Bq} / \mathrm{g}$ ), and a set of factors for building occupancy (accounting for surface sources), relating the TEDE in mrem per $\mathrm{dpm} / 100 \mathrm{~cm}^{2}$ (or $\mu \mathrm{Sv}$ per $\mathrm{Bq} / 100 \mathrm{~cm}^{2}$ ) of surface activity. Each of the derived sets of annual TEDE factors for buildings is developed in a manner designed to independently describe a specific situation (i.e., volume or surface contamination). The resulting unit concentration annual TEDE factors are listed by radionuclide and exposure pathway. Modifications can be made to the scenario assumptions, including the exposure durations, ingestion rates, or air concentrations to account for site-specific conditions.

For unrestricted use of land, residual radioactive soil contamination may be described in a thin or thick soil layer, on the surface or stabilized under a clean soil layer, over a large or small area, and may consist of many different radionuclides or mixtures of radionuclides. The derived unit soil concentration annual TEDE factors provided in this document, in units of mrem per $\mathrm{pCi} / \mathrm{g}$ (or $\mu \mathrm{Sv}$ per $\mathrm{Bq} / \mathrm{g}$ ) of soil, relate to surface soils and are based on 
a residential use scenario that considers a combination of the following pathways: inhalation, external exposure, and agricultural food product ingestion. Additional annual TEDE factors related to potential ingestion of contaminated drinking water are also provided. These factors account for the potential effect of the total radionuclide inventory at the site, in the soil or in building materials left onsite that potentially may be demolished and disposed of as buried rubble. Because of the generic treatment of potentially complex ground-water systems, the annual TEDE for the drinking water scenario for a given inventory may only indicate when additional site data or more sophisticated modeling are warranted. Modifications can be made to the TEDE factors to account for contaminated soil that is isolated from the surface by a layer of clean soil or overburden, or to account for sitespecific ground-water conditions.

Example calculations are provided using the annual TEDE factors. Example TEDE values are calculated for representative mixtures and quantities of radionuclides associated with radiopharmaceutical/industrial-source manufacturing plants, uranium fuel fabrication facilities, depleted and enriched uranium sources, and light-water power reactors. The examples consider residual radioactive contamination in both buildings and soils. Finally, a summary discussion of the scenario pathway analysis and the resulting unit concentration annual TEDE factors is provided. 


\section{ACKNOWLEDGMENTS}

The authors would like to thank all of those individuals who helped contribute to the development, production, and publication of this report. We greatly appreciate the guidance, assistance, technical review, and encouragement provided by Dr. Stan Neuder, Harvey Scott, Dr. Robert A. Meck and Dr. Donald A. Cool of the U.S. Nuclear Regulatory Commission (NRC), and Joe Soldat and Bruce Napier of Pacific Northwest Laboratory (PNL). We would like to recognize the technical contributions of Dr. Richard B. Codell (NRC), Dr. Donald W. Lee (Oak Ridge National Laboratory), and Dr. R. William Nelson (PNL) in the development of the generic drinking-water model. We would also like to thank Carolyn Schauls for her contribution in typing, organizing, and correcting the draft and final manuscripts. 


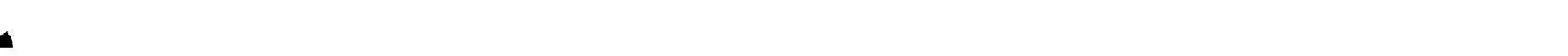




\section{CONTENTS}

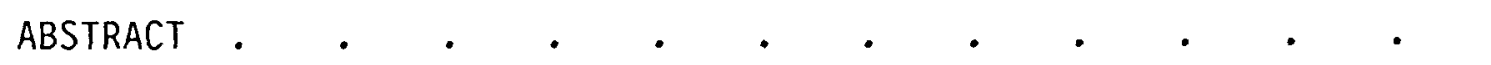

i i i

FOREWORD

EXECUTIVE SUMMARY

ACKNOWLEDGMENTS

1.0 INTRODUCTION

2.0 MODELING APPROACH

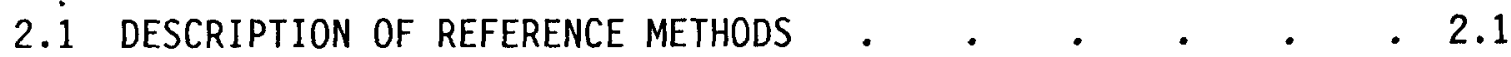

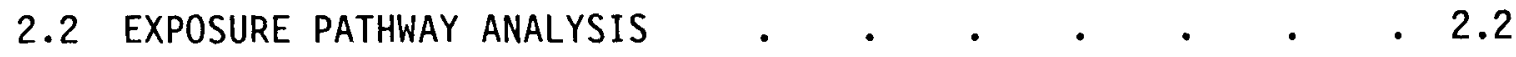

2.2.1 External Exposure to Penetrating Radiation . . . 2.3

2.2.2 Inhalation . . . . . . . . . . 2.15

2.2.3 Secondary Ingestion . . . . . . . 2.25

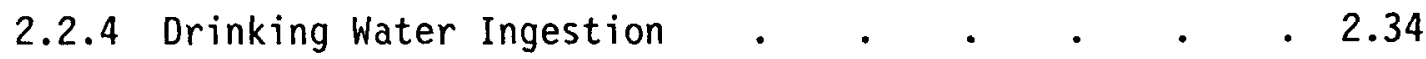

2.2.5 Agricultural Pathways . . . . . . . 2.43

3.0 SCENARIO ANALYSIS FOR RESIDUAL RADIOACTIVE MATERIALS • • • • 3.1

3.1 SCENARIO DESCRIPTIONS FOR THE RELEASE OF BUILOINGS • • 3.1

3.1.1 Building Renovation (Volume Activity) Scenario • $\quad 3.2$

3.1.2 Building Occupancy (Surface Activity) Scenario • . 3.13

3.2 SCENARIO DESCRIPTIONS FOR THE RELEASE OF
CONTAMINATED LAND..
.

3.2.1 Residential Use (Surface Soil) Scenario . . . 3.23

3.2.2 Drinking Water Use Scenario . . . . . . . 3.34

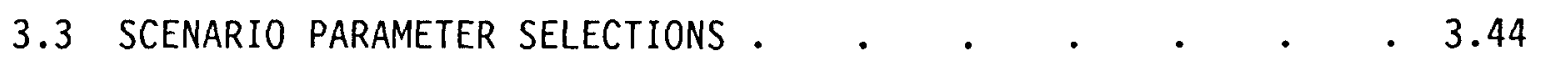

4.0 EXAMPLE DOSE CALCULATIONS FOR REFERENCE BUILDINGS AND LAND • • 4.1

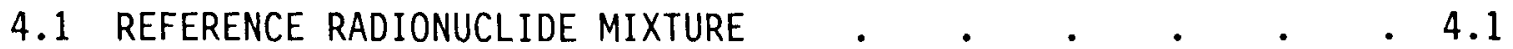

4.2 EXAMPLE DOSE CALCULATIONS FOR THE BUILDING

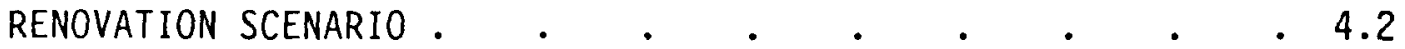


4.3 EXAMPLE DOSE CALCULATIONS FOR THE BUILDING

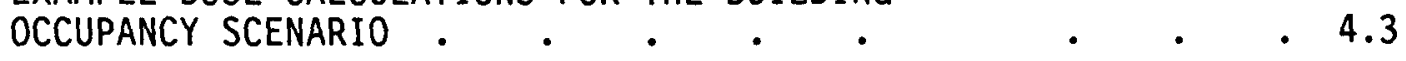

4.4 EXAMPLE DOSE CALCULATIONS FOR THE SURFACE SOIL

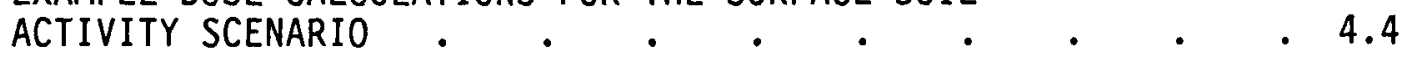

4.5 EXAMPLE DOSE CALCULATIONS FOR THE DRINKING WATER SCENARIO • 4.5

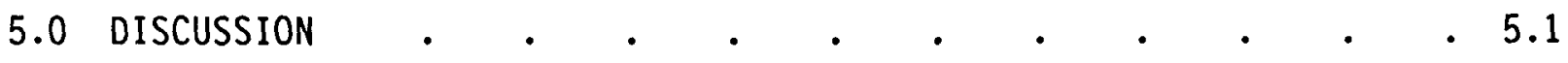

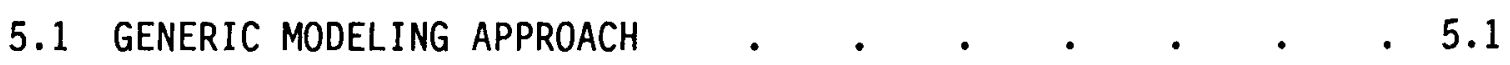

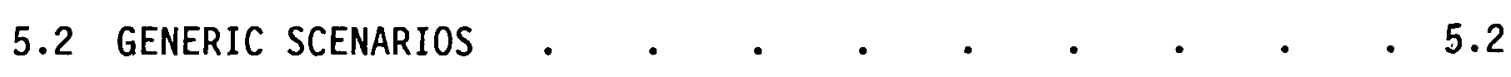

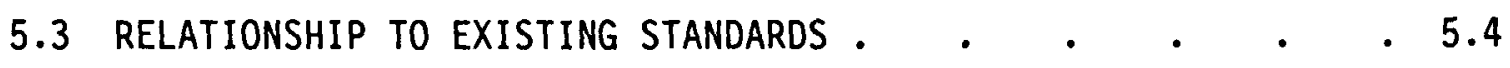

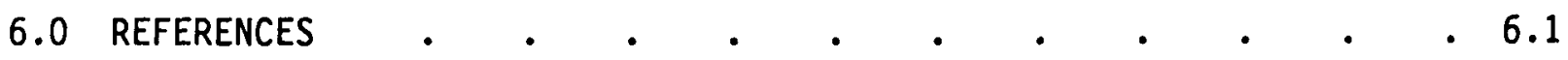
APPENDIX A - EXTERNAL EXPOSURE MODELING SENSITIVITY STUDIES • • • A.1

A.1 WHOLE BODY DOSE RATE VERSUS SOURCE AREA . . . . . . A.1

A.2 EXTERNAL DOSE RATE VERSUS ROOM VOLUME • • • • • . A.2

A.3 EXTERNAL DOSE RATE VERSUS SOURCE CONFIGURATION • • . A.4

A.4 EXTERNAL DOSE RATES FOR SOIL CONTAMINATION CONDITIONS • . A.4

A.5 EXTERNAL DOSE RATE MODELING APPROACH

A.6 SUMMARY 6 .

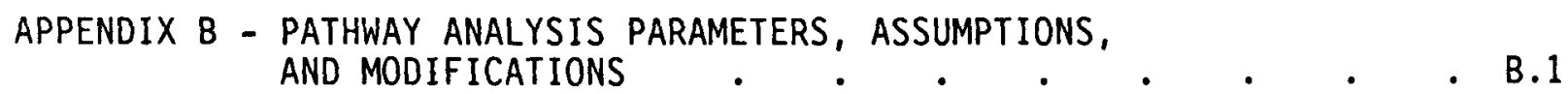

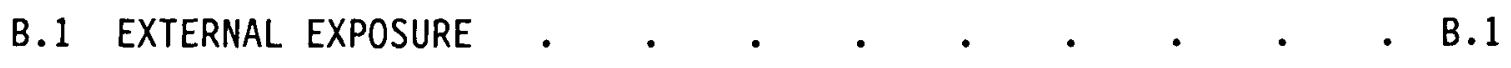

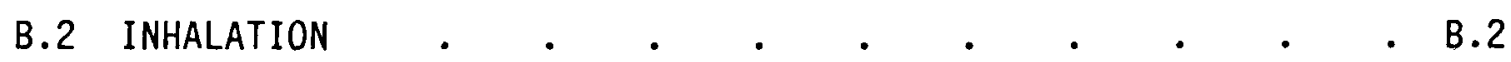

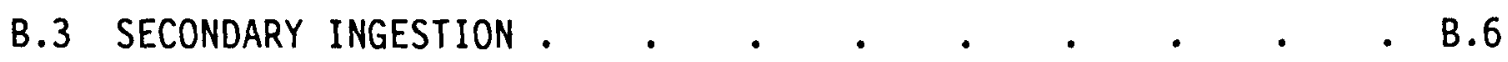

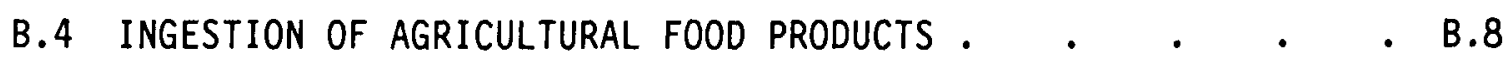

B.5 DRINKING WATER INGESTION • • • • • • • • • • • • B.8

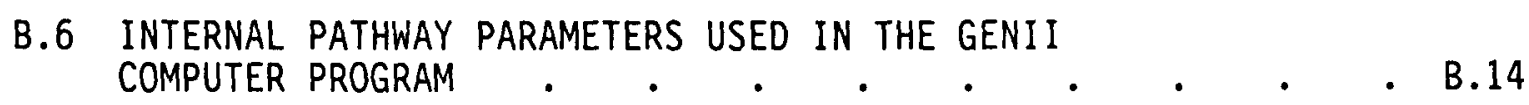

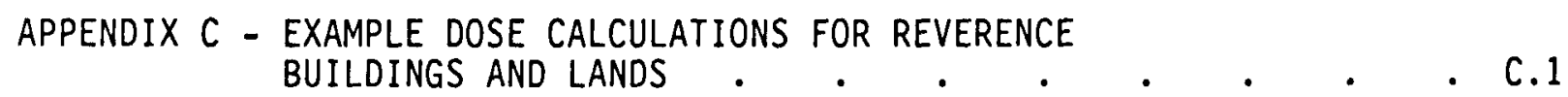

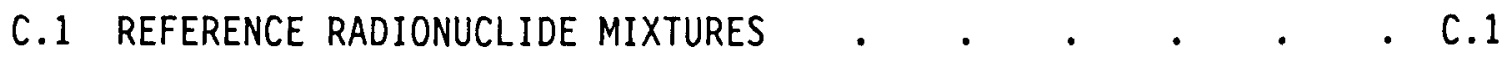


C.2 EXAMPLE DOSE CALCULATIONS FOR THE BUILDING RENOVATION SCENARIO • $\cdot$ •

C.3 EXAMPLE DOSE CALCULATIONS FOR THE BUILDING

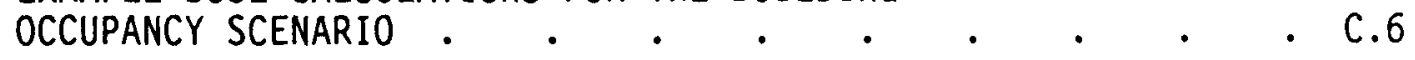

C.4 EXAMPLE DOSE CALCULATIONS FOR THE RESIDENTIAL USE (SURFACE SOIL) SCENARIO $\cdot \cdot \cdot \cdot \cdot \cdot \cdot \cdot \cdot \cdot \cdot$ C. .14

C.5 EXAMPLE DOSE CALCULATIONS FOR THE DRINKING

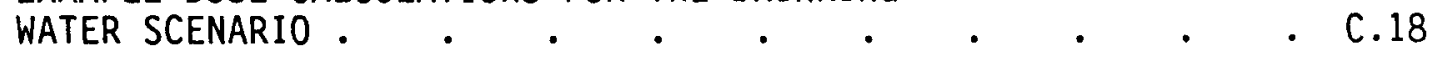

APPENDIX D - COMPUTERIZED MODEL INPUT/OUTPUT . • • • • • • • 0.1

D.1 RADIOACTIVE CHAIN DECAY • . . . . . . . . . . . D.1

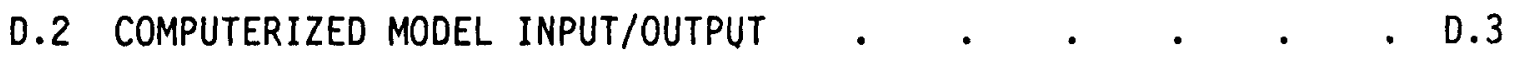
APPENDIX E - GLOSSARY • • • • . • • • • • • E.1 


\section{FIGURES}

A.1 Estimated Tissue Dose Rate Versus Source Area . . . . . A.2

A.2 Estimated Tissue Dose Rate at $1 \mathrm{~m}$ Above a Floor Having $1 \mathrm{Ci} / \mathrm{m}^{2}$ as a Function of Room Volume $. . . . \quad . \quad . \quad A .3$

A.3 Estimated Tissue Dose Rate for Selected Source Configurations Versus Room Volume for an Effective

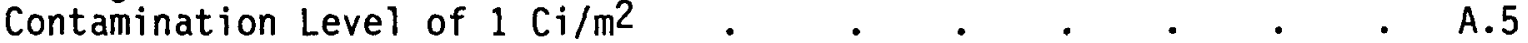

A.4 Estimated Tissue Dose Rate for Selected Conditions Versus Area of Soil Contamination for an Effective Concentration of $1 \mathrm{Ci} / \mathrm{m}^{2} \quad$. A.6

B.1 Two-Box Representation of a Simple Leach-Rate System . . B.10 


\section{TABLES}

2.1 External Dose Rate Conversion Factors for Exposure to

Residual Radioactive Materials . . . . . • . . . 2.6

2.2 Inhalation Committed Effective Dose Rate Conversion

Factors for Exposure to Residual Radioactive Materials . . 2.16

2.3 Ingestion Committed Effective Dose Rate Conversion Factors

for Exposure to Residual Radioactive Materials . . . . 2.26

2.4 Maximum Organ Dose Rate Conversion Factors for Ingestion

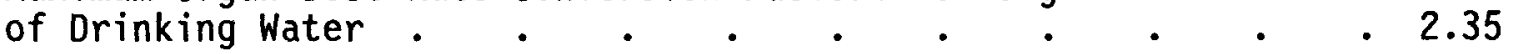

2.5 Agricultural Food Product Ingestion Committed Effective Dose Rate Conversion Factors for Exposure to Residual Radioactive

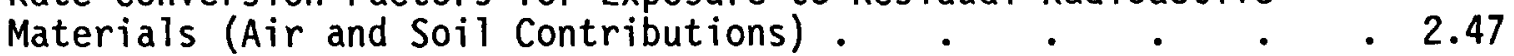

3.1 Annual Total Effective Dose Equivalent Factors for the Building

Renovation (Volume Activity) Scenario . . . . . . 3.4

3.2 Annual Total Effective Dose Equivalent Factors for the Building Occupancy (Surface Activity) Scenario . . . . . . 3.14

3.3 Annual Total Effective Dose Equivalent Factors for the $\quad 3.25$

3.4 Drinking Water Scenario Annual Committed Effective Dose Equivalent Factors for Exposure to Residual Radioactive Materials . . . . . . . . . . 3.35

3.5 Expected Ranges for Major Scenario or Pathway Parameters
and the Selected Values Used in This Study . . . . 3.45

Estimated Relative Activity of Radionuclides in Deposited
Surface Contamination at the Reference Power Reactor . . 4.2

4.2 Estimated Annual Total Effective Dose Equivalents for the Building Renovation (Volume Activity) Scenario Using the Light-Water Power Reactor Surface Contamination Inventory . • 4.3

4.3 Estimated Annual Total Effective Dose Equivalents for the Building Occupancy (Surface Activity) Scenario Using the Light-Water Power Reactor Surface Contamination Inventory • . 4.4

4.4 Estimated Annual Total Effective Dose Equivalents for the Residential Use (Surface Soil) Scenario Using the Light-Water Power Reactor Surface Contamination Inventory • . 4.5 
4.5 Estimated Annual Total Effective Dose Equivalents for the Drinking Water Scenario Using the Light-Water Power Reactor

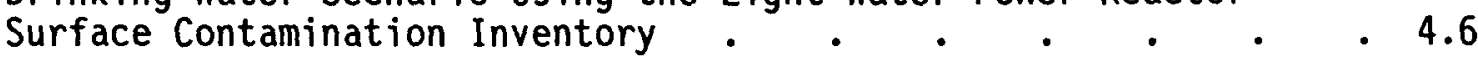

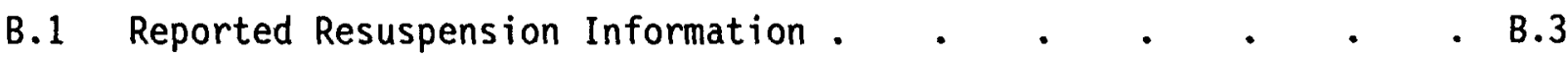

B.2 Inhalation Solubility Class Information . . . . . . B.5

B.3 Referenced Surface-Contamination Ingestion Data - • • • - B.7

B.4 Ground Water Partition Coefficient Information . . . • . B.13

B.5 Parameter Values Used by the GENII Computer Program
for Calculation of Internal Dose Conversion Factors $~ . ~$

C.1 Estimated Relative Activity of Radionuclides at a Reference Uranium Fuel Fabrication Facility 10 Years After Shutdown . . C.3

C.2 Estimated Relative Activity of Radionuclides in Depleted and Enriched Uranium Mixtures . . . . . . . . C.3

C.3 Estimated Relative Activity of Radionuclides in Neutron-
Activated Concrete at the Reference Power Reactor . . . C.4

C.4 Estimated Relative Activity of Radionuclides at the

Reference Radiopharmaceutical/Industrial-Source Facility . • C.5

C.5 Estimated Annual Total Effective Dose Equivalents for the Building Renovation (Volume Activity) Scenario Using the Uranium Fuel Fabrication Facility Inventory 10 Years After Shutdown.

C.6 Estimated Annual Total Effective Dose Equivalents for the Building Renovation (Volume Activity) Scenario Using the Depleted and Enriched Uranium Mixture . . . . . . C.8

C.7 Estimated Annual Total Effective Dose Equivalents for the Building Renovation (Volume Activity) Scenario Using the Neutron-Activated Power Reactor Concrete Inventory 30 Years After Shutdown

C.8 Estimated Annual Total Effective Dose Equivalents for the Building Renovation (Volume Activity) Scenario Using the Reference Radiopharmaceutical/Industrial-Source Inventory 5 Years After Shutdown

C.9 Estimated Annual Total Effective Dose Equivalents for the Building Occupancy (Surface Activity) Scenario Using the Uranium Fuel Fabrication Facility Inventory 10 Years After Shutdown. 
C.10 Estimated Annual Total Effective Dose Equivalents for the Building Occupancy (Surface Activity) Scenario Using the Depleted and Enriched Uranium Mixture . . . . . . C.11

C.11 Estimated Annual Total Effective Dose Equivalents for the Building Occupancy (Surface Activity) Scenario Using the Neutron-Activated Power Reactor Concrete Inventory

C.12 Estimated Annual Total Effective Dose Equivalents for the Building Occupancy (Surface Activity) Scenario Using the Reference Radiopharmaceutical/Industrial-Source Inventory 5 Years After Shutdown.$\quad \cdot \quad \cdot \quad \cdot \quad \cdot \quad \cdot \quad \cdot \quad \cdot \quad$.

C.13 Estimated Annual Total Effective Dose Equivalents for the Residential Use (Surface Soil) Scenario Using the Uranium Fuel Fabrication Facility Inventory 10 Years After Shutdown

C.14 Estimated Annual Total Effective Dose Equivalents for Residential Use (Surface Soil) Scenario Using the Depleted

C.15 Estimated Annual Total Effective Dose Equivalents for the Residential Use (Surface Soil) Scenario Using the NeutronActivated Power Reactor Concrete Inventory 30 Years After Shutdown .

C.16 Estimated Annual Total Effective Dose Equivalents for the Residential Use (Surface Soil) Scenario Using the Reference Radiopharmaceutical/Industrial-Source Inventory 5 Years After Shutdown

C.17 Estimated Annual Total Effective Dose Equivalents for the Drinking Water Scenario Using the Uranium Fuel Fabrication Facility Inventory 10 Years After Shutdown . . . . . . . C.19

C.18 Estimated Annual Total Effective Dose Equivalents for the Drinking Water Scenario Using the Depleted and Enriched Uranium Mixtures

C.19 Estimated Annual Total Effective Dose Equivalents for the Drinking Water Scenario Using the Neutron-Activated Power Reactor Concrete Inventory 30 Years After Shutdown

C.20 Estimated Annual Total Effective Dose Equivalents for the Drinking Water Scenario Using the Reference Radiopharmaceutical/ Industrial-Source Inventory 5 Years After Shutdown

D.1 Radionuclide Decay and Daughter Ingrowth Chain Organization in the Radionuclide Master Library . . . . D.6

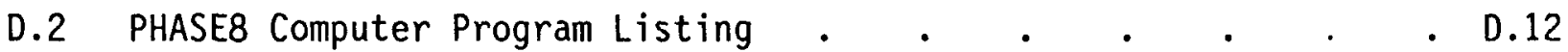


D.3 Radionuclide Control List (RMDLIBDA.SHT) • • • • • • D.38

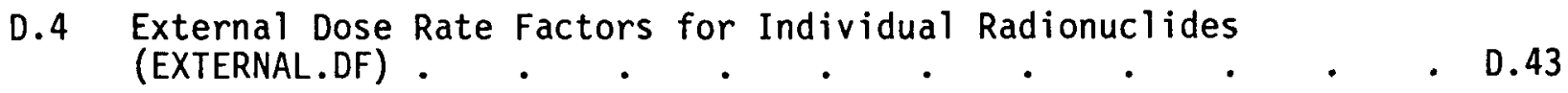

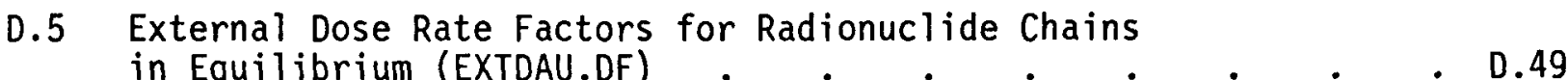

D.6 Internal Dose Rate Factors for Radionuclide Chains in Equilibrium (INTERNAL.DF) . . . . . . . . . D.54

D.7 Internal Dose Rate Factors for Individual

Radionuclides (INTNODAU.DF) .

D.8 EXTDF Output for Surface Source Configuration . • . • . D.64

D.9 EXTDF Output for Volume Source Configuration . . . . 0.70

D.10 EXTDF Output for Soil Source Configuration . . . . . D.76

D.11 GENEXT1.BAS Computer Program Listing . . . • • • D.82

D.12 EXTOPT.TPL GENII Input File Template . • . • • • • D.84

D.13 EXTPAR.TPL GENII Input File Template . • . • • • • D.85

D.14 Control Program Usage for Generating Internal Dose Factors
Contained in Files INTERNAL.DF and INTNODAU.DF . • . . D.88

D.15 GENALL.BAS Computer Program Listing . . . . . . D.89

D.16 GENALL2.BAS Computer Program Listing • • • • • • • . D.91

D.17 GENALL3.BAS Computer Program Listing . • . • . . D.93

D.18 GENALL4.BAS Computer Program Listing . • • • • • D.95

D.19 SOILOPT.TPL GENII Input File Template • • • • • • • • D.97

D.20 SOILPAR.TPL GENII Input File Template . . . • • • . D.98

D.21 FACILOPT.TPL GENII Input File Template . . • . . . D.101

D.22 FACILPAR.TPL GENII Input file Template • • • • • • . D.102

D.23 Radionuclide Master Library - Radionuclide Chains in . . . D.105
Equilibrium (RMDLIB.DAT)

D.24 Radionuclide Master Library - Radionuclides Without
Explicit Daughters. . . . . . . . . D.111 
D.25 Food Transfer Coefficient Data for Soil Contribution to Food Product Ingestion . . . . . . . . D.117

D.26 Food Transfer Coefficient Data for Air Contribution to Food Product Ingestion . . . . . . . . . . D.119 


\subsection{INTRODUCTION}

The U.S. Nuclear Regulatory Commission (NRC) is considering an expansion of its current criteria governing the release of slightly radioactive property for unrestricted use. Current NRC staff criteria are in two forms: 1) acceptable volumetric concentrations of source material contamination in soil, provided in $\mathrm{pCi} / \mathrm{g}$ (Federal Register 1981), and 2) acceptable levels of surface contamination, provided in units of $\mathrm{dpm} / 100 \mathrm{~cm} 2$ for average, maximum, and removable contamination conditions (NRC 1974). Both of these NRC staff criteria for release of slightly radioactive material have limitations. The criteria for volumetric concentrations in soil, for instance, are limited to the uranium and thorium chain of radionuclides and are only applicable to current contamination resulting from past operations. The NRC staff criteria governing surface contamination, although appropriate for all radionuclides, are often difficult to apply because of the wide variation in surface and volumetric contamination conditions and the varying mixture of radionuclides usually found in many licensed facilities. In addition, the values found in both sets of criteria are not consistent with the revised dosimetry system recommended by the International Commission on Radiological Protection (ICRP) in Publications $26(1977)$ and $30(1979-1988)$.

To ensure a uniform approach and consistent regulatory decisions, therefore, comprehensive guidance needs to be developed to include release criteria for radioactive contamination found within soil, structures, materials, and equipment. Initial guidance developed by the NRC wili cover the unrestricted release of buildings and land; future NRC efforts will be devoted to developing a system for releasing contaminated tools and equipment and recycling other materials. All of this new guidance is being developed in terms of radiation dose and will be consistent with the revised dose system recommended by the ICRP.

As a contribution to the development of revised guidance by the NRC, this document provides information on the generic pathway model used to derive the potential total effective dose equivalent (TEDE)(a) to an average individual in a given population group from unit radionuclide concentrations of residual contamination. The methodology used to calculate doses from unit concentrations of radionuclides is consistent with the recommendations of the ICRP in Publication No. 30 (1979-1988). The input parameters for each exposure pathway and scenario are selected to provide a reasonable estimate of the likely radiation dose to an average individual in a limited population exposed to residual contamination in buildings or soil. They are not selected to perform a worst case (overly conservative) analys is of the potential radiation dose, and do not represent the average dose to all members of the public. Still, the resulting annual TEDE factors likely provide an overestimate of the actual dose that individuals may receive. The models, pathways, scenarios, and parameters given here can be modified and used to develop screening values for the release of buildings and soils.

(a) As defined in Appendix E--Glossary. 
They should also be useful in determining when more detailed site-specific assessments or modifications to the generic scenarios are required.

Two sets of derived unit concentration annual TEDE factors for residual radioactive materials in buildings are needed to adequately describe the potential contamination conditions found at actual facilities. These include derived unit concentration annual TEDE factors accounting for: 1) building renovation (subsurface or volume sources), and 2) normal building occupancy (thin layer and removable surface contamination). For building renovation (volume sources), the derived unit concentration annual TEDE factors, in mrem per $\mathrm{pCi} / \mathrm{g}$ (and $\mu \mathrm{Sv}$ per $\mathrm{Bq} / \mathrm{g}$ ), account for residual contamination contained within walls or floors. Estimates of the subsurface inventory are important to account for residual inventories of difficult-to-measure alpha-emitters or beta-emitters. The set of building occupancy (surface activity) TEDE factors (in units of mrem per dpm $/ 100 \mathrm{~cm}^{2}$ and $\mu \mathrm{Sv}$ per $\mathrm{Bq} / 100 \mathrm{~cm}{ }^{2}$ ) is intended to model the situation where the residual inventory is present as a thin layer of fixed and removable surface contamination.

The two sets of generically derived annual TEDE factors for soil contained in this document may be applied to contaminated surface soil conditions and total inventory conditions that lead to potential drinkingwater contamination. Methods are also provided to modify the factors to account for dilution by clean overburden soil, modified dietary intakes, modified air concentrations, and more sophisticated ground-water modeling efforts. The derived unit concentration annual TEDE factors for soil are in units of mrem per $\mathrm{pCi} / \mathrm{g}$ (and $\mu \mathrm{Sv}$ per $\mathrm{Bq} / \mathrm{g}$ ) of surface soil, and the inventory factors are in units of mrem per $\mathrm{pCi}$ (and $\mu \mathrm{Sv}$ per $\mathrm{Bq}$ ) for the total inventory through the potential pathway of drinking contaminated ground water. Maximum organ-specific dose conversion factors are also provided (using ICRP 30 methods) for the drinking water ingestion pathway to permit a rough comparison with current drinking water standards.

As a demonstration of the use of the derived unit concentration annual TEDE factors, example TEDEs are calculated for representative mixtures and quantities for selected reference licensed facilities. For these calculations, annual TEDEs for residual contamination in both buildings and soil are included. The reference facilities considered include a light-water power reactor, an industrial source and radiopharmaceutical manufacturing facility, a uranium fuel fabrication facility, and reference depleted and enriched uranium sources. Finally, a discussion of the information contained in this document is provided. 


\subsection{MODELING APPROACH}

A radiation exposure pathway analys is is required before calculation of the annual TEDE factors describing exposure to residual radioactivity present at the time of unrestricted release of decommissioned buildings and land. In this analysis, computerized models are used with radiation exposure scenarios that describe conditions that may exist after the release of buildings or land for unrestricted use. This section discusses the computerized models, the equations used to describe the significant radiation exposure pathways, and the calculated dose conversion or committed effective dose conversion factors for those exposure pathways. The pathway dose rate conversion factors are used to develop the scenario-specific annual TEDE factors discussed in Section 3.0. Additional information on a sensitivity study for determining external exposure dose rate conversion factors and more detailed descriptions of modeling other exposure pathways are found in Appendixes $A$ and $B$, respectively.

\subsection{DESCRIPTION OF REFERENCE METHODS}

This section describes the computerized model used in calculating unitconcentration pathway dose conversion factors. Dosimetric information for the reference (adult) man, as described by the ICRP (1975), was used in all calculations. After an initial review of existing radiation pathway analys is models, the ONSITE/MAXI1 (Kennedy et al. 1987) computer program was initially selected because of its flexibility in allowing the user to define various exposure scenarios. This flexibility extends to scenarios describing exposures within structures and to user-defined scenarios. During the development of this report, the capabilities of the ONSITE/MAXI1 computer program were included as part of a newer computerized model, the GENII software package (Napier et al. 1988). The GENII software is designed for estimating individual and population doses from releases of radionuclides to air, water, or soil and includes an enhanced user-defined scenario capability. The software package was developed and documented under a strict quality assurance (QA) program based on the American National Standards Institute (ANSI) standard NQA-1 (ASME 1986). All steps of the code development have been extensively tested. The tests have included comparison of results generated by hand calculations and other computer software. Hand calculation worksheets are provided in Volume 3 of the documentation (Napier et al. 1988). These worksheets were used to benchmark the code output to other environmental dosimetry programs, including ONSITE.MAXI1. A 10-volume set of test documentation is available for review from the authors, upon request. During development, the program was reviewed by an external peerreview panel of national and international environmental health physicists with pathway modeling experience. Following this review, the code was placed under configuration management providing for change control and documentation updates to all identified users.

The GENII software is described in three volumes of documentation (Napier et al. 1988). The first volume describes the theoretical considerations of the system, including conceptual diagrams, mathematical 
representations of the solutions. Descriptions of the solution techniques are also included, where appropriate. The second volume is a user manual, which provides code structure, user instructions, required system configurations, and topics related to $Q A$. The third volume is a code maintenance manual for the user who may require knowledge of the code details, including code logic diagrams, global dictionary, worksheets, example hand calculations, and listings of the code and its associated data libraries.

The GENII software includes modules for calculating environmental transport and dilution of and/or accumulation of radionuclides in environmental media, external radiation dose rate factors for various source geometries, and internal radiation dose rate factors for inhalation and ingestion exposures consistent with those recommended by the ICRP (19791988). The software package comprises several computer programs and data libraries. The computer programs fall into three categories: user interface (i.e., interactive menu-driven program to assist the user with scenario generation), internal and external dose factor generators, and the environmental dosimetry programs. APPRENTICE is the user interface for the short-term environmental dosimetry programs. INDIF and EXTDF are programs that generate internal and external dose rate factors, respectively. For maximum flexibility, the short-term environmental dosimetry portion has been divided into three interrelated but separate programs (ENVIN, ENV, and DOSE) that handle input organization and checking, environmental exposure, and dose calculations, respectively. The software package can be used for routine or accidental releases, or for exposures to contaminated surface soil or subsoil. It also permits the user to define scenario conditions that can be used to model exposures in structures after their unrestricted release.

\subsection{EXPOSURE PATHWAY ANALYSIS}

The potential routes through which people may be exposed to radiation or radionuclides are called exposure pathways. A collection of exposure pathways, with specific modeling assumptions, are used to construct radiation exposure scenarios. These scenarios are designed to be conceptual models of potential patterns of human activity (actions, events, and other processes) that result in radiation exposures to individuals or groups of people.

The exposure pathways considered for buildings include:

- direct external exposure to penetrating radiation

- inhalation of airborne materials

- secondary ingestion.

The exposure pathways considered for residual radioactivity in soil include:

- direct external exposure to penetrating radiation

- inhalation of airborne materials

- ingestion of agricultural food products grown in contaminated soil

- ingestion of drinking water from a ground-water source. 
This section describes the mathematical equations and parameters necessary to calculate radiation dose conversion factors for each of the major exposure pathways. Listings by radionuclide are provided of the pathway-specific dose conversion or committed effective dose conversion factors used to construct scenario-specific TEDE factors. Daughters with half-lives less than 10 minutes are always assumed to be in equilibrium with their parent and are referred to as implicit daughters. Because the pathwayspecific dose conversion or committed effective dose conversion factors are based on unit activity, it is important to note that the unit activity includes contributions from both the parent and implicit daughters. To accomplish this, a +1 notation for the radionuclide listings and an appropriate footnote are included with all dose factor tables. Daughters with half-lives greater than 10 minutes that may not be in equilibrium with their parents are referred to as explicit daughters. These are shown in equilibrium conditions with the parent by $a+D$ listing and footnote in the dose factor tables. Separate values are also included for parents and daughters so that values for non-equilibrium cases can be determined. For cases where there is a short-lived parent with a long-lived daughter, separate listings for parent and daughter are included so that any initial ratio may be evaluated. Finally, for decay chains, the listings include parents alone, parents with $+D$ and $+I$ notations (to include both implicit and explicit daughters) and daughters alone so that equilibrium and nonequilibrium cases can be considered. This notation is used consistently in this document to provide maximum flexibility for consideration of all parentdaughter and decay chain situations. The dose factor and annual TEDE listings contain over 200 entries for individual radionuclides and parentdaughter decay chains. For completeness, several short-lived radionuclides that are considered by the ICRP $(1979-1988)$ are also considered in the listings in this document. A further description of chain decay and implicit and explicit daughters is found in Appendix D.

\subsubsection{External Exposure To Penetrating Radiation}

The first pathway considered is external exposure to direct penetrating radiation. The fundamental relationship for calculating radiation dose rates to people from any radionuclide in an exposure pathway is given in Equation 2.1 (Soldat, Robinson, and Baker 1974).

$$
R_{i p}=C_{i p} U_{p} D_{i p}
$$

where $R_{i p}=$ the radiation dose equivalent or committed radiation dose equivalent from radionuclide $i$ via exposure pathway $p$, in mrem $(\mu \mathrm{Sv})$ per year of exposure

$C_{i p}=$ concentration of radionuclide $i$ in the media of exposure in pathway $\mathrm{p}$, in $\mathrm{pCi} / \mathrm{L}(\mathrm{Bg} / \mathrm{L})$ for water or $\mathrm{milk}, \mathrm{pCi} / \mathrm{kg}(\mathrm{Bq} / \mathrm{kg})$ for food, $\mathrm{pCi} / \mathrm{m}^{3}\left(\mathrm{~Bq} / \mathrm{m}^{3}\right)$ for air, $\mathrm{pCi} / \mathrm{g}(\mathrm{Bq} / \mathrm{g})$ for volume contamination in buildings and soil, and $\mathrm{dpm} / 100 \mathrm{~cm} 2(\mathrm{~Bq} / 100$ $\mathrm{cm}^{2}$ ) for surface contamination in buildings 


\section{$U_{p}=$ usage parameter (exposure rate or intake rate) associated with exposure pathway $p$, in $h / y r$ for external, L/yr for water or milk, or $\mathrm{kg} / \mathrm{yr}$ for food \\ $D_{i p}=$ dose rate equivalent factor or the committed effective dose equivalent factor for radionuclide $i$ and exposure pathway $p$, used to convert the concentration and usage parameters to the radiation dose equivalent or to the committed effective dose equivalent, in $\mathrm{mrem} / \mathrm{pC} i \cdot h(\mu \mathrm{Sv} / \mathrm{Bq} \bullet \mathrm{h})$ for external and $\mathrm{mrem} / \mathrm{pCi}$ $(\mu \mathrm{Sv} / \mathrm{Bq})$ for internal pathways.}

For external exposure, $U_{p}$ is given in units of $h / y r$ and $D_{i p}$ in $\mathrm{mrem} / \mathrm{pCi} \bullet \mathrm{h}(\mu \mathrm{Sv} / \mathrm{Bq} \bullet \mathrm{h})$ or $\mathrm{mrem} / \mathrm{dpm} \bullet \mathrm{h} / 100 \mathrm{~cm} 2\left(\mu \mathrm{Sv} / \mathrm{Bq} \bullet \mathrm{h} / 100 \mathrm{~cm}^{2}\right)$. Preliminary studies, as discussed in Appendix A, determined that:

- for buildings with non-homogeneous distributions of source materials (i.e., higher concentrations on the floor than on the walls or ceiling), dose factors for an infinite flat plane provide an approximate solution over a wide variation of room volumes--for situations where the ceiling may be a significant source, the infinite flat plane conversion factors may be doubled to account for two-plane sources.

- for surface soils, the use of a large cylinder source solution, with an assumed radius of $5 \mathrm{~m}$ and a contamination depth of $15 \mathrm{~cm}$ (the assumed plow layer) will provide an adequate modeling bas is for source areas greater than about $80 \mathrm{~m}^{2}$. Although subsurface sources may be important, for this study the external exposure pathway dose rate factors are derived to represent a surface soil, volume source.

The external dose rate conversion factors are calculated using the GENII software package (Napier et al. 1988). They are given in terms of the deep dose equivalent (i.e., photon energies that penetrate to a depth of $1 \mathrm{~cm}$ of tissue) using the methods of Kocher (1981a). Table 2.1 shows external dose rate conversion factors for surface and volume sources in buildings and for a surface layer of soil to a depth of $15 \mathrm{~cm}$ (the approximate plow layer). For surface sources in buildings, the dose rate conversion factors are in units of $\mathrm{mrem} / \mathrm{h}$ of exposure per $\mathrm{dpm} / 100 \mathrm{~cm} 2$ and $\mu \mathrm{Sv} / \mathrm{h}$ of exposure per $\mathrm{Bq} / 100 \mathrm{~cm} 2$. For volume sources in buildings or soils, the dose rate conversion factors are in units of mrem/h of exposure per $\mathrm{pCi} / \mathrm{g}$ and $\mu \mathrm{Sv} / \mathrm{h}$ of exposure per $\mathrm{Bq} / \mathrm{g}$. These factors can be multiplied by the scenario-specific exposure duration $(\mathrm{h} / \mathrm{yr})$ and the initial activity level $(\mathrm{dpm} / 100 \mathrm{~cm} 2, \mathrm{~Bq} / 100 \mathrm{~cm} 2, \mathrm{pCi} / \mathrm{g}$, or $\mathrm{Bq} / \mathrm{g}$ ) to obtain the annual external dose for a given exposure scenario.

External exposure rates for unit quantities of individual radionuclides are calculated considering contributions from electromagnetic radiation from gamma- and $x$-rays; bremsstralung from the slowing-down of beta particles, auger electrons, and conversion electrons; and annihilation radiation from positrons. Alpha particles are not calculated to contribute to the exposure rates. The source of the radionuclide data is the DRALIST file (Oak Ridge National Laboratory 1981), which was adapted for GENII by including implicit daughter energies with their parents. This is an extremely comprehensive list, containing most known radiations, including many with very low 
Frequencies of occurrence (e.g. a $0.8 \mathrm{MeV}$ gamma ray from Po-210 which only sccurs once in every 105 decays). Some radionuclides are pure alpha emitters (e.g. Sm-147). These nuclides will have a calculated exposure rate of zero. jome radionuclides are considered to have "no" emissions, but in actuality lave very infrequent or very low-energy radiations. Examples of these types ire $\mathrm{Fe}-55$ or $\mathrm{Ni}-59$, which usually decay via internal conversion. Each of these two radionuclides actually has a number of low-energy auger or internal zonversion electrons, which may lead to low-energy bremsstralung, and also lave a few very-low-energy gamma or $x$-rays associated with their decays. 3remsstralung is calculated as a function of the energy of the electron emitted, and the atomic number of the source matrix, using the procedure of the ISOSHLD shielding code (Enge1, Greenborg, and Hendrickson 1966). This technique assigns a number and energy of emitted photons as a function of the electron energy. Energies less than $5 \mathrm{keV}$ do not result in emitted photons. An example radionuclide with a beta particle emitted $100 \%$ of the time, but at a very low energy, is Re-187. The maximum beta energy per decay of $\operatorname{Re}-187$ is $0.0026 \mathrm{keV}$, an thus no contribution from bremsstralung is included. For radionuclides which emit positrons, the energy of the annihilation gamma is treated as if the gamma were from any other source.

The ISOSHLD model works on 25 discrete energy groups, rather than individual energies. The lowest energy group is from 0.01 to $0.02 \mathrm{Mev}$ (10 to $20 \mathrm{keV}$ ), with photon radiations of energies lower than this value lumped into the 0.01 to $0.02 \mathrm{MeV}$ group. This tends to overestimate the resultant exposure rate, because the average energy of this group, $0.015 \mathrm{MeV}$, may overrepresent the actual value for a given radionuclide. This procedure provides a conservative value of the dose response. However, for such radionuclides, even the overestimation will be a very small number, relative to those radionuclides with significant emissions.

Hand calculations were conducted for key radionuclides, including $60 \mathrm{Co}$ $137 \mathrm{Cs}, 152 \mathrm{Eu}$, and $154 \mathrm{Eu}$ to compare with the external dose conversion factors used in other pathway analys is studies. The calculations consisted mainly of unit conversions. When compared to the data base used for surface contamination supporting 10 CFR 61 (Oztunali and Roles 1986), the values used in this study were lower by factors ranging from 2 to 3 . For example, for 60 Co the 10 CFR 61 data base reported $2.14 \mathrm{E}-04$ and our value is $8.2 \mathrm{E}-05$ $\mathrm{mrem} / \mathrm{yr}$ per $\mathrm{pCi} / \mathrm{m}^{2}$. For volume soil sources, the values used in this study were all very close to the 10 CFR 61 data, lower within a factor of 1.2 to 2 . For example, for 60 Co the 10 CFR 61 data base reported $1.6 \mathrm{E}-05$ and our value is $1.3 \mathrm{E}-05 \mathrm{mrem} / \mathrm{yr}$ per $\mathrm{pCi} / \mathrm{g}$. Other comparisons of the factors used in this study with the external exposure factors found in Regulatory Guide 1.109 (NRC 1977) were conducted and showed similar results: the external dose factors used in this study were consistently about a factor of 2 lower than the Regulatory Guide 1.109 values. For example, for 60 Co the Regulatory Guide 1.109 surface soil value was $1.7 \mathrm{E}-08$ and our value is $9.4 \mathrm{E}-09 \mathrm{mrem} / \mathrm{h}$ per $\mathrm{pCi} / \mathrm{m}^{2}$. For completeness, surface values from Regulatory Guide 1.109 and the 10 CFR 61 data base were also compared. The results were within factors of 2, with the Regulatory Guide 1.109 values being lower. For example, for 60 Co Regulatory Guide 1.109 reported $1.7 \mathrm{E}-08$ and the data base for 10 CFR 61 reported $2.4 \mathrm{E}-08 \mathrm{mrem} / \mathrm{h}$ per $\mathrm{pCi} / \mathrm{m}^{2}$. The results of these calculations show close agreement for all three data sets for this type of modeling analysis. 
TABLE 2.1. External Dose Rate Conversion Factors for Exposure to Residual Radioactive Materials

Building

Surface Sources

Radio- $\quad \overline{\mathrm{mrem}} / \mathrm{h}$ per $\mu \mathrm{Sv} / \mathrm{h}$ per

nuclide $\mathrm{dpm} / 100 \mathrm{~cm}^{2} \mathrm{~Bq} / 100 \mathrm{~cm}^{2}$

${ }^{3} \mathrm{H}$

${ }^{10} \mathrm{Be}$

${ }^{14} \mathrm{C}$

${ }^{18} \mathrm{~F}$

${ }^{22} \mathrm{Na}$

${ }^{32} \mathrm{p}$

${ }^{35} \mathrm{~S}$

${ }^{36} \mathrm{Cl}$

${ }^{46} \mathrm{~K}$

${ }^{41} \mathrm{Ca}$

${ }^{48} \mathrm{SC}$

${ }^{51} \mathrm{Cr}$

${ }^{54} \mathrm{Mn}$

${ }^{56} \mathrm{Fe}$

${ }_{58}^{57} \mathrm{Co}$

${ }^{68} \mathrm{Co}$

${ }_{63}^{59} \mathrm{~N}$

${ }^{63} \mathrm{Ni}$

${ }^{84} \mathrm{Cu}$

${ }^{85} \mathrm{Zn}$
$2.4 \mathrm{E}-16$

4.0E-17

8.5E-11 1.4E-11

$5.0 \mathrm{E}-12$

1.6E-07

3. $6 \mathrm{E}-07$

6.7E-07

8.4E-13

2.7E-08

$6.0 \mathrm{E}-08$

$1.1 \mathrm{E}-07$

7.7E-10 $\quad 1.3 \mathrm{E}-10$

1.5E-11 2.5E-12

$5.7 \mathrm{E}-12 \quad 9.5 \mathrm{E}-13$

1. $3 \mathrm{E}-10$

2.2E-11

2.7E-08

4.5E-09

$3.2 \mathrm{E}-10$

1.6E-11

$5.4 \mathrm{E}-11$

2.6E-12

3. $6 \mathrm{E}-07$

6.7E-09

1.5E-07

2.9E-07

7. $4 \mathrm{E}-10$

1. $9 \mathrm{E}-07$

2. $4 \mathrm{E}-08$

1.7E-07

4.2E-07

$6.0 \mathrm{E}-08$

1.1E-09

2. $5 \mathrm{E}-08$

4. $9 \mathrm{E}-08$

1. $2 \mathrm{E}-10$

3. $2 \mathrm{E}-08$

3. $9 \mathrm{E}-09$

2. $9 \mathrm{E}-08$

7. $0 \mathrm{E}-08$

9.0E-10

4. $0 \mathrm{E}-13$

9.7E-08

1. $5 \mathrm{E}-10$

$6.7 \mathrm{E}-14$

1. $6 \mathrm{E}-08$

3.1E-08

5.2E-09

1.1E-07 1.8E-08
Building Volume Sources mrem/h per $\mu \mathrm{Sv} / \mathrm{h}$ per $\mathrm{pCi} / \mathrm{g}$ $\mathrm{Bq} / \mathrm{g}$

1.0E-14 2.7E-12

1. $4 \mathrm{E}-08$

3.8E-06

1.1E-07

$1.6 \mathrm{E}-02$

3.7E-02

7. $4 \mathrm{E}-02$

2.7E-04

6.1E-05

4.1E-07

$2.9 \mathrm{E}-06$

$1.0 \mathrm{E}-08$

2.1E-03

4.1E-03

$5.8 \mathrm{E}-01$

2.3E-07

1.5E-09

1.3E-07

2.7E-09

6.9E-06

2.6E-07

2.9E-03

1.6E-04

3.7E-06

1. $4 \mathrm{E}-08$

1.1E-08

3.8E-02

2. $0 \mathrm{E}-03$

6.0E-04

3. $5 \mathrm{E}-05$

9.5E-03

2.2E-06

5.6E-05

1.2E-04

1.5E-02

3.1E-02

7. $9 \mathrm{E}-04$

1.7E-03

2.1E-01 3.1E-08

7. $5 \mathrm{E}-05$

8.5E-06

2.0E-02

3. $3 E-08$

1.1E-03

8.8E-06 $3.0 \mathrm{E}-01$

6.8E-06

1. 8E-03

7.6E-05

2.0E-02

$6.6 \mathrm{E}-05$

1.7E-04

$1.8 \mathrm{E}-02$

$9.6 \mathrm{E}-04$

2. $4 \mathrm{E}-03$

. $6 \mathrm{E}-01$

4.5E-02

3. $8 \mathrm{E}-08$

2.0E-11

1.0E-05

4.0E-08

5.3E-09

5.7E-11

5. 5E-04

$1.1 \mathrm{E}-05$

3. $8 \mathrm{E}-05$

1.0E-02

1.1E-05

3.1E-03

1. $9 \mathrm{E}-04$

5.2E-02

4.3E-05

1.2E-02

6.2E-04

1.7E-01 
TABLE 2.1. External Dose Rate Conversion Factors for

Exposure to Residual Radioactive Materials (contd)

Building

Surface Sources

Radionuclide

${ }^{69 m} \mathrm{Zn+D}$
${ }^{69} \mathrm{~m} \mathrm{Zn}$
${ }^{69} \mathrm{Zn}$
${ }^{78} \mathrm{As}$
${ }^{75} \mathrm{Se}$
${ }^{79} \mathrm{Se}$

${ }^{82} \mathrm{Br}$
${ }^{83} \mathrm{Br}+\mathrm{D}$
${ }^{84} \mathrm{Br}$

${ }^{88} \mathrm{Rb}$
${ }^{89} \mathrm{Rb}$
${ }_{\mathrm{Rb}}^{89} \mathrm{Rb}$
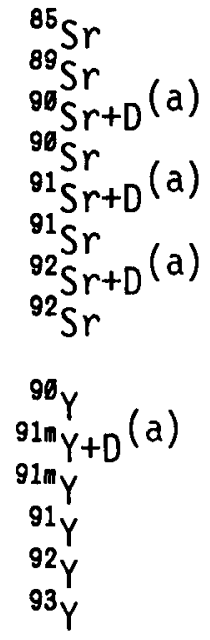

$1.6 \mathrm{E}-09$
$5.4 \mathrm{E}-08$
$1.1 \mathrm{E}-07$
$1.3 \mathrm{E}-09$
$5.0 \mathrm{E}-08$
$1.8 \mathrm{E}-08$

${ }^{93} \mathrm{Zr+D}(\mathrm{a})$

${ }^{93} \mathrm{Zr}$ Zr+D (a)

${ }_{95} \mathrm{Zr}$ (a)

${ }^{97} \mathrm{Zr+D}{ }^{(a)}$

${ }^{93 \mathrm{~m}^{\mathrm{m}}} \mathrm{Nb}$
1.7E-10

$3.2 \mathrm{E}-13$

$3.7 \mathrm{E}-08$

$7.9 \mathrm{E}-08$

2. $2 \mathrm{E}-10$

8.6E-08

6. $6 \mathrm{E}-08$

$3.9 \mathrm{E}-12$

4.8E-07

$9.8 \mathrm{E}-10$

$1.4 \mathrm{E}-09$

2.8E-07

1.6E-08

1.7 E-07

3. $4 \mathrm{E}-07$

8. $4 \mathrm{E}-08$

6. $4 \mathrm{E}-10$

7. $8 \mathrm{E}-10$

7. $2 \mathrm{E}-11$

$6.8 \mathrm{E}-08$

1.2E-07

1. $4 \mathrm{E}-07$

2. $4 \mathrm{E}-07$

6.2E-09

1. $3 \mathrm{E}-08$

3. $6 \mathrm{E}-11$

$1.4 \mathrm{E}-08$

1.1E-08

6. $5 \mathrm{E}-13$

8.0E-08

1. $6 \mathrm{E}-10$

2. $3 \mathrm{E}-10$

4.7E-08

2.7E-09

1. $9 \mathrm{E}-08$

2.8E-08

5.7E-08

1. $4 \mathrm{E}-08$

1. $1 \mathrm{E}-10$

1.3E-10

1. $2 \mathrm{E}-11$

$1.1 \mathrm{E}-08$

2. $0 \mathrm{E}-08$

2. $4 \mathrm{E}-08$

4. $0 \mathrm{E}-08$

2. $6 \mathrm{E}-10$

9.0E-09

1. $9 \mathrm{E}-08$

2. $2 \mathrm{E}-10$

8.3E-09

2. $9 \mathrm{E}-09$

2. $9 \mathrm{E}-11$

5. $3 \mathrm{E}-14$

2. $2 \mathrm{E}-08$

$1.3 \mathrm{E}-07$

2. $2 \mathrm{E}-08$

8.7E-08

3. $2 \mathrm{E}-08$

3. $6 \mathrm{E}-10$

2. $6 \mathrm{E}-07$

5. $.5 \mathrm{E}-08$

6.0E-11

4. $4 \mathrm{E}-08$
$1.1 \mathrm{E}-07$
Building

Volume Sources $\mathrm{mrem} / \mathrm{h}$ per $\mu \mathrm{Sv} / \mathrm{h}$ per $\mathrm{pCi} / \mathrm{g}$ $\mathrm{Bq} / \mathrm{g}$

1. $4 \mathrm{E}-05$

2. $9 \mathrm{E}-05$

4. $6 \mathrm{E}-08$

3. $9 \mathrm{E}-03$

7.9E-03

1.3E-05

3.3E-05

8. $8 \mathrm{E}-03$

2.2E-05

5. $9 \mathrm{E}-03$

3. $0 \mathrm{E}-10$

8. $2 \mathrm{E}-08$

1.9E-04

5.0E-02

2.5E-07

4.8E-07

6.7E-05

1.3E-04

1.1E-04

3.1E-02

6.3E-06

4. $6 \mathrm{E}-05$

$1.7 \mathrm{E}-03$

1.2E-02

6.7E-05

1. $4 \mathrm{E}-04$

$1.8 \mathrm{E}-02$

3.7E-02

3.1E-05

8.3E-03

1.7E-07

2.2E-07

1.2E-08

2.7E-05

4. 5E-05

5.7E-05

9.7E-05

4. $6 \mathrm{E}-05$

6.1E-05

3.2E-06

7.2E -03

1.2E-02

$1.5 \mathrm{E}-02$

2.6E-02

4. $6 \mathrm{E}-07$

1.2E-04

2.1E-05

4. $2 \mathrm{E}-05$

4.2E-07

1.9E-05

6.6E-06

5. $6 \mathrm{E}-03$

1.1E-02

1.1E-04

5. $3 \mathrm{E}-03$

$1.8 \mathrm{E}-03$

7.5E-09

2. $0 E-06$

1. $5 \mathrm{E}-11$

4. $1 \mathrm{E}-09$

5.2E-05

1. $4 \mathrm{E}-02$

5.0E-05

3. $3 \mathrm{E}-05$

1.2E-05

$1.4 \mathrm{E}-02$

$8.8 \mathrm{E}-03$

$3.4 \mathrm{E}-03$
1. $5 \mathrm{E}-08$

1. $0 \mathrm{E}-04$
4.1E-06

2.7E-02
Soil Volume Sources $\mathrm{mrem} / \mathrm{h}$ per $\mu \mathrm{Sv} / \mathrm{h}$ per

$\begin{array}{ccc}\mathrm{pCi} / \mathrm{g} & & \mathrm{Bq} / \mathrm{g} \\ 2.4 \mathrm{E}-04 & & 6.5 \mathrm{E}-02 \\ 5.0 \mathrm{E}-04 & & 1.3 \mathrm{E}-01 \\ 5.0 \mathrm{E}-07 & 1.4 \mathrm{E}-04\end{array}$

4.9E-04 1.3E-01

2.9E-04 7.9E-02

1.6E-09 4.3E-07

2.7E-03 7.3E-01

3.9E-06 1.0E-03

7.9E-06 2.1E-03

1.7E-03 4.7E-01

9.2E-05 2.5E-02

7.0E-04 1.9E-01

9.6E-04 2.6E-01

2.0E-03 5.5E-01

5.2E-04 1.4E-01

2.1E-06 5.7E-04

3.0E-06 8.0E-04

$1.1 \mathrm{E}-07 \quad 2.9 \mathrm{E}-05$

4.1E-04 1.1E-01

$6.7 \mathrm{E}-04 \quad 1.8 \mathrm{E}-01$

8.0E-04 2.2E-01

1.4E-03 3.8E-01

6.0E-06 1.6E-03

3.1E-04 8.5E-02

6.4E-04 1.7E-01

5.7E-06 1.5E-03

$2.9 \mathrm{E}-04 \quad 7.8 \mathrm{E}-02$

9.7E-05 2.6E-02

7.7E-09 2.1E-06

4.0E-11 1.1E-08

7.4E-04 2.0E-01

7.3E-04 2.0E-01

5.0E-04 1.3E-01

$1.9 \mathrm{E}-04 \quad 5.0 \mathrm{E}-02$

$1.6 \mathrm{E}-08 \quad 4.3 \mathrm{E}-06$

$1.5 \mathrm{E}-03 \quad 4.0 \mathrm{E}-01$ 
TABLE 2.1. External Dose Rate Conversion Factors for

Exposure to Residual Radioactive Materials (contd)

Building

Surface Sources

Radionuclide

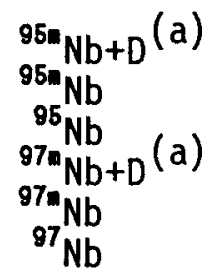

${ }^{93} \mathrm{Mo}_{\mathrm{D}}(\mathrm{a})$
${ }^{93} \mathrm{Mo}$
${ }^{99} \mathrm{Mo+D}$
${ }^{99} \mathrm{Mo}$

${ }^{99 m} \mathrm{TC}$
${ }^{99} \mathrm{TC}$
${ }^{161} \mathrm{TC}$

${ }_{163} \mathrm{Ru}+\mathrm{D}(\mathrm{a})$
${ }_{103} \mathrm{Ru}$ (a)
${ }_{105} \mathrm{Ru}+\mathrm{D}$
${ }_{105} \mathrm{Ru}+\mathrm{I}(\mathrm{b})$
${ }_{108} \mathrm{Ru}+\mathrm{I}$
(b)

${ }^{105} \mathrm{Rh}$

${ }_{{ }^{163}}^{169} \mathrm{Pd}+\mathrm{D}$
${ }^{167} \mathrm{Pd}$
${ }^{167} \mathrm{Pd}$
${ }^{169} \mathrm{Pd}+\mathrm{I}$ (b)
${ }^{{ }^{116} \mathrm{~m}} \mathrm{Ag}+\mathrm{I}$
${ }^{111} \mathrm{Ag}$

${ }^{1109} \mathrm{Cd}$
${ }^{113 n} \mathrm{Cd}$
${ }^{115 n} \mathrm{Cd}(\mathrm{d}+\mathrm{D}$
${ }^{115 n} \mathrm{Cd}$
${ }^{115} \mathrm{Cd}$

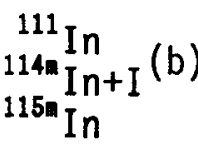

${ }^{117 m} \mathrm{Sn}$

8.0E-08

1.2E-08

$1.5 \mathrm{E}-07$

1.1E-07

$1.2 \mathrm{E}-07$

$1.2 \mathrm{E}-07$

$1.0 \mathrm{E}-09$

$1.7 \mathrm{E}-09$

2.3E-08

$2.6 \mathrm{E}-08$

2. $0 \mathrm{E}-08$

$1.9 \mathrm{E}-11$

$6.6 \mathrm{E}-08$

4.1E-08

8. $2 \mathrm{E}-08$

$5.0 \mathrm{E}-08$

1. $4 \mathrm{E}-07$

3. $9 \mathrm{E}-08$

$6.1 \mathrm{E}-10$

1. $5 \mathrm{E}-08$

2.6E-09

4.7E-09

2.5E-14

3.2E-09

4. $9 \mathrm{E}-07$

5.0E-09

4. 4E-09

8.1E-11

2. $2 E-09$

4. $6 \mathrm{E}-09$

3. $3 \mathrm{E}-08$

$6.6 \mathrm{E}-08$

2. $4 \mathrm{E}-08$

3.1E-08

2. $4 \mathrm{E}-08$
Building

Volume Sources

mrem/h per $\mu \mathrm{Sv} / \mathrm{h}$ per $\mathrm{pCi} / \mathrm{g}$ $\mathrm{dpm} / 100 \mathrm{~cm}^{2} \mathrm{~Bq} / 100 \mathrm{~cm}^{2}$

1.3E-08

2.0E-09

2. $4 \mathrm{E}-08$

1. $9 \mathrm{E}-08$

1. $9 \mathrm{E}-08$

2. $0 \mathrm{E}-08$

1.7E-10

2. $9 \mathrm{E}-10$

3.8E-09

4.3E-09

3. $4 \mathrm{E}-09$

$3.1 \mathrm{E}-12$

$1.1 \mathrm{E}-08$

$6.8 \mathrm{E}-09$

1. $4 \mathrm{E}-08$

8.3E-09

2. $4 \mathrm{E}-08$

$6.6 \mathrm{E}-09$

1. $0 \mathrm{E}-10$

2.5E-09

4.3E-10

7. $8 \mathrm{E}-10$

$4.1 \mathrm{E}-15$

5.3E-10

8.1E-08

8.3E-10

7.4E-10

1. $4 \mathrm{E}-11$

3.7E-10

$7.7 \mathrm{E}-10$

$5.5 \mathrm{E}-09$

$1.1 \mathrm{E}-08$

4.0E-09

5.2E-09

4.1E-09
2. $9 \mathrm{E}-05$

3.7E-06

$5.6 \mathrm{E}-05$

4.3E-05

4.3E-05

$4.5 \mathrm{E}-05$

4.3E-08 1.2E-05

7. $4 \mathrm{E}-08$

7.8E-06

9.5E-06

6.2E-06

2.1E-09

2. $4 \mathrm{E}-05$

$1.5 \mathrm{E}-05$

3. $0 \mathrm{E}-05$

1.9E-05

5.1E-05

1.5E-05

2.7E-08

5. $5 \mathrm{E}-06$

7.4E-06

1. $5 \mathrm{E}-03$

1.2E-07

2.1E-07

1.0E-12

3. $2 \mathrm{E}-07$

3.3E-05

5.8E-05

2. $8 \mathrm{E}-10$

8.7E-05

1.9E-04

1.8E-06

5.1E-02

4.8E-04

2. $0 \mathrm{E}-07$

5.3E-05

1. $4 \mathrm{E}-08$

8.0E-07

3.7E-06

2. $2 \mathrm{E}-04$

$1.7 \mathrm{E}-06$

1. $2 \mathrm{E}-05$

4.6E-04

3. $3 \mathrm{E}-03$

2.0E-05

7.8E-06

5.4E-03

2.1E-03

2. $9 \mathrm{E}-03$

6.4E-06

1.7E-03

2.5E-04

5.1E-04

2.8E-04

7.8E-04

2.3E-04 $\mathrm{pCi} / \mathrm{g}$

ume Sources

$\mathrm{Bq} / \mathrm{g}$

4.0E-04 1.1E-01

$5.0 \mathrm{E}-05 \quad 1.3 \mathrm{E}-02$

7.9E-04 2.1E-01

$6.5 \mathrm{E}-04 \quad 1.8 \mathrm{E}-01$

$6.6 \mathrm{E}-04 \quad 1.8 \mathrm{E}-01$

$6.8 \mathrm{E}-04 \quad 1.8 \mathrm{E}-01$

4.5E-08 1.2E-05

$7.6 \mathrm{E}-08$

$2.1 \mathrm{E}-05$

2.8E-02

3.7E-02

$6.9 \mathrm{E}-05 \quad 1.9 \mathrm{E}-02$

1. $5 \mathrm{E}-08$

4.1E-06

$1.0 \mathrm{E}-01$

$6.7 \mathrm{E}-02$

1.4E-01

7.7E-02

2.1E-01

$6.2 \mathrm{E}-02$

1.0E-07

2.7E-05

8.7E-05

$2.4 \mathrm{E}-02$

5.1E-07

$1.4 \mathrm{E}-04$

$9.5 \mathrm{E}-07$

2.6E-04

$1.3 \mathrm{E}-12$

$3.4 \mathrm{E}-10$

$7.9 \mathrm{E}-04$

2. $8 \mathrm{E}-03$

7.5E-01

2.7E-05

7.4E-03

7.9E-07

2.1E-04

1.3E-07

3. $5 \mathrm{E}-05$

1.2E-05

3. $3 \mathrm{E}-03$

2.5E-05

6.7E-03

2.0E-04

5.5E-02

2.6E-04

6.9E-02

1.1E-04

3. $0 \mathrm{E}-02$

1.7E-04

4. 5E-02

$6.9 \mathrm{E}-05$

1. $9 \mathrm{E}-02$ 
TABLE 2.1. External Dose Rate Conversion Factors for

Exposure to Residual Radioactive Materials (contd)

Building

Surface Sources

Radio- $\quad \frac{\mathrm{mrem} / \mathrm{h} \text { per } \mu \mathrm{Sv} / \mathrm{h} \text { per }}{2}$

nuclide

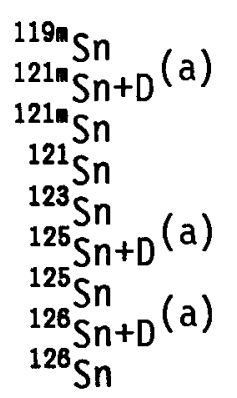

${ }^{124} \mathrm{Sb}$
${ }^{125} \mathrm{Sb}+\mathrm{D}$
${ }^{1264} \mathrm{Sb}(\mathrm{a})$
${ }^{126 n} \mathrm{Sb}+\mathrm{D}$
${ }^{126} \mathrm{Sb}$
${ }^{127} \mathrm{Sb} \mathrm{Sb}$

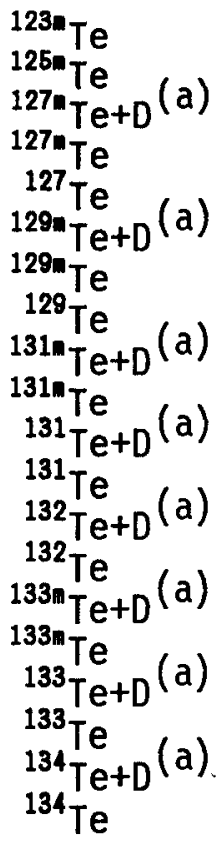

${ }^{125} \mathrm{I}$
${ }^{136} \mathrm{I}$
${ }^{13} \mathrm{I}+\mathrm{D}$

$5.3 \mathrm{E}-10$

$0.0 \mathrm{E}+00$

$0.0 \mathrm{E}+00$

$0.0 \mathrm{E}+00$

2.7E-10

$1.0 \mathrm{E}-08$

8. $9 \mathrm{E}-09$

2.7E-08

$1.4 \mathrm{E}-09$

8.6E-09

3. $3 \mathrm{E}-07$

6. $6 \mathrm{E}-08$

8. $2 \mathrm{E}-08$

3. $0 \mathrm{E}-07$

2.8E-07

4. $8 \mathrm{E}-07$

5. $3 \mathrm{E}-08$

1.2E-07

2.3E-08

8. $6 \mathrm{E}-09$

1. $9 \mathrm{E}-09$

2.8E-09

1.0E-09

8.7E-09

7.5E-09

1.1E-08

1. $5 \mathrm{E}-07$

2.6E-07

6.7E-08

7. $4 \mathrm{E}-08$

2. $2 \mathrm{E}-07$

4. $4 \mathrm{E}-08$

1. $6 \mathrm{E}-07$

4.0E-07

8.7E-08

1.7E-07

3. $0 \mathrm{E}-07$

1.6E-07

5.6E-08

1.1E-08

1. $4 \mathrm{E}-08$

5. $0 \mathrm{E}-08$

4.7E-08

8.0E-08

8. $9 \mathrm{E}-09$

2.0E-08

3.8E-09

1.4E-09

3.1E-10

4. $6 \mathrm{E}-10$

1. $8 \mathrm{E}-10$

1. $5 \mathrm{E}-09$

1.3E-09

1. $9 \mathrm{E}-09$

2.5E-08

4.3E-08

1.1E-08

1.2E-08

3.7E-08

7.3E-09

2.7E-08

6.7E-08

1.5E-08

2. $8 \mathrm{E}-08$

5. $0 \mathrm{E}-08$

2. $6 \mathrm{E}-08$

1.0E-08

5.5E-09

3. $6 \mathrm{E}-07$

6.2E-08
Building

$\mathrm{mrem} / \mathrm{h}$ per $\mu \mathrm{Sv} / \mathrm{h}$ per
1.7E-09

9. $2 \mathrm{E}-10$

$6.0 \mathrm{E}-08$

1.0E-08

$\begin{array}{ll}1.4 \mathrm{E}-07 & 3.8 \mathrm{E}-05 \\ 0.0 \mathrm{E}+00 & 0.0 \mathrm{E}+00 \\ 0.0 \mathrm{E}+00 & 0.0 \mathrm{E}+00 \\ 0.0 \mathrm{E}+00 & 0.0 \mathrm{E}+00 \\ 5.7 \mathrm{E}-07 & 1.5 \mathrm{E}-04 \\ 2.2 \mathrm{E}-05 & 6.0 \mathrm{E}-03 \\ 2.1 \mathrm{E}-05 & 5.6 \mathrm{E}-03 \\ 6.1 \mathrm{E}-05 & 1.6 \mathrm{E}-02 \\ 1.7 \mathrm{E}-06 & 4.7 \mathrm{E}-04\end{array}$

1.3E-04

2.3E-05

3. $0 \mathrm{E}-05$

$1.1 \mathrm{E}-04$

1.1E-04

1.8E-04

2.0E-05

4. $4 \mathrm{E}-05$

3.5E-02

$6.3 \mathrm{E}-03$

8.0E-03

3. $0 \mathrm{E}-02$

2. $9 \mathrm{E}-02$

4. $9 \mathrm{E}-02$

$1.2 \mathrm{E}-02$

6.0 E-06

5.2E-07

2.6E-07

1.7E-07

3. $6 \mathrm{E}-07$

2.8E-06

2.2E-06

3.7E-06

$5.6 \mathrm{E}-05$

9. $9 \mathrm{E}-05$

2.5E-05

2.7E-05

8.0E-05

$1.3 \mathrm{E}-05$

$6.1 \mathrm{E}-05$

1. $6 \mathrm{E}-04$

3. $3 \mathrm{E}-05$

$6.5 \mathrm{E}-05$

1.1E-04

5.8E-05

$1.6 \mathrm{E}-03$

1. $4 \mathrm{E}-04$

7.0E-05

4.5E-05

9.8E-05

7.5E-04

5.8E-04

$1.0 \mathrm{E}-03$

1.5E-02

2.7E-02

$6.7 \mathrm{E}-03$

7.2E-03

2.2E-02

3. $6 \mathrm{E}-03$

$1.6 \mathrm{E}-02$

4.2E-02

8.9E-03

1.7E-02

3. $0 \mathrm{E}-02$

1. $6 \mathrm{E}-02$

5.9E-07

1.6E-04

3. $4 \mathrm{E}-07$

9.1E-05

1.3E-04

$3.6 \mathrm{E}-02$

$6.0 \mathrm{E}-03$

2. $2 \mathrm{E}-05$
5. $4 \mathrm{E}-03$
Volume Sources $\mathrm{pCi} / \mathrm{g}$

Soil Volume Sources mrem $/ \mathrm{h}$ per $\mu \mathrm{Sv} / \mathrm{h}$ per

\begin{tabular}{|c|c|}
\hline $\begin{array}{l}5.4 \mathrm{E}-07 \\
0.0 \mathrm{E}+00 \\
0.0 \mathrm{E}+00 \\
0.0 \mathrm{E}+00 \\
8.1 \mathrm{E}-06 \\
3.4 \mathrm{E}-04 \\
3.1 \mathrm{E}-04 \\
9.8 \mathrm{E}-04 \\
1.6 \mathrm{E}-05\end{array}$ & $\begin{array}{l}1.5 \mathrm{E}-04 \\
0.0 \mathrm{E}+00 \\
0.0 \mathrm{E}+00 \\
0.0 \mathrm{E}+00 \\
2.2 \mathrm{E}-03 \\
9.1 \mathrm{E}-02 \\
8.4 \mathrm{E}-02 \\
2.6 \mathrm{E}-01 \\
4.2 \mathrm{E}-03\end{array}$ \\
\hline
\end{tabular}

1. $9 \mathrm{E}-03$

$5.2 \mathrm{E}-01$

3.7E-04

9.9E-02

$1.3 \mathrm{E}-01$

4. $6 \mathrm{E}-01$

$1.7 \mathrm{E}-03$

4.5E-01

$1.7 \mathrm{E}-03$

7.5E-01

.8E-03

8. $2 \mathrm{E}-02$

6.7E-04 1.8E-01

6.6E-05

$1.8 \mathrm{E}-02$

2.6E-06 7.1E-04

3. $4 \mathrm{E}-06$

9.2E-04

8.6E-07

2. $3 \mathrm{E}-04$

6.0E-06

$1.6 \mathrm{E}-03$

4.1E-05

1.1E-02

3.1E-05

$8.5 \mathrm{E}-03$

5.9E-05

1. $6 \mathrm{E}-02$

7.9E-04

2.1E-01

1. $4 \mathrm{E}-03$

3. $8 \mathrm{E}-01$

3.7E-04

9.9E-02

3. $9 \mathrm{E}-04$

1.1E-01

$1.2 \mathrm{E}-03 \quad 3.3 \mathrm{E}-01$

1.8E-04

4.8E-02

9.7E-04

$2.6 \mathrm{E}-01$

2.3E-03

6.2E-01

5.1E-04

$1.4 \mathrm{E}-01$

9.9E-04

2.7E-01

1.7E-03

4.6E-01

2.3E-01
2.9E-06

$1.7 \mathrm{E}-06$

2.1E-03

3. $5 \mathrm{E}-04$
7.8E-04

4.5E-04

5.7E-01

9.4E-02 
TABLE 2.1. External Dose Rate Conversion Factors for

Exposure to Residual Radioactive Materials (contd)

\begin{tabular}{|c|c|c|c|c|c|c|}
\hline \multirow[b]{2}{*}{$\begin{array}{l}\text { Radio- } \\
\text { nuclide }\end{array}$} & \multicolumn{2}{|c|}{$\begin{array}{c}\text { Building } \\
\text { Surface Sources }\end{array}$} & \multicolumn{2}{|c|}{$\begin{array}{l}\text { Building } \\
\text { Volume Sources }\end{array}$} & \multicolumn{2}{|c|}{ Soil Volume Sources } \\
\hline & $\begin{array}{l}\text { mrem/h per } \\
\mathrm{dpm} / 100 \mathrm{~cm} 2\end{array}$ & $\begin{array}{l}\mu \mathrm{Sv} / \mathrm{h} \text { per } \\
\mathrm{Bq} / 100 \mathrm{~cm}^{2}\end{array}$ & $\begin{array}{c}\text { mrem/h per } \\
\mathrm{pCi} / \mathrm{g} \\
\end{array}$ & $\begin{array}{c}\mu \mathrm{Sv} / \mathrm{h} \text { per } \\
\mathrm{Bq} / \mathrm{g}\end{array}$ & $\begin{array}{c}\text { mrem } / \mathrm{h} \text { per } \\
\mathrm{pCi} / \mathrm{g} \\
\end{array}$ & $\begin{array}{c}\mu \mathrm{Sv} / \mathrm{h} \text { per } \\
\mathrm{Bq} / \mathrm{g}\end{array}$ \\
\hline $\begin{array}{l}{ }^{131} \mathrm{I} \\
132 \mathrm{I} \\
133 \mathrm{I}+\mathrm{D}(\mathrm{a}) \\
{ }_{133} \mathrm{I} \\
134 \mathrm{I} \\
135 \mathrm{I}+\mathrm{D}(\mathrm{a}) \\
{ }^{135} \mathrm{I}\end{array}$ & $\begin{array}{l}6.5 \mathrm{E}-08 \\
4.1 \mathrm{E}-07 \\
5.0 \mathrm{E}-08 \\
9.7 \mathrm{E}-08 \\
4.5 \mathrm{E}-07 \\
1.4 \mathrm{E}-07 \\
2.7 \mathrm{E}-07\end{array}$ & $\begin{array}{l}1.1 \mathrm{E}-08 \\
6.9 \mathrm{E}-08 \\
8.3 \mathrm{E}-09 \\
1.6 \mathrm{E}-08 \\
7.6 \mathrm{E}-08 \\
2.4 \mathrm{E}-08 \\
4.5 \mathrm{E}-08\end{array}$ & $\begin{array}{l}2.4 \mathrm{E}-05 \\
1.6 \mathrm{E}-04 \\
1.8 \mathrm{E}-05 \\
3.6 \mathrm{E}-05 \\
1.8 \mathrm{E}-04 \\
5.7 \mathrm{E}-05 \\
1.1 \mathrm{E}-04\end{array}$ & $\begin{array}{l}6.4 \mathrm{E}-03 \\
4.3 \mathrm{E}-02 \\
4.9 \mathrm{E}-03 \\
9.8 \mathrm{E}-03 \\
4.7 \mathrm{E}-02 \\
1.5 \mathrm{E}-02 \\
2.9 \mathrm{E}-02\end{array}$ & $\begin{array}{l}3.7 \mathrm{E}-04 \\
2.3 \mathrm{E}-03 \\
2.9 \mathrm{E}-04 \\
6.0 \mathrm{E}-04 \\
2.6 \mathrm{E}-03 \\
8.2 \mathrm{E}-04 \\
1.6 \mathrm{E}-03\end{array}$ & $\begin{array}{l}1.0 \mathrm{E}-01 \\
6.3 \mathrm{E}-01 \\
7.9 \mathrm{E}-02 \\
1.6 \mathrm{E}-01 \\
6.9 \mathrm{E}-01 \\
2.2 \mathrm{E}-01 \\
4.2 \mathrm{E}-01\end{array}$ \\
\hline 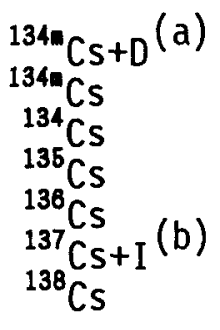 & $\begin{array}{l}2.7 \mathrm{E}-09 \\
5.7 \mathrm{E}-09 \\
3.0 \mathrm{E}-07 \\
7.5 \mathrm{E}-12 \\
3.7 \mathrm{E}-07 \\
1.0 \mathrm{E}-07 \\
4.1 \mathrm{E}-07\end{array}$ & $\begin{array}{l}4.6 \mathrm{E}-10 \\
9.5 \mathrm{E}-10 \\
4.9 \mathrm{E}-08 \\
1.3 \mathrm{E}-12 \\
6.2 \mathrm{E}-08 \\
1.7 \mathrm{E}-08 \\
6.8 \mathrm{E}-08\end{array}$ & $\begin{array}{l}5.7 \mathrm{E}-07 \\
1.2 \mathrm{E}-06 \\
1.1 \mathrm{E}-04 \\
7.1 \mathrm{E}-10 \\
1.4 \mathrm{E}-04 \\
3.8 \mathrm{E}-05 \\
1.6 \mathrm{E}-04\end{array}$ & $\begin{array}{l}1.5 \mathrm{E}-04 \\
3.1 \mathrm{E}-04 \\
3.0 \mathrm{E}-02 \\
1.9 \mathrm{E}-07 \\
3.9 \mathrm{E}-02 \\
1.0 \mathrm{E}-02 \\
4.4 \mathrm{E}-02\end{array}$ & $\begin{array}{l}5.6 \mathrm{E}-06 \\
1.1 \mathrm{E}-05 \\
1.6 \mathrm{E}-03 \\
4.4 \mathrm{E}-09 \\
2.1 \mathrm{E}-03 \\
5.7 \mathrm{E}-04 \\
2.4 \mathrm{E}-03\end{array}$ & $\begin{array}{l}1.5 \mathrm{E}-03 \\
3.1 \mathrm{E}-03 \\
4.4 \mathrm{E}-01 \\
1.2 \mathrm{E}-06 \\
5.6 \mathrm{E}-01 \\
1.5 \mathrm{E}-01 \\
6.5 \mathrm{E}-01\end{array}$ \\
\hline $\begin{array}{l}{ }^{139} \mathrm{Ba} \\
{ }^{146} \mathrm{Ba}+\mathrm{D} \\
{ }^{146} \mathrm{Ba} \\
{ }^{141} \mathrm{Ba}{ }^{\mathrm{Ba}}(\mathrm{a}) \\
{ }^{141} \mathrm{Ba} \\
{ }^{142} \mathrm{Ba} \mathrm{Ba}(\mathrm{a}) \\
{ }^{142} \mathrm{Ba}\end{array}$ & $\begin{array}{l}6.6 \mathrm{E}-09 \\
2.0 \mathrm{E}-07 \\
3.2 \mathrm{E}-08 \\
5.6 \mathrm{E}-08 \\
1.5 \mathrm{E}-07 \\
2.9 \mathrm{E}-07 \\
1.6 \mathrm{E}-07\end{array}$ & $\begin{array}{l}1.1 \mathrm{E}-09 \\
3.3 \mathrm{E}-08 \\
5.3 \mathrm{E}-09 \\
9.4 \mathrm{E}-09 \\
2.5 \mathrm{E}-08 \\
4.8 \mathrm{E}-08 \\
2.6 \mathrm{E}-08\end{array}$ & $\begin{array}{l}2.0 \mathrm{E}-06 \\
7.8 \mathrm{E}-05 \\
1.1 \mathrm{E}-05 \\
2.1 \mathrm{E}-05 \\
5.7 \mathrm{E}-05 \\
1.2 \mathrm{E}-04 \\
6.0 \mathrm{E}-05\end{array}$ & $\begin{array}{l}5.5 \mathrm{E}-04 \\
2.1 \mathrm{E}-02 \\
3.0 \mathrm{E}-03 \\
5.6 \mathrm{E}-03 \\
1.5 \mathrm{E}-02 \\
3.3 \mathrm{E}-02 \\
1.6 \mathrm{E}-02\end{array}$ & $\begin{array}{l}2.4 \mathrm{E}-05 \\
1.2 \mathrm{E}-03 \\
1.8 \mathrm{E}-04 \\
3.0 \mathrm{E}-04 \\
8.4 \mathrm{E}-04 \\
1.8 \mathrm{E}-03 \\
8.8 \mathrm{E}-04\end{array}$ & $\begin{array}{l}6.6 \mathrm{E}-03 \\
3.3 \mathrm{E}-01 \\
5.0 \mathrm{E}-02 \\
8.1 \mathrm{E}-02 \\
2.3 \mathrm{E}-01 \\
4.8 \mathrm{E}-01 \\
2.4 \mathrm{E}-01\end{array}$ \\
\hline $\begin{array}{l}{ }^{146} \mathrm{La} \\
{ }^{141} \mathrm{La}+{ }^{1}(a) \\
{ }^{141} \mathrm{La} \\
{ }^{142} \mathrm{La}\end{array}$ & $\begin{array}{l}3.8 \mathrm{E}-07 \\
1.0 \mathrm{E}-08 \\
9.5 \mathrm{E}-09 \\
4.4 \mathrm{E}-07\end{array}$ & $\begin{array}{l}6.3 \mathrm{E}-08 \\
1.7 \mathrm{E}-09 \\
1.6 \mathrm{E}-09 \\
7.3 \mathrm{E}-08\end{array}$ & $\begin{array}{l}1.5 \mathrm{E}-04 \\
3.5 \mathrm{E}-06 \\
3.6 \mathrm{E}-06 \\
1.8 \mathrm{E}-04\end{array}$ & $\begin{array}{l}4.0 \mathrm{E}-02 \\
9.5 \mathrm{E}-04 \\
9.8 \mathrm{E}-04 \\
4.8 \mathrm{E}-02\end{array}$ & $\begin{array}{l}2.2 \mathrm{E}-03 \\
4.4 \mathrm{E}-05 \\
5.2 \mathrm{E}-05 \\
2.7 \mathrm{E}-03\end{array}$ & $\begin{array}{l}5.9 \mathrm{E}-01 \\
1.2 \mathrm{E}-02 \\
1.4 \mathrm{E}-02 \\
7.4 \mathrm{E}-01\end{array}$ \\
\hline $\begin{array}{l}{ }^{141} \mathrm{Ce} \\
{ }^{143} \mathrm{Ce}+\mathrm{D}(\mathrm{a}) \\
{ }^{143} \mathrm{Ce} \\
{ }^{144} \mathrm{Ce}+\mathrm{D} \\
{ }^{144} \mathrm{Ce}\end{array}$ & $\begin{array}{l}1.2 E-08 \\
2.2 E-08 \\
4.5 E-08 \\
5.4 E-09 \\
3.4 E-09\end{array}$ & $\begin{array}{l}2.1 \mathrm{E}-09 \\
3.6 \mathrm{E}-09 \\
7.5 \mathrm{E}-09 \\
9.0 \mathrm{E}-10 \\
5.6 \mathrm{E}-10\end{array}$ & $\begin{array}{l}3.5 \mathrm{E}-06 \\
7.2 \mathrm{E}-06 \\
1.5 \mathrm{E}-05 \\
1.8 \mathrm{E}-06 \\
8.9 \mathrm{E}-07\end{array}$ & $\begin{array}{l}9.5 \mathrm{E}-04 \\
2.0 \mathrm{E}-03 \\
4.0 \mathrm{E}-03 \\
5.0 \mathrm{E}-04 \\
2.4 \mathrm{E}-04\end{array}$ & $\begin{array}{l}3.8 E-05 \\
1.0 E-04 \\
2.1 E-04 \\
2.5 E-05 \\
9.4 E-06\end{array}$ & $\begin{array}{l}1.0 \mathrm{E}-02 \\
2.8 \mathrm{E}-02 \\
5.6 \mathrm{E}-02 \\
6.7 \mathrm{E}-03 \\
2.5 \mathrm{E}-03\end{array}$ \\
\hline $\begin{array}{l}{ }_{143}^{144} \mathrm{Pr} \\
{ }_{144 m} \mathrm{Pr}+\mathrm{D}^{14} \mathrm{Pr} \\
{ }^{144} \mathrm{Pr}\end{array}$ & $\begin{array}{l}2.2 \mathrm{E}-10 \\
5.1 \mathrm{E}-09 \\
2.6 \mathrm{E}-09 \\
7.8 \mathrm{E}-09\end{array}$ & $\begin{array}{l}3.6 \mathrm{E}-11 \\
8.5 \mathrm{E}-10 \\
4.3 \mathrm{E}-10 \\
1.3 \mathrm{E}-09\end{array}$ & $\begin{array}{l}4.6 \mathrm{E}-08 \\
1.5 \mathrm{E}-06 \\
2.7 \mathrm{E}-07 \\
2.9 \mathrm{E}-06\end{array}$ & $\begin{array}{l}1.3 \mathrm{E}-05 \\
4.1 \mathrm{E}-04 \\
7.4 \mathrm{E}-05 \\
7.8 \mathrm{E}-04\end{array}$ & $\begin{array}{l}5.1 E-07 \\
2.2 E-05 \\
1.6 E-06 \\
4.2 E-05\end{array}$ & $\begin{array}{l}1.4 \mathrm{E}-04 \\
5.9 \mathrm{E}-03 \\
4.3 \mathrm{E}-04 \\
1.1 \mathrm{E}-02\end{array}$ \\
\hline${ }^{147} \mathrm{Nd}+\mathrm{D}(\mathrm{a})$ & $\begin{array}{l}1.1 \mathrm{E}-08 \\
2.3 \mathrm{E}-08\end{array}$ & $\begin{array}{l}1.9 \mathrm{E}-09 \\
3.8 \mathrm{E}-09\end{array}$ & $\begin{array}{l}3.5 \mathrm{E}-06 \\
7.3 \mathrm{E}-06\end{array}$ & $\begin{array}{l}9.5 \mathrm{E}-04 \\
2.0 \mathrm{E}-03\end{array}$ & $\begin{array}{l}5.4 E-05 \\
1.1 E-04\end{array}$ & $\begin{array}{l}1.5 \mathrm{E}-02 \\
3.0 \mathrm{E}-02\end{array}$ \\
\hline${ }^{147} \mathrm{Pm}$ & $1.1 E-11$ & $1.8 E-12$ & $1.2 \mathrm{E}-09$ & $3.1 \mathrm{E}-07$ & $8.3 E-09$ & $2.2 E-06$ \\
\hline
\end{tabular}


TABLE 2.1. External Dose Rate Conversion Factors for

Exposure to Residual Radioactive Materials (contd)

Building

Surface Sources

Radionuclide

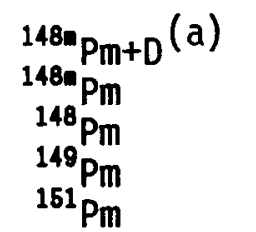

${ }_{{ }^{151} \mathrm{Sm}} \mathrm{Sm}^{153} \mathrm{Sm}$

${ }^{152} \mathrm{Eu}$
${ }^{154} \mathrm{Eu}$
${ }^{155} \mathrm{Eu}$
${ }^{158} \mathrm{Eu}$

${ }^{153} \mathrm{Gd}$

${ }^{186} \mathrm{~Tb}$

${ }^{188 \mathrm{~m}_{\mathrm{HO}}}$

${ }^{181} \mathrm{~W}$
${ }^{185} \mathrm{~W}$
${ }^{187} \mathrm{~W}$

${ }^{187} \mathrm{Re}$

${ }^{185}$ Os

${ }^{192} \mathrm{Ir}$

${ }^{203} \mathrm{Hg}$

${ }^{210} \mathrm{~Pb}+\mathrm{D}(\mathrm{a})$
${ }^{210} \mathrm{~Pb}$
${ }^{212} \mathrm{~Pb}+\mathrm{D}(\mathrm{a})$
${ }^{212} \mathrm{~Pb}$

${ }^{210} \mathrm{Bi}+\mathrm{D}(\mathrm{a})$

${ }^{212} \mathrm{Bi}+\mathrm{I}(b)$

${ }^{216} \mathrm{Po}$

3.6E-07

3.8E-07

$1.1 \mathrm{E}-07$

2.1E-09

5.9E-08

$0.0 \mathrm{E}+00$

$5.4 \mathrm{E}-12$

$1.2 \mathrm{E}-08$

2.1E-07

2.1E-07

1. $0 \mathrm{E}-08$

2.3E-07

2.0E-08

1.9E-07

2.7E-07

6.0E-09

4. $6 \mathrm{E}-11$

8.4E-08

1.3E-07

$1.2 \mathrm{E}-08$

1.5E-07

3. $5 \mathrm{E}-08$

4.1E-10

9.5E-10

8. $3 \mathrm{E}-08$

2.5E-08

1. $6 \mathrm{E}-10$

3. $2 \mathrm{E}-10$

1.6E-12
Building

Volume Sources mrem/h per $\mu \mathrm{Sv} / \mathrm{h}$ per $\mathrm{pCi} / \mathrm{g}$ $\mathrm{dpm} / 100 \mathrm{~cm}^{2} \mathrm{~Bq} / 100 \mathrm{~cm}^{2}$

$6.0 \mathrm{E}-08$

$6.3 \mathrm{E}-08$

1.8E-08

3.5E-10

9. $9 \mathrm{E}-09$

$0.0 E+00$

8.9E-13

2.1E-09

3. $4 \mathrm{E}-08$

3. $6 \mathrm{E}-08$

1.7E-09

3. $8 \mathrm{E}-08$

3.3E-09

3.1E-08

4.6E-08

1.0E-09

7.7E-12

1.4E-08

$0.0 E+00 \quad 0.0 E+00$

2.1E-08

2.0E-09

2.5E-08

5.8E-09

$6.8 \mathrm{E}-11$

1. $6 \mathrm{E}-10$

1. $4 \mathrm{E}-08$

4. $2 E-09$

2.6E-11

5.3E-11

3. $9 \mathrm{E}-08$

2.6E-13
1.4E-04

1. $4 \mathrm{E}-04$

4.2E-05

7.0E-07

2.1E-05

$0.0 \mathrm{E}+00$

2. $4 \mathrm{E}-10$

2.9E-06

7.8E-05

8.2E-05

2.7E-06

9.1E-05

4.2E-06

7.1E-05

1.0E-04

1.1E-06

7.2E-09

3.1E-05

2.0E-06

8.3E-03

$0.0 \mathrm{E}+00$

4. $6 \mathrm{E}-05$

3. $0 \mathrm{E}-06$

$0.0 \mathrm{E}+00$

1.2E-02

8.2E-04

5.5E-05

1.5E-02

1.2E-05

3.1E-03

4. $8 \mathrm{E}-08$

1. $3 \mathrm{E}-05$

7.2E-08

3.3E-05

$1.9 \mathrm{E}-05$

9.0E-03

2. $2 \mathrm{E}-03$

8. 3E-06

3.7E-08

1. $0 \mathrm{E}-05$

7. $4 \mathrm{E}-08$

9. $4 \mathrm{E}-05$

$2.0 \mathrm{E}-05$

2.5E-02

$6.0 \mathrm{E}-10$

1. $6 \mathrm{E}-07$

Soil Volume Sources $\mathrm{mrem} / \mathrm{h}$ per $\mu \mathrm{Sv} / \mathrm{h}$ per

$\mathrm{pCi} / \mathrm{q}$

2.0E-03

2.2E-03

$6.1 \mathrm{E}-04$

9.6E-06

3.1E-04

$0.0 E+00$

$5.9 \mathrm{E}-10$

2.9E-05

$1.1 \mathrm{E}-03$

$1.2 \mathrm{E}-03$

2.7E-05

1.3E-03

3. $9 \mathrm{E}-05$

1.0E-03

1.5E-03

8. 3E-06

6.5E-08

4.7E-04

$0.0 \mathrm{E}+00$

6.8E-04

3. $0 \mathrm{E}-05$

8.7E-04

1.6E-04

3. $9 \mathrm{E}-07$

3.1E-07

5. $0 \mathrm{E}-04$

1.1E-04

4.3E-07

8. $6 \mathrm{E}-07$

1. $4 \mathrm{E}-03$

8.4E-09
5.4E-01

$5.8 \mathrm{E}-01$

$1.6 \mathrm{E}-01$

2.6E-03

8.4E-02

$0.0 \mathrm{E}+00$

1. $6 \mathrm{E}-07$

$7.8 \mathrm{E}-03$

3.1E-01

3. $2 \mathrm{E}-01$

7.3E-03

3. $6 \mathrm{E}-01$

1.0E-02

2.8E-01

4.0E-01

2. $2 \mathrm{E}-03$

1.7E-05

1.3E-01

$0.0 E+00$

1.8E-01

8.0E-03

2. $4 \mathrm{E}-01$

4.2E-02

1.0E-04

8.5E-05

1. $4 \mathrm{E}-01$

3. $0 \mathrm{E}-02$

1.1E-04

2. $3 E-04$

3. $9 \mathrm{E}-01$

2.3E-06 
TABLE 2.1. External Dose Rate Conversion Factors for

Exposure to Residual Radioactive Materials (contd)

Building

Surface Sources

Radio-

nuclide

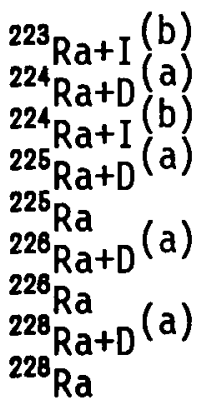

${ }^{225} \mathrm{Ac}+\mathrm{I}(\mathrm{b})$
${ }^{227} \mathrm{AC+D}(\mathrm{a})$
${ }^{227} \mathrm{AC}$
${ }^{228} \mathrm{AC+D}(\mathrm{a})$
$\mathrm{AC}$

${ }^{227} T h+D^{(a)}$

${ }^{227} \mathrm{Th}$ (a)

${ }^{228} \mathrm{Th}$ Th+D (a)

${ }^{2239} \mathrm{Th}$ Th+D $(a)$

${ }^{239} \mathrm{Th}_{\mathrm{Th}+\mathrm{D}}(\mathrm{a})$

${ }^{231} \mathrm{Th}$ Th+D (a)

232 Th-Nat (c)

${ }^{232} \mathrm{Th}$
${ }^{234} \mathrm{~T} h+\mathrm{D}+\mathrm{I}(\mathrm{a})$

${ }^{231} \mathrm{~Pa}+\mathrm{D}(\mathrm{a})$
${ }^{231} \mathrm{~Pa}$
${ }^{233} \mathrm{~Pa}$
${ }^{23} \mathrm{~Pa}$

${ }^{232} U+D(a)$
${ }^{232} U$ (a)
${ }^{233} U+D(a)$
${ }^{233} U$
${ }^{234} U$ (a)
${ }^{235} U$ U

$5.0 \mathrm{E}-08$
$4.2 \mathrm{E}-08$
$1.8 \mathrm{E}-09$
$6.2 \mathrm{E}-09$
$2.8 \mathrm{E}-09$
$3.3 \mathrm{E}-08$
$8.4 \mathrm{E}-10$
$4.6 \mathrm{E}-08$
$5.4 \mathrm{E}-14$

4.2E-08

8.4E-09

$5.4 \mathrm{E}-11$

5.2E-08

$1.7 \mathrm{E}-07$

9.6E-09

$1.9 \mathrm{E}-08$

3. $6 \mathrm{E}-08$

$5.4 \mathrm{E}-10$

7.3E-09

1. $5 \mathrm{E}-08$

3. $0 \mathrm{E}-08$

2.7E-10

4.1E-10

4.3E-09

4. $1 E-08$

4.1E-08

2. $4 \mathrm{E}-10$

2.6E-09

4.7E-09

8.3E-09

7.0E-09

4.1E-08

3.5E-07

4.8E-11

3. $5 \mathrm{E}-10$

$6.5 \mathrm{E}-09$

1. $4 \mathrm{E}-10$

2.9E-10

1.2E-08

2.1E-08
Building

Volume Sources

mrem/h per $\mu \mathrm{Sv} / \mathrm{h}$ per $\mathrm{dpm} / 100 \mathrm{~cm}^{2} \mathrm{~Bq} / 100 \mathrm{~cm}^{2}$

8.4E-09

7.0E-09

3.1E-10

1.0E-09

4.7E-10

5.5E-09

1. $4 \mathrm{E}-10$

7.7E-09

8.9E-15
7.1E-09

1. $4 \mathrm{E}-09$

8.9E-12

8.6E-09

2.8E-08

1. $6 \mathrm{E}-09$

3. $2 \mathrm{E}-09$

6.0E-09

9.0E-11

1.2E-09

2.5E-09

5.0E-09

4.5E-11

6.9E-11

6.9E-09

6.9E-09

4.1E-11

4.3E-10

7.9E-10

1. $4 \mathrm{E}-09$

1.2E-09

6.8E-09

5.8E-08

8.1E-12

5.8E-11

1.1E-09

2. $4 \mathrm{E}-11$

4. $9 \mathrm{E}-11$

2.0E-09

3. 5E-09
7.2E-10 $\mathrm{pCi} / \mathrm{g}$

\begin{tabular}{ccc}
$\mathrm{yC} / \mathrm{g}$ & & $\mathrm{Bq} / \mathrm{g}$ \\
\cline { 1 - 1 } $1.7 \mathrm{E}-05$ & & $4.7 \mathrm{E}-03$ \\
$1.7 \mathrm{E}-05$ & & $4.6 \mathrm{E}-03$ \\
$6.2 \mathrm{E}-07$ & & $1.7 \mathrm{E}-04$ \\
$2.2 \mathrm{E}-06$ & & $5.9 \mathrm{E}-04$ \\
$3.6 \mathrm{E}-07$ & & $9.8 \mathrm{E}-05$ \\
$1.3 \mathrm{E}-05$ & & $3.6 \mathrm{E}-03$ \\
$2.5 \mathrm{E}-07$ & & $6.7 \mathrm{E}-05$ \\
$1.8 \mathrm{E}-05$ & & $4.8 \mathrm{E}-03$ \\
$2.3 \mathrm{E}-12$ & & $6.2 \mathrm{E}-10$
\end{tabular}

1.5E-05

2.8E-06

8.2E-09

2.0E-05

6.5E-05

3.2E-06

6.0E-06

1. $4 \mathrm{E}-05$

9. $9 \mathrm{E}-08$

2.4E-06

3. $9 \mathrm{E}-06$

1.2E-05

2.2E-08

5.3E-08

5. $4 \mathrm{E}-07$

1.6E-05

1. $6 \mathrm{E}-05$

1.5E-08

8.0E-07

1.5E-06

4.1E-03

7. $6 \mathrm{E}-04$

2. $2 \mathrm{E}-06$

5. 4E-03

1.8E-02

8.7E-04

1. $6 \mathrm{E}-03$

3. $9 \mathrm{E}-03$

2.7E-05

6.5E-04

1.1E-03

3. $2 \mathrm{E}-03$

5. $9 \mathrm{E}-06$

1. $4 \mathrm{E}-05$

1. $5 \mathrm{E}-04$

4. $3 \mathrm{E}-03$

4.3E-03

4.1E-06

2.2E-04

4.0E-04

2.7E-06

1.9E-06

7.2E-04

1. $4 \mathrm{E}-05$

1.3E-04

5. $2 \mathrm{E}-04$

3.8E-03

3. $6 \mathrm{E}-02$

3. $0 \mathrm{E}-09$

2. $2 \mathrm{E}-08$

2.1E-06

1.7E-08

$1.6 \mathrm{E}-08$

3. $4 \mathrm{E}-06$

$6.3 \mathrm{E}-06$
8.1E-07

5. $9 \mathrm{E}-06$

$5.8 \mathrm{E}-04$

4.6E-06

4. $4 \mathrm{E}-06$

9.1E-04

1.7E-03
Soil Volume Sources mrem $/ \mathrm{h}$ per $\mu \mathrm{Sv} / \mathrm{h}$ per $\mathrm{pCi} / \mathrm{g} \quad \mathrm{Bq} / \mathrm{g}$

$\begin{array}{ll}2.5 \mathrm{E}-04 & 6.8 \mathrm{E}-02 \\ 2.5 \mathrm{E}-04 & 6.9 \mathrm{E}-02 \\ 8.5 \mathrm{E}-06 & 2.3 \mathrm{E}-03 \\ 3.4 \mathrm{E}-05 & 9.3 \mathrm{E}-03 \\ 2.2 \mathrm{E}-06 & 6.0 \mathrm{E}-04 \\ 2.0 \mathrm{E}-04 & 5.3 \mathrm{E}-02 \\ 2.8 \mathrm{E}-06 & 7.5 \mathrm{E}-04 \\ 2.7 \mathrm{E}-04 & 7.2 \mathrm{E}-02 \\ 3.3 \mathrm{E}-12 & 8.8 \mathrm{E}-10\end{array}$

2.4E-04

6.4E-02

4.0E-05

7. $8 \mathrm{E}-08$

3. $0 \mathrm{E}-04$

$1.1 \mathrm{E}-02$

2.1E-05

8.1E-02

2.6E-01

4.6E-05

1.2E-02

8.3E-05

2. $2 \mathrm{E}-02$

2.2E-04

5. $9 \mathrm{E}-02$

$1.0 \mathrm{E}-06$

2.8E-04

3. $4 \mathrm{E}-05$

9.2E-03

4.2E-05

$1.1 \mathrm{E}-02$

$1.8 \mathrm{E}-04$

4.8E-02

1. $2 \mathrm{E}-07$

3. $3 \mathrm{E}-05$

4.3E-07

$1.2 \mathrm{E}-04$

4. $4 \mathrm{E}-06$

$1.2 \mathrm{E}-03$

2. $4 \mathrm{E}-04$

$6.5 \mathrm{E}-02$

2. $4 \mathrm{E}-04$

$6.5 \mathrm{E}-02$

$6.4 \mathrm{E}-08$

1.7E-05

1.1E-05

3.0E-03

5.3E-03

\subsection{E-05}

1.1E-02

2.8E-05

7. $6 \mathrm{E}-03$

2.1E-04

5.7E-02

1. $9 \mathrm{E}-03$

5.2E-01

1. $3 \mathrm{E}-08$

3.5E-06

9. $4 \mathrm{E}-08$

2.5E-05

3. $0 \mathrm{E}-05$

8.2E-03

1.5E-07

3.9E-05

5.7E-08

$1.5 \mathrm{E}-05$

3.8E-05

1.0E-02

7.2E-05

1.9E-02 
TABLE 2.1. External Dose Rate Conversion Factors for

Exposure to Residual Radioactive Materials (contd)

Building

Surface Sources

Radio-

nuclide

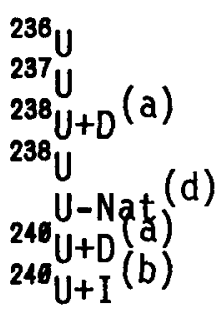

${ }^{237} \mathrm{~Np}+\mathrm{D}(\mathrm{a})$
$237 \mathrm{~Np}$
$238 \mathrm{~Np}+\mathrm{D}(\mathrm{a})$
$238 \mathrm{~Np}$
${ }^{238} \mathrm{~Np}+\mathrm{D}(\mathrm{a})$
${ }^{239} \mathrm{~Np}$

${ }^{238} \mathrm{Pu}$
${ }^{237} \mathrm{Pu}$
${ }^{238} \mathrm{Pu}$
${ }^{239} \mathrm{Pu}$
${ }^{248} \mathrm{Pu}$
${ }^{241} \mathrm{Pu}+\mathrm{D}(\mathrm{a})$
${ }^{241} \mathrm{Pu}$
${ }^{242} \mathrm{Pu}$
${ }^{243} \mathrm{Pu}+\mathrm{D}(\mathrm{a})$
${ }^{243} \mathrm{Pu}(\mathrm{a}$
${ }^{244} \mathrm{Pu}+\mathrm{D}$
${ }^{244} \mathrm{Pu}$

${ }^{241} \mathrm{Am}$
${ }^{2421} \mathrm{Am}+\mathrm{D}$
${ }^{2424} \mathrm{Am}$
${ }^{242} \mathrm{Am}+\mathrm{D}(\mathrm{a})$
${ }^{242} \mathrm{Am}$
${ }^{243} \mathrm{Am}+\mathrm{D}(\mathrm{a})$
${ }^{243} \mathrm{Am}$

${ }^{242} \mathrm{Cm}+\mathrm{D}(\mathrm{a})$
${ }^{242} \mathrm{Cm}$
${ }^{244} \mathrm{Cm}$
${ }^{244} \mathrm{Cm}+\mathrm{D}$
${ }^{245} \mathrm{Cm}$
${ }^{245} \mathrm{Cm}+\mathrm{D}$
${ }^{246} \mathrm{Cm}$

2.7E-10

$2.7 \mathrm{E}-08$

$1.8 \mathrm{E}-09$

2. $4 \mathrm{E}-10$

$7.3 \mathrm{E}-10$

2.2E-08

$6.7 \mathrm{E}-08$

2.2E-08

$5.4 \mathrm{E}-09$

4.7E-08

9.7E-08

1. $5 \mathrm{E}-08$

3.1E-08

$3.5 \mathrm{E}-10$

1.0E-08

3.1E-10

1. $3 \mathrm{E}-10$

2.9E-10

2.1E-09

7.9E-16

2. $4 \mathrm{E}-10$

$1.1 \mathrm{E}-08$

3. $\mathrm{E}-09$

1. $6 \mathrm{E}-08$

2.1E-10

4.2E-09

1.2E-09

8. $5 \mathrm{E}-10$

1.3E-09

3. $3 \mathrm{E}-09$

1.3E-08

8.1E-09

3. $3 \mathrm{E}-10$

3. $1 \mathrm{E}-10$

2.3E-08

1. $3 \mathrm{E}-10$

2.7E-10

5. $6 \mathrm{E}-09$

1. $4 \mathrm{E}-08$

2. $4 E-10$
Building

Volume Sources mrem/h per $\mu \mathrm{Sv} / \mathrm{h}$ per $\mathrm{dpm} / 100 \mathrm{~cm}^{2} \mathrm{~Bq} / 100 \mathrm{~cm}^{2}$

\subsection{E-11 \\ 4.5E-09 \\ 3. $0 \mathrm{E}-10$ \\ 4. $0 \mathrm{E}-11$ \\ 1.2E-10 \\ 3. $6 \mathrm{E}-09$ \\ 1.1E-08}

3.7E-09

8. $9 \mathrm{E}-10$

$7.9 \mathrm{E}-09$

1. $6 \mathrm{E}-08$

2.5E-09

5.2E-09

5.8E-11

1.7E-09

5.1E-11

2.1E-11

4. $9 \mathrm{E}-11$

3. $4 \mathrm{E}-10$

1.3E-16

4.1E-11

1. $9 \mathrm{E}-09$

5. $9 \mathrm{E}-10$

2.7E-09

3. $5 \mathrm{E}-11$

7.1E-10

2. $0 \mathrm{E}-10$

1. $4 \mathrm{E}-10$

2.2E-10

5. $6 \mathrm{E}-10$

2.1E-09

1.4E-09

5. $4 \mathrm{E}-11$

5.1E-11

3. $9 \mathrm{E}-09$

2.2E-11

4.6E-11

9. $4 \mathrm{E}-10$

2.3E-09

4.1E-11

\section{$\mathrm{pCi} / \mathrm{g}$}

1. $3 \mathrm{E}-08$

7.7E-06

5.4E-07

1.2E-08

1. $6 \mathrm{E}-07$

8.0E-06

2.5E-05

7.3E-06

9. $5 \mathrm{E}-07$

$1.8 \mathrm{E}-05$

3.8E-05

4.7E-06

9.7E-06

1. $6 \mathrm{E}-08$

2.7E-06

1. $4 \mathrm{E}-08$

8.3E-09

1. $3 \mathrm{E}-08$

3.1E-07

3. $4 \mathrm{E}-14$

1.1E-08

3. $5 \mathrm{E}-06$

9.4E-07

6.0E-06

9.2E-09

6.4E-07

2. $2 \mathrm{E}-07$

4. $8 \mathrm{E}-08$

2.8E-07

7. $9 \mathrm{E}-07$

3.7E-06

1.8E-06

1.5E-08

1. $4 \mathrm{E}-08$

7.2E-06

5. $9 \mathrm{E}-09$

1.2E-08

1. $4 \mathrm{E}-06$

3.7E-06

1.1E-08
$\mathrm{Bq} / \mathrm{g}$

2.1E-03

1. $4 \mathrm{E}-04$

3.2E-06

4.2E-05

2.2E-03

6.7E-03

2. $0 \mathrm{E}-03$

2. $6 \mathrm{E}-04$

5.0E-03

1.0E-02

1.3E-03

2. $6 \mathrm{E}-03$

4. $4 \mathrm{E}-06$

7.3E-04

3.7E-06

2. $2 \mathrm{E}-06$

3.6E-06

8.5E-05

$9.1 \mathrm{E}-12$

2. $9 \mathrm{E}-06$

9.3E-04

2.5E-04

1. $6 \mathrm{E}-03$

2.5E-06

1.7E-04

5.9E-05

1.3E-05

7.5E-05

2.1E-04

1.0E-03

4. $9 \mathrm{E}-04$

4.0E-06

3.7E-06

$1.9 \mathrm{E}-03$

1. $6 \mathrm{E}-06$

3.2E-06

$3.8 \mathrm{E}-04$

$1.0 \mathrm{E}-03$

2.9E-06
3. $6 \mathrm{E}-06$
Soil Volume Sources mrem/h per $\mu \mathrm{Sv} / \mathrm{h}$ per

\begin{tabular}{|c|c|}
\hline $\begin{array}{l}3.0 \mathrm{E}-08 \\
9.2 \mathrm{E}-05 \\
7.5 \mathrm{E}-06 \\
2.6 \mathrm{E}-08 \\
1.7 \mathrm{E}-06 \\
1.2 \mathrm{E}-04 \\
3.7 \mathrm{E}-04\end{array}$ & $\begin{array}{l}8.0 \mathrm{E}-06 \\
2.5 \mathrm{E}-02 \\
2.0 \mathrm{E}-03 \\
7.0 \mathrm{E}-06 \\
4.5 \mathrm{E}-04 \\
3.2 \mathrm{E}-02 \\
9.9 \mathrm{E}-02\end{array}$ \\
\hline
\end{tabular}

1.0E-04

2.8E-02

8. $9 \mathrm{E}-06$

2. $4 \mathrm{E}-03$

$7.4 \mathrm{E}-02$

5.6E-04 1.5E-01

5.9E-05 1.6E-02

1.2E-04 3.3E-02

$2.8 \mathrm{E}-08 \quad 7.6 \mathrm{E}-06$

2.9E-05 7.8E-03

$1.9 \mathrm{E}-08 \quad 5.1 \mathrm{E}-06$

4.2E-08 1.1E-05

$1.9 \mathrm{E}-08 \quad 5.1 \mathrm{E}-06$

2.1E-06 5.6E-04

3.5E-14 9.5E-12

$1.6 \mathrm{E}-08 \quad 4.3 \mathrm{E}-06$

4.3E-05 1.2E-02

$1.0 \mathrm{E}-05 \quad 2.7 \mathrm{E}-03$

8.8E-05 2.4E-02

$1.1 \mathrm{E}-08 \quad 3.0 \mathrm{E}-06$

4.3E-06 1.2E-03

2.2E-06 5.9E-04

$1.8 \mathrm{E}-07 \quad 4.8 \mathrm{E}-05$

2.9E-06 7.8E-04

8.3E-06 2.2E-03

4.5E-05 $1.2 \mathrm{E}-02$

1.6E-05 4.3E-03

$1.9 \mathrm{E}-08 \quad 5.2 \mathrm{E}-06$

$1.8 \mathrm{E}-08 \quad 4.8 \mathrm{E}-06$

8.9E-05 2.4E-02

7.5E-09 2.0E-06

$1.5 \mathrm{E}-08 \quad 4.1 \mathrm{E}-06$

$1.4 \mathrm{E}-05 \quad 3.9 \mathrm{E}-03$

4.0E-05 1.1E-02

1.3E-08 3.4E-06 
TABLE 2.1. External Dose Rate Conversion Factors for

Exposure to Residual Radioactive Materials (contd)

Building

Surface Sources

Radionuclide

${ }^{247} \mathrm{Cm}+\mathrm{D}(\mathrm{a})$
${ }^{247} \mathrm{Cm}$
${ }^{248} \mathrm{Cm}$

${ }^{252} \mathrm{Cf}$

$\mathrm{mrem} / \mathrm{h}$ per $\mu$ Sv $/ \mathrm{h}$ per

$\mathrm{dpm} / 100 \mathrm{~cm}^{2} \mathrm{~Bq} / 100 \mathrm{~cm}^{2}$

4.4E-08

6.4E-08

7.3E-09

$1.9 \mathrm{E}-10$

$1.1 \mathrm{E}-08$

$3.2 \mathrm{E}-11$

1.9E-10

3.2E-11
Building Volume Sources mrem/h per $\mu$ Sv/h per $\mathrm{pCi} / \mathrm{g} \quad \mathrm{Bq} / \mathrm{g}$

$1.5 \mathrm{E}-05$

4.1E-03

$6.4 \mathrm{E}-03$

2.3E-06

$8.5 \mathrm{E}-09$

8.7E-09

2.3E-06
Soil Volume Sources

mrem/h per $\mu \mathrm{Sv} / \mathrm{h}$ per $\mathrm{pCi} / \mathrm{g}$ $\mathrm{Bg} / \mathrm{g}$

2. $4 \mathrm{E}-04$

6.5E-02

4.0E-04 1.1E-01

$1.1 \mathrm{E}-08 \quad 3.1 \mathrm{E}-06$

1.3E-08 3.4E-06

(a) Where $+D$ means "plus daughters in equilibrium" referring to explicit daughters, or daughters with half-lives greater than 10 minutes. Separate values are also included for parents and daughters so that combined factors for non-equilibrium cases can be determined. Note: in some cases $+D$ also includes implicit daughters (i.e., uranium and thorium decay chains).

(b) Where +I means "plus implicit daughters in equilibrium" referring to daughters with half-lives less than 10 minutes. The decay energies associated with these daughters have been assigned to the parent, and the appropriate branching ratios (described in Appendix D) have been considered.

(c) Where Th-Nat includes an equilibrium mixture of ${ }^{232}$ Th plus 10 daughters in the decay chain, as discussed in Appendix $D$.

(d) Where $1 \mathrm{Ci} \mathrm{U}$-Nat equals $48.9 \%{ }^{238} \mathrm{U}$ plus $48.9 \%{ }^{234} \mathrm{U}$ plus $2.25 \%{ }^{235} \mathrm{U}$. 


\subsubsection{Inhalation}

The dose rate conversion factors for inhalation generated using the GENII somputer program (Napier et al., Vol. 1 1988) are based on the models, metabolic Jata, and recommendations found in ICRP 30 (1979-1988). The general equation for estimating the committed effective dose rate equivalent by inhalation received by individuals for radionuclide $i$ is:

$$
H_{I N H, i}=(V t) C_{d} C_{w, i} D_{I N H, i}
$$

where $H_{I N H, i}=$ the committed effective dose equivalent from a 1-yr intake of radionuclide $i$ by inhalation, in mrem $(\mu \mathrm{Sv})$ per year of intake

$V=$ the ventilation rate of the individual, in $\mathrm{m} 3 / \mathrm{h}$ (Note: For this study, a constant ventilation rate of $0.97 \mathrm{~m} 3 / \mathrm{h}$ is assumed from ICRP 1975).

$t=$ the duration of exposure for the individual, in $h / y r$

$C_{d}=$ concentration of respirable dust in air, in $\mathrm{g} / \mathrm{m}^{3}$

$C_{w, i}=$ the concentration of radionuclide $i$ in the contaminated material, in $\mathrm{pCi} / \mathrm{g}(\mathrm{Bq} / \mathrm{g})$

$\mathrm{DF}_{\mathrm{INH}, i}=$ the committed effective dose from inhalation of radionuclide $i$, in $\mathrm{mrem} / \mathrm{pCi}(\mu \mathrm{Sv} / \mathrm{Bq})$.

The term in parentheses in Equation 2.2 is equivalent to the usage parameter defined in Equation 2.1. The values used for the parameters shown in Equation 2.2 are either scenario specific, or are based on the data requirements of the ICRP dosimetry system, as discussed in Appendix B. For the solubility class, assumptions were made by element to maximize the expected dose using the ICRP 30 lung model, as discussed in Appendix B.

Inhalation committed effective dose conversion factors are shown in Table 2.2. These factors are in units of mrem per hour of exposure per $\mathrm{pCi} / \mathrm{m}^{3}$ in air and $\mu \mathrm{Sv}$ per hour of exposure per $\mathrm{Bq} / \mathrm{m}^{3}$ in air. The factors in Table 2.2 can be multiplied by the exposure duration and the estimated air concentration to obtain an estimate of the committed effective dose equivalent for inhalation.

A comparison of the inhalation committed effective dose conversion factors for selected radionuclides used in this study with those found in the literature was next conducted. The radionuclides considered included $90 \mathrm{Sr}, 134 \mathrm{Cs}, 235 \mathrm{U}$, $238 \mathrm{U}$ and $239 \mathrm{Pu}$. Comparisons with the dose per unit intake factors from ICRP 30 (1979-1988) and from Federal Guidance Report No. 11 (EPA 1988) showed agreement within a few percent. This agreement verifies that the basic inhalation dose conversion factors used in this study are consistent with the ICRP methods and with other Federal guidance. 
TABLE 2.2. Inhalation Committed Effective Dose Rate Conversion Factors for Exposure to Residual Radioactive Materials

\begin{tabular}{|c|c|c|}
\hline Radionuclide & $\begin{array}{c}\text { mrem } / \mathrm{h} \text { per } \\
\mathrm{pCi} / \mathrm{m}^{3} \\
\end{array}$ & $\begin{array}{c}\mu \mathrm{Sv} / \mathrm{h} \text { per } \\
\mathrm{Bq} / \mathrm{m}^{3} \\
\end{array}$ \\
\hline${ }^{3} \mathrm{H}$ & $8.2 E-08$ & $2.2 E-05$ \\
\hline${ }^{10} \mathrm{Be}$ & $3.4 \mathrm{E}-04$ & $9.2 \mathrm{E}-02$ \\
\hline${ }^{14} \mathrm{C}$ & $1.9 E-06$ & $5.1 E-04$ \\
\hline${ }^{18} \mathrm{~F}$ & $7.7 E-08$ & $2.1 E-05$ \\
\hline${ }_{{ }^{24} \mathrm{Na}}^{22} \mathrm{Na}$ & $\begin{array}{l}8.6 \mathrm{E}-06 \\
1.1 \mathrm{E}-06\end{array}$ & $\begin{array}{l}2.3 E-03 \\
3.0 E-04\end{array}$ \\
\hline $\begin{array}{l}{ }^{32} \mathrm{p} \\
{ }^{33}\end{array}$ & $\begin{array}{l}5.5 \mathrm{E}-06 \\
5.6 \mathrm{E}-07\end{array}$ & $\begin{array}{l}1.5 \mathrm{E}-03 \\
1.5 \mathrm{E}-04\end{array}$ \\
\hline${ }^{35} \mathrm{~S}$ & $2.4 \mathrm{E}-06$ & $6.5 E-04$ \\
\hline${ }^{36} \mathrm{Cl}$ & $1.8 \mathrm{E}-06$ & $4.9 E-04$ \\
\hline${ }^{16} \mathrm{~K}$ & $9.9 E-06$ & $2.7 E-03$ \\
\hline${ }^{41} \mathrm{Ca}$ & $\begin{array}{l}1.2 \mathrm{E}-06 \\
6.4 \mathrm{E}-06\end{array}$ & $\begin{array}{l}3.2 E-04 \\
1.7 E-03\end{array}$ \\
\hline${ }^{48} \mathrm{Sc}$ & $2.8 E-05$ & $7.6 \mathrm{E}-03$ \\
\hline${ }^{51} \mathrm{Cr}$ & $3.1 E-07$ & $8.4 E-05$ \\
\hline $\begin{array}{l}{ }^{54} \mathrm{Mn} \\
{ }^{58} \mathrm{Mn}\end{array}$ & $\begin{array}{l}6.1 \mathrm{E}-06 \\
3.3 \mathrm{E}-07\end{array}$ & $\begin{array}{l}1.6 \mathrm{E}-03 \\
8.9 \mathrm{E}-05\end{array}$ \\
\hline $\begin{array}{l}{ }^{65} \mathrm{Fe} \\
{ }^{59} \mathrm{Fe}\end{array}$ & $\begin{array}{l}1.2 E-06 \\
1.2 E-05\end{array}$ & $\begin{array}{l}3.2 E-04 \\
3.2 E-03\end{array}$ \\
\hline $\begin{array}{l}{ }^{58} \mathrm{Co} \\
{ }^{58} \mathrm{Co} \\
{ }_{\mathrm{Co}}\end{array}$ & $\begin{array}{l}8.3 \mathrm{E}-06 \\
1.0 \mathrm{E}-05 \\
1.9 \mathrm{E}-04\end{array}$ & $\begin{array}{l}2.2 E-03 \\
2.7 E-03 \\
5.1 E-02\end{array}$ \\
\hline $\begin{array}{l}{ }^{59} \mathrm{Ni} \\
{ }^{63} \mathrm{Ni} \\
{ }^{65} \mathrm{Ni}\end{array}$ & $\begin{array}{l}7.9 \mathrm{E}-07 \\
1.9 \mathrm{E}-06 \\
2.3 \mathrm{E}-07\end{array}$ & $\begin{array}{l}2.1 \mathrm{E}-04 \\
5.1 \mathrm{E}-04 \\
6.2 \mathrm{E}-05\end{array}$ \\
\hline${ }^{64} \mathrm{Cu}$ & $1.9 \mathrm{E}-07$ & $5.1 E-05$ \\
\hline 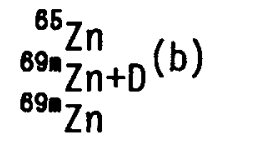 & $\begin{array}{l}1.7 \mathrm{E}-05 \\
4.1 \mathrm{E}-07 \\
7.8 \mathrm{E}-07\end{array}$ & $\begin{array}{l}4.6 \mathrm{E}-03 \\
1.1 \mathrm{E}-04 \\
2.1 \mathrm{E}-04\end{array}$ \\
\hline
\end{tabular}


TABLE 2.2. Inhalation Committed Effective Dose Rate Conversion Factors for Exposure to Residual Radioactive Materials (contd)

\begin{tabular}{|c|c|c|}
\hline Radionuclide & $\begin{array}{c}\text { mrem/h per } \\
\mathrm{pCi} / \mathrm{m}^{3}\end{array}$ & $\begin{array}{c}\mu \mathrm{Sv} / \mathrm{h} \text { per } \\
\mathrm{Bq} / \mathrm{m}^{3}\end{array}$ \\
\hline${ }^{69} \mathrm{Zn}$ & $3.8 E-08$ & $1.0 \mathrm{E}-05$ \\
\hline${ }^{76}$ As & $3.8 E-06$ & $1.0 \mathrm{E}-03$ \\
\hline${ }^{75} \mathrm{Se}$ & $\begin{array}{l}7.6 \mathrm{E}-06 \\
8.8 \mathrm{E}-06\end{array}$ & $\begin{array}{l}2.1 \mathrm{E}-03 \\
2.4 \mathrm{E}-03\end{array}$ \\
\hline $\begin{array}{l}{ }^{82} \mathrm{Br} \\
{ }^{83}{ }_{\mathrm{Br}+\mathrm{D}}(\mathrm{b}) \\
{ }^{83} \mathrm{Br} \\
{ }^{84} \mathrm{Br}\end{array}$ & $\begin{array}{l}1.1 \mathrm{E}-06 \\
4.5 \mathrm{E}-08 \\
8.9 \mathrm{E}-08 \\
9.4 \mathrm{E}-08\end{array}$ & $\begin{array}{l}3.0 \mathrm{E}-04 \\
1.2 \mathrm{E}-05 \\
2.4 \mathrm{E}-05 \\
2.5 \mathrm{E}-05\end{array}$ \\
\hline $\begin{array}{l}{ }^{86} \mathrm{Rb} \\
{ }^{88}{ }_{\mathrm{Rb}} \\
{ }^{89} \mathrm{Rb}+\mathrm{R} \\
{ }^{89} \mathrm{Rb}\end{array}$ & $\begin{array}{l}5.8 \mathrm{E}-06 \\
8.3 \mathrm{E}-08 \\
2.6 \mathrm{E}-06 \\
4.2 \mathrm{E}-08\end{array}$ & $\begin{array}{l}1.6 \mathrm{E}-03 \\
2.2 \mathrm{E}-05 \\
7.2 \mathrm{E}-04 \\
1.1 \mathrm{E}-05\end{array}$ \\
\hline $\begin{array}{l}{ }^{85} \mathrm{Sr} \\
{ }^{89} \mathrm{Sr} \\
{ }^{96} \mathrm{Sr+D} \\
{ }^{96} \mathrm{Sr} \\
{ }^{91} \mathrm{Sr} \quad(\mathrm{b}) \\
{ }^{91} \mathrm{Sr} \\
{ }^{92} \mathrm{Sr} \mathrm{Sr+D} \\
{ }^{92} \mathrm{Sr}\end{array}$ & $\begin{array}{l}1.4 \mathrm{E}-06 \\
5.3 \mathrm{E}-06 \\
1.0 \mathrm{E}-04 \\
2.0 \mathrm{E}-04 \\
1.9 \mathrm{E}-05 \\
8.6 \mathrm{E}-07 \\
7.0 \mathrm{E}-07 \\
5.9 \mathrm{E}-07\end{array}$ & $\begin{array}{l}3.8 \mathrm{E}-04 \\
1.4 \mathrm{E}-03 \\
2.8 \mathrm{E}-02 \\
5.4 \mathrm{E}-02 \\
5.0 \mathrm{E}-03 \\
2.3 \mathrm{E}-04 \\
1.9 \mathrm{E}-04 \\
1.6 \mathrm{E}-04\end{array}$ \\
\hline $\begin{array}{l}{ }^{96}{ }_{Y} \\
{ }^{91 n_{Y}} Y+D \\
{ }^{91 n_{Y}}(b) \\
{ }^{91} Y \\
{ }^{92} Y \\
{ }^{93} Y\end{array}$ & $\begin{array}{l}8.5 \mathrm{E}-06 \\
2.4 \mathrm{E}-05 \\
3.6 \mathrm{E}-08 \\
4.7 \mathrm{E}-05 \\
8.0 \mathrm{E}-07 \\
2.2 \mathrm{E}-06\end{array}$ & $\begin{array}{l}2.3 \mathrm{E}-03 \\
6.3 \mathrm{E}-03 \\
9.7 \mathrm{E}-06 \\
1.3 \mathrm{E}-02 \\
2.2 \mathrm{E}-04 \\
5.9 \mathrm{E}-04\end{array}$ \\
\hline $\begin{array}{l}{ }^{93} \mathrm{Zr+D}(\mathrm{b}) \\
{ }^{93} \mathrm{Zr} \\
{ }^{95} \mathrm{Zr+D}(\mathrm{b}) \\
{ }^{95} \mathrm{Zr} \\
{ }^{97} \mathrm{Zr+D} \\
{ }^{97} \mathrm{Zr}\end{array}$ & $\begin{array}{l}5.5 \mathrm{E}-05 \\
7.9 \mathrm{E}-05 \\
1.0 \mathrm{E}-05 \\
1.5 \mathrm{E}-05 \\
1.3 \mathrm{E}-06 \\
3.8 \mathrm{E}-06\end{array}$ & $\begin{array}{l}1.5 \mathrm{E}-02 \\
2.1 \mathrm{E}-02 \\
2.7 \mathrm{E}-03 \\
4.1 \mathrm{E}-03 \\
3.6 \mathrm{E}-04 \\
1.0 \mathrm{E}-03\end{array}$ \\
\hline $\begin{array}{l}{ }_{94}^{93} \mathrm{Nb} \\
{ }_{\mathrm{Nb}} \\
{ }_{95}{ }_{\mathrm{Nb}+\mathrm{D}}(\mathrm{b}) \\
{ }_{95}{ }_{\mathrm{Nb}}^{\mathrm{Nb}} \\
{ }_{97 \mathrm{Nb}} \mathrm{Nb+0}\end{array}$ & $\begin{array}{l}2.9 \mathrm{E}-05 \\
3.7 \mathrm{E}-04 \\
3.9 \mathrm{E}-06 \\
2.4 \mathrm{E}-06 \\
5.5 \mathrm{E}-06 \\
4.2 \mathrm{E}-08\end{array}$ & $\begin{array}{l}7.8 \mathrm{E}-03 \\
1.0 \mathrm{E}-01 \\
1.1 \mathrm{E}-03 \\
6.5 \mathrm{E}-04 \\
1.5 \mathrm{E}-03 \\
1.1 \mathrm{E}-05\end{array}$ \\
\hline
\end{tabular}


TABLE 2.2. Inhalation Committed Effective Dose Rate Conversion Factors for Exposure to Residual Radioactive Materials (contd)

\begin{tabular}{|c|c|c|}
\hline Radionuclide & $\begin{array}{c}\text { mrem } / \mathrm{h} \text { per } \\
\mathrm{pCi} / \mathrm{m}^{3} \\
\end{array}$ & $\begin{array}{c}\mu \mathrm{Sv} / \mathrm{h} \text { per } \\
\mathrm{Bq} / \mathrm{m}^{3}\end{array}$ \\
\hline $\begin{array}{l}97 m \\
{ }^{97} \\
\mathrm{Nb}\end{array}$ & $\begin{array}{l}1.2 \mathrm{E}-09 \\
8.3 \mathrm{E}-08\end{array}$ & $\begin{array}{l}3.2 \mathrm{E}-07 \\
2.2 \mathrm{E}-05\end{array}$ \\
\hline $\begin{array}{l}{ }^{93} \mathrm{Mo}_{\mathrm{D}}(\mathrm{b}) \\
{ }^{93}{ }_{\mathrm{Mo}}(\mathrm{b}) \\
{ }^{99} \mathrm{Mo+D} \\
{ }^{99}{ }_{\mathrm{Mo}}\end{array}$ & $\begin{array}{l}1.5 \mathrm{E}-05 \\
9.5 \mathrm{E}-07 \\
1.1 \mathrm{E}-06 \\
1.9 \mathrm{E}-06\end{array}$ & $\begin{array}{l}4.1 \mathrm{E}-03 \\
2.6 \mathrm{E}-04 \\
2.9 \mathrm{E}-04 \\
5.1 \mathrm{E}-04\end{array}$ \\
\hline $\begin{array}{l}{ }^{99 m} \mathrm{Tc} \\
{ }^{99} \mathrm{Tc} \\
{ }^{101} \mathrm{Tc}\end{array}$ & $\begin{array}{l}3.1 \mathrm{E}-08 \\
8.9 \mathrm{E}-06 \\
1.5 \mathrm{E}-08\end{array}$ & $\begin{array}{l}8.4 \mathrm{E}-06 \\
2.4 \mathrm{E}-03 \\
4.0 \mathrm{E}-06\end{array}$ \\
\hline $\begin{array}{l}{ }_{{ }^{103} \mathrm{Ru}+\mathrm{D}}(\mathrm{b}) \\
{ }_{103} \mathrm{Ru} \\
{ }^{105} \mathrm{Ru} \text { (b) } \\
{ }^{105} \mathrm{Ru} \text { (b) }(c) \\
{ }^{106} \mathrm{Ru+I}(\mathrm{c})\end{array}$ & $\begin{array}{l}4.4 \mathrm{E}-06 \\
8.7 \mathrm{E}-06 \\
5.0 \mathrm{E}-07 \\
5.2 \mathrm{E}-07 \\
2.2 \mathrm{E}-04\end{array}$ & $\begin{array}{l}1.2 \mathrm{E}-03 \\
2.3 \mathrm{E}-03 \\
1.3 \mathrm{E}-04 \\
1.4 \mathrm{E}-04 \\
6.1 \mathrm{E}-02\end{array}$ \\
\hline $\begin{array}{l}{ }^{103 m} \mathrm{Rh} \\
{ }^{105} \mathrm{Rh}\end{array}$ & $\begin{array}{l}4.8 \mathrm{E}-09 \\
9.6 \mathrm{E}-07\end{array}$ & $\begin{array}{l}\text { 1.3E-06 } \\
2.6 \mathrm{E}-04\end{array}$ \\
\hline $\begin{array}{l}{ }^{103} \mathrm{Pd}+\mathrm{D} \\
{ }_{103} \mathrm{Pd} \\
{ }_{107} \mathrm{Pd} \\
{ }_{109} \mathrm{Pd}+\mathrm{I}(\mathrm{c})\end{array}$ & $\begin{array}{l}7.5 \mathrm{E}-07 \\
1.5 \mathrm{E}-06 \\
1.2 \mathrm{E}-05 \\
6.5 \mathrm{E}-07\end{array}$ & $\begin{array}{l}2.0 \mathrm{E}-04 \\
4.1 \mathrm{E}-04 \\
3.2 \mathrm{E}-03 \\
1.8 \mathrm{E}-04\end{array}$ \\
\hline${ }^{11 \theta_{\mathrm{m}}} \mathrm{Ag}+\mathrm{I}(\mathrm{C})$ & $\begin{array}{l}4.1 E-05 \\
3.3 E-06\end{array}$ & $\begin{array}{l}1.1 E-02 \\
8.9 E-04\end{array}$ \\
\hline $\begin{array}{l}{ }^{169} \mathrm{Cd} \\
{ }_{113{ }^{m}} \mathrm{Cd} \\
{ }_{115 m} \mathrm{Cd} \\
{ }^{115} \mathrm{Cd}+\mathrm{D} \\
\mathrm{Cd}\end{array}$ & $\begin{array}{l}1.1 \mathrm{E}-04 \\
1.4 \mathrm{E}-03 \\
3.5 \mathrm{E}-05 \\
6.9 \mathrm{E}-05 \\
3.9 \mathrm{E}-06\end{array}$ & $\begin{array}{l}3.0 \mathrm{E}-02 \\
3.8 \mathrm{E}-01 \\
9.3 \mathrm{E}-03 \\
1.9 \mathrm{E}-02 \\
1.1 \mathrm{E}-03\end{array}$ \\
\hline $\begin{array}{l}{ }^{111} \operatorname{In}_{\operatorname{In}}(\mathrm{In}+\mathrm{I} \\
{ }_{115 \mathrm{~m}}(\mathrm{In}\end{array}$ & $\begin{array}{l}5.4 \mathrm{E}-07 \\
2.2 \mathrm{E}-05 \\
1.2 \mathrm{E}-07\end{array}$ & $\begin{array}{l}1.5 \mathrm{E}-04 \\
6.1 \mathrm{E}-03 \\
3.2 \mathrm{E}-05\end{array}$ \\
\hline $\begin{array}{l}{ }^{117 m} \mathrm{Sn} \\
{ }^{119 m} \mathrm{Sn} \\
{ }^{121 m} \mathrm{Sn+D} \\
{ }^{121 m} \mathrm{Sn} \\
{ }^{121} \mathrm{Sn} \\
{ }^{123} \mathrm{Sn} \\
{ }^{125} \mathrm{Sn} \mathrm{Sn}_{\mathrm{D}}(\mathrm{b})\end{array}$ & $\begin{array}{l}4.2 E-06 \\
5.8 E-06 \\
6.2 E-06 \\
1.1 E-05 \\
4.8 E-07 \\
3.1 E-05 \\
1.3 E-05\end{array}$ & $\begin{array}{l}1.1 \mathrm{E}-03 \\
1.6 \mathrm{E}-03 \\
1.7 \mathrm{E}-03 \\
3.0 \mathrm{E}-03 \\
1.3 \mathrm{E}-04 \\
8.4 \mathrm{E}-03 \\
3.5 \mathrm{E}-03\end{array}$ \\
\hline
\end{tabular}


TABLE 2.2. Inhalation Committed Effective Dose Rate Conversion Factors for Exposure to Residual Radioactive Materials (contd)

\begin{tabular}{|c|c|c|}
\hline Radionuclide & $\begin{array}{c}\text { mrem } / \mathrm{h} \text { per } \\
\mathrm{pCi} / \mathrm{m}^{3} \\
\end{array}$ & $\begin{array}{c}\mu \mathrm{Sv} / \mathrm{h} \text { per } \\
\mathrm{Bq} / \mathrm{m}^{3} \\
\end{array}$ \\
\hline 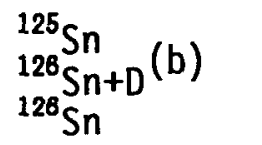 & $\begin{array}{l}1.5 E-05 \\
4.5 E-05 \\
9.4 E-05\end{array}$ & $\begin{array}{l}4.1 \mathrm{E}-03 \\
1.2 \mathrm{E}-02 \\
2.5 \mathrm{E}-02\end{array}$ \\
\hline $\begin{array}{l}{ }^{124} \mathrm{Sb} \\
{ }^{125} \mathrm{Sb}+\mathrm{D} \\
{ }^{125} \mathrm{Sb} \\
{ }^{126}{ }_{\mathrm{Sb}} \mathrm{Sb}+\mathrm{D} \\
{ }^{126} \mathrm{Sb} \\
{ }^{127} \mathrm{Sb} \\
{ }_{\mathrm{Sb}}(\mathrm{b} b\end{array}$ & $\begin{array}{l}2.5 \mathrm{E}-05 \\
1.1 \mathrm{E}-05 \\
1.2 \mathrm{E}-05 \\
1.5 \mathrm{E}-06 \\
2.9 \mathrm{E}-08 \\
1.1 \mathrm{E}-05 \\
4.4 \mathrm{E}-06 \\
5.8 \mathrm{E}-06\end{array}$ & $\begin{array}{l}6.8 \mathrm{E}-03 \\
2.9 \mathrm{E}-03 \\
3.2 \mathrm{E}-03 \\
4.0 \mathrm{E}-04 \\
7.8 \mathrm{E}-06 \\
3.0 \mathrm{E}-03 \\
1.2 \mathrm{E}-03 \\
1.6 \mathrm{E}-03\end{array}$ \\
\hline 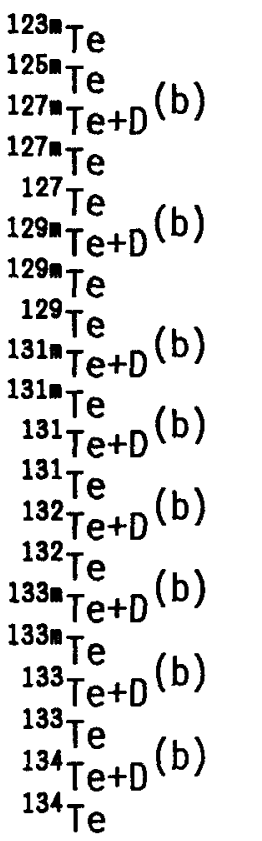 & $\begin{array}{l}1.0 \mathrm{E}-05 \\
7.0 \mathrm{E}-06 \\
1.1 \mathrm{E}-05 \\
2.1 \mathrm{E}-05 \\
3.2 \mathrm{E}-07 \\
2.9 \mathrm{E}-04 \\
4.8 \mathrm{E}-04 \\
1.5 \mathrm{E}-07 \\
1.9 \mathrm{E}-05 \\
1.2 \mathrm{E}-05 \\
1.5 \mathrm{E}-05 \\
1.2 \mathrm{E}-07 \\
6.5 \mathrm{E}-06 \\
1.2 \mathrm{E}-05 \\
1.8 \mathrm{E}-06 \\
4.3 \mathrm{E}-07 \\
1.8 \mathrm{E}-06 \\
8.7 \mathrm{E}-08 \\
1.2 \mathrm{E}-07 \\
1.0 \mathrm{E}-07\end{array}$ & $\begin{array}{l}2.7 \mathrm{E}-03 \\
1.9 \mathrm{E}-03 \\
2.9 \mathrm{E}-03 \\
5.7 \mathrm{E}-03 \\
8.6 \mathrm{E}-05 \\
8.0 \mathrm{E}-02 \\
1.3 \mathrm{E}-01 \\
4.1 \mathrm{E}-05 \\
5.1 \mathrm{E}-03 \\
3.2 \mathrm{E}-03 \\
4.0 \mathrm{E}-03 \\
3.2 \mathrm{E}-05 \\
1.8 \mathrm{E}-03 \\
3.2 \mathrm{E}-03 \\
5.0 \mathrm{E}-04 \\
1.2 \mathrm{E}-04 \\
4.8 \mathrm{E}-04 \\
2.3 \mathrm{E}-05 \\
3.2 \mathrm{E}-05 \\
2.7 \mathrm{E}-05\end{array}$ \\
\hline $\begin{array}{l}{ }^{125} \mathrm{I} \\
{ }^{129} \mathrm{I} \\
{ }^{130} \mathrm{I} \\
{ }_{131} \mathrm{I}+\mathrm{D}^{(\mathrm{b})} \\
{ }^{132} \mathrm{I} \\
133 \mathrm{I}+\mathrm{D}^{(\mathrm{b})} \\
{ }^{133} \mathrm{I} \\
{ }^{134} \mathrm{I} \\
{ }_{135} \mathrm{I}+\mathrm{D}^{(\mathrm{b})}\end{array}$ & $\begin{array}{l}2.2 \mathrm{E}-05 \\
1.5 \mathrm{E}-04 \\
2.4 \mathrm{E}-06 \\
3.0 \mathrm{E}-05 \\
3.0 \mathrm{E}-05 \\
3.8 \mathrm{E}-07 \\
2.6 \mathrm{E}-06 \\
5.3 \mathrm{E}-06 \\
1.4 \mathrm{E}-07 \\
5.1 \mathrm{E}-07 \\
1.1 \mathrm{E}-06\end{array}$ & $\begin{array}{l}5.9 \mathrm{E}-03 \\
4.1 \mathrm{E}-02 \\
6.5 \mathrm{E}-04 \\
8.0 \mathrm{E}-03 \\
8.1 \mathrm{E}-03 \\
1.0 \mathrm{E}-04 \\
7.1 \mathrm{E}-04 \\
1.4 \mathrm{E}-03 \\
3.8 \mathrm{E}-05 \\
1.4 \mathrm{E}-04 \\
3.0 \mathrm{E}-04\end{array}$ \\
\hline
\end{tabular}


TABLE 2.2. Inhalation Committed Effective Dose Rate Conversion Factors for Exposure to Residual Radioactive Materials (contd)

\begin{tabular}{|c|c|c|}
\hline Radionuclide & $\begin{array}{c}\text { mrem } / \mathrm{h} \text { per } \\
\mathrm{pCi} / \mathrm{m}^{3} \\
\end{array}$ & $\begin{array}{c}\mu \mathrm{Sv} / \mathrm{h} \text { per } \\
\mathrm{Bq} / \mathrm{m}^{3}\end{array}$ \\
\hline $\begin{array}{l}{ }^{134 m} \mathrm{CS}+\mathrm{D}(\mathrm{b}) \\
{ }^{134} \mathrm{CS} \\
{ }^{134} \mathrm{Cs} \\
{ }^{135} \mathrm{Cs} \\
{ }^{136} \mathrm{Cs} \\
{ }^{137} \mathrm{Cs} \\
{ }^{138} \mathrm{Cs}+\mathrm{I} \\
\mathrm{Cs}\end{array}$ & $\begin{array}{l}1.8 \mathrm{E}-08 \\
3.6 \mathrm{E}-08 \\
4.0 \mathrm{E}-05 \\
3.6 \mathrm{E}-06 \\
6.4 \mathrm{E}-06 \\
1.3 \mathrm{E}-05 \\
9.6 \mathrm{E}-08\end{array}$ & $\begin{array}{l}4.9 \mathrm{E}-06 \\
9.7 \mathrm{E}-06 \\
1.1 \mathrm{E}-02 \\
9.7 \mathrm{E}-04 \\
1.7 \mathrm{E}-03 \\
3.6 \mathrm{E}-03 \\
2.6 \mathrm{E}-05\end{array}$ \\
\hline $\begin{array}{l}{ }^{139} \mathrm{Ba} \\
{ }^{146} \mathrm{Ba}+\mathrm{D} \\
{ }^{148} \mathrm{Ba} \\
\left.{ }^{141} \mathrm{Ba}+\mathrm{b}\right) \\
{ }^{141} \mathrm{Ba} \\
\left.{ }^{142} \mathrm{Ba}+\mathrm{b}\right) \\
{ }^{142} \mathrm{Ba}\end{array}$ & $\begin{array}{l}1.7 \mathrm{E}-07 \\
3.2 \mathrm{E}-06 \\
2.9 \mathrm{E}-06 \\
3.0 \mathrm{E}-06 \\
7.8 \mathrm{E}-08 \\
1.5 \mathrm{E}-07 \\
3.9 \mathrm{E}-08\end{array}$ & $\begin{array}{l}4.6 \mathrm{E}-05 \\
8.6 \mathrm{E}-04 \\
7.8 \mathrm{E}-04 \\
8.1 \mathrm{E}-04 \\
2.1 \mathrm{E}-05 \\
4.1 \mathrm{E}-05 \\
1.1 \mathrm{E}-05\end{array}$ \\
\hline $\begin{array}{l}{ }^{146} \mathrm{La} \\
{ }^{141} \mathrm{La}+\mathrm{D} \\
{ }^{141} \mathrm{La} \\
{ }^{142} \mathrm{La} \\
{ }^{2} \mathrm{ba}\end{array}$ & $\begin{array}{l}3.4 \mathrm{E}-06 \\
4.5 \mathrm{E}-06 \\
5.8 \mathrm{E}-07 \\
2.6 \mathrm{E}-07\end{array}$ & $\begin{array}{l}9.2 \mathrm{E}-04 \\
1.2 \mathrm{E}-03 \\
1.6 \mathrm{E}-04 \\
7.0 \mathrm{E}-05\end{array}$ \\
\hline $\begin{array}{l}{ }^{141} \mathrm{Ce} \\
{ }^{143} \mathrm{Ce}+\mathrm{D}^{(b)} \\
{ }^{144} \mathrm{Ce} \\
{ }^{144} \mathrm{Ce}+\mathrm{D}^{(b)}\end{array}$ & $\begin{array}{l}8.4 \mathrm{E}-06 \\
5.5 \mathrm{E}-06 \\
3.4 \mathrm{E}-06 \\
1.8 \mathrm{E}-04 \\
3.6 \mathrm{E}-04\end{array}$ & $\begin{array}{l}2.3 \mathrm{E}-03 \\
1.5 \mathrm{E}-03 \\
9.2 \mathrm{E}-04 \\
4.8 \mathrm{E}-02 \\
9.7 \mathrm{E}-02\end{array}$ \\
\hline $\begin{array}{l}{ }^{143} \mathrm{Pr} \\
{ }^{144 m} \mathrm{Pr}+\mathrm{D}\end{array}$ (b) & $\begin{array}{l}7.8 \mathrm{E}-06 \\
3.1 \mathrm{E}-08 \\
1.8 \mathrm{E}-08 \\
4.3 \mathrm{E}-08\end{array}$ & $\begin{array}{l}2.1 \mathrm{E}-03 \\
8.4 \mathrm{E}-06 \\
4.9 \mathrm{E}-06 \\
1.2 \mathrm{E}-05\end{array}$ \\
\hline${ }^{147} \mathrm{Nd}+\mathrm{D}(\mathrm{b})$ & $\begin{array}{l}2.2 \mathrm{E}-05 \\
6.5 \mathrm{E}-06\end{array}$ & $\begin{array}{l}5.9 \mathrm{E}-03 \\
1.8 \mathrm{E}-03\end{array}$ \\
\hline $\begin{array}{l}{ }_{147}^{148 m} \mathrm{Pm} \\
{ }_{148}{ }_{\mathrm{Pm}+\mathrm{D}}\end{array}$ & $\begin{array}{l}3.8 \mathrm{E}-05 \\
2.1 \mathrm{E}-05 \\
2.2 \mathrm{E}-05 \\
1.1 \mathrm{E}-05 \\
2.9 \mathrm{E}-06 \\
1.8 \mathrm{E}-06\end{array}$ & $\begin{array}{l}1.0 \mathrm{E}-02 \\
5.7 \mathrm{E}-03 \\
5.9 \mathrm{E}-03 \\
3.0 \mathrm{E}-03 \\
7.8 \mathrm{E}-04 \\
4.9 \mathrm{E}-04\end{array}$ \\
\hline $\begin{array}{l}{ }^{147} \mathrm{Sm} \\
{ }^{151} \mathrm{Sm} \\
{ }^{153} \mathrm{Sm}\end{array}$ & $\begin{array}{l}7.3 E-02 \\
2.9 E-05 \\
1.9 E-06\end{array}$ & $\begin{array}{l}2.0 \mathrm{E}+01 \\
7.8 \mathrm{E}-03 \\
5.1 \mathrm{E}-04\end{array}$ \\
\hline
\end{tabular}


TABLE 2.2. Inhalation Committed Effective Dose Rate Conversion Factors for Exposure to Residual Radioactive Materials (contd)

\begin{tabular}{|c|c|c|}
\hline Radionuclide & $\begin{array}{l}\mathrm{mrem} / \mathrm{h} \text { per } \\
\mathrm{pCi} / \mathrm{m}^{3}\end{array}$ & $\begin{array}{c}\mu \mathrm{Sv} / \mathrm{h} \text { per } \\
\mathrm{Bg} / \mathrm{m}^{3}\end{array}$ \\
\hline $\begin{array}{l}{ }^{152} \mathrm{Eu} \\
154 \mathrm{Eu} \\
156 \mathrm{Eu} \\
{ }^{156} \mathrm{Eu}\end{array}$ & $\begin{array}{l}2.0 \mathrm{E}-04 \\
2.6 \mathrm{E}-04 \\
3.9 \mathrm{E}-05 \\
1.4 \mathrm{E}-05\end{array}$ & $\begin{array}{l}5.4 \mathrm{E}-02 \\
7.0 \mathrm{E}-02 \\
1.1 \mathrm{E}-02 \\
3.8 \mathrm{E}-03\end{array}$ \\
\hline${ }^{153} \mathrm{Gd}$ & $2.3 E-05$ & $6.2 E-03$ \\
\hline${ }^{168} \mathrm{~Tb}$ & $2.4 \mathrm{E}-05$ & $6.5 E-03$ \\
\hline${ }^{166 m} \mathrm{HO}$ & $7.2 E-04$ & $1.9 \mathrm{E}-01$ \\
\hline $\begin{array}{l}181 \mathrm{~W} \\
185 \mathrm{~W} \\
187 \mathrm{~W}\end{array}$ & $\begin{array}{l}1.5 \mathrm{E}-07 \\
7.5 \mathrm{E}-07 \\
6.5 \mathrm{E}-07\end{array}$ & $\begin{array}{l}4.1 E-05 \\
2.0 E-04 \\
1.8 E-04\end{array}$ \\
\hline${ }^{187} \mathrm{Re}$ & $5.6 E-08$ & $1.5 E-05$ \\
\hline $\begin{array}{l}{ }^{185} 0 \mathrm{~s} \\
{ }^{191} \mathrm{Os}\end{array}$ & $\begin{array}{l}9.2 \mathrm{E}-06 \\
2.2 \mathrm{E}-06\end{array}$ & $\begin{array}{l}2.5 \mathrm{E}-03 \\
5.9 \mathrm{E}-04\end{array}$ \\
\hline${ }^{192} \mathrm{Ir}$ & $2.6 \mathrm{E}-05$ & $7.0 \mathrm{E}-03$ \\
\hline${ }^{263} \mathrm{Hg}$ & $6.8 \mathrm{E}-06$ & $1.8 \mathrm{E}-03$ \\
\hline $\begin{array}{l}{ }^{216} \mathrm{~Pb}+\mathrm{D} \\
{ }^{210} \mathrm{~Pb} \\
{ }^{212} \mathrm{~Pb} \\
{ }^{212} \mathrm{~Pb}+\mathrm{D} \\
{ }^{212} \mathrm{~Pb}\end{array}$ & $\begin{array}{l}7.3 \mathrm{E}-03 \\
1.3 \mathrm{E}-02 \\
6.0 \mathrm{E}-05 \\
1.6 \mathrm{E}-04\end{array}$ & $\begin{array}{l}2.0 \mathrm{E}+00 \\
3.5 \mathrm{E}+00 \\
1.6 \mathrm{E}-02 \\
4.3 \mathrm{E}-02\end{array}$ \\
\hline $\begin{array}{l}{ }^{218} \mathrm{Bi}+\mathrm{D}(\mathrm{b}) \\
{ }^{2111} \mathrm{Bi} \\
{ }^{218} \mathrm{Bi}+\mathrm{I}\end{array}$ & $\begin{array}{l}4.3 \mathrm{E}-03 \\
1.9 \mathrm{E}-04 \\
9.0 \mathrm{E}-06\end{array}$ & $\begin{array}{l}1.2 \mathrm{E}+00 \\
5.1 \mathrm{E}-02 \\
2.4 \mathrm{E}-03\end{array}$ \\
\hline${ }^{216} \mathrm{Po}$ & $8.4 \mathrm{E}-03$ & $2.3 E+00$ \\
\hline 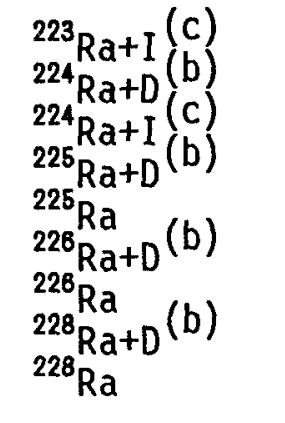 & $\begin{array}{l}1.2 \mathrm{E}-03 \\
4.8 \mathrm{E}-04 \\
2.7 \mathrm{E}-03 \\
2.1 \mathrm{E}-03 \\
7.1 \mathrm{E}-03 \\
3.2 \mathrm{E}-03 \\
7.9 \mathrm{E}-03 \\
3.6 \mathrm{E}-02 \\
3.7 \mathrm{E}-03\end{array}$ & $\begin{array}{l}3.4 \mathrm{E}-01 \\
1.3 \mathrm{E}-01 \\
7.3 \mathrm{E}-01 \\
5.8 \mathrm{E}-01 \\
1.9 \mathrm{E}+00 \\
8.7 \mathrm{E}-01 \\
2.1 \mathrm{E}+00 \\
9.6 \mathrm{E}+00 \\
1.0 \mathrm{E}+00\end{array}$ \\
\hline${ }^{225} \mathrm{AC}+\mathrm{I}^{(\mathrm{c})}$ & $1.3 \mathrm{E}-03$ & $3.6 \mathrm{E}-01$ \\
\hline
\end{tabular}


TABLE 2.2. Inhalation Committed Effective Dose Rate Conversion Factors for Exposure to Residual Radioactive Materials (contd)

\begin{tabular}{|c|c|c|}
\hline Radionuclide & $\begin{array}{l}\text { mrem/h per } \\
\mathrm{pCi} / \mathrm{m}^{3} \\
\end{array}$ & $\begin{array}{c}\mu \mathrm{Sv} / \mathrm{h} \text { per } \\
\mathrm{Bq} / \mathrm{m}^{3} \\
\end{array}$ \\
\hline $\begin{array}{l}{ }^{227} \mathrm{AC}+\mathrm{D}(\mathrm{b}) \\
{ }^{227} \mathrm{AC} \\
{ }^{228} \mathrm{AC}+\mathrm{D} \\
{ }^{228} \mathrm{AC}\end{array}$ & $\begin{array}{l}1.6 \mathrm{E}-01 \\
1.3 \mathrm{E}+00 \\
4.0 \mathrm{E}-02 \\
1.1 \mathrm{E}-04\end{array}$ & $\begin{array}{l}4.4 E+01 \\
3.5 E+02 \\
1.1 E+01 \\
3.0 E-02\end{array}$ \\
\hline 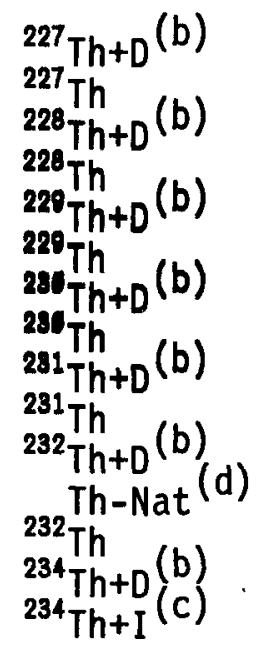 & $\begin{array}{l}3.3 \mathrm{E}-03 \\
1.6 \mathrm{E}-02 \\
4.6 \mathrm{E}-02 \\
3.1 \mathrm{E}-01 \\
2.1 \mathrm{E}-01 \\
1.7 \mathrm{E}+00 \\
2.8 \mathrm{E}-02 \\
2.5 \mathrm{E}-01 \\
8.1 \mathrm{E}-08 \\
8.1 \mathrm{E}-07 \\
1.4 \mathrm{E}-01 \\
1.4 \mathrm{E}-01 \\
1.1 \mathrm{E}+00 \\
1.7 \mathrm{E}-05 \\
3.4 \mathrm{E}-05\end{array}$ & $\begin{array}{l}8.9 E-01 \\
4.3 E+00 \\
1.2 E+01 \\
8.4 E+01 \\
5.7 E+01 \\
4.6 E+02 \\
7.6 E+00 \\
6.8 E+01 \\
2.2 E-05 \\
2.2 E-04 \\
3.8 E+01 \\
3.8 E+01 \\
3.0 E+02 \\
4.6 E-03 \\
9.2 E-03\end{array}$ \\
\hline $\begin{array}{l}{ }^{231} \mathrm{~Pa}+\mathrm{D} \\
{ }^{231} \mathrm{~Pa} \\
{ }^{233} \mathrm{~Pa} \\
{ }^{234} \mathrm{~Pa} \\
{ }^{\mathrm{Pa}}\end{array}$ & $\begin{array}{l}2.3 \mathrm{E}-01 \\
8.4 \mathrm{E}-01 \\
9.2 \mathrm{E}-06 \\
8.2 \mathrm{E}-07\end{array}$ & $\begin{array}{l}6.3 \mathrm{E}+01 \\
2.3 \mathrm{E}+02 \\
2.5 \mathrm{E}-03 \\
2.2 \mathrm{E}-04\end{array}$ \\
\hline 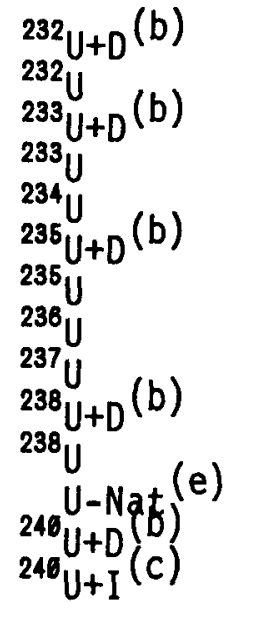 & $\begin{array}{l}8.6 \mathrm{E}-02 \\
6.0 \mathrm{E}-01 \\
2.0 \mathrm{E}-01 \\
1.3 \mathrm{E}-01 \\
1.3 \mathrm{E}-01 \\
6.0 \mathrm{E}-02 \\
1.2 \mathrm{E}-01 \\
1.2 \mathrm{E}-01 \\
3.5 \mathrm{E}-06 \\
3.7 \mathrm{E}-02 \\
1.1 \mathrm{E}-01 \\
1.2 \mathrm{E}-01 \\
7.3 \mathrm{E}-07 \\
2.2 \mathrm{E}-06\end{array}$ & $\begin{array}{l}2.3 \mathrm{E}+01 \\
1.6 \mathrm{E}+02 \\
5.4 \mathrm{E}+01 \\
3.5 \mathrm{E}+01 \\
3.5 \mathrm{E}+01 \\
1.6 \mathrm{E}+01 \\
3.2 \mathrm{E}+01 \\
3.2 \mathrm{E}+01 \\
9.5 \mathrm{E}-04 \\
9.9 \mathrm{E}+00 \\
3.0 \mathrm{E}+01 \\
3.2 \mathrm{E}+01 \\
2.0 \mathrm{E}-04 \\
5.9 \mathrm{E}-04\end{array}$ \\
\hline $\begin{array}{l}{ }^{237} \mathrm{~Np}+\mathrm{D}(\mathrm{b}) \\
{ }^{237} \mathrm{~Np} \\
{ }^{238} \mathrm{~Np}+\mathrm{D} \\
{ }^{238} \mathrm{~Np} \\
{ }^{238} \mathrm{~Np}+\mathrm{D} \\
\mathrm{Np}\end{array}$ & $\begin{array}{l}3.1 \mathrm{E}-01 \\
6.1 \mathrm{E}-01 \\
1.4 \mathrm{E}-01 \\
4.0 \mathrm{E}-05 \\
1.2 \mathrm{E}-06\end{array}$ & $\begin{array}{l}8.2 E+01 \\
1.6 E+02 \\
3.8 E+01 \\
1.1 E-02 \\
3.2 E-04\end{array}$ \\
\hline
\end{tabular}


TABLE 2.2. Inhalation Committed Effective Dose Rate Conversion Factors for Exposure to Residual Radioactive Materials (contd)

\begin{tabular}{|c|c|c|}
\hline Radionuclide & $\begin{array}{l}\mathrm{mrem} / \mathrm{h} \text { per } \\
\mathrm{pCi} / \mathrm{m}^{3}\end{array}$ & $\begin{array}{c}\mu \mathrm{Sv} / \mathrm{h} \text { per } \\
\mathrm{Bg} / \mathrm{m}^{3}\end{array}$ \\
\hline${ }^{239} \mathrm{~Np}$ & $2.4 \mathrm{E}-06$ & $6.5 \mathrm{E}-04$ \\
\hline 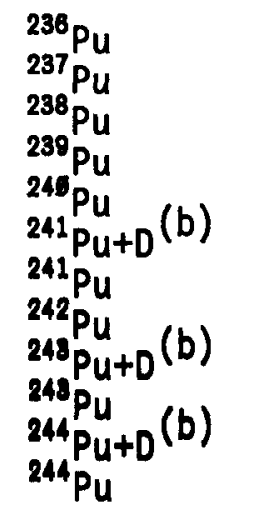 & $\begin{array}{l}7.2 \mathrm{E}-02 \\
1.8 \mathrm{E}-06 \\
2.8 \mathrm{E}-01 \\
2.9 \mathrm{E}-01 \\
2.9 \mathrm{E}-01 \\
2.1 \mathrm{E}-01 \\
4.7 \mathrm{E}-03 \\
2.7 \mathrm{E}-01 \\
7.0 \mathrm{E}-08 \\
1.4 \mathrm{E}-07 \\
6.5 \mathrm{E}-02 \\
2.6 \mathrm{E}-01\end{array}$ & $\begin{array}{l}1.9 \mathrm{E}+01 \\
4.9 \mathrm{E}-04 \\
7.6 \mathrm{E}+01 \\
7.8 \mathrm{E}+01 \\
7.8 \mathrm{E}+01 \\
5.8 \mathrm{E}+01 \\
1.3 \mathrm{E}+00 \\
7.3 \mathrm{E}+01 \\
1.9 \mathrm{E}-05 \\
3.8 \mathrm{E}=05 \\
1.8 \mathrm{E}+01 \\
7.0 \mathrm{E}+01\end{array}$ \\
\hline $\begin{array}{l}{ }^{241} \mathrm{Am} \\
{ }^{2424} \mathrm{Am}+\mathrm{D} \\
{ }^{242 \pi} \mathrm{Am} \\
{ }^{242} \mathrm{Am}+\mathrm{D} \\
{ }^{242} \mathrm{Bm} \\
{ }^{243} \mathrm{Am}+\mathrm{D} \\
{ }^{243} \mathrm{Am}\end{array}$ & $\begin{array}{l}4.3 \mathrm{E}-01 \\
1.6 \mathrm{E}-01 \\
3.9 \mathrm{E}-01 \\
8.5 \mathrm{E}-02 \\
5.2 \mathrm{E}-05 \\
1.4 \mathrm{E}-01 \\
4.1 \mathrm{E}-01\end{array}$ & $\begin{array}{l}1.2 E+02 \\
4.4 E+01 \\
1.1 E+02 \\
2.3 E+01 \\
1.4 E-02 \\
3.7 E+01 \\
1.1 E+02\end{array}$ \\
\hline $\begin{array}{l}{ }^{242} \mathrm{Cm}+\mathrm{D} \\
{ }^{242} \mathrm{Cm} \\
{ }^{243} \mathrm{Cm} \\
{ }^{244} \mathrm{Cm}+\mathrm{D} \\
{ }^{244} \mathrm{Cm} \\
{ }^{246} \mathrm{Cm}+\mathrm{D} \\
{ }^{245} \mathrm{Cm} \\
{ }^{246} \mathrm{Cm} \\
{ }^{247} \mathrm{Cm} \text { (b) } \\
{ }^{247} \mathrm{Cm} \\
{ }^{248} \mathrm{Cm}\end{array}$ & $\begin{array}{l}1.6 \mathrm{E}-01 \\
1.5 \mathrm{E}-02 \\
5.8 \mathrm{E}-01 \\
1.2 \mathrm{E}-01 \\
2.3 \mathrm{E}-01 \\
2.8 \mathrm{E}-01 \\
4.2 \mathrm{E}-01 \\
4.2 \mathrm{E}-01 \\
2.0 \mathrm{E}-01 \\
3.9 \mathrm{E}-01 \\
1.5 \mathrm{E}+00\end{array}$ & $\begin{array}{l}4.3 \mathrm{E}+01 \\
4.0 \mathrm{E}+00 \\
1.6 \mathrm{E}+02 \\
3.1 \mathrm{E}+01 \\
6.2 \mathrm{E}+01 \\
7.7 \mathrm{E}+01 \\
1.1 \mathrm{E}+02 \\
1.1 \mathrm{E}+02 \\
5.3 \mathrm{E}+01 \\
1.1 \mathrm{E}+02 \\
4.0 \mathrm{E}+02\end{array}$ \\
\hline${ }^{252} \mathrm{Cf}$ & $1.3 \mathrm{E}-01$ & $3.5 E+01$ \\
\hline
\end{tabular}

(a) Includes a $50 \%$ contribution from skin absorption, as recommended by the ICRP (1979-1988).

(b) Where $+D$ means "plus daughters in equilibrium" referring to explicit daughters, or daughters with half-lives greater than 10 minutes. Separate values are also included for parents and daughters so that combined factors for non-equilibrium cases can be determined. Note: in some cases $+D$ also includes implicit daughters (i.e., uranium and thorium decay chains). 
TABLE 2.2. Inhalation Committed Effective Dose Rate Conversion Factors for Exposure to Residual Radioactive Materials (contd)

(c) Where +I means "plus implicit daughters in equilibrium" referring to daughters with half-lives less than 10 minutes. The decay energies associated with these daughters have been assigned to the parent, and the appropriate branching ratios (described in Appendix D) have been considered.

(d) Where Th-Nat includes an equilibrium mixture of ${ }^{232}$ Th plus 10 daughters in the decay chain, as discussed in Appendix $D$.

(e) Where $1 \mathrm{Ci} U$-Nat equals $48.9 \%{ }^{238} \mathrm{U}$ plus $48.9 \%{ }^{234} \mathrm{U}$ plus $2.25 \%{ }^{235} \mathrm{U}$. 


\subsubsection{Secondary Ingestion}

Ingestion of removable radioactive contamination found on facility surfaces can occur when the contamination is transferred from a surface to hands, foodstuffs, cigarettes, or other items that enter the mouth. These pathways of potential ingestion are referred to as secondary ingestion pathways.

The general equation for estimating the committed dose rate by secondary ingestion received by the individuals defined in the exposure scenarios for radionuclide $i$ is:

$$
H_{I N G, i}=\left(\begin{array}{ll}
I & t
\end{array}\right) C_{I N G, i} D F_{I N G, i}
$$

where $H_{I N G, i}=$ the committed effective dose equivalent from a $1-y r$ intake of radionuclide $i$ by ingestion, in mrem $(\mu S v)$ per year of exposure

$I=$ the rate of secondary ingestion of removable surface contamination, in $\mathrm{g} / \mathrm{h}$

$t=$ the duration of exposure for the individual, in $h / y r$ $C_{I N G, i}=\begin{aligned} & \text { the concentration of radionuclide } i \text { in the removable dust, } \\ & \text { in } \mathrm{pC} i / g(\mathrm{~Bq} / \mathrm{g})\end{aligned}$

$\begin{aligned} & D F_{I N G, i}= \text { the committed effective dose equivalent rate from ingestion } \\ & \text { of radionuclide } i \text {, in } \mathrm{mrem} / \mathrm{pC} i(\mu \mathrm{Sv} / \mathrm{Bq}) \text {. }\end{aligned}$

The term in parentheses in Equation 2.3 is equivalent to the usage parameter defined in Equation 2.1. Potential values for the ingestion rate, I, from literature sources are discussed in Appendix B. Ingestion committed effective dose conversion factors are shown in Table 2.3. These factors apply to both secondary ingestion and ingestion of drinking water and are in units of mrem per $\mathrm{pCi}$ ingested and $\mu \mathrm{Sv}$ per $\mathrm{Bq}$ ingested. These factors can be multiplied by the exposure duration $(h)$ and secondary ingestion rate in $\mathrm{pCi} / \mathrm{h}$ or $\mathrm{Bq} / \mathrm{h}$ to obtain scenario-specific estimates of the committed effective dose equivalent rate from the secondary ingestion pathway.

A comparison of the ingestion committed effective dose conversion factors for selected radionuclides used in this study with those found in the literature was next conducted. The radionuclides considered included $90 \mathrm{Sr}$, $134 \mathrm{Cs}, 235 \mathrm{U}, 238 \mathrm{U}$ and $239 \mathrm{Pu}$. Comparisons with the dose per unit intake factors from ICRP 30 (1979-1988) and from Federal Guidance Report No. 11 (EPA 1988) showed agreement within a few percent. This agreement verifies that the basic ingestion dose conversion factors used in this study are consistent with the ICRP methods and with other Federal guidance. 
IABLE 2.3. Ingestion Committed Effective Dose Rate Conversion Factors for Exposure to Residual Radioactive Materials

\begin{tabular}{|c|c|c|}
\hline Radionuclide & mrem/pCi & $\mu \mathrm{Sv} / \mathrm{Bq}$ \\
\hline${ }^{3} \mathrm{H}$ & $5.7 E-08$ & $1.5 \mathrm{E}-05$ \\
\hline${ }^{10} \mathrm{Be}$ & $4.6 \mathrm{E}-06$ & $1.2 \mathrm{E}-03$ \\
\hline${ }^{14} \mathrm{C}$ & $1.9 \mathrm{E}-06$ & 5.1E-04 \\
\hline${ }^{18} \mathrm{~F}$ & $1.1 \mathrm{E}-07$ & $3.0 \mathrm{E}-05$ \\
\hline${ }^{22} \mathrm{Na}$ & $\begin{array}{l}1.4 \mathrm{E}-05 \\
1.4 \mathrm{E}-06\end{array}$ & $\begin{array}{l}3.8 \mathrm{E}-03 \\
3.8 \mathrm{E}-04\end{array}$ \\
\hline $\begin{array}{l}32 p \\
{ }_{33} p\end{array}$ & $\begin{array}{l}8.5 \mathrm{E}-06 \\
8.7 \mathrm{E}-07\end{array}$ & $\begin{array}{l}2.3 \mathrm{E}-03 \\
2.3 \mathrm{E}-04\end{array}$ \\
\hline${ }^{35} \mathrm{~S}$ & $4.0 E-07$ & $1.1 E-04$ \\
\hline${ }^{36} \mathrm{Cl}$ & $2.8 E-06$ & $7.6 \mathrm{E}-04$ \\
\hline${ }^{48} K$ & $1.7 \mathrm{E}-05$ & $4.6 \mathrm{E}-03$ \\
\hline $\begin{array}{l}{ }^{41} \mathrm{Ca} \\
{ }^{45} \mathrm{Ca}\end{array}$ & $\begin{array}{l}\text { 1.2E-06 } \\
3.1 \mathrm{E}-06\end{array}$ & $\begin{array}{l}3.2 \mathrm{E}-04 \\
8.4 \mathrm{E}-04\end{array}$ \\
\hline${ }^{46} \mathrm{SC}$ & $6.2 E-06$ & $1.7 \mathrm{E}-03$ \\
\hline${ }^{51} \mathrm{Cr}$ & $1.4 \mathrm{E}-07$ & $3.8 \mathrm{E}-05$ \\
\hline $\begin{array}{l}{ }^{54} \mathrm{Mn} \\
{ }^{60} \mathrm{Mn}\end{array}$ & $\begin{array}{l}2.8 \mathrm{E}-06 \\
1.0 \mathrm{E}-06\end{array}$ & $\begin{array}{l}7.6 \mathrm{E}-04 \\
2.7 \mathrm{E}-04\end{array}$ \\
\hline $\begin{array}{l}{ }^{55} \mathrm{Fe} \\
{ }^{59} \mathrm{Fe}\end{array}$ & $\begin{array}{l}5.8 \mathrm{E}-07 \\
6.5 \mathrm{E}-06\end{array}$ & $\begin{array}{l}1.6 \mathrm{E}-04 \\
1.8 \mathrm{E}-03\end{array}$ \\
\hline $\begin{array}{l}{ }^{57} \mathrm{Co} \\
{ }^{58} \mathrm{CO} \\
{ }^{80} \mathrm{CO}\end{array}$ & $\begin{array}{l}7.5 \mathrm{E}-07 \\
2.9 \mathrm{E}-06 \\
1.0 \mathrm{E}-05\end{array}$ & $\begin{array}{l}2.0 \mathrm{E}-04 \\
7.8 \mathrm{E}-04 \\
2.7 \mathrm{E}-03\end{array}$ \\
\hline $\begin{array}{l}{ }^{59} \mathrm{Ni} \\
{ }^{63} \mathrm{Ni} \\
{ }^{65} \mathrm{Ni}\end{array}$ & $\begin{array}{l}1.9 \mathrm{E}-07 \\
5.1 \mathrm{E}-07 \\
6.5 \mathrm{E}-07\end{array}$ & $\begin{array}{l}5.1 \mathrm{E}-05 \\
1.4 \mathrm{E}-04 \\
1.8 \mathrm{E}-04\end{array}$ \\
\hline${ }^{84} \mathrm{Cu}$ & $4.8 E-07$ & $1.3 E-04$ \\
\hline $\begin{array}{l}{ }^{65} \mathrm{Zn} \\
{ }^{69 n} \mathrm{Zn}+\mathrm{D} \\
{ }^{69 n} \mathrm{Zn} \\
{ }^{69} \mathrm{Zn}\end{array}$ & $\begin{array}{l}1.3 E-05 \\
7.0 E-07 \\
1.3 E-06 \\
8.9 E-08\end{array}$ & $\begin{array}{l}3.5 E-03 \\
1.9 E-04 \\
3.5 E-04 \\
2.4 E-05\end{array}$ \\
\hline & 2.26 & \\
\hline
\end{tabular}


TABLE 2.3. Ingestion Committed Effective Dose Rate Conversion Factors for Exposure to Residual Radioactive Materials (contd)

\begin{tabular}{|c|c|c|}
\hline Radionuclide & mrem/pCi & $\mu \mathrm{Sv} / \mathrm{Bq}$ \\
\hline${ }^{78}$ As & $5.3 E-06$ & $1.4 \mathrm{E}-03$ \\
\hline${ }^{75} \mathrm{Se}$ & $\begin{array}{l}8.9 \mathrm{E}-06 \\
8.0 \mathrm{E}-06\end{array}$ & $\begin{array}{l}2.4 \mathrm{E}-03 \\
2.2 \mathrm{E}-03\end{array}$ \\
\hline $\begin{array}{l}{ }^{82} \mathrm{Br} \\
{ }^{83} \mathrm{Br} \\
{ }^{83} \mathrm{Br}+\mathrm{D} \\
{ }^{84} \mathrm{Br} \\
{ }_{\mathrm{Br}}\end{array}$ & $\begin{array}{l}1.6 \mathrm{E}-06 \\
4.7 \mathrm{E}-08 \\
9.4 \mathrm{E}-08 \\
1.8 \mathrm{E}-07\end{array}$ & $\begin{array}{l}4.3 \mathrm{E}-04 \\
1.3 \mathrm{E}-05 \\
2.5 \mathrm{E}-05 \\
4.9 \mathrm{E}-05\end{array}$ \\
\hline $\begin{array}{l}{ }^{86} \mathrm{Rb} \\
{ }^{88}{ }_{\mathrm{Rb}} \\
{ }^{89} \mathrm{Rb}+\mathrm{D} \\
{ }^{89} \mathrm{Rb}\end{array}$ & $\begin{array}{l}9.1 \mathrm{E}-06 \\
1.8 \mathrm{E}-07 \\
4.4 \mathrm{E}-06 \\
9.5 \mathrm{E}-08\end{array}$ & $\begin{array}{l}2.5 \mathrm{E}-03 \\
4.9 \mathrm{E}-05 \\
1.2 \mathrm{E}-03 \\
2.6 \mathrm{E}-05\end{array}$ \\
\hline $\begin{array}{l}{ }^{85} \mathrm{Sr} \\
{ }^{89} \mathrm{Sr} \\
{ }^{90} \mathrm{Sr+D}(\mathrm{a}) \\
{ }^{90} \mathrm{Sr} \\
{ }^{91} \mathrm{Sr+D}(\mathrm{a}) \\
{ }^{91} \mathrm{Sr} \\
{ }^{92} \mathrm{Sr+D}(\mathrm{a}) \\
{ }^{92} \mathrm{Sr}\end{array}$ & $\begin{array}{l}1.7 \mathrm{E}-06 \\
8.7 \mathrm{E}-06 \\
6.5 \mathrm{E}-05 \\
1.2 \mathrm{E}-04 \\
4.7 \mathrm{E}-06 \\
2.4 \mathrm{E}-06 \\
1.8 \mathrm{E}-06 \\
1.6 \mathrm{E}-06\end{array}$ & $\begin{array}{l}4.6 E-04 \\
2.3 E-03 \\
1.8 E-02 \\
3.2 E-02 \\
1.3 E-03 \\
6.5 E-04 \\
4.9 E-04 \\
4.3 E-04\end{array}$ \\
\hline 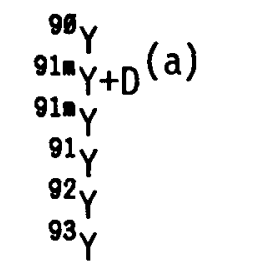 & $\begin{array}{l}1.1 \mathrm{E}-05 \\
4.7 \mathrm{E}-06 \\
4.1 \mathrm{E}-08 \\
9.4 \mathrm{E}-06 \\
2.0 \mathrm{E}-06 \\
4.8 \mathrm{E}-06\end{array}$ & $\begin{array}{l}3.0 \mathrm{E}-03 \\
1.3 \mathrm{E}-03 \\
1.1 \mathrm{E}-05 \\
2.5 \mathrm{E}-03 \\
5.4 \mathrm{E}-04 \\
1.3 \mathrm{E}-03\end{array}$ \\
\hline $\begin{array}{l}{ }^{93} \mathrm{Zr+D}(\mathrm{a}) \\
{ }^{93} \mathrm{Zr} \\
{ }^{95} \mathrm{Zr+D}(\mathrm{a}) \\
{ }^{95} \mathrm{Zr} \\
{ }^{97} \mathrm{Zr+D}(\mathrm{a}) \\
{ }^{97} \mathrm{Zr}\end{array}$ & $\begin{array}{l}1.0 \mathrm{E}-06 \\
1.6 \mathrm{E}-06 \\
3.1 \mathrm{E}-06 \\
3.7 \mathrm{E}-06 \\
2.9 \mathrm{E}-06 \\
8.4 \mathrm{E}-06\end{array}$ & $\begin{array}{l}2.8 \mathrm{E}-04 \\
4.3 \mathrm{E}-04 \\
8.5 \mathrm{E}-04 \\
1.0 \mathrm{E}-03 \\
7.9 \mathrm{E}-04 \\
2.3 \mathrm{E}-03\end{array}$ \\
\hline 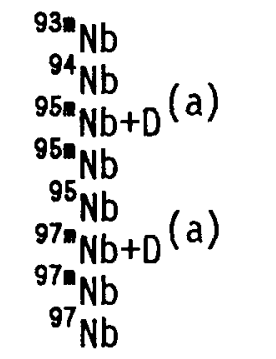 & $\begin{array}{l}5.0 \mathrm{E}-07 \\
7.2 \mathrm{E}-06 \\
2.4 \mathrm{E}-06 \\
2.3 \mathrm{E}-06 \\
2.5 \mathrm{E}-06 \\
1.2 \mathrm{E}-07 \\
3.9 \mathrm{E}-09 \\
2.4 \mathrm{E}-07\end{array}$ & $\begin{array}{l}1.3 E-04 \\
1.9 E-03 \\
6.5 E-04 \\
6.2 E-04 \\
6.7 E-04 \\
3.2 E-05 \\
1.1 E-06 \\
6.5 E-05\end{array}$ \\
\hline
\end{tabular}


TABLE 2.3. Ingestion Committed Effective Dose Rate Conversion Factors for Exposure to Residual Radioactive Materials (contd)

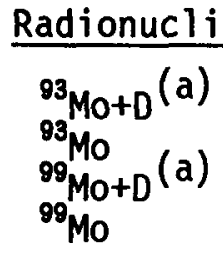

$\underline{\mathrm{mrem} / \mathrm{pCi}}$

9.0E-07

$1.4 E-06$

$1.6 \mathrm{E}-06$

3.1E-06

8.3E-08

$3.1 \mathrm{E}-06$

$7.5 \mathrm{E}-08$

$1.5 \mathrm{E}-06$

3.0E-06

9.0E-07

1.3E-06

1.3E-05

1.2E-08

$1.5 \mathrm{E}-06$

4.0E-07

7.8E-07

1.5E-07

1.3E-06

1.7E-05

5.1E-06

1.3E-05

$1.6 \mathrm{E}-04$

8.0E-06

1.6E-05

6.1E-06

1.3E-06

$7.7 \mathrm{E}-06$

3.5E-07

2. $9 \mathrm{E}-06$

$1.4 \mathrm{E}-06$

$1.2 \mathrm{E}-06$

1.5E-06

9.1E-07

8.4E-06

7.2E-06

1.2E-05

9.8E-06

1. $9 \mathrm{E}-05$ $\mu \mathrm{Sv} / \mathrm{Bq}$

2. $4 \mathrm{E}-04$

$3.8 \mathrm{E}-04$

$4.4 \mathrm{E}-04$

8.4E-04

2.2E-05

$8.4 \mathrm{E}-04$

2.0E-05

4.1E-04

8.1E-04

2.4E-04

3.5E-04

3.5E-03

3.2E-06

4.1E-04

1.1E-04

2.1E-04

4.1E-05

3.5E-04

4.5E-03

1. $4 \mathrm{E}-03$

3. $5 \mathrm{E}-03$

4. 3E-02

2. $2 \mathrm{E}-03$

4. $3 \mathrm{E}-03$

1.6E-03

3. 5E-04

2.1E-03

9.4E-05

$7.8 \mathrm{E}-04$

3.8E-04

3. $3 \mathrm{E}-04$

4.1E-04

2.5E-04

2.3E-03

1. $9 \mathrm{E}-03$

3. $2 \mathrm{E}-03$

2.6E-03

5.1E-03 
TABLE 2.3. Ingestion Committed Effective Dose Rate Conversion Factors for Exposure to Residual Radioactive Materials (contd)

\begin{tabular}{|c|c|c|}
\hline Radionuclide & $\mathrm{mrem} / \mathrm{pCi}$ & $\mu \mathrm{Sv} / \mathrm{Bq}$ \\
\hline $\begin{array}{l}{ }^{124} \mathrm{Sb} \\
{ }^{125} \mathrm{Sb}+\mathrm{D} \\
{ }^{125} \mathrm{Sb} \\
{ }^{126 \mathrm{~m}} \mathrm{Sb} \\
{ }^{126 \mathrm{~m}} \mathrm{Sb} \\
{ }^{126} \mathrm{Sb} \\
{ }^{127} \mathrm{Sb} \\
{ }^{127} \mathrm{Sb}+\mathrm{d} \\
\mathrm{Sb}\end{array}$ & $\begin{array}{l}9.9 \mathrm{E}-06 \\
2.9 \mathrm{E}-06 \\
2.8 \mathrm{E}-06 \\
1.3 \mathrm{E}-06 \\
9.1 \mathrm{E}-08 \\
1.0 \mathrm{E}-05 \\
4.3 \mathrm{E}-06 \\
7.2 \mathrm{E}-06\end{array}$ & $\begin{array}{l}2.7 \mathrm{E}-03 \\
7.9 \mathrm{E}-04 \\
7.6 \mathrm{E}-04 \\
3.6 \mathrm{E}-04 \\
2.5 \mathrm{E}-05 \\
2.7 \mathrm{E}-03 \\
1.2 \mathrm{E}-03 \\
1.9 \mathrm{E}-03\end{array}$ \\
\hline 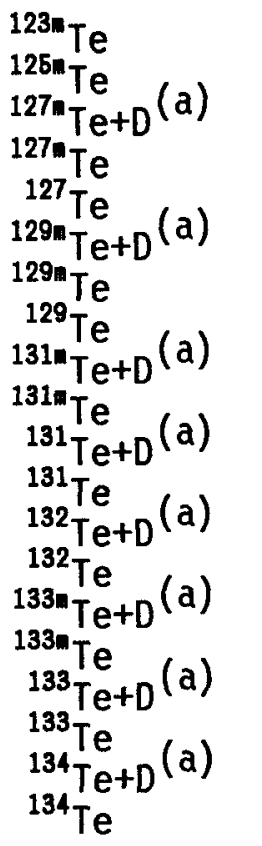 & $\begin{array}{l}5.7 \mathrm{E}-06 \\
3.7 \mathrm{E}-06 \\
4.5 \mathrm{E}-06 \\
8.2 \mathrm{E}-06 \\
7.1 \mathrm{E}-07 \\
3.3 \mathrm{E}-04 \\
5.4 \mathrm{E}-04 \\
2.6 \mathrm{E}-07 \\
3.0 \mathrm{E}-05 \\
1.7 \mathrm{E}-05 \\
2.5 \mathrm{E}-05 \\
2.1 \mathrm{E}-07 \\
7.5 \mathrm{E}-06 \\
1.5 \mathrm{E}-05 \\
3.5 \mathrm{E}-06 \\
7.8 \mathrm{E}-07 \\
3.3 \mathrm{E}-06 \\
1.6 \mathrm{E}-07 \\
2.3 \mathrm{E}-07 \\
1.9 \mathrm{E}-07\end{array}$ & $\begin{array}{l}1.5 \mathrm{E}-03 \\
1.0 \mathrm{E}-03 \\
1.2 \mathrm{E}-03 \\
2.2 \mathrm{E}-03 \\
1.9 \mathrm{E}-04 \\
9.0 \mathrm{E}-02 \\
1.5 \mathrm{E}-01 \\
7.0 \mathrm{E}-05 \\
8.1 \mathrm{E}-03 \\
4.6 \mathrm{E}-03 \\
6.7 \mathrm{E}-03 \\
5.7 \mathrm{E}-05 \\
2.0 \mathrm{E}-03 \\
4.1 \mathrm{E}-03 \\
9.4 \mathrm{E}-04 \\
2.1 \mathrm{E}-04 \\
8.8 \mathrm{E}-04 \\
4.3 \mathrm{E}-05 \\
6.1 \mathrm{E}-05 \\
5.1 \mathrm{E}-05\end{array}$ \\
\hline $\begin{array}{l}{ }^{125} \mathrm{I} \\
129 \mathrm{I} \\
130 \\
{ }_{1}^{131} \mathrm{I}+\mathrm{D}(\mathrm{a}) \\
{ }^{131} \mathrm{I} \\
{ }^{132} \mathrm{I} \\
{ }^{133} \mathrm{I}+\mathrm{D}(\mathrm{a}) \\
{ }^{133} \mathrm{I} \\
{ }_{134} \mathrm{I} \\
{ }_{136} \mathrm{I}+\mathrm{D}(\mathrm{a}) \\
{ }^{135} \mathrm{I}\end{array}$ & $\begin{array}{l}3.6 \mathrm{E}-05 \\
2.5 \mathrm{E}-04 \\
4.4 \mathrm{E}-06 \\
4.9 \mathrm{E}-05 \\
5.0 \mathrm{E}-05 \\
6.9 \mathrm{E}-07 \\
4.8 \mathrm{E}-06 \\
9.7 \mathrm{E}-06 \\
2.6 \mathrm{E}-07 \\
9.7 \mathrm{E}-07 \\
2.1 \mathrm{E}-06\end{array}$ & $\begin{array}{l}9.7 \mathrm{E}-03 \\
6.8 \mathrm{E}-02 \\
1.2 \mathrm{E}-03 \\
1.3 \mathrm{E}-02 \\
1.4 \mathrm{E}-02 \\
1.9 \mathrm{E}-04 \\
1.3 \mathrm{E}-03 \\
2.6 \mathrm{E}-03 \\
7.0 \mathrm{E}-05 \\
2.6 \mathrm{E}-04 \\
5.7 \mathrm{E}-04\end{array}$ \\
\hline $\begin{array}{l}{ }^{134 m} \mathrm{Cs}+\mathrm{D}(\mathrm{a}) \\
{ }^{134 m} \mathrm{Cs} \\
{ }^{134} \mathrm{Cs} \\
{ }^{135} \mathrm{Cs} \\
{ }^{136} \mathrm{Cs}\end{array}$ & $\begin{array}{l}1.8 E-08 \\
3.6 E-08 \\
6.7 E-05 \\
6.4 E-06 \\
1.0 E-05\end{array}$ & $\begin{array}{l}4.9 \mathrm{E}-06 \\
9.7 \mathrm{E}-06 \\
1.8 \mathrm{E}-02 \\
1.7 \mathrm{E}-03 \\
2.7 \mathrm{E}-03\end{array}$ \\
\hline
\end{tabular}


TABLE 2.3. Ingestion Committed Effective Dose Rate Conversion Factors for Exposure to Residual Radioactive Materials (contd)

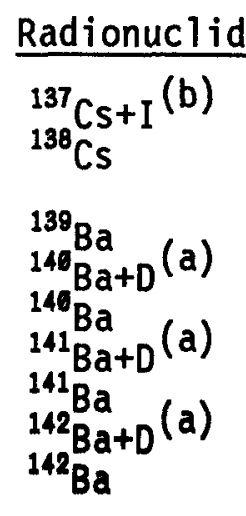

\begin{tabular}{|c|c|}
\hline $\mathrm{em} / \mathrm{pC} \mathbf{i}$ & $\mu \mathrm{Sv} / \mathrm{Bq}$ \\
\hline $\begin{array}{l}2.4 \mathrm{E}-05 \\
1.9 \mathrm{E}-07\end{array}$ & $5.1 \mathrm{E}$ \\
\hline
\end{tabular}

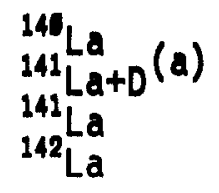

4.1E-07

8.5E-06

9.1E-06

$1.5 \mathrm{E}-06$

2.1E-07

4.1E-07

1.1E-07

$1.1 \mathrm{E}-04$

$2.3 \mathrm{E}-03$

2.5E-03

4.1E-04

5.7E-05

1.1E-04

3. OE-05

8.3E-06

2.2E-06

1.5E-06

7.0E-07

2.2E-03

5.9E-04

4.1E-04

1.9E-04

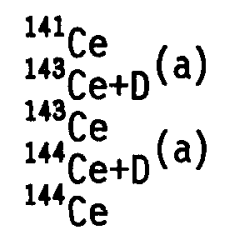

2. $9 \mathrm{E}-06$

7.8E-04

4. $6 \mathrm{E}-06$

1. $3 \mathrm{E}-03$

4.6E-06

1. $2 \mathrm{E}-03$

1.0E-05

2.8E-03

2.1E-05

5.7E-03

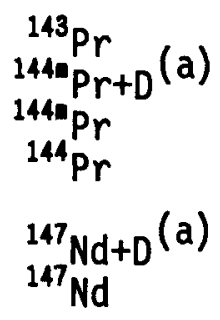

4.7E-06

8.5E-08

1.3E-03

4.8E-08

2.3E-05

1.2E-07

1.3E-05

3.2E-05

2.6E-06

4.2E-06

7.0E-04

1.1E-03

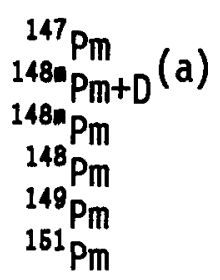

1. OE-06

2.7E-04

7.8E-06

2.1E-03

7. $6 \mathrm{E}-06$

2.1E-03

1.1E-05

3. $0 \mathrm{E}-03$

4.1E-06

1.1E-03

3. $0 \mathrm{E}-06$

8.1E-04

${ }^{147} \mathrm{Sm}$
${ }^{151} \mathrm{Sm}$
${ }^{153} \mathrm{Sm}$

1. $9 \mathrm{E}-04$

3. $8 \mathrm{E}-07$

5.1E-02

3. OE-06

1.0E-04

8.1E-04

${ }^{152} \mathrm{Eu}$
${ }^{154} \mathrm{Eu}$
${ }^{165} \mathrm{Eu}$
${ }^{158} \mathrm{Eu}$
${ }^{163} \mathrm{Gd}$

6.3E-06

1.7E-03

9. $2 \mathrm{E}-06$

2.5E-03

1.5E-06

4.1E-04

9.1E-06

2.5E-03

1.2E-06

3.2E-04 
TABLE 2.3. Ingestion Committed Effective Dose Rate Conversion Factors for Exposure to Residual Radioactive Materials (contd)

\begin{tabular}{|c|c|c|}
\hline Radionuclide & mrem/pCi & $\mu \mathrm{Sv} / \mathrm{Bq}$ \\
\hline${ }^{168} \mathrm{~Tb}$ & $6.6 \mathrm{E}-06$ & $1.8 \mathrm{E}-03$ \\
\hline${ }^{188 n} \mathrm{Ho}$ & $7.7 E-06$ & $2.1 E-03$ \\
\hline $\begin{array}{l}181 \mathrm{~W} \\
185 \mathrm{~W} \\
187 \mathrm{~W}\end{array}$ & $\begin{array}{l}2.9 \mathrm{E}-07 \\
1.6 \mathrm{E}-06 \\
2.1 \mathrm{E}-06\end{array}$ & $\begin{array}{l}7.8 \mathrm{E}-05 \\
4.3 \mathrm{E}-04 \\
5.7 \mathrm{E}-04\end{array}$ \\
\hline${ }^{187} \mathrm{Re}$ & $1.4 E-08$ & $3.8 \mathrm{E}-06$ \\
\hline $\begin{array}{l}1050 \mathrm{~S} \\
{ }^{101} \mathrm{OS}\end{array}$ & $\begin{array}{l}2.2 E=06 \\
2.3 E=06\end{array}$ & $\begin{array}{l}5.9 E-04 \\
6.2 E-04\end{array}$ \\
\hline${ }^{102}$ Ir & $5.7 E-06$ & $1.5 \mathrm{E}-03$ \\
\hline${ }^{203} \mathrm{Hg}$ & $1.1 \mathrm{E}-05$ & 3.0E-03 \\
\hline $\begin{array}{l}{ }^{216} \mathrm{~Pb}+\mathrm{D} \\
{ }_{216}(\mathrm{a}) \\
{ }^{212} \mathrm{~Pb} \\
{ }^{212} \mathrm{~Pb}+\mathrm{D}\end{array}$ & $\begin{array}{l}2.4 \mathrm{E}-03 \\
5.4 \mathrm{E}-03 \\
1.7 \mathrm{E}-05 \\
4.9 \mathrm{E}-05\end{array}$ & $\begin{array}{l}6.6 \mathrm{E}-01 \\
1.5 \mathrm{E}+00 \\
4.5 \mathrm{E}-03 \\
1.3 \mathrm{E}-02\end{array}$ \\
\hline $\begin{array}{l}{ }^{216} B i+D(a) \\
{ }^{210} B j \quad(b) \\
{ }^{212} B i+I\end{array}$ & $\begin{array}{l}9.5 \mathrm{E}-04 \\
6.3 \mathrm{E}-06 \\
5.5 \mathrm{E}-07\end{array}$ & $\begin{array}{l}2.6 \mathrm{E}-01 \\
1.7 \mathrm{E}-03 \\
1.5 \mathrm{E}-04\end{array}$ \\
\hline${ }^{216} \mathrm{Po}$ & $1.9 \mathrm{E}-03$ & 5.1E-01 \\
\hline 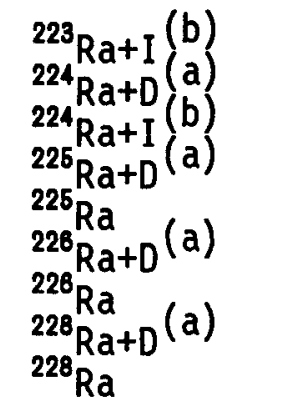 & $\begin{array}{l}4.5 \mathrm{E}-05 \\
3.0 \mathrm{E}-05 \\
1.3 \mathrm{E}-04 \\
4.7 \mathrm{E}-05 \\
2.2 \mathrm{E}-04 \\
9.1 \mathrm{E}-04 \\
9.5 \mathrm{E}-04 \\
1.6 \mathrm{E}-04 \\
8.4 \mathrm{E}-04\end{array}$ & $\begin{array}{l}1.2 \mathrm{E}-02 \\
8.1 \mathrm{E}-03 \\
3.5 \mathrm{E}-02 \\
1.3 \mathrm{E}-02 \\
5.9 \mathrm{E}-02 \\
2.5 \mathrm{E}-01 \\
2.6 \mathrm{E}-01 \\
4.2 \mathrm{E}-02 \\
2.3 \mathrm{E}-01\end{array}$ \\
\hline $\begin{array}{l}{ }^{225} \mathrm{Ac}+\mathrm{I}(\mathrm{b}) \\
{ }^{227} \mathrm{Ac}+\mathrm{D}(\mathrm{a}) \\
{ }^{227} \mathrm{AC} \\
{ }^{228} \mathrm{AC}+\mathrm{D}(\mathrm{a}) \\
{ }^{228} \mathrm{AC}\end{array}$ & $\begin{array}{l}1.8 \mathrm{E}-05 \\
1.8 \mathrm{E}-03 \\
1.4 \mathrm{E}-02 \\
7.2 \mathrm{E}-05 \\
2.1 \mathrm{E}-06\end{array}$ & $\begin{array}{l}4.9 E-03 \\
4.7 E-01 \\
3.8 E+00 \\
2.0 E-02 \\
5.7 E-04\end{array}$ \\
\hline $\begin{array}{l}{ }^{227} \mathrm{Th+D}(\mathrm{a}) \\
{ }^{227} \mathrm{Th} \\
{ }^{228} \mathrm{Th}+\mathrm{D} \\
(\mathrm{a})\end{array}$ & $\begin{array}{l}4.4 \mathrm{E}-05 \\
3.8 \mathrm{E}-05 \\
8.1 \mathrm{E}-05\end{array}$ & $\begin{array}{l}1.2 \mathrm{E}-02 \\
1.0 \mathrm{E}-02 \\
2.2 \mathrm{E}-02\end{array}$ \\
\hline
\end{tabular}


TABLE 2.3. Ingestion Committed Effective Dose Rate Conversion Factors for Exposure to Residual Radioactive Materials (contd)

\begin{tabular}{|c|c|c|}
\hline Radionuclide & $\mathrm{mrem} / \mathrm{pCi}$ & $\mu \mathrm{Sv} / \mathrm{Bq}$ \\
\hline $\begin{array}{l}{ }^{228} \mathrm{Th} \\
{ }^{229} \mathrm{Th}+\mathrm{D}(\mathrm{a}) \\
{ }^{229} \mathrm{Th} \\
{ }^{236} \mathrm{Th+D}(\mathrm{a}) \\
{ }^{236} \mathrm{Th} \\
{ }^{231} \mathrm{Th} \text { (a) } \\
{ }^{231} \mathrm{Th} \\
{ }^{232} \mathrm{Th} \text { (a) } \\
{ }_{\text {Th-Nat }}(\mathrm{c}) \\
{ }^{232} \mathrm{Th} \\
{ }^{234} \mathrm{Th}_{\mathrm{T}} \text { (a) } \\
{ }^{234} \mathrm{Th+I} \text { (b) }\end{array}$ & $\begin{array}{l}4.0 \mathrm{E}-04 \\
4.8 \mathrm{E}-04 \\
3.5 \mathrm{E}-03 \\
8.8 \mathrm{E}-04 \\
5.4 \mathrm{E}-04 \\
1.3 \mathrm{E}-07 \\
1.3 \mathrm{E}-06 \\
4.2 \mathrm{E}-04 \\
4.2 \mathrm{E}-04 \\
2.8 \mathrm{E}-03 \\
6.5 \mathrm{E}-06 \\
1.3 \mathrm{E}-05\end{array}$ & $\begin{array}{l}1.1 \mathrm{E}-01 \\
1.3 \mathrm{E}-01 \\
9.5 \mathrm{E}-01 \\
2.4 \mathrm{E}-01 \\
1.5 \mathrm{E}-01 \\
3.5 \mathrm{E}-05 \\
3.5 \mathrm{E}-04 \\
1.1 \mathrm{E}-01 \\
1.1 \mathrm{E}-01 \\
7.6 \mathrm{E}-01 \\
1.8 \mathrm{E}-03 \\
3.5 \mathrm{E}-03\end{array}$ \\
\hline $\begin{array}{l}{ }^{231} \mathrm{~Pa}+\mathrm{D} \\
{ }^{231} \mathrm{~Pa} \\
{ }^{233} \mathrm{~Pa} \\
{ }^{234} \mathrm{~Pa} \\
{ }^{\mathrm{Pa}}\end{array}$ & $\begin{array}{l}2.8 \mathrm{E}-03 \\
1.1 \mathrm{E}-02 \\
3.6 \mathrm{E}-06 \\
2.2 \mathrm{E}-06\end{array}$ & $\begin{array}{l}7.5 \mathrm{E}-01 \\
3.0 \mathrm{E}+00 \\
9.7 \mathrm{E}-04 \\
5.9 \mathrm{E}-04\end{array}$ \\
\hline 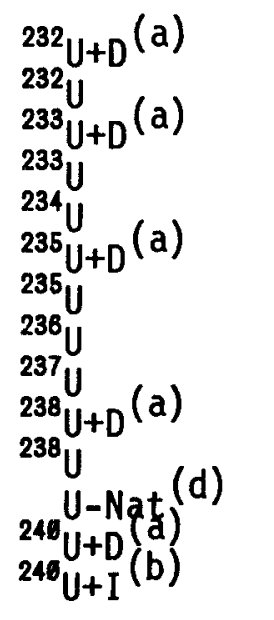 & $\begin{array}{l}9.7 \mathrm{E}-06 \\
6.8 \mathrm{E}-05 \\
4.3 \mathrm{E}-04 \\
2.6 \mathrm{E}-05 \\
2.6 \mathrm{E}-05 \\
1.4 \mathrm{E}-05 \\
2.6 \mathrm{E}-05 \\
2.4 \mathrm{E}-05 \\
3.2 \mathrm{E}-06 \\
1.2 \mathrm{E}-05 \\
2.4 \mathrm{E}-05 \\
2.5 \mathrm{E}-05 \\
1.6 \mathrm{E}-06 \\
4.7 \mathrm{E}-06\end{array}$ & $\begin{array}{l}2.6 \mathrm{E}-03 \\
1.8 \mathrm{E}-02 \\
1.2 \mathrm{E}-01 \\
7.0 \mathrm{E}-03 \\
7.0 \mathrm{E}-03 \\
3.8 \mathrm{E}-03 \\
7.0 \mathrm{E}-03 \\
6.5 \mathrm{E}-03 \\
8.6 \mathrm{E}-04 \\
3.3 \mathrm{E}-03 \\
6.5 \mathrm{E}-03 \\
6.8 \mathrm{E}-03 \\
4.2 \mathrm{E}-04 \\
1.3 \mathrm{E}-03\end{array}$ \\
\hline $\begin{array}{l}{ }^{237} \mathrm{~Np}+\mathrm{D}(\mathrm{a}) \\
{ }^{237} \mathrm{~Np} \\
{ }^{238} \mathrm{Np+D}(\mathrm{a}) \\
{ }^{238} \mathrm{~Np} \\
{ }^{238} \mathrm{Np+D} \\
{ }_{\mathrm{Np}}(\mathrm{a})\end{array}$ & $\begin{array}{l}2.6 \mathrm{E}-03 \\
5.2 \mathrm{E}-03 \\
1.7 \mathrm{E}-04 \\
4.0 \mathrm{E}-06 \\
1.7 \mathrm{E}-06 \\
3.3 \mathrm{E}-06\end{array}$ & $\begin{array}{l}7.0 \mathrm{E}-01 \\
1.4 \mathrm{E}+00 \\
4.6 \mathrm{E}-02 \\
1.1 \mathrm{E}-03 \\
4.5 \mathrm{E}-04 \\
8.9 \mathrm{E}-04\end{array}$ \\
\hline $\begin{array}{l}{ }^{236} \mathrm{Pu} \\
{ }^{237} \mathrm{Pu} \\
{ }^{238} \mathrm{Pu} \\
{ }^{239} \mathrm{Pu} \\
{ }_{240} \mathrm{Pu} \\
{ }^{241} \mathrm{Pu}+\mathrm{D}(\mathrm{a}) \\
{ }^{241} \mathrm{Pu}\end{array}$ & $\begin{array}{l}6.3 \mathrm{E}-05 \\
4.3 \mathrm{E}-07 \\
3.4 \mathrm{E}-04 \\
3.7 \mathrm{E}-04 \\
3.7 \mathrm{E}-04 \\
1.8 \mathrm{E}-03 \\
6.8 \mathrm{E}-06\end{array}$ & $\begin{array}{l}1.7 \mathrm{E}-02 \\
1.2 \mathrm{E}-04 \\
9.2 \mathrm{E}-02 \\
1.0 \mathrm{E}-01 \\
1.0 \mathrm{E}-01 \\
4.9 \mathrm{E}-01 \\
1.8 \mathrm{E}-03\end{array}$ \\
\hline
\end{tabular}


TABLE 2.3. Ingestion Committed Effective Dose Rate Conversion Factors for Exposure to Residual Radioactive Materials (contd)

\begin{tabular}{|c|c|c|}
\hline Radionuclide & $\mathrm{mrem} / \mathrm{pCi}$ & $\mu \mathrm{Sv} / \mathrm{Bq}$ \\
\hline $\begin{array}{l}{ }_{242}^{243} \mathrm{Pu} \\
{ }^{243} \mathrm{Pu}+\mathrm{D} \\
{ }^{244} \mathrm{Pu} \\
{ }^{244} \mathrm{Pu} \text { (a) } \\
\text { (a) }\end{array}$ & $\begin{array}{l}3.4 \mathrm{E}-04 \\
1.6 \mathrm{E}-07 \\
3.2 \mathrm{E}-07 \\
9.0 \mathrm{E}-05 \\
3.5 \mathrm{E}-04\end{array}$ & $\begin{array}{l}9.2 \mathrm{E}-02 \\
4.3 \mathrm{E}-05 \\
8.6 \mathrm{E}-05 \\
2.4 \mathrm{E}-02 \\
9.4 \mathrm{E}-02\end{array}$ \\
\hline $\begin{array}{l}{ }^{241} \mathrm{Am} \\
{ }^{2422} \mathrm{Am}+\mathrm{D} \\
{ }^{242} \mathrm{Am} \\
{ }^{242} \mathrm{Am}+\mathrm{D} \\
{ }^{242} \mathrm{Am} \\
{ }^{243} \mathrm{Am}+\mathrm{am} \\
{ }^{243} \mathrm{Am}\end{array}$ & $\begin{array}{l}3.6 \mathrm{E}-03 \\
9.9 \mathrm{E}-04 \\
3.5 \mathrm{E}-03 \\
1.3 \mathrm{E}-04 \\
1.4 \mathrm{E}-06 \\
1.2 \mathrm{E}-03 \\
3.6 \mathrm{E}-03\end{array}$ & $\begin{array}{l}9.7 \mathrm{E}-01 \\
2.7 \mathrm{E}-01 \\
9.5 \mathrm{E}-01 \\
3.5 \mathrm{E}-02 \\
3.8 \mathrm{E}-04 \\
3.2 \mathrm{E}-01 \\
9.7 \mathrm{E}-01\end{array}$ \\
\hline $\begin{array}{l}{ }^{242} \mathrm{Cm}+\mathrm{D}(\mathrm{a}) \\
{ }^{242} \mathrm{Cm} \\
{ }^{243} \mathrm{Cm} \\
{ }^{244} \mathrm{Cm} \text { ( } \mathrm{D}(\mathrm{a}) \\
{ }^{244} \mathrm{Cm} \\
{ }^{245} \mathrm{Cm}+\mathrm{D}(\mathrm{a}) \\
{ }^{245} \mathrm{Cm} \\
{ }^{248} \mathrm{Cm} \\
247 \mathrm{Cm} \text { (a) } \\
{ }^{247} \mathrm{Cm} \\
{ }^{248} \mathrm{Cm}\end{array}$ & $\begin{array}{l}2.5 \mathrm{E}-04 \\
1.1 \mathrm{E}-04 \\
2.9 \mathrm{E}-03 \\
1.0 \mathrm{E}-03 \\
2.0 \mathrm{E}-03 \\
2.4 \mathrm{E}-03 \\
3.7 \mathrm{E}-03 \\
3.7 \mathrm{E}-03 \\
1.7 \mathrm{E}-03 \\
3.4 \mathrm{E}-03 \\
1.3 \mathrm{E}-02\end{array}$ & $\begin{array}{l}6.7 \mathrm{E}-02 \\
3.0 \mathrm{E}-02 \\
7.8 \mathrm{E}-01 \\
2.7 \mathrm{E}-01 \\
5.4 \mathrm{E}-01 \\
6.6 \mathrm{E}-01 \\
1.0 \mathrm{E}+00 \\
1.0 \mathrm{E}+00 \\
4.6 \mathrm{E}-01 \\
9.2 \mathrm{E}-01 \\
3.5 \mathrm{E}+00\end{array}$ \\
\hline${ }^{252} \mathrm{Cf}$ & $1.1 \mathrm{E}-03$ & $3.0 \mathrm{E}-01$ \\
\hline
\end{tabular}

(a) Where $+D$ means "plus daughters in equilibrium" referring to explicit daughters, or daughters with half-lives greater than 10 minutes. Separate values are also included for parents and daughters so that combined factors for non-equilibrium cases can be determined. Note: in some cases $+D$ also includes implicit daughters (i.e., uranium and thorium decay chains).

(b) Where +I means "plus implicit daughters in equilibrium" referring to daughters with half-lives less than 10 minutes. The decay energies associated with these daughters have been assigned to the parent, and the appropriate branching ratios (described in Appendix D) have been considered.

(c) Where Th-Nat includes an equilibrium mixture of ${ }^{232}$ Th plus 10 daughters in the decay chain, as discussed in Appendix $D$.

(d) Where $1 \mathrm{Ci} \mathrm{U}$-Nat equals $48.9 \%{ }^{238} \mathrm{U}$ plus $48.9 \%{ }^{234} \mathrm{U}$ plus $2.25 \%{ }^{235} \mathrm{U}$. 


\subsubsection{Drinking Water Ingestion}

For sites contaminated with residual radioactivity in soils, the potential exists for contamination of ground-water sources. Appendix B contains a brief discussion of models that could apply to generic drinkingwater contamination situations. These models can be used to estimate the concentration of radionuclides in a ground-water source that could provide drinking water through a well. The concentration is calculated based on the entire inventory ( $\mathrm{pC} i$ or $\mathrm{Bq}$ ) in soils or in buildings that could be demolished as left as buried rubble, not the concentration $(\mathrm{pCi} / \mathrm{g}$ or $\mathrm{Bq} / \mathrm{g}$ ) in the soil or building materials. The general equation for estimating the committed dose rate by ingestion of drinking water containing radionuclide $i$ is:

$$
H_{D W, i}=\text { QDW CDW, } i \text { DFING, } i
$$

where HDW, $i=$ the committed effective dose equivalent from a $1-y r$ intake of radionuclide $i$ by ingestion of drinking water, in mrem ( $\mu \mathrm{Sv})$ per year of intake

QDW = the quantity of drinking water ingested during a year, in $L$

CDW, $i=$ the concentration of radionuclide $i$ in the drinking water, in $\mathrm{pCi} / \mathrm{L}(\mathrm{Bq} / \mathrm{L})$

DFING, $i=$ the committed effective dose equivalent factor from ingestion of radionuclide $i$, in $\mathrm{mrem} / \mathrm{pC} i(\mu \mathrm{Sv} / \mathrm{Bq})$ per year of intake.

Parameter values for the quantity of drinking water ingested during a year and the concentration of radionuclides in the drinking water are scenario specific, as discussed in Appendix B. Committed effective dose conversion factors for ingestion are shown in Table 2.3. These factors apply to both secondary ingestion and drinking water and are in units of mrem/pCi and $\mu \mathrm{Sv} / \mathrm{Bq}$ ingested. Scenario-specific estimates of the committed effective dose equivalent from ingestion of drinking water can be obtained by multiplying the factors shown in Table 2.3 by the quantity of drinking water consumed during a year $(L)$ and the concentration of radionuclides in that water source ( $\mathrm{pCi} / \mathrm{L}$ or $\mathrm{Bq} / \mathrm{L}$ ). For comparison with U.S. Environmental Protection Agency (EPA) drinking water standards, expressed in terms of organ dose limits, maximum organ doses by radionuclide are needed. Table 2.4 lists the maximum organ dose per unit of intake (mrem/pCi and $\mu \mathrm{Sv} / \mathrm{Bq}$ ) and the limiting organ for each radionuclide. It should be noted that the maximum organ doses shown in Table 2.4 are based on the dosimetry models, metabolic data, and recommendations of the ICRP in Publication 30 (1977-1988). 
TABLE 2.4. Maximum Organ Dose Rate Conversion Factors for Ingestion of Drinking Water

\begin{tabular}{|c|c|c|c|}
\hline Radionuclide & $\mathrm{mrem} / \mathrm{pCi}$ & $\mu \mathrm{Sv} / \mathrm{Bq}$ & Limiting Organ(a) \\
\hline${ }^{3} \mathrm{H}$ & $6.7 \mathrm{E}-08$ & $1.8 \mathrm{E}-05$ & SI Wall \\
\hline${ }^{16} \mathrm{Be}$ & $4.8 E-05$ & $1.3 \mathrm{E}-02$ & LLI Wall \\
\hline${ }^{14} \mathrm{C}$ & $2.3 E-06$ & $6.2 E-04$ & LLI Wall \\
\hline${ }^{18} \mathrm{~F}$ & $1.1 \mathrm{E}-06$ & $3.0 \mathrm{E}-04$ & Stomach Wall \\
\hline $\begin{array}{l}{ }^{22} \mathrm{Na} \\
{ }^{24} \mathrm{Na}\end{array}$ & $\begin{array}{l}2.3 \mathrm{E}-05 \\
4.4 \mathrm{E}-06\end{array}$ & $\begin{array}{l}6.2 \mathrm{E}-03 \\
1.2 \mathrm{E}-03\end{array}$ & $\begin{array}{l}\text { Bone Surface } \\
\text { Stomach Wall }\end{array}$ \\
\hline $\begin{array}{l}{ }^{32} \mathrm{p} \\
{ }^{33} \mathrm{p}\end{array}$ & $\begin{array}{l}3.1 \mathrm{E}-05 \\
4.8 \mathrm{E}-06\end{array}$ & $\begin{array}{l}8.4 \mathrm{E}-03 \\
1.3 \mathrm{E}-03\end{array}$ & $\begin{array}{l}\text { Red Marrow } \\
\text { Bone Surface }\end{array}$ \\
\hline${ }^{35} \mathrm{~S}$ & $2.0 E-06$ & $5.4 \mathrm{E}-04$ & LLI Wall \\
\hline${ }^{38} \mathrm{Cl}$ & $3.2 E-06$ & $8.6 E-04$ & SI Wall \\
\hline${ }^{48} \mathrm{~K}$ & $2.0 \mathrm{E}-05$ & $5.4 \mathrm{E}-03$ & SI Wall \\
\hline${ }^{41} \mathrm{Ca}$ & $\begin{array}{l}1.5 \mathrm{E}-05 \\
1.9 \mathrm{E}-05\end{array}$ & $\begin{array}{l}4.1 \mathrm{E}-03 \\
5.1 \mathrm{E}-03\end{array}$ & $\begin{array}{l}\text { Bone Surface } \\
\text { Bone Surface }\end{array}$ \\
\hline${ }^{46} \mathrm{SC}$ & $3.7 E-05$ & $1.0 \mathrm{E}-02$ & LLI Wall \\
\hline${ }^{51} \mathrm{Cr}$ & $9.2 \mathrm{E}-07$ & $2.5 \mathrm{E}-04$ & LLI Wall \\
\hline $\begin{array}{l}{ }^{54} \mathrm{Mn} \\
{ }^{56} \mathrm{Mn}\end{array}$ & $\begin{array}{l}8.5 \mathrm{E}-06 \\
5.2 \mathrm{E}-06\end{array}$ & $\begin{array}{l}2.3 \mathrm{E}-03 \\
1.4 \mathrm{E}-03\end{array}$ & $\begin{array}{l}\text { LLI Wall } \\
\text { ULI Wall }\end{array}$ \\
\hline $\begin{array}{l}{ }^{55} \mathrm{Fe} \\
{ }^{59} \mathrm{Fe}\end{array}$ & $\begin{array}{l}2.1 \mathrm{E}-06 \\
3.1 \mathrm{E}-05\end{array}$ & $\begin{array}{l}5.7 \mathrm{E}-04 \\
8.4 \mathrm{E}-03\end{array}$ & $\begin{array}{l}\text { Spleen } \\
\text { LLI Wall }\end{array}$ \\
\hline $\begin{array}{l}{ }^{57} \mathrm{Co} \\
{ }^{58} \mathrm{Co} \\
{ }^{86} \mathrm{Co}\end{array}$ & $\begin{array}{l}4.8 E-06 \\
1.4 E-05 \\
4.3 E-05\end{array}$ & $\begin{array}{l}\text { 1.3E-03 } \\
3.8 \mathrm{E}-03 \\
1.2 \mathrm{E}-02\end{array}$ & $\begin{array}{l}\text { LLI Wall } \\
\text { LLI Wall } \\
\text { LLI Wall }\end{array}$ \\
\hline $\begin{array}{l}{ }^{69} \mathrm{Ni} \\
{ }^{63} \mathrm{Ni} \\
{ }^{85} \mathrm{Ni}\end{array}$ & $\begin{array}{l}9.7 \mathrm{E}-07 \\
3.2 \mathrm{E}-06 \\
3.6 \mathrm{E}-06\end{array}$ & $\begin{array}{l}2.6 \mathrm{E}-04 \\
8.6 \mathrm{E}-04 \\
9.7 \mathrm{E}-04\end{array}$ & $\begin{array}{l}\text { LLI Wall } \\
\text { LLI WaIl } \\
\text { ULI Wall }\end{array}$ \\
\hline${ }^{84} \mathrm{Cu}$ & $2.9 E-06$ & $7.8 \mathrm{E}-04$ & LLI Wall \\
\hline $\begin{array}{l}{ }^{65} \mathrm{Zn} \\
{ }^{69 m} \mathrm{Zn}+\mathrm{D} \\
\left.{ }^{69 m} \mathrm{~m}\right) \\
{ }^{69} \mathrm{Zn} \\
{ }^{\mathrm{Zn}}\end{array}$ & $\begin{array}{l}1.7 \mathrm{E}-05 \\
4.4 \mathrm{E}-06 \\
8.9 \mathrm{E}-06 \\
7.8 \mathrm{E}-07\end{array}$ & $\begin{array}{l}4.6 \mathrm{E}-03 \\
1.2 \mathrm{E}-03 \\
2.4 \mathrm{E}-03 \\
2.1 \mathrm{E}-04\end{array}$ & $\begin{array}{l}\text { LLI Wall } \\
\text { LLI Wall } \\
\text { LLI Wall } \\
\text { Stomach Wall }\end{array}$ \\
\hline
\end{tabular}


TABLE 2.4. Maximum Organ Dose Rate Conversion Factors for Ingestion of Drinking Water (contd)

\begin{tabular}{|c|c|c|c|}
\hline Radionuclide & $\mathrm{mrem} / \mathrm{pCi}$ & $\mu \mathrm{Sv} / \mathrm{Bq}$ & Limiting Organ (a) \\
\hline${ }^{78}$ As & 4. $4 \mathrm{E}-05$ & $1.2 \mathrm{E}-02$ & LLI Wall \\
\hline $\begin{array}{l}{ }^{75} \mathrm{Se} \\
{ }^{79} \mathrm{Se}\end{array}$ & $\begin{array}{l}2.6 \mathrm{E}-05 \\
4.8 \mathrm{E}-05\end{array}$ & $\begin{array}{l}\text { 7.0E-03 } \\
1.3 \mathrm{E}-02\end{array}$ & $\begin{array}{l}\text { Kidneys } \\
\text { Kidneys }\end{array}$ \\
\hline $\begin{array}{l}{ }^{82} \mathrm{Br} \\
{ }^{83} \mathrm{Br}+\mathrm{D}(\mathrm{b}) \\
{ }^{83} \mathrm{Br} \\
{ }^{84} \mathrm{Br}\end{array}$ & $\begin{array}{l}3.0 \mathrm{E}-06 \\
5.5 \mathrm{E}-07 \\
1.1 \mathrm{E}-06 \\
2.6 \mathrm{E}-06\end{array}$ & $\begin{array}{l}8.1 \mathrm{E}-04 \\
1.5 \mathrm{E}-04 \\
3.0 \mathrm{E}-04 \\
7.0 \mathrm{E}-04\end{array}$ & $\begin{array}{l}\text { Stomach Wall } \\
\text { Stomach Wall } \\
\text { Stomach Wall } \\
\text { Stomach Wall }\end{array}$ \\
\hline $\begin{array}{l}{ }^{86} \mathrm{Rb} \\
{ }^{88} \mathrm{Rb} \\
{ }^{89} \mathrm{Rb}+\mathrm{Db} \\
{ }_{\mathrm{Bg}}^{\mathrm{Rb}}\end{array}$ & $\begin{array}{l}2.7 \mathrm{E}-05 \\
2.8 \mathrm{E}-06 \\
3.9 \mathrm{E}-05 \\
1.4 \mathrm{E}-06\end{array}$ & $\begin{array}{l}7.3 \mathrm{E}-03 \\
7.6 \mathrm{E}-04 \\
1.1 \mathrm{E}-02 \\
3.8 \mathrm{E}-04\end{array}$ & $\begin{array}{l}\text { Bone Surface } \\
\text { Stomach Wall } \\
\text { LLI Wall } \\
\text { Stomach Wall }\end{array}$ \\
\hline $\begin{array}{l}{ }^{85} \mathrm{Sr} \\
{ }^{89} \mathrm{Sr} \\
{ }^{96} \mathrm{~S} r+D(b) \\
{ }^{98} \mathrm{Sr} \\
{ }^{91} \mathrm{Sr}+\mathrm{D} \\
{ }^{91} \mathrm{Sr} \\
{ }^{92} \mathrm{Sr} \\
{ }^{92} \mathrm{Sr}\end{array}$ & $\begin{array}{l}5.2 \mathrm{E}-06 \\
7.8 \mathrm{E}-05 \\
7.0 \mathrm{E}-04 \\
1.4 \mathrm{E}-03 \\
5.1 \mathrm{E}-05 \\
1.4 \mathrm{E}-05 \\
1.2 \mathrm{E}-05 \\
1.1 \mathrm{E}-05\end{array}$ & $\begin{array}{l}1.4 \mathrm{E}-03 \\
2.1 \mathrm{E}-02 \\
1.9 \mathrm{E}-01 \\
3.8 \mathrm{E}-01 \\
1.4 \mathrm{E}-02 \\
3.8 \mathrm{E}-03 \\
3.2 \mathrm{E}-03 \\
3.0 \mathrm{E}-03\end{array}$ & $\begin{array}{l}\text { LLI Wall } \\
\text { LLI Wall } \\
\text { Bone Surface } \\
\text { Bone Surface } \\
\text { LLI Wall } \\
\text { LLI Wall } \\
\text { ULI Wall } \\
\text { ULI Wall }\end{array}$ \\
\hline $\begin{array}{l}{ }^{96} Y \\
{ }^{91} Y \\
{ }^{91 m} Y \\
\\
{ }^{91} Y \\
{ }^{92} Y \\
{ }^{93} Y\end{array}$ & $\begin{array}{l}1.2 \mathrm{E}-04 \\
5.5 \mathrm{E}-05 \\
1.9 \mathrm{E}-07 \\
1.1 \mathrm{E}-04 \\
1.3 \mathrm{E}-05 \\
3.5 \mathrm{E}-05\end{array}$ & $\begin{array}{l}3.2 \mathrm{E}-02 \\
1.5 \mathrm{E}-02 \\
5.1 \mathrm{E}-05 \\
3.0 \mathrm{E}-02 \\
3.5 \mathrm{E}-03 \\
9.5 \mathrm{E}-03\end{array}$ & $\begin{array}{l}\text { LLI Wall } \\
\text { LLI Wall } \\
\text { Stomach Wal1 } \\
\text { LLI Wall } \\
\text { ULI Wall } \\
\text { LLI Wall }\end{array}$ \\
\hline $\begin{array}{l}{ }^{93} \mathrm{Zr+D} \\
{ }^{93} \mathrm{Zr} \\
{ }^{95} \mathrm{Zr} \\
{ }^{95} \mathrm{Zr+D} \\
{ }^{97} \mathrm{Zr} \\
{ }^{97} \mathrm{Zr+D} \\
{ }^{97} \mathrm{Zr}\end{array}$ & $\begin{array}{l}1.7 \mathrm{E}-05 \\
3.3 \mathrm{E}-05 \\
2.2 \mathrm{E}-05 \\
2.9 \mathrm{E}-05 \\
2.3 \mathrm{E}-05 \\
6.7 \mathrm{E}-05\end{array}$ & $\begin{array}{l}4.5 \mathrm{E}-03 \\
8.9 \mathrm{E}-03 \\
5.9 \mathrm{E}-03 \\
7.8 \mathrm{E}-03 \\
6.1 \mathrm{E}-03 \\
1.8 \mathrm{E}-02\end{array}$ & $\begin{array}{l}\text { Bone Surface } \\
\text { Bone Surface } \\
\text { LLI Wall } \\
\text { LLI Wall } \\
\text { LLI Wall } \\
\text { LLI Wall }\end{array}$ \\
\hline 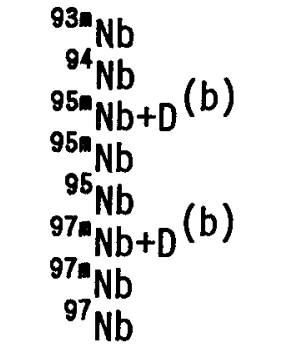 & $\begin{array}{l}5.6 \mathrm{E}-06 \\
4.8 \mathrm{E}-05 \\
2.0 \mathrm{E}-05 \\
2.4 \mathrm{E}-05 \\
1.5 \mathrm{E}-05 \\
7.0 \mathrm{E}-07 \\
2.7 \mathrm{E}-08 \\
1.4 \mathrm{E}-06\end{array}$ & $\begin{array}{l}1.5 \mathrm{E}-03 \\
1.3 \mathrm{E}-02 \\
5.3 \mathrm{E}-03 \\
6.5 \mathrm{E}-03 \\
4.1 \mathrm{E}-03 \\
1.9 \mathrm{E}-04 \\
7.3 \mathrm{E}-06 \\
3.8 \mathrm{E}-04\end{array}$ & $\begin{array}{l}\text { LLI Wall } \\
\text { LLI Wall } \\
\text { LLI Wall } \\
\text { LLI Wall } \\
\text { LLI Wall } \\
\text { Stomach Wall } \\
\text { Stomach Wall } \\
\text { Stomach Wall }\end{array}$ \\
\hline
\end{tabular}


TABLE 2.4. Maximum Organ Dose Rate Conversion Factors for Ingestion of Drinking Water (contd)

\begin{tabular}{|c|c|c|c|}
\hline Radionuclide & $\mathrm{mrem} / \mathrm{pCi}$ & $\mu \mathrm{Sv} / \mathrm{Bq}$ & Limiting Organ (a) \\
\hline $\begin{array}{l}{ }^{93} \mathrm{Mo}+\mathrm{D} \\
{ }^{93}{ }_{\mathrm{Mo}}(\mathrm{b}) \\
{ }^{99} \mathrm{Mo+D} \\
{ }^{99} \mathrm{Mo}\end{array}$ & $\begin{array}{l}3.4 \mathrm{E}-06 \\
6.7 \mathrm{E}-06 \\
6.4 \mathrm{E}-06 \\
1.1 \mathrm{E}-05\end{array}$ & $\begin{array}{l}9.0 \mathrm{E}-04 \\
1.8 \mathrm{E}-03 \\
1.7 \mathrm{E}-03 \\
3.0 \mathrm{E}-03\end{array}$ & $\begin{array}{l}\text { Liver } \\
\text { Liver } \\
\text { LLI Wall } \\
\text { LLI Wall }\end{array}$ \\
\hline $\begin{array}{l}{ }^{9911} \mathrm{Tc} \\
{ }^{99} \mathrm{Tc} \\
{ }^{161} \mathrm{TC} \\
\mathrm{TC}\end{array}$ & $\begin{array}{l}4.1 E-07 \\
2.7 E-05 \\
5.7 E-07\end{array}$ & $\begin{array}{l}1.1 \mathrm{E}-04 \\
7.3 \mathrm{E}-03 \\
1.5 \mathrm{E}-04\end{array}$ & $\begin{array}{l}\text { ULI Wall } \\
\text { Thyroid } \\
\text { ULI Wall }\end{array}$ \\
\hline 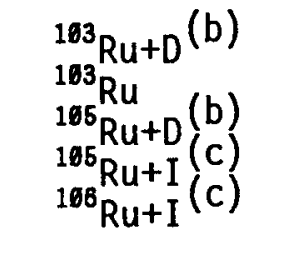 & $\begin{array}{l}1.2 \mathrm{E}-05 \\
2.4 \mathrm{E}-05 \\
6.7 \mathrm{E}-06 \\
7.0 \mathrm{E}-06 \\
1.3 \mathrm{E}-04\end{array}$ & $\begin{array}{l}3.2 \mathrm{E}-03 \\
6.5 \mathrm{E}-03 \\
1.8 \mathrm{E}-03 \\
1.9 \mathrm{E}-03 \\
3.5 \mathrm{E}-02\end{array}$ & $\begin{array}{l}\text { LLI Wall } \\
\text { LLI Wall } \\
\text { LLI Wall } \\
\text { ULI Wall } \\
\text { LLI Wall }\end{array}$ \\
\hline $\begin{array}{l}{ }^{103 !} \mathrm{Rh} \\
{ }^{105} \mathrm{Rh}\end{array}$ & $\begin{array}{l}9.6 \mathrm{E}-08 \\
1.4 \mathrm{E}-05\end{array}$ & $\begin{array}{l}2.6 \mathrm{E}-05 \\
3.8 \mathrm{E}-03\end{array}$ & $\begin{array}{l}\text { Stomach Wall } \\
\text { LLI WaIl }\end{array}$ \\
\hline $\begin{array}{l}{ }_{103}^{103} \mathrm{Pd}+\mathrm{D} \\
{ }_{103} \mathrm{Pd} \\
{ }_{107} \mathrm{Pd} \\
{ }^{169} \mathrm{Pd}+\mathrm{I} \\
{ }^{2}(\mathrm{c})\end{array}$ & $\begin{array}{l}4.3 \mathrm{E}-06 \\
8.5 \mathrm{E}-06 \\
1.7 \mathrm{E}-06 \\
1.0 \mathrm{E}-05\end{array}$ & $\begin{array}{l}1.1 \mathrm{E}-03 \\
2.3 \mathrm{E}-03 \\
4.6 \mathrm{E}-04 \\
2.8 \mathrm{E}-03\end{array}$ & $\begin{array}{l}\text { LLI Wall } \\
\text { LLI Wal1 } \\
\text { LLI Wal1 } \\
\text { LLI Wal1 }\end{array}$ \\
\hline${ }^{1116 \mathrm{n}} \mathrm{Ag}+\mathrm{I}(\mathrm{c})$ & $\begin{array}{l}1.3 \mathrm{E}-04 \\
5.5 \mathrm{E}-05\end{array}$ & $\begin{array}{l}3.4 \mathrm{E}-02 \\
1.5 \mathrm{E}-02\end{array}$ & $\begin{array}{l}\text { LLI Wal1 } \\
\text { LLI Wal1 }\end{array}$ \\
\hline $\begin{array}{l}{ }^{169} \mathrm{Cd} \\
{ }^{113 n} \mathrm{Cd} \\
{ }^{115 m} \mathrm{Cd}+\mathrm{D} \\
\left.{ }^{115} \mathrm{Cd}\right) \\
{ }^{115} \mathrm{Cd} \\
{ }^{15} \mathrm{Cd}\end{array}$ & $\begin{array}{l}1.5 \mathrm{E}-04 \\
2.1 \mathrm{E}-03 \\
5.0 \mathrm{E}-05 \\
1.0 \mathrm{E}-04 \\
5.7 \mathrm{E}-05\end{array}$ & $\begin{array}{l}4.1 \mathrm{E}-02 \\
5.7 \mathrm{E}-01 \\
1.4 \mathrm{E}-02 \\
2.7 \mathrm{E}-02 \\
1.5 \mathrm{E}-02\end{array}$ & $\begin{array}{l}\text { Kidneys } \\
\text { Kidneys } \\
\text { LLI Wall } \\
\text { LLI Wall } \\
\text { LLI Wall }\end{array}$ \\
\hline $\begin{array}{l}{ }_{114}^{111} \operatorname{In} \\
{ }_{115 n} \text { In+I }(c) \\
\text { In }\end{array}$ & $\begin{array}{l}7.4 \mathrm{E}-06 \\
8.2 \mathrm{E}-05 \\
2.1 \mathrm{E}-06\end{array}$ & $\begin{array}{l}2.0 \mathrm{E}-03 \\
2.2 \mathrm{E}-02 \\
5.7 \mathrm{E}-04\end{array}$ & $\begin{array}{l}\text { LLI Wall } \\
\text { LLI Wall } \\
\text { ULI Wall }\end{array}$ \\
\hline 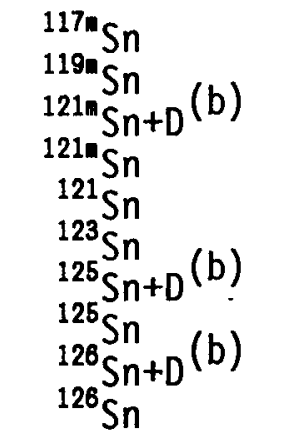 & $\begin{array}{l}3.0 \mathrm{E}-05 \\
1.5 \mathrm{E}-05 \\
1.3 \mathrm{E}-05 \\
1.6 \mathrm{E}-05 \\
8.9 \mathrm{E}-06 \\
9.6 \mathrm{E}-05 \\
7.2 \mathrm{E}-05 \\
1.3 \mathrm{E}-04 \\
7.9 \mathrm{E}-05 \\
1.6 \mathrm{E}-04\end{array}$ & $\begin{array}{l}8.1 \mathrm{E}-03 \\
4.1 \mathrm{E}-03 \\
3.5 \mathrm{E}-03 \\
4.3 \mathrm{E}-03 \\
2.4 \mathrm{E}-03 \\
2.6 \mathrm{E}-02 \\
1.9 \mathrm{E}-02 \\
3.5 \mathrm{E}-02 \\
2.1 \mathrm{E}-02 \\
4.3 \mathrm{E}-02\end{array}$ & $\begin{array}{l}\text { LLI Wal1 } \\
\text { LLI Wal1 } \\
\text { LLI Wal1 } \\
\text { LLI Wal1 } \\
\text { LLI Wal1 } \\
\text { LLI Wal1 } \\
\text { LLI Wall } \\
\text { LLI Wal1 } \\
\text { LLI Wal1 } \\
\text { LLI Wall }\end{array}$ \\
\hline
\end{tabular}


TABLE 2.4. Maximum Organ Dose Rate Conversion Factors for Ingestion of Drinking Water (contd)

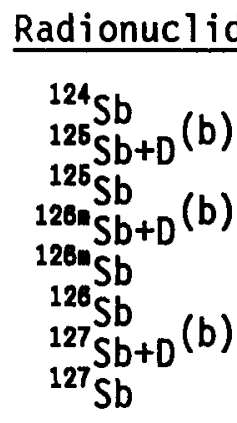

$\underline{\mathrm{mrem} / \mathrm{pCi}} \quad \underline{\mu \mathrm{Sv} / \mathrm{Bq}}$

8.5E-05

2.3E-05

$2.4 \mathrm{E}-05$

8.7E-06

9.6E-07

$7.0 \mathrm{E}-05$

4.0E-05

$7.4 \mathrm{E}-05$

9.2E-05

$4.9 \mathrm{E}-05$

$3.9 \mathrm{E}-05$

$7.8 \mathrm{E}-05$

$4.8 \mathrm{E}-06$

1. $3 \mathrm{E}-03$

2.1E-03

1.5E-06

8.1E-04

1.4E-04

8.0E-04

3.3E-06

8.0E-05

1.5E-04

9.8E-05

1.3E-05

9. $9 \mathrm{E}-05$

3. $0 \mathrm{E}-06$

1. $9 \mathrm{E}-06$

2.1E-06

1.2E-03

8. $3 \mathrm{E}-03$

1.2E-04

1. $6 \mathrm{E}-03$

1. $6 \mathrm{E}-03$

1.0E-05

1.5E-04

3. $0 \mathrm{E}-04$

2.1E-06

2. $4 \mathrm{E}-05$

5.2E-05

2.1E-07

4.1E-07

8.8E-05

7.8E-06

1. $4 \mathrm{E}-05$
2.3E-02

6.1E-03

6.5E-03

2.3E-03

2.6E-04

1.9E-02

1.1E-02

2. $0 \mathrm{E}-02$

2.5E-02

1.3E-02

1.1E-02

2.1E-02

1.3E-03

3.5E-01

5.7E-01

4.1E-04

2.2E-01

3.8E-02

2.1E-01

8.9E-04

2.2E-02

4.1E-02

2.6E-02

3. $5 \mathrm{E}-03$

2.7E-02

8.1E-04

5.1E-04

5.7E-04

3. $2 \mathrm{E}-01$

$2.2 \mathrm{E}+00$

3.2E-02

4.3E-01

4.3E-01

2.7E-03

4.0E-02

8.1E-02

5.7E-04

6.5E-03

1. $4 \mathrm{E}-02$

5.5E-05

1.1E-04

2. $4 \mathrm{E}-02$

2.1E-03

3. $8 \mathrm{E}-03$
Limiting $\operatorname{Organ}(a)$

LLI Wall

LLI Wall

LLI Wall

LLI Wall

Stomach Wall

LLI Wall

LLI Wal1

LLI Wall

Bone Surface

Bone Surface

Bone Surface

Bone Surface

LLI Wall

Testes

Testes

Stomach Wall

Thyroid

Thyroid

Thyroid

Thyroid

Thyroid

Thyroid

Thyroid

Thyroid

Thyroid

Thyroid

Thyroid

Thyroid

Thyroid

Thyroid

Thyroid

Thyroid

Thyroid

Thyroid

Thyroid

Thyroid

Stomach Wall

Thyroid

Thyroid

Stomach Wall

Stomach Wall

SI Wall

SI Wall

SI Wall 
TABLE 2.4. Maximum Organ Dose Rate Conversion Factors for Ingestion of Drinking Water (contd)

\begin{tabular}{|c|c|c|c|}
\hline Radionuclide & mrem/pCi & $\mu \mathrm{Sv} / \mathrm{Bq}$ & Limiting Organ (a) \\
\hline $\begin{array}{l}{ }^{137} \mathrm{Cs}+\mathrm{I} \\
{ }^{138} \mathrm{Cs}\end{array}$ & $\begin{array}{l}2.9 E-05 \\
2.7 E-06\end{array}$ & $\begin{array}{l}7.9 \mathrm{E}-03 \\
7.3 \mathrm{E}-04\end{array}$ & $\begin{array}{l}\text { SI Wall } \\
\text { Stomach Wall }\end{array}$ \\
\hline $\begin{array}{l}{ }^{139} \mathrm{Ba} \\
{ }^{140} \mathrm{Ba}+\mathrm{D} \\
{ }^{140} \mathrm{Ba} \\
{ }^{141} \mathrm{Ba}+\mathrm{b}(\mathrm{b}) \\
{ }^{141} \mathrm{Ba} \\
{ }^{142} \mathrm{Ba}+\mathrm{D} \\
{ }^{142} \mathrm{Ba}\end{array}$ & $\begin{array}{l}2.6 \mathrm{E}-06 \\
8.0 \mathrm{E}-05 \\
9.6 \mathrm{E}-05 \\
1.3 \mathrm{E}-05 \\
1.4 \mathrm{E}-06 \\
2.0 \mathrm{E}-06 \\
7.4 \mathrm{E}-07\end{array}$ & $\begin{array}{l}7.0 \mathrm{E}-04 \\
2.2 \mathrm{E}-02 \\
2.6 \mathrm{E}-02 \\
3.5 \mathrm{E}-03 \\
3.8 \mathrm{E}-04 \\
5.4 \mathrm{E}-04 \\
2.0 \mathrm{E}-04\end{array}$ & $\begin{array}{l}\text { Stomach Wall } \\
\text { LLI Wal1 } \\
\text { LLI Wal1 } \\
\text { LLI Wall } \\
\text { Stomach Wall } \\
\text { Stomach Wal1 } \\
\text { Stomach Wall }\end{array}$ \\
\hline $\begin{array}{l}{ }_{141}^{140} \mathrm{La} \\
{ }_{141} \mathrm{La}+\mathrm{D} \\
{ }^{142} \mathrm{La} \\
{ }^{\mathrm{La}}\end{array}$ & $\begin{array}{l}6.3 \mathrm{E}-05 \\
1.9 \mathrm{E}-05 \\
9.6 \mathrm{E}-06 \\
3.3 \mathrm{E}-06\end{array}$ & $\begin{array}{l}1.7 \mathrm{E}-02 \\
5.1 \mathrm{E}-03 \\
2.6 \mathrm{E}-03 \\
8.9 \mathrm{E}-04\end{array}$ & $\begin{array}{l}\text { LLI Wall } \\
\text { LLI Wal1 } \\
\text { ULI Wal1 } \\
\text { Stomach Wall }\end{array}$ \\
\hline $\begin{array}{l}{ }^{141} \mathrm{Ce} \\
{ }_{143} \mathrm{Ce}+\mathrm{D} \\
{ }^{143} \mathrm{Ce} \\
\left.{ }^{144} \mathrm{Ce}+\mathrm{D}\right) \\
{ }^{144} \mathrm{Ce}\end{array}$ & $\begin{array}{l}3.2 E-05 \\
5.0 E-05 \\
4.4 E-05 \\
1.2 E-04 \\
2.5 E-04\end{array}$ & $\begin{array}{l}8.6 \mathrm{E}-03 \\
1.4 \mathrm{E}-02 \\
1.2 \mathrm{E}-02 \\
3.4 \mathrm{E}-02 \\
6.8 \mathrm{E}-02\end{array}$ & $\begin{array}{l}\text { LLI Wa11 } \\
\text { LLI Wal1 } \\
\text { LLI } \text { Wal1 } \\
\text { LLI Wal1 } \\
\text { LLI Wal1 }\end{array}$ \\
\hline $\begin{array}{l}{ }_{143}^{143} \mathrm{Pr} \\
{ }_{144} \mathrm{Pr}+\mathrm{D} \\
{ }_{144 m} \mathrm{Pr} \\
{ }^{144} \mathrm{Pr}\end{array}$ & $\begin{array}{l}5.5 \mathrm{E}-05 \\
1.0 \mathrm{E}-06 \\
5.5 \mathrm{E}-07 \\
1.6 \mathrm{E}-06\end{array}$ & $\begin{array}{l}1.5 E-02 \\
2.8 E-04 \\
1.5 E-04 \\
4.3 E-04\end{array}$ & $\begin{array}{l}\text { LLI Wall } \\
\text { Stomach Wall } \\
\text { Stomach Wall } \\
\text { Stomach Wall }\end{array}$ \\
\hline 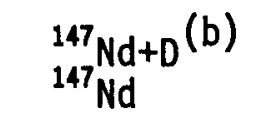 & $\begin{array}{l}2.8 \mathrm{E}-05 \\
4.4 \mathrm{E}-05\end{array}$ & $\begin{array}{l}7.6 \mathrm{E}-03 \\
1.2 \mathrm{E}-02\end{array}$ & $\begin{array}{l}\text { LLI Wall } \\
\text { LLI Wal1 }\end{array}$ \\
\hline $\begin{array}{l}{ }^{147} \mathrm{Pm} \\
{ }^{148 m} \mathrm{Pm}+\mathrm{D} \\
{ }^{148 m} \mathrm{Pm} \\
{ }^{148} \mathrm{Pm} \\
{ }^{149} \mathrm{Pm} \\
{ }^{161} \mathrm{Pm} \\
{ }_{\mathrm{Pm}}\end{array}$ & $\begin{array}{l}1.2 \mathrm{E}-05 \\
5.5 \mathrm{E}-05 \\
5.2 \mathrm{E}-05 \\
1.2 \mathrm{E}-04 \\
4.4 \mathrm{E}-05 \\
2.7 \mathrm{E}-05\end{array}$ & $\begin{array}{l}3.2 \mathrm{E}-03 \\
1.5 \mathrm{E}-02 \\
1.4 \mathrm{E}-02 \\
3.2 \mathrm{E}-02 \\
1.2 \mathrm{E}-02 \\
7.3 \mathrm{E}-03\end{array}$ & $\begin{array}{l}\text { LLI Wal1 } \\
\text { LLI Wal1 } \\
\text { LLI Wal1 } \\
\text { LLI Wal1 } \\
\text { LLI Wal1 } \\
\text { LLI Wal1 }\end{array}$ \\
\hline $\begin{array}{l}{ }^{147} \mathrm{Sm} \\
{ }^{151} \mathrm{Sm} \\
{ }^{15 m}\end{array}$ & $\begin{array}{l}3.2 E-03 \\
3.7 E-06 \\
3.0 E-05\end{array}$ & $\begin{array}{l}8.6 E-01 \\
1.0 E-03 \\
8.1 E-03\end{array}$ & $\begin{array}{l}\text { Bone Surface } \\
\text { LLI Wal1 } \\
\text { LLI Wal1 }\end{array}$ \\
\hline $\begin{array}{l}{ }^{152} \mathrm{Eu} \\
{ }^{154} \mathrm{Eu} \\
{ }_{155} \mathrm{Eu} \\
{ }^{156} \mathrm{Eu}\end{array}$ & $\begin{array}{l}3.7 E-05 \\
6.7 E-05 \\
1.3 E-05 \\
8.5 E-05\end{array}$ & $\begin{array}{l}1.0 \mathrm{E}-02 \\
1.8 \mathrm{E}-02 \\
3.5 \mathrm{E}-03 \\
2.3 \mathrm{E}-02\end{array}$ & $\begin{array}{ll}\text { LLI } & \text { Wal1 } \\
\text { LLI } & \text { Wal1 } \\
\text { LLI } & \text { Wal1 } \\
\text { LLI } & \text { Wall }\end{array}$ \\
\hline${ }^{153} \mathrm{Gd}$ & $1.0 \mathrm{E}-05$ & $2.7 E-03$ & LLI Wall \\
\hline
\end{tabular}


TABLE 2.4. Maximum Organ Dose Rate Conversion Factors for Ingestion of Drinking Water (contd)

\begin{tabular}{|c|c|c|c|}
\hline Radionuclide & $\mathrm{mrem} / \mathrm{pC} \mathrm{i}$ & $\mu \mathrm{Sv} / \mathrm{Bq}$ & Limiting Organ(a) \\
\hline${ }^{168} \mathrm{~Tb}$ & $5.5 \mathrm{E}-05$ & $1.5 \mathrm{E}-02$ & LLI Wall \\
\hline${ }^{166 m_{\mathrm{HO}}}$ & $4.1 \mathrm{E}-05$ & $1.1 \mathrm{E}-02$ & LLI Wall \\
\hline $\begin{array}{l}181 \mathrm{~W} \\
185 \mathrm{~W} \\
187 \mathrm{~W}\end{array}$ & $\begin{array}{l}1.9 \mathrm{E}-06 \\
1.7 \mathrm{E}-05 \\
1.7 \mathrm{E}-05\end{array}$ & $\begin{array}{l}5.1 \mathrm{E}-04 \\
4.6 \mathrm{E}-03 \\
4.6 \mathrm{E}-03\end{array}$ & $\begin{array}{l}\text { LLI Wa11 } \\
\text { LLI WaI1 } \\
\text { LLI Wal1 }\end{array}$ \\
\hline${ }^{187} \operatorname{Re}$ & $9.0 \mathrm{E}-08$ & $2.4 \mathrm{E}-05$ & ULI Wall \\
\hline $\begin{array}{l}{ }^{185} 0 \mathrm{~s} \\
{ }^{191} 0 \mathrm{~s}\end{array}$ & $\begin{array}{l}1.0 \mathrm{E}-05 \\
2.4 \mathrm{E}-05\end{array}$ & $\begin{array}{l}2.7 \mathrm{E}-03 \\
6.5 \mathrm{E}-03\end{array}$ & $\begin{array}{l}\text { LLI Wall } \\
\text { LLI Wall }\end{array}$ \\
\hline${ }^{192} \mathrm{Ir}$ & $4.8 \mathrm{E}-05$ & $1.3 \mathrm{E}-02$ & LLI WaTl \\
\hline${ }^{263} \mathrm{Hg}$ & $7.0 \mathrm{E}-05$ & $1.9 \mathrm{E}-02$ & Kidneys \\
\hline $\begin{array}{l}{ }^{216} \mathrm{~Pb}+\mathrm{D} \\
{ }^{216} \mathrm{~Pb} \\
{ }^{212} \mathrm{~Pb} \\
{ }^{212} \mathrm{~Pb}+\mathrm{Db}\end{array}$ & $\begin{array}{l}2.7 E-02 \\
8.0 E-02 \\
2.2 E-04 \\
6.7 E-04\end{array}$ & $\begin{array}{l}7.2 \mathrm{E}+00 \\
2.2 \mathrm{E}+01 \\
6.0 \mathrm{E}-02 \\
1.8 \mathrm{E}-01\end{array}$ & $\begin{array}{l}\text { Bone Surface } \\
\text { Bone Surface } \\
\text { Bone Surface } \\
\text { Bone Surface }\end{array}$ \\
\hline $\begin{array}{l}{ }^{216} \mathrm{Bi}+\mathrm{D} \\
{ }^{216} \mathrm{Bi}^{(b)} \\
{ }^{212} \mathrm{Bi}+\mathrm{I}\end{array}$ & $\begin{array}{l}8.0 \mathrm{E}-03 \\
5.5 \mathrm{E}-05 \\
2.9 \mathrm{E}-06\end{array}$ & $\begin{array}{l}2.2 \mathrm{E}+00 \\
1.5 \mathrm{E}-02 \\
8.0 \mathrm{E}-04\end{array}$ & $\begin{array}{l}\text { Spleen } \\
\text { LLI Wall } \\
\text { Stomach Wall }\end{array}$ \\
\hline${ }^{218} \mathrm{Po}$ & $1.6 \mathrm{E}-02$ & $4.3 E+00$ & Spleen \\
\hline 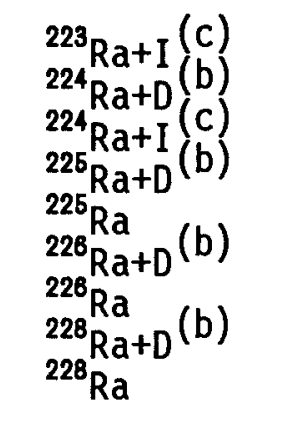 & $\begin{array}{l}6.8 \mathrm{E}-04 \\
3.2 \mathrm{E}-04 \\
1.2 \mathrm{E}-03 \\
8.4 \mathrm{E}-04 \\
5.6 \mathrm{E}-03 \\
1.1 \mathrm{E}-02 \\
2.3 \mathrm{E}-02 \\
3.3 \mathrm{E}-03 \\
2.0 \mathrm{E}-02\end{array}$ & $\begin{array}{l}1.8 \mathrm{E}-01 \\
8.6 \mathrm{E}-02 \\
3.2 \mathrm{E}-01 \\
2.3 \mathrm{E}-01 \\
1.5 \mathrm{E}+00 \\
3.0 \mathrm{E}+00 \\
6.2 \mathrm{E}+00 \\
9.0 \mathrm{E}-01 \\
5.4 \mathrm{E}+00\end{array}$ & $\begin{array}{l}\text { Bone Surface } \\
\text { Bone Surface } \\
\text { Bone Surface } \\
\text { Bone Surface } \\
\text { Bone Surface } \\
\text { Bone Surface } \\
\text { Bone Surface } \\
\text { Bone Surface } \\
\text { Bone Surface }\end{array}$ \\
\hline $\begin{array}{l}{ }^{225} \mathrm{Ac}+\mathrm{I}(\mathrm{c}) \\
{ }^{227} \mathrm{Ac}(\mathrm{b}) \\
{ }^{227} \mathrm{Ac} \\
{ }^{228} \mathrm{Ac}(\mathrm{b}) \\
{ }^{228} \mathrm{Ac}\end{array}$ & $\begin{array}{l}1.7 \mathrm{E}-04 \\
3.1 \mathrm{E}-02 \\
2.5 \mathrm{E}-01 \\
1.4 \mathrm{E}-03 \\
1.1 \mathrm{E}-05\end{array}$ & $\begin{array}{l}4.5 \mathrm{E}-02 \\
8.4 \mathrm{E}+00 \\
6.8 \mathrm{E}+01 \\
3.7 \mathrm{E}-01 \\
3.0 \mathrm{E}-03\end{array}$ & $\begin{array}{l}\text { LLI Wall } \\
\text { Bone Surface } \\
\text { Bone Surface } \\
\text { Bone Surface } \\
\text { Bone Surface }\end{array}$ \\
\hline $\begin{array}{l}{ }^{227} T h+D(b) \\
{ }^{227} T h \\
{ }^{228} T h+D \\
T(b)\end{array}$ & $\begin{array}{l}6.1 \mathrm{E}-04 \\
3.4 \mathrm{E}-04 \\
1.6 \mathrm{E}-03\end{array}$ & $\begin{array}{l}1.7 \mathrm{E}-01 \\
9.2 \mathrm{E}-02 \\
4.2 \mathrm{E}-01\end{array}$ & $\begin{array}{l}\text { Bone Surface } \\
\text { LLI Wall } \\
\text { Bone Surface }\end{array}$ \\
\hline
\end{tabular}


TABLE 2.4. Maximum Organ Dose Rate Conversion Factors for Ingestion of Drinking Water (contd)

\begin{tabular}{|c|c|c|c|}
\hline Radionuclide & mrem/pCi & $\mu \mathrm{Sv} / \mathrm{Bq}$ & Limiting Organ (a) \\
\hline 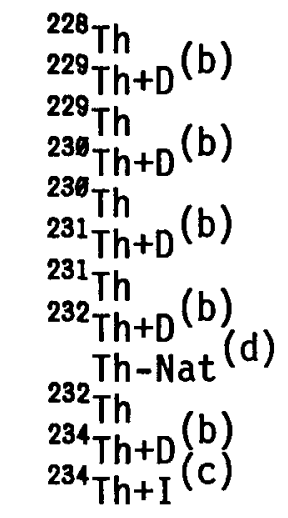 & $\begin{array}{l}8.9 \mathrm{E}-03 \\
1.2 \mathrm{E}-02 \\
8.8 \mathrm{E}-02 \\
1.2 \mathrm{E}-02 \\
1.3 \mathrm{E}-02 \\
1.3 \mathrm{E}-06 \\
1.3 \mathrm{E}-05 \\
9.9 \mathrm{E}-03 \\
9.9 \mathrm{E}-03 \\
6.9 \mathrm{E}-02 \\
8.0 \mathrm{E}-05 \\
1.6 \mathrm{E}-04\end{array}$ & $\begin{array}{l}2.4 \mathrm{E}+00 \\
3.2 \mathrm{E}+00 \\
2.4 \mathrm{E}+01 \\
3.2 \mathrm{E}+00 \\
3.5 \mathrm{E}+00 \\
3.5 \mathrm{E}-04 \\
3.5 \mathrm{E}-03 \\
2.7 \mathrm{E}+00 \\
2.7 \mathrm{E}+00 \\
1.9 \mathrm{E}+01 \\
2.2 \mathrm{E}-02 \\
4.3 \mathrm{E}-02\end{array}$ & $\begin{array}{l}\text { Bone Surface } \\
\text { Bone Surface } \\
\text { Bone Surface } \\
\text { Bone Surface } \\
\text { Bone Surface } \\
\text { LLI Wall } \\
\text { LLI Wall } \\
\text { Bone Surface } \\
\text { Bone Surface } \\
\text { Bone Surface } \\
\text { LLI Wall } \\
\text { LLI Wall }\end{array}$ \\
\hline $\begin{array}{l}{ }^{231}{ }^{231} \mathrm{~Pa}+\mathrm{D}^{(\mathrm{b})} \\
{ }^{233} \mathrm{~Pa} \\
{ }^{234} \mathrm{~Pa} \\
{ }^{23 a}\end{array}$ & $\begin{array}{l}5.8 E-02 \\
2.7 E-01 \\
3.7 E-05 \\
1.2 E-05\end{array}$ & $\begin{array}{l}1.6 \mathrm{E}+01 \\
7.3 \mathrm{E}+01 \\
1.0 \mathrm{E}-02 \\
3.2 \mathrm{E}-03\end{array}$ & $\begin{array}{l}\text { Bone Surface } \\
\text { Bone Surface } \\
\text { LLI Wall } \\
\text { ULI Wall }\end{array}$ \\
\hline 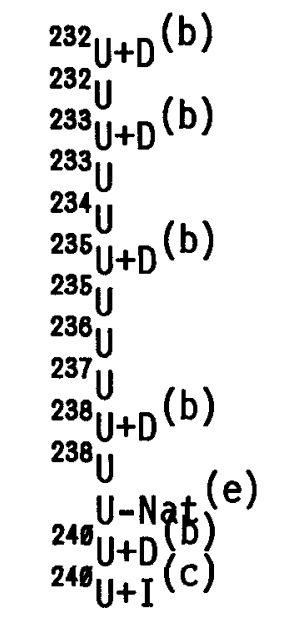 & $\begin{array}{l}1.4 \mathrm{E}-04 \\
9.7 \mathrm{E}-04 \\
1.0 \mathrm{E}-02 \\
1.9 \mathrm{E}-04 \\
1.9 \mathrm{E}-04 \\
1.0 \mathrm{E}-04 \\
2.0 \mathrm{E}-04 \\
1.7 \mathrm{E}-04 \\
3.3 \mathrm{E}-05 \\
1.1 \mathrm{E}-04 \\
1.7 \mathrm{E}-04 \\
1.8 \mathrm{E}-04 \\
1.2 \mathrm{E}-05 \\
3.7 \mathrm{E}-05\end{array}$ & $\begin{array}{l}3.7 \mathrm{E}-02 \\
2.6 \mathrm{E}-01 \\
2.8 \mathrm{E}+00 \\
5.1 \mathrm{E}-02 \\
5.1 \mathrm{E}-02 \\
2.8 \mathrm{E}-02 \\
5.4 \mathrm{E}-02 \\
4.6 \mathrm{E}-02 \\
8.9 \mathrm{E}-03 \\
3.0 \mathrm{E}-02 \\
4.6 \mathrm{E}-02 \\
4.9 \mathrm{E}-02 \\
3.3 \mathrm{E}-03 \\
1.0 \mathrm{E}-02\end{array}$ & $\begin{array}{l}\text { Bone Surface } \\
\text { Bone Surface } \\
\text { Bone Surface } \\
\text { LLI Wal1 } \\
\text { LLI Wall } \\
\text { LLI Wall } \\
\text { LLI Wall } \\
\text { LLI Wall } \\
\text { LLI Wall } \\
\text { LLI Wall } \\
\text { LLI Wal1 } \\
\text { LLI Wal1 } \\
\text { LLI Wal1 } \\
\text { LLI Wal1 }\end{array}$ \\
\hline $\begin{array}{l}{ }^{237} \mathrm{Np+D}(b) \\
{ }^{237} \mathrm{~Np} \\
238{ }_{N p+D}(b) \\
238{ }_{N p}(b) \\
{ }^{239}{ }_{N p+D}(b) \\
{ }_{N p}\end{array}$ & $\begin{array}{l}6.0 \mathrm{E}-02 \\
1.2 \mathrm{E}-01 \\
2.9 \mathrm{E}-03 \\
3.4 \mathrm{E}-05 \\
1.7 \mathrm{E}-05 \\
3.3 \mathrm{E}-05\end{array}$ & $\begin{array}{l}1.6 \mathrm{E}+01 \\
3.2 \mathrm{E}+01 \\
8.0 \mathrm{E}-01 \\
9.2 \mathrm{E}-03 \\
4.5 \mathrm{E}-03 \\
8.9 \mathrm{E}-03\end{array}$ & $\begin{array}{l}\text { Bone Surface } \\
\text { Bone Surface } \\
\text { Bone Surface } \\
\text { LLI Wall } \\
\text { LLI Wall } \\
\text { LLI Wall }\end{array}$ \\
\hline $\begin{array}{l}{ }^{236} \mathrm{Pu} \\
{ }^{237} \mathrm{Pu} \\
{ }^{238} \mathrm{Pu} \\
{ }^{239} \mathrm{Pu} \\
{ }^{248} \mathrm{Pu} \\
{ }^{241} \mathrm{Pu}+\mathrm{D} \\
{ }^{241} \mathrm{Pu}\end{array}$ & $\begin{array}{l}7.8 \mathrm{E}-04 \\
3.7 \mathrm{E}-06 \\
5.9 \mathrm{E}-03 \\
6.7 \mathrm{E}-03 \\
6.7 \mathrm{E}-03 \\
3.4 \mathrm{E}-02 \\
1.3 \mathrm{E}-04\end{array}$ & $\begin{array}{l}2.1 \mathrm{E}-01 \\
1.0 \mathrm{E}-03 \\
1.6 \mathrm{E}+00 \\
1.8 \mathrm{E}+00 \\
1.8 \mathrm{E}+00 \\
9.2 \mathrm{E}+00 \\
3.5 \mathrm{E}-02\end{array}$ & $\begin{array}{l}\text { Bone Surface } \\
\text { LLI Wall } \\
\text { Bone Surface } \\
\text { Bone Surface } \\
\text { Bone Surface } \\
\text { Bone Surface } \\
\text { Bone Surface }\end{array}$ \\
\hline
\end{tabular}


TABLE 2.4. Maximum Organ Dose Rate Conversion Factors for Ingestion of Drinking Water (contd)

\begin{tabular}{|c|c|c|c|}
\hline Radionuclide & mrem/pCi & $\mu \mathrm{Sv} / \mathrm{Bq}$ & Limiting $\operatorname{Organ}(a)$ \\
\hline $\begin{array}{l}{ }^{242} \mathrm{Pu}^{243} \mathrm{Pu+D} \\
{ }_{243} \mathrm{Pu} \\
\left.{ }_{244} \mathrm{Pu}^{2} \mathrm{~b}\right) \\
{ }^{244} \mathrm{Pu}\end{array}$ & $\begin{array}{l}6.1 \mathrm{E}-03 \\
1.0 \mathrm{E}-06 \\
2.1 \mathrm{E}-06 \\
1.5 \mathrm{E}-03 \\
6.1 \mathrm{E}-03\end{array}$ & $\begin{array}{l}1.6 \mathrm{E}+00 \\
2.8 \mathrm{E}-04 \\
5.7 \mathrm{E}-04 \\
4.1 \mathrm{E}-01 \\
1.6 \mathrm{E}+00\end{array}$ & $\begin{array}{l}\text { Bone Surface } \\
\text { ULI Wall } \\
\text { ULI Wall } \\
\text { Bone Surface } \\
\text { Bone Surface }\end{array}$ \\
\hline $\begin{array}{l}{ }^{241} \mathrm{Am} \\
{ }^{242 \times} \mathrm{Am}+\mathrm{D} \\
{ }^{2424} \mathrm{Am} \\
{ }^{242} \mathrm{Am}+\mathrm{D} \\
{ }^{242} \mathrm{Am} \\
{ }^{243} \mathrm{Am}+\mathrm{D} \\
{ }^{243} \mathrm{Am}\end{array}$ & $\begin{array}{l}6.7 \mathrm{E}-02 \\
1.9 \mathrm{E}-02 \\
6.4 \mathrm{E}-02 \\
2.2 \mathrm{E}-03 \\
9.6 \mathrm{E}-06 \\
2.2 \mathrm{E}-02 \\
6.7 \mathrm{E}-02\end{array}$ & $\begin{array}{l}1.8 \mathrm{E}+01 \\
5.0 \mathrm{E}+00 \\
1.7 \mathrm{E}+01 \\
6.0 \mathrm{E}-01 \\
2.6 \mathrm{E}-03 \\
6.0 \mathrm{E}+00 \\
1.8 \mathrm{E}+01\end{array}$ & $\begin{array}{l}\text { Bone Surface } \\
\text { Bone Surface } \\
\text { Bone Surface } \\
\text { Bone Surface } \\
\text { LLI Wall } \\
\text { Bone Surface } \\
\text { Bone Surface }\end{array}$ \\
\hline $\begin{array}{l}{ }^{242} \mathrm{Cm}+\mathrm{D} \\
{ }^{242} \mathrm{Cm} \\
{ }^{243} \mathrm{Cm} \\
{ }^{244} \mathrm{Cm} \text {-D } \\
{ }^{244} \mathrm{Cm} \\
{ }^{245} \mathrm{Cm} \text { (b) } \\
{ }^{245} \mathrm{Cm} \\
{ }^{246} \mathrm{Cm} \\
{ }^{247} \mathrm{Cm} \\
{ }^{247} \mathrm{Cm}+\mathrm{D} \\
{ }^{248} \mathrm{Cm} \\
\mathrm{Cm}\end{array}$ & $\begin{array}{l}4.1 \mathrm{E}-03 \\
1.6 \mathrm{E}-03 \\
5.2 \mathrm{E}-02 \\
1.8 \mathrm{E}-02 \\
3.6 \mathrm{E}-02 \\
4.7 \mathrm{E}-02 \\
6.8 \mathrm{E}-02 \\
7.0 \mathrm{E}-02 \\
3.2 \mathrm{E}-02 \\
6.4 \mathrm{E}-02 \\
2.5 \mathrm{E}-01\end{array}$ & $\begin{array}{l}1.1 \mathrm{E}+00 \\
4.3 \mathrm{E}-01 \\
1.4 \mathrm{E}+01 \\
4.9 \mathrm{E}+00 \\
9.7 \mathrm{E}+00 \\
1.3 \mathrm{E}+01 \\
1.8 \mathrm{E}+01 \\
1.9 \mathrm{E}+01 \\
8.6 \mathrm{E}+00 \\
1.7 \mathrm{E}+01 \\
6.8 \mathrm{E}+01\end{array}$ & $\begin{array}{l}\text { Bone Surface } \\
\text { Bone Surface } \\
\text { Bone Surface } \\
\text { Bone Surface } \\
\text { Bone Surface } \\
\text { Bone Surface } \\
\text { Bone Surface } \\
\text { Bone Surface } \\
\text { Bone Surface } \\
\text { Bone Surface } \\
\text { Bone Surface }\end{array}$ \\
\hline${ }^{252} \mathrm{Cf}$ & $2.2 \mathrm{E}-02$ & $5.9 E+00$ & Bone Surface \\
\hline
\end{tabular}

(a) Where $\mathrm{Si}$ is Small Intestine, LLI is Lower Large Intestine, and ULI is Upper Large Intestine.

(b) Where $+D$ means "plus daughters in equilibrium" referring to explicit daughters, or daughters with half-lives greater than 10 minutes. Separate values are also included for parents and daughters so that combined factors for non-equilibrium cases can be determined. Note: in some cases $+D$ also includes implicit daughters (i.e., uranium and thorium decay chains.

(c) Where +I means "plus implicit daughters in equilibrium" referring to daughters with half-lives less than 10 minutes. The decay energies associated with these daughters have been assigned to the parent, and the appropriate branching ratios (described in Appendix $D$ ) have been considered.

(d) Where Th-Nat includes an equilibrium mixture of ${ }^{232}$ Th plus 10 daughters in the decay chain, as discussed in Appendix $D$.

(e) Where $1 \mathrm{Ci} \mathrm{U}$-Nat equals $48.9 \%{ }^{238} \mathrm{U}$ plus $48.9 \%{ }^{234} \mathrm{U}$ plus $2.25 \%{ }^{235} \mathrm{U}$. 


\subsubsection{Agricultural Pathways}

Food crops may become contaminated by radionuclides deposited directly on plant surfaces from the air or from irrigation water, or by radionuclides taken up from soil previously contaminated via air or water. Many factors must be considered when calculating doses or dose rates from ingestion of these foods. These factors account for the movement of radionuclides from soil to the receptor in a complex sequence (Soldat and Harr 1971; Baker et al. 1976).

Equations used to calculate concentrations in various plant food products (i.e., the $C_{i p}$ terms in Equation 2.1) are given in two parts: the first part accounts for direct deposition onto leaves and translocation to the edible parts of the plant, and the second accounts for long-term accumulation in the soil and consequent root uptake. The equations do not account for the rainsplash of soil onto plant surfaces or washing plant materials before ingestion. The concentration of radioactive material in vegetation resulting from direct deposition onto plant foliage and uptake of radionuclides previously deposited in the soil is determined by:

$c_{i v}=\left\{\frac{\left[d_{i}^{a}+d_{i}^{I}\right] r T_{v}\left[1-\exp \left[-\lambda_{E i} t\right]\right]}{Y_{v} \lambda_{E i}}+\frac{\left[d_{i}+d_{i}\right] f_{t i v}{ }_{i v}\left[1-\exp \left[-\lambda_{i} t_{b}\right]\right]}{p \lambda_{i}}\right.$

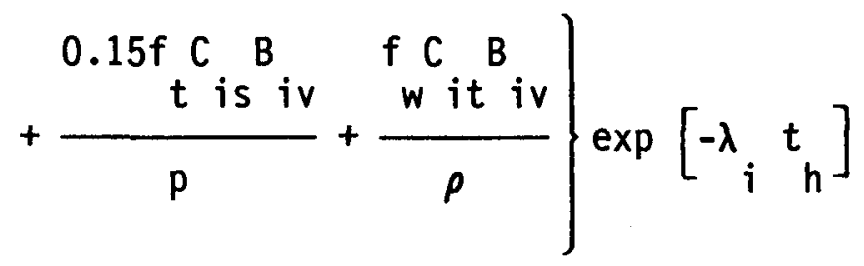

where $C_{i v}=\begin{aligned} & \text { concentration of radionuclide } i \text { in the edible portion of the } \\ & \text { vegetation, in } \mathrm{pCi} / \mathrm{kg}(\mathrm{Bq} / \mathrm{kg})\end{aligned}$

$$
\begin{aligned}
& d_{i}^{a}=\text { deposition rate of radionuclide } i \text {, in } \mathrm{pCi} / \mathrm{m}^{2}-\text { day }\left(\mathrm{Bq} / \mathrm{m}^{2}-\text { day }\right) \text { : } \\
& \quad d_{i}^{a}=86,400 \chi_{i} v_{d i}
\end{aligned}
$$


where $86,400=$ dimensional conversion factor, in sec/day

$\chi_{i}=\underset{\text { in } \mathrm{pC} \mathrm{i} / \mathrm{m}^{3}\left(\mathrm{~Bq} / \mathrm{m}^{3}\right)}{\text { a r a }}$

$V=$ deposition velocity of radionuclide $i$, in $\mathrm{m} / \mathrm{sec}$ di

$\mathrm{I}$
$\mathrm{i}$

$$
d_{i}^{I}=C_{i w} I
$$

where $C_{i w}=$ the concentration of radionuclide $i$ in the water used for irrigation, in $\mathrm{pCi} / \mathrm{L}(\mathrm{Bq} / \mathrm{L})$, and $\mathrm{I}$ is the irrigation rate (the amount of water sprinkled on a unit area of field in 1 day), in $\mathrm{L} / \mathrm{m}^{2}$ day

$r=$ fraction of initially deposited material retained on the vegetation (dimensionless)

$T_{v}=$ factor for translocation of externally deposited radionuclides to the edible parts of the vegetation (dimensionless). For simplicity, this parameter is assumed to be independent of the radionuclide and is assigned values of 1 for leafy vegetables and fresh forage, and 0.1 for all other produce, including grain.

$\lambda_{E i}=$ the effective removal constant for radionuclide $i$, in days -1 : $\lambda_{E i}=\lambda_{i}+\lambda_{W}$

where $\lambda_{i}=$ the radiological decay constant for radionuclide $i$, in days -1 , and $\lambda_{w}$ is the weathering removal constant for vegetation, days $^{-1}$, taken to be $(0.693 / 14$ ) days -1 (i.e., a weathering halftime of $14 \mathrm{~d}$ )

$t_{e}=t$ time of above-ground vegetation exposure to contamination during growing season, in days

$Y_{V}=$ vegetation yield, in $\mathrm{kg} / \mathrm{m}^{2}$

$f_{t}=$ fraction of the roots in the plow layer of soil (dimensionless)

$B_{i v}=$ concentration factor for uptake of radionuclide $i$ from the soil in vegetation $\mathrm{v}$, in $\mathrm{pCi} / \mathrm{kg}(\mathrm{Bq} / \mathrm{kg})$ wet weight $\mathrm{per} \mathrm{pCi} / \mathrm{kg}(\mathrm{Bq} / \mathrm{kg})$ dry soil

$t_{b}=$ time for buildup of radionuclides in the soil, in days 
$p=$ soil "surface density," in $\mathrm{kg}$ dry soil $/ \mathrm{m}^{2}$. A value of $224 \mathrm{~kg} / \mathrm{m}^{2}$ is used, assuming the contaminated ground is plowed to a depth of $15 \mathrm{~cm}$.

$0.15=$ thickness of plow layer

$C_{i s}=$ concentration of radionuclide $i$ in soil available for plant uptake from residual contamination in the soil plow layer (top $15 \mathrm{~cm}$ of soil), in $\mathrm{pCi} / \mathrm{m}^{2}\left(\mathrm{~Bq} / \mathrm{m}^{2}\right)$

$f_{W}=$ fraction of the roots that penetrate the residual contamination (dimensionless)

$C_{i t}=$ concentration of radionuclide $i$ available for plant uptake from subsurface contamination, in $\mathrm{pCi} / \mathrm{m}^{3}\left(\mathrm{~Bq} / \mathrm{m}^{3}\right)$

$\rho=$ bulk density of subsurface soil, in $\mathrm{kg} / \mathrm{m}^{3}$ (taken to be $1600 \mathrm{~kg} / \mathrm{m}^{3}$ )

$t_{h}=$ holdup time between harvest and food consumption, in days.

The first term inside the brackets of Equation 2.5 relates to the concentration resulting from direct deposition of airborne and irrigationborne material on foliage during the growing season. The second term relates to plant uptake from the soil of material previously deposited. The third and fourth terms account for uptake of radionuclides contained in the top $0.15 \mathrm{~m}$ of soil and below this layer, respectively. Specific values used for the parameters in Equation 2.5 are published in NRC (1977), Oztunali et. al. (1981), and Napier et al. (Vol. 1, 1988), and are discussed in Appendix B. These concentrations and intake rates by type of plant crop are used with ingestion dose conversion factors to perform ingestion dose calculations using equations similar to Equations 2.1 and 2.4 to obtain the committed effective dose equivalent.

The radionuclide concentration in animal products such as meat, milk, and eggs depends on the amount of contaminated forage or feed eaten by the animal. This concentration is described by:

$$
C_{i a}=S_{i a}\left[C_{i f} Q_{f}+C_{i a w} Q_{a w}\right]
$$

where $C_{i a}=$ concentration of radionuclide $i$ in the animal product, in $\mathrm{pCi} / \mathrm{kg}(\mathrm{Bq} / \mathrm{kg})$ or $\mathrm{pCi} / \mathrm{L}(\mathrm{Bq} / \mathrm{L})$

$S_{i a}=$ equilibrium transfer coefficient of radionuclide $i$ from daily intake of the animal to the edible portion of the animal product, in $\mathrm{pCi} / \mathrm{L}(\mathrm{Bq} / \mathrm{L}) \mathrm{milk}$, or $\mathrm{pCi} / \mathrm{kg}(\mathrm{Bq} / \mathrm{kg})$ meat or eggs, per $\mathrm{pCi} / \mathrm{d}(\mathrm{Bq} / \mathrm{d})$ intake by animal 


$$
\begin{aligned}
& C_{i f}=\text { concentration of radionuclide } i \text { in feed or forage, in } \mathrm{pCi} / \mathrm{kg} \\
& Q_{f}=\text { animal consumption rate of contaminated feed or forage, in }
\end{aligned}
$$

Specific values of the parameters used in Equation 2.6 are described in Napier et al. (Vol. 1, 1988) and obtained from Oztunali et al. (1981) and NRC (1977), as discussed in Appendix B. Committed effective dose conversion factors for the ingestion of agricultural food products are shown for over 200 radionuclides in Table 2.5. These factors account for both deposition of resuspended contaminated soil on leaves and root uptake from soil and do not account for rainsplash of soil onto plant surfaces. They are in units of mrem per year of exposure per $\mathrm{pCi} / \mathrm{g}$ in soil and $\mu \mathrm{Sv}$ per year of exposure per $\mathrm{Bq} / \mathrm{g}$ in soil. They are for ingestion of agricultural foods only and do not include other pathways such as external or inhalation exposure. The factors are based on ingestion of the total diet (NRC 1977) grown in contaminated soil, including vegetable produce and animal products. Scenario-specific estimates of the committed effective dose equivalent from ingestion of agricultural products can be obtained by multiplying the factors shown in Table 2.5 by the fraction of the total diet assumed to be grown on a site and the site-specific soil concentration of radionuclides. 
TABLE 2.5. Agricultural Food Product Ingestion Committed Effective Dose Rate Conversion Factors for Exposure to Residual Radioactive Materials (Air and Soil Contributions)

\begin{tabular}{|c|c|c|c|c|}
\hline Radionuclide & $\begin{array}{l}\text { mrem per } \\
\mathrm{pCi} / \mathrm{m}^{3}\end{array}$ & $\begin{array}{l}\mu \mathrm{SV} p \mathrm{per}^{3} \\
\mathrm{~Bq} / \mathrm{m}^{3}\end{array}$ & $\begin{array}{l}\text { mrem per } \\
\text { pCi } / g\end{array}$ & $\begin{array}{l}\mu \text { Sv per } \\
\mathrm{Bq} / \mathrm{g}\end{array}$ \\
\hline${ }^{3} \mathrm{H}$ & $2.3 \mathrm{E}-03$ & $6.2 \mathrm{E}-01$ & $0.0 \mathrm{E}+00$ & $0.0 \mathrm{E}+00$ \\
\hline${ }^{16} \mathrm{Be}$ & $5.7 \mathrm{E}-02$ & $1.5 \mathrm{E}+01$ & $3.7 E-04$ & $1.0 \mathrm{E}-01$ \\
\hline${ }^{14} \mathrm{C}$ & $7.2 \mathrm{E}-01$ & $1.9 E+02$ & $0.0 \mathrm{E}+00$ & $0.0 E+00$ \\
\hline${ }^{18} \mathrm{~F}$ & $7.8 \mathrm{E}-06$ & $2.1 E-03$ & $8.9 E-05$ & $2.4 \mathrm{E}-02$ \\
\hline${ }^{22} \mathrm{Na}$ & $\begin{array}{l}1.7 \mathrm{E}+00 \\
1.6 \mathrm{E}-03\end{array}$ & $\begin{array}{l}4.6 E+02 \\
4.3 E-01\end{array}$ & $\begin{array}{l}5.7 \mathrm{E}-01 \\
1.7 \mathrm{E}-02\end{array}$ & $\begin{array}{l}\text { 1. } 5 \mathrm{E}+02 \\
4.6 \mathrm{E}+00\end{array}$ \\
\hline $\begin{array}{l}{ }^{32} \mathrm{p} \\
{ }^{33} \mathrm{p}\end{array}$ & $\begin{array}{l}3.5 \mathrm{E}-01 \\
4.6 \mathrm{E}-02\end{array}$ & $\begin{array}{l}9.5 \mathrm{E}+01 \\
1.2 \mathrm{E}+01\end{array}$ & $\begin{array}{l}4.3 \mathrm{E}+00 \\
4.6 \mathrm{E}-01\end{array}$ & $\begin{array}{l}1.2 E+03 \\
1.2 E+02\end{array}$ \\
\hline${ }^{35} \mathrm{~S}$ & $2.6 \mathrm{E}-02$ & $7.0 E+00$ & $1.0 \mathrm{E}-01$ & 2.7E+01 \\
\hline${ }^{36} \mathrm{Cl}$ & 2.1E-01 & $5.7 E+01$ & $1.1 \mathrm{E}+01$ & $3.0 E+03$ \\
\hline${ }^{46} \mathrm{~K}$ & $5.9 \mathrm{E}-01$ & $1.6 \mathrm{E}+02$ & $3.2 E+00$ & $8.6 E+02$ \\
\hline $\begin{array}{l}{ }^{41} \mathrm{Ca} \\
{ }^{45} \mathrm{Ca}\end{array}$ & $\begin{array}{l}6.1 \mathrm{E}-02 \\
1.4 \mathrm{E}-01\end{array}$ & $\begin{array}{l}1.6 \mathrm{E}+01 \\
3.8 \mathrm{E}+01\end{array}$ & $\begin{array}{l}2.3 \mathrm{E}-02 \\
5.0 \mathrm{E}-02\end{array}$ & $\begin{array}{l}6.2 E+00 \\
1.3 E+01\end{array}$ \\
\hline${ }^{48} \mathrm{SC}$ & $6.9 \mathrm{E}-02$ & $1.9 E+01$ & $2.5 \mathrm{E}-03$ & $6.8 \mathrm{E}-01$ \\
\hline${ }^{51} \mathrm{Cr}$ & $1.8 \mathrm{E}-03$ & 4.9E-01 & $7.6 \mathrm{E}-06$ & 2.1E-03 \\
\hline $\begin{array}{l}{ }^{54} \mathrm{Mn} \\
{ }^{58} \mathrm{Mn}\end{array}$ & $\begin{array}{l}3.5 \mathrm{E}-02 \\
9.7 \mathrm{E}-05\end{array}$ & $\begin{array}{l}9.4 \mathrm{E}+00 \\
2.6 \mathrm{E}-02\end{array}$ & $\begin{array}{l}1.6 \mathrm{E}-02 \\
5.5 \mathrm{E}-03\end{array}$ & $\begin{array}{l}4.3 E+00 \\
1.5 E+00\end{array}$ \\
\hline $\begin{array}{l}{ }^{55} \mathrm{Fe} \\
{ }^{59} \mathrm{Fe}\end{array}$ & $\begin{array}{l}1.3 \mathrm{E}-02 \\
7.3 \mathrm{E}-02\end{array}$ & $\begin{array}{l}3.5 E+00 \\
2.0 E+01\end{array}$ & $\begin{array}{l}1.4 \mathrm{E}-04 \\
9.5 \mathrm{E}-04\end{array}$ & $\begin{array}{l}3.8 \mathrm{E}-02 \\
2.6 \mathrm{E}-01\end{array}$ \\
\hline $\begin{array}{l}{ }^{57} \mathrm{Co} \\
{ }^{58} \mathrm{CO} \\
{ }^{68} \mathrm{CO}\end{array}$ & $\begin{array}{l}1.2 \mathrm{E}-02 \\
3.9 \mathrm{E}-02 \\
1.8 \mathrm{E}-01\end{array}$ & $\begin{array}{l}3.2 E+00 \\
1.1 E+01 \\
4.9 E+01\end{array}$ & $\begin{array}{l}1.7 \mathrm{E}-03 \\
5.9 \mathrm{E}-03 \\
2.4 \mathrm{E}-02\end{array}$ & $\begin{array}{l}4.6 \mathrm{E}-01 \\
1.6 \mathrm{E}+00 \\
6.5 \mathrm{E}+00\end{array}$ \\
\hline $\begin{array}{l}{ }^{69} \mathrm{Ni} \\
{ }^{63} \mathrm{Ni} \\
{ }^{65} \mathrm{Ni}\end{array}$ & $\begin{array}{l}8.1 \mathrm{E}-03 \\
2.2 \mathrm{E}-02 \\
6.2 \mathrm{E}-05\end{array}$ & $\begin{array}{l}2.2 E+00 \\
5.9 E+00 \\
1.7 E-02\end{array}$ & $\begin{array}{l}1.9 \mathrm{E}-03 \\
5.1 \mathrm{E}-03 \\
2.3 \mathrm{E}-03\end{array}$ & $\begin{array}{l}5.1 \mathrm{E}-01 \\
1.4 \mathrm{E}+00 \\
6.2 \mathrm{E}-01\end{array}$ \\
\hline${ }^{84} \mathrm{Cu}$ & $2.8 \mathrm{E}-04$ & $7.6 \mathrm{E}-02$ & $1.2 \mathrm{E}-02$ & $3.2 E+00$ \\
\hline${ }^{85} \mathrm{Zn}$ & $1.4 E+00$ & $3.8 E+02$ & $3.8 E+00$ & $1.0 E+03$ \\
\hline
\end{tabular}


TABLE 2.5. Agricultural Food Product Ingestion Committed Effective Dose Rate Conversion Factors for Exposure to Residual Radioactive Materials (Air and Soil Contributions) (contd)

\begin{tabular}{|c|c|c|c|c|}
\hline \multirow[b]{2}{*}{ Radionuclide } & \multicolumn{2}{|c|}{ Air Deposition } & \multicolumn{2}{|c|}{ Soil Uptake } \\
\hline & $\begin{array}{l}\text { mrem per } \\
\mathrm{pCi} / \mathrm{m}^{3}\end{array}$ & $\begin{array}{c}\mu \mathrm{Sv} p e r \\
\mathrm{~Bq} / \mathrm{m}^{3} \\
\end{array}$ & $\begin{array}{l}\text { mrem per } \\
\mathrm{pCi} / \mathrm{g}\end{array}$ & $\begin{array}{c}\mu \mathrm{Sv} \text { per } \\
\mathrm{Bg} / \mathrm{g}\end{array}$ \\
\hline $\begin{array}{l}{ }^{69 m} \mathrm{Zn+D}(\mathrm{a}) \\
{ }^{69} \mathrm{Zn} \mathrm{Zn}\end{array}$ & $\begin{array}{l}6.5 E-04 \\
1.2 E-03 \\
3.2 E-06\end{array}$ & $\begin{array}{l}1.8 E-01 \\
3.2 E-01 \\
8.6 E-04\end{array}$ & $\begin{array}{l}6.5 E-02 \\
1.2 E-01 \\
6.6 E-03\end{array}$ & $\begin{array}{l}1.8 E+01 \\
3.2 E+01 \\
1.8 E+00\end{array}$ \\
\hline & $5.0 \mathrm{E}-03$ & $1.4 E+00$ & $2.1 E-03$ & $5.7 \mathrm{E}-01$ \\
\hline${ }^{79} \mathrm{Se}$ & $\begin{array}{l}1.7 \mathrm{E}+00 \\
2.7 \mathrm{E}+00\end{array}$ & $\begin{array}{l}4.6 \mathrm{E}+02 \\
7.3 \mathrm{E}+02\end{array}$ & $\begin{array}{l}4.6 E-01 \\
5.7 E-01\end{array}$ & $\begin{array}{l}1.2 E+02 \\
1.5 E+02\end{array}$ \\
\hline $\begin{array}{l}{ }^{82} \mathrm{Br} \\
{ }^{83} \mathrm{Br}+\mathrm{D} \\
{ }^{83} \mathrm{Br} \\
{ }^{84} \mathrm{Br} \\
{ }_{\mathrm{Br}}\end{array}$ & $\begin{array}{l}5.9 \mathrm{E}-03 \\
4.3 \mathrm{E}-06 \\
8.5 \mathrm{E}-06 \\
3.6 \mathrm{E}-06\end{array}$ & $\begin{array}{l}1.6 \mathrm{E}+00 \\
1.1 \mathrm{E}-03 \\
2.3 \mathrm{E}-03 \\
9.7 \mathrm{E}-04\end{array}$ & $\begin{array}{l}6.0 \mathrm{E}-02 \\
1.4 \mathrm{E}-03 \\
2.8 \mathrm{E}-03 \\
5.2 \mathrm{E}-03\end{array}$ & $\begin{array}{l}1.6 \mathrm{E}+01 \\
3.8 \mathrm{E}-01 \\
7.6 \mathrm{E}-01 \\
1.4 \mathrm{E}+00\end{array}$ \\
\hline $\begin{array}{l}{ }^{86} \mathrm{Rb} \\
{ }^{88} \mathrm{Rb} \\
{ }^{89}{ }_{\mathrm{Rb}+\mathrm{D}}(\mathrm{a}) \\
{ }_{\mathrm{Bg}}^{\mathrm{B}} \mathrm{Rb}\end{array}$ & $\begin{array}{l}5.0 \mathrm{E}-01 \\
2.0 \mathrm{E}-06 \\
6.5 \mathrm{E}-02 \\
9.3 \mathrm{E}-07\end{array}$ & $\begin{array}{l}1.3 \mathrm{E}+02 \\
5.4 \mathrm{E}-04 \\
1.8 \mathrm{E}+01 \\
2.5 \mathrm{E}-04\end{array}$ & $\begin{array}{l}6.2 \mathrm{E}-01 \\
4.3 \mathrm{E}-03 \\
2.8 \mathrm{E}-01 \\
2.3 \mathrm{E}-03\end{array}$ & $\begin{array}{l}1.7 \mathrm{E}+02 \\
1.2 \mathrm{E}+00 \\
7.4 \mathrm{E}+01 \\
6.2 \mathrm{E}-01\end{array}$ \\
\hline 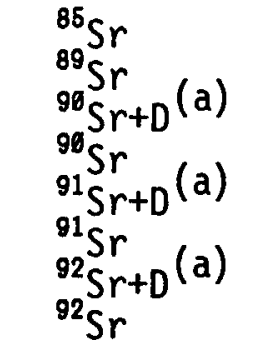 & $\begin{array}{l}2.7 \mathrm{E}-02 \\
1.3 \mathrm{E}-01 \\
1.2 \mathrm{E}+00 \\
2.2 \mathrm{E}+00 \\
3.8 \mathrm{E}-02 \\
8.9 \mathrm{E}-04 \\
3.1 \mathrm{E}-04 \\
1.6 \mathrm{E}-04\end{array}$ & $\begin{array}{l}7.3 \mathrm{E}+00 \\
3.5 \mathrm{E}+01 \\
3.2 \mathrm{E}+02 \\
5.9 \mathrm{E}+02 \\
1.0 \mathrm{E}+01 \\
2.4 \mathrm{E}-01 \\
8.5 \mathrm{E}-02 \\
4.3 \mathrm{E}-02\end{array}$ & $\begin{array}{l}1.1 \mathrm{E}-01 \\
5.5 \mathrm{E}-01 \\
3.8 \mathrm{E}+00 \\
7.7 \mathrm{E}+00 \\
5.4 \mathrm{E}-02 \\
1.3 \mathrm{E}-01 \\
4.5 \mathrm{E}-02 \\
8.8 \mathrm{E}-02\end{array}$ & $\begin{array}{l}3.0 \mathrm{E}+01 \\
1.5 \mathrm{E}+02 \\
1.0 \mathrm{E}+03 \\
2.1 \mathrm{E}+03 \\
1.5 \mathrm{E}+01 \\
3.5 \mathrm{E}+01 \\
1.2 \mathrm{E}+01 \\
2.4 \mathrm{E}+01\end{array}$ \\
\hline $\begin{array}{l}{ }^{96} Y \\
{ }^{91 n_{Y}} Y+D \\
{ }^{91 n_{Y}}(a) \\
{ }^{91} Y \\
92 Y \\
{ }^{93} Y \\
{ }^{93} Y\end{array}$ & $\begin{array}{l}2.2 \mathrm{E}-02 \\
4.9 \mathrm{E}-02 \\
1.3 \mathrm{E}-06 \\
9.8 \mathrm{E}-02 \\
2.6 \mathrm{E}-04 \\
1.8 \mathrm{E}-03\end{array}$ & $\begin{array}{l}5.9 \mathrm{E}+00 \\
1.3 \mathrm{E}+01 \\
3.5 \mathrm{E}-04 \\
2.6 \mathrm{E}+01 \\
7.0 \mathrm{E}-02 \\
4.9 \mathrm{E}-01\end{array}$ & $\begin{array}{l}5.0 \mathrm{E}-03 \\
2.3 \mathrm{E}-03 \\
1.9 \mathrm{E}-05 \\
4.5 \mathrm{E}-03 \\
9.2 \mathrm{E}-04 \\
2.2 \mathrm{E}-03\end{array}$ & $\begin{array}{l}1.4 E+00 \\
6.1 E-01 \\
5.1 E-03 \\
1.2 E+00 \\
2.5 E-01 \\
5.9 E-01\end{array}$ \\
\hline $\begin{array}{l}{ }^{93} Z r+D \\
{ }^{93} \mathrm{Zr} \\
{ }^{95} \mathrm{Zr} \\
{ }^{95} \mathrm{Zr+D} \\
{ }^{97} \mathrm{Zr} \\
{ }^{97} \mathrm{Zr+D} \\
{ }^{\mathrm{Zr}}(\mathrm{a})\end{array}$ & $\begin{array}{l}4.1 \mathrm{E}-02 \\
3.5 \mathrm{E}-02 \\
8.0 \mathrm{E}-02 \\
4.6 \mathrm{E}-02 \\
1.8 \mathrm{E}-03 \\
5.0 \mathrm{E}-03\end{array}$ & $\begin{array}{l}1.1 \mathrm{E}+01 \\
9.4 \mathrm{E}+00 \\
2.2 \mathrm{E}+01 \\
1.2 \mathrm{E}+01 \\
4.8 \mathrm{E}-01 \\
1.4 \mathrm{E}+00\end{array}$ & $\begin{array}{l}3.6 \mathrm{E}-03 \\
9.5 \mathrm{E}-05 \\
4.5 \mathrm{E}-03 \\
1.5 \mathrm{E}-04 \\
2.3 \mathrm{E}-04 \\
2.6 \mathrm{E}-04\end{array}$ & $\begin{array}{l}9.6 \mathrm{E}-01 \\
2.6 \mathrm{E}-02 \\
1.2 \mathrm{E}+00 \\
4.1 \mathrm{E}-02 \\
6.3 \mathrm{E}-02 \\
7.0 \mathrm{E}-02\end{array}$ \\
\hline $\begin{array}{l}93{ }^{93} \\
{ }_{94}\end{array}$ & $\begin{array}{l}4.6 \mathrm{E}-02 \\
6.8 \mathrm{E}-01\end{array}$ & $\begin{array}{l}1.2 E+01 \\
1.8 E+02\end{array}$ & $\begin{array}{l}\text { 7.0E-03 } \\
1.0 \mathrm{E}-01\end{array}$ & $\begin{array}{l}1.9 \mathrm{E}+00 \\
2.7 \mathrm{E}+01\end{array}$ \\
\hline
\end{tabular}


TABLE 2.5. Agricultural Food Product Ingestion Committed Effective Dose Rate Conversion Factors for Exposure to Residual Radioactive Materials (Air and Soil Contributions) (contd)

\begin{tabular}{|c|c|c|c|c|}
\hline Radionuclide & $\begin{array}{l}\text { mrem per } \\
\mathrm{pCi} / \mathrm{m}^{3} \\
\end{array}$ & $\begin{array}{l}\mu \mathrm{Sv} \text { per } \\
\mathrm{Bg} / \mathrm{m}^{3} \\
\end{array}$ & $\begin{array}{l}\text { mrem per } \\
\mathrm{pCi} / \mathrm{g}\end{array}$ & $\begin{array}{l}\mu \text { Sv per } \\
\mathrm{Bq} / \mathrm{g}\end{array}$ \\
\hline $\begin{array}{l}{ }^{95 m} \mathrm{Nb}+\mathrm{D} \\
{ }^{96} \mathrm{Nb} \\
\mathrm{Nb} \\
{ }^{95} \mathrm{Nb} \\
{ }^{971} \mathrm{Nb}+\mathrm{D} \\
97 \mathrm{a}) \\
{ }^{97} \mathrm{Nb} \\
\mathrm{Nb}\end{array}$ & $\begin{array}{l}3.1 \mathrm{E}-02 \\
8.7 \mathrm{E}-03 \\
5.1 \mathrm{E}-02 \\
5.5 \mathrm{E}-06 \\
2.5 \mathrm{E}-09 \\
1.1 \mathrm{E}-05\end{array}$ & $\begin{array}{l}8.5 \mathrm{E}+00 \\
2.3 \mathrm{E}+00 \\
1.4 \mathrm{E}+01 \\
1.5 \mathrm{E}-03 \\
6.8 \mathrm{E}-07 \\
3.0 \mathrm{E}-03\end{array}$ & $\begin{array}{l}6.7 \mathrm{E}-03 \\
4.5 \mathrm{E}-03 \\
8.7 \mathrm{E}-03 \\
2.2 \mathrm{E}-04 \\
6.9 \mathrm{E}-06 \\
4.2 \mathrm{E}-04\end{array}$ & $\begin{array}{l}1.8 \mathrm{E}+00 \\
1.2 \mathrm{E}+00 \\
2.3 \mathrm{E}+00 \\
5.8 \mathrm{E}-02 \\
1.9 \mathrm{E}-03 \\
1.1 \mathrm{E}-01\end{array}$ \\
\hline $\begin{array}{l}{ }^{93} \mathrm{Mo}+\mathrm{D} \\
{ }^{93} \mathrm{Mo} \\
{ }^{99} \mathrm{Mo} \\
{ }^{99} \mathrm{Mo+D} \\
{ }^{99} \mathrm{Mo}\end{array}$ & $\begin{array}{l}3.5 \mathrm{E}-02 \\
2.4 \mathrm{E}-02 \\
4.2 \mathrm{E}-03 \\
7.8 \mathrm{E}-03\end{array}$ & $\begin{array}{l}9.4 \mathrm{E}+00 \\
6.5 \mathrm{E}+00 \\
1.1 \mathrm{E}+00 \\
2.1 \mathrm{E}+00\end{array}$ & $\begin{array}{l}2.4 \mathrm{E}-02 \\
4.0 \mathrm{E}-02 \\
4.7 \mathrm{E}-02 \\
7.4 \mathrm{E}-02\end{array}$ & $\begin{array}{l}6.5 \mathrm{E}+00 \\
1.1 \mathrm{E}+01 \\
1.3 \mathrm{E}+01 \\
2.0 \mathrm{E}+01\end{array}$ \\
\hline $\begin{array}{l}{ }^{99 n} \mathrm{Tc} \\
{ }^{99} \mathrm{Tc} \\
{ }^{101} \mathrm{TC} \\
\mathrm{TC}\end{array}$ & $\begin{array}{l}1.9 \mathrm{E}-05 \\
1.2 \mathrm{E}-01 \\
6.8 \mathrm{E}-07\end{array}$ & $\begin{array}{l}5.1 \mathrm{E}-03 \\
3.2 \mathrm{E}+01 \\
1.8 \mathrm{E}-04\end{array}$ & $\begin{array}{l}1.7 \mathrm{E}-02 \\
1.2 \mathrm{E}+00 \\
1.6 \mathrm{E}-02\end{array}$ & $\begin{array}{l}4.6 E+00 \\
3.2 E+02 \\
4.3 E+00\end{array}$ \\
\hline 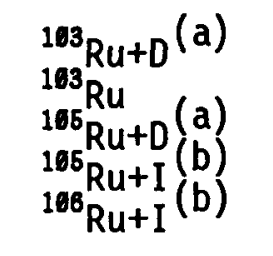 & $\begin{array}{l}3.2 \mathrm{E}-02 \\
6.3 \mathrm{E}-02 \\
8.3 \mathrm{E}-04 \\
2.1 \mathrm{E}-04 \\
1.3 \mathrm{E}+00\end{array}$ & $\begin{array}{l}8.5 \mathrm{E}+00 \\
1.7 \mathrm{E}+01 \\
2.2 \mathrm{E}-01 \\
5.7 \mathrm{E}-02 \\
3.5 \mathrm{E}+02\end{array}$ & $\begin{array}{l}3.5 \mathrm{E}-02 \\
6.9 \mathrm{E}-02 \\
2.8 \mathrm{E}-02 \\
1.2 \mathrm{E}-02 \\
1.1 \mathrm{E}+00\end{array}$ & $\begin{array}{l}9.5 \mathrm{E}+00 \\
1.9 \mathrm{E}+01 \\
7.5 \mathrm{E}+00 \\
3.2 \mathrm{E}+00 \\
3.1 \mathrm{E}+02\end{array}$ \\
\hline $\begin{array}{r}{ }^{10310} \mathrm{Rh} \\
{ }^{106 h}\end{array}$ & $\begin{array}{l}\text { 4. } 3 E-07 \\
2.1 E-03\end{array}$ & $\begin{array}{l}1.2 \mathrm{E}-04 \\
5.7 \mathrm{E}-01\end{array}$ & $\begin{array}{l}5.6 \mathrm{E}-04 \\
7.2 \mathrm{E}-02\end{array}$ & $\begin{array}{l}1.5 \mathrm{E}-01 \\
1.9 \mathrm{E}+01\end{array}$ \\
\hline $\begin{array}{l}{ }^{163} \mathrm{Pd}+{ }(\mathrm{a}) \\
{ }_{103} \mathrm{Pd} \\
{ }_{107} \mathrm{Pd} \\
{ }^{109} \mathrm{Pd}+\mathrm{I}\end{array}$ & $\begin{array}{l}5.5 \mathrm{E}-03 \\
1.1 \mathrm{E}-02 \\
3.8 \mathrm{E}-03 \\
7.0 \mathrm{E}-04\end{array}$ & $\begin{array}{l}1.5 \mathrm{E}+00 \\
3.0 \mathrm{E}+00 \\
1.0 \mathrm{E}+00 \\
1.9 \mathrm{E}-01\end{array}$ & $\begin{array}{l}4.7 \mathrm{E}-03 \\
8.8 \mathrm{E}-03 \\
1.7 \mathrm{E}-03 \\
1.3 \mathrm{E}-02\end{array}$ & $\begin{array}{l}1.3 E+00 \\
2.4 E+00 \\
4.6 E-01 \\
3.5 E+00\end{array}$ \\
\hline${ }^{116 \mathrm{~m}} \mathrm{Ag}+\mathrm{I}(\mathrm{b})$ & $\begin{array}{l}2.3 \mathrm{E}+00 \\
2.6 \mathrm{E}-01\end{array}$ & $\begin{array}{l}6.1 \mathrm{E}+02 \\
7.0 \mathrm{E}+01\end{array}$ & $\begin{array}{l}2.1 \mathrm{E}+00 \\
5.2 \mathrm{E}-01\end{array}$ & $\begin{array}{l}5.7 E+02 \\
1.4 E+02\end{array}$ \\
\hline $\begin{array}{l}{ }_{169}^{169} \mathrm{Cd} \\
{ }^{113} \mathrm{Cd} \\
{ }_{115} \mathrm{Cd} \\
{ }^{115} \mathrm{Cd}+\mathrm{D} \\
{ }^{115} \mathrm{Cd} \\
{ }^{115} \mathrm{Cd}\end{array}$ & $\begin{array}{l}1.6 \mathrm{E}-01 \\
2.0 \mathrm{E}+00 \\
8.0 \mathrm{E}-02 \\
1.6 \mathrm{E}-01 \\
1.1 \mathrm{E}-02\end{array}$ & $\begin{array}{l}4.3 E+01 \\
5.4 E+02 \\
2.2 E+01 \\
4.3 E+01 \\
3.0 E+00\end{array}$ & $\begin{array}{l}\text { 8.7E-01 } \\
1.1 \mathrm{E}+01 \\
5.5 \mathrm{E}-01 \\
1.1 \mathrm{E}+00 \\
3.8 \mathrm{E}-01\end{array}$ & $\begin{array}{l}2.3 \mathrm{E}+02 \\
3.0 \mathrm{E}+03 \\
1.5 \mathrm{E}+02 \\
3.0 \mathrm{E}+02 \\
1.0 \mathrm{E}+02\end{array}$ \\
\hline 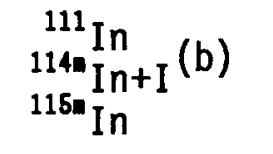 & $\begin{array}{l}2.9 \mathrm{E}-03 \\
8.2 \mathrm{E}-02 \\
5.7 \mathrm{E}-05\end{array}$ & $\begin{array}{l}7.8 \mathrm{E}-01 \\
2.2 \mathrm{E}+01 \\
1.5 \mathrm{E}-02\end{array}$ & $\begin{array}{l}4.1 \mathrm{E}-04 \\
2.5 \mathrm{E}-03 \\
1.1 \mathrm{E}-04\end{array}$ & $\begin{array}{l}1.1 \mathrm{E}-01 \\
6.6 \mathrm{E}-01 \\
3.0 \mathrm{E}-02\end{array}$ \\
\hline $117 \mathrm{~m} n$ & $2.3 \mathrm{E}-02$ & $6.2 E+00$ & $9.5 \mathrm{E}-03$ & $2.6 \mathrm{E}+0$ \\
\hline
\end{tabular}

$\frac{\text { Air Deposition }}{\text { mremper } \mu \text { Sv per }} \frac{\text { Soil Uptake }}{\text { mremper } \mu \text { Sv per }}$

$3.5 \mathrm{E}-02 \quad 9.4 \mathrm{E}+00$

$.4 \mathrm{E}-02$

$1.1 \mathrm{E}+01$

$4.2 \mathrm{E}-03 \quad 1.1 \mathrm{E}+00$

4.7E-02 $1.3 \mathrm{E}+01$

$1.7 \mathrm{E}-02 \quad 4.6 \mathrm{E}+00$

$1.2 \mathrm{E}-01$

$3.1 \mathrm{E}-03$

$1.2 \mathrm{E}+00$

3. $2 \mathrm{E}+02$

3. $5 \mathrm{E}-02$

9. $5 \mathrm{E}+00$

6.3E-02

1. $7 \mathrm{E}+01$

6.9E-02

7. $5 \mathrm{E}+00$

8.3E-04

5.7E-02

$3.2 \mathrm{E}+00$

2.1E-04

$3.5 \mathrm{E}+02$

$1.2 \mathrm{E}-02$

(1)


TABLE 2.5. Agricultural Food Product Ingestion Committed Effective Dose Rate Conversion Factors for Exposure to Residual Radioactive Materials (Air and Soil Contributions) (contd)

\begin{tabular}{|c|c|c|c|c|}
\hline \multirow[b]{2}{*}{ Radionuclide } & \multicolumn{2}{|c|}{ Air Deposition } & \multicolumn{2}{|c|}{ Soil Uptake } \\
\hline & $\begin{array}{l}\text { mrem per } \\
\mathrm{pCi} / \mathrm{m}^{3}\end{array}$ & $\begin{array}{c}\mu \mathrm{Sv} p \mathrm{per} \\
\mathrm{Bq} / \mathrm{m}^{3} \\
\end{array}$ & $\begin{array}{l}\text { mrem per } \\
\mathrm{pCi} / \mathrm{g}\end{array}$ & $\begin{array}{c}\mu \mathrm{Sv} \text { per } \\
\mathrm{Bg} / \mathrm{g}\end{array}$ \\
\hline $\begin{array}{l}{ }^{119 m} \mathrm{Sn} \\
{ }^{121 m} \mathrm{Sn}+\mathrm{D}(\mathrm{a}) \\
{ }^{121} \mathrm{Sn}_{\mathrm{Sn}} \\
{ }^{121} \mathrm{Sn} \\
{ }^{123} \mathrm{Sn} \\
{ }^{125} \mathrm{Sn+D} \\
{ }^{125} \mathrm{Sn} \\
{ }^{126} \mathrm{~S} \mathrm{~S}+\mathrm{D} \\
{ }^{126} \mathrm{Sn}\end{array}$ & $\begin{array}{l}2.2 \mathrm{E}-02 \\
2.1 \mathrm{E}-02 \\
2.5 \mathrm{E}-02 \\
9.3 \mathrm{E}-04 \\
1.2 \mathrm{E}-01 \\
6.3 \mathrm{E}-02 \\
7.6 \mathrm{E}-02 \\
1.7 \mathrm{E}-01 \\
3.3 \mathrm{E}-01\end{array}$ & $\begin{array}{l}5.9 E+00 \\
5.8 E+00 \\
6.7 E+00 \\
2.5 E-01 \\
3.2 E+01 \\
1.7 E+01 \\
2.1 E+01 \\
4.5 E+01 \\
8.9 E+01\end{array}$ & $\begin{array}{l}4.6 \mathrm{E}-03 \\
4.1 \mathrm{E}-03 \\
4.9 \mathrm{E}-03 \\
2.9 \mathrm{E}-03 \\
2.8 \mathrm{E}-02 \\
1.7 \mathrm{E}-01 \\
3.9 \mathrm{E}-02 \\
3.1 \mathrm{E}-02 \\
6.4 \mathrm{E}-02\end{array}$ & $\begin{array}{l}1.2 \mathrm{E}+00 \\
1.1 \mathrm{E}+00 \\
1.3 \mathrm{E}+00 \\
7.8 \mathrm{E}-01 \\
7.6 \mathrm{E}+00 \\
4.6 \mathrm{E}+01 \\
1.1 \mathrm{E}+01 \\
8.5 \mathrm{E}+00 \\
1.7 \mathrm{E}+01\end{array}$ \\
\hline $\begin{array}{l}{ }^{124} \mathrm{Sb} \\
{ }^{125} \mathrm{Sb}+\mathrm{D} \\
{ }^{125} \mathrm{Sb} \\
{ }^{126 m} \mathrm{Sb} \\
{ }^{128 m} \mathrm{Sb}+\mathrm{D} \\
{ }^{126} \mathrm{Sb} \\
{ }^{127} \mathrm{Sb} \\
{ }^{127} \mathrm{Sb}+\mathrm{Sb}\end{array}$ & $\begin{array}{l}1.2 \mathrm{E}-01 \\
5.3 \mathrm{E}-02 \\
3.9 \mathrm{E}-02 \\
8.8 \mathrm{E}-03 \\
1.1 \mathrm{E}-06 \\
7.2 \mathrm{E}-02 \\
2.7 \mathrm{E}-02 \\
2.2 \mathrm{E}-02\end{array}$ & $\begin{array}{l}3.2 \mathrm{E}+01 \\
1.4 \mathrm{E}+01 \\
1.1 \mathrm{E}+01 \\
2.4 \mathrm{E}+00 \\
3.0 \mathrm{E}-04 \\
1.9 \mathrm{E}+01 \\
7.4 \mathrm{E}+00 \\
5.9 \mathrm{E}+00\end{array}$ & $\begin{array}{l}2.0 \mathrm{E}-02 \\
2.8 \mathrm{E}-01 \\
5.6 \mathrm{E}-03 \\
2.7 \mathrm{E}-03 \\
1.8 \mathrm{E}-04 \\
2.0 \mathrm{E}-02 \\
4.2 \mathrm{E}-01 \\
1.4 \mathrm{E}-02\end{array}$ & $\begin{array}{l}5.4 \mathrm{E}+00 \\
7.7 \mathrm{E}+01 \\
1.5 \mathrm{E}+00 \\
7.3 \mathrm{E}-01 \\
4.9 \mathrm{E}-02 \\
5.4 \mathrm{E}+00 \\
1.1 \mathrm{E}+02 \\
3.8 \mathrm{E}+00\end{array}$ \\
\hline 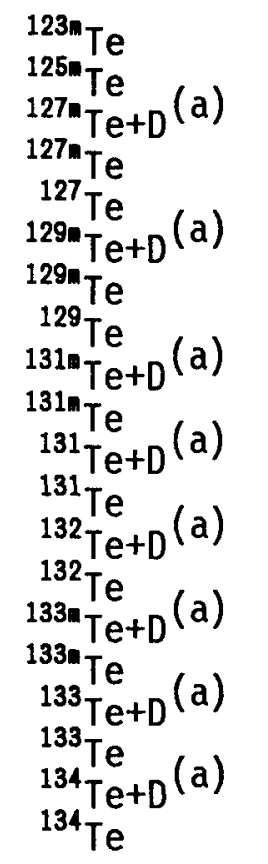 & $\begin{array}{l}1.3 \mathrm{E}-01 \\
6.3 \mathrm{E}-02 \\
1.0 \mathrm{E}-01 \\
1.9 \mathrm{E}-01 \\
2.4 \mathrm{E}-04 \\
4.1 \mathrm{E}+00 \\
6.7 \mathrm{E}+00 \\
1.1 \mathrm{E}-05 \\
4.0 \mathrm{E}+00 \\
2.0 \mathrm{E}-02 \\
4.4 \mathrm{E}+00 \\
3.4 \mathrm{E}-06 \\
2.2 \mathrm{E}-02 \\
4.2 \mathrm{E}-02 \\
4.4 \mathrm{E}-02 \\
2.8 \mathrm{E}-05 \\
4.6 \mathrm{E}-02 \\
1.3 \mathrm{E}-06 \\
6.0 \mathrm{E}-05 \\
4.9 \mathrm{E}-06\end{array}$ & $\begin{array}{l}3.5 \mathrm{E}+01 \\
1.7 \mathrm{E}+01 \\
2.7 \mathrm{E}+01 \\
5.1 \mathrm{E}+01 \\
6.5 \mathrm{E}-02 \\
1.1 \mathrm{E}+03 \\
1.8 \mathrm{E}+03 \\
3.0 \mathrm{E}-03 \\
1.1 \mathrm{E}+03 \\
5.4 \mathrm{E}+00 \\
1.2 \mathrm{E}+03 \\
9.2 \mathrm{E}-04 \\
5.9 \mathrm{E}+00 \\
1.1 \mathrm{E}+01 \\
1.2 \mathrm{E}+01 \\
7.6 \mathrm{E}-03 \\
1.2 \mathrm{E}+01 \\
3.5 \mathrm{E}-04 \\
1.6 \mathrm{E}-02 \\
1.3 \mathrm{E}-03\end{array}$ & $\begin{array}{l}2.9 \mathrm{E}+00 \\
1.4 \mathrm{E}+00 \\
2.2 \mathrm{E}+00 \\
4.0 \mathrm{E}+00 \\
1.7 \mathrm{E}-01 \\
1.0 \mathrm{E}+02 \\
1.7 \mathrm{E}+02 \\
6.3 \mathrm{E}-02 \\
2.3 \mathrm{E}+00 \\
4.3 \mathrm{E}+00 \\
4.2 \mathrm{E}-01 \\
5.3 \mathrm{E}-02 \\
1.9 \mathrm{E}+00 \\
3.8 \mathrm{E}+00 \\
9.8 \mathrm{E}-02 \\
1.9 \mathrm{E}-01 \\
5.0 \mathrm{E}-02 \\
4.0 \mathrm{E}-02 \\
2.4 \mathrm{E}-02 \\
4.6 \mathrm{E}-02\end{array}$ & $\begin{array}{l}7.8 \mathrm{E}+02 \\
3.8 \mathrm{E}+02 \\
6.0 \mathrm{E}+02 \\
1.1 \mathrm{E}+03 \\
4.6 \mathrm{E}+01 \\
2.8 \mathrm{E}+04 \\
4.6 \mathrm{E}+04 \\
1.7 \mathrm{E}+01 \\
6.3 \mathrm{E}+02 \\
1.2 \mathrm{E}+03 \\
1.1 \mathrm{E}+02 \\
1.4 \mathrm{E}+01 \\
5.1 \mathrm{E}+02 \\
1.0 \mathrm{E}+03 \\
2.6 \mathrm{E}+01 \\
5.1 \mathrm{E}+01 \\
1.3 \mathrm{E}+01 \\
1.1 \mathrm{E}+01 \\
6.5 \mathrm{E}+00 \\
1.2 \mathrm{E}+01\end{array}$ \\
\hline $\begin{array}{l}{ }^{125} \mathrm{I} \\
{ }^{129}{ }_{\mathrm{I}} \\
{ }^{13 \theta_{I}}{ }_{\mathrm{I}}(\mathrm{a})\end{array}$ & $\begin{array}{l}1.4 \mathrm{E}+01 \\
1.2 \mathrm{E}+02 \\
2.9 \mathrm{E}-02 \\
8.8 \mathrm{E}+00\end{array}$ & $\begin{array}{l}3.8 \mathrm{E}+03 \\
3.2 \mathrm{E}+04 \\
7.8 \mathrm{E}+00 \\
2.4 \mathrm{E}+03\end{array}$ & $\begin{array}{l}6.2 \mathrm{E}-01 \\
4.7 \mathrm{E}+00 \\
4.8 \mathrm{E}-02 \\
7.8 \mathrm{E}-01\end{array}$ & $\begin{array}{l}1.7 E+02 \\
1.3 E+03 \\
1.3 E+01 \\
2.1 E+02\end{array}$ \\
\hline
\end{tabular}


TABLE 2.5. Agricultural Food Product Ingestion Committed Effective Dose Rate Conversion Factors for Exposure to Residual Radioactive Materials (Air and Soil Contributions) (contd)

\begin{tabular}{|c|c|c|c|c|}
\hline \multirow[b]{2}{*}{ Radionuclide } & \multicolumn{2}{|c|}{ Air Deposition } & \multicolumn{2}{|c|}{ Soil Uptake } \\
\hline & $\begin{array}{l}\text { mrem per } \\
\mathrm{pCi} / \mathrm{m}^{3}\end{array}$ & $\begin{array}{c}\mu \mathrm{SV} \text { per } \\
\mathrm{Bq} / \mathrm{m}^{3} \\
\end{array}$ & $\begin{array}{l}\text { mrem per } \\
\mathrm{pCi} / \mathrm{g}\end{array}$ & $\begin{array}{l}\mu \mathrm{Sv} p e r \\
\mathrm{~Bq} / \mathrm{g}\end{array}$ \\
\hline $\begin{array}{l}131 \mathrm{I} \\
132 \mathrm{I} \\
133 \mathrm{I}+\mathrm{I}(\mathrm{a}) \\
133 \mathrm{I}+\mathrm{I} \\
134 \mathrm{I} \\
135 \mathrm{I}+\mathrm{D}(\mathrm{a}) \\
{ }^{135} \mathrm{I}\end{array}$ & $\begin{array}{l}8.9 \mathrm{E}+00 \\
7.5 \mathrm{E}-04 \\
6.9 \mathrm{E}-02 \\
1.4 \mathrm{E}-01 \\
1.1 \mathrm{E}-04 \\
3.0 \mathrm{E}-03 \\
6.6 \mathrm{E}-03\end{array}$ & $\begin{array}{l}2.4 \mathrm{E}+03 \\
2.0 \mathrm{E}-01 \\
1.9 \mathrm{E}+01 \\
3.8 \mathrm{E}+01 \\
3.0 \mathrm{E}-02 \\
8.2 \mathrm{E}-01 \\
1.8 \mathrm{E}+00\end{array}$ & $\begin{array}{l}7.9 \mathrm{E}-01 \\
7.1 \mathrm{E}-03 \\
5.4 \mathrm{E}-02 \\
1.1 \mathrm{E}-01 \\
2.7 \mathrm{E}-03 \\
1.0 \mathrm{E}-02 \\
2.2 \mathrm{E}-02\end{array}$ & $\begin{array}{l}2.1 \mathrm{E}+02 \\
1.9 \mathrm{E}+00 \\
1.5 \mathrm{E}+01 \\
3.0 \mathrm{E}+01 \\
7.3 \mathrm{E}-01 \\
2.7 \mathrm{E}+00 \\
5.9 \mathrm{E}+00\end{array}$ \\
\hline $\begin{array}{l}{ }^{1344} C s+D(a) \\
{ }^{134} C S C s \\
{ }^{134} C S \\
{ }^{135} C S \\
{ }^{138} C S \\
{ }^{137} C S \\
{ }^{138} C S+I\end{array}$ & $\begin{array}{l}2.0 \mathrm{E}-06 \\
4.0 \mathrm{E}-06 \\
2.0 \mathrm{E}+00 \\
1.9 \mathrm{E}-01 \\
1.4 \mathrm{E}-01 \\
7.2 \mathrm{E}-01 \\
3.8 \mathrm{E}-06\end{array}$ & $\begin{array}{l}5.4 \mathrm{E}-04 \\
1.1 \mathrm{E}-03 \\
5.4 \mathrm{E}+02 \\
5.1 \mathrm{E}+01 \\
3.8 \mathrm{E}+01 \\
1.9 \mathrm{E}+02 \\
1.0 \mathrm{E}-03\end{array}$ & $\begin{array}{l}3.1 \mathrm{E}-05 \\
6.3 \mathrm{E}-05 \\
2.0 \mathrm{E}-01 \\
1.9 \mathrm{E}-02 \\
2.4 \mathrm{E}-02 \\
1.2 \mathrm{E}-02 \\
3.2 \mathrm{E}-04\end{array}$ & $\begin{array}{l}8.5 \mathrm{E}-03 \\
1.7 \mathrm{E}-02 \\
5.4 \mathrm{E}+01 \\
5.1 \mathrm{E}+00 \\
6.5 \mathrm{E}+00 \\
3.3 \mathrm{E}+00 \\
8.6 \mathrm{E}-02\end{array}$ \\
\hline $\begin{array}{l}{ }^{139} \mathrm{Ba} \\
{ }^{144} \mathrm{Ba}+\mathrm{D} \\
{ }^{141} \mathrm{Ba} \\
{ }^{141} \mathrm{Ba}+\mathrm{D} \\
{ }^{142} \mathrm{Ba} \\
{ }^{142} \mathrm{Ba} \mathrm{Ba}\end{array}$ & $\begin{array}{l}2.1 \mathrm{E}-05 \\
6.0 \mathrm{E}-02 \\
5.9 \mathrm{E}-02 \\
9.0 \mathrm{E}-03 \\
2.4 \mathrm{E}-06 \\
2.4 \mathrm{E}-05 \\
7.4 \mathrm{E}-07\end{array}$ & $\begin{array}{l}5.7 E-03 \\
1.6 E+01 \\
1.6 E+01 \\
2.4 E+00 \\
6.5 E-04 \\
6.5 E-03 \\
2.0 E-04\end{array}$ & $\begin{array}{l}3.9 \mathrm{E}-04 \\
6.5 \mathrm{E}-03 \\
8.7 \mathrm{E}-03 \\
7.3 \mathrm{E}-04 \\
2.0 \mathrm{E}-04 \\
2.2 \mathrm{E}-04 \\
1.0 \mathrm{E}-04\end{array}$ & $\begin{array}{l}1.1 \mathrm{E}-01 \\
1.8 \mathrm{E}+00 \\
2.3 \mathrm{E}+00 \\
2.0 \mathrm{E}-01 \\
5.4 \mathrm{E}-02 \\
5.8 \mathrm{E}-02 \\
2.7 \mathrm{E}-02\end{array}$ \\
\hline $\begin{array}{l}148 \mathrm{La} \\
141 \mathrm{La}(\mathrm{a}) \\
141 \mathrm{La} \\
142 \mathrm{La}\end{array}$ & $\begin{array}{l}1.1 \mathrm{E}-02 \\
1.4 \mathrm{E}-02 \\
2.2 \mathrm{E}-04 \\
4.2 \mathrm{E}-05\end{array}$ & & $\begin{array}{l}3.8 E-03 \\
1.0 E-03 \\
6.8 E-04 \\
3.3 E-04\end{array}$ & $\begin{array}{l}1.0 \mathrm{E}+00 \\
2.7 \mathrm{E}-01 \\
1.8 \mathrm{E}-01 \\
8.9 \mathrm{E}-02\end{array}$ \\
\hline $\begin{array}{l}{ }^{141} \mathrm{Ce} \\
{ }^{143} \mathrm{Ce}+\mathrm{D} \\
{ }^{14} \mathrm{Ce} \\
{ }^{144} \mathrm{Ce}+\mathrm{D} \\
{ }^{144} \mathrm{Ce}\end{array}$ & $\begin{array}{l}2.6 \mathrm{E}-02 \\
1.9 \mathrm{E}-02 \\
5.3 \mathrm{E}-03 \\
1.2 \mathrm{E}-01 \\
2.5 \mathrm{E}-01\end{array}$ & $\begin{array}{l}7.0 E+00 \\
5.1 E+00 \\
1.4 E+00 \\
3.4 E+01 \\
6.8 E+01\end{array}$ & $\begin{array}{l}1.4 \mathrm{E}-03 \\
2.2 \mathrm{E}-03 \\
2.1 \mathrm{E}-03 \\
5.0 \mathrm{E}-03 \\
1.0 \mathrm{E}-02\end{array}$ & $\begin{array}{l}3.8 \mathrm{E}-01 \\
5.8 \mathrm{E}-01 \\
5.7 \mathrm{E}-01 \\
1.3 \mathrm{E}+00 \\
2.7 \mathrm{E}+00\end{array}$ \\
\hline $\begin{array}{l}{ }^{143} \mathrm{Pr} \\
{ }^{1444} \mathrm{Pr}+\mathrm{D} \\
{ }^{144} \mathrm{Pr} \\
{ }^{144} \mathrm{Pr}\end{array}$ & $\begin{array}{l}3.0 \mathrm{E}-02 \\
1.0 \mathrm{E}-06 \\
2.2 \mathrm{E}-07 \\
1.3 \mathrm{E}-06\end{array}$ & $\begin{array}{l}8.1 E+00 \\
2.8 E-04 \\
5.9 E-05 \\
3.5 E-04\end{array}$ & $\begin{array}{l}2.2 \mathrm{E}-03 \\
3.9 \mathrm{E}-05 \\
2.2 \mathrm{E}-05 \\
5.6 \mathrm{E}-05\end{array}$ & $\begin{array}{l}5.9 \mathrm{E}-01 \\
1.1 \mathrm{E}-02 \\
5.9 \mathrm{E}-03 \\
1.5 \mathrm{E}-02\end{array}$ \\
\hline $\begin{array}{l}{ }^{147} \mathrm{Nd+D} \\
{ }^{147} \mathrm{Nd}\end{array}$ & $\begin{array}{l}1.9 \mathrm{E}-02 \\
2.3 \mathrm{E}-02\end{array}$ & $\begin{array}{l}5.0 E+00 \\
6.2 E+00\end{array}$ & $\begin{array}{l}1.1 \mathrm{E}-03 \\
1.9 \mathrm{E}-03\end{array}$ & $\begin{array}{l}3.0 \mathrm{E}-01 \\
5.1 \mathrm{E}-01\end{array}$ \\
\hline${ }^{47} \mathrm{Pm}$ & $4 \mathrm{E}-02$ & $.8 E+00$ & $3.3 E-04$ & 8.9E-02 \\
\hline
\end{tabular}


TABLE 2.5. Agricultural Food Product Ingestion Committed Effective Dose Rate Conversion Factors for Exposure to Residual Radioactive Materials (Air and Soil Contributions) (contd)

\begin{tabular}{|c|c|c|c|c|}
\hline Radionuclide & $\begin{array}{l}\text { mrem per } \\
\mathrm{pCi} / \mathrm{m}^{3}\end{array}$ & $\begin{array}{l}\mu \mathrm{Sv} \text { per } \\
\mathrm{Bq} / \mathrm{m}^{3}\end{array}$ & $\begin{array}{l}\text { mrem per } \\
\mathrm{pCi} / \mathrm{g}\end{array}$ & $\begin{array}{c}\mu S v \text { per } \\
\mathrm{Bq} / \mathrm{g}\end{array}$ \\
\hline $\begin{array}{l}{ }_{148 m} \mathrm{Pm}+\mathrm{D}(\mathrm{a}) \\
{ }^{148 \mathrm{~m}} \mathrm{Pm} \\
{ }_{148} \mathrm{Pm} \\
{ }^{149} \mathrm{Pm} \\
{ }^{161} \mathrm{Pm}\end{array}$ & $\begin{array}{l}7.4 \mathrm{E}-02 \\
7.2 \mathrm{E}-02 \\
3.9 \mathrm{E}-02 \\
7.2 \mathrm{E}-03 \\
3.0 \mathrm{E}-03\end{array}$ & $\begin{array}{l}2.0 \mathrm{E}+01 \\
1.9 \mathrm{E}+01 \\
1.1 \mathrm{E}+01 \\
1.9 \mathrm{E}+00 \\
8.1 \mathrm{E}-01\end{array}$ & $\begin{array}{l}2.4 E-03 \\
2.4 E-03 \\
3.5 E-03 \\
1.3 E-03 \\
9.5 E-04\end{array}$ & $\begin{array}{l}6.5 \mathrm{E}-01 \\
6.5 \mathrm{E}-01 \\
9.5 \mathrm{E}-01 \\
3.5 \mathrm{E}-01 \\
2.6 \mathrm{E}-01\end{array}$ \\
\hline $\begin{array}{l}{ }^{147} \mathrm{Sm} \\
{ }^{151} \mathrm{Sm} \\
{ }^{15 m}\end{array}$ & $\begin{array}{l}2.5 E+00 \\
5.1 E-03 \\
4.7 E-03\end{array}$ & $\begin{array}{l}6.7 \mathrm{E}+02 \\
1.4 \mathrm{E}+00 \\
1.3 \mathrm{E}+00\end{array}$ & $\begin{array}{l}8.0 \mathrm{E}-02 \\
1.6 \mathrm{E}-04 \\
1.2 \mathrm{E}-03\end{array}$ & $\begin{array}{l}2.2 E+01 \\
4.3 E-02 \\
3.2 E-01\end{array}$ \\
\hline $\begin{array}{l}{ }^{152} \mathrm{Eu} \\
{ }^{154} \mathrm{Eu} \\
{ }^{165} \mathrm{Eu} \\
{ }^{156} \mathrm{Eu}\end{array}$ & $\begin{array}{l}8.4 E-02 \\
1.2 E-01 \\
2.0 E-02 \\
6.1 E-02\end{array}$ & $\begin{array}{l}2.3 \mathrm{E}+01 \\
3.2 \mathrm{E}+01 \\
5.4 \mathrm{E}+00 \\
1.6 \mathrm{E}+01\end{array}$ & $\begin{array}{l}3.3 \mathrm{E}-03 \\
4.8 \mathrm{E}-03 \\
7.8 \mathrm{E}-04 \\
4.2 \mathrm{E}-03\end{array}$ & $\begin{array}{l}8.9 \mathrm{E}-01 \\
1.3 \mathrm{E}+00 \\
2.1 \mathrm{E}-01 \\
1.1 \mathrm{E}+00\end{array}$ \\
\hline${ }^{153} \mathrm{Gd}$ & $1.4 \mathrm{E}-02$ & $3.8 E+00$ & $1.8 \mathrm{E}-03$ & 4.9E-01 \\
\hline${ }^{160} \mathrm{~Tb}$ & $7.1 E-02$ & $1.9 E+01$ & $6.9 \mathrm{E}-04$ & $1.9 \mathrm{E}-01$ \\
\hline${ }^{168 m} \mathrm{Ho}$ & $1.0 \mathrm{E}-01$ & 2. $7 E+01$ & $4.4 E-03$ & $1.2 \mathrm{E}+00$ \\
\hline $\begin{array}{l}{ }^{181} \mathrm{~W} \\
{ }^{185} \mathrm{~W} \\
{ }^{187} \mathrm{~W}\end{array}$ & $\begin{array}{l}3.6 \mathrm{E}-03 \\
1.9 \mathrm{E}-02 \\
1.8 \mathrm{E}-03\end{array}$ & $\begin{array}{l}9.7 \mathrm{E}-01 \\
5.1 \mathrm{E}+00 \\
4.9 \mathrm{E}-01\end{array}$ & $\begin{array}{l}1.0 \mathrm{E}-03 \\
5.6 \mathrm{E}-03 \\
7.2 \mathrm{E}-03\end{array}$ & $\begin{array}{l}2.7 E-01 \\
1.5 E+00 \\
1.9 E+00\end{array}$ \\
\hline${ }^{187} \mathrm{Re}$ & $2.1 E-04$ & $5.7 \mathrm{E}-02$ & $5.8 \mathrm{E}-07$ & $1.6 \mathrm{E}-04$ \\
\hline $\begin{array}{l}{ }^{186} \text { Os } \\
{ }^{191} \text { Os }\end{array}$ & $\begin{array}{l}0.0 E+00 \\
0.0 E+00\end{array}$ & $\begin{array}{l}0.0 \mathrm{E}+00 \\
0.0 \mathrm{E}+00\end{array}$ & $\begin{array}{l}0.0 E+00 \\
0.0 E+00\end{array}$ & $\begin{array}{l}0.0 E+00 \\
0.0 E+00\end{array}$ \\
\hline${ }^{192} \mathrm{Ir}$ & $6.1 E-02$ & $1.6 \mathrm{E}+01$ & $1.8 \mathrm{E}-02$ & $4.9 E+00$ \\
\hline${ }^{203} \mathrm{Hg}$ & $1.7 \mathrm{E}-01$ & $4.6 \mathrm{E}+01$ & $3.8 \mathrm{E}-01$ & $1.0 E+02$ \\
\hline $\begin{array}{l}{ }_{210}^{210} \mathrm{~Pb}+\mathrm{D}(\mathrm{a}) \\
{ }^{210} \mathrm{~Pb} \\
{ }^{212} \mathrm{~Pb}+\mathrm{Pb}\end{array}$ & $\begin{array}{l}3.1 \mathrm{E}+01 \\
6.7 \mathrm{E}+01 \\
6.3 \mathrm{E}-03 \\
1.9 \mathrm{E}-02\end{array}$ & $\begin{array}{l}8.4 E+03 \\
1.8 E+04 \\
1.7 E+00 \\
5.1 E+00\end{array}$ & $\begin{array}{l}1.7 \mathrm{E}+00 \\
4.1 \mathrm{E}+00 \\
2.1 \mathrm{E}-02 \\
3.6 \mathrm{E}-02\end{array}$ & $\begin{array}{l}4.5 E+02 \\
1.1 E+03 \\
5.7 E+00 \\
9.7 E+00\end{array}$ \\
\hline $\begin{array}{l}{ }^{216} \mathrm{Bi}+\mathrm{D}(\mathrm{a}) \\
{ }^{210_{\mathrm{Bi}}} \mathrm{Bi}(\mathrm{b}) \\
{ }^{212} \mathrm{Bi+ \textrm {I }}\end{array}$ & $\begin{array}{l}1.2 \mathrm{E}+01 \\
2.3 \mathrm{E}-02 \\
2.1 \mathrm{E}-05\end{array}$ & $\begin{array}{l}3.2 E+03 \\
6.2 E+00 \\
5.7 E-03\end{array}$ & $\begin{array}{l}4.6 \mathrm{E}-01 \\
1.8 \mathrm{E}-01 \\
1.5 \mathrm{E}-02\end{array}$ & $\begin{array}{l}1.2 E+02 \\
4.9 E+01 \\
4.0 E+00\end{array}$ \\
\hline${ }^{218} \mathrm{PO}$ & $2.3 E+01$ & $6.2 E+03$ & $6.1 E-01$ & $1.6 \mathrm{E}+02$ \\
\hline
\end{tabular}


TABLE 2.5. Agricultural Food Product Ingestion Committed Effective Dose Rate Conversion Factors for Exposure to Residual Radioactive Materials (Air and Soil Contributions) (contd)

\begin{tabular}{|c|c|c|c|c|}
\hline adionuclide & $\begin{array}{l}\text { mrem per } \\
\mathrm{pCi} / \mathrm{m}^{3}\end{array}$ & $\begin{array}{c}\mu \mathrm{Sv} p \mathrm{per} \\
\mathrm{Bq} / \mathrm{m}^{3} \\
\end{array}$ & $\begin{array}{l}\text { mrem per } \\
\mathrm{pCi} / \mathrm{g}\end{array}$ & $\begin{array}{c}\mu \mathrm{Sv} \text { per } \\
\mathrm{Bq} / \mathrm{g} \\
\end{array}$ \\
\hline $\begin{array}{l}{ }^{223} \mathrm{Ra}+\mathrm{I}(\mathrm{b}) \\
{ }^{224} \mathrm{Ra}+\mathrm{D}(\mathrm{a}) \\
{ }^{224} \mathrm{Ra} \mathrm{C}(\mathrm{b}) \\
{ }^{225} \mathrm{Ra} \text { (a) } \\
{ }^{225} \mathrm{Ra} \mathrm{Ra} \\
{ }^{228} \mathrm{Ra}(\mathrm{a}) \\
{ }^{228} \mathrm{Ra} \mathrm{Ra} \\
{ }^{228} \mathrm{Ra}(\mathrm{a}) \\
{ }^{228} \mathrm{Ra} \mathrm{Ra}\end{array}$ & $\begin{array}{l}2.8 \mathrm{E}-01 \\
8.8 \mathrm{E}-02 \\
3.8 \mathrm{E}-01 \\
3.9 \mathrm{E}-01 \\
1.6 \mathrm{E}+00 \\
1.2 \mathrm{E}+01 \\
1.3 \mathrm{E}+01 \\
2.1 \mathrm{E}+00 \\
1.1 \mathrm{E}+01\end{array}$ & $\begin{array}{l}7.7 E+01 \\
2.4 E+01 \\
1.0 E+02 \\
1.0 E+02 \\
4.3 E+02 \\
3.3 E+03 \\
3.5 E+03 \\
5.7 E+02 \\
3.0 E+03\end{array}$ & $\begin{array}{l}1.2 \mathrm{E}-01 \\
7.0 \mathrm{E}-02 \\
3.6 \mathrm{E}-01 \\
9.1 \mathrm{E}-02 \\
6.1 \mathrm{E}-01 \\
8.6 \mathrm{E}-01 \\
2.7 \mathrm{E}+00 \\
3.4 \mathrm{E}-01 \\
2.3 \mathrm{E}+00\end{array}$ & $\begin{array}{l}3.3 \mathrm{E}+01 \\
1.9 \mathrm{E}+01 \\
9.7 \mathrm{E}+01 \\
2.5 \mathrm{E}+01 \\
1.6 \mathrm{E}+02 \\
2.3 \mathrm{E}+02 \\
7.3 \mathrm{E}+02 \\
9.3 \mathrm{E}+01 \\
6.2 \mathrm{E}+02\end{array}$ \\
\hline $\begin{array}{l}{ }^{225} \mathrm{AC}+\mathrm{I}(\mathrm{b}) \\
{ }^{227} \mathrm{AC}+\mathrm{D}(\mathrm{a}) \\
{ }^{227} \mathrm{AC} \\
{ }^{228} \mathrm{AC}+\mathrm{D}(\mathrm{a}) \\
{ }^{228} \mathrm{AC}\end{array}$ & $\begin{array}{l}1.0 \mathrm{E}-01 \\
2.1 \mathrm{E}+01 \\
1.7 \mathrm{E}+02 \\
8.9 \mathrm{E}-01 \\
4.9 \mathrm{E}-04\end{array}$ & $\begin{array}{l}2.7 E+01 \\
5.7 E+03 \\
4.6 E+04 \\
2.4 E+02 \\
1.3 E-01\end{array}$ & $\begin{array}{l}4.7 \mathrm{E}-03 \\
5.4 \mathrm{E}-01 \\
3.6 \mathrm{E}+00 \\
9.1 \mathrm{E}-02 \\
5.4 \mathrm{E}-04\end{array}$ & $\begin{array}{l}1.3 E+00 \\
1.5 E+02 \\
9.7 E+02 \\
2.5 E+01 \\
1.5 E-01\end{array}$ \\
\hline $\begin{array}{l}{ }^{227} T h+D(a) \\
{ }^{227} T h \\
{ }^{228} T h+D(a) \\
{ }^{228} T h \\
{ }^{228} T h+D(a) \\
{ }^{229} T h \\
{ }^{236} T h+D(a) \\
{ }^{236} T h \\
{ }^{231} T h+D(a) \\
{ }^{231} T h \\
{ }^{232} T h+D(a) \\
{ }^{232} T h-N a t(c) \\
{ }^{232} T h \\
{ }^{234} T h+D(a) \\
{ }^{234} T h+I \\
T h)\end{array}$ & $\begin{array}{l}4.6 \mathrm{E}-01 \\
2.8 \mathrm{E}-01 \\
1.0 \mathrm{E}+00 \\
4.8 \mathrm{E}+00 \\
5.9 \mathrm{E}+00 \\
4.3 \mathrm{E}+01 \\
1.1 \mathrm{E}+01 \\
6.5 \mathrm{E}+00 \\
1.2 \mathrm{E}-04 \\
1.2 \mathrm{E}-03 \\
5.2 \mathrm{E}+00 \\
5.2 \mathrm{E}+00 \\
3.3 \mathrm{E}+01 \\
5.5 \mathrm{E}-02 \\
1.1 \mathrm{E}-01\end{array}$ & $\begin{array}{l}1.2 E+02 \\
7.6 E+01 \\
2.7 E+02 \\
1.3 E+03 \\
1.6 E+03 \\
1.2 E+04 \\
3.0 E+03 \\
1.8 E+03 \\
3.2 E-02 \\
3.2 E-01 \\
1.4 E+03 \\
1.4 E+03 \\
8.9 E+03 \\
1.5 E+01 \\
3.0 E+01\end{array}$ & $\begin{array}{l}1.1 \mathrm{E}-01 \\
3.0 \mathrm{E}-02 \\
1.0 \mathrm{E}-01 \\
3.1 \mathrm{E}-01 \\
4.3 \mathrm{E}-01 \\
2.8 \mathrm{E}+00 \\
8.1 \mathrm{E}-01 \\
4.2 \mathrm{E}-01 \\
1.0 \mathrm{E}-04 \\
1.0 \mathrm{E}-03 \\
5.2 \mathrm{E}-01 \\
5.2 \mathrm{E}-01 \\
2.2 \mathrm{E}+00 \\
5.5 \mathrm{E}-03 \\
1.1 \mathrm{E}-02\end{array}$ & $\begin{array}{l}2.9 \mathrm{E}+01 \\
8.1 \mathrm{E}+00 \\
2.8 \mathrm{E}+01 \\
8.4 \mathrm{E}+01 \\
1.1 \mathrm{E}+02 \\
7.6 \mathrm{E}+02 \\
2.2 \mathrm{E}+02 \\
1.1 \mathrm{E}+02 \\
2.7 \mathrm{E}-02 \\
2.7 \mathrm{E}-01 \\
1.4 \mathrm{E}+02 \\
1.4 \mathrm{E}+02 \\
5.9 \mathrm{E}+02 \\
1.5 \mathrm{E}+00 \\
3.0 \mathrm{E}+00\end{array}$ \\
\hline $\begin{array}{l}{ }^{231} \mathrm{~Pa}+\mathrm{D}(\mathrm{a}) \\
{ }^{231} \mathrm{~Pa} \\
{ }^{233} \mathrm{~Pa} \\
{ }^{234} \mathrm{~Pa}\end{array}$ & $\begin{array}{l}3.6 \mathrm{E}+01 \\
1.4 \mathrm{E}+02 \\
3.0 \mathrm{E}-02 \\
5.5 \mathrm{E}-04\end{array}$ & $\begin{array}{l}9.6 \mathrm{E}+03 \\
3.8 \mathrm{E}+04 \\
8.1 \mathrm{E}+00 \\
1.5 \mathrm{E}-01\end{array}$ & $\begin{array}{l}2.6 \mathrm{E}+00 \\
1.9 \mathrm{E}+01 \\
6.0 \mathrm{E}-03 \\
3.7 \mathrm{E}-03\end{array}$ & $\begin{array}{l}6.9 \mathrm{E}+02 \\
5.1 \mathrm{E}+03 \\
1.6 \mathrm{E}+00 \\
1.0 \mathrm{E}+00\end{array}$ \\
\hline $\begin{array}{l}{ }^{232} U+D^{(a)} \\
{ }^{232} U \\
{ }^{233} U+D(a) \\
{ }^{233} U \\
{ }^{234} U \\
{ }^{235} U+D(a) \\
{ }^{235} U\end{array}$ & $\begin{array}{l}1.3 \mathrm{E}-01 \\
9.3 \mathrm{E}-01 \\
5.2 \mathrm{E}+00 \\
3.6 \mathrm{E}-01 \\
3.5 \mathrm{E}-01 \\
1.9 \mathrm{E}-01 \\
3.6 \mathrm{E}-01\end{array}$ & $\begin{array}{l}3.6 \mathrm{E}+01 \\
2.5 \mathrm{E}+02 \\
1.4 \mathrm{E}+03 \\
9.7 \mathrm{E}+01 \\
9.5 \mathrm{E}+01 \\
5.1 \mathrm{E}+01 \\
9.7 \mathrm{E}+01\end{array}$ & $\begin{array}{l}4.7 \mathrm{E}-03 \\
3.3 \mathrm{E}-02 \\
3.8 \mathrm{E}-01 \\
1.3 \mathrm{E}-02 \\
1.3 \mathrm{E}-02 \\
7.0 \mathrm{E}-03 \\
1.3 \mathrm{E}-02\end{array}$ & $\begin{array}{l}1.3 \mathrm{E}+00 \\
8.9 \mathrm{E}+00 \\
1.0 \mathrm{E}+02 \\
3.5 \mathrm{E}+00 \\
3.5 \mathrm{E}+00 \\
1.9 \mathrm{E}+00 \\
3.5 \mathrm{E}+00\end{array}$ \\
\hline
\end{tabular}


TABLE 2.5. Agricultural Food Product Ingestion Committed Effective Dose Rate Conversion Factors for Exposure to Residual Radioactive Materials (Air and Soil Contributions) (contd)

\begin{tabular}{|c|c|c|c|c|}
\hline \multirow[b]{2}{*}{ Radionuclide } & \multicolumn{2}{|c|}{ Air Deposition } & \multicolumn{2}{|c|}{ Soil Uptake } \\
\hline & $\begin{array}{l}\text { mrem per } \\
\mathrm{pCi} / \mathrm{m}^{3} \\
\end{array}$ & $\begin{array}{l}\mu \mathrm{Sv} \text { per } \\
\mathrm{Bq} / \mathrm{m}^{3} \\
\end{array}$ & $\begin{array}{l}\text { mrem per } \\
\mathrm{pCi} / \mathrm{g}\end{array}$ & $\begin{array}{l}\mu \mathrm{Sv} \text { per } \\
\mathrm{Bq} / \mathrm{g}\end{array}$ \\
\hline 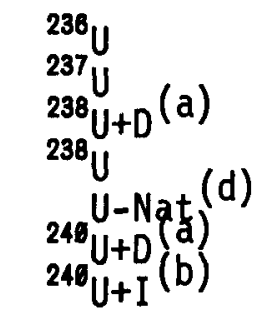 & $\begin{array}{l}3.3 \mathrm{E}-01 \\
1.5 \mathrm{E}-02 \\
1.6 \mathrm{E}-01 \\
3.2 \mathrm{E}-01 \\
3.4 \mathrm{E}-01 \\
8.0 \mathrm{E}-04 \\
2.4 \mathrm{E}-03\end{array}$ & $\begin{array}{l}8.9 \mathrm{E}+01 \\
4.0 \mathrm{E}+00 \\
4.4 \mathrm{E}+01 \\
8.6 \mathrm{E}+01 \\
9.1 \mathrm{E}+01 \\
2.2 \mathrm{E}-01 \\
6.5 \mathrm{E}-01\end{array}$ & $\begin{array}{l}1.2 \mathrm{E}-02 \\
1.5 \mathrm{E}-03 \\
7.3 \mathrm{E}-03 \\
1.1 \mathrm{E}-02 \\
1.2 \mathrm{E}-02 \\
7.3 \mathrm{E}-04 \\
2.2 \mathrm{E}-03\end{array}$ & $\begin{array}{l}3.2 E+00 \\
4.1 E-01 \\
2.0 E+00 \\
3.0 E+00 \\
3.2 E+00 \\
2.0 E-01 \\
5.9 E-01\end{array}$ \\
\hline $\begin{array}{l}{ }^{237} \underset{N p+D}{ }(a) \\
287 \underset{N p}{N}(a) \\
288 N_{N p+D}(a) \\
288{ }_{N p}(a) \\
280{ }_{N p+D}(a) \\
280 \\
N p\end{array}$ & $\begin{array}{l}3.2 E+01 \\
6.4 E+01 \\
2.0 E+00 \\
6.8 E-03 \\
3.0 E-03 \\
6.1 E-03\end{array}$ & $\begin{array}{l}8.6 \mathrm{E}+03 \\
1.7 \mathrm{E}+04 \\
5.5 \mathrm{E}+02 \\
1.8 \mathrm{E}+00 \\
8.2 \mathrm{E}-01 \\
1.6 \mathrm{E}+00\end{array}$ & $\begin{array}{l}1.2 \mathrm{E}+00 \\
2.4 \mathrm{E}+00 \\
1.9 \mathrm{E}-02 \\
1.9 \mathrm{E}-03 \\
7.5 \mathrm{E}-04 \\
1.5 \mathrm{E}-03\end{array}$ & $\begin{array}{l}3.2 \mathrm{E}+02 \\
6.5 \mathrm{E}+02 \\
5.0 \mathrm{E}+00 \\
5.1 \mathrm{E}-01 \\
2.0 \mathrm{E}=01 \\
4.1 \mathrm{E}-01\end{array}$ \\
\hline $\begin{array}{l}{ }^{236} \mathrm{Pu} \\
{ }^{237} \mathrm{Pu} \\
{ }^{238} \mathrm{Pu} \\
{ }^{239} \mathrm{Pu} \\
{ }^{248} \mathrm{Pu} \\
{ }_{241} \mathrm{Pu}+\mathrm{D}(\mathrm{a}) \\
{ }^{241} \mathrm{Pu} \\
{ }^{242} \mathrm{Pu} \\
{ }^{243} \mathrm{Pu}+\mathrm{D}(\mathrm{a}) \\
{ }_{243} \mathrm{Pu} \\
{ }_{244} \mathrm{Pu}+\mathrm{D}(\mathrm{a}) \\
{ }_{244} \mathrm{Pu}\end{array}$ & $\begin{array}{l}7.6 \mathrm{E}-01 \\
4.1 \mathrm{E}-03 \\
4.1 \mathrm{E}+00 \\
4.5 \mathrm{E}+00 \\
4.5 \mathrm{E}+00 \\
2.4 \mathrm{E}+01 \\
8.3 \mathrm{E}-02 \\
4.2 \mathrm{E}+00 \\
2.8 \mathrm{E}-05 \\
5.6 \mathrm{E}-05 \\
1.1 \mathrm{E}+00 \\
4.3 \mathrm{E}+00\end{array}$ & $\begin{array}{l}2.1 \mathrm{E}+02 \\
1.1 \mathrm{E}+00 \\
1.1 \mathrm{E}+03 \\
1.2 \mathrm{E}+03 \\
1.2 \mathrm{E}+03 \\
6.3 \mathrm{E}+03 \\
2.2 \mathrm{E}+01 \\
1.1 \mathrm{E}+03 \\
7.6 \mathrm{E}-03 \\
1.5 \mathrm{E}-02 \\
3.0 \mathrm{E}+02 \\
1.2 \mathrm{E}+03\end{array}$ & $\begin{array}{l}6.6 \mathrm{E}-03 \\
4.4 \mathrm{E}-05 \\
3.5 \mathrm{E}-02 \\
3.9 \mathrm{E}-02 \\
3.8 \mathrm{E}-02 \\
2.0 \mathrm{E}+00 \\
7.1 \mathrm{E}-04 \\
3.6 \mathrm{E}-02 \\
1.7 \mathrm{E}-05 \\
3.3 \mathrm{E}-05 \\
9.8 \mathrm{E}-03 \\
3.7 \mathrm{E}-02\end{array}$ & $\begin{array}{l}1.8 \mathrm{E}+00 \\
1.2 \mathrm{E}-02 \\
9.4 \mathrm{E}+00 \\
1.1 \mathrm{E}+01 \\
1.0 \mathrm{E}+01 \\
5.5 \mathrm{E}+02 \\
1.9 \mathrm{E}-01 \\
9.7 \mathrm{E}+00 \\
4.5 \mathrm{E}-03 \\
8.9 \mathrm{E}-03 \\
2.6 \mathrm{E}+00 \\
1.0 \mathrm{E}+01\end{array}$ \\
\hline $\begin{array}{l}{ }^{241} \mathrm{Am} \\
242 \mathrm{Am}(\mathrm{am}+\mathrm{a} \\
242 \mathrm{~m} \mathrm{Am} \\
{ }^{242} \mathrm{Am}+\mathrm{D}(\mathrm{a}) \\
{ }^{242} \mathrm{Am} \\
{ }^{243} \mathrm{Am}+\mathrm{D}(\mathrm{a}) \\
{ }^{243} \mathrm{Am}\end{array}$ & $\begin{array}{l}4.7 \mathrm{E}+01 \\
1.3 \mathrm{E}+01 \\
4.5 \mathrm{E}+01 \\
1.6 \mathrm{E}+00 \\
8.2 \mathrm{E}-04 \\
1.6 \mathrm{E}+01 \\
4.7 \mathrm{E}+01\end{array}$ & $\begin{array}{l}1.3 \mathrm{E}+04 \\
3.5 \mathrm{E}+03 \\
1.2 \mathrm{E}+04 \\
4.3 \mathrm{E}+02 \\
2.2 \mathrm{E}-01 \\
4.2 \mathrm{E}+03 \\
1.3 \mathrm{E}+04\end{array}$ & $\begin{array}{l}4.1 E+00 \\
1.0 E+00 \\
3.9 E+00 \\
2.7 E-02 \\
1.4 E-03 \\
1.4 E+00 \\
4.1 E+00\end{array}$ & $\begin{array}{l}1.1 \mathrm{E}+03 \\
2.8 \mathrm{E}+02 \\
1.1 \mathrm{E}+03 \\
7.3 \mathrm{E}+00 \\
3.8 \mathrm{E}-01 \\
3.7 \mathrm{E}+02 \\
1.1 \mathrm{E}+03\end{array}$ \\
\hline $\begin{array}{l}{ }^{242} \mathrm{Cm}+D^{(a)} \\
{ }^{242} \mathrm{Cm} \\
{ }^{243} \mathrm{Cm} \\
{ }^{244} \mathrm{Cm}+\mathrm{D} \\
{ }^{246} \mathrm{Cm} \\
{ }^{246} \mathrm{Cm}+\mathrm{C} \\
{ }^{246} \mathrm{Cm} \\
\mathrm{Cm}\end{array}$ & $\begin{array}{l}3.0 \mathrm{E}+00 \\
1.3 \mathrm{E}+00 \\
3.5 \mathrm{E}+01 \\
1.2 \mathrm{E}+01 \\
2.4 \mathrm{E}+01 \\
3.1 \mathrm{E}+01 \\
4.5 \mathrm{E}+01 \\
4.5 \mathrm{E}+01\end{array}$ & $\begin{array}{l}8.0 \mathrm{E}+02 \\
3.5 \mathrm{E}+02 \\
9.4 \mathrm{E}+03 \\
3.2 \mathrm{E}+03 \\
6.5 \mathrm{E}+03 \\
8.3 \mathrm{E}+03 \\
1.2 \mathrm{E}+04 \\
1.2 \mathrm{E}+04\end{array}$ & $\begin{array}{l}4.7 \mathrm{E}-02 \\
5.1 \mathrm{E}-02 \\
1.2 \mathrm{E}+00 \\
4.7 \mathrm{E}-01 \\
9.3 \mathrm{E}-01 \\
1.9 \mathrm{E}+00 \\
1.7 \mathrm{E}+00 \\
1.7 \mathrm{E}+00\end{array}$ & $\begin{array}{l}1.3 \mathrm{E}+01 \\
1.4 \mathrm{E}+01 \\
3.2 \mathrm{E}+02 \\
1.3 \mathrm{E}+02 \\
2.5 \mathrm{E}+02 \\
5.2 \mathrm{E}+02 \\
4.6 \mathrm{E}+02 \\
4.6 \mathrm{E}+02\end{array}$ \\
\hline
\end{tabular}


TABLE 2.5. Agricultural Food Product Ingestion Committed Effective Dose Rate Conversion Factors for Exposure to Residual Radioactive Materials (Air and Soil Contributions) (contd)

\begin{tabular}{|c|c|c|c|c|}
\hline \multirow[b]{2}{*}{ Radionuclide } & \multicolumn{2}{|c|}{ Air Deposition } & \multicolumn{2}{|c|}{ Soil Uptake } \\
\hline & $\begin{array}{l}\text { mrem per } \\
\mathrm{pCi} / \mathrm{m}^{3}\end{array}$ & $\begin{array}{c}\mu \mathrm{SV} \text { per } \\
\mathrm{Bg} / \mathrm{m}^{3}\end{array}$ & $\begin{array}{l}\text { mrem per } \\
\mathrm{pCi} / \mathrm{g}\end{array}$ & $\begin{array}{l}\mu \mathrm{Sv} \text { per } \\
\mathrm{Bq} / \mathrm{g}\end{array}$ \\
\hline $\begin{array}{l}{ }^{247} \mathrm{Cm}+\mathrm{D}^{(a)} \\
{ }^{247} \mathrm{Cm} \\
{ }^{44 m} \mathrm{Cm}\end{array}$ & $\begin{array}{l}2.1 E+01 \\
4.2 E+01 \\
1.6 E+02\end{array}$ & $\begin{array}{l}5.7 \mathrm{E} \\
1.1 \mathrm{E} \\
4.3 \mathrm{E}\end{array}$ & $\begin{array}{l}8.0 \\
1.6 \\
6.2\end{array}$ & $\begin{array}{l}2.2 \mathrm{E}+02 \\
4.3 \mathrm{E}+02 \\
1.7 \mathrm{E}+03\end{array}$ \\
\hline${ }^{252} \mathrm{Cf}$ & $1.4 \mathrm{E}+01$ & $3.8 E+03$ & $1.1 \mathrm{E}-01$ & $3.0 E+01$ \\
\hline
\end{tabular}

(a) Where $+D$ means "plus daughters in equilibrium" referring to explicit daughters, or daughters with half-lives greater than 10 minutes. Separate values are also included for parents and daughters so that combined factors for non-equilibrium cases can be determined. Note: in some cases $+D$ also includes implicit daughters (i.e., uranium and thorium decay chains).

(b) Where +I means "plus implicit daughters in equilibrium" referring to daughters with half-lives less than 10 minutes. The decay energies associated with these daughters have been assigned to the parent, and the appropriate branching ratios (described in Appendix D) have been considered.

(c) Where Th-Nat includes an equilibrium mixture of ${ }^{232}$ Th plus 10 daughters in the decay chain, as discussed in Appendix $D$.

(d) Where $1 \mathrm{Ci} U$-Nat equals $48.9 \%{ }^{238} \mathrm{U}$ plus $48.9 \%{ }^{234} \mathrm{U}$ plus $2.25 \%{ }^{235} \mathrm{U}$. 



\subsection{SCENARIO ANALYSIS FOR RESIDUAL RADIOACTIVE MATERIALS}

Radiation exposure scenarios represent a combination of radiation exposure pathways in an effort to conceptually model the potential conditions, events, and processes that result in radiation exposure to individuals or groups of people. Residual contamination can exist in buildings as volume or surface sources and as volume sources in soils. To adequately model these conditions, a series of scenarios for volume and surface source relationships were developed. The scenarios combine the pathway dose rate conversion factors and committed effective dose rate conversion factors described in Section 2.0 with scenario-specific assumptions to produce annual TEDE factors. The scenario-specific assumptions include the exposure durations, quantities inhaled or ingested, and media concentrations (i.e., surface, air, food product, or drinking water concentrations). Listings of the annual TEDE are provided for each scenario considered for over 200 radionuclides or parent-daughter mixtures. For this study, daughters with half-lives less than 10 minutes are always assumed to be in equilibrium with their parent and are referred to as implicit daughters. It is important to note that the unit activity shown in the TEDE tables includes contributions from both the parent and daughters. To accomplish this, a +I notation has been included in the TEDE listings with an appropriate footnote describing the meaning. Daughters with half-lives greater than 10 minutes that may not be in equilibrium with their parents are referred to as explicit daughters. These are shown in equilibrium conditions with the parent by a $+D$ listing in the TEDE tables. Separate values are also included for parents and daughters so that values for non-equilibrium cases can be determined. For cases where there is a short-lived parent with a long-lived daughter, no attempt is made to combine the parent and daughter. Instead, separate listings for parent and daughter are included so that any equilibrium condition may be evaluated. Finally, for decay chains, the listing include parents alone, parents with +0 and $+I$ notations (to include both implicit and explicit daughters) and daughters alone so that equilibrium and non-equilibrium cases can be considered. A further description of the parent-daughter and chain decay relationships used in this study is included in Appendix $D$. The following sections describe the scenarios for residual radioactive materials in buildings and in soils, and the resulting annual TEDE factors for volume and surface activity.

\subsection{SCENARIO DESCRIPTIONS FOR THE RELEASE OF BUILDINGS}

Two scenarios are defined for facilities to account for 1) building renovation (sub-surface or volume sources), and 2) normal building occupancy (thin-layer and removable surface sources). The building renovation scenario accounts for an average volumetric concentration of material in building surfaces. The building occupancy scenario accounts for contamination located in a thin surface layer, with a small fraction that is removable. This approach is needed to permit the calculation of unit concentration annual TEDE factors for residual radioactivity in structures. The resulting generically derived unit concentration annual TEDE factors should serve as a basis for developing screening levels for simple situations and should help 
determine when more detailed site-specific data, models, and assessments are required. For this study, it is assumed that the facility is used as a commercial building after license termination. Sections 3.1 .1 and 3.1 .2 describe the scenarios, parameters, and calculations developed to model the unrestricted release of buildings. The results of the dose calculations are provided in terms of unit concentration annual TEDE factors for each scenario. Additional information regarding potential modifications to the calculations to account for site-specific conditions and a further discussion of the selection of parameter values are found in Appendix B.

\subsubsection{Building Renovation (Volume Activity) Scenario}

Decontamination operations are designed to reduce or eliminate the surface inventory through process including surface washing, sandblasting, or concrete spalling. For the building renovation scenario, it is assumed that the residual contamination in a building is associated with volume sources; that is, radionuclides are assumed to be distributed within an effective volume of material and are not confined to a thin surface layer. This assumption also anticipates that there could be subsurface sources or volume sources, for example, around floor drains or cracks that could contain significant quantities of radioactive material. It also anticipates the variability among types of surfaces and materials that may be encountered. To permit the derivation of unit concentration annual TEDE factors for volume sources in buildings, the building renovation scenario was defined accounting for exposures to individuals in a facility by external penetrating radiation, inhalation, and secondary ingestion. Typical licensed facilities are likely to continue to be used as commercial buildings. To do so, it is anticipated that they will require some renovation after unrestricted release with disturbance of volume sources associated with floors and walls. For this scenario, it is assumed that there are no significant removable contamination sources at the time of license termination (i.e., only a small amount of loose material is present after decontamination efforts). However, volume contamination sources are assumed to be disturbed during building renovation resulting in loose contamination available for resuspension and secondary ingestion. After renovation, the loose contamination is assumed to be removed and the surfaces stabilized through the application of fresh paint, new floor surfaces, and other methods. For completeness, long-term chronic exposure to low levels of loose surface contamination is considered in the building occupancy scenario discussed in Section 3.1.2.

For this analysis, the residual contamination is assumed to be homogeneously distributed in the top $1 \mathrm{~cm}$ of building surfaces. Building renovation operations are also assumed to uniformly mix materials within the top $1 \mathrm{~cm}$ of building surface as they are removed. Building renovation is assumed to last for $500 \mathrm{~h}$ (about 3 work-months) and produce an average air concentration of dust of $10^{-4} \mathrm{~g} / \mathrm{m}^{3}$ in air. For secondary ingestion, the individual is assumed to ingest $0.01 \mathrm{~g} / \mathrm{h}$ during $500 \mathrm{~h}$ of exposure. These parameters and their expected ranges are summarized in Section 3.3 and are more fully discussed in Appendix B. 
To develop a renovation scenario with a balanced set of exposure pathways, it is assumed that the same individual is exposed by all three pathways: external, inhalation, and secondary ingestion. This scenario does not include consideration of indoor radon from residual uranium in building materials. Building renovation is assumed to occur immediately after release of the building, before significant radioactive decay occurs, in an attempt to maximize the potential impact of renovation. The results of unit concentration dose rate calculations for this scenario (mrem per $\mathrm{pCi} / \mathrm{g}$ and $\mu$ Sv per $\mathrm{Bq} / \mathrm{g}$ ), accounting for volume sources in the facility, are shown in Table 3.1. This table contains the annual TEDE factors for the building renovation scenario and lists the contribution by exposure pathway for about 200 radionuclides, including key parent-daughter decay chains. Again, the $+I$ (implicit daughter) and $+D$ (explicit daughter) notation has been used to provide maximum flexibility for consideration of all parent-daughter and decay chain situations. Further description of the parent-daughter and chain decay relationships used in this study is provided in Appendix $D$.

Hand calculations were performed to verify the computer calculations for selected radionuclides using the pathway factors from Section 2.0 and the Building renovation scenario assumptions for the external exposure, inhalation, and secondary ingestion pathways. The results for $60 \mathrm{co}, 90 \mathrm{Sr}$, $137 \mathrm{Cs}, 235 \mathrm{U}$, and $239 \mathrm{Pu}$ indicated that the values in Table 3.1 were calculated correctly. 
TABLE 3.1. Annual Total Effective Dose Equivalent Factors for the Building Renovation (Volume Activity) Scenario(a)

\begin{tabular}{|c|c|c|c|c|c|}
\hline Radionuclide & $\begin{array}{l}\text { External } \\
\text { (mrem) }\end{array}$ & $\begin{array}{l}\text { Inhalation } \\
\text { (mrem) }\end{array}$ & $\begin{array}{c}\text { Secondary } \\
\text { Ingestion } \\
\text { (mrem) }\end{array}$ & $\begin{array}{c}\text { TEDE } \\
\text { (mrem) }\end{array}$ & $\begin{array}{l}\text { TEDE } \\
(\mu S V)\end{array}$ \\
\hline${ }^{3} \mathrm{H}$ & $5.1 E-12$ & $4.1 \mathrm{E}-09$ & $2.8 \mathrm{E}-07$ & $2.9 E-07$ & $7.8 \mathrm{E}-05$ \\
\hline${ }^{16} \mathrm{Be}$ & $7.0 \mathrm{E}-06$ & $1.7 E-05$ & $2.3 \mathrm{E}-05$ & 4.7E-05 & 1. $3 E-02$ \\
\hline${ }^{14} \mathrm{C}$ & $2.1 E-07$ & $9.5 E-08$ & $9.5 \mathrm{E}-06$ & $9.8 \mathrm{E}-06$ & $2.6 \mathrm{E}-03$ \\
\hline${ }^{18} \mathrm{~F}$ & $3.0 \mathrm{E}-02$ & $3.8 \mathrm{E}-09$ & $5.5 \mathrm{E}-07$ & $3.0 \mathrm{E}-02$ & $8.1 E+00$ \\
\hline $\begin{array}{l}{ }^{22} \mathrm{Na} \\
{ }^{24} \mathrm{Na}\end{array}$ & $\begin{array}{l}6.9 \mathrm{E}-02 \\
1.4 \mathrm{E}-01\end{array}$ & $\begin{array}{l}4.3 \mathrm{E}-07 \\
5.5 \mathrm{E}-08\end{array}$ & $\begin{array}{l}7.0 \mathrm{E}-05 \\
7.0 \mathrm{E}-06\end{array}$ & $\begin{array}{l}6.9 \mathrm{E}-02 \\
1.4 \mathrm{E}-01\end{array}$ & $\begin{array}{l}1.9 E+01 \\
3.7 E+01\end{array}$ \\
\hline $\begin{array}{l}{ }^{32} \mathrm{p} \\
{ }^{33} \mathrm{p}\end{array}$ & $\begin{array}{l}1.1 \mathrm{E}-04 \\
7.7 \mathrm{E}-07\end{array}$ & $\begin{array}{l}2.7 \mathrm{E}-07 \\
2.8 \mathrm{E}-08\end{array}$ & $\begin{array}{l}4.2 E-05 \\
4.3 E-06\end{array}$ & $\begin{array}{l}1.6 \mathrm{E}-04 \\
5.1 \mathrm{E}-06\end{array}$ & $\begin{array}{l}4.2 \mathrm{E}-02 \\
1.4 \mathrm{E}-03\end{array}$ \\
\hline${ }^{35} \mathrm{~S}$ & $2.4 \mathrm{E}-07$ & $1.2 E-07$ & $2.0 \mathrm{E}-06$ & 2.4E-06 & $6.4 \mathrm{E}-04$ \\
\hline${ }^{36} \mathrm{Cl}$ & $1.3 \mathrm{E}-05$ & $9.0 \mathrm{E}-08$ & $1.4 \mathrm{E}-05$ & $2.7 E-05$ & $7.2 E-03$ \\
\hline${ }^{46} \mathrm{~K}$ & $5.4 \mathrm{E}-03$ & $4.9 \mathrm{E}-07$ & $8.5 E-05$ & $5.5 E-03$ & $1.5 \mathrm{E}+00$ \\
\hline${ }^{41} \mathrm{Ca}$ & $\begin{array}{l}6.9 \mathrm{E}-06 \\
8.1 \mathrm{E}-07\end{array}$ & $\begin{array}{l}6.0 \mathrm{E}-08 \\
3.2 \mathrm{E}-07\end{array}$ & $\begin{array}{l}6.0 \mathrm{E}-06 \\
1.5 \mathrm{E}-05\end{array}$ & $\begin{array}{l}1.3 \mathrm{E}-05 \\
1.7 \mathrm{E}-05\end{array}$ & $\begin{array}{l}3.5 \mathrm{E}-03 \\
4.5 \mathrm{E}-03\end{array}$ \\
\hline${ }^{46} \mathrm{SC}$ & $7.0 \mathrm{E}-02$ & $1.4 \mathrm{E}-06$ & $3.1 E-05$ & $7.0 \mathrm{E}-02$ & $1.9 \mathrm{E}+01$ \\
\hline${ }^{51} \mathrm{Cr}$ & $1.1 \mathrm{E}-03$ & $1.5 \mathrm{E}-08$ & $7.0 \mathrm{E}-07$ & $1.1 \mathrm{E}-03$ & $3.0 \mathrm{E}-01$ \\
\hline $\begin{array}{l}{ }^{54} \mathrm{Mn} \\
{ }^{56} \mathrm{Mn}\end{array}$ & $\begin{array}{l}2.8 \mathrm{E}-02 \\
5.8 \mathrm{E}-02\end{array}$ & $\begin{array}{l}\text { 3.0E }-07 \\
1.6 \mathrm{E}-08\end{array}$ & $\begin{array}{l}1.4 \mathrm{E}-05 \\
5.0 \mathrm{E}-06\end{array}$ & $\begin{array}{l}2.8 \mathrm{E}-02 \\
5.8 \mathrm{E}-02\end{array}$ & $\begin{array}{l}7.6 \mathrm{E}+00 \\
1.6 \mathrm{E}+01\end{array}$ \\
\hline $\begin{array}{l}{ }^{55} \mathrm{Fe} \\
{ }^{59} \mathrm{Fe}\end{array}$ & $\begin{array}{l}1.6 \mathrm{E}-05 \\
3.8 \mathrm{E}-02\end{array}$ & $\begin{array}{l}6.0 \mathrm{E}-08 \\
6.0 \mathrm{E}-07\end{array}$ & $\begin{array}{l}2.9 E-06 \\
3.3 E-05\end{array}$ & $\begin{array}{l}1.9 \mathrm{E}-05 \\
3.8 \mathrm{E}-02\end{array}$ & $\begin{array}{l}5.0 \mathrm{E}-03 \\
1.0 \mathrm{E}+01\end{array}$ \\
\hline $\begin{array}{l}{ }^{57} \mathrm{Co} \\
{ }^{58} \mathrm{Co} \\
{ }^{68} \mathrm{Co}\end{array}$ & $\begin{array}{l}3.4 E-03 \\
3.3 E-02 \\
8.3 E-02\end{array}$ & $\begin{array}{l}4.1 \mathrm{E}-07 \\
5.0 \mathrm{E}-07 \\
9.5 \mathrm{E}-06\end{array}$ & $\begin{array}{l}3.8 E-06 \\
1.4 E-05 \\
5.0 E-05\end{array}$ & $\begin{array}{l}3.4 \mathrm{E}-03 \\
3.3 \mathrm{E}-02 \\
8.3 \mathrm{E}-02\end{array}$ & $\begin{array}{l}\text { 9.2E-01 } \\
8.9 \mathrm{E}+00 \\
2.3 \mathrm{E}+01\end{array}$ \\
\hline $\begin{array}{l}{ }^{59} \mathrm{Ni} \\
{ }^{63} \mathrm{Ni} \\
{ }^{85} \mathrm{Ni}\end{array}$ & $\begin{array}{l}1.9 \mathrm{E}-05 \\
9.9 \mathrm{E}-09 \\
1.9 \mathrm{E}-02\end{array}$ & $\begin{array}{l}3.9 \mathrm{E}-08 \\
9.5 \mathrm{E}-08 \\
1.2 \mathrm{E}-08\end{array}$ & $\begin{array}{l}9.5 \mathrm{E}-07 \\
2.5 \mathrm{E}-06 \\
3.2 \mathrm{E}-06\end{array}$ & $\begin{array}{l}2.0 \mathrm{E}-05 \\
2.7 \mathrm{E}-06 \\
1.9 \mathrm{E}-02\end{array}$ & $\begin{array}{l}5.5 E-03 \\
7.2 E-04 \\
5.2 E+00\end{array}$ \\
\hline${ }^{64} \mathrm{Cu}$ & $5.7 \mathrm{E}-03$ & $9.5 \mathrm{E}-09$ & $2.4 \mathrm{E}-06$ & $5.7 \mathrm{E}-03$ & $1.5 \mathrm{E}+00$ \\
\hline${ }^{65} \mathrm{Zn} \mathrm{Zn} \mathrm{Zn+D}(\mathrm{b})$ & $\begin{array}{l}2.2 \mathrm{E}-02 \\
7.2 \mathrm{E}-03\end{array}$ & $\begin{array}{l}8.5 \mathrm{E}-07 \\
2.0 \mathrm{E}-08\end{array}$ & $\begin{array}{l}6.5 \mathrm{E}-05 \\
3.5 \mathrm{E}-06\end{array}$ & $\begin{array}{l}2.2 \mathrm{E}-02 \\
7.2 \mathrm{E}-03\end{array}$ & $\begin{array}{l}5.8 E+00 \\
2.0 E+00\end{array}$ \\
\hline
\end{tabular}


TABLE 3.1. Annual Total Effective Dose Equivalent Factors for the Building Renovation (Volume Activity) Scenario(a) (contd)

\begin{tabular}{|c|c|c|c|c|c|}
\hline Radionuclide & $\begin{array}{c}\text { External } \\
\text { (mrem) }\end{array}$ & $\begin{array}{c}\text { Inhalation } \\
\text { (mrem) }\end{array}$ & $\begin{array}{c}\text { Secondary } \\
\text { Ingestion } \\
\text { (mrem) }\end{array}$ & $\begin{array}{c}\text { TEDE } \\
\text { (mrem) }\end{array}$ & $\begin{array}{l}\text { TEDE } \\
(\mu \mathrm{SV})\end{array}$ \\
\hline${ }^{89 m} \mathrm{Zn}$ & $\begin{array}{l}1.5 \mathrm{E}-02 \\
2.3 \mathrm{E}-05\end{array}$ & $\begin{array}{l}3.9 \mathrm{E}-08 \\
1.9 \mathrm{E}-09\end{array}$ & $\begin{array}{l}6.5 \mathrm{E}-06 \\
4.5 \mathrm{E}-07\end{array}$ & $\begin{array}{l}1.5 \mathrm{E}-02 \\
2.4 \mathrm{E}-05\end{array}$ & $\begin{array}{l}4.0 \mathrm{E}+00 \\
6.4 \mathrm{E}-03\end{array}$ \\
\hline${ }^{76}$ As & $1.6 \mathrm{E}-02$ & $1.9 \mathrm{E}-07$ & $2.6 \mathrm{E}-05$ & $1.6 \mathrm{E}-02$ & $4.4 \mathrm{E}+00$ \\
\hline $\begin{array}{l}{ }^{75} \mathrm{Se} \\
{ }^{79} \mathrm{Se}\end{array}$ & $\begin{array}{l}1.1 \mathrm{E}-02 \\
1.5 \mathrm{E}-07\end{array}$ & $\begin{array}{l}3.8 \mathrm{E}-07 \\
4.4 \mathrm{E}-07\end{array}$ & $\begin{array}{l}4.4 \mathrm{E}-05 \\
4.0 \mathrm{E}-05\end{array}$ & $\begin{array}{l}1.1 \mathrm{E}-02 \\
4.1 \mathrm{E}-05\end{array}$ & $\begin{array}{l}2.9 \mathrm{E}+00 \\
1.1 \mathrm{E}-02\end{array}$ \\
\hline $\begin{array}{l}{ }^{82} \mathrm{Br} \\
{ }^{83} \mathrm{Br}+\mathrm{D} \\
{ }^{83} \mathrm{Br} \\
{ }^{84} \mathrm{Br} \\
{ }_{\mathrm{Br}}\end{array}$ & $\begin{array}{l}9.3 \mathrm{E}-02 \\
1.2 \mathrm{E}-04 \\
2.4 \mathrm{E}-04 \\
5.7 \mathrm{E}-02\end{array}$ & $\begin{array}{l}5.5 \mathrm{E}-08 \\
2.2 \mathrm{E}-09 \\
4.4 \mathrm{E}-09 \\
4.7 \mathrm{E}-09\end{array}$ & $\begin{array}{l}8.0 \mathrm{E}-06 \\
2.4 \mathrm{E}-07 \\
4.7 \mathrm{E}-07 \\
9.0 \mathrm{E}-07\end{array}$ & $\begin{array}{l}9.3 \mathrm{E}-02 \\
1.2 \mathrm{E}-04 \\
2.4 \mathrm{E}-04 \\
5.7 \mathrm{E}-02\end{array}$ & $\begin{array}{l}2.5 \mathrm{E}+01 \\
3.4 \mathrm{E}-02 \\
6.5 \mathrm{E}-02 \\
1.5 \mathrm{E}+01\end{array}$ \\
\hline $\begin{array}{l}{ }^{86} \mathrm{Rb} \\
{ }^{88} \mathrm{Rb} \\
{ }^{89} \mathrm{Rb}+\mathrm{D} \\
{ }^{89} \mathrm{Rb}\end{array}$ & $\begin{array}{l}3.1 \mathrm{E}-03 \\
2.3 \mathrm{E}-02 \\
3.4 \mathrm{E}-02 \\
6.9 \mathrm{E}-02\end{array}$ & $\begin{array}{l}2.9 \mathrm{E}-07 \\
4.1 \mathrm{E}-09 \\
1.3 \mathrm{E}-07 \\
2.1 \mathrm{E}-09\end{array}$ & $\begin{array}{l}4.6 \mathrm{E}-05 \\
9.0 \mathrm{E}-07 \\
2.2 \mathrm{E}-05 \\
4.8 \mathrm{E}-07\end{array}$ & $\begin{array}{l}3.2 \mathrm{E}-03 \\
2.3 \mathrm{E}-02 \\
3.4 \mathrm{E}-02 \\
6.9 \mathrm{E}-02\end{array}$ & $\begin{array}{l}8.6 \mathrm{E}-01 \\
6.2 \mathrm{E}+00 \\
9.1 \mathrm{E}+00 \\
1.9 \mathrm{E}+01\end{array}$ \\
\hline $\begin{array}{l}{ }^{85} \mathrm{Sr} \\
{ }^{89} \mathrm{Sr} \\
{ }^{98} \mathrm{Sr+D} \\
{ }^{98} \mathrm{Sr} \text { (b) } \\
{ }^{91} \mathrm{Sr} \\
{ }^{91} \mathrm{Sr+D} \\
{ }^{92} \mathrm{Sr} \\
{ }^{92} \mathrm{Sr}+\mathrm{D}\end{array}$ & $\begin{array}{l}1.5 \mathrm{E}-02 \\
8.5 \mathrm{E}-05 \\
1.1 \mathrm{E}-04 \\
5.8 \mathrm{E}-06 \\
1.3 \mathrm{E}-02 \\
2.3 \mathrm{E}-02 \\
2.9 \mathrm{E}-02 \\
4.8 \mathrm{E}-02\end{array}$ & $\begin{array}{l}7.0 \mathrm{E}-08 \\
2.6 \mathrm{E}-07 \\
5.2 \mathrm{E}-06 \\
1.0 \mathrm{E}-05 \\
9.3 \mathrm{E}-07 \\
4.3 \mathrm{E}-08 \\
3.5 \mathrm{E}-08 \\
3.0 \mathrm{E}-08\end{array}$ & $\begin{array}{l}8.5 \mathrm{E}-06 \\
4.3 \mathrm{E}-05 \\
3.3 \mathrm{E}-04 \\
6.0 \mathrm{E}-04 \\
2.3 \mathrm{E}-05 \\
1.2 \mathrm{E}-05 \\
9.0 \mathrm{E}-06 \\
8.0 \mathrm{E}-06\end{array}$ & $\begin{array}{l}1.5 \mathrm{E}-02 \\
1.3 \mathrm{E}-04 \\
4.4 \mathrm{E}-04 \\
6.2 \mathrm{E}-04 \\
1.3 \mathrm{E}-02 \\
2.3 \mathrm{E}-02 \\
2.9 \mathrm{E}-02 \\
4.8 \mathrm{E}-02\end{array}$ & $\begin{array}{l}4.1 \mathrm{E}+0 \mathrm{C} \\
3.5 \mathrm{E}-02 \\
1.2 \mathrm{E}-01 \\
1.7 \mathrm{E}-01 \\
3.6 \mathrm{E}+0 \mathrm{C} \\
6.1 \mathrm{E}+0 \mathrm{C} \\
7.7 \mathrm{E}+0 \mathrm{C} \\
1.3 \mathrm{E}+01\end{array}$ \\
\hline 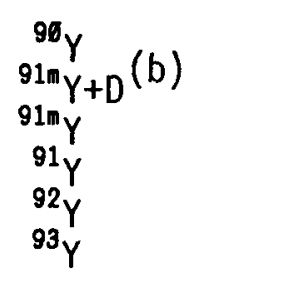 & $\begin{array}{l}2.3 \mathrm{E}-04 \\
1.0 \mathrm{E}-02 \\
2.1 \mathrm{E}-02 \\
2.1 \mathrm{E}-04 \\
9.7 \mathrm{E}-03 \\
3.3 \mathrm{E}-03\end{array}$ & $\begin{array}{l}4.2 \mathrm{E}-07 \\
1.2 \mathrm{E}-06 \\
1.8 \mathrm{E}-09 \\
2.3 \mathrm{E}-06 \\
4.0 \mathrm{E}-08 \\
1.1 \mathrm{E}-07\end{array}$ & $\begin{array}{l}5.5 \mathrm{E}-05 \\
2.4 \mathrm{E}-05 \\
2.0 \mathrm{E}-07 \\
4.7 \mathrm{E}-05 \\
1.0 \mathrm{E}-05 \\
2.4 \mathrm{E}-05\end{array}$ & $\begin{array}{l}2.8 \mathrm{E}-04 \\
1.0 \mathrm{E}-02 \\
2.1 \mathrm{E}-02 \\
2.6 \mathrm{E}-04 \\
9.7 \mathrm{E}-03 \\
3.3 \mathrm{E}-03\end{array}$ & $\begin{array}{l}7.7 \mathrm{E}-02 \\
2.8 \mathrm{E}+00 \\
5.7 \mathrm{E}+00 \\
7.0 \mathrm{E}-02 \\
2.6 \mathrm{E}+00 \\
9.0 \mathrm{E}-01\end{array}$ \\
\hline $\begin{array}{l}{ }^{93} \mathrm{Zr+D} \text { (b) } \\
{ }^{93} \mathrm{Zr} \\
{ }^{95} \mathrm{Zr+D}(\mathrm{b}) \\
{ }^{95} \mathrm{Zr} \\
{ }^{97} \mathrm{Zr+D} \\
{ }^{97} \mathrm{Zr}\end{array}$ & $\begin{array}{l}3.7 \mathrm{E}-06 \\
7.6 \mathrm{E}-09 \\
2.6 \mathrm{E}-02 \\
2.5 \mathrm{E}-02 \\
1.6 \mathrm{E}-02 \\
6.2 \mathrm{E}-03\end{array}$ & $\begin{array}{l}2.8 \mathrm{E}-06 \\
4.0 \mathrm{E}-06 \\
5.0 \mathrm{E}-07 \\
7.5 \mathrm{E}-07 \\
6.6 \mathrm{E}-08 \\
1.9 \mathrm{E}-07\end{array}$ & $\begin{array}{l}5.2 \mathrm{E}-06 \\
8.0 \mathrm{E}-06 \\
1.6 \mathrm{E}-05 \\
1.8 \mathrm{E}-05 \\
1.5 \mathrm{E}-05 \\
4.2 \mathrm{E}-05\end{array}$ & $\begin{array}{l}1.2 \mathrm{E}-05 \\
1.2 \mathrm{E}-05 \\
2.6 \mathrm{E}-02 \\
2.5 \mathrm{E}-02 \\
1.6 \mathrm{E}-02 \\
6.3 \mathrm{E}-03\end{array}$ & $\begin{array}{l}3.2 \mathrm{E}-03 \\
3.2 \mathrm{E}-03 \\
7.0 \mathrm{E}+00 \\
6.8 \mathrm{E}+00 \\
4.4 \mathrm{E}+00 \\
1.7 \mathrm{E}+00\end{array}$ \\
\hline $\begin{array}{l}{ }_{93 m_{\mathrm{m}}} \mathrm{Nb} \\
{ }^{95} \mathrm{Nb} \\
{ }^{95 \mathrm{~m}_{\mathrm{mb}}} \mathrm{Nb}\end{array}$ & $\begin{array}{l}7.6 \mathrm{E}-06 \\
5.0 \mathrm{E}-02 \\
1.4 \mathrm{E}-02 \\
1.8 \mathrm{E}-03\end{array}$ & $\begin{array}{l}1.5 E-06 \\
1.9 E-05 \\
2.0 E-07 \\
1.2 E-07\end{array}$ & $\begin{array}{l}2.5 \mathrm{E}-06 \\
3.6 \mathrm{E}-05 \\
1.2 \mathrm{E}-05 \\
1.1 \mathrm{E}-05\end{array}$ & $\begin{array}{l}1.2 \mathrm{E}-05 \\
5.0 \mathrm{E}-02 \\
1.4 \mathrm{E}-02 \\
1.9 \mathrm{E}-03\end{array}$ & $\begin{array}{l}3.1 \mathrm{E}-03 \\
1.4 \mathrm{E}+01 \\
3.9 \mathrm{E}+00 \\
5.0 \mathrm{E}-01\end{array}$ \\
\hline
\end{tabular}


TABLE 3.1. Annual Total Effective Dose Equivalent Factors for the Building Renovation (Volume Activity) Scenario(a) (contd)

\begin{tabular}{|c|c|c|c|c|c|}
\hline Radionuclide & $\begin{array}{c}\text { External } \\
\text { (mrem) } \\
\end{array}$ & $\begin{array}{l}\text { Inhalation } \\
\text { (mrem) }\end{array}$ & $\begin{array}{c}\text { Secondary } \\
\text { Ingestion } \\
\text { (mrem) }\end{array}$ & $\begin{array}{c}\text { TEDE } \\
\text { (mrem) }\end{array}$ & $\begin{array}{l}\text { TEDE } \\
(\mu S v)\end{array}$ \\
\hline $\begin{array}{l}{ }^{95} \mathrm{Nb} \\
{ }_{97}^{97}{ }_{\mathrm{Nb}}+\mathrm{D} \\
{ }_{97}^{97} \mathrm{Nb} \\
{ }_{\mathrm{Nb}}\end{array}$ & $\begin{array}{l}2.8 \mathrm{E}-02 \\
2.2 \mathrm{E}-02 \\
2.2 \mathrm{E}-02 \\
2.2 \mathrm{E}-02\end{array}$ & $\begin{array}{l}2.7 \mathrm{E}-07 \\
2.1 \mathrm{E}-09 \\
6.0 \mathrm{E}-11 \\
4.1 \mathrm{E}-09\end{array}$ & $\begin{array}{l}1.2 \mathrm{E}-05 \\
6.0 \mathrm{E}-07 \\
2.0 \mathrm{E}-08 \\
1.2 \mathrm{E}-06\end{array}$ & $\begin{array}{l}2.8 \mathrm{E}-02 \\
2.2 \mathrm{E}-02 \\
2.2 \mathrm{E}-02 \\
2.2 \mathrm{E}-02\end{array}$ & $\begin{array}{l}7.6 \mathrm{E}+00 \\
5.9 \mathrm{E}+00 \\
5.8 \mathrm{E}+00 \\
6.0 \mathrm{E}+00\end{array}$ \\
\hline $\begin{array}{l}{ }^{93} \mathrm{Mo+D} \\
{ }_{93} \mathrm{Mo}(b) \\
{ }^{98}{ }_{\mathrm{Mo+D}}(\mathrm{b}) \\
{ }^{98} \mathrm{Mo}\end{array}$ & $\begin{array}{l}2.2 E-05 \\
3.7 E-05 \\
3.9 E-03 \\
4.7 E-03\end{array}$ & $\begin{array}{l}7.5 \mathrm{E}-07 \\
4.7 \mathrm{E}-08 \\
5.3 \mathrm{E}-08 \\
9.5 \mathrm{E}-08\end{array}$ & $\begin{array}{l}4.5 \mathrm{E}-06 \\
7.0 \mathrm{E}-06 \\
8.2 \mathrm{E}-06 \\
1.5 \mathrm{E}-05\end{array}$ & $\begin{array}{l}2.7 E-05 \\
4.4 E-05 \\
3.9 E-03 \\
4.7 E-03\end{array}$ & $\begin{array}{l}7.3 E-03 \\
1.2 E-02 \\
1.1 E+00 \\
1.3 E+00\end{array}$ \\
\hline $\begin{array}{l}{ }^{99 \pi} \mathrm{Tc} \\
{ }^{90} \mathrm{TC} \\
{ }^{101} \mathrm{TC} \\
\end{array}$ & $\begin{array}{l}3.1 E-03 \\
1.1 E-06 \\
1.2 E-02\end{array}$ & $\begin{array}{l}1.6 E-09 \\
4.4 E-07 \\
7.5 E-10\end{array}$ & $\begin{array}{l}4.2 E-07 \\
1.5 E-05 \\
3.8 E-07\end{array}$ & $\begin{array}{l}3.1 E-03 \\
1.7 E-05 \\
1.2 E=02\end{array}$ & $\begin{array}{l}8.4 E-01 \\
4.6 E-03 \\
3.2 E+00\end{array}$ \\
\hline 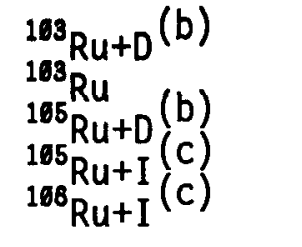 & $\begin{array}{l}7.6 \mathrm{E}-03 \\
1.5 \mathrm{E}-02 \\
9.4 \mathrm{E}-03 \\
2.6 \mathrm{E}-02 \\
7.3 \mathrm{E}-03\end{array}$ & $\begin{array}{l}2.2 \mathrm{E}-07 \\
4.3 \mathrm{E}-07 \\
2.5 \mathrm{E}-08 \\
2.6 \mathrm{E}-08 \\
1.1 \mathrm{E}-05\end{array}$ & $\begin{array}{l}7.5 \mathrm{E}-06 \\
1.5 \mathrm{E}-05 \\
4.5 \mathrm{E}-06 \\
6.5 \mathrm{E}-06 \\
6.5 \mathrm{E}-05\end{array}$ & $\begin{array}{l}7.7 \mathrm{E}-03 \\
1.5 \mathrm{E}-02 \\
9.4 \mathrm{E}-03 \\
2.6 \mathrm{E}-02 \\
7.4 \mathrm{E}-03\end{array}$ & $\begin{array}{l}2.1 E+00 \\
4.1 E+00 \\
2.5 E+00 \\
6.9 E+00 \\
2.0 E+00\end{array}$ \\
\hline $\begin{array}{l}{ }_{103}^{163} \mathrm{Rh} \\
{ }_{\mathrm{Rh}}\end{array}$ & $\begin{array}{l}1.4 \mathrm{E}-05 \\
2.7 \mathrm{E}-03\end{array}$ & $\begin{array}{l}2.4 E-10 \\
4.8 E-08\end{array}$ & $\begin{array}{l}6.0 \mathrm{E}-08 \\
7.5 \mathrm{E}-06\end{array}$ & $\begin{array}{l}1.4 \mathrm{E}-05 \\
2.8 \mathrm{E}-03\end{array}$ & $\begin{array}{l}3.7 E-03 \\
7.4 E-01\end{array}$ \\
\hline $\begin{array}{l}{ }_{103} \mathrm{Pd}+\mathrm{D} \\
{ }_{103} \mathrm{Pd} \\
{ }_{107} \mathrm{Pd} \\
{ }_{109} \mathrm{Pd}(\mathrm{Pd}+\mathrm{I}\end{array}$ & $\begin{array}{l}6.0 \mathrm{E}-05 \\
1.1 \mathrm{E}-04 \\
5.2 \mathrm{E}-10 \\
1.6 \mathrm{E}-04\end{array}$ & $\begin{array}{l}3.8 \mathrm{E}-08 \\
7.5 \mathrm{E}-08 \\
6.0 \mathrm{E}-07 \\
3.3 \mathrm{E}-08\end{array}$ & $\begin{array}{l}2.0 \mathrm{E}-06 \\
3.9 \mathrm{E}-06 \\
7.5 \mathrm{E}-07 \\
6.5 \mathrm{E}-06\end{array}$ & $\begin{array}{l}6.2 \mathrm{E}-05 \\
1.1 \mathrm{E}-04 \\
1.4 \mathrm{E}-06 \\
1.7 \mathrm{E}-04\end{array}$ & $\begin{array}{l}1.7 \mathrm{E}-02 \\
3.0 \mathrm{E}-02 \\
3.6 \mathrm{E}-04 \\
4.6 \mathrm{E}-02\end{array}$ \\
\hline${ }^{1116 \mathrm{Ag}} \mathrm{Ag}+\mathrm{I}(\mathrm{c})$ & $\begin{array}{l}9.4 E-02 \\
8.8 E-04\end{array}$ & $\begin{array}{l}2.0 \mathrm{E}-06 \\
1.7 \mathrm{E}-07\end{array}$ & $\begin{array}{l}8.3 E-05 \\
2.5 E-05\end{array}$ & $\begin{array}{l}9.4 \mathrm{E}-02 \\
9.1 \mathrm{E}-04\end{array}$ & $\begin{array}{l}2.5 E+01 \\
2.4 E-01\end{array}$ \\
\hline $\begin{array}{l}{ }^{169} \mathrm{Cd} \\
{ }_{113 m} \mathrm{Cd} \\
{ }_{116 m} \mathrm{Cd}(\mathrm{b}) \\
{ }_{115{ }^{m}} \mathrm{Cd}+\mathrm{D} \\
{ }^{116} \mathrm{Cd}\end{array}$ & $\begin{array}{l}9.8 E-05 \\
6.9 E-06 \\
4.0 E-04 \\
8.6 E-04 \\
6.1 E-03\end{array}$ & $\begin{array}{l}5.5 \mathrm{E}-06 \\
7.0 \mathrm{E}-05 \\
1.7 \mathrm{E}-06 \\
3.4 \mathrm{E}-06 \\
1.9 \mathrm{E}-07\end{array}$ & $\begin{array}{l}6.5 E-05 \\
8.0 E-04 \\
4.0 E-05 \\
8.0 E-05 \\
3.0 E-05\end{array}$ & $\begin{array}{l}1.7 \mathrm{E}-04 \\
8.8 \mathrm{E}-04 \\
4.4 \mathrm{E}-04 \\
9.4 \mathrm{E}-04 \\
6.1 \mathrm{E}-03\end{array}$ & $\begin{array}{l}4.5 \mathrm{E}-02 \\
2.4 \mathrm{E}-01 \\
1.2 \mathrm{E}-01 \\
2.5 \mathrm{E}-01 \\
1.6 \mathrm{E}+00\end{array}$ \\
\hline $\begin{array}{l}{ }_{114 m}^{111} \operatorname{In} \\
{ }^{115 m} \operatorname{In}+I \\
I n\end{array}$ & $\begin{array}{l}1.0 \mathrm{E}-02 \\
3.9 \mathrm{E}-03 \\
5.3 \mathrm{E}-03\end{array}$ & $\begin{array}{l}2.7 E-08 \\
1.1 E-06 \\
6.0 E-09\end{array}$ & $\begin{array}{l}6.5 \mathrm{E}-06 \\
3.8 \mathrm{E}-05 \\
1.7 \mathrm{E}-06\end{array}$ & $\begin{array}{l}1.0 \mathrm{E}-02 \\
3.9 \mathrm{E}-03 \\
5.3 \mathrm{E}-03\end{array}$ & $\begin{array}{l}2.7 E+00 \\
1.1 E+00 \\
1.4 E+00\end{array}$ \\
\hline $\begin{array}{l}{ }^{117 m} \mathrm{~m} n \\
{ }_{119 m} \mathrm{Sn} \\
{ }_{121 \mathrm{~m}} \mathrm{Sn+D} \\
{ }_{121 \mathrm{~m}} \mathrm{Sn}\end{array}$ & $\begin{array}{l}3.2 E-03 \\
7.0 E-05 \\
0.0 E+00 \\
0.0 E+00\end{array}$ & $\begin{array}{l}2.1 E-07 \\
2.9 E-07 \\
3.1 E-07 \\
5.5 E-07\end{array}$ & $\begin{array}{l}1.4 \mathrm{E}-05 \\
7.0 \mathrm{E}-06 \\
6.2 \mathrm{E}-06 \\
7.5 \mathrm{E}-06\end{array}$ & $\begin{array}{l}3.2 E-03 \\
7.8 E-05 \\
6.5 E-06 \\
8.1 E-06\end{array}$ & $\begin{array}{l}8.6 \mathrm{E}-01 \\
2.1 \mathrm{E}-02 \\
1.8 \mathrm{E}-03 \\
2.2 \mathrm{E}-03\end{array}$ \\
\hline
\end{tabular}


TABLE 3.1. Annual Total Effective Dose Equivalent Factors for the Building Renovation (Volume Activity) Scenario(a) (contd)

\begin{tabular}{|c|c|c|c|c|c|}
\hline Radionuclide & $\begin{array}{c}\text { External } \\
\text { (mrem) }\end{array}$ & $\begin{array}{l}\text { Inhalation } \\
\text { (mrem) }\end{array}$ & $\begin{array}{l}\text { Secondary } \\
\text { Ingestion } \\
\text { (mrem) } \\
\end{array}$ & $\begin{array}{c}\text { TEDE } \\
\text { (mrem) }\end{array}$ & $\begin{array}{l}\text { TEDE } \\
(\mu S v)\end{array}$ \\
\hline $\begin{array}{l}{ }^{121} \mathrm{Sn} \\
{ }^{123} \mathrm{Sn} \\
{ }^{125} \mathrm{Sn+D} \\
{ }^{125} \mathrm{Sn} \\
{ }^{126} \mathrm{Sn}_{\mathrm{Sn}}(\mathrm{b}) \\
{ }^{126} \mathrm{Sn}\end{array}$ & $\begin{array}{l}0.0 \mathrm{E}+00 \\
2.8 \mathrm{E}-04 \\
1.1 \mathrm{E}-02 \\
1.0 \mathrm{E}-02 \\
3.0 \mathrm{E}-02 \\
8.7 \mathrm{E}-04\end{array}$ & $\begin{array}{l}2.4 E-08 \\
1.5 E-06 \\
6.5 E-07 \\
7.5 E-07 \\
2.2 E-06 \\
4.7 E-06\end{array}$ & $\begin{array}{l}4.6 \mathrm{E}-06 \\
4.2 \mathrm{E}-05 \\
3.6 \mathrm{E}-05 \\
6.0 \mathrm{E}-05 \\
4.9 \mathrm{E}-05 \\
9.5 \mathrm{E}-05\end{array}$ & $\begin{array}{l}4.6 \mathrm{E}-06 \\
3.3 \mathrm{E}-04 \\
1.1 \mathrm{E}-02 \\
1.0 \mathrm{E}-02 \\
3.0 \mathrm{E}-02 \\
9.6 \mathrm{E}-04\end{array}$ & $\begin{array}{l}1.2 \mathrm{E}-03 \\
8.9 \mathrm{E}-02 \\
3.0 \mathrm{E}+00 \\
2.8 \mathrm{E}+00 \\
8.2 \mathrm{E}+00 \\
2.6 \mathrm{E}-01\end{array}$ \\
\hline $\begin{array}{l}{ }^{124} \mathrm{Sb} \\
{ }^{125} \mathrm{Sb}+\mathrm{D} \\
{ }^{125} \mathrm{Sb} \\
{ }^{126 \mathrm{~m}} \mathrm{Sb} \text { (b) } \\
{ }^{126 m} \mathrm{Sb} \\
{ }^{126} \mathrm{Sb} \\
{ }^{127} \mathrm{Sb} \\
{ }^{127} \mathrm{Sb}+\mathrm{Db}\end{array}$ & $\begin{array}{l}6.5 \mathrm{E}-02 \\
1.2 \mathrm{E}-02 \\
1.5 \mathrm{E}-02 \\
5.6 \mathrm{E}-02 \\
5.3 \mathrm{E}-02 \\
9.1 \mathrm{E}-02 \\
1.0 \mathrm{E}-02 \\
2.2 \mathrm{E}-02\end{array}$ & $\begin{array}{l}1.3 \mathrm{E}-06 \\
5.3 \mathrm{E}-07 \\
6.0 \mathrm{E}-07 \\
7.5 \mathrm{E}-08 \\
1.5 \mathrm{E}-09 \\
5.5 \mathrm{E}-07 \\
2.2 \mathrm{E}-07 \\
2.9 \mathrm{E}-07\end{array}$ & $\begin{array}{l}4.9 E-05 \\
1.5 E-05 \\
1.4 E-05 \\
6.6 E-06 \\
4.5 E-07 \\
5.0 E-05 \\
2.1 E-05 \\
3.6 E-05\end{array}$ & $\begin{array}{l}6.5 \mathrm{E}-02 \\
1.2 \mathrm{E}-02 \\
1.5 \mathrm{E}-02 \\
5.6 \mathrm{E}-02 \\
5.3 \mathrm{E}-02 \\
9.1 \mathrm{E}-02 \\
1.0 \mathrm{E}-02 \\
2.2 \mathrm{E}-02\end{array}$ & $\begin{array}{l}1.8 E+01 \\
3.2 E+00 \\
4.0 E+00 \\
1.5 E+01 \\
1.4 E+01 \\
2.5 E+01 \\
2.7 E+00 \\
5.9 E+00\end{array}$ \\
\hline 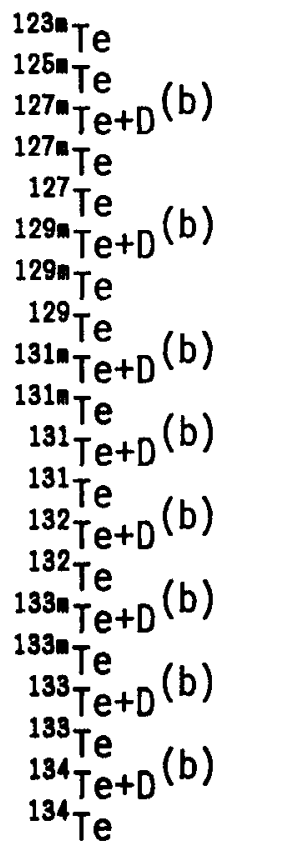 & $\begin{array}{l}3.0 \mathrm{E}-03 \\
2.6 \mathrm{E}-04 \\
1.3 \mathrm{E}-04 \\
8.3 \mathrm{E}-05 \\
1.8 \mathrm{E}-04 \\
1.4 \mathrm{E}-03 \\
1.1 \mathrm{E}-03 \\
1.8 \mathrm{E}-03 \\
2.8 \mathrm{E}-02 \\
5.0 \mathrm{E}-02 \\
1.2 \mathrm{E}-02 \\
1.3 \mathrm{E}-02 \\
4.0 \mathrm{E}-02 \\
6.7 \mathrm{E}-03 \\
3.1 \mathrm{E}-02 \\
7.8 \mathrm{E}-02 \\
1.6 \mathrm{E}-02 \\
3.2 \mathrm{E}-02 \\
5.6 \mathrm{E}-02 \\
2.9 \mathrm{E}-02\end{array}$ & $\begin{array}{l}5.0 \mathrm{E}-07 \\
3.5 \mathrm{E}-07 \\
5.3 \mathrm{E}-07 \\
1.0 \mathrm{E}-06 \\
1.6 \mathrm{E}-08 \\
1.5 \mathrm{E}-05 \\
2.4 \mathrm{E}-05 \\
7.5 \mathrm{E}-09 \\
9.4 \mathrm{E}-07 \\
6.0 \mathrm{E}-07 \\
7.5 \mathrm{E}-07 \\
6.0 \mathrm{E}-09 \\
3.3 \mathrm{E}-07 \\
6.0 \mathrm{E}-07 \\
9.2 \mathrm{E}-08 \\
2.2 \mathrm{E}-08 \\
8.9 \mathrm{E}-08 \\
4.3 \mathrm{E}-09 \\
6.0 \mathrm{E}-09 \\
5.0 \mathrm{E}-09\end{array}$ & $\begin{array}{l}2.9 \mathrm{E}-05 \\
1.8 \mathrm{E}-05 \\
2.2 \mathrm{E}-05 \\
4.1 \mathrm{E}-05 \\
3.5 \mathrm{E}-06 \\
1.7 \mathrm{E}-03 \\
2.7 \mathrm{E}-03 \\
1.3 \mathrm{E}-06 \\
1.5 \mathrm{E}-04 \\
8.5 \mathrm{E}-05 \\
1.2 \mathrm{E}-04 \\
1.1 \mathrm{E}-06 \\
3.7 \mathrm{E}-05 \\
7.5 \mathrm{E}-05 \\
1.7 \mathrm{E}-05 \\
3.9 \mathrm{E}-06 \\
1.6 \mathrm{E}-05 \\
8.0 \mathrm{E}-07 \\
1.1 \mathrm{E}-06 \\
9.5 \mathrm{E}-07\end{array}$ & $\begin{array}{l}3.1 \mathrm{E}-03 \\
2.8 \mathrm{E}-04 \\
1.5 \mathrm{E}-04 \\
1.3 \mathrm{E}-04 \\
1.9 \mathrm{E}-04 \\
3.1 \mathrm{E}-03 \\
3.8 \mathrm{E}-03 \\
1.8 \mathrm{E}-03 \\
2.8 \mathrm{E}-02 \\
5.0 \mathrm{E}-02 \\
1.3 \mathrm{E}-02 \\
1.3 \mathrm{E}-02 \\
4.0 \mathrm{E}-02 \\
6.8 \mathrm{E}-03 \\
3.1 \mathrm{E}-02 \\
7.8 \mathrm{E}-02 \\
1.6 \mathrm{E}-02 \\
3.2 \mathrm{E}-02 \\
5.6 \mathrm{E}-02 \\
2.9 \mathrm{E}-02\end{array}$ & $\begin{array}{l}8.2 \mathrm{E}-01 \\
7.5 \mathrm{E}-02 \\
4.1 \mathrm{E}-02 \\
3.4 \mathrm{E}-02 \\
5.0 \mathrm{E}-02 \\
8.2 \mathrm{E}-01 \\
1.0 \mathrm{E}+00 \\
5.0 \mathrm{E}-01 \\
7.6 \mathrm{E}+00 \\
1.3 \mathrm{E}+01 \\
3.4 \mathrm{E}+00 \\
3.6 \mathrm{E}+00 \\
1.1 \mathrm{E}+01 \\
1.8 \mathrm{E}+00 \\
8.2 \mathrm{E}+00 \\
2.1 \mathrm{E}+01 \\
4.4 \mathrm{E}+00 \\
8.7 \mathrm{E}+00 \\
1.5 \mathrm{E}+01 \\
7.8 \mathrm{E}+00\end{array}$ \\
\hline $\begin{array}{l}{ }^{125} \mathrm{I} \\
{ }_{129} \mathrm{I} \\
136 \mathrm{I} \\
{ }_{131} \mathrm{I}+\mathrm{D}(\mathrm{b}) \\
{ }_{131} \mathrm{I} \\
{ }^{132} \mathrm{I} \\
{ }^{133} \mathrm{I}+\mathrm{D}(\mathrm{b}) \\
{ }_{133} \mathrm{I}\end{array}$ & $\begin{array}{l}3.0 \mathrm{E}-04 \\
1.7 \mathrm{E}-04 \\
6.7 \mathrm{E}-02 \\
1.1 \mathrm{E}-02 \\
1.2 \mathrm{E}-02 \\
7.9 \mathrm{E}-02 \\
9.1 \mathrm{E}-03 \\
1.8 \mathrm{E}-02\end{array}$ & $\begin{array}{l}1.1 \mathrm{E}-06 \\
7.5 \mathrm{E}-06 \\
1.2 \mathrm{E}-07 \\
1.5 \mathrm{E}-06 \\
1.5 \mathrm{E}-06 \\
1.9 \mathrm{E}-08 \\
1.3 \mathrm{E}-07 \\
2.6 \mathrm{E}-07\end{array}$ & $\begin{array}{l}1.8 \mathrm{E}-04 \\
1.3 \mathrm{E}-03 \\
2.2 \mathrm{E}-05 \\
2.5 \mathrm{E}-04 \\
2.5 \mathrm{E}-04 \\
3.4 \mathrm{E}-06 \\
2.4 \mathrm{E}-05 \\
4.8 \mathrm{E}-05\end{array}$ & $\begin{array}{l}4.8 \mathrm{E}-04 \\
1.4 \mathrm{E}-03 \\
6.7 \mathrm{E}-02 \\
1.1 \mathrm{E}-02 \\
1.2 \mathrm{E}-02 \\
7.9 \mathrm{E}-02 \\
9.1 \mathrm{E}-03 \\
1.8 \mathrm{E}-02\end{array}$ & $\begin{array}{l}1.3 E-01 \\
3.8 E-01 \\
1.8 E+01 \\
3.1 E+00 \\
3.2 E+00 \\
2.1 E+01 \\
2.5 E+00 \\
4.9 E+00\end{array}$ \\
\hline
\end{tabular}


TABLE 3.1. Annual Total Effective Dose Equivalent Factors for the Building Renovation (Volume Activity) Scenario(a) (contd)

\begin{tabular}{|c|c|c|c|c|c|}
\hline Radionuclide & $\begin{array}{c}\text { External } \\
\text { (mrem) }\end{array}$ & $\begin{array}{c}\text { Inhalation } \\
\text { (mrem) }\end{array}$ & $\begin{array}{l}\text { Secondary } \\
\text { Ingestion } \\
\text { (mrem) }\end{array}$ & $\begin{array}{c}\text { TEDE } \\
\text { (mrem) }\end{array}$ & $\begin{array}{l}\text { TEDE } \\
(\mu S v)\end{array}$ \\
\hline${ }^{135}{ }_{\mathrm{I}}^{134} \mathrm{I}+\mathrm{D}(\mathrm{b})$ & $\begin{array}{l}8.8 \mathrm{E}-02 \\
2.9 \mathrm{E}-02 \\
5.4 \mathrm{E}-02\end{array}$ & $\begin{array}{l}7.0 \mathrm{E}-09 \\
2.5 \mathrm{E}-08 \\
5.5 \mathrm{E}-08\end{array}$ & $\begin{array}{l}1.3 \mathrm{E}-06 \\
4.8 \mathrm{E}-06 \\
1.0 \mathrm{E}-05\end{array}$ & $\begin{array}{l}8.8 \mathrm{E}-02 \\
2.9 \mathrm{E}-02 \\
5.4 \mathrm{E}-02\end{array}$ & $\begin{array}{l}2.4 E+01 \\
7.7 E+00 \\
1.5 E+01\end{array}$ \\
\hline $\begin{array}{l}{ }^{134 m} \mathrm{Cs}+\mathrm{D}^{(b)} \\
{ }^{134 \mathrm{~m}} \mathrm{Cs} \\
{ }^{134} \mathrm{Cs} \\
{ }^{135} \mathrm{CS} \\
{ }^{136} \mathrm{Cs} \\
{ }^{137} \mathrm{Cs}(\mathrm{Cs}+\mathrm{C}) \\
{ }^{138} \mathrm{Cs}\end{array}$ & $\begin{array}{l}2.9 \mathrm{E}-04 \\
5.8 \mathrm{E}-04 \\
5.6 \mathrm{E}-02 \\
3.5 \mathrm{E}-07 \\
7.2 \mathrm{E}-02 \\
1.9 \mathrm{E}-02 \\
8.1 \mathrm{E}-02\end{array}$ & $\begin{array}{l}9.0 \mathrm{E}-10 \\
1.8 \mathrm{E}-09 \\
2.0 \mathrm{E}-06 \\
1.8 \mathrm{E}-07 \\
3.2 \mathrm{E}-07 \\
6.7 \mathrm{E}-07 \\
4.8 \mathrm{E}-09\end{array}$ & $\begin{array}{l}9.0 \mathrm{E}-08 \\
1.8 \mathrm{E}-07 \\
3.3 \mathrm{E}-04 \\
3.2 \mathrm{E}-05 \\
5.0 \mathrm{E}-05 \\
1.2 \mathrm{E}-04 \\
9.5 \mathrm{E}-07\end{array}$ & $\begin{array}{l}2.9 \mathrm{E}-04 \\
5.8 \mathrm{E}-04 \\
5.6 \mathrm{E}-02 \\
3.3 \mathrm{E}-05 \\
7.2 \mathrm{E}-02 \\
1.9 \mathrm{E}-02 \\
8.1 \mathrm{E}-02\end{array}$ & $\begin{array}{l}7.7 \mathrm{E}-02 \\
1.6 \mathrm{E}-01 \\
1.5 \mathrm{E}+01 \\
8.8 \mathrm{E}-03 \\
1.9 \mathrm{E}+01 \\
5.1 \mathrm{E}+00 \\
2.2 \mathrm{E}+01\end{array}$ \\
\hline $\begin{array}{l}{ }^{139} \mathrm{Ba} \\
{ }^{140} \mathrm{Ba}+\mathrm{D} \\
141 \mathrm{Ba} \\
{ }^{141} \mathrm{Ba}+\mathrm{D}(\mathrm{b}) \\
{ }^{142} \mathrm{Ba} \\
{ }^{142} \mathrm{Ba} \mathrm{Ba}\end{array}$ & $\begin{array}{l}1.0 \mathrm{E}-03 \\
3.9 \mathrm{E}-02 \\
5.6 \mathrm{E}-03 \\
1.0 \mathrm{E}-02 \\
2.8 \mathrm{E}-02 \\
6.0 \mathrm{E}-02 \\
3.0 \mathrm{E}-02\end{array}$ & $\begin{array}{l}8.5 \mathrm{E}-09 \\
1.6 \mathrm{E}-07 \\
1.5 \mathrm{E}-07 \\
1.5 \mathrm{E}-07 \\
3.9 \mathrm{E}-09 \\
7.5 \mathrm{E}-09 \\
1.9 \mathrm{E}-09\end{array}$ & $\begin{array}{l}2.0 \mathrm{E}-06 \\
4.2 \mathrm{E}-05 \\
4.6 \mathrm{E}-05 \\
7.7 \mathrm{E}-06 \\
1.1 \mathrm{E}-06 \\
2.0 \mathrm{E}-06 \\
5.5 \mathrm{E}-07\end{array}$ & $\begin{array}{l}1.0 \mathrm{E}-03 \\
3.9 \mathrm{E}-02 \\
5.6 \mathrm{E}-03 \\
1.0 \mathrm{E}-02 \\
2.8 \mathrm{E}-02 \\
6.0 \mathrm{E}-02 \\
3.0 \mathrm{E}-02\end{array}$ & $\begin{array}{l}2.7 E-01 \\
1.1 E+01 \\
1.5 E+00 \\
2.8 E+00 \\
7.6 E+00 \\
1.6 E+01 \\
8.2 E+00\end{array}$ \\
\hline $\begin{array}{l}{ }^{140} \mathrm{La} \\
{ }^{141} \mathrm{La}+D \\
{ }^{141} \mathrm{La} \\
{ }^{142} \mathrm{La}\end{array}$ & $\begin{array}{l}7.5 \mathrm{E}-02 \\
1.8 \mathrm{E}-03 \\
1.8 \mathrm{E}-03 \\
8.9 \mathrm{E}-02\end{array}$ & $\begin{array}{l}1.7 \mathrm{E}-07 \\
2.2 \mathrm{E}-07 \\
2.9 \mathrm{E}-08 \\
1.3 \mathrm{E}-08\end{array}$ & $\begin{array}{l}4.1 \mathrm{E}-05 \\
1.1 \mathrm{E}-05 \\
7.5 \mathrm{E}-06 \\
3.5 \mathrm{E}-06\end{array}$ & $\begin{array}{l}7.5 \mathrm{E}-02 \\
1.8 \mathrm{E}-03 \\
1.8 \mathrm{E}-03 \\
8.9 \mathrm{E}-02\end{array}$ & $\begin{array}{l}2.0 \mathrm{E}+01 \\
4.8 \mathrm{E}-01 \\
4.9 \mathrm{E}-01 \\
2.4 \mathrm{E}+01\end{array}$ \\
\hline $\begin{array}{l}{ }^{141} \mathrm{Ce} \\
{ }^{143} \mathrm{Ce}+\mathrm{D} \\
{ }^{143} \mathrm{Ce} \\
\left.{ }^{144} \mathrm{Ce}+\mathrm{b}\right) \\
{ }^{144} \mathrm{Ce}\end{array}$ & $\begin{array}{l}1.8 \mathrm{E}-03 \\
3.6 \mathrm{E}-03 \\
7.3 \mathrm{E}-03 \\
9.2 \mathrm{E}-04 \\
4.4 \mathrm{E}-04\end{array}$ & $\begin{array}{l}4.2 E-07 \\
2.7 E-07 \\
1.7 E-07 \\
8.9 E-06 \\
1.8 E-05\end{array}$ & $\begin{array}{l}1.4 \mathrm{E}-05 \\
2.3 \mathrm{E}-05 \\
2.3 \mathrm{E}-05 \\
5.2 \mathrm{E}-05 \\
1.0 \mathrm{E}-04\end{array}$ & $\begin{array}{l}1.8 \mathrm{E}-03 \\
3.6 \mathrm{E}-03 \\
7.3 \mathrm{E}-03 \\
9.8 \mathrm{E}-04 \\
5.7 \mathrm{E}-04\end{array}$ & $\begin{array}{l}4.8 E-01 \\
9.8 E-01 \\
2.0 E+00 \\
2.6 E-01 \\
1.5 E-01\end{array}$ \\
\hline $\begin{array}{l}{ }^{143} \mathrm{Pr} \\
{ }_{144 \mathrm{~m}} \mathrm{Pr}+\mathrm{D} \\
{ }^{144} \mathrm{~m} \\
{ }^{144} \mathrm{Pr}\end{array}$ & $\begin{array}{l}2.3 \mathrm{E}-05 \\
7.6 \mathrm{E}-04 \\
1.4 \mathrm{E}-04 \\
1.4 \mathrm{E}-03\end{array}$ & $\begin{array}{l}3.9 \mathrm{E}-07 \\
1.6 \mathrm{E}-09 \\
9.0 \mathrm{E}-10 \\
2.1 \mathrm{E}-09\end{array}$ & $\begin{array}{l}2.3 E-05 \\
4.2 E-07 \\
2.4 E-07 \\
6.0 E-07\end{array}$ & $\begin{array}{l}4.7 \mathrm{E}-05 \\
7.6 \mathrm{E}-04 \\
1.4 \mathrm{E}-04 \\
1.4 \mathrm{E}-03\end{array}$ & $\begin{array}{l}1.3 E-02 \\
2.1 E-01 \\
3.7 E-02 \\
3.9 E-01\end{array}$ \\
\hline $\begin{array}{l}{ }^{147} \mathrm{Nd}+\mathrm{D} \\
{ }^{147} \mathrm{Nd}\end{array}$ & $\begin{array}{l}1.8 \mathrm{E}-03 \\
3.6 \mathrm{E}-03\end{array}$ & $\begin{array}{l}1.1 E-06 \\
3.3 E-07\end{array}$ & $\begin{array}{l}1.3 E-05 \\
2.1 E-05\end{array}$ & $\begin{array}{l}1.8 \mathrm{E}-03 \\
3.7 \mathrm{E}-03\end{array}$ & $\begin{array}{l}4.8 E-01 \\
9.9 E-01\end{array}$ \\
\hline $\begin{array}{l}{ }_{147}^{148 m} \mathrm{Pm} \\
{ }^{148 m} \mathrm{Pm}+\mathrm{D} \\
{ }_{148 \mathrm{~m}}{ }^{\mathrm{Pm}} \\
{ }^{148} \mathrm{Pm} \\
{ }_{149} \mathrm{Pm} \\
{ }^{151} \mathrm{Pm} \\
{ }_{\mathrm{Pm}}\end{array}$ & $\begin{array}{l}5.8 \mathrm{E}-07 \\
6.8 \mathrm{E}-02 \\
7.1 \mathrm{E}-02 \\
2.1 \mathrm{E}-02 \\
3.5 \mathrm{E}-04 \\
1.0 \mathrm{E}-02\end{array}$ & $\begin{array}{l}1.9 \mathrm{E}-06 \\
1.1 \mathrm{E}-06 \\
1.1 \mathrm{E}-06 \\
5.5 \mathrm{E}-07 \\
1.5 \mathrm{E}-07 \\
9.0 \mathrm{E}-08\end{array}$ & $\begin{array}{l}5.0 \mathrm{E}-06 \\
3.9 \mathrm{E}-05 \\
3.8 \mathrm{E}-05 \\
5.5 \mathrm{E}-05 \\
2.0 \mathrm{E}-05 \\
1.5 \mathrm{E}-05\end{array}$ & $\begin{array}{l}7.5 \mathrm{E}-06 \\
6.8 \mathrm{E}-02 \\
7.1 \mathrm{E}-02 \\
2.1 \mathrm{E}-02 \\
3.7 \mathrm{E}-04 \\
1.0 \mathrm{E}-02\end{array}$ & $\begin{array}{l}2.0 \mathrm{E}-03 \\
1.8 \mathrm{E}+01 \\
1.9 \mathrm{E}+01 \\
5.7 \mathrm{E}+00 \\
1.0 \mathrm{E}-01 \\
2.8 \mathrm{E}+00\end{array}$ \\
\hline
\end{tabular}


TABLE 3.1. Annual Total Effective Dose Equivalent Factors for the Building Renovation (Volume Activity) Scenario(a) (contd)

\begin{tabular}{|c|c|c|c|c|c|}
\hline Radionuclide & $\begin{array}{c}\text { External } \\
\text { (mrem) }\end{array}$ & $\begin{array}{c}\text { Inhalation } \\
\text { (mrem) } \\
\end{array}$ & $\begin{array}{l}\text { Secondary } \\
\text { Ingestion } \\
\text { (mrem) }\end{array}$ & $\begin{array}{c}\text { TEDE } \\
\text { (mrem) }\end{array}$ & $\begin{array}{l}\text { TEDE } \\
(\mu S v)\end{array}$ \\
\hline $\begin{array}{l}{ }^{147} \mathrm{Sm} \\
{ }^{151} \mathrm{Sm} \\
{ }^{153} \mathrm{Sm}\end{array}$ & $\begin{array}{l}0.0 \mathrm{E}+00 \\
1.2 \mathrm{E}-07 \\
1.5 \mathrm{E}-03\end{array}$ & $\begin{array}{l}3.7 \mathrm{E}-03 \\
1.5 \mathrm{E}-06 \\
9.5 \mathrm{E}-08\end{array}$ & $\begin{array}{l}9.5 E-04 \\
1.9 E-06 \\
1.5 E-05\end{array}$ & $\begin{array}{l}4.6 \mathrm{E}-03 \\
3.5 \mathrm{E}-06 \\
1.5 \mathrm{E}-03\end{array}$ & $\begin{array}{l}1.2 E+00 \\
9.4 E-04 \\
4.0 E-01\end{array}$ \\
\hline $\begin{array}{l}{ }^{152} \mathrm{Eu} \\
{ }^{154} \mathrm{Eu} \\
{ }^{156} \mathrm{Eu} \\
{ }^{158} \mathrm{Eu}\end{array}$ & $\begin{array}{l}3.9 \mathrm{E}-02 \\
4.1 \mathrm{E}-02 \\
1.3 \mathrm{E}-03 \\
4.5 \mathrm{E}-02\end{array}$ & $\begin{array}{l}1.0 \mathrm{E}-05 \\
1.3 \mathrm{E}-05 \\
1.9 \mathrm{E}-06 \\
7.0 \mathrm{E}-07\end{array}$ & $\begin{array}{l}3.1 \mathrm{E}-05 \\
4.6 \mathrm{E}-05 \\
7.5 \mathrm{E}-06 \\
4.6 \mathrm{E}-05\end{array}$ & $\begin{array}{l}3.9 \mathrm{E}-02 \\
4.1 \mathrm{E}-02 \\
1.4 \mathrm{E}-03 \\
4.6 \mathrm{E}-02\end{array}$ & $\begin{array}{l}1.1 \mathrm{E}+01 \\
1.1 \mathrm{E}+01 \\
3.7 \mathrm{E}-01 \\
1.2 \mathrm{E}+01\end{array}$ \\
\hline${ }^{153} \mathrm{Gd}$ & $2.1 \mathrm{E}-03$ & $1.2 E-06$ & $6.0 \mathrm{E}-06$ & $2.1 E-03$ & $5.7 \mathrm{E}-01$ \\
\hline${ }^{186} \mathrm{~Tb}$ & $3.6 \mathrm{E}-02$ & $1.2 E-06$ & $3.3 E-05$ & $3.6 \mathrm{E}-02$ & $9.6 \mathrm{E}+00$ \\
\hline${ }^{168 m_{m}} \mathrm{Ho}$ & $5.1 E-02$ & $3.6 E-05$ & $3.8 \mathrm{E}-05$ & $5.1 E-02$ & $1.4 E+01$ \\
\hline $\begin{array}{l}{ }^{181} W \\
185 W \\
187 W\end{array}$ & $\begin{array}{l}5.7 E-04 \\
3.6 E-06 \\
1.5 E-02\end{array}$ & $\begin{array}{l}7.5 \mathrm{E}-09 \\
3.8 \mathrm{E}-08 \\
3.3 \mathrm{E}-08\end{array}$ & $\begin{array}{l}1.5 \mathrm{E}-06 \\
8.0 \mathrm{E}-06 \\
1.0 \mathrm{E}-05\end{array}$ & $\begin{array}{l}5.7 \mathrm{E}-04 \\
1.2 \mathrm{E}-05 \\
1.5 \mathrm{E}-02\end{array}$ & $\begin{array}{l}1.5 E-01 \\
3.1 E-03 \\
4.2 E+00\end{array}$ \\
\hline${ }^{187} \mathrm{Re}$ & $0.0 \mathrm{E}+00$ & $2.8 E-09$ & $7.0 E-08$ & $7.3 \mathrm{E}-08$ & $2.0 E-05$ \\
\hline $\begin{array}{l}{ }^{185} 0 \mathrm{~s} \\
{ }^{191} 0 \mathrm{~s}\end{array}$ & $\begin{array}{l}2.3 E-02 \\
1.5 E-03\end{array}$ & $\begin{array}{l}4.6 \mathrm{E}-07 \\
1.1 \mathrm{E}-07\end{array}$ & $\begin{array}{l}1.1 \mathrm{E}-05 \\
1.1 \mathrm{E}-05\end{array}$ & $\begin{array}{l}2.3 \mathrm{E}-02 \\
1.5 \mathrm{E}-03\end{array}$ & $\begin{array}{l}6.2 E+00 \\
4.1 E-01\end{array}$ \\
\hline & $2.7 \mathrm{E}-02$ & $1.3 \mathrm{E}-06$ & $2.9 \mathrm{E}-05$ & $2.8 \mathrm{E}-02$ & $7.4 E+00$ \\
\hline${ }^{203} \mathrm{Hg}$ & $5.8 E-03$ & $3.4 \mathrm{E}-07$ & $5.5 E-05$ & $5.9 E-03$ & $1.6 \mathrm{E}+00$ \\
\hline $\begin{array}{l}{ }^{210} \mathrm{~Pb}+\mathrm{D}(\mathrm{b}) \\
{ }^{210} \mathrm{~Pb} \\
{ }^{212} \mathrm{~Pb}+\mathrm{D} \\
{ }^{212} \mathrm{~Pb}\end{array}$ & $\begin{array}{l}2.4 \mathrm{E}-05 \\
3.6 \mathrm{E}-05 \\
1.7 \mathrm{E}-02 \\
4.1 \mathrm{E}-03\end{array}$ & $\begin{array}{l}3.7 \mathrm{E}-04 \\
6.5 \mathrm{E}-04 \\
3.0 \mathrm{E}-06 \\
8.0 \mathrm{E}-06\end{array}$ & $\begin{array}{l}1.2 \mathrm{E}-02 \\
2.7 \mathrm{E}-02 \\
8.3 \mathrm{E}-05 \\
2.5 \mathrm{E}-04\end{array}$ & $\begin{array}{l}1.3 \mathrm{E}-02 \\
2.8 \mathrm{E}-02 \\
1.7 \mathrm{E}-02 \\
4.4 \mathrm{E}-03\end{array}$ & $\begin{array}{l}3.4 \mathrm{E}+00 \\
7.5 \mathrm{E}+00 \\
4.5 \mathrm{E}+00 \\
1.2 \mathrm{E}+00\end{array}$ \\
\hline $\begin{array}{l}{ }^{210} \mathrm{Bi}+\mathrm{D}(\mathrm{b}) \\
{ }^{210} \mathrm{Bi} \\
{ }^{212} \mathrm{Bi}+\mathrm{I} \\
{ }^{2}(\mathrm{c})\end{array}$ & $\begin{array}{l}1.8 \mathrm{E}-05 \\
3.7 \mathrm{E}-05 \\
4.7 \mathrm{E}-02\end{array}$ & $\begin{array}{l}2.2 \mathrm{E}-04 \\
9.5 \mathrm{E}-06 \\
4.5 \mathrm{E}-07\end{array}$ & $\begin{array}{l}4.7 \mathrm{E}-03 \\
3.1 \mathrm{E}-05 \\
2.7 \mathrm{E}-06\end{array}$ & $\begin{array}{l}5.0 \mathrm{E}-03 \\
7.8 \mathrm{E}-05 \\
4.7 \mathrm{E}-02\end{array}$ & $\begin{array}{l}1.3 E+00 \\
2.1 E-02 \\
1.3 E+01\end{array}$ \\
\hline${ }^{210} \mathrm{Po}$ & $3.0 \mathrm{E}-07$ & $4.2 E-04$ & $9.5 E-03$ & $9.9 \mathrm{E}-03$ & $2.7 E+00$ \\
\hline $\begin{array}{l}{ }^{223} \mathrm{Ra}+\mathrm{I}(\mathrm{c}) \\
224 \mathrm{Ra} \text { (b) } \\
{ }^{224} \mathrm{Ra} \text { (c) }(\mathrm{c}) \\
{ }^{225} \mathrm{Ra} \mathrm{Ra} \text { (b) } \\
{ }^{225} \mathrm{Ba} \\
{ }^{226} \mathrm{Ra} \mathrm{Ra}(\mathrm{b})\end{array}$ & $\begin{array}{l}8.6 \mathrm{E}-03 \\
8.4 \mathrm{E}-03 \\
3.1 \mathrm{E}-04 \\
1.1 \mathrm{E}-03 \\
1.8 \mathrm{E}-04 \\
6.6 \mathrm{E}-03\end{array}$ & $\begin{array}{l}6.2 \mathrm{E}-05 \\
2.4 \mathrm{E}-05 \\
1.3 \mathrm{E}-04 \\
1.1 \mathrm{E}-04 \\
3.5 \mathrm{E}-04 \\
1.6 \mathrm{E}-04\end{array}$ & $\begin{array}{l}2.2 \mathrm{E}-04 \\
1.5 \mathrm{E}-04 \\
6.5 \mathrm{E}-04 \\
2.4 \mathrm{E}-04 \\
1.1 \mathrm{E}-03 \\
4.6 \mathrm{E}-03\end{array}$ & $\begin{array}{l}8.9 \mathrm{E}-03 \\
8.6 \mathrm{E}-03 \\
1.1 \mathrm{E}-03 \\
1.4 \mathrm{E}-03 \\
1.6 \mathrm{E}-03 \\
1.1 \mathrm{E}-02\end{array}$ & $\begin{array}{l}2.4 E+00 \\
2.3 E+00 \\
3.0 E-01 \\
3.9 E-01 \\
4.4 E-01 \\
3.1 E+00\end{array}$ \\
\hline
\end{tabular}


TABLE 3.1. Annual Total Effective Dose Equivalent Factors for the Building Renovation (Volume Activity) Scenario(a) (contd)

\begin{tabular}{|c|c|c|c|c|c|}
\hline Radionuclide & $\begin{array}{c}\text { External } \\
\text { (mrem) }\end{array}$ & $\begin{array}{c}\text { Inhalation } \\
\text { (mrem) }\end{array}$ & $\begin{array}{l}\text { Secondary } \\
\text { Ingestion } \\
\text { (mrem) } \\
\end{array}$ & $\begin{array}{l}\text { TEDE } \\
\text { (mrem) }\end{array}$ & $\begin{array}{l}\text { TEDE } \\
(\mu \mathrm{SV})\end{array}$ \\
\hline $\begin{array}{l}{ }^{228} \mathrm{Ra} \\
{ }^{228}{ }_{\mathrm{Ra}} \mathrm{Ra}+\mathrm{Da}\end{array}(\mathrm{b})$ & $\begin{array}{l}1.2 \mathrm{E}-04 \\
8.9 \mathrm{E}-03 \\
1.1 \mathrm{E}-09\end{array}$ & $\begin{array}{l}4.0 E-04 \\
1.8 E-03 \\
1.8 E-04\end{array}$ & $\begin{array}{l}4.7 E-03 \\
7.8 E-04 \\
4.2 E-03\end{array}$ & $\begin{array}{l}5.3 \mathrm{E}-03 \\
1.1 \mathrm{E}-02 \\
4.4 \mathrm{E}-03\end{array}$ & $\begin{array}{l}1.4 E+00 \\
3.1 E+00 \\
1.2 E+00\end{array}$ \\
\hline $\begin{array}{l}{ }^{225} \mathrm{Ac}+\mathrm{I}(\mathrm{c}) \\
{ }^{227} \mathrm{Ac}+\mathrm{D}(\mathrm{b}) \\
{ }^{227} \mathrm{Ac} \\
{ }^{228} \mathrm{Ac}+\mathrm{D}(\mathrm{b})\end{array}$ & $\begin{array}{l}7.6 \mathrm{E}-03 \\
1.4 \mathrm{E}-03 \\
4.1 \mathrm{E}-06 \\
1.0 \mathrm{E}-02 \\
3.3 \mathrm{E}-02\end{array}$ & $\begin{array}{l}6.6 \mathrm{E}-05 \\
8.1 \mathrm{E}-03 \\
6.5 \mathrm{E}-02 \\
2.0 \mathrm{E}-03 \\
5.5 \mathrm{E}-06\end{array}$ & $\begin{array}{l}9.2 \mathrm{E}-05 \\
8.8 \mathrm{E}-03 \\
7.0 \mathrm{E}-02 \\
3.6 \mathrm{E}-04 \\
1.0 \mathrm{E}-05\end{array}$ & $\begin{array}{l}7.8 \mathrm{E}-03 \\
1.8 \mathrm{E}-02 \\
1.4 \mathrm{E}-01 \\
1.2 \mathrm{E}-02 \\
3.3 \mathrm{E}-02\end{array}$ & $\begin{array}{l}2.1 E+00 \\
4.9 E+00 \\
3.6 E+01 \\
3.3 E+00 \\
8.8 E+00\end{array}$ \\
\hline 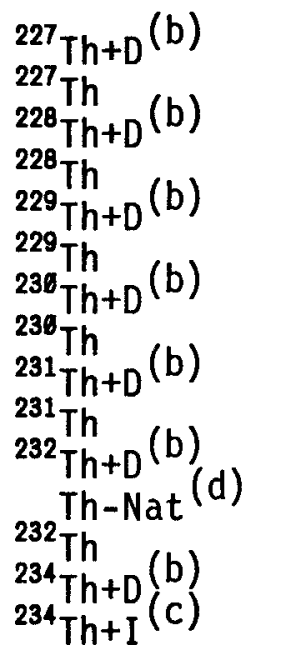 & $\begin{array}{l}1.6 \mathrm{E}-03 \\
3.0 \mathrm{E}-03 \\
7.2 \mathrm{E}-03 \\
5.0 \mathrm{E}-05 \\
1.2 \mathrm{E}-03 \\
2.0 \mathrm{E}-03 \\
5.9 \mathrm{E}-03 \\
1.1 \mathrm{E}-05 \\
2.7 \mathrm{E}-05 \\
2.7 \mathrm{E}-04 \\
8.0 \mathrm{E}-03 \\
8.0 \mathrm{E}-03 \\
7.7 \mathrm{E}-06 \\
4.0 \mathrm{E}-04 \\
7.5 \mathrm{E}-04\end{array}$ & $\begin{array}{l}1.6 \mathrm{E}-04 \\
8.0 \mathrm{E}-04 \\
2.3 \mathrm{E}-03 \\
1.5 \mathrm{E}-02 \\
1.1 \mathrm{E}-02 \\
8.5 \mathrm{E}-02 \\
1.4 \mathrm{E}-03 \\
1.2 \mathrm{E}-02 \\
4.0 \mathrm{E}-09 \\
4.1 \mathrm{E}-08 \\
7.0 \mathrm{E}-03 \\
7.0 \mathrm{E}-03 \\
5.5 \mathrm{E}-02 \\
8.5 \mathrm{E}-07 \\
1.7 \mathrm{E}-06\end{array}$ & $\begin{array}{l}2.2 \mathrm{E}-04 \\
1.9 \mathrm{E}-04 \\
4.1 \mathrm{E}-04 \\
2.0 \mathrm{E}-03 \\
2.4 \mathrm{E}-03 \\
1.8 \mathrm{E}-02 \\
4.4 \mathrm{E}-03 \\
2.7 \mathrm{E}-03 \\
6.5 \mathrm{E}-07 \\
6.5 \mathrm{E}-06 \\
2.1 \mathrm{E}-03 \\
2.1 \mathrm{E}-03 \\
1.4 \mathrm{E}-02 \\
3.3 \mathrm{E}-05 \\
6.5 \mathrm{E}-05\end{array}$ & $\begin{array}{l}2.0 \mathrm{E}-03 \\
4.0 \mathrm{E}-03 \\
9.9 \mathrm{E}-03 \\
1.8 \mathrm{E}-02 \\
1.4 \mathrm{E}-02 \\
1.0 \mathrm{E}-01 \\
1.2 \mathrm{E}-02 \\
1.5 \mathrm{E}-02 \\
2.7 \mathrm{E}-05 \\
2.8 \mathrm{E}-04 \\
1.7 \mathrm{E}-02 \\
1.7 \mathrm{E}-02 \\
6.9 \mathrm{E}-02 \\
4.4 \mathrm{E}-04 \\
8.1 \mathrm{E}-04\end{array}$ & $\begin{array}{l}5.4 \mathrm{E}-01 \\
1.1 \mathrm{E}+00 \\
2.7 \mathrm{E}+00 \\
4.7 \mathrm{E}+00 \\
3.8 \mathrm{E}+00 \\
2.8 \mathrm{E}+01 \\
3.2 \mathrm{E}+00 \\
4.1 \mathrm{E}+00 \\
7.3 \mathrm{E}-03 \\
7.5 \mathrm{E}-02 \\
4.6 \mathrm{E}+00 \\
4.6 \mathrm{E}+00 \\
1.9 \mathrm{E}+01 \\
1.2 \mathrm{E}-01 \\
2.2 \mathrm{E}-01\end{array}$ \\
\hline $\begin{array}{l}{ }^{231} \mathrm{~Pa}+\mathrm{D} \\
{ }^{231} \mathrm{~Pa} \\
{ }^{233} \mathrm{~Pa} \\
{ }^{234} \mathrm{~Pa} \\
{ }^{\mathrm{Pa}}\end{array}$ & $\begin{array}{l}1.3 \mathrm{E}-03 \\
9.7 \mathrm{E}-04 \\
7.0 \mathrm{E}-03 \\
6.6 \mathrm{E}-02\end{array}$ & $\begin{array}{l}1.2 \mathrm{E}-02 \\
4.2 \mathrm{E}-02 \\
4.6 \mathrm{E}-07 \\
4.1 \mathrm{E}-08\end{array}$ & $\begin{array}{l}1.4 \mathrm{E}-02 \\
5.5 \mathrm{E}-02 \\
1.8 \mathrm{E}-05 \\
1.1 \mathrm{E}-05\end{array}$ & $\begin{array}{l}2.7 \mathrm{E}-02 \\
9.8 \mathrm{E}-02 \\
7.0 \mathrm{E}-03 \\
6.6 \mathrm{E}-02\end{array}$ & $\begin{array}{l}7.3 E+00 \\
2.6 E+01 \\
1.9 E+00 \\
1.8 E+01\end{array}$ \\
\hline $\begin{array}{l}{ }^{232} U+D \\
{ }^{232} U \\
{ }^{233} U+D \\
{ }^{233} U \\
{ }^{234} U \\
{ }^{235} U+D \\
{ }^{235} U \\
{ }^{238} U \\
{ }^{237} U \\
{ }^{238} U+D \\
{ }^{238}{ }_{U}(b) \\
{ }^{246} U-N a t(b) \\
{ }^{246} U+D \\
U+I\end{array}$ & $\begin{array}{l}1.5 \mathrm{E}-06 \\
1.1 \mathrm{E}-05 \\
1.1 \mathrm{E}-03 \\
8.5 \mathrm{E}-06 \\
8.2 \mathrm{E}-06 \\
1.7 \mathrm{E}-03 \\
3.2 \mathrm{E}-03 \\
6.7 \mathrm{E}-06 \\
3.9 \mathrm{E}-03 \\
2.7 \mathrm{E}-04 \\
7.8 \mathrm{E}-05 \\
1.1 \mathrm{E}-04 \\
4.0 \mathrm{E}-03 \\
1.2 \mathrm{E}-02\end{array}$ & $\begin{array}{l}4.3 \mathrm{E}-03 \\
3.0 \mathrm{E}-02 \\
1.0 \mathrm{E}-02 \\
6.5 \mathrm{E}-03 \\
6.5 \mathrm{E}-03 \\
3.0 \mathrm{E}-03 \\
6.0 \mathrm{E}-03 \\
6.0 \mathrm{E}-03 \\
1.7 \mathrm{E}-07 \\
1.8 \mathrm{E}-03 \\
6.0 \mathrm{E}-03 \\
6.2 \mathrm{E}-03 \\
3.7 \mathrm{E}-08 \\
1.1 \mathrm{E}-07\end{array}$ & $\begin{array}{l}4.9 \mathrm{E}-05 \\
3.4 \mathrm{E}-04 \\
2.2 \mathrm{E}-03 \\
1.3 \mathrm{E}-04 \\
1.3 \mathrm{E}-04 \\
7.0 \mathrm{E}-05 \\
1.3 \mathrm{E}-04 \\
1.2 \mathrm{E}-04 \\
1.6 \mathrm{E}-05 \\
6.2 \mathrm{E}-05 \\
1.3 \mathrm{E}-04 \\
1.3 \mathrm{E}-04 \\
7.8 \mathrm{E}-06 \\
2.3 \mathrm{E}-05\end{array}$ & $\begin{array}{l}4.3 \mathrm{E}-03 \\
3.0 \mathrm{E}-02 \\
1.3 \mathrm{E}-02 \\
6.6 \mathrm{E}-03 \\
6.6 \mathrm{E}-03 \\
4.8 \mathrm{E}-03 \\
9.3 \mathrm{E}-03 \\
6.1 \mathrm{E}-03 \\
3.9 \mathrm{E}-03 \\
2.2 \mathrm{E}-03 \\
6.2 \mathrm{E}-03 \\
6.5 \mathrm{E}-03 \\
4.0 \mathrm{E}-03 \\
1.2 \mathrm{E}-02\end{array}$ & $\begin{array}{l}1.2 \mathrm{E}+00 \\
8.2 \mathrm{E}+00 \\
3.6 \mathrm{E}+00 \\
1.8 \mathrm{E}+00 \\
1.8 \mathrm{E}+00 \\
1.3 \mathrm{E}+00 \\
2.5 \mathrm{E}+00 \\
1.7 \mathrm{E}+00 \\
1.0 \mathrm{E}+00 \\
5.8 \mathrm{E}-01 \\
1.7 \mathrm{E}+00 \\
1.8 \mathrm{E}+00 \\
1.1 \mathrm{E}+00 \\
3.3 \mathrm{E}+00\end{array}$ \\
\hline
\end{tabular}


TABLE 3.1. Annual Total Effective Dose Equivalent Factors for the Building Renovation (Volume Activity) Scenario(a) (contd)

\begin{tabular}{|c|c|c|c|c|c|}
\hline Radionuclide & $\begin{array}{c}\text { External } \\
\text { (mrem) }\end{array}$ & $\begin{array}{l}\text { Inhalation } \\
\text { (mrem) }\end{array}$ & $\begin{array}{l}\text { Secondary } \\
\text { Ingestion } \\
\text { (mrem) }\end{array}$ & $\begin{array}{c}\text { TEDE } \\
\text { (mrem) }\end{array}$ & $\begin{array}{l}\text { TEDE } \\
(\mu \text { Sv }) \\
\end{array}$ \\
\hline $\begin{array}{l}{ }^{237} \mathrm{~Np}+\mathrm{D} \\
{ }^{237} \mathrm{~Np} \\
{ }^{233} \mathrm{~Np} \\
{ }^{238} \mathrm{~Np} \\
{ }_{\mathrm{Np}} \\
{ }^{239} \mathrm{~Np}+\mathrm{D} \\
{ }^{239} \mathrm{~Np}\end{array}$ & $\begin{array}{l}3.7 \mathrm{E}-03 \\
4.7 \mathrm{E}-04 \\
9.2 \mathrm{E}-03 \\
1.9 \mathrm{E}-02 \\
2.4 \mathrm{E}-03 \\
4.9 \mathrm{E}-03\end{array}$ & $\begin{array}{l}1.5 \mathrm{E}-02 \\
3.1 \mathrm{E}-02 \\
7.0 \mathrm{E}-03 \\
2.0 \mathrm{E}-06 \\
6.0 \mathrm{E}-08 \\
1.2 \mathrm{E}-07\end{array}$ & $\begin{array}{l}1.3 \mathrm{E}-02 \\
2.6 \mathrm{E}-02 \\
8.5 \mathrm{E}-04 \\
2.0 \mathrm{E}-05 \\
8.3 \mathrm{E}-06 \\
1.7 \mathrm{E}-05\end{array}$ & $\begin{array}{l}3.2 \mathrm{E}-02 \\
5.7 \mathrm{E}-02 \\
1.7 \mathrm{E}-02 \\
1.9 \mathrm{E}-02 \\
2.4 \mathrm{E}-03 \\
4.9 \mathrm{E}-03\end{array}$ & $\begin{array}{l}8.6 \mathrm{E}+00 \\
1.5 \mathrm{E}+01 \\
4.6 \mathrm{E}+00 \\
5.1 \mathrm{E}+00 \\
6.4 \mathrm{E}-01 \\
1.3 \mathrm{E}+00\end{array}$ \\
\hline $\begin{array}{l}{ }^{236} \mathrm{Pu} \\
{ }^{237} \mathrm{Pu} \\
{ }^{238} \mathrm{Pu} \\
{ }^{239} \mathrm{Pu} \\
{ }^{248} \mathrm{Pu} \\
{ }^{241} \mathrm{Pu}+\mathrm{Pu} \\
{ }^{241} \mathrm{Pu} \\
{ }^{242} \mathrm{Pu} \\
{ }^{243} \mathrm{Pu}+\mathrm{bu} \\
{ }^{243} \mathrm{Pu} \\
{ }^{244} \mathrm{Pu} \text { (b) } \\
{ }^{244} \mathrm{Pu} \mathrm{Pu}\end{array}$ & $\begin{array}{l}8.1 \mathrm{E}-06 \\
1.3 \mathrm{E}-03 \\
6.9 \mathrm{E}-06 \\
4.1 \mathrm{E}-06 \\
6.6 \mathrm{E}-06 \\
1.6 \mathrm{E}-04 \\
1.7 \mathrm{E}-11 \\
5.5 \mathrm{E}-06 \\
1.7 \mathrm{E}-03 \\
4.7 \mathrm{E}-04 \\
3.0 \mathrm{E}-03 \\
4.6 \mathrm{E}-06\end{array}$ & $\begin{array}{l}3.6 \mathrm{E}-03 \\
9.0 \mathrm{E}-08 \\
1.4 \mathrm{E}-02 \\
1.4 \mathrm{E}-02 \\
1.4 \mathrm{E}-02 \\
1.1 \mathrm{E}-02 \\
2.3 \mathrm{E}-04 \\
1.3 \mathrm{E}-02 \\
3.5 \mathrm{E}-09 \\
7.0 \mathrm{E}-09 \\
3.3 \mathrm{E}-03 \\
1.3 \mathrm{E}-02\end{array}$ & $\begin{array}{l}3.1 \mathrm{E}-04 \\
2.2 \mathrm{E}-06 \\
1.7 \mathrm{E}-03 \\
1.9 \mathrm{E}-03 \\
1.9 \mathrm{E}-03 \\
9.0 \mathrm{E}-03 \\
3.4 \mathrm{E}-05 \\
1.7 \mathrm{E}-03 \\
8.0 \mathrm{E}-07 \\
1.6 \mathrm{E}-06 \\
4.5 \mathrm{E}-04 \\
1.7 \mathrm{E}-03\end{array}$ & $\begin{array}{l}3.9 \mathrm{E}-03 \\
1.3 \mathrm{E}-03 \\
1.6 \mathrm{E}-02 \\
1.6 \mathrm{E}-02 \\
1.6 \mathrm{E}-02 \\
2.0 \mathrm{E}-02 \\
2.7 \mathrm{E}-04 \\
1.5 \mathrm{E}-02 \\
1.7 \mathrm{E}-03 \\
4.7 \mathrm{E}-04 \\
6.7 \mathrm{E}-03 \\
1.5 \mathrm{E}-02\end{array}$ & $\begin{array}{l}1.1 \mathrm{E}+00 \\
3.6 \mathrm{E}-01 \\
4.2 \mathrm{E}+00 \\
4.4 \mathrm{E}+00 \\
4.4 \mathrm{E}+00 \\
5.4 \mathrm{E}+00 \\
7.3 \mathrm{E}-02 \\
4.1 \mathrm{E}+00 \\
4.7 \mathrm{E}-01 \\
1.3 \mathrm{E}-01 \\
1.8 \mathrm{E}+00 \\
4.0 \mathrm{E}+00\end{array}$ \\
\hline $\begin{array}{l}{ }^{241} \mathrm{Am} \\
{ }^{242 m} \mathrm{Am}+\mathrm{D} \\
{ }^{242} \mathrm{Am} \\
{ }^{242} \mathrm{Am}+\mathrm{D} \\
{ }^{242} \mathrm{Am} \\
{ }^{243} \mathrm{Am}+\mathrm{D} \\
{ }^{243} \mathrm{Am}\end{array}$ & $\begin{array}{l}3.2 \mathrm{E}-04 \\
1.1 \mathrm{E}-04 \\
2.4 \mathrm{E}-05 \\
1.4 \mathrm{E}-04 \\
3.9 \mathrm{E}-04 \\
1.9 \mathrm{E}-03 \\
9.1 \mathrm{E}-04\end{array}$ & $\begin{array}{l}2.1 \mathrm{E}-02 \\
8.2 \mathrm{E}-03 \\
2.0 \mathrm{E}-02 \\
4.2 \mathrm{E}-03 \\
2.6 \mathrm{E}-06 \\
6.8 \mathrm{E}-03 \\
2.0 \mathrm{E}-02\end{array}$ & $\begin{array}{l}1.8 \mathrm{E}-02 \\
5.0 \mathrm{E}-03 \\
1.8 \mathrm{E}-02 \\
6.5 \mathrm{E}-04 \\
7.0 \mathrm{E}-06 \\
6.0 \mathrm{E}-03 \\
1.8 \mathrm{E}-02\end{array}$ & $\begin{array}{l}4.0 \mathrm{E}-02 \\
1.3 \mathrm{E}-02 \\
3.7 \mathrm{E}-02 \\
5.0 \mathrm{E}-03 \\
4.0 \mathrm{E}-04 \\
1.5 \mathrm{E}-02 \\
3.9 \mathrm{E}-02\end{array}$ & $\begin{array}{l}1.1 \mathrm{E}+01 \\
3.6 \mathrm{E}+00 \\
1.0 \mathrm{E}+01 \\
1.4 \mathrm{E}+00 \\
1.1 \mathrm{E}-01 \\
4.0 \mathrm{E}+00 \\
1.1 \mathrm{E}+01\end{array}$ \\
\hline $\begin{array}{l}{ }^{242} \mathrm{Cm}+D \text { (b) } \\
242 \mathrm{Cm} \\
{ }^{243} \mathrm{Cm} \\
{ }^{244} \mathrm{Cm}+\mathrm{D} \\
\left.{ }^{244} \mathrm{~b}\right) \\
{ }^{245} \mathrm{Cm} \\
{ }^{245} \mathrm{Cm}+\mathrm{D} \\
{ }^{246} \mathrm{Cm} \\
{ }^{247} \mathrm{Cm} \\
{ }^{247} \mathrm{Cm}+\mathrm{D} \\
{ }^{248} \mathrm{Cm} \\
{ }^{448} \mathrm{Cm}\end{array}$ & $\begin{array}{l}7.5 \mathrm{E}-06 \\
6.8 \mathrm{E}-06 \\
3.6 \mathrm{E}-03 \\
2.9 \mathrm{E}-06 \\
6.0 \mathrm{E}-06 \\
7.0 \mathrm{E}-04 \\
1.8 \mathrm{E}-03 \\
5.3 \mathrm{E}-06 \\
7.6 \mathrm{E}-03 \\
1.2 \mathrm{E}-02 \\
4.3 \mathrm{E}-06\end{array}$ & $\begin{array}{l}7.9 \mathrm{E}-03 \\
7.5 \mathrm{E}-04 \\
2.9 \mathrm{E}-02 \\
5.8 \mathrm{E}-03 \\
1.2 \mathrm{E}-02 \\
1.4 \mathrm{E}-02 \\
2.1 \mathrm{E}-02 \\
2.1 \mathrm{E}-02 \\
9.8 \mathrm{E}-03 \\
2.0 \mathrm{E}-02 \\
7.5 \mathrm{E}-02\end{array}$ & $\begin{array}{l}1.2 \mathrm{E}-03 \\
5.5 \mathrm{E}-04 \\
1.4 \mathrm{E}-02 \\
5.0 \mathrm{E}-03 \\
1.0 \mathrm{E}-02 \\
1.2 \mathrm{E}-02 \\
1.9 \mathrm{E}-02 \\
1.9 \mathrm{E}-02 \\
8.5 \mathrm{E}-03 \\
1.7 \mathrm{E}-02 \\
6.5 \mathrm{E}-02\end{array}$ & $\begin{array}{l}9.2 \mathrm{E}-03 \\
1.3 \mathrm{E}-03 \\
4.7 \mathrm{E}-02 \\
1.1 \mathrm{E}-02 \\
2.2 \mathrm{E}-02 \\
2.7 \mathrm{E}-02 \\
4.1 \mathrm{E}-02 \\
4.0 \mathrm{E}-02 \\
2.6 \mathrm{E}-02 \\
4.8 \mathrm{E}-02 \\
1.4 \mathrm{E}-01\end{array}$ & $\begin{array}{l}2.5 \mathrm{E}+00 \\
3.5 \mathrm{E}-01 \\
1.3 \mathrm{E}+01 \\
2.9 \mathrm{E}+00 \\
5.8 \mathrm{E}+00 \\
7.3 \mathrm{E}+00 \\
1.1 \mathrm{E}+01 \\
1.1 \mathrm{E}+01 \\
7.0 \mathrm{E}+00 \\
1.3 \mathrm{E}+01 \\
3.8 \mathrm{E}+01\end{array}$ \\
\hline${ }^{252} \mathrm{Cf}$ & $4.3 E-06$ & $6.5 \mathrm{E}-03$ & $5.5 E-03$ & $1.2 \mathrm{E}-02$ & $3.2 \mathrm{E}+00$ \\
\hline
\end{tabular}

(a) Based on $500 \mathrm{~h}$ of exposure during building renovation with a contamination level of $1 \mathrm{pCi} / \mathrm{g}(1 \mathrm{~Bq} / \mathrm{g})$. 
TABLE 3.1. Annual Total Effective Dose Equivalent Factors for the Building Renovation (Volume Activity) Scenario(a) (contd)

(b) Where $+D$ means "plus daughters in equilibrium" referring to explicit daughters, or daughters with half-lives greater than 10 minutes. Separate values are also included for parents and daughters so that combined factors for non-equilibrium cases can be determined. Note: in some cases $+D$ also includes implicit daughters (i.e., uranium and thorium decay chains).

(c) Where +I means "plus implicit daughters in equilibrium" referring to daughters with half-lives less than 10 minutes. The decay energies associated with these daughters have been assigned to the parent, and the appropriate branching ratios (described in Appendix D) have been considered.

(d) Where Th-Nat includes an equilibrium mixture of ${ }^{232}$ Th plus 10 daughters in the decay chain, as discussed in Appendix $D$.

(e) Where $1 \mathrm{Ci}$ U-Nat equals $48.9 \%{ }^{238} \mathrm{U}$ plus $48.9 \%{ }^{234} \mathrm{U}$ plus $2.25 \%{ }^{235} \mathrm{U}$. 


\subsubsection{Building 0ccupancy (Surface Activity) Scenario}

At many facilities, most of the residual radioactive inventory may be associated with surface or thin-layer surface sources. Because surface decontamination operations may not remove all of the surface sources, a scenario describing surface activity must be considered. For this analysis, the building occupancy scenario is defined to account for potential exposure to both fixed and removable thin layer or surface contamination sources. This scenario is used to derive surface activity annual TEDE factors.

This scenario considers chronic exposure to an individual for $2000 \mathrm{~h} / \mathrm{yr}$, which represents occupancy during a full work year (i.e., 50 weeks at $8 \mathrm{~h} / \mathrm{d}$ ) in a commercial facility. The exposure pathways considered include direct external exposure from surface sources, inhalation of resuspended removable contamination, and secondary ingestion of removable contamination. This scenario does not include consideration of indoor radon gas that may evolve from residual 226 Ra in the building. The air concentration and secondary ingestion rates are assumed to be $10 \%$ of those encountered during building renovation, or $10^{-5} \mathrm{~g} / \mathrm{m}^{3}$ and $0.001 \mathrm{~g} / \mathrm{h}$, respectively. These parameter values and their expected ranges are summarized in Section 3.3 and are more fully discussed in Appendix B. These assumptions and parameter values are used to modify the pathway dose rate conversion and committed effective dose rate conversion factors listed in Section 2.0 to obtain the scenario-specific annual TEDE factors (shown in Table 3.2), in units of mrem per dpm/100 cm2 (and $\mu \mathrm{Sv}$ per $\mathrm{Bq} / 100 \mathrm{~cm}^{2}$ ). Table 3.2 ists the contribution by exposure pathway for about 200 radionuclides including key parent-daughter relationships. Again, the $+I$ (implicit daughter) and $+D$ (explicit daughter) notation has been used to provide maximum flexibility for consideration of all parent-daughter and decay chain situations. Further description of the parent-daughter and chain decay relationships used in this study is provided in Appendix $D$.

Hand calculations were performed to verify the computer calculations for selected radionuclides using the pathway factors from Section 2.0 and the building occupancy scenario assumptions for the external exposure, inhalation, and secondary ingestion pathways. The results for $60 \mathrm{Co}, 90 \mathrm{sr}$, $137 \mathrm{Cs}, 235 \mathrm{U}^{\prime}$, and $239 \mathrm{Pu}$ indicated that the values in Table 3.2 were calculated correctly. 
TABLE 3.2. Annual Total Effective Dose Equivalent Factors for the Building Occupancy (Surface Activity) Scenario(a)

\begin{tabular}{|c|c|c|c|c|c|}
\hline Radionuclide & $\begin{array}{c}\text { External } \\
\text { (mrem) } \\
\end{array}$ & $\begin{array}{l}\text { Inhalation } \\
\text { (mrem) } \\
\end{array}$ & $\begin{array}{l}\text { Secondary } \\
\text { Ingestion } \\
\text { (mrem) }\end{array}$ & $\begin{array}{c}\text { TEDE } \\
\text { (mrem) }\end{array}$ & $\begin{array}{l}\text { TEDE } \\
(\mu \mathrm{SV}) \\
\end{array}$ \\
\hline${ }^{3} \mathrm{H}$ & $4.8 \mathrm{E}-13$ & $1.6 \mathrm{E}-09$ & $1.1 E-07$ & $1.2 E-07$ & $3.1 E-05$ \\
\hline${ }^{16} \mathrm{Be}$ & $1.7 \mathrm{E}-07$ & $6.8 E-06$ & $9.2 \mathrm{E}-06$ & $1.6 \mathrm{E}-05$ & $4.4 E-03$ \\
\hline${ }^{14} \mathrm{C}$ & $1.0 \mathrm{E}-08$ & $3.8 E-08$ & $3.8 E-06$ & $3.8 E-06$ & $1.0 E-03$ \\
\hline${ }^{18} \mathrm{~F}$ & $3.2 \mathrm{E}-04$ & $1.5 \mathrm{E}-09$ & $2.2 \mathrm{E}-07$ & $3.2 E-04$ & $1.9 E-01$ \\
\hline${ }^{24} \mathrm{Na}$ & $\begin{array}{l}\text { 7.2E-04 } \\
1.3 \mathrm{E}-03\end{array}$ & $\begin{array}{l}1.7 \mathrm{E}-07 \\
2.2 \mathrm{E}-08\end{array}$ & $\begin{array}{l}2.8 \mathrm{E}-05 \\
2.8 \mathrm{E}-06\end{array}$ & $\begin{array}{l}7.5 \mathrm{E}-04 \\
1.3 \mathrm{E}-03\end{array}$ & $\begin{array}{l}4.4 E-01 \\
8.0 E-01\end{array}$ \\
\hline${ }^{32} \mathrm{p}$ & $\begin{array}{l}1.5 E-06 \\
3.0 E-08\end{array}$ & $\begin{array}{l}1.1 \mathrm{E}-07 \\
1.1 \mathrm{E}-08\end{array}$ & $\begin{array}{l}1.7 \mathrm{E}-05 \\
1.7 \mathrm{E}-06\end{array}$ & $\begin{array}{l}1.9 \mathrm{E}-05 \\
1.8 \mathrm{E}-06\end{array}$ & $\begin{array}{l}5.5 E-03 \\
4.9 E-04\end{array}$ \\
\hline${ }^{35} \mathrm{~S}$ & $1.1 \mathrm{E}-08$ & $4.8 \mathrm{E}-08$ & 8.0E-07 & $8.6 \mathrm{E}-07$ & $2.4 \mathrm{E}-04$ \\
\hline${ }^{36} \mathrm{Cl}$ & $2.6 \mathrm{E}-07$ & $3.6 E-08$ & $5.6 \mathrm{E}-06$ & $5.9 \mathrm{E}-06$ & $1.7 E-03$ \\
\hline${ }^{46} \mathrm{~K}$ & $5.4 \mathrm{E}-05$ & $2.0 \mathrm{E}-07$ & $3.4 \mathrm{E}-05$ & $8.8 \mathrm{E}-05$ & $4.2 \mathrm{E}-02$ \\
\hline $\begin{array}{l}{ }^{41} \mathrm{Ca} \\
{ }^{45} \mathrm{Ca}\end{array}$ & $\begin{array}{l}6.5 E-07 \\
3.1 E-08\end{array}$ & $\begin{array}{l}2.4 \mathrm{E}-08 \\
1.3 \mathrm{E}-07\end{array}$ & $\begin{array}{l}2.4 E-06 \\
6.2 E-06\end{array}$ & $\begin{array}{l}3.1 \mathrm{E}-06 \\
6.4 \mathrm{E}-06\end{array}$ & $\begin{array}{l}1.0 \mathrm{E}-03 \\
1.7 \mathrm{E}-03\end{array}$ \\
\hline${ }^{46} \mathrm{SC}$ & $7.1 \mathrm{E}-04$ & $5.6 \mathrm{E}-07$ & $1.2 E-05$ & $7.3 E-04$ & 4.3E-01 \\
\hline${ }^{51} \mathrm{Cr}$ & $1.3 \mathrm{E}-05$ & $6.2 E-09$ & $2.8 E-07$ & $1.4 E-05$ & $8.1 E-03$ \\
\hline $\begin{array}{l}{ }^{54} \mathrm{Mn} \\
{ }^{56} \mathrm{Mn}\end{array}$ & $\begin{array}{l}2.9 \mathrm{E}-04 \\
5.8 \mathrm{E}-04\end{array}$ & $\begin{array}{l}1.2 E-07 \\
6.6 E-09\end{array}$ & $\begin{array}{l}5.6 E-06 \\
2.0 E-06\end{array}$ & $\begin{array}{l}3.0 \mathrm{E}-04 \\
5.9 \mathrm{E}-04\end{array}$ & $\begin{array}{l}1.8 \mathrm{E}-01 \\
3.5 \mathrm{E}-01\end{array}$ \\
\hline $\begin{array}{l}{ }^{55} \mathrm{Fe} \\
{ }^{59} \mathrm{Fe}\end{array}$ & $\begin{array}{l}1.5 E-06 \\
3.8 E-04\end{array}$ & $\begin{array}{l}2.4 E-08 \\
2.4 E-07\end{array}$ & $\begin{array}{l}1.2 E-06 \\
1.3 E-05\end{array}$ & $\begin{array}{l}2.7 E-06 \\
3.9 E-04\end{array}$ & $\begin{array}{l}1.2 \mathrm{E}-03 \\
2.3 \mathrm{E}-01\end{array}$ \\
\hline $\begin{array}{l}{ }^{57} \mathrm{Co} \\
{ }^{68} \mathrm{Co} \\
{ }^{50}\end{array}$ & $\begin{array}{l}4.7 E-05 \\
3.5 E-04 \\
8.4 E-04\end{array}$ & $\begin{array}{l}1.7 E-07 \\
2.0 E-07 \\
3.8 E-06\end{array}$ & $\begin{array}{l}1.5 \mathrm{E}-06 \\
5.8 \mathrm{E}-06 \\
2.0 \mathrm{E}-05\end{array}$ & $\begin{array}{l}4.9 \mathrm{E}-05 \\
3.5 \mathrm{E}-04 \\
8.6 \mathrm{E}-04\end{array}$ & $\begin{array}{l}2.9 E-02 \\
2.1 E-01 \\
5.1 E-01\end{array}$ \\
\hline $\begin{array}{l}{ }^{58} \mathrm{Ni} \\
{ }^{63} \mathrm{Ni} \\
{ }_{\mathrm{Ni}}\end{array}$ & $\begin{array}{l}1.8 \mathrm{E}-06 \\
8.0 \mathrm{E}-10 \\
1.9 \mathrm{E}-04\end{array}$ & $\begin{array}{l}1.6 \mathrm{E}-08 \\
3.8 \mathrm{E}-08 \\
4.6 \mathrm{E}-09\end{array}$ & $\begin{array}{l}3.8 \mathrm{E}-07 \\
1.0 \mathrm{E}-06 \\
1.3 \mathrm{E}-06\end{array}$ & $\begin{array}{l}2.2 E-06 \\
1.1 E-06 \\
1.9 E-04\end{array}$ & $\begin{array}{l}1.2 E-03 \\
2.9 E-04 \\
1.2 E-01\end{array}$ \\
\hline${ }^{84} \mathrm{Cu}$ & $6.2 E-05$ & $3.8 E-09$ & $9.6 E-07$ & $6.3 E-05$ & $3.8 E-02$ \\
\hline${ }^{65} \mathrm{Zn}_{\mathrm{Zn}+\mathrm{D}}(\mathrm{b})$ & $\begin{array}{l}2.2 \mathrm{E}-04 \\
7.4 \mathrm{E}-05\end{array}$ & $\begin{array}{l}3.4 E-07 \\
8.2 E-09\end{array}$ & $\begin{array}{l}2.6 \mathrm{E}-05 \\
1.4 \mathrm{E}-06\end{array}$ & $\begin{array}{l}2.5 E-04 \\
7.6 E-05\end{array}$ & $\begin{array}{l}1.4 E-01 \\
4.5 E-02\end{array}$ \\
\hline
\end{tabular}


TABLE 3.2. Annual Total Effective Dose Equivalent Factors for the Building Occupancy (Surface Activity) Scenario(a) (contd)

\begin{tabular}{|c|c|c|c|c|c|}
\hline Radionuclide & $\begin{array}{c}\text { External } \\
\text { (mrem) } \\
\end{array}$ & $\begin{array}{c}\text { Inhalation } \\
\text { (mrem) }\end{array}$ & $\begin{array}{l}\text { Secondary } \\
\text { Ingestion } \\
\text { (mrem) }\end{array}$ & $\begin{array}{c}\text { TEDE } \\
\text { (mrem) }\end{array}$ & $\begin{array}{l}\text { TEDE } \\
(\mu \mathrm{SV}) \\
\end{array}$ \\
\hline${ }^{80} \mathrm{Zn} Z \mathrm{Zn}$ & $\begin{array}{l}1.6 \mathrm{E}-04 \\
4.3 \mathrm{E}-07\end{array}$ & $\begin{array}{l}1.6 \mathrm{E}-08 \\
7.6 \mathrm{E}-10\end{array}$ & $\begin{array}{l}2.6 \mathrm{E}-06 \\
1.8 \mathrm{E}-07\end{array}$ & $\begin{array}{l}1.6 \mathrm{E}-04 \\
6.1 \mathrm{E}-07\end{array}$ & $\begin{array}{l}9.5 \mathrm{E}-02 \\
3.1 \mathrm{E}-04\end{array}$ \\
\hline${ }^{78} \mathrm{As}$ & $1.7 \mathrm{E}-04$ & $7.6 \mathrm{E}-08$ & $1.1 \mathrm{E}-05$ & $1.8 \mathrm{E}-04$ & $1.1 \mathrm{E}-01$ \\
\hline${ }^{75} \mathrm{Se}$ & $\begin{array}{l}1.3 \mathrm{E}-04 \\
7.8 \mathrm{E}-09\end{array}$ & $\begin{array}{l}1.5 \mathrm{E}-07 \\
1.8 \mathrm{E}-07\end{array}$ & $\begin{array}{l}1.8 \mathrm{E}-05 \\
1.6 \mathrm{E}-05\end{array}$ & $\begin{array}{l}1.5 \mathrm{E}-04 \\
1.6 \mathrm{E}-05\end{array}$ & $\begin{array}{l}\text { 8. } 4 \mathrm{E}-02 \\
4.4 \mathrm{E}-03\end{array}$ \\
\hline $\begin{array}{l}{ }^{82} \mathrm{Br} \\
{ }^{83} \mathrm{Br}+\mathrm{D} \\
{ }^{83} \mathrm{Br} \\
{ }^{84} \mathrm{Br}\end{array}$ & $\begin{array}{l}9.6 \mathrm{E}-04 \\
2.0 \mathrm{E}-06 \\
2.8 \mathrm{E}-06 \\
5.6 \mathrm{E}-04\end{array}$ & $\begin{array}{l}2.2 E-08 \\
8.9 E-10 \\
1.8 E-09 \\
1.9 E-09\end{array}$ & $\begin{array}{l}3.2 \mathrm{E}-06 \\
9.4 \mathrm{E}-08 \\
1.9 \mathrm{E}-07 \\
3.6 \mathrm{E}-07\end{array}$ & $\begin{array}{l}9.7 \mathrm{E}-04 \\
2.1 \mathrm{E}-06 \\
3.0 \mathrm{E}-06 \\
5.6 \mathrm{E}-04\end{array}$ & $\begin{array}{l}5.8 \mathrm{E}-01 \\
1.2 \mathrm{E}-03 \\
1.7 \mathrm{E}-03 \\
3.4 \mathrm{E}-01\end{array}$ \\
\hline 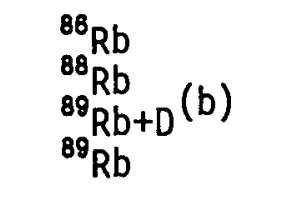 & $\begin{array}{l}3.2 \mathrm{E}-05 \\
2.3 \mathrm{E}-04 \\
3.3 \mathrm{E}-04 \\
6.9 \mathrm{E}-04\end{array}$ & $\begin{array}{l}1.2 \mathrm{E}-07 \\
1.7 \mathrm{E}-09 \\
5.3 \mathrm{E}-08 \\
8.4 \mathrm{E}-10\end{array}$ & $\begin{array}{l}1.8 \mathrm{E}-05 \\
3.6 \mathrm{E}-07 \\
8.8 \mathrm{E}-06 \\
1.9 \mathrm{E}-07\end{array}$ & $\begin{array}{l}5.1 \mathrm{E}-05 \\
2.3 \mathrm{E}-04 \\
3.4 \mathrm{E}-04 \\
6.9 \mathrm{E}-04\end{array}$ & $\begin{array}{l}2.4 \mathrm{E}-02 \\
1.4 \mathrm{E}-01 \\
2.0 \mathrm{E}-01 \\
4.1 \mathrm{E}-01\end{array}$ \\
\hline $\begin{array}{l}{ }^{85} S r \\
{ }^{89} S r \\
{ }^{96} S r+D \\
{ }^{96} S r \\
{ }^{91} S r \\
{ }^{91} S r+D \\
{ }^{92} S r \\
{ }^{92} S r+D \\
{ }^{92} S r\end{array}$ & $\begin{array}{l}1.7 \mathrm{E}-04 \\
1.3 \mathrm{E}-06 \\
1.6 \mathrm{E}-06 \\
1.4 \mathrm{E}-07 \\
1.4 \mathrm{E}-04 \\
2.3 \mathrm{E}-04 \\
2.8 \mathrm{E}-04 \\
4.8 \mathrm{E}-04\end{array}$ & $\begin{array}{l}2.8 \mathrm{E}-08 \\
1.1 \mathrm{E}-07 \\
2.1 \mathrm{E}-06 \\
4.0 \mathrm{E}-06 \\
3.7 \mathrm{E}-07 \\
1.7 \mathrm{E}-08 \\
1.4 \mathrm{E}-08 \\
1.2 \mathrm{E}-08\end{array}$ & $\begin{array}{l}3.4 \mathrm{E}-06 \\
1.7 \mathrm{E}-05 \\
1.3 \mathrm{E}-04 \\
2.4 \mathrm{E}-04 \\
9.3 \mathrm{E}-06 \\
4.8 \mathrm{E}-06 \\
3.6 \mathrm{E}-06 \\
3.2 \mathrm{E}-06\end{array}$ & $\begin{array}{l}1.7 \mathrm{E}-04 \\
1.9 \mathrm{E}-05 \\
1.3 \mathrm{E}-04 \\
2.4 \mathrm{E}-04 \\
1.5 \mathrm{E}-04 \\
2.4 \mathrm{E}-04 \\
2.9 \mathrm{E}-04 \\
4.9 \mathrm{E}-04\end{array}$ & $\begin{array}{l}1.0 \mathrm{E}-01 \\
5.5 \mathrm{E}-03 \\
3.7 \mathrm{E}-02 \\
6.6 \mathrm{E}-02 \\
8.5 \mathrm{E}-02 \\
1.4 \mathrm{E}-01 \\
1.7 \mathrm{E}-01 \\
2.9 \mathrm{E}-01\end{array}$ \\
\hline $\begin{array}{l}{ }^{96} Y \\
{ }^{911} Y \\
\\
91 Y_{Y} \\
{ }^{91} Y \\
{ }^{92} Y \\
{ }^{93} Y\end{array}$ & $\begin{array}{l}3.1 \mathrm{E}-06 \\
1.1 \mathrm{E}-04 \\
2.2 \mathrm{E}-04 \\
2.6 \mathrm{E}-06 \\
1.0 \mathrm{E}-04 \\
3.5 \mathrm{E}-05\end{array}$ & $\begin{array}{l}1.7 \mathrm{E}-07 \\
4.7 \mathrm{E}-07 \\
7.2 \mathrm{E}-10 \\
9.4 \mathrm{E}-07 \\
1.6 \mathrm{E}-08 \\
4.4 \mathrm{E}-08\end{array}$ & $\begin{array}{l}2.2 \mathrm{E}-05 \\
9.5 \mathrm{E}-06 \\
8.2 \mathrm{E}-08 \\
1.9 \mathrm{E}-05 \\
4.0 \mathrm{E}-06 \\
9.6 \mathrm{E}-06\end{array}$ & $\begin{array}{l}2.5 \mathrm{E}-05 \\
1.2 \mathrm{E}-04 \\
2.2 \mathrm{E}-04 \\
2.2 \mathrm{E}-05 \\
1.0 \mathrm{E}-04 \\
4.5 \mathrm{E}-05\end{array}$ & $\begin{array}{l}7.9 \mathrm{E}-03 \\
6.8 \mathrm{E}-02 \\
1.3 \mathrm{E}-01 \\
6.9 \mathrm{E}-03 \\
6.1 \mathrm{E}-02 \\
2.4 \mathrm{E}-02\end{array}$ \\
\hline $\begin{array}{l}{ }^{93} \mathrm{Zr+D}(\mathrm{b}) \\
{ }^{93} \mathrm{Zr} \\
{ }^{95} \mathrm{Zr+D}(\mathrm{b}) \\
{ }^{95} \mathrm{Zr} \\
{ }^{97} \mathrm{Zr+D}(\mathrm{b}) \\
{ }^{97} \mathrm{Zr}\end{array}$ & $\begin{array}{l}3.4 \mathrm{E}-07 \\
6.4 \mathrm{E}-10 \\
2.7 \mathrm{E}-04 \\
2.7 \mathrm{E}-04 \\
1.7 \mathrm{E}-04 \\
6.5 \mathrm{E}-05\end{array}$ & $\begin{array}{l}1.1 \mathrm{E}-06 \\
1.6 \mathrm{E}-06 \\
2.0 \mathrm{E}-07 \\
3.0 \mathrm{E}-07 \\
2.6 \mathrm{E}-08 \\
7.6 \mathrm{E}-08\end{array}$ & $\begin{array}{l}2.1 \mathrm{E}-06 \\
3.2 \mathrm{E}-06 \\
6.3 \mathrm{E}-06 \\
7.4 \mathrm{E}-06 \\
5.8 \mathrm{E}-06 \\
1.7 \mathrm{E}-05\end{array}$ & $\begin{array}{l}3.5 \mathrm{E}-06 \\
4.8 \mathrm{E}-06 \\
2.8 \mathrm{E}-04 \\
2.7 \mathrm{E}-04 \\
1.8 \mathrm{E}-04 \\
8.1 \mathrm{E}-05\end{array}$ & $\begin{array}{l}1.1 \mathrm{E}-03 \\
1.3 \mathrm{E}-03 \\
1.6 \mathrm{E}-01 \\
1.6 \mathrm{E}-01 \\
1.1 \mathrm{E}-01 \\
4.3 \mathrm{E}-02\end{array}$ \\
\hline 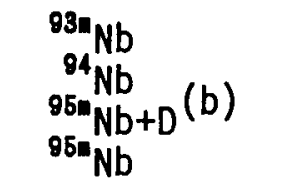 & $\begin{array}{l}7.2 \mathrm{E}-07 \\
5.3 \mathrm{E}-04 \\
1.6 \mathrm{E}-04 \\
2.4 \mathrm{E}-05\end{array}$ & $\begin{array}{l}5.8 \mathrm{E}-07 \\
7.4 \mathrm{E}-06 \\
7.8 \mathrm{E}-08 \\
4.8 \mathrm{E}-08\end{array}$ & $\begin{array}{l}1.0 \mathrm{E}-06 \\
1.4 \mathrm{E}-05 \\
4.8 \mathrm{E}-06 \\
4.6 \mathrm{E}-06\end{array}$ & $\begin{array}{l}2.3 \mathrm{E}-06 \\
5.5 \mathrm{E}-04 \\
1.6 \mathrm{E}-04 \\
2.8 \mathrm{E}-05\end{array}$ & $\begin{array}{l}\text { 8.6E-04 } \\
3.2 \mathrm{E}-01 \\
9.7 \mathrm{E}-02 \\
1.5 \mathrm{E}-02\end{array}$ \\
\hline
\end{tabular}


TABLE 3.2. Annual Total Effective Dose Equivalent Factors for the Building Occupancy (Surface Activity) Scenario(a) (contd)

\begin{tabular}{|c|c|c|c|c|c|}
\hline Radionuclide & $\begin{array}{c}\text { External } \\
\text { (mrem) }\end{array}$ & $\begin{array}{c}\text { Inhalation } \\
\text { (mrem) }\end{array}$ & $\begin{array}{l}\text { Secondary } \\
\text { Ingestion } \\
\text { (mrem) }\end{array}$ & $\begin{array}{c}\text { TEDE } \\
\text { (mrem) }\end{array}$ & $\begin{array}{l}\text { TEDE } \\
(\mu \mathrm{SV}) \\
\end{array}$ \\
\hline $\begin{array}{l}{ }_{95}^{95} \mathrm{Nb} \\
{ }_{97} \\
977_{N b} \\
97 \\
{ }_{N b}\end{array}$ & $\begin{array}{l}2.9 \mathrm{E}-04 \\
2.3 \mathrm{E}-04 \\
2.3 \mathrm{E}-04 \\
2.4 \mathrm{E}-04\end{array}$ & $\begin{array}{l}1.1 \mathrm{E}-07 \\
8.4 \mathrm{E}-10 \\
2.4 \mathrm{E}-11 \\
1.7 \mathrm{E}-09\end{array}$ & $\begin{array}{l}5.0 \mathrm{E}-06 \\
2.4 \mathrm{E}-07 \\
7.8 \mathrm{E}-09 \\
4.8 \mathrm{E}-07\end{array}$ & $\begin{array}{l}3.0 \mathrm{E}-04 \\
2.3 \mathrm{E}-04 \\
2.3 \mathrm{E}-04 \\
2.4 \mathrm{E}-04\end{array}$ & $\begin{array}{l}1.8 \mathrm{E}-01 \\
1.4 \mathrm{E}-01 \\
1.4 \mathrm{E}-01 \\
1.4 \mathrm{E}-01\end{array}$ \\
\hline $\begin{array}{l}{ }^{93} \mathrm{Mo}+\mathrm{D} \\
{ }^{93} \mathrm{Mo} \\
{ }^{99} \mathrm{Mo}+\mathrm{b} \\
{ }^{99} \mathrm{Mo}\end{array}$ & $\begin{array}{l}2.0 \mathrm{E}-06 \\
3.5 \mathrm{E}-06 \\
4.5 \mathrm{E}-05 \\
5.1 \mathrm{E}-05\end{array}$ & $\begin{array}{l}3.0 \mathrm{E}-07 \\
1.9 \mathrm{E}-08 \\
2.1 \mathrm{E}-08 \\
3.8 \mathrm{E}-08\end{array}$ & $\begin{array}{l}1.8 \mathrm{E}-06 \\
2.8 \mathrm{E}-06 \\
3.3 \mathrm{E}-06 \\
6.2 \mathrm{E}-06\end{array}$ & $\begin{array}{l}4.1 \mathrm{E}-06 \\
6.3 \mathrm{E}-06 \\
4.8 \mathrm{E}-05 \\
5.8 \mathrm{E}-05\end{array}$ & $\begin{array}{l}1.8 \mathrm{E}-03 \\
2.8 \mathrm{E}-03 \\
2.8 \mathrm{E}-02 \\
3.3 \mathrm{E}-02\end{array}$ \\
\hline $\begin{array}{l}{ }^{99 m} \mathrm{TC} \\
{ }^{99} \mathrm{TC} \\
{ }^{101} \mathrm{TC}\end{array}$ & $\begin{array}{l}4.1 \mathrm{E}-05 \\
3.8 \mathrm{E}-08 \\
1.3 \mathrm{E}-04\end{array}$ & $\begin{array}{l}6.2 \mathrm{E}-10 \\
1.8 \mathrm{E}-07 \\
3.0 \mathrm{E}-10\end{array}$ & $\begin{array}{l}1.7 \mathrm{E}-07 \\
6.2 \mathrm{E}-06 \\
1.5 \mathrm{E}-07\end{array}$ & $\begin{array}{l}4.1 \mathrm{E}-05 \\
6.4 \mathrm{E}-06 \\
1.3 \mathrm{E}-04\end{array}$ & $\begin{array}{l}2.4 \mathrm{E}-02 \\
1.7 \mathrm{E}-03 \\
7.9 \mathrm{E}-02\end{array}$ \\
\hline $\begin{array}{l}{ }^{103} \mathrm{Ru+D}(\mathrm{b}) \\
{ }_{103} \mathrm{Ru} \\
{ }^{105} \mathrm{Ru}+\mathrm{D}(\mathrm{b}) \\
{ }^{105} \mathrm{Ru+I}(\mathrm{c}) \\
{ }^{106} \mathrm{Ru+I}(\mathrm{c})\end{array}$ & $\begin{array}{l}8.1 \mathrm{E}-05 \\
1.6 \mathrm{E}-04 \\
9.9 \mathrm{E}-05 \\
2.8 \mathrm{E}-04 \\
7.8 \mathrm{E}-05\end{array}$ & $\begin{array}{l}\text { 8.7E-08 } \\
1.7 \mathrm{E}-07 \\
1.0 \mathrm{E}-08 \\
1.0 \mathrm{E}-08 \\
4.5 \mathrm{E}-06\end{array}$ & $\begin{array}{l}3.0 \mathrm{E}-06 \\
6.0 \mathrm{E}-06 \\
1.8 \mathrm{E}-06 \\
2.6 \mathrm{E}-06 \\
2.6 \mathrm{E}-05\end{array}$ & $\begin{array}{l}8.4 \mathrm{E}-05 \\
1.7 \mathrm{E}-04 \\
1.0 \mathrm{E}-04 \\
2.8 \mathrm{E}-04 \\
1.1 \mathrm{E}-04\end{array}$ & $\begin{array}{l}5.0 \mathrm{E}-02 \\
1.0 \mathrm{E}-01 \\
6.0 \mathrm{E}-02 \\
1.7 \mathrm{E}-01 \\
5.5 \mathrm{E}-02\end{array}$ \\
\hline $\begin{array}{l}{ }^{103 m} \mathrm{Rh} \\
{ }^{105} \mathrm{Rh} \\
\mathrm{Rh}\end{array}$ & $\begin{array}{l}1.2 E-06 \\
3.0 E-05\end{array}$ & $\begin{array}{l}9.6 \mathrm{E}-11 \\
1.9 \mathrm{E}-08\end{array}$ & $\begin{array}{l}2.4 \mathrm{E}-08 \\
3.0 \mathrm{E}-06\end{array}$ & $\begin{array}{l}1.2 E-06 \\
3.3 E-05\end{array}$ & $\begin{array}{l}7.4 \mathrm{E}-04 \\
1.9 \mathrm{E}-02\end{array}$ \\
\hline $\begin{array}{l}{ }^{163} \mathrm{Pd}+\mathrm{D}(\mathrm{b}) \\
{ }_{103} \mathrm{Pd} \\
{ }^{167} \mathrm{Pd} \\
{ }^{169} \mathrm{Pd}+\mathrm{I}\end{array}$ & $\begin{array}{l}5.1 \mathrm{E}-06 \\
9.4 \mathrm{E}-06 \\
4.9 \mathrm{E}-11 \\
6.4 \mathrm{E}-06\end{array}$ & $\begin{array}{l}1.5 \mathrm{E}-08 \\
3.0 \mathrm{E}-08 \\
2.4 \mathrm{E}-07 \\
1.3 \mathrm{E}-08\end{array}$ & $\begin{array}{l}7.9 \mathrm{E}-07 \\
1.6 \mathrm{E}-06 \\
3.0 \mathrm{E}-07 \\
2.6 \mathrm{E}-06\end{array}$ & $\begin{array}{l}5.9 \mathrm{E}-06 \\
1.1 \mathrm{E}-05 \\
5.4 \mathrm{E}-07 \\
9.0 \mathrm{E}-06\end{array}$ & $\begin{array}{l}3.3 \mathrm{E}-03 \\
6.0 \mathrm{E}-03 \\
1.5 \mathrm{E}-04 \\
4.5 \mathrm{E}-03\end{array}$ \\
\hline${ }^{116 \mathrm{~m}} \mathrm{Ag}+\mathrm{I}(\mathrm{c})$ & $\begin{array}{l}9.7 E-04 \\
1.0 E-05\end{array}$ & $\begin{array}{l}8.2 \mathrm{E}-07 \\
6.6 \mathrm{E}-08\end{array}$ & $\begin{array}{l}3.3 \mathrm{E}-05 \\
1.0 \mathrm{E}-05\end{array}$ & $\begin{array}{l}1.0 \mathrm{E}-03 \\
2.0 \mathrm{E}-05\end{array}$ & $\begin{array}{l}5.9 \mathrm{E}-01 \\
8.8 \mathrm{E}-03\end{array}$ \\
\hline $\begin{array}{l}{ }^{169} \mathrm{Cd} \\
{ }_{1131} \mathrm{Cd} \\
{ }_{1161} \mathrm{Cd}+\mathrm{D} \\
{ }^{115 n} \mathrm{Cd} \\
{ }^{115} \mathrm{Cd}\end{array}$ & $\begin{array}{l}8.8 \mathrm{E}-06 \\
1.6 \mathrm{E}-07 \\
4.5 \mathrm{E}-06 \\
9.2 \mathrm{E}-06 \\
6.6 \mathrm{E}-05\end{array}$ & $\begin{array}{l}2.2 \mathrm{E}-06 \\
2.8 \mathrm{E}-05 \\
6.9 \mathrm{E}-07 \\
1.4 \mathrm{E}-06 \\
7.8 \mathrm{E}-08\end{array}$ & $\begin{array}{l}2.6 \mathrm{E}-05 \\
3.2 \mathrm{E}-04 \\
1.6 \mathrm{E}-05 \\
3.2 \mathrm{E}-05 \\
1.2 \mathrm{E}-05\end{array}$ & $\begin{array}{l}3.7 \mathrm{E}-05 \\
3.5 \mathrm{E}-04 \\
2.1 \mathrm{E}-05 \\
4.3 \mathrm{E}-05 \\
7.8 \mathrm{E}-05\end{array}$ & $\begin{array}{l}1.3 \mathrm{E}-02 \\
9.4 \mathrm{E}-02 \\
7.2 \mathrm{E}-03 \\
1.5 \mathrm{E}-02 \\
4.3 \mathrm{E}-02\end{array}$ \\
\hline $\begin{array}{l}{ }_{111}^{111} \text { In } \\
{ }_{116 n} \text { In+I } \\
{ }^{11 n}\end{array}$ & $\begin{array}{l}1.3 \mathrm{E}-04 \\
4.8 \mathrm{E}-05 \\
6.2 \mathrm{E}-05\end{array}$ & $\begin{array}{l}1.1 \mathrm{E}-08 \\
4.5 \mathrm{E}-07 \\
2.4 \mathrm{E}-09\end{array}$ & $\begin{array}{l}2.6 \mathrm{E}-06 \\
1.5 \mathrm{E}-05 \\
7.0 \mathrm{E}-07\end{array}$ & $\begin{array}{l}1.3 \mathrm{E}-04 \\
6.3 \mathrm{E}-05 \\
6.3 \mathrm{E}-05\end{array}$ & $\begin{array}{l}8.0 \mathrm{E}-02 \\
3.3 \mathrm{E}-02 \\
3.7 \mathrm{E}-02\end{array}$ \\
\hline 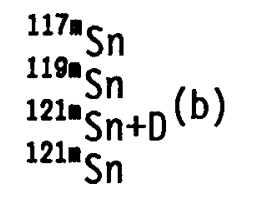 & $\begin{array}{l}4.9 E-05 \\
6.3 E-06 \\
0.0 E+00 \\
0.0 E+00\end{array}$ & $\begin{array}{l}8.4 \mathrm{E}-08 \\
1.2 \mathrm{E}-07 \\
1.2 \mathrm{E}-07 \\
2.2 \mathrm{E}-07\end{array}$ & $\begin{array}{l}5.8 E-06 \\
2.8 E-06 \\
2.5 E-06 \\
3.0 E-06\end{array}$ & $\begin{array}{l}5.5 E-05 \\
9.3 E-06 \\
2.6 E-06 \\
3.2 E-06\end{array}$ & $\begin{array}{l}3.1 \mathrm{E}-02 \\
4.6 \mathrm{E}-03 \\
7.0 \mathrm{E}-04 \\
8.7 \mathrm{E}-04\end{array}$ \\
\hline
\end{tabular}


TABLE 3.2. Annual Total Effective Dose Equivalent Factors for the Building Occupancy (Surface Activity) Scenario(a) (contd)

\begin{tabular}{|c|c|c|c|c|c|}
\hline Radionuclide & $\begin{array}{c}\text { External } \\
\text { (mrem) } \\
\end{array}$ & $\begin{array}{c}\text { Inhalation } \\
\text { (mrem) } \\
\end{array}$ & $\begin{array}{l}\text { Secondary } \\
\text { Ingestion } \\
\text { (mrem) }\end{array}$ & $\begin{array}{c}\text { TEDE } \\
\text { (mrem) }\end{array}$ & $\begin{array}{l}\text { TEDE } \\
(\mu \mathrm{Sv})\end{array}$ \\
\hline $\begin{array}{l}{ }^{121} S n \\
{ }^{123} S n \\
{ }^{125} S n+D \\
{ }^{125} S n \\
{ }^{126} S_{S n+D}(b) \\
{ }^{126} S n\end{array}$ & $\begin{array}{l}0.0 \mathrm{E}+00 \\
3.3 \mathrm{E}-06 \\
1.2 \mathrm{E}-04 \\
1.1 \mathrm{E}-04 \\
3.3 \mathrm{E}-04 \\
1.7 \mathrm{E}-05\end{array}$ & $\begin{array}{l}9.6 \mathrm{E}-09 \\
6.2 \mathrm{E}-07 \\
2.6 \mathrm{E}-07 \\
3.0 \mathrm{E}-07 \\
9.0 \mathrm{E}-07 \\
1.9 \mathrm{E}-06\end{array}$ & $\begin{array}{l}1.8 \mathrm{E}-06 \\
1.7 \mathrm{E}-05 \\
1.4 \mathrm{E}-05 \\
2.4 \mathrm{E}-05 \\
2.0 \mathrm{E}-05 \\
3.8 \mathrm{E}-05\end{array}$ & $\begin{array}{l}1.8 \mathrm{E}-06 \\
2.1 \mathrm{E}-05 \\
1.4 \mathrm{E}-04 \\
1.3 \mathrm{E}-04 \\
3.5 \mathrm{E}-04 \\
5.7 \mathrm{E}-05\end{array}$ & $\begin{array}{l}4.9 \mathrm{E}-04 \\
6.7 \mathrm{E}-03 \\
7.7 \mathrm{E}-02 \\
7.0 \mathrm{E}-02 \\
2.0 \mathrm{E}-01 \\
2.1 \mathrm{E}-02\end{array}$ \\
\hline $\begin{array}{l}{ }^{124} \mathrm{Sb} \\
{ }^{125} \mathrm{Sb}+\mathrm{D} \\
{ }^{125} \mathrm{Sb} \\
{ }^{126 m} \mathrm{Sb} \\
{ }^{126 m} \mathrm{Sb} \\
{ }^{126} \mathrm{Sb} \\
{ }^{127} \mathrm{Sb} \\
{ }^{127} \mathrm{Sb}+\mathrm{Db}\end{array}$ & $\begin{array}{l}6.7 \mathrm{E}-04 \\
1.3 \mathrm{E}-04 \\
1.6 \mathrm{E}-04 \\
5.9 \mathrm{E}-04 \\
5.7 \mathrm{E}-04 \\
9.6 \mathrm{E}-04 \\
1.1 \mathrm{E}-04 \\
2.3 \mathrm{E}-04\end{array}$ & $\begin{array}{l}5.0 \mathrm{E}-07 \\
2.1 \mathrm{E}-07 \\
2.4 \mathrm{E}-07 \\
3.0 \mathrm{E}-08 \\
5.8 \mathrm{E}-10 \\
2.2 \mathrm{E}-07 \\
8.9 \mathrm{E}-08 \\
1.2 \mathrm{E}-07\end{array}$ & $\begin{array}{l}2.0 \mathrm{E}-05 \\
5.8 \mathrm{E}-06 \\
5.6 \mathrm{E}-06 \\
2.6 \mathrm{E}-06 \\
1.8 \mathrm{E}-07 \\
2.0 \mathrm{E}-05 \\
8.6 \mathrm{E}-06 \\
1.4 \mathrm{E}-05\end{array}$ & $\begin{array}{l}6.9 \mathrm{E}-04 \\
1.4 \mathrm{E}-04 \\
1.7 \mathrm{E}-04 \\
6.0 \mathrm{E}-04 \\
5.7 \mathrm{E}-04 \\
9.8 \mathrm{E}-04 \\
1.1 \mathrm{E}-04 \\
2.5 \mathrm{E}-04\end{array}$ & $\begin{array}{l}4.1 \mathrm{E}-01 \\
8.1 \mathrm{E}-02 \\
1.0 \mathrm{E}-01 \\
3.6 \mathrm{E}-01 \\
3.4 \mathrm{E}-01 \\
5.8 \mathrm{E}-01 \\
6.6 \mathrm{E}-02 \\
1.4 \mathrm{E}-01\end{array}$ \\
\hline 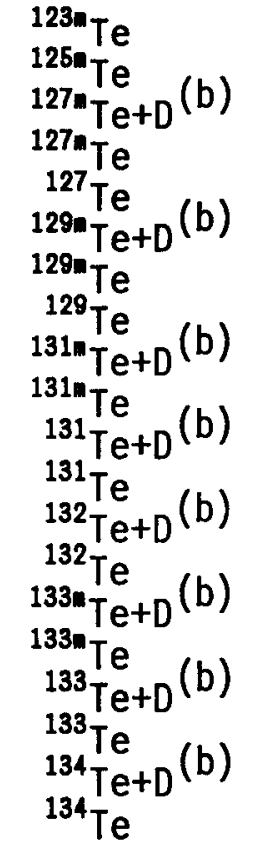 & $\begin{array}{l}4.5 \mathrm{E}-05 \\
1.7 \mathrm{E}-05 \\
3.8 \mathrm{E}-06 \\
5.5 \mathrm{E}-06 \\
2.1 \mathrm{E}-06 \\
1.7 \mathrm{E}-05 \\
1.5 \mathrm{E}-05 \\
2.2 \mathrm{E}-05 \\
3.0 \mathrm{E}-04 \\
5.2 \mathrm{E}-04 \\
1.3 \mathrm{E}-04 \\
1.5 \mathrm{E}-04 \\
4.4 \mathrm{E}-04 \\
8.7 \mathrm{E}-05 \\
3.3 \mathrm{E}-04 \\
8.0 \mathrm{E}-04 \\
1.7 \mathrm{E}-04 \\
3.4 \mathrm{E}-04 \\
5.9 \mathrm{E}-04 \\
3.2 \mathrm{E}-04\end{array}$ & $\begin{array}{l}2.0 \mathrm{E}-07 \\
1.4 \mathrm{E}-07 \\
2.1 \mathrm{E}-07 \\
4.2 \mathrm{E}-07 \\
6.4 \mathrm{E}-09 \\
5.9 \mathrm{E}-06 \\
9.6 \mathrm{E}-06 \\
3.0 \mathrm{E}-09 \\
3.8 \mathrm{E}-07 \\
2.4 \mathrm{E}-07 \\
3.0 \mathrm{E}-07 \\
2.4 \mathrm{E}-09 \\
1.3 \mathrm{E}-07 \\
2.4 \mathrm{E}-07 \\
3.7 \mathrm{E}-08 \\
8.6 \mathrm{E}-09 \\
3.6 \mathrm{E}-08 \\
1.7 \mathrm{E}-09 \\
2.4 \mathrm{E}-09 \\
2.0 \mathrm{E}-09\end{array}$ & $\begin{array}{l}1.1 \mathrm{E}-05 \\
7.4 \mathrm{E}-06 \\
9.0 \mathrm{E}-06 \\
1.6 \mathrm{E}-05 \\
1.4 \mathrm{E}-06 \\
6.6 \mathrm{E}-04 \\
1.1 \mathrm{E}-03 \\
5.2 \mathrm{E}-07 \\
6.0 \mathrm{E}-05 \\
3.4 \mathrm{E}-05 \\
5.0 \mathrm{E}-05 \\
4.2 \mathrm{E}-07 \\
1.5 \mathrm{E}-05 \\
3.0 \mathrm{E}-05 \\
7.0 \mathrm{E}-06 \\
1.6 \mathrm{E}-06 \\
6.5 \mathrm{E}-06 \\
3.2 \mathrm{E}-07 \\
4.5 \mathrm{E}-07 \\
3.8 \mathrm{E}-07\end{array}$ & $\begin{array}{l}5.7 \mathrm{E}-05 \\
2.5 \mathrm{E}-05 \\
1.3 \mathrm{E}-05 \\
2.2 \mathrm{E}-05 \\
3.5 \mathrm{E}-06 \\
6.9 \mathrm{E}-04 \\
1.1 \mathrm{E}-03 \\
2.3 \mathrm{E}-05 \\
3.6 \mathrm{E}-04 \\
5.5 \mathrm{E}-04 \\
1.8 \mathrm{E}-04 \\
1.5 \mathrm{E}-04 \\
4.5 \mathrm{E}-04 \\
1.2 \mathrm{E}-04 \\
3.3 \mathrm{E}-04 \\
8.0 \mathrm{E}-04 \\
1.8 \mathrm{E}-04 \\
3.4 \mathrm{E}-04 \\
6.0 \mathrm{E}-04 \\
3.2 \mathrm{E}-04\end{array}$ & $\begin{array}{l}3.0 \mathrm{E}-02 \\
1.2 \mathrm{E}-02 \\
4.7 \mathrm{E}-03 \\
7.9 \mathrm{E}-03 \\
1.6 \mathrm{E}-03 \\
1.9 \mathrm{E}-01 \\
3.0 \mathrm{E}-01 \\
1.3 \mathrm{E}-02 \\
1.9 \mathrm{E}-01 \\
3.2 \mathrm{E}-01 \\
9.4 \mathrm{E}-02 \\
8.9 \mathrm{E}-02 \\
2.7 \mathrm{E}-01 \\
6.0 \mathrm{E}-02 \\
2.0 \mathrm{E}-01 \\
4.8 \mathrm{E}-01 \\
1.1 \mathrm{E}-01 \\
2.0 \mathrm{E}-01 \\
3.6 \mathrm{E}-01 \\
1.9 \mathrm{E}-01\end{array}$ \\
\hline $\begin{array}{l}{ }^{125} \mathrm{I} \\
{ }^{129} \mathrm{I} \\
{ }^{130} \mathrm{I} \\
{ }^{131} \mathrm{I}+\mathrm{D} \\
{ }^{131} \mathrm{I} \\
{ }_{1}^{132} \mathrm{~b} \\
{ }^{133} \mathrm{I} \mathrm{I}+\mathrm{D} \\
{ }^{133} \mathrm{I}\end{array}$ & $\begin{array}{l}2.0 \mathrm{E}-05 \\
1.1 \mathrm{E}-05 \\
7.2 \mathrm{E}-04 \\
1.2 \mathrm{E}-04 \\
1.3 \mathrm{E}-04 \\
8.2 \mathrm{E}-04 \\
1.0 \mathrm{E}-04 \\
1.9 \mathrm{E}-04\end{array}$ & $\begin{array}{l}4.4 \mathrm{E}-07 \\
3.0 \mathrm{E}-06 \\
4.8 \mathrm{E}-08 \\
5.9 \mathrm{E}-07 \\
6.0 \mathrm{E}-07 \\
7.6 \mathrm{E}-09 \\
5.2 \mathrm{E}-08 \\
1.1 \mathrm{E}-07\end{array}$ & $\begin{array}{l}7.2 \mathrm{E}-05 \\
5.0 \mathrm{E}-04 \\
8.8 \mathrm{E}-06 \\
9.9 \mathrm{E}-05 \\
1.0 \mathrm{E}-04 \\
1.4 \mathrm{E}-06 \\
9.6 \mathrm{E}-06 \\
1.9 \mathrm{E}-05\end{array}$ & $\begin{array}{l}9.3 \mathrm{E}-05 \\
5.1 \mathrm{E}-04 \\
7.3 \mathrm{E}-04 \\
2.2 \mathrm{E}-04 \\
2.3 \mathrm{E}-04 \\
8.2 \mathrm{E}-04 \\
1.1 \mathrm{E}-04 \\
2.1 \mathrm{E}-04\end{array}$ & $\begin{array}{l}3.2 \mathrm{E}-02 \\
1.4 \mathrm{E}-01 \\
4.3 \mathrm{E}-01 \\
1.0 \mathrm{E}-01 \\
1.0 \mathrm{E}-01 \\
4.9 \mathrm{E}-01 \\
6.3 \mathrm{E}-02 \\
1.2 \mathrm{E}-01\end{array}$ \\
\hline
\end{tabular}


TABLE 3.2. Annual Total Effective Dose Equivalent Factors for the Building Occupancy (Surface Activity) Scenario(a) (contd)

\begin{tabular}{|c|c|c|c|c|c|}
\hline Radionuclide & $\begin{array}{c}\text { External } \\
\text { (mrem) } \\
\end{array}$ & $\begin{array}{c}\text { Inhalation } \\
\text { (mrem) } \\
\end{array}$ & $\begin{array}{l}\text { Secondary } \\
\text { Ingestion } \\
\text { (mrem) } \\
\end{array}$ & $\begin{array}{c}\text { TEDE } \\
\text { (mrem) }\end{array}$ & $\begin{array}{l}\text { TEDE } \\
(\mu S v)\end{array}$ \\
\hline${ }^{135}{ }_{I}^{134} \mathrm{I}+\mathrm{D}$ & $\begin{array}{l}9.1 \mathrm{E}-04 \\
2.9 \mathrm{E}-04 \\
5.4 \mathrm{E}-04\end{array}$ & $\begin{array}{l}2.8 \mathrm{E}-09 \\
1.0 \mathrm{E}-08 \\
2.2 \mathrm{E}-08\end{array}$ & $\begin{array}{l}5.2 E-07 \\
1.9 E-06 \\
4.2 E-06\end{array}$ & $\begin{array}{l}9.1 \mathrm{E}-04 \\
2.9 \mathrm{E}-04 \\
5.5 \mathrm{E}-04\end{array}$ & $\begin{array}{l}5.5 E-01 \\
1.7 E-01 \\
3.3 E-01\end{array}$ \\
\hline $\begin{array}{l}{ }^{134 m} \mathrm{Cs}+\mathrm{D} \\
\left.{ }^{134} \mathrm{CS}\right) \\
{ }^{134} \mathrm{Cs} \\
{ }^{135} \mathrm{Cs} \\
{ }^{136} \mathrm{Cs} \\
{ }^{137} \mathrm{Cs} \quad(\mathrm{Cs}) \\
{ }^{138} \mathrm{Cs}\end{array}$ & $\begin{array}{l}5.5 \mathrm{E}-06 \\
1.1 \mathrm{E}-05 \\
5.9 \mathrm{E}-04 \\
1.5 \mathrm{E}-08 \\
7.5 \mathrm{E}-04 \\
2.0 \mathrm{E}-04 \\
8.1 \mathrm{E}-04\end{array}$ & $\begin{array}{l}3.6 \mathrm{E}-10 \\
7.2 \mathrm{E}-10 \\
8.0 \mathrm{E}-07 \\
7.2 \mathrm{E}-08 \\
1.3 \mathrm{E}-07 \\
2.7 \mathrm{E}-07 \\
1.9 \mathrm{E}-09\end{array}$ & $\begin{array}{l}3.6 \mathrm{E}-08 \\
7.2 \mathrm{E}-08 \\
1.3 \mathrm{E}-04 \\
1.3 \mathrm{E}-05 \\
2.0 \mathrm{E}-05 \\
4.7 \mathrm{E}-05 \\
3.8 \mathrm{E}-07\end{array}$ & $\begin{array}{l}5.5 \mathrm{E}-06 \\
1.1 \mathrm{E}-05 \\
7.3 \mathrm{E}-04 \\
1.3 \mathrm{E}-05 \\
7.7 \mathrm{E}-04 \\
2.5 \mathrm{E}-04 \\
8.1 \mathrm{E}-04\end{array}$ & $\begin{array}{l}3.3 \mathrm{E}-03 \\
6.8 \mathrm{E}-03 \\
3.9 \mathrm{E}-01 \\
3.5 \mathrm{E}-03 \\
4.5 \mathrm{E}-01 \\
1.3 \mathrm{E}-01 \\
4.9 \mathrm{E}-01\end{array}$ \\
\hline $\begin{array}{l}{ }^{139} \mathrm{Ba} \\
{ }^{148} \mathrm{Ba}+\mathrm{D} \\
{ }^{148} \mathrm{Ba} \\
\left.{ }^{141} \mathrm{Ba}+\mathrm{b}\right) \\
{ }^{141} \mathrm{Ba} \\
\left.{ }^{142} \mathrm{Ba}+\mathrm{b}\right) \\
{ }^{142} \mathrm{Ba}\end{array}$ & $\begin{array}{l}1.3 \mathrm{E}-05 \\
4.0 \mathrm{E}-04 \\
6.3 \mathrm{E}-05 \\
1.1 \mathrm{E}-04 \\
3.0 \mathrm{E}-04 \\
5.7 \mathrm{E}-04 \\
3.2 \mathrm{E}-04\end{array}$ & $\begin{array}{l}3.4 \mathrm{E}-09 \\
6.4 \mathrm{E}-08 \\
5.8 \mathrm{E}-08 \\
6.0 \mathrm{E}-08 \\
1.6 \mathrm{E}-09 \\
3.0 \mathrm{E}-09 \\
7.8 \mathrm{E}-10\end{array}$ & $\begin{array}{l}8.2 E-07 \\
1.7 E-05 \\
1.8 E-05 \\
3.1 E-06 \\
4.2 E-07 \\
8.1 E-07 \\
2.2 E-07\end{array}$ & $\begin{array}{l}1.4 \mathrm{E}-05 \\
4.2 \mathrm{E}-04 \\
8.2 \mathrm{E}-05 \\
1.2 \mathrm{E}-04 \\
3.0 \mathrm{E}-04 \\
5.8 \mathrm{E}-04 \\
3.2 \mathrm{E}-04\end{array}$ & $\begin{array}{l}8.1 \mathrm{E}-03 \\
2.4 \mathrm{E}-01 \\
4.3 \mathrm{E}-02 \\
6.8 \mathrm{E}-02 \\
1.8 \mathrm{E}-01 \\
3.4 \mathrm{E}-01 \\
1.9 \mathrm{E}-01\end{array}$ \\
\hline $\begin{array}{l}{ }^{140} \mathrm{La} \\
{ }_{141} \mathrm{La}+D \\
141 \mathrm{La} \\
142 \mathrm{La}\end{array}$ & $\begin{array}{l}7.6 \mathrm{E}-04 \\
2.1 \mathrm{E}-05 \\
1.9 \mathrm{E}-05 \\
8.8 \mathrm{E}-04\end{array}$ & $\begin{array}{l}6.8 \mathrm{E}-08 \\
9.0 \mathrm{E}-08 \\
1.2 \mathrm{E}-08 \\
5.2 \mathrm{E}-09\end{array}$ & $\begin{array}{l}1.7 \mathrm{E}-05 \\
4.4 \mathrm{E}-06 \\
3.0 \mathrm{E}-06 \\
1.4 \mathrm{E}-06\end{array}$ & $\begin{array}{l}7.7 E-04 \\
2.5 E-05 \\
2.2 E-05 \\
8.8 E-04\end{array}$ & $\begin{array}{l}4.6 \mathrm{E}-01 \\
1.4 \mathrm{E}-02 \\
1.2 \mathrm{E}-02 \\
5.3 \mathrm{E}-01\end{array}$ \\
\hline $\begin{array}{l}{ }^{141} \mathrm{Ce} \\
{ }^{143} \mathrm{Ce}+\mathrm{D} \\
{ }^{143} \mathrm{Ce} \\
\left.{ }^{144} \mathrm{Ce}+\mathrm{D}\right) \\
{ }^{144} \mathrm{Ce}\end{array}$ & $\begin{array}{l}2.5 E-05 \\
4.3 E-05 \\
8.9 E-05 \\
1.1 E-05 \\
6.7 E-06\end{array}$ & $\begin{array}{l}1.7 \mathrm{E}-07 \\
1.1 \mathrm{E}-07 \\
6.8 \mathrm{E}-08 \\
3.6 \mathrm{E}-06 \\
7.2 \mathrm{E}-06\end{array}$ & $\begin{array}{l}5.8 E-06 \\
9.3 E-06 \\
9.2 E-06 \\
2.1 E-05 \\
4.2 E-05\end{array}$ & $\begin{array}{l}3.1 \mathrm{E}-05 \\
5.3 \mathrm{E}-05 \\
9.9 \mathrm{E}-05 \\
3.5 \mathrm{E}-05 \\
5.6 \mathrm{E}-05\end{array}$ & $\begin{array}{l}1.6 \mathrm{E}-02 \\
2.8 \mathrm{E}-02 \\
5.6 \mathrm{E}-02 \\
1.3 \mathrm{E}-02 \\
1.7 \mathrm{E}-02\end{array}$ \\
\hline $\begin{array}{l}{ }_{143}^{143} \mathrm{Pr} \\
{ }_{144 m} \mathrm{Pr}+\mathrm{D} \\
{ }^{144} \mathrm{Pr} \\
\mathrm{Pr}\end{array}$ & $\begin{array}{l}4.3 E-07 \\
1.0 E-05 \\
5.1 E-06 \\
1.6 E-05\end{array}$ & $\begin{array}{l}1.6 \mathrm{E}-07 \\
6.2 \mathrm{E}-10 \\
3.6 \mathrm{E}-10 \\
8.6 \mathrm{E}-10\end{array}$ & $\begin{array}{l}9.4 \mathrm{E}-06 \\
1.7 \mathrm{E}-07 \\
9.6 \mathrm{E}-08 \\
2.4 \mathrm{E}-07\end{array}$ & $\begin{array}{l}1.0 \mathrm{E}-05 \\
1.0 \mathrm{E}-05 \\
5.2 \mathrm{E}-06 \\
1.6 \mathrm{E}-05\end{array}$ & $\begin{array}{l}2.8 E-03 \\
6.1 E-03 \\
3.1 E-03 \\
9.4 E-03\end{array}$ \\
\hline $\begin{array}{l}{ }^{147} \mathrm{Nd}+\mathrm{D} \\
{ }^{147} \mathrm{Nd}\end{array}$ & $\begin{array}{l}2.2 \mathrm{E}-05 \\
4.6 \mathrm{E}-05\end{array}$ & $\begin{array}{l}4.4 \mathrm{E}-07 \\
1.3 \mathrm{E}-07\end{array}$ & $\begin{array}{l}5.2 E-06 \\
8.4 E-06\end{array}$ & $\begin{array}{l}2.8 E-05 \\
5.5 E-05\end{array}$ & $\begin{array}{l}1.5 E-02 \\
3.0 E-02\end{array}$ \\
\hline $\begin{array}{l}{ }^{147} \mathrm{Pm} \\
{ }_{148 m}{ }_{\mathrm{Pm}+\mathrm{D}}(\mathrm{b}) \\
{ }^{148} \mathrm{~m} \mathrm{Pm} \\
{ }^{148} \mathrm{Pm} \\
{ }^{149} \mathrm{Pm} \\
{ }^{151} \mathrm{Pm}\end{array}$ & $\begin{array}{l}2.1 \mathrm{E}-08 \\
7.1 \mathrm{E}-04 \\
7.6 \mathrm{E}-04 \\
2.1 \mathrm{E}-04 \\
4.2 \mathrm{E}-06 \\
1.2 \mathrm{E}-04\end{array}$ & $\begin{array}{l}7.6 \mathrm{E}-07 \\
4.2 \mathrm{E}-07 \\
4.4 \mathrm{E}-07 \\
2.2 \mathrm{E}-07 \\
5.8 \mathrm{E}-08 \\
3.6 \mathrm{E}-08\end{array}$ & $\begin{array}{l}2.0 \mathrm{E}-06 \\
1.6 \mathrm{E}-05 \\
1.5 \mathrm{E}-05 \\
2.2 \mathrm{E}-05 \\
8.2 \mathrm{E}-06 \\
6.0 \mathrm{E}-06\end{array}$ & $\begin{array}{l}2.8 \mathrm{E}-06 \\
7.3 \mathrm{E}-04 \\
7.7 \mathrm{E}-04 \\
2.3 \mathrm{E}-04 \\
1.2 \mathrm{E}-05 \\
1.2 \mathrm{E}-04\end{array}$ & $\begin{array}{l}7.6 \mathrm{E}-04 \\
4.3 \mathrm{E}-01 \\
4.6 \mathrm{E}-01 \\
1.3 \mathrm{E}-01 \\
4.8 \mathrm{E}-03 \\
7.3 \mathrm{E}-02\end{array}$ \\
\hline
\end{tabular}


TABLE 3.2. Annual Total Effective Dose Equivalent Factors for the Building Occupancy (Surface Activity) Scenario(a) (contd)

\begin{tabular}{|c|c|c|c|c|c|}
\hline Zadionuclide & $\begin{array}{c}\text { External } \\
\text { (mrem) } \\
\end{array}$ & $\begin{array}{l}\text { Inhalation } \\
\text { (mrem) }\end{array}$ & $\begin{array}{l}\text { Secondary } \\
\text { Ingestion } \\
\text { (mrem) } \\
\end{array}$ & $\begin{array}{c}\text { TEDE } \\
\text { (mrem) }\end{array}$ & $\begin{array}{l}\text { TEDE } \\
(\mu \mathrm{SV}) \\
\end{array}$ \\
\hline $\begin{array}{l}{ }^{147} \mathrm{Sm} \\
{ }^{153} \mathrm{Sm} \\
{ }^{15 m}\end{array}$ & $\begin{array}{l}0.0 E+00 \\
1.1 E-08 \\
2.5 E-05\end{array}$ & $\begin{array}{l}1.5 \mathrm{E}-03 \\
5.8 \mathrm{E}-07 \\
3.8 \mathrm{E}-08\end{array}$ & $\begin{array}{l}3.8 \mathrm{E}-04 \\
7.6 \mathrm{E}-07 \\
6.0 \mathrm{E}-06\end{array}$ & $\begin{array}{l}1.8 E-03 \\
1.4 E-06 \\
3.1 E-05\end{array}$ & $\begin{array}{l}5.0 \mathrm{E}-01 \\
3.7 \mathrm{E}-04 \\
1.6 \mathrm{E}-02\end{array}$ \\
\hline $\begin{array}{l}{ }^{152} \mathrm{Eu} \\
{ }^{154} \mathrm{Eu} \\
155 \mathrm{Eu} \\
{ }^{158} \mathrm{Eu}\end{array}$ & $\begin{array}{l}4.1 \mathrm{E}-04 \\
4.3 \mathrm{E}-04 \\
2.1 \mathrm{E}-05 \\
4.6 \mathrm{E}-04\end{array}$ & $\begin{array}{l}4.0 \mathrm{E}-06 \\
5.2 \mathrm{E}-06 \\
7.8 \mathrm{E}-07 \\
2.8 \mathrm{E}-07\end{array}$ & $\begin{array}{l}1.3 \mathrm{E}-05 \\
1.8 \mathrm{E}-05 \\
3.0 \mathrm{E}-06 \\
1.8 \mathrm{E}-05\end{array}$ & $\begin{array}{l}4.3 \mathrm{E}-04 \\
4.5 \mathrm{E}-04 \\
2.4 \mathrm{E}-05 \\
4.8 \mathrm{E}-04\end{array}$ & $\begin{array}{l}2.5 \mathrm{E}-01 \\
2.6 \mathrm{E}-01 \\
1.3 \mathrm{E}-02 \\
2.8 \mathrm{E}-01\end{array}$ \\
\hline${ }^{153} \mathrm{Gd}$ & $3.9 E-05$ & 4.6E-07 & $2.4 E-06$ & $4.2 \mathrm{E}-05$ & $2.4 E-02$ \\
\hline${ }^{160} \mathrm{~Tb}$ & $3.7 \mathrm{E}-04$ & $4.8 E-07$ & $1.3 E-05$ & $3.8 E-04$ & $2.3 E-01$ \\
\hline${ }^{168 \mathbf{m}_{\mathrm{Ho}}}$ & $5.5 \mathrm{E}-04$ & $1.4 \mathrm{E}-05$ & $1.5 \mathrm{E}-05$ & $5.8 \mathrm{E}-04$ & $3.4 E-01$ \\
\hline $\begin{array}{l}{ }^{181} \mathrm{~W} \\
{ }^{185} \mathrm{~W} \\
{ }^{187}\end{array}$ & $\begin{array}{l}1.2 \mathrm{E}-05 \\
9.3 \mathrm{E}-08 \\
1.7 \mathrm{E}-04\end{array}$ & $\begin{array}{l}3.0 \mathrm{E}-09 \\
1.5 \mathrm{E}-08 \\
1.3 \mathrm{E}-08\end{array}$ & $\begin{array}{l}5.8 E-07 \\
3.2 E-06 \\
4.2 E-06\end{array}$ & $\begin{array}{l}1.3 E-05 \\
3.3 E-06 \\
1.7 E-04\end{array}$ & $\begin{array}{l}7.4 \mathrm{E}-03 \\
9.2 \mathrm{E}-04 \\
1.0 \mathrm{E}-01\end{array}$ \\
\hline${ }^{187} \mathrm{Re}$ & $0.0 \mathrm{E}+00$ & $1.1 \mathrm{E}-09$ & $2.8 \mathrm{E}-08$ & $2.9 E-08$ & $7.9 \mathrm{E}-06$ \\
\hline $\begin{array}{l}{ }^{185} \text { Os } \\
{ }^{191} \text { Os }\end{array}$ & $\begin{array}{l}2.5 E-04 \\
2.4 E-05\end{array}$ & $\begin{array}{l}1.8 \mathrm{E}-07 \\
4.4 \mathrm{E}-08\end{array}$ & $\begin{array}{l}4.4 \mathrm{E}-06 \\
4.6 \mathrm{E}-06\end{array}$ & $\begin{array}{l}2.5 \mathrm{E}-04 \\
2.8 \mathrm{E}-05\end{array}$ & $\begin{array}{l}1.5 \mathrm{E}-01 \\
1.6 \mathrm{E}-02\end{array}$ \\
\hline & $3.0 \mathrm{E}-04$ & $5.2 E-07$ & $1.1 \mathrm{E}-05$ & $3.1 \mathrm{E}-04$ & $1.8 \mathrm{E}-01$ \\
\hline & $6.9 \mathrm{E}-05$ & $1.4 \mathrm{E}-07$ & $2.2 E-05$ & $9.1 E-05$ & $4.7 \mathrm{E}-02$ \\
\hline $\begin{array}{l}{ }^{216} \mathrm{~Pb}+\mathrm{D} \\
{ }^{210} \mathrm{~Pb} \\
\left.{ }^{212} \mathrm{~Pb}+\mathrm{b}\right) \\
{ }^{212} \mathrm{~Pb}\end{array}$ & $\begin{array}{l}8.1 \mathrm{E}-07 \\
1.9 \mathrm{E}-06 \\
1.7 \mathrm{E}-04 \\
5.0 \mathrm{E}-05\end{array}$ & $\begin{array}{l}1.5 \mathrm{E}-04 \\
2.6 \mathrm{E}-04 \\
1.2 \mathrm{E}-06 \\
3.2 \mathrm{E}-06\end{array}$ & $\begin{array}{l}4.9 \mathrm{E}-03 \\
1.1 \mathrm{E}-02 \\
3.3 \mathrm{E}-05 \\
9.8 \mathrm{E}-05\end{array}$ & $\begin{array}{l}5.0 \mathrm{E}-03 \\
1.1 \mathrm{E}-02 \\
2.0 \mathrm{E}-04 \\
1.5 \mathrm{E}-04\end{array}$ & $\begin{array}{l}1.4 \mathrm{E}+00 \\
3.0 \mathrm{E}+00 \\
1.1 \mathrm{E}-01 \\
5.8 \mathrm{E}-02\end{array}$ \\
\hline $\begin{array}{l}{ }^{216} \mathrm{Bi}+\mathrm{D}(\mathrm{b}) \\
{ }^{216}{ }_{\mathrm{Bi}} \\
{ }^{212} \mathrm{Bi}+\mathrm{I} \\
\left.{ }^{\mathrm{C}} \mathrm{c}\right)\end{array}$ & $\begin{array}{l}3.1 \mathrm{E}-07 \\
6.4 \mathrm{E}-07 \\
4.7 \mathrm{E}-04\end{array}$ & $\begin{array}{l}8.6 \mathrm{E}-05 \\
3.8 \mathrm{E}-06 \\
1.8 \mathrm{E}-07\end{array}$ & $\begin{array}{l}1.9 \mathrm{E}-03 \\
1.3 \mathrm{E}-05 \\
1.1 \mathrm{E}-06\end{array}$ & $\begin{array}{l}2.0 \mathrm{E}-03 \\
1.7 \mathrm{E}-05 \\
4.7 \mathrm{E}-04\end{array}$ & $\begin{array}{l}5.4 E-01 \\
4.8 E-03 \\
2.8 E-01\end{array}$ \\
\hline${ }^{210} \mathrm{Po}$ & $3.1 E-09$ & $1.7 \mathrm{E}-04$ & $3.8 E-03$ & $4.0 \mathrm{E}-03$ & $1.1 E+00$ \\
\hline $\begin{array}{l}{ }^{223} \mathrm{Ra}+\mathrm{I}(\mathrm{c}) \\
{ }^{224} \mathrm{Ra} \text { (b) } \\
{ }^{224} \mathrm{Ra}(\mathrm{I}) \\
{ }^{225} \mathrm{Ra}(\mathrm{C}) \\
{ }^{226} \mathrm{Ra} \mathrm{C}(\mathrm{b}) \\
{ }^{226} \mathrm{Ra} \\
\mathrm{Ra}+\mathrm{D}\end{array}$ & $\begin{array}{l}1.0 \mathrm{E}-04 \\
8.3 \mathrm{E}-05 \\
3.7 \mathrm{E}-06 \\
1.2 \mathrm{E}-05 \\
5.6 \mathrm{E}-06 \\
6.6 \mathrm{E}-05\end{array}$ & $\begin{array}{l}2.5 \mathrm{E}-05 \\
9.7 \mathrm{E}-06 \\
5.4 \mathrm{E}-05 \\
4.3 \mathrm{E}-05 \\
1.4 \mathrm{E}-04 \\
6.4 \mathrm{E}-05\end{array}$ & $\begin{array}{l}9.0 \mathrm{E}-05 \\
6.0 \mathrm{E}-05 \\
2.6 \mathrm{E}-04 \\
9.4 \mathrm{E}-05 \\
4.4 \mathrm{E}-04 \\
1.8 \mathrm{E}-03\end{array}$ & $\begin{array}{l}2.2 \mathrm{E}-04 \\
1.5 \mathrm{E}-04 \\
3.2 \mathrm{E}-04 \\
1.5 \mathrm{E}-04 \\
5.9 \mathrm{E}-04 \\
2.0 \mathrm{E}-03\end{array}$ & $\begin{array}{l}9.2 \mathrm{E}-02 \\
6.9 \mathrm{E}-02 \\
8.7 \mathrm{E}-02 \\
4.4 \mathrm{E}-02 \\
1.6 \mathrm{E}-01 \\
5.5 \mathrm{E}-01\end{array}$ \\
\hline
\end{tabular}


TABLE 3.2. Annual Total Effective Dose Equivalent Factors for the Building Occupancy (Surface Activity) Scenario(a) (contd)

\begin{tabular}{|c|c|c|c|c|c|}
\hline Radionuclide & $\begin{array}{c}\text { External } \\
\text { (mrem) }\end{array}$ & $\begin{array}{l}\text { Inhalation } \\
\text { (mrem) }\end{array}$ & $\begin{array}{l}\text { Secondary } \\
\text { Ingestion } \\
\text { (mrem) }\end{array}$ & $\begin{array}{c}\text { TEDE } \\
\text { (mrem) }\end{array}$ & $\begin{array}{l}\text { TEDE } \\
(\mu \mathrm{S} v) \\
\end{array}$ \\
\hline $\begin{array}{l}{ }^{226}{ }^{228} \mathrm{Ra} \\
{ }^{228} \\
{ }_{\mathrm{Ra}}\end{array}$ & $\begin{array}{l}1.7 \mathrm{E}-06 \\
9.2 \mathrm{E}-05 \\
1.1 \mathrm{E}-10\end{array}$ & $\begin{array}{l}1.6 \mathrm{E}-04 \\
7.1 \mathrm{E}-04 \\
7.4 \mathrm{E}-05\end{array}$ & $\begin{array}{l}1.9 \mathrm{E}-03 \\
3.1 \mathrm{E}-04 \\
1.7 \mathrm{E}-03\end{array}$ & $\begin{array}{l}2.1 \mathrm{E}-03 \\
1.1 \mathrm{E}-03 \\
1.8 \mathrm{E}-03\end{array}$ & $\begin{array}{l}5.6 E-01 \\
3.3 E-01 \\
4.7 E-01\end{array}$ \\
\hline $\begin{array}{l}{ }^{225} \mathrm{Ac}+\mathrm{I}(\mathrm{c}) \\
{ }^{227} \mathrm{Ac}+\mathrm{D}(\mathrm{b}) \\
{ }^{227} \mathrm{Ac} \\
{ }^{228} \mathrm{AC}+\mathrm{D} \\
\mathrm{AC}\end{array}$ & $\begin{array}{l}8.5 \mathrm{E}-05 \\
1.7 \mathrm{E}-05 \\
1.1 \mathrm{E}-07 \\
1.0 \mathrm{E}-04 \\
3.4 \mathrm{E}-04\end{array}$ & $\begin{array}{l}2.6 \mathrm{E}-05 \\
3.2 \mathrm{E}-03 \\
2.6 \mathrm{E}-02 \\
8.0 \mathrm{E}-04 \\
2.2 \mathrm{E}-06\end{array}$ & $\begin{array}{l}3.7 \mathrm{E}-05 \\
3.5 \mathrm{E}-03 \\
2.8 \mathrm{E}-02 \\
1.4 \mathrm{E}-04 \\
4.2 \mathrm{E}-06\end{array}$ & $\begin{array}{l}1.5 \mathrm{E}-04 \\
6.8 \mathrm{E}-03 \\
5.4 \mathrm{E}-02 \\
1.0 \mathrm{E}-03 \\
3.4 \mathrm{E}-04\end{array}$ & $\begin{array}{l}6.8 E-02 \\
1.8 E+00 \\
1.5 E+01 \\
3.2 E-01 \\
2.0 E-01\end{array}$ \\
\hline 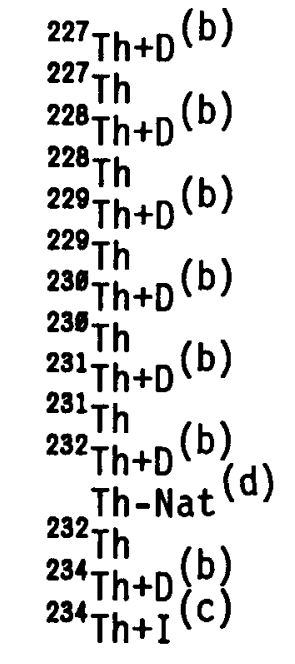 & $\begin{array}{l}1.9 \mathrm{E}-05 \\
3.8 \mathrm{E}-05 \\
7.1 \mathrm{E}-05 \\
1.1 \mathrm{E}-06 \\
1.5 \mathrm{E}-05 \\
3.0 \mathrm{E}-05 \\
5.9 \mathrm{E}-05 \\
5.4 \mathrm{E}-07 \\
8.2 \mathrm{E}-07 \\
8.6 \mathrm{E}-06 \\
8.2 \mathrm{E}-05 \\
8.2 \mathrm{E}-05 \\
4.9 \mathrm{E}-07 \\
5.1 \mathrm{E}-06 \\
9.5 \mathrm{E}-06\end{array}$ & $\begin{array}{l}6.6 \mathrm{E}-05 \\
3.2 \mathrm{E}-04 \\
9.1 \mathrm{E}-04 \\
6.2 \mathrm{E}-03 \\
4.3 \mathrm{E}-03 \\
3.4 \mathrm{E}-02 \\
5.6 \mathrm{E}-04 \\
5.0 \mathrm{E}-03 \\
1.6 \mathrm{E}-09 \\
1.6 \mathrm{E}-08 \\
2.8 \mathrm{E}-03 \\
2.8 \mathrm{E}-03 \\
2.2 \mathrm{E}-02 \\
3.4 \mathrm{E}-07 \\
6.8 \mathrm{E}-07\end{array}$ & $\begin{array}{l}8.9 \mathrm{E}-05 \\
7.6 \mathrm{E}-05 \\
1.6 \mathrm{E}-04 \\
8.0 \mathrm{E}-04 \\
9.5 \mathrm{E}-04 \\
7.0 \mathrm{E}-03 \\
1.8 \mathrm{E}-03 \\
1.1 \mathrm{E}-03 \\
2.6 \mathrm{E}-07 \\
2.6 \mathrm{E}-06 \\
8.4 \mathrm{E}-04 \\
8.4 \mathrm{E}-04 \\
5.6 \mathrm{E}-03 \\
1.3 \mathrm{E}-05 \\
2.6 \mathrm{E}-05\end{array}$ & $\begin{array}{l}1.7 \mathrm{E}-04 \\
4.3 \mathrm{E}-04 \\
1.1 \mathrm{E}-03 \\
7.0 \mathrm{E}-03 \\
5.2 \mathrm{E}-03 \\
4.1 \mathrm{E}-02 \\
2.4 \mathrm{E}-03 \\
6.1 \mathrm{E}-03 \\
1.1 \mathrm{E}-06 \\
1.1 \mathrm{E}-05 \\
3.7 \mathrm{E}-03 \\
3.7 \mathrm{E}-03 \\
2.8 \mathrm{E}-02 \\
1.8 \mathrm{E}-05 \\
3.6 \mathrm{E}-05\end{array}$ & $\begin{array}{l}5.3 \mathrm{E}-02 \\
1.3 \mathrm{E}-01 \\
3.3 \mathrm{E}-01 \\
1.9 \mathrm{E}+00 \\
1.4 \mathrm{E}+00 \\
1.1 \mathrm{E}+01 \\
6.6 \mathrm{E}-01 \\
1.6 \mathrm{E}+00 \\
5.7 \mathrm{E}-04 \\
5.9 \mathrm{E}-03 \\
1.0 \mathrm{E}+00 \\
1.0 \mathrm{E}+00 \\
7.5 \mathrm{E}+00 \\
6.7 \mathrm{E}-03 \\
1.3 \mathrm{E}-02\end{array}$ \\
\hline $\begin{array}{l}{ }^{231} \mathrm{~Pa} \\
{ }^{231} \mathrm{~Pa} \\
{ }^{233} \mathrm{~Pa} \\
{ }^{234} \mathrm{~Pa}\end{array}$ & $\begin{array}{l}1.7 E-05 \\
1.4 E-05 \\
8.1 E-05 \\
6.9 E-04\end{array}$ & $\begin{array}{l}4.7 \mathrm{E}-03 \\
1.7 \mathrm{E}-02 \\
1.8 \mathrm{E}-07 \\
1.6 \mathrm{E}-08\end{array}$ & $\begin{array}{l}5.6 \mathrm{E}-03 \\
2.2 \mathrm{E}-02 \\
7.2 \mathrm{E}-06 \\
4.4 \mathrm{E}-06\end{array}$ & $\begin{array}{l}1.0 \mathrm{E}-02 \\
3.9 \mathrm{E}-02 \\
8.8 \mathrm{E}-05 \\
7.0 \mathrm{E}-04\end{array}$ & $\begin{array}{l}2.8 \mathrm{E}+00 \\
1.0 \mathrm{E}+01 \\
5.1 \mathrm{E}-02 \\
4.2 \mathrm{E}-01\end{array}$ \\
\hline 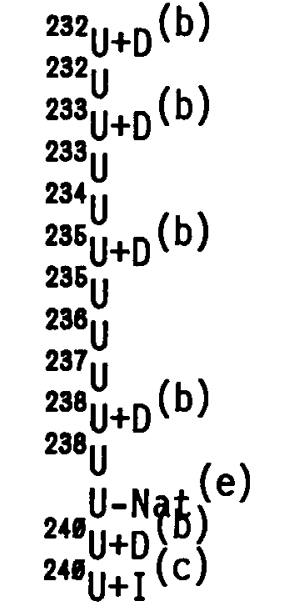 & $\begin{array}{l}9.7 \mathrm{E}-08 \\
7.0 \mathrm{E}-07 \\
1.3 \mathrm{E}-05 \\
2.9 \mathrm{E}-07 \\
5.9 \mathrm{E}-07 \\
2.4 \mathrm{E}-05 \\
4.2 \mathrm{E}-05 \\
5.4 \mathrm{E}-07 \\
5.3 \mathrm{E}-05 \\
3.6 \mathrm{E}-06 \\
1.5 \mathrm{E}-06 \\
2.0 \mathrm{E}-06 \\
4.3 \mathrm{E}-05 \\
1.3 \mathrm{E}-04\end{array}$ & $\begin{array}{l}1.7 \mathrm{E}-03 \\
1.2 \mathrm{E}-02 \\
4.0 \mathrm{E}-03 \\
2.6 \mathrm{E}-03 \\
2.6 \mathrm{E}-03 \\
1.2 \mathrm{E}-03 \\
2.4 \mathrm{E}-03 \\
2.4 \mathrm{E}-03 \\
7.0 \mathrm{E}-08 \\
7.3 \mathrm{E}-04 \\
2.4 \mathrm{E}-03 \\
2.5 \mathrm{E}-03 \\
1.5 \mathrm{E}-08 \\
4.4 \mathrm{E}-08\end{array}$ & $\begin{array}{l}1.9 \mathrm{E}-05 \\
1.4 \mathrm{E}-04 \\
8.7 \mathrm{E}-04 \\
5.2 \mathrm{E}-05 \\
5.2 \mathrm{E}-05 \\
2.8 \mathrm{E}-05 \\
5.2 \mathrm{E}-05 \\
4.8 \mathrm{E}-05 \\
6.4 \mathrm{E}-06 \\
2.5 \mathrm{E}-05 \\
5.0 \mathrm{E}-05 \\
5.1 \mathrm{E}-05 \\
3.1 \mathrm{E}-06 \\
9.4 \mathrm{E}-06\end{array}$ & $\begin{array}{l}1.7 \mathrm{E}-03 \\
1.2 \mathrm{E}-02 \\
4.9 \mathrm{E}-03 \\
2.7 \mathrm{E}-03 \\
2.7 \mathrm{E}-03 \\
1.3 \mathrm{E}-03 \\
2.5 \mathrm{E}-03 \\
2.4 \mathrm{E}-03 \\
6.0 \mathrm{E}-05 \\
7.6 \mathrm{E}-04 \\
2.5 \mathrm{E}-03 \\
2.6 \mathrm{E}-03 \\
4.6 \mathrm{E}-05 \\
1.4 \mathrm{E}-04\end{array}$ & $\begin{array}{l}4.7 \mathrm{E}-01 \\
3.3 \mathrm{E}+00 \\
1.3 \mathrm{E}+00 \\
7.2 \mathrm{E}-01 \\
7.2 \mathrm{E}-01 \\
3.5 \mathrm{E}-01 \\
6.9 \mathrm{E}-01 \\
6.6 \mathrm{E}-01 \\
3.4 \mathrm{E}-02 \\
2.1 \mathrm{E}-01 \\
6.6 \mathrm{E}-01 \\
6.9 \mathrm{E}-01 \\
2.7 \mathrm{E}-02 \\
8.3 \mathrm{E}-02\end{array}$ \\
\hline
\end{tabular}


TABLE 3.2. Annual Total Effective Dose Equivalent Factors for the Building 0ccupancy (Surface Activity) Scenario(a) (contd)

\begin{tabular}{|c|c|c|c|c|c|}
\hline Radionuclide & $\begin{array}{c}\text { External } \\
\text { (mrem) }\end{array}$ & $\begin{array}{c}\text { Inhalation } \\
\text { (mrem) }\end{array}$ & $\begin{array}{l}\text { Secondary } \\
\text { Ingestion } \\
\text { (mrem) } \\
\end{array}$ & $\begin{array}{c}\text { TEDE } \\
\text { (mrem) }\end{array}$ & $\begin{array}{l}\text { TEDE } \\
(\mu S v) \\
\end{array}$ \\
\hline $\begin{array}{l}{ }^{237} \mathrm{Np+D}(\mathrm{b}) \\
{ }^{237} \mathrm{~Np} \\
{ }^{238} \mathrm{Np+D}(\mathrm{b}) \\
{ }^{238} \mathrm{~Np} \\
{ }^{238} \mathrm{Np+D}(\mathrm{b}) \\
{ }^{239} \mathrm{~Np}\end{array}$ & $\begin{array}{l}4.5 \mathrm{E}-05 \\
1.1 \mathrm{E}-05 \\
9.5 \mathrm{E}-05 \\
1.9 \mathrm{E}-04 \\
3.0 \mathrm{E}-05 \\
6.3 \mathrm{E}-05\end{array}$ & $\begin{array}{l}6.1 \mathrm{E}-03 \\
1.2 \mathrm{E}-02 \\
2.8 \mathrm{E}-03 \\
8.0 \mathrm{E}-07 \\
2.4 \mathrm{E}-08 \\
4.8 \mathrm{E}-08\end{array}$ & $\begin{array}{l}5.2 \mathrm{E}-03 \\
1.0 \mathrm{E}-02 \\
3.4 \mathrm{E}-04 \\
8.0 \mathrm{E}-06 \\
3.3 \mathrm{E}-06 \\
6.6 \mathrm{E}-06\end{array}$ & $\begin{array}{l}1.1 \mathrm{E}-02 \\
2.3 \mathrm{E}-02 \\
3.2 \mathrm{E}-03 \\
2.0 \mathrm{E}-04 \\
3.4 \mathrm{E}-05 \\
6.9 \mathrm{E}-05\end{array}$ & $\begin{array}{l}3.1 \mathrm{E}+00 \\
6.1 \mathrm{E}+00 \\
9.0 \mathrm{E}-01 \\
1.2 \mathrm{E}-01 \\
1.9 \mathrm{E}-02 \\
3.9 \mathrm{E}-02\end{array}$ \\
\hline $\begin{array}{l}{ }^{236} \mathrm{Pu} \\
{ }^{237} \mathrm{Pu} \\
{ }^{238} \mathrm{Pu} \\
{ }^{239} \mathrm{Pu} \\
{ }^{246} \mathrm{Pu} \\
{ }_{241} \mathrm{Pu}+\mathrm{D} \\
{ }^{241} \mathrm{Pu} \\
{ }^{242} \mathrm{Pu} \\
\left.{ }^{243} \mathrm{Pu}+\mathrm{D}\right) \\
{ }^{243} \mathrm{Pu} \\
\left.{ }^{244} \mathrm{Pu}+\mathrm{b}\right) \\
{ }^{244} \mathrm{Pu}\end{array}$ & $\begin{array}{l}7.0 \mathrm{E}-07 \\
2.0 \mathrm{E}-05 \\
6.1 \mathrm{E}-07 \\
2.5 \mathrm{E}-07 \\
5.9 \mathrm{E}-07 \\
4.1 \mathrm{E}-06 \\
1.6 \mathrm{E}-12 \\
4.9 \mathrm{E}-07 \\
2.2 \mathrm{E}-05 \\
7.1 \mathrm{E}-06 \\
3.2 \mathrm{E}-05 \\
4.2 \mathrm{E}-07\end{array}$ & $\begin{array}{l}1.4 \mathrm{E}-03 \\
3.6 \mathrm{E}-08 \\
5.6 \mathrm{E}-03 \\
5.8 \mathrm{E}-03 \\
5.8 \mathrm{E}-03 \\
4.3 \mathrm{E}-03 \\
9.4 \mathrm{E}-05 \\
5.4 \mathrm{E}-03 \\
1.4 \mathrm{E}-09 \\
2.8 \mathrm{E}-09 \\
1.3 \mathrm{E}-03 \\
5.2 \mathrm{E}-03\end{array}$ & $\begin{array}{l}1.3 \mathrm{E}-04 \\
8.6 \mathrm{E}-07 \\
6.8 \mathrm{E}-04 \\
7.4 \mathrm{E}-04 \\
7.4 \mathrm{E}-04 \\
3.6 \mathrm{E}-03 \\
1.4 \mathrm{E}-05 \\
6.8 \mathrm{E}-04 \\
3.2 \mathrm{E}-07 \\
6.4 \mathrm{E}-07 \\
1.8 \mathrm{E}-04 \\
7.0 \mathrm{E}-04\end{array}$ & $\begin{array}{l}1.6 \mathrm{E}-03 \\
2.1 \mathrm{E}-05 \\
6.3 \mathrm{E}-03 \\
6.5 \mathrm{E}-03 \\
6.5 \mathrm{E}-03 \\
7.9 \mathrm{E}-03 \\
1.1 \mathrm{E}-04 \\
6.1 \mathrm{E}-03 \\
2.3 \mathrm{E}-05 \\
7.7 \mathrm{E}-06 \\
1.5 \mathrm{E}-03 \\
5.9 \mathrm{E}-03\end{array}$ & $\begin{array}{l}4.2 \mathrm{E}-01 \\
1.2 \mathrm{E}-02 \\
1.7 \mathrm{E}+00 \\
1.8 \mathrm{E}+00 \\
1.8 \mathrm{E}+00 \\
2.1 \mathrm{E}+00 \\
2.9 \mathrm{E}-02 \\
1.6 \mathrm{E}+00 \\
1.3 \mathrm{E}-02 \\
4.4 \mathrm{E}-03 \\
4.2 \mathrm{E}-01 \\
1.6 \mathrm{E}+00\end{array}$ \\
\hline $\begin{array}{l}{ }^{241} \mathrm{Am} \\
{ }^{242 m} \mathrm{Am}+\mathrm{D} \\
2420 \mathrm{Am} \\
{ }^{242} \mathrm{Am}+\mathrm{D}(\mathrm{b}) \\
{ }^{242} \mathrm{Am} \\
{ }^{243} \mathrm{Am}+\mathrm{D}(\mathrm{b}) \\
{ }^{243} \mathrm{Am}\end{array}$ & $\begin{array}{l}8.5 \mathrm{E}-06 \\
2.4 \mathrm{E}-06 \\
1.7 \mathrm{E}-06 \\
2.6 \mathrm{E}-06 \\
6.7 \mathrm{E}-06 \\
2.5 \mathrm{E}-05 \\
1.6 \mathrm{E}-05\end{array}$ & $\begin{array}{l}8.6 \mathrm{E}-03 \\
3.3 \mathrm{E}-03 \\
7.8 \mathrm{E}-03 \\
1.7 \mathrm{E}-03 \\
1.0 \mathrm{E}-06 \\
2.7 \mathrm{E}-03 \\
8.2 \mathrm{E}-03\end{array}$ & $\begin{array}{l}7.2 \mathrm{E}-03 \\
2.0 \mathrm{E}-03 \\
7.0 \mathrm{E}-03 \\
2.6 \mathrm{E}-04 \\
2.8 \mathrm{E}-06 \\
2.4 \mathrm{E}-03 \\
7.2 \mathrm{E}-03\end{array}$ & $\begin{array}{l}1.6 \mathrm{E}-02 \\
5.3 \mathrm{E}-03 \\
1.5 \mathrm{E}-02 \\
2.0 \mathrm{E}-03 \\
1.1 \mathrm{E}-05 \\
5.2 \mathrm{E}-03 \\
1.5 \mathrm{E}-02\end{array}$ & $\begin{array}{l}4.3 E+00 \\
1.4 E+00 \\
4.0 E+00 \\
5.3 E-01 \\
5.1 E-03 \\
1.4 E+00 \\
4.2 E+00\end{array}$ \\
\hline $\begin{array}{l}{ }^{242} \mathrm{Cm}+\mathrm{D}^{(b)} \\
{ }^{242} \mathrm{Cm} \\
{ }^{243} \mathrm{Cm} \\
{ }^{244} \mathrm{Cm}+\mathrm{D} \\
{ }^{244} \mathrm{Cm} \\
\left.{ }^{245} \mathrm{Cm}+\mathrm{D}\right) \\
{ }^{245} \mathrm{Cm} \\
{ }^{246} \mathrm{Cm} \\
{ }^{247} \mathrm{Cm}{ }^{247} \mathrm{Cm} \\
{ }^{248} \mathrm{Cm}\end{array}$ & $\begin{array}{l}6.5 \mathrm{E}-07 \\
6.1 \mathrm{E}-07 \\
4.7 \mathrm{E}-05 \\
2.6 \mathrm{E}-07 \\
5.5 \mathrm{E}-07 \\
1.1 \mathrm{E}-05 \\
2.7 \mathrm{E}-05 \\
4.9 \mathrm{E}-07 \\
8.8 \mathrm{E}-05 \\
1.3 \mathrm{E}-04 \\
3.9 \mathrm{E}-07\end{array}$ & $\begin{array}{l}3.2 \mathrm{E}-03 \\
3.0 \mathrm{E}-04 \\
1.2 \mathrm{E}-02 \\
2.3 \mathrm{E}-03 \\
4.6 \mathrm{E}-03 \\
5.7 \mathrm{E}-03 \\
8.4 \mathrm{E}-03 \\
8.4 \mathrm{E}-03 \\
3.9 \mathrm{E}-03 \\
7.8 \mathrm{E}-03 \\
3.0 \mathrm{E}-02\end{array}$ & $\begin{array}{l}4.9 \mathrm{E}-04 \\
2.2 \mathrm{E}-04 \\
5.8 \mathrm{E}-03 \\
2.0 \mathrm{E}-03 \\
4.0 \mathrm{E}-03 \\
4.9 \mathrm{E}-03 \\
7.4 \mathrm{E}-03 \\
7.4 \mathrm{E}-03 \\
3.4 \mathrm{E}-03 \\
6.8 \mathrm{E}-03 \\
2.6 \mathrm{E}-02\end{array}$ & $\begin{array}{l}3.7 \mathrm{E}-03 \\
5.2 \mathrm{E}-04 \\
1.7 \mathrm{E}-02 \\
4.3 \mathrm{E}-03 \\
8.6 \mathrm{E}-03 \\
1.1 \mathrm{E}-02 \\
1.6 \mathrm{E}-02 \\
1.6 \mathrm{E}-02 \\
7.4 \mathrm{E}-03 \\
1.5 \mathrm{E}-02 \\
5.6 \mathrm{E}-02\end{array}$ & $\begin{array}{l}9.9 \mathrm{E}-01 \\
1.4 \mathrm{E}-01 \\
4.7 \mathrm{E}+00 \\
1.2 \mathrm{E}+00 \\
2.3 \mathrm{E}+00 \\
2.9 \mathrm{E}+00 \\
4.3 \mathrm{E}+00 \\
4.3 \mathrm{E}+00 \\
2.0 \mathrm{E}+00 \\
4.0 \mathrm{E}+00 \\
1.5 \mathrm{E}+01\end{array}$ \\
\hline${ }^{252} \mathrm{Cf}$ & $3.9 \mathrm{E}-07$ & $2.6 \mathrm{E}-03$ & $2.2 \mathrm{E}-03$ & $4.8 \mathrm{E}-03$ & $1.3 \mathrm{E}+00$ \\
\hline
\end{tabular}

(a) Based on $2000 \mathrm{~h}$ of exposure to removable surface activity at an activity level of $1 \mathrm{dpm} / 100 \mathrm{~cm}^{2}\left(1 \mathrm{~Bq} / 100 \mathrm{~cm}^{2}\right)$. 
TABLE 3.2. Annual Total Effective Dose Equivalent Factors for the Building Occupancy (Surface Activity) Scenario(a) (contd)

(b) Where +D means "plus daughters in equilibrium" referring to explicit daughters, or daughters with half-lives greater than 10 minutes. Separate values are also included for parents and daughters so that combined factors for non-equilibrium cases can be determined. Note: in some cases +D also includes implicit daughters (i.e, uranium and thorium decay chains).

(c) Where +I means "plus implicit daughters in equilibrium" referring to daughters with half-lives less than 10 minutes. The decay energies associated with these daughters have been assigned to the parent, and the appropriate branching ratios (described in Appendix D) have been considered.

(d) Where Th-Nat includes an equilibrium mixture of ${ }^{232}$ Th plus 10 daughters in the decay chain, as discussed in Appendix $D$.

(e) Where $1 \mathrm{Ci} \mathrm{U}$-Nat equals $48.9 \%{ }^{238} \mathrm{U}$ plus $48.9 \%$ ${ }^{234} \mathrm{U}$ plus $2.25 \%{ }^{235} \mathrm{U}$. 


\subsection{SCENARIO DESCRIPTIONS FOR THE RELEASE OF CONTAMINATED LAND}

A wide variety of conditions may exist for contaminated land: e.g., contaminated soil may: 1) be in a thick or thin layer, 2) reside on the soil surface or be buried under clean soi1,3) cover a small or large area, or 4) consist of many different radionuclides or mixtures of radionuclides. Two generic scenarios are provided that will address contaminated land in a conservative manner and account for near-term residential (surface soil) and long-term (drinking-water) use. These two scenarios are considered to be mutually exclusive; that is, each is designed to model a specific situation independently. To avoid accounting for the residual activity twice, the inventory is considered to be either in the surface soils available for agricultural activities, or in deeper soils migrating to the ground water. To further avoid accounting for the inventory twice, irrigation of the site with contaminated ground water is not considered. The residential use scenario is designed to provide an estimate of the potential dose resulting from specific concentrations of radionuclides $(\mathrm{pCi} / \mathrm{g}$ or $\mathrm{Bq} / \mathrm{g}$ ) in surface soils. The drinking water scenario is designed to provide an estimate of the potential dose from the entire inventory of radioactive materials (the total $\mathrm{pC} \mathrm{i}$ or $\mathrm{Bq}$ ) estimated to be on a site as soil contamination or as a residual inventory in building materials that could be left as buried rubble. The scenarios are used to derive annual TEDE factors, expressed in mrem $\mathrm{per} p \mathrm{pi} / \mathrm{g}$ (and $\mu \mathrm{Sv}$ per $\mathrm{Bq} / \mathrm{g}$ ) of surface soil and in mrem per $\mathrm{pCi}$ (and $\mu \mathrm{Sv}$ per $\mathrm{Bq}$ ) in soil for drinking-water consumption. The following sections describe the generic scenarios used to develop the annual TEDE factors.

\subsubsection{Residential Use (Surface Soil) Scenario}

The generically derived unit concentration committed effective dose equivalent conversion factors for surface soil, in units of mrem per $\mathrm{pCi} / \mathrm{g}$ (and $\mu \mathrm{Sv}$ per $\mathrm{Bq} / \mathrm{g}$ ), are based on a scenario accounting for reasonable use of the land. Previous studies for low-level radioactive waste management and decommissioning have provided an evaluation of this type of scenario (Healy, Rodgers, and Wienke 1979; Witherspoon 1982; Oztunali, et. al. 1981). Perhaps the most detailed evaluation was conducted for the intruder-agriculture scenario described in the Draft Environmental Impact Statement on 10 CFR Part 61, "Licensing Requirements for Land Disposal of Radioactive Waste" (NRC 1981) and a supplementary document entitled Update of Part 61 Impacts Analys is Methodology (Oztunali and Roles 1986). This scenario accounts for residential use of the land with backyard gardening activities. The three major radiation exposure pathways considered for this scenario include inhalation of air contaminated by the resuspended soil, ingestion of agricultural products grown in the contaminated soil, and direct exposure to penetrating gamma radiation. Rainsplash of contaminated soil onto plant surfaces and indoor radon from residual $226 \mathrm{Ra}$ in soils are not considered. For this scenario, an accounting is made of the various activities of the individual, in $\mathrm{h} / \mathrm{yr}$. The individual is assumed to spend $4380 \mathrm{~h} / \mathrm{yr}$ residing at home, indoors, $1700 \mathrm{~h} / \mathrm{yr}$ outdoors, and $100 \mathrm{~h} / \mathrm{yr}$ outdoors in gardening activities on the site. The individual is assumed to breath air with an indoor dust loading of $5 \times 10^{-5} \mathrm{~g} / \mathrm{m}^{3}$, and average outdoor dust loading of 
$1 \times 10^{-4} \mathrm{~g} / \mathrm{m}^{3}$ from dusty work conditions, and an airborne dust loading while gardening of $5 \times 10^{-4} \mathrm{~g} / \mathrm{m}^{3}$. The individual is assumed to ingest $25 \%$ of his total diet, as defined in Regulatory Guide 1.109 (NRC 1977), from food grown in the contaminated soil at the site. Soil-to-plant transfer factors and other data for this pathway are consistent with data found in 0ztunali et al. (1981), as discussed in Appendix B. For external exposure, the shielding factor afforded by the house is dependent on the type of house construction and the nature of the contaminated soil (i.e., the source thickness and size). Previous studies have considered shielding factors associated with the atmospheric deposition of radioactive material from passing plumes (Aldrich et al. 1978; Kocher 1978; Jensen 1985). Estimated shielding factors from these studies range from about 0.02 to 0.6 , with the majority of values reported from 0.04 to about 0.4 (Aldrich et al. 1978). For this study, a representative shielding factor within this range of 0.33 is assumed.

These parameters and their expected ranges are summarized in Section 3.3 and are more fully discussed in Appendix B. The limited diet is used, assuming that the site is of limited size and that only backyard gardening is attempted. Even if larger sites are encountered where more vigorous agricultural practices may be possible, large-area farming in the United States usually results in the production of a single crop, which is not anticipated to increase to total dietary consumption of radioactive materials by an average individual. The diet for this analysis consists of $47.5 \mathrm{~kg} / \mathrm{yr}$ of vegetable produce, $27.5 \mathrm{~L} / \mathrm{yr}$ of milk, $19.2 \mathrm{~kg} / \mathrm{yr}$ of meats (beef, pork, and poultry), and $4.8 \mathrm{~kg} / \mathrm{yr}$ of eggs. Again, indoor radon is not considered.

The annual TEDE factors for the residential use scenario are derived by assuming that the land is uniformly contaminated to a depth of $15 \mathrm{~cm}$ (the assumed plow layer) over a relatively large area (in excess on $0.15 \mathrm{ha}$ ). No further dilution by mixing with non-contaminated soils is assumed. Doses from external exposure are estimated using a cylinder source solution with a radius of $5 \mathrm{~m}$ and a thickness of $15 \mathrm{~cm}$. For radionuclides that have significant inhalation dose conversion factors, or for those that are mobile in the food chain, the derived surface soil annual TEDE factors are more restrictive than factors derived solely on the external dose rate. The annual TEDE factors derived for this scenario are designed to provide an estimate of the potential dose resulting from specific surface soil concentrations of radionuclides (in $\mathrm{pCi} / \mathrm{g}$ or $\mathrm{Bq} / \mathrm{g}$ ). These factors are necessarily conservative because it is impossible to accurately account for the potential end-use of a site without including detailed site-specific data. However, the parameters used in this scenario have been selected to predict the potential dose to an average (not maximum) individual, as discussed in Section 3.3. The resulting annual TEDE factors for the residential use scenario are shown in Table 3.3 in units of mrem per $\mathrm{pCi} / \mathrm{g}$ (and $\mu \mathrm{Sv}$ per $\mathrm{Bq} / \mathrm{g}$ ) in soil. Table 3.3 lists the contribution by exposure pathway for about 200 radionuclides including key parent-daughter relationships. Again, the $+I$ (implicit daughter) and $+D$ (explicit daughter) notation has been used to provide maximum flexibility for consideration of all parent-daughter and decay chain situations. Further description of the parent-daughter and chain decay relationships used in this study is provided in Appendix D. Hand calculations were performed for selected radionuclides to verify that the pathway calculations were performed correctly. 
TABLE 3.3. Annual Total Effective Dose Equivalent Factors for the Residential Use (Surface Soil) Scenario(a)

\begin{tabular}{|c|c|c|c|c|c|}
\hline Radionuclide & $\begin{array}{c}\text { External } \\
\text { (mrem) }\end{array}$ & $\begin{array}{c}\text { Inhalation } \\
\text { (mrem) }\end{array}$ & $\begin{array}{c}\text { Food } \\
\text { Ingestion } \\
\text { (mrem) } \\
\end{array}$ & $\begin{array}{c}\text { TEDE } \\
\text { (mrem) }\end{array}$ & $\begin{array}{l}\text { TEDE } \\
(\mu \mathrm{Sv})\end{array}$ \\
\hline${ }^{3} \mathrm{H}$ & $3.4 \mathrm{E}-11$ & $3.6 \mathrm{E}-08$ & $2.9 \mathrm{E}-08$ & $6.5 E-08$ & $1.7 E-05$ \\
\hline${ }^{16} \mathrm{Be}$ & $4.3 \mathrm{E}-04$ & $1.5 \mathrm{E}-04$ & $9.3 \mathrm{E}-05$ & $6.7 E-04$ & $1.8 \mathrm{E}-01$ \\
\hline${ }^{14} \mathrm{C}$ & $7.3 \mathrm{E}-06$ & $8.3 E-07$ & $9.0 \mathrm{E}-06$ & $1.7 \mathrm{E}-05$ & $4.6 \mathrm{E}-03$ \\
\hline${ }^{18} \mathrm{~F}$ & $3.3 E+00$ & $3.4 \mathrm{E}-08$ & $2.2 E-05$ & $3.3 E+00$ & $8.9 E+02$ \\
\hline $\begin{array}{l}{ }^{22} \mathrm{Na} \\
{ }^{24} \mathrm{Na}\end{array}$ & $\begin{array}{l}6.9 \mathrm{E}+00 \\
1.3 \mathrm{E}+01\end{array}$ & $\begin{array}{l}3.8 \mathrm{E}-06 \\
4.8 \mathrm{E}-07\end{array}$ & $\begin{array}{l}1.4 \mathrm{E}-01 \\
4.3 \mathrm{E}-03\end{array}$ & $\begin{array}{l}7.1 \mathrm{E}+00 \\
1.3 \mathrm{E}+01\end{array}$ & $\begin{array}{l}1.9 E+03 \\
3.6 E+03\end{array}$ \\
\hline $\begin{array}{l}32 \mathrm{p} \\
{ }^{33} \mathrm{p}\end{array}$ & $\begin{array}{l}9.5 \mathrm{E}-03 \\
3.3 \mathrm{E}-05\end{array}$ & $\begin{array}{l}2.4 \mathrm{E}-06 \\
2.5 \mathrm{E}-07\end{array}$ & $\begin{array}{l}1.1 \mathrm{E}+00 \\
1.2 \mathrm{E}-01\end{array}$ & $\begin{array}{l}1.1 \mathrm{E}+00 \\
1.2 \mathrm{E}-01\end{array}$ & $\begin{array}{l}2.9 \mathrm{E}+02 \\
3.1 \mathrm{E}+01\end{array}$ \\
\hline${ }^{35} \mathrm{~S}$ & $8.8 \mathrm{E}-06$ & $1.1 \mathrm{E}-06$ & $2.5 \mathrm{E}-02$ & $2.5 E-02$ & $6.8 E+00$ \\
\hline${ }^{36} \mathrm{Cl}$ & $8.5 E-04$ & $7.9 E-07$ & $2.8 \mathrm{E}+00$ & $2.8 E+00$ & $7.4 E+02$ \\
\hline${ }^{46} K$ & $5.0 E-01$ & $4.3 E-06$ & $8.0 E-01$ & $1.3 E+00$ & $3.5 E+02$ \\
\hline $\begin{array}{l}{ }^{41} \mathrm{Ca} \\
{ }^{45} \mathrm{Ca}\end{array}$ & $\begin{array}{l}4.6 \mathrm{E}-05 \\
3.5 \mathrm{E}-05\end{array}$ & $\begin{array}{l}5.3 \mathrm{E}-07 \\
2.8 \mathrm{E}-06\end{array}$ & $\begin{array}{l}5.8 \mathrm{E}-03 \\
1.3 \mathrm{E}-02\end{array}$ & $\begin{array}{l}5.8 \mathrm{E}-03 \\
1.3 \mathrm{E}-02\end{array}$ & $\begin{array}{l}1.6 \mathrm{E}+00 \\
3.4 \mathrm{E}+00\end{array}$ \\
\hline${ }^{46} \mathrm{Sc}$ & $6.4 \mathrm{E}+00$ & $1.2 \mathrm{E}-05$ & $6.3 E-04$ & $6.4 E+00$ & $1.7 E+03$ \\
\hline${ }^{51} \mathrm{Cr}$ & $1.1 \mathrm{E}-01$ & $1.4 \mathrm{E}-07$ & $1.9 \mathrm{E}-06$ & $1.1 \mathrm{E}-01$ & $3.1 E+01$ \\
\hline $\begin{array}{l}{ }^{54} \mathrm{Mn} \\
{ }^{58} \mathrm{Mn}\end{array}$ & $\begin{array}{l}2.6 \mathrm{E}+00 \\
5.6 \mathrm{E}+00\end{array}$ & $\begin{array}{l}2.7 \mathrm{E}-06 \\
1.4 \mathrm{E}-07\end{array}$ & $\begin{array}{l}4.0 \mathrm{E}-03 \\
1.4 \mathrm{E}-03\end{array}$ & $\begin{array}{l}2.6 E+00 \\
5.6 E+00\end{array}$ & $\begin{array}{l}7.0 \mathrm{E}+02 \\
1.5 \mathrm{E}+03\end{array}$ \\
\hline $\begin{array}{l}{ }^{55} \mathrm{Fe} \\
{ }^{59} \mathrm{Fe}\end{array}$ & $\begin{array}{l}1.1 \mathrm{E}-04 \\
3.6 \mathrm{E}+00\end{array}$ & $\begin{array}{l}5.3 \mathrm{E}-07 \\
5.3 \mathrm{E}-06\end{array}$ & $\begin{array}{l}3.5 \mathrm{E}-05 \\
2.4 \mathrm{E}-04\end{array}$ & $\begin{array}{l}1.4 \mathrm{E}-04 \\
3.6 \mathrm{E}+00\end{array}$ & $\begin{array}{l}3.8 \mathrm{E}-02 \\
9.6 \mathrm{E}+02\end{array}$ \\
\hline $\begin{array}{l}{ }^{57} \mathrm{Co} \\
{ }^{58} \mathrm{Co} \\
{ }^{60} \mathrm{Co}\end{array}$ & $\begin{array}{l}2.5 \mathrm{E}-01 \\
3.1 \mathrm{E}+00 \\
7.7 \mathrm{E}+00\end{array}$ & $\begin{array}{l}3.6 \mathrm{E}-06 \\
4.4 \mathrm{E}-06 \\
8.3 \mathrm{E}-05\end{array}$ & $\begin{array}{l}4.3 \mathrm{E}-04 \\
1.5 \mathrm{E}-03 \\
6.0 \mathrm{E}-03\end{array}$ & $\begin{array}{l}2.5 \mathrm{E}-01 \\
3.1 \mathrm{E}+00 \\
7.7 \mathrm{E}+00\end{array}$ & $\begin{array}{l}6.6 \mathrm{E}+01 \\
8.4 \mathrm{E}+02 \\
2.1 \mathrm{E}+03\end{array}$ \\
\hline $\begin{array}{l}{ }^{59} \mathrm{Ni} \\
{ }^{83} \mathrm{Ni} \\
{ }^{65} \mathrm{Ni}\end{array}$ & $\begin{array}{l}1.3 \mathrm{E}-04 \\
1.9 \mathrm{E}-07 \\
1.8 \mathrm{E}+00\end{array}$ & $\begin{array}{l}3.5 \mathrm{E}-07 \\
8.3 \mathrm{E}-07 \\
1.0 \mathrm{E}-07\end{array}$ & $\begin{array}{l}4.8 \mathrm{E}-04 \\
1.3 \mathrm{E}-03 \\
5.8 \mathrm{E}-04\end{array}$ & $\begin{array}{l}6.0 \mathrm{E}-04 \\
1.3 \mathrm{E}-03 \\
1.8 \mathrm{E}+00\end{array}$ & $\begin{array}{l}1.6 \mathrm{E}-01 \\
3.4 \mathrm{E}-01 \\
4.9 \mathrm{E}+02\end{array}$ \\
\hline${ }^{84} \mathrm{Cu}$ & $6.3 \mathrm{E}-01$ & $8.3 E-08$ & $3.0 \mathrm{E}-03$ & $6.3 E-01$ & $1.7 E+02$ \\
\hline${ }^{65} \mathrm{Zn} \mathrm{Zn+D}(\mathrm{c})$ & $\begin{array}{l}2.0 \mathrm{E}+00 \\
7.8 \mathrm{E}-01\end{array}$ & $\begin{array}{l}7.5 \mathrm{E}-06 \\
1.8 \mathrm{E}-07\end{array}$ & $\begin{array}{l}9.5 \mathrm{E}-01 \\
1.6 \mathrm{E}-02\end{array}$ & $\begin{array}{l}3.0 \mathrm{E}+00 \\
8.0 \mathrm{E}-01\end{array}$ & $\begin{array}{l}8.0 E+02 \\
2.2 E+02\end{array}$ \\
\hline
\end{tabular}


IABLE 3.3. Annual Total Effective Dose Equivalent Factors for the Residential Use (Surface Soil) Scenario(a) (contd)

\begin{tabular}{|c|c|c|c|c|c|}
\hline Radionuclide & $\begin{array}{c}\text { External } \\
\text { (mrem) } \\
\end{array}$ & $\begin{array}{c}\text { Inhalation } \\
\text { (mrem) }\end{array}$ & $\begin{array}{c}\text { Food } \\
\text { Ingestion } \\
\text { (mrem) }\end{array}$ & $\begin{array}{c}\text { TEDE } \\
\text { (mrem) }\end{array}$ & $\begin{array}{l}\text { TEDE } \\
(\mu \text { S })\end{array}$ \\
\hline${ }^{69 m} \mathrm{Zn}$ & $\begin{array}{l}1.6 \mathrm{E}+00 \\
1.6 \mathrm{E}-03\end{array}$ & $\begin{array}{l}3.4 \mathrm{E}-07 \\
1.7 \mathrm{E}-08\end{array}$ & $\begin{array}{l}3.0 \mathrm{E}-02 \\
1.6 \mathrm{E}-03\end{array}$ & $\begin{array}{l}1.6 E+00 \\
3.3 E-03\end{array}$ & $\begin{array}{l}4.4 E+02 \\
8.9 E-01\end{array}$ \\
\hline${ }^{76}$ As & $1.6 \mathrm{E}+00$ & $1.7 \mathrm{E}-06$ & $5.3 E-04$ & $1.6 \mathrm{E}+00$ & $4.3 E+02$ \\
\hline $\begin{array}{l}{ }^{75} \mathrm{Se} \\
{ }^{79} \mathrm{Se}\end{array}$ & $\begin{array}{l}9.5 \mathrm{E}-01 \\
5.2 \mathrm{E}-06\end{array}$ & $\begin{array}{l}3.3 E-06 \\
3.9 E-06\end{array}$ & $\begin{array}{l}1.2 \mathrm{E}-01 \\
1.4 \mathrm{E}-01\end{array}$ & $\begin{array}{l}1.1 \mathrm{E}+00 \\
1.4 \mathrm{E}-01\end{array}$ & $\begin{array}{l}2.9 E+02 \\
3.8 E+01\end{array}$ \\
\hline $\begin{array}{l}{ }^{82} \mathrm{Br} \\
{ }^{83} \mathrm{Br}+\mathrm{D} \\
{ }^{83} \mathrm{Br} \\
{ }^{84} \mathrm{Br}\end{array}$ & $\begin{array}{l}8.8 \mathrm{E}+00 \\
1.3 \mathrm{E}-02 \\
2.6 \mathrm{E}-02 \\
5.6 \mathrm{E}+00\end{array}$ & $\begin{array}{l}4.8 \mathrm{E}-07 \\
2.0 \mathrm{E}-08 \\
3.9 \mathrm{E}-08 \\
4.1 \mathrm{E}-08\end{array}$ & $\begin{array}{l}1.5 \mathrm{E}-02 \\
3.5 \mathrm{E}-04 \\
7.0 \mathrm{E}-04 \\
1.3 \mathrm{E}-03\end{array}$ & $\begin{array}{l}8.8 \mathrm{E}+00 \\
1.3 \mathrm{E}-02 \\
2.6 \mathrm{E}-02 \\
5.6 \mathrm{E}+00\end{array}$ & $\begin{array}{l}2.4 \mathrm{E}+03 \\
3.5 \mathrm{E}+00 \\
7.1 \mathrm{E}+00 \\
1.5 \mathrm{E}+03\end{array}$ \\
\hline $\begin{array}{l}{ }^{86} \mathrm{Rb} \\
{ }^{88} \mathrm{Rb} \\
{ }^{89} \mathrm{Rb}+\mathrm{D} \\
{ }^{89} \mathrm{Rb}\end{array}$ & $\begin{array}{l}3.0 \mathrm{E}-01 \\
2.3 \mathrm{E}+00 \\
3.1 \mathrm{E}+00 \\
6.6 \mathrm{E}+00\end{array}$ & $\begin{array}{l}2.5 \mathrm{E}-06 \\
3.6 \mathrm{E}-08 \\
1.2 \mathrm{E}-06 \\
1.8 \mathrm{E}-08\end{array}$ & $\begin{array}{l}1.6 \mathrm{E}-01 \\
1.1 \mathrm{E}-03 \\
6.9 \mathrm{E}-02 \\
5.8 \mathrm{E}-04\end{array}$ & $\begin{array}{l}4.5 \mathrm{E}-01 \\
2.3 \mathrm{E}+00 \\
3.2 \mathrm{E}+00 \\
6.6 \mathrm{E}+00\end{array}$ & $\begin{array}{l}1.2 \mathrm{E}+02 \\
6.1 \mathrm{E}+02 \\
8.6 \mathrm{E}+02 \\
1.8 \mathrm{E}+03\end{array}$ \\
\hline $\begin{array}{l}{ }^{85} \mathrm{Sr} \\
{ }^{89} \mathrm{Sr} \\
{ }^{96} \mathrm{Sr+D}(\mathrm{C}) \\
{ }^{96} \mathrm{Sr} \\
{ }^{91} \mathrm{Sr+D}(\mathrm{C}) \\
{ }^{91} \mathrm{Sr} \\
{ }^{92} \mathrm{Sr+D}(\mathrm{C}) \\
{ }^{92} \mathrm{Sr}\end{array}$ & $\begin{array}{l}1.7 E+00 \\
6.9 E-03 \\
9.6 E-03 \\
3.5 E-04 \\
1.3 E+00 \\
2.2 E+00 \\
2.6 E+00 \\
4.5 E+00\end{array}$ & $\begin{array}{l}6.1 \mathrm{E}-07 \\
2.3 \mathrm{E}-06 \\
4.6 \mathrm{E}-05 \\
8.8 \mathrm{E}-05 \\
8.2 \mathrm{E}-06 \\
3.8 \mathrm{E}-07 \\
3.1 \mathrm{E}-07 \\
2.6 \mathrm{E}-07\end{array}$ & $\begin{array}{l}2.8 \mathrm{E}-02 \\
1.4 \mathrm{E}-01 \\
9.6 \mathrm{E}-01 \\
1.9 \mathrm{E}+00 \\
1.4 \mathrm{E}-02 \\
3.3 \mathrm{E}-02 \\
1.1 \mathrm{E}-02 \\
2.2 \mathrm{E}-02\end{array}$ & $\begin{array}{l}1.7 \mathrm{E}+00 \\
1.4 \mathrm{E}-01 \\
9.7 \mathrm{E}-01 \\
1.9 \mathrm{E}+00 \\
1.3 \mathrm{E}+00 \\
2.2 \mathrm{E}+00 \\
2.6 \mathrm{E}+00 \\
4.5 \mathrm{E}+00\end{array}$ & $\begin{array}{l}4.6 E+02 \\
3.9 E+01 \\
2.6 E+02 \\
5.2 E+02 \\
3.6 E+02 \\
6.0 E+02 \\
7.1 E+02 \\
1.2 E+03\end{array}$ \\
\hline $\begin{array}{l}{ }^{96}{ }_{Y} \\
{ }^{91 m_{Y}} Y+D \\
{ }^{91 m_{Y}}(c) \\
{ }^{91} Y \\
{ }^{92} Y \\
{ }^{93} Y\end{array}$ & $\begin{array}{l}1.9 \mathrm{E}-02 \\
1.0 \mathrm{E}+00 \\
2.1 \mathrm{E}+00 \\
1.9 \mathrm{E}-02 \\
9.3 \mathrm{E}-01 \\
3.2 \mathrm{E}-01\end{array}$ & $\begin{array}{l}3.7 \mathrm{E}-06 \\
1.0 \mathrm{E}-05 \\
1.6 \mathrm{E}-08 \\
2.1 \mathrm{E}-05 \\
3.5 \mathrm{E}-07 \\
9.7 \mathrm{E}-07\end{array}$ & $\begin{array}{l}1.3 \mathrm{E}-03 \\
5.6 \mathrm{E}-04 \\
4.8 \mathrm{E}-06 \\
1.1 \mathrm{E}-03 \\
2.3 \mathrm{E}-04 \\
5.5 \mathrm{E}-04\end{array}$ & $\begin{array}{l}2.1 \mathrm{E}-02 \\
1.0 \mathrm{E}+00 \\
2.1 \mathrm{E}+00 \\
2.0 \mathrm{E}-02 \\
9.3 \mathrm{E}-01 \\
3.2 \mathrm{E}-01\end{array}$ & $\begin{array}{l}5.6 \mathrm{E}+00 \\
2.7 \mathrm{E}+02 \\
5.6 \mathrm{E}+02 \\
5.3 \mathrm{E}+00 \\
2.5 \mathrm{E}+02 \\
8.5 \mathrm{E}+01\end{array}$ \\
\hline $\begin{array}{l}{ }^{93} \mathrm{Zr+D}(\mathrm{c}) \\
{ }^{93} \mathrm{Zr} \\
{ }^{95} \mathrm{Zr+D}(\mathrm{c}) \\
{ }^{95} \mathrm{Zr} \\
{ }^{97} \mathrm{Zr}(\mathrm{c}) \\
{ }^{97} \mathrm{Zr}\end{array}$ & $\begin{array}{l}2.5 \mathrm{E}-05 \\
1.3 \mathrm{E}-07 \\
2.4 \mathrm{E}+00 \\
2.4 \mathrm{E}+00 \\
1.6 \mathrm{E}+00 \\
6.0 \mathrm{E}-01\end{array}$ & $\begin{array}{l}2.4 \mathrm{E}-05 \\
3.5 \mathrm{E}-05 \\
4.4 \mathrm{E}-06 \\
6.6 \mathrm{E}-06 \\
5.8 \mathrm{E}-07 \\
1.7 \mathrm{E}-06\end{array}$ & $\begin{array}{l}8.9 \mathrm{E}-04 \\
2.4 \mathrm{E}-05 \\
1.1 \mathrm{E}-03 \\
3.8 \mathrm{E}-05 \\
5.9 \mathrm{E}-05 \\
6.5 \mathrm{E}-05\end{array}$ & $\begin{array}{l}9.4 \mathrm{E}-04 \\
5.9 \mathrm{E}-05 \\
2.4 \mathrm{E}+00 \\
2.4 \mathrm{E}+00 \\
1.6 \mathrm{E}+00 \\
6.0 \mathrm{E}-01\end{array}$ & $\begin{array}{l}2.5 \mathrm{E}-01 \\
1.6 \mathrm{E}-02 \\
6.5 \mathrm{E}+02 \\
6.4 \mathrm{E}+02 \\
4.3 \mathrm{E}+02 \\
1.6 \mathrm{E}+02\end{array}$ \\
\hline $\begin{array}{l}{ }^{93 m} \mathrm{Nb} \\
{ }_{94}^{94} \mathrm{Nb} \\
{ }^{95 m} \mathrm{Nb}+\mathrm{D} \\
\mathrm{Nb}\end{array}$ & $\begin{array}{l}5.1 E-05 \\
4.8 E+00 \\
1.3 E+00 \\
1.6 E-01\end{array}$ & $\begin{array}{l}1.3 \mathrm{E}-05 \\
1.6 \mathrm{E}-04 \\
1.7 \mathrm{E}-06 \\
1.1 \mathrm{E}-06\end{array}$ & $\begin{array}{l}1.8 E-03 \\
2.5 E-02 \\
1.7 E-03 \\
1.1 E-03\end{array}$ & $\begin{array}{l}1.8 \mathrm{E}-03 \\
4.8 \mathrm{E}+00 \\
1.3 \mathrm{E}+00 \\
1.6 \mathrm{E}-01\end{array}$ & $\begin{array}{l}4.9 E-01 \\
1.3 E+03 \\
3.6 E+02 \\
4.4 E+01\end{array}$ \\
\hline
\end{tabular}


TABLE 3.3. Annual Total Effective Dose Equivalent Factors for the Residential Use (Surface Soil) Scenario(a) (contd)

\begin{tabular}{|c|c|c|c|c|c|}
\hline Radionuclide & $\begin{array}{c}\text { External } \\
\text { (mrem) }\end{array}$ & $\begin{array}{c}\text { Inhalation } \\
\text { (mrem) } \\
\end{array}$ & $\begin{array}{c}\text { Food (b) } \\
\text { Ingestion } \\
\text { (mrem) } \\
\end{array}$ & $\begin{array}{c}\text { TEDE } \\
\text { (mrem) }\end{array}$ & $\begin{array}{l}\text { TEDE } \\
(\mu S v)\end{array}$ \\
\hline $\begin{array}{l}{ }^{95} \mathrm{Nb} \\
{ }_{97 m}^{97} \mathrm{Nb}+\mathrm{D} \\
{ }^{97} \mathrm{Nb} \\
{ }_{\mathrm{Nb}}\end{array}$ & $\begin{array}{l}2.6 E+00 \\
2.1 E+00 \\
2.1 E+00 \\
2.2 E+00\end{array}$ & $\begin{array}{l}2.4 \mathrm{E}-06 \\
1.8 \mathrm{E}-08 \\
5.3 \mathrm{E}-10 \\
3.6 \mathrm{E}-08\end{array}$ & $\begin{array}{l}2.2 \mathrm{E}-03 \\
5.4 \mathrm{E}-05 \\
1.7 \mathrm{E}-06 \\
1.1 \mathrm{E}-04\end{array}$ & $\begin{array}{l}2.6 \mathrm{E}+00 \\
2.1 \mathrm{E}+00 \\
2.1 \mathrm{E}+00 \\
2.2 \mathrm{E}+00\end{array}$ & $\begin{array}{l}7.0 \mathrm{E}+02 \\
5.7 \mathrm{E}+02 \\
5.7 \mathrm{E}+02 \\
5.9 \mathrm{E}+02\end{array}$ \\
\hline $\begin{array}{l}{ }^{93} \mathrm{Mo+D} \\
{ }^{83} \mathrm{Mo} \\
{ }^{99} \mathrm{Mo} \\
{ }_{99}{ }_{\mathrm{Mo}}(\mathrm{C})\end{array}$ & $\begin{array}{l}1.5 \mathrm{E}-04 \\
2.5 \mathrm{E}-04 \\
3.3 \mathrm{E}-01 \\
4.5 \mathrm{E}-01\end{array}$ & $\begin{array}{l}6.6 \mathrm{E}-06 \\
4.2 \mathrm{E}-07 \\
4.7 \mathrm{E}-07 \\
8.3 \mathrm{E}-07\end{array}$ & $\begin{array}{l}6.0 \mathrm{E}-03 \\
1.0 \mathrm{E}-02 \\
1.2 \mathrm{E}-02 \\
1.9 \mathrm{E}-02\end{array}$ & $\begin{array}{l}6.2 \mathrm{E}-03 \\
1.0 \mathrm{E}-02 \\
3.4 \mathrm{E}-01 \\
4.7 \mathrm{E}-01\end{array}$ & $\begin{array}{l}1.7 E+00 \\
2.8 E+00 \\
9.3 E+01 \\
1.3 E+02\end{array}$ \\
\hline $\begin{array}{l}{ }^{99 m} \mathrm{Tc} \\
{ }_{99}^{99} \mathrm{TC} \\
{ }^{161} \mathrm{TC}\end{array}$ & $\begin{array}{l}2.2 E-01 \\
4.9 E-05 \\
1.2 E+00\end{array}$ & $\begin{array}{l}1.4 \mathrm{E}-08 \\
3.9 \mathrm{E}-06 \\
6.6 \mathrm{E}-09\end{array}$ & $\begin{array}{l}\text { 4. 3E-03 } \\
3.0 E-01 \\
4.0 E-03\end{array}$ & $\begin{array}{l}2.3 E-01 \\
3.0 E-01 \\
1.2 E+00\end{array}$ & $\begin{array}{l}6.2 E+01 \\
8.1 E+01 \\
3.4 E+02\end{array}$ \\
\hline $\begin{array}{l}{ }_{163}^{16 u+D}(c) \\
{ }^{163} \mathrm{Ru} \\
{ }^{105} \mathrm{Ru+D}(\mathrm{c}) \\
{ }^{165} \mathrm{Ru+I}(\mathrm{d}) \\
{ }^{160} \mathrm{Ru+I}(\mathrm{d})\end{array}$ & $\begin{array}{l}8.1 E-01 \\
1.7 E+00 \\
9.2 E-01 \\
2.5 E+00 \\
7.5 E-01\end{array}$ & $\begin{array}{l}1.9 E-06 \\
3.8 E-06 \\
2.2 E-07 \\
2.3 E-07 \\
9.9 E-05\end{array}$ & $\begin{array}{l}8.8 E-03 \\
1.7 E-02 \\
6.9 E-03 \\
3.0 E-03 \\
2.9 E-01\end{array}$ & $\begin{array}{l}8.2 E-01 \\
1.7 E+00 \\
9.3 E-01 \\
2.5 E+00 \\
1.0 E+00\end{array}$ & $\begin{array}{l}2.2 E+02 \\
4.5 E+02 \\
2.5 E+02 \\
6.9 E+02 \\
2.8 E+02\end{array}$ \\
\hline $\begin{array}{l}{ }^{103 m} \mathrm{Rh} \\
{ }^{105} \mathrm{Rh}\end{array}$ & $\begin{array}{l}3.3 E-04 \\
2.8 E-01\end{array}$ & $\begin{array}{l}2.1 E-09 \\
4.2 E-07\end{array}$ & $\begin{array}{l}1.4 \mathrm{E}-04 \\
1.8 \mathrm{E}-02\end{array}$ & $\begin{array}{l}4.7 E-04 \\
3.0 E-01\end{array}$ & $\begin{array}{l}1.3 E-01 \\
8.1 E+01\end{array}$ \\
\hline $\begin{array}{l}{ }_{{ }_{103}^{103} \mathrm{Pd}+\mathrm{D}}(\mathrm{c}) \\
{ }_{107} \mathrm{Pd} \\
{ }_{109} \mathrm{Pd} \\
{ }^{16 d+I}(\mathrm{~d})\end{array}$ & $\begin{array}{l}1.7 \mathrm{E}-03 \\
3.1 \mathrm{E}-03 \\
4.1 \mathrm{E}-09 \\
9.5 \mathrm{E}-03\end{array}$ & $\begin{array}{l}3.3 E-07 \\
6.6 E-07 \\
5.3 E-06 \\
2.9 E-07\end{array}$ & $\begin{array}{l}1.2 \mathrm{E}-03 \\
2.2 \mathrm{E}-03 \\
4.3 \mathrm{E}-04 \\
3.3 \mathrm{E}-03\end{array}$ & $\begin{array}{l}2.8 E-03 \\
5.3 E-03 \\
4.3 E-04 \\
1.3 E-02\end{array}$ & $\begin{array}{l}7.6 \mathrm{E}-01 \\
1.4 \mathrm{E}+00 \\
1.2 \mathrm{E}-01 \\
3.4 \mathrm{E}+00\end{array}$ \\
\hline 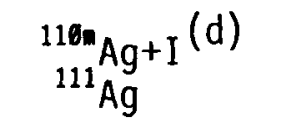 & $\begin{array}{l}9.0 E+00 \\
8.9 E-02\end{array}$ & $\begin{array}{l}1.8 E-05 \\
1.4 E-06\end{array}$ & $\begin{array}{l}5.3 \mathrm{E}-01 \\
1.3 \mathrm{E}-01\end{array}$ & $\begin{array}{l}9.5 \mathrm{E}+00 \\
2.2 \mathrm{E}-01\end{array}$ & $\begin{array}{l}2.6 E+03 \\
5.9 E+01\end{array}$ \\
\hline $\begin{array}{l}{ }^{169} \mathrm{Cd} \\
{ }_{113 m} \mathrm{Cd} \\
{ }_{115 m} \mathrm{Cd}(\mathrm{C}) \\
{ }^{116{ }^{m}} \mathrm{Cd} \\
{ }^{115} \mathrm{Cd}\end{array}$ & $\begin{array}{l}2.6 \mathrm{E}-03 \\
4.2 \mathrm{E}-04 \\
3.9 \mathrm{E}-02 \\
8.1 \mathrm{E}-02 \\
6.6 \mathrm{E}-01\end{array}$ & $\begin{array}{l}4.8 E-05 \\
6.1 E-04 \\
1.5 E-05 \\
3.0 E-05 \\
1.7 E-06\end{array}$ & $\begin{array}{l}2.2 \mathrm{E}-01 \\
2.8 \mathrm{E}+00 \\
1.4 \mathrm{E}-01 \\
2.8 \mathrm{E}-01 \\
9.5 \mathrm{E}-02\end{array}$ & $\begin{array}{l}2.2 \mathrm{E}-01 \\
2.8 \mathrm{E}+00 \\
1.8 \mathrm{E}-01 \\
3.6 \mathrm{E}-01 \\
7.6 \mathrm{E}-01\end{array}$ & $\begin{array}{l}5.9 E+01 \\
7.4 E+02 \\
4.8 E+01 \\
9.6 E+01 \\
2.0 E+02\end{array}$ \\
\hline $\begin{array}{l}{ }^{111} \operatorname{In}_{\ln }(d) \\
{ }_{115 m} \operatorname{In}+I\end{array}$ & $\begin{array}{l}8.3 E-01 \\
3.6 E-01 \\
5.4 E-01\end{array}$ & $\begin{array}{l}2.4 \mathrm{E}-07 \\
9.9 \mathrm{E}-06 \\
5.3 \mathrm{E}-08\end{array}$ & $\begin{array}{l}1.0 E-04 \\
6.1 E-04 \\
2.8 E-05\end{array}$ & $\begin{array}{l}8.3 E-01 \\
3.6 E-01 \\
5.4 E-01\end{array}$ & $\begin{array}{l}2.3 E+02 \\
9.8 E+01 \\
1.5 E+02\end{array}$ \\
\hline $\begin{array}{l}{ }_{119 m}^{11 m} S n \\
{ }^{119 m} S n \\
{ }^{121 m} S n+D \\
S n\end{array}$ & $\begin{array}{l}2.3 \mathrm{E}-01 \\
1.8 \mathrm{E}-03 \\
0.0 \mathrm{E}+00 \\
0.0 \mathrm{E}+00\end{array}$ & $\begin{array}{l}1.8 \mathrm{E}-06 \\
2.5 \mathrm{E}-06 \\
2.7 \mathrm{E}-06 \\
4.8 \mathrm{E}-06\end{array}$ & $\begin{array}{l}2.4 \mathrm{E}-03 \\
1.2 \mathrm{E}-03 \\
1.0 \mathrm{E}-03 \\
1.2 \mathrm{E}-03\end{array}$ & $\begin{array}{l}2.3 \mathrm{E}-01 \\
2.9 \mathrm{E}-03 \\
1.0 \mathrm{E}-03 \\
1.2 \mathrm{E}-03\end{array}$ & $\begin{array}{l}6.1 \mathrm{E}+01 \\
7.9 \mathrm{E}-01 \\
2.7 \mathrm{E}-01 \\
3.3 \mathrm{E}-01\end{array}$ \\
\hline
\end{tabular}


TABLE 3.3. Annual Total Effective Dose Equivalent Factors for the Residential Use (Surface Soil) Scenario(a) (contd)

\begin{tabular}{|c|c|c|c|c|c|}
\hline Radionuclide & $\begin{array}{c}\text { External } \\
\text { (mrem) }\end{array}$ & $\begin{array}{c}\text { Inhalation } \\
\text { (mrem) }\end{array}$ & $\begin{array}{c}\text { Food } \\
\text { Ingestion } \\
\text { (mrem) }\end{array}$ & $\begin{array}{c}\text { TEDE } \\
\text { (mrem) }\end{array}$ & $\begin{array}{l}\text { TEDE } \\
(\mu \text { Sv) }\end{array}$ \\
\hline $\begin{array}{l}{ }^{121} S_{n} \\
{ }^{123} S_{n} \\
{ }^{125} S_{n+D}(c) \\
{ }^{126}{ }_{S n+D}(c) \\
{ }^{126} S_{n}\end{array}$ & $\begin{array}{l}0.0 \mathrm{E}+00 \\
2.6 \mathrm{E}-02 \\
1.1 \mathrm{E}+00 \\
1.0 \mathrm{E}+00 \\
3.2 \mathrm{E}+00 \\
5.1 \mathrm{E}-02\end{array}$ & $\begin{array}{l}2.1 \mathrm{E}-07 \\
1.4 \mathrm{E}-05 \\
5.7 \mathrm{E}-06 \\
6.6 \mathrm{E}-06 \\
2.0 \mathrm{E}-05 \\
4.1 \mathrm{E}-05\end{array}$ & $\begin{array}{l}7.3 \mathrm{E}-04 \\
7.0 \mathrm{E}-03 \\
4.3 \mathrm{E}-02 \\
9.8 \mathrm{E}-03 \\
7.8 \mathrm{E}-03 \\
1.6 \mathrm{E}-02\end{array}$ & $\begin{array}{l}7.3 E-04 \\
3.3 E-02 \\
1.1 E+00 \\
1.0 E+00 \\
3.2 E+00 \\
6.7 E-02\end{array}$ & $\begin{array}{l}2.0 \mathrm{E}-01 \\
9.0 \mathrm{E}+00 \\
3.1 \mathrm{E}+02 \\
2.7 \mathrm{E}+02 \\
8.6 \mathrm{E}+02 \\
1.8 \mathrm{E}+01\end{array}$ \\
\hline $\begin{array}{l}{ }^{124} \mathrm{Sb} \\
{ }^{125} \mathrm{Sb}+\mathrm{D} \\
{ }^{125} \mathrm{Sb} \\
{ }^{126 m} \mathrm{Sb}+\mathrm{c}(\mathrm{c}) \\
{ }^{126 \mathrm{~m}} \mathrm{Sb} \\
{ }^{126} \mathrm{Sb} \\
{ }^{127} \mathrm{Sb}+\mathrm{D} \\
{ }_{\mathrm{Sb}}(\mathrm{c})\end{array}$ & $\begin{array}{l}6.3 \mathrm{E}+00 \\
1.2 \mathrm{E}+00 \\
1.5 \mathrm{E}+00 \\
5.5 \mathrm{E}+00 \\
5.4 \mathrm{E}+00 \\
9.0 \mathrm{E}+00 \\
9.9 \mathrm{E}-01 \\
2.2 \mathrm{E}+00\end{array}$ & $\begin{array}{l}1.1 \mathrm{E}-05 \\
4.6 \mathrm{E}-06 \\
5.3 \mathrm{E}-06 \\
6.5 \mathrm{E}-07 \\
1.3 \mathrm{E}-08 \\
4.8 \mathrm{E}-06 \\
1.9 \mathrm{E}-06 \\
2.5 \mathrm{E}-06\end{array}$ & $\begin{array}{l}5.0 \mathrm{E}-03 \\
7.1 \mathrm{E}-02 \\
1.4 \mathrm{E}-03 \\
6.8 \mathrm{E}-04 \\
4.5 \mathrm{E}-05 \\
5.0 \mathrm{E}-03 \\
1.1 \mathrm{E}-01 \\
3.5 \mathrm{E}-03\end{array}$ & $\begin{array}{l}6.3 \mathrm{E}+00 \\
1.3 \mathrm{E}+00 \\
1.5 \mathrm{E}+00 \\
5.5 \mathrm{E}+00 \\
5.4 \mathrm{E}+00 \\
9.0 \mathrm{E}+00 \\
1.1 \mathrm{E}+00 \\
2.2 \mathrm{E}+00\end{array}$ & $\begin{array}{l}1.7 \mathrm{E}+03 \\
3.4 \mathrm{E}+02 \\
4.1 \mathrm{E}+02 \\
1.5 \mathrm{E}+03 \\
1.4 \mathrm{E}+03 \\
2.4 \mathrm{E}+03 \\
2.9 \mathrm{E}+02 \\
5.9 \mathrm{E}+02\end{array}$ \\
\hline 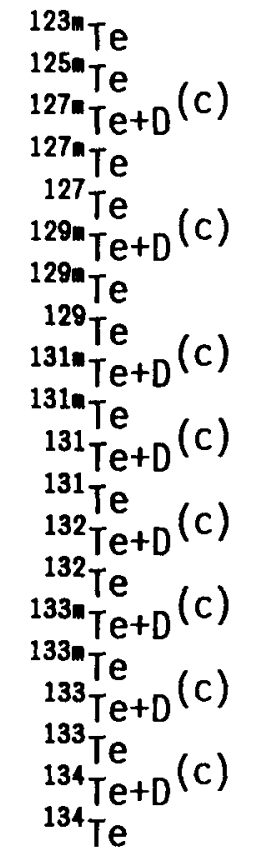 & $\begin{array}{l}2.1 \mathrm{E}-01 \\
8.6 \mathrm{E}-03 \\
1.1 \mathrm{E}-02 \\
2.8 \mathrm{E}-03 \\
1.9 \mathrm{E}-02 \\
1.3 \mathrm{E}-01 \\
1.0 \mathrm{E}-01 \\
1.9 \mathrm{E}-01 \\
2.6 \mathrm{E}+00 \\
4.6 \mathrm{E}+00 \\
1.2 \mathrm{E}+00 \\
1.3 \mathrm{E}+00 \\
3.9 \mathrm{E}+00 \\
5.8 \mathrm{E}-01 \\
3.1 \mathrm{E}+00 \\
7.5 \mathrm{E}+00 \\
1.7 \mathrm{E}+00 \\
3.2 \mathrm{E}+00 \\
5.5 \mathrm{E}+00 \\
2.8 \mathrm{E}+00\end{array}$ & $\begin{array}{l}4.4 \mathrm{E}-06 \\
3.1 \mathrm{E}-06 \\
4.7 \mathrm{E}-06 \\
9.2 \mathrm{E}-06 \\
1.4 \mathrm{E}-07 \\
1.3 \mathrm{E}-04 \\
2.1 \mathrm{E}-04 \\
6.6 \mathrm{E}-08 \\
8.3 \mathrm{E}-06 \\
5.3 \mathrm{E}-06 \\
6.5 \mathrm{E}-06 \\
5.3 \mathrm{E}-08 \\
2.9 \mathrm{E}-06 \\
5.3 \mathrm{E}-06 \\
8.1 \mathrm{E}-07 \\
1.9 \mathrm{E}-07 \\
7.8 \mathrm{E}-07 \\
3.8 \mathrm{E}-08 \\
5.3 \mathrm{E}-08 \\
4.4 \mathrm{E}-08\end{array}$ & $\begin{array}{l}7.3 \mathrm{E}-01 \\
3.5 \mathrm{E}-01 \\
5.5 \mathrm{E}-01 \\
1.0 \mathrm{E}+00 \\
4.3 \mathrm{E}-02 \\
2.6 \mathrm{E}+01 \\
4.3 \mathrm{E}+01 \\
1.6 \mathrm{E}-02 \\
5.8 \mathrm{E}-01 \\
1.1 \mathrm{E}+00 \\
1.0 \mathrm{E}-01 \\
1.3 \mathrm{E}-02 \\
4.8 \mathrm{E}-01 \\
9.5 \mathrm{E}-01 \\
2.5 \mathrm{E}-02 \\
4.8 \mathrm{E}-02 \\
1.2 \mathrm{E}-02 \\
1.0 \mathrm{E}-02 \\
6.0 \mathrm{E}-03 \\
1.2 \mathrm{E}-02\end{array}$ & $\begin{array}{l}9.4 \mathrm{E}-01 \\
3.6 \mathrm{E}-01 \\
5.7 \mathrm{E}-01 \\
1.0 \mathrm{E}+00 \\
6.2 \mathrm{E}-02 \\
2.6 \mathrm{E}+01 \\
4.3 \mathrm{E}+01 \\
2.1 \mathrm{E}-01 \\
3.2 \mathrm{E}+00 \\
5.7 \mathrm{E}+00 \\
1.3 \mathrm{E}+00 \\
1.3 \mathrm{E}+00 \\
4.4 \mathrm{E}+00 \\
1.5 \mathrm{E}+00 \\
3.2 \mathrm{E}+00 \\
7.5 \mathrm{E}+00 \\
1.7 \mathrm{E}+00 \\
3.2 \mathrm{E}+00 \\
5.5 \mathrm{E}+00 \\
2.8 \mathrm{E}+00\end{array}$ & $\begin{array}{l}2.5 \mathrm{E}+02 \\
9.7 \mathrm{E}+01 \\
1.5 \mathrm{E}+02 \\
2.7 \mathrm{E}+02 \\
1.7 \mathrm{E}+01 \\
7.1 \mathrm{E}+03 \\
1.2 \mathrm{E}+04 \\
5.6 \mathrm{E}+01 \\
8.5 \mathrm{E}+02 \\
1.5 \mathrm{E}+03 \\
3.5 \mathrm{E}+02 \\
3.5 \mathrm{E}+02 \\
1.2 \mathrm{E}+03 \\
4.1 \mathrm{E}+02 \\
8.5 \mathrm{E}+02 \\
2.0 \mathrm{E}+03 \\
4.5 \mathrm{E}+02 \\
8.7 \mathrm{E}+02 \\
1.5 \mathrm{E}+03 \\
7.5 \mathrm{E}+02\end{array}$ \\
\hline $\begin{array}{l}{ }^{125} \mathrm{I} \\
{ }^{129} \mathrm{I} \\
{ }^{136} \mathrm{I} \\
{ }^{131} \mathrm{I}+\mathrm{D}(\mathrm{c}) \\
{ }^{131} \mathrm{I} \\
{ }^{132} \mathrm{I} \\
{ }^{133} \mathrm{I}+\mathrm{D}^{(\mathrm{c})} \\
{ }_{\mathrm{I}}^{133}\end{array}$ & $\begin{array}{l}9.3 \mathrm{E}-03 \\
5.4 \mathrm{E}-03 \\
6.9 \mathrm{E}+00 \\
1.1 \mathrm{E}+00 \\
1.2 \mathrm{E}+00 \\
7.6 \mathrm{E}+00 \\
9.5 \mathrm{E}-01 \\
1.9 \mathrm{E}+00\end{array}$ & $\begin{array}{l}9.7 \mathrm{E}-06 \\
6.6 \mathrm{E}-05 \\
1.1 \mathrm{E}-06 \\
1.3 \mathrm{E}-05 \\
1.3 \mathrm{E}-05 \\
1.7 \mathrm{E}-07 \\
1.1 \mathrm{E}-06 \\
2.3 \mathrm{E}-06\end{array}$ & $\begin{array}{l}1.6 \mathrm{E}-01 \\
1.2 \mathrm{E}+00 \\
1.2 \mathrm{E}-02 \\
2.0 \mathrm{E}-01 \\
2.0 \mathrm{E}-01 \\
1.8 \mathrm{E}-03 \\
1.4 \mathrm{E}-02 \\
2.8 \mathrm{E}-02\end{array}$ & $\begin{array}{l}1.6 \mathrm{E}-01 \\
1.2 \mathrm{E}+00 \\
6.9 \mathrm{E}+00 \\
1.3 \mathrm{E}+00 \\
1.4 \mathrm{E}+00 \\
7.6 \mathrm{E}+00 \\
9.6 \mathrm{E}-01 \\
2.0 \mathrm{E}+00\end{array}$ & $\begin{array}{l}4.4 E+01 \\
3.2 E+02 \\
1.9 E+03 \\
3.6 E+02 \\
3.8 E+02 \\
2.0 E+03 \\
2.6 E+02 \\
5.3 E+02\end{array}$ \\
\hline
\end{tabular}


TABLE 3.3. Annual Total Effective Dose Equivalent Factors for the Residential Use (Surface Soil) Scenario(a) (contd)

\begin{tabular}{|c|c|c|c|c|c|}
\hline Radionuclide & $\begin{array}{c}\text { External } \\
\text { (mrem) }\end{array}$ & $\begin{array}{c}\text { Inhalation } \\
\text { (mrem) }\end{array}$ & $\begin{array}{c}\text { Food }(b) \\
\text { Ingestion } \\
\text { (mrem) }\end{array}$ & $\begin{array}{c}\text { TEDE } \\
\text { (mrem) }\end{array}$ & $\begin{array}{l}\text { TEDE } \\
(\mu S v)\end{array}$ \\
\hline 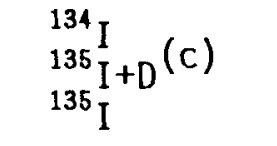 & $\begin{array}{l}8.3 E+00 \\
2.6 E+00 \\
5.1 E+00\end{array}$ & $\begin{array}{l}6.1 E-08 \\
2.2 E-07 \\
4.8 E-07\end{array}$ & $\begin{array}{l}6.8 E-04 \\
2.5 E-03 \\
5.5 E-03\end{array}$ & $\begin{array}{l}8.3 E+00 \\
2.7 E+00 \\
5.1 E+00\end{array}$ & $\begin{array}{l}2.3 E+03 \\
7.2 E+02 \\
1.4 E+03\end{array}$ \\
\hline $\begin{array}{l}{ }^{134 m} \mathrm{Cs}+\mathrm{D}^{(\mathrm{C})} \\
{ }^{134 \mathrm{~m}} \mathrm{Cs} \\
{ }^{134} \mathrm{Cs} \\
{ }^{136} \mathrm{Cs} \\
{ }^{138} \mathrm{Cs} \\
{ }^{137} \mathrm{Cs} \quad(\mathrm{d}) \\
{ }^{138} \mathrm{Cs}\end{array}$ & $\begin{array}{l}1.8 \mathrm{E}-02 \\
3.7 \mathrm{E}-02 \\
5.3 \mathrm{E}+00 \\
1.4 \mathrm{E}-05 \\
6.7 \mathrm{E}+00 \\
1.9 \mathrm{E}+00 \\
7.8 \mathrm{E}+00\end{array}$ & $\begin{array}{l}7.9 \mathrm{E}-09 \\
1.6 \mathrm{E}-08 \\
1.8 \mathrm{E}-05 \\
1.6 \mathrm{E}-06 \\
2.8 \mathrm{E}-06 \\
5.9 \mathrm{E}-06 \\
4.2 \mathrm{E}-08\end{array}$ & $\begin{array}{l}7.9 \mathrm{E}-06 \\
1.6 \mathrm{E}-05 \\
5.0 \mathrm{E}-02 \\
4.8 \mathrm{E}-03 \\
6.0 \mathrm{E}-03 \\
3.1 \mathrm{E}-03 \\
8.0 \mathrm{E}-05\end{array}$ & $\begin{array}{l}1.8 \mathrm{E}-02 \\
3.7 \mathrm{E}-02 \\
5.4 \mathrm{E}+00 \\
4.8 \mathrm{E}-03 \\
6.7 \mathrm{E}+00 \\
1.9 \mathrm{E}+00 \\
7.8 \mathrm{E}+00\end{array}$ & $\begin{array}{l}4.9 E+00 \\
1.0 E+01 \\
1.5 E+03 \\
1.3 E+00 \\
1.8 E+03 \\
5.0 E+02 \\
2.1 E+03\end{array}$ \\
\hline $\begin{array}{l}{ }^{139} \mathrm{Ba} \\
{ }^{140} \mathrm{Ba}+\mathrm{D} \\
{ }^{141} \mathrm{Ba} \\
{ }^{141} \mathrm{Ba}+\mathrm{C} \\
{ }^{141} \mathrm{Ba} \\
\left.{ }^{142} \mathrm{Ba}+\mathrm{C}\right) \\
{ }^{142} \mathrm{Ba}\end{array}$ & $\begin{array}{l}7.9 \mathrm{E}-02 \\
3.9 \mathrm{E}+00 \\
6.0 \mathrm{E}-01 \\
9.7 \mathrm{E}-01 \\
2.7 \mathrm{E}+00 \\
5.7 \mathrm{E}+00 \\
2.8 \mathrm{E}+00\end{array}$ & $\begin{array}{l}7.5 \mathrm{E}-08 \\
1.4 \mathrm{E}-06 \\
1.3 \mathrm{E}-06 \\
1.3 \mathrm{E}-06 \\
3.4 \mathrm{E}-08 \\
6.6 \mathrm{E}-08 \\
1.7 \mathrm{E}-08\end{array}$ & $\begin{array}{l}9.8 \mathrm{E}-05 \\
1.6 \mathrm{E}-03 \\
2.2 \mathrm{E}-03 \\
1.8 \mathrm{E}-04 \\
5.0 \mathrm{E}-05 \\
5.4 \mathrm{E}-05 \\
2.5 \mathrm{E}-05\end{array}$ & $\begin{array}{l}8.0 \mathrm{E}-02 \\
3.9 \mathrm{E}+00 \\
6.0 \mathrm{E}-01 \\
9.7 \mathrm{E}-01 \\
2.7 \mathrm{E}+00 \\
5.7 \mathrm{E}+00 \\
2.8 \mathrm{E}+00\end{array}$ & $\begin{array}{l}2.1 E+01 \\
1.1 E+03 \\
1.6 E+02 \\
2.6 E+02 \\
7.3 E+02 \\
1.5 E+03 \\
7.7 E+02\end{array}$ \\
\hline $\begin{array}{l}{ }^{146} \mathrm{La} \\
141 \mathrm{La}+\mathrm{D} \\
141 \mathrm{La} \\
{ }^{142} \mathrm{La}\end{array}$ & $\begin{array}{l}7.1 \mathrm{E}+00 \\
1.4 \mathrm{E}-01 \\
1.7 \mathrm{E}-01 \\
8.9 \mathrm{E}+00\end{array}$ & $\begin{array}{l}1.5 \mathrm{E}-06 \\
2.0 \mathrm{E}-06 \\
2.5 \mathrm{E}-07 \\
1.1 \mathrm{E}-07\end{array}$ & $\begin{array}{l}9.5 E-04 \\
2.5 E-04 \\
1.7 E-04 \\
8.3 E-05\end{array}$ & $\begin{array}{l}7.1 \mathrm{E}+00 \\
1.4 \mathrm{E}-01 \\
1.7 \mathrm{E}-01 \\
8.9 \mathrm{E}+00\end{array}$ & $\begin{array}{l}1.9 E+03 \\
3.9 E+01 \\
4.5 E+01 \\
2.4 E+03\end{array}$ \\
\hline $\begin{array}{l}{ }^{141} \mathrm{Ce} \\
{ }_{13}^{143} \mathrm{Ce}+\mathrm{D} \\
{ }_{143} \mathrm{Ce} \\
{ }^{144} \mathrm{Ce} \text { (c) } \\
{ }^{144} \mathrm{Ce}\end{array}$ & $\begin{array}{l}1.2 E-01 \\
3.4 E-01 \\
6.7 E-01 \\
8.0 E-02 \\
3.0 E-02\end{array}$ & $\begin{array}{l}3.7 \mathrm{E}-06 \\
2.4 \mathrm{E}-06 \\
1.5 \mathrm{E}-06 \\
7.8 \mathrm{E}-05 \\
1.6 \mathrm{E}-04\end{array}$ & $\begin{array}{l}3.5 E-04 \\
5.4 E-04 \\
5.3 E-04 \\
1.2 E-03 \\
2.5 E-03\end{array}$ & $\begin{array}{l}1.2 \mathrm{E}-01 \\
3.4 \mathrm{E}-01 \\
6.7 \mathrm{E}-01 \\
8.2 \mathrm{E}-02 \\
3.3 \mathrm{E}-02\end{array}$ & $\begin{array}{l}3.4 E+01 \\
9.2 E+01 \\
1.8 E+02 \\
2.2 E+01 \\
8.9 E+00\end{array}$ \\
\hline $\begin{array}{l}{ }_{143}^{144} \mathrm{Pr} \\
{ }_{144 m} \mathrm{Pr}+\mathrm{D} \\
{ }_{144} \mathrm{Pr}\end{array}$ & $\begin{array}{l}1.6 \mathrm{E}-03 \\
7.0 \mathrm{E}-02 \\
5.2 \mathrm{E}-03 \\
1.4 \mathrm{E}-01\end{array}$ & $\begin{array}{l}3.4 \mathrm{E}-06 \\
1.4 \mathrm{E}-08 \\
7.9 \mathrm{E}-09 \\
1.9 \mathrm{E}-08\end{array}$ & $\begin{array}{l}5.5 E-04 \\
9.8 E-06 \\
5.5 E-06 \\
1.4 E-05\end{array}$ & $\begin{array}{l}2.2 \mathrm{E}-03 \\
7.0 \mathrm{E}-02 \\
5.2 \mathrm{E}-03 \\
1.4 \mathrm{E}-01\end{array}$ & $\begin{array}{l}5.9 E-01 \\
1.9 E+01 \\
1.4 E+00 \\
3.7 E+01\end{array}$ \\
\hline${ }^{147} \mathrm{Nd}+\mathrm{D}^{(\mathrm{c})}$ & $\begin{array}{l}1.7 \mathrm{E}-01 \\
3.6 \mathrm{E}-01\end{array}$ & $\begin{array}{l}9.7 E-06 \\
2.9 E-06\end{array}$ & $\begin{array}{l}2.8 E-04 \\
4.8 E-04\end{array}$ & $\begin{array}{l}1.7 \mathrm{E}-01 \\
3.6 \mathrm{E}-01\end{array}$ & $\begin{array}{l}4.7 E+01 \\
9.6 E+01\end{array}$ \\
\hline $\begin{array}{l}{ }^{147} \mathrm{Pm} \\
{ }_{148 m} \mathrm{Pm}+\mathrm{D} \\
{ }_{148 m} \mathrm{Pm} \\
{ }^{148} \mathrm{Pm} \\
{ }^{149} \mathrm{Pm} \\
{ }^{151} \mathrm{Pm}\end{array}$ & $\begin{array}{l}2.7 \mathrm{E}-05 \\
6.5 \mathrm{E}+00 \\
7.0 \mathrm{E}+00 \\
2.0 \mathrm{E}+00 \\
3.1 \mathrm{E}-02 \\
1.0 \mathrm{E}+00\end{array}$ & $\begin{array}{l}1.7 \mathrm{E}-05 \\
9.3 \mathrm{E}-06 \\
9.7 \mathrm{E}-06 \\
4.8 \mathrm{E}-06 \\
1.3 \mathrm{E}-06 \\
7.9 \mathrm{E}-07\end{array}$ & $\begin{array}{l}8.3 E-05 \\
6.0 E-04 \\
6.0 E-04 \\
8.8 E-04 \\
3.3 E-04 \\
2.4 E-04\end{array}$ & $\begin{array}{l}1.3 E-04 \\
6.5 E+00 \\
7.0 E+00 \\
2.0 E+00 \\
3.2 E-02 \\
1.0 E+00\end{array}$ & $\begin{array}{l}3.4 E-02 \\
1.8 E+03 \\
1.9 E+03 \\
5.3 E+02 \\
8.5 E+00 \\
2.7 E+02\end{array}$ \\
\hline
\end{tabular}


TABLE 3.3. Annual Total Effective Dose Equivalent Factors for the Residential Use (Surface Soi1) Scenario(a) (contd)

\begin{tabular}{|c|c|c|c|c|c|}
\hline Radionuclide & $\begin{array}{c}\text { External } \\
\text { (mrem) }\end{array}$ & $\begin{array}{c}\text { Inhalation } \\
\text { (mrem) }\end{array}$ & $\begin{array}{c}\text { Food }(b) \\
\text { Ingestion } \\
\text { (mrem) }\end{array}$ & $\begin{array}{c}\text { TEDE } \\
\text { (mrem) }\end{array}$ & $\begin{array}{l}\text { TEDE } \\
(\mu S v)\end{array}$ \\
\hline $\begin{array}{l}{ }^{147} \mathrm{Sm} \\
{ }^{153} \mathrm{Sm} \\
{ }^{15 m}\end{array}$ & $\begin{array}{l}0.0 E+00 \\
1.9 E-06 \\
9.4 E-02\end{array}$ & $\begin{array}{l}3.2 E-02 \\
1.3 E-05 \\
8.3 E-07\end{array}$ & $\begin{array}{l}2.0 E-02 \\
4.0 E-05 \\
3.0 E-04\end{array}$ & $\begin{array}{l}5.2 E-02 \\
5.5 E-05 \\
9.5 E-02\end{array}$ & $\begin{array}{l}1.4 E+01 \\
1.5 E-02 \\
2.6 E+01\end{array}$ \\
\hline $\begin{array}{l}{ }^{152} \mathrm{Eu} \\
{ }^{154} \mathrm{Eu} \\
{ }^{156} \mathrm{Eu} \\
{ }^{156} \mathrm{Eu}\end{array}$ & $\begin{array}{l}3.7 \mathrm{E}+00 \\
3.8 \mathrm{E}+00 \\
8.8 \mathrm{E}-02 \\
4.4 \mathrm{E}+00\end{array}$ & $\begin{array}{l}8.8 E-05 \\
1.1 E-04 \\
1.7 E-05 \\
6.1 E-06\end{array}$ & $\begin{array}{l}8.3 E-04 \\
1.2 E-03 \\
2.0 E-04 \\
1.1 E-03\end{array}$ & $\begin{array}{l}3.7 \mathrm{E}+00 \\
3.8 \mathrm{E}+00 \\
8.8 \mathrm{E}-02 \\
4.4 \mathrm{E}+00\end{array}$ & $\begin{array}{l}9.9 E+02 \\
1.0 E+03 \\
2.4 E+01 \\
1.2 E+03\end{array}$ \\
\hline${ }^{163} \mathrm{Gd}$ & $1.3 E-01$ & $1.0 E-05$ & $4.5 E-04$ & $1.3 \mathrm{E}-01$ & $3.4 E+01$ \\
\hline${ }^{160} \mathrm{~Tb}$ & $3.3 E+00$ & $1.1 E-05$ & $1.7 E-04$ & $3.3 E+00$ & $8.9 E+02$ \\
\hline${ }^{186 m} \mathrm{Ho}$ & $4.8 E+00$ & $3.2 E-04$ & $1.1 E-03$ & $4.8 E+00$ & $1.3 E+03$ \\
\hline $\begin{array}{l}{ }^{181} \mathrm{~W} \\
{ }^{185} \mathrm{~W} \\
{ }^{187} \mathrm{~W}\end{array}$ & $\begin{array}{l}2.7 E-02 \\
2.1 E-04 \\
1.5 E+00\end{array}$ & $\begin{array}{l}6.6 E-08 \\
3.3 E-07 \\
2.9 E-07\end{array}$ & $\begin{array}{l}2.5 E-04 \\
1.4 E-03 \\
1.8 E-03\end{array}$ & $\begin{array}{l}2.7 \mathrm{E}-02 \\
1.6 \mathrm{E}-03 \\
1.5 \mathrm{E}+00\end{array}$ & $\begin{array}{l}7.4 E+00 \\
4.3 E-01 \\
4.1 E+02\end{array}$ \\
\hline${ }^{187} \mathrm{Re}$ & $0.0 E+00$ & $2.5 E-08$ & $1.5 E-07$ & 1.7E-07 & $4.6 E-05$ \\
\hline $\begin{array}{l}{ }^{185} \mathrm{OS} \\
{ }^{191} \mathrm{OS}\end{array}$ & $\begin{array}{l}2.2 E+00 \\
9.7 E-02\end{array}$ & $\begin{array}{l}4.0 \mathrm{E}-06 \\
9.7 \mathrm{E}-07\end{array}$ & $\begin{array}{l}0.0 E+00 \\
0.0 E+00\end{array}$ & $\begin{array}{l}2.2 E+00 \\
9.7 E-02\end{array}$ & $\begin{array}{l}5.9 E+02 \\
2.6 E+01\end{array}$ \\
\hline${ }^{192} \mathrm{Ir}$ & $2.8 \mathrm{E}+00$ & $1.1 \mathrm{E}-05$ & $4.5 E-03$ & $2.8 E+00$ & $7.6 E+02$ \\
\hline${ }^{203} \mathrm{Hg}$ & $5.1 E-01$ & $3.0 E-06$ & $9.5 E-02$ & $6.0 E-01$ & $1.6 \mathrm{E}+02$ \\
\hline $\begin{array}{l}{ }^{210} \mathrm{~Pb}+\mathrm{D}(\mathrm{c}) \\
{ }^{210} \mathrm{~Pb} \\
{ }^{212} \mathrm{~Pb}+\mathrm{D} \\
{ }^{212} \mathrm{~Pb}\end{array}$ & $\begin{array}{l}1.3 \mathrm{E}-03 \\
1.0 \mathrm{E}-03 \\
1.6 \mathrm{E}+00 \\
3.6 \mathrm{E}-01\end{array}$ & $\begin{array}{l}3.2 \mathrm{E}-03 \\
5.7 \mathrm{E}-03 \\
2.6 \mathrm{E}-05 \\
7.0 \mathrm{E}-05\end{array}$ & $\begin{array}{l}4.2 E-01 \\
1.0 E+00 \\
5.3 E-03 \\
9.0 E-03\end{array}$ & $\begin{array}{l}4.2 E-01 \\
1.0 E+00 \\
1.6 E+00 \\
3.6 E-01\end{array}$ & $\begin{array}{l}1.1 E+02 \\
2.8 E+02 \\
4.4 E+02 \\
9.8 E+01\end{array}$ \\
\hline $\begin{array}{l}{ }^{210} \mathrm{Bi+D}(\mathrm{c}) \\
{ }^{210} \mathrm{Bi} \\
{ }^{212} \mathrm{Bi}+\mathrm{I}\end{array}$ & $\begin{array}{l}1.4 E-03 \\
2.8 E-03 \\
4.7 E+00\end{array}$ & $\begin{array}{l}1.9 E-03 \\
8.3 E-05 \\
4.0 E-06\end{array}$ & $\begin{array}{l}1.2 E-01 \\
4.5 E-02 \\
3.8 E-03\end{array}$ & $\begin{array}{l}1.2 E-01 \\
4.8 E-02 \\
4.7 E+00\end{array}$ & $\begin{array}{l}3.2 E+01 \\
1.3 E+01 \\
1.3 E+03\end{array}$ \\
\hline${ }^{210} \mathrm{Po}$ & $2.7 \mathrm{E}-05$ & $3.7 E-03$ & $1.5 E-01$ & $1.6 E-01$ & $4.2 E+01$ \\
\hline $\begin{array}{l}{ }^{223} \mathrm{Ra}+\mathrm{I}(\mathrm{d}) \\
224 \mathrm{Ra}(\mathrm{c}) \\
{ }^{224}{ }_{\mathrm{Ra}}(\mathrm{I}) \\
\left.{ }^{225} \mathrm{~d}\right) \\
{ }^{225} \mathrm{Ra}+\mathrm{D}(\mathrm{c}) \\
{ }^{226} \mathrm{Ra} \\
\mathrm{Ra}+\mathrm{D}\end{array}$ & $\begin{array}{l}8.2 \mathrm{E}-01 \\
8.3 \mathrm{E}-01 \\
2.8 \mathrm{E}-02 \\
1.1 \mathrm{E}-01 \\
7.2 \mathrm{E}-03 \\
6.4 \mathrm{E}-01\end{array}$ & $\begin{array}{l}5.5 \mathrm{E}-04 \\
2.1 \mathrm{E}-04 \\
1.2 \mathrm{E}-03 \\
9.4 \mathrm{E}-04 \\
3.1 \mathrm{E}-03 \\
1.4 \mathrm{E}-03\end{array}$ & $\begin{array}{l}3.0 \mathrm{E}-02 \\
1.8 \mathrm{E}-02 \\
9.0 \mathrm{E}-02 \\
2.3 \mathrm{E}-02 \\
1.5 \mathrm{E}-01 \\
2.1 \mathrm{E}-01\end{array}$ & $\begin{array}{l}8.5 \mathrm{E}-01 \\
8.4 \mathrm{E}-01 \\
1.2 \mathrm{E}-01 \\
1.4 \mathrm{E}-01 \\
1.6 \mathrm{E}-01 \\
8.5 \mathrm{E}-01\end{array}$ & $\begin{array}{l}2.3 E+02 \\
2.3 E+02 \\
3.2 E+01 \\
3.7 E+01 \\
4.4 E+01 \\
2.3 E+02\end{array}$ \\
\hline
\end{tabular}


TABLE 3.3. Annual Total Effective Dose Equivalent Factors for the Residential Use (Surface Soil) Scenario(a) (contd)

\begin{tabular}{|c|c|c|c|c|c|}
\hline Radionuclide & $\begin{array}{c}\text { External } \\
\text { (mrem) }\end{array}$ & $\begin{array}{c}\text { Inhalation } \\
\text { (mrem) } \\
\end{array}$ & $\begin{array}{l}\text { Food }(b) \\
\text { Ingestion } \\
\text { (mrem) }\end{array}$ & $\begin{array}{c}\text { TEDE } \\
\text { (mrem) }\end{array}$ & $\begin{array}{l}\text { TEDE } \\
\text { ( } \mu \text { SV })\end{array}$ \\
\hline $\begin{array}{l}{ }^{228} \mathrm{Ra} \\
{ }^{228} \mathrm{Ra}+\mathrm{Da}\end{array}$ & $\begin{array}{l}9.0 \mathrm{E}-03 \\
8.7 \mathrm{E}-01 \\
1.1 \mathrm{E}-08\end{array}$ & $\begin{array}{l}3.5 \mathrm{E}-03 \\
1.6 \mathrm{E}-02 \\
1.6 \mathrm{E}-03\end{array}$ & $\begin{array}{l}6.8 E-01 \\
8.6 E-02 \\
5.8 E-01\end{array}$ & $\begin{array}{l}6.9 \mathrm{E}-01 \\
9.7 \mathrm{E}-01 \\
5.8 \mathrm{E}-01\end{array}$ & $\begin{array}{l}1.9 \mathrm{E}+02 \\
2.6 \mathrm{E}+02 \\
1.6 \mathrm{E}+02\end{array}$ \\
\hline $\begin{array}{l}{ }^{225} A c+I(d) \\
{ }^{227} A c+D(c) \\
{ }^{227} A C=A C+D(c) \\
{ }^{228} A C\end{array}$ & $\begin{array}{l}7.7 \mathrm{E}-01 \\
1.3 \mathrm{E}-01 \\
2.5 \mathrm{E}-04 \\
9.8 \mathrm{E}-01 \\
3.1 \mathrm{E}+00\end{array}$ & $\begin{array}{l}5.8 \mathrm{E}-04 \\
7.1 \mathrm{E}-02 \\
5.7 \mathrm{E}-01 \\
1.8 \mathrm{E}-02 \\
4.8 \mathrm{E}-05\end{array}$ & $\begin{array}{l}1.2 \mathrm{E}-03 \\
1.3 \mathrm{E}-01 \\
9.0 \mathrm{E}-01 \\
2.3 \mathrm{E}-02 \\
1.4 \mathrm{E}-04\end{array}$ & $\begin{array}{l}7.7 E-01 \\
3.4 E-01 \\
1.5 E+00 \\
1.0 E+00 \\
3.1 E+00\end{array}$ & $\begin{array}{l}2.1 E+02 \\
9.1 E+01 \\
4.0 E+02 \\
2.7 E+02 \\
8.4 E+02\end{array}$ \\
\hline 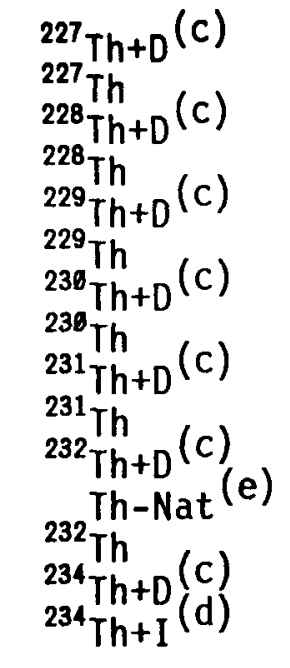 & $\begin{array}{l}1.5 \mathrm{E}-01 \\
2.7 \mathrm{E}-01 \\
7.1 \mathrm{E}-01 \\
3.3 \mathrm{E}-03 \\
1.1 \mathrm{E}-01 \\
1.3 \mathrm{E}-01 \\
5.7 \mathrm{E}-01 \\
4.0 \mathrm{E}-04 \\
1.4 \mathrm{E}-03 \\
1.4 \mathrm{E}-02 \\
7.8 \mathrm{E}-01 \\
7.8 \mathrm{E}-01 \\
2.1 \mathrm{E}-04 \\
3.7 \mathrm{E}-02 \\
6.4 \mathrm{E}-02\end{array}$ & $\begin{array}{l}1.4 \mathrm{E}-03 \\
7.0 \mathrm{E}-03 \\
2.0 \mathrm{E}-02 \\
1.4 \mathrm{E}-01 \\
9.3 \mathrm{E}-02 \\
7.5 \mathrm{E}-01 \\
1.2 \mathrm{E}-02 \\
1.1 \mathrm{E}-01 \\
3.6 \mathrm{E}-08 \\
3.6 \mathrm{E}-07 \\
6.1 \mathrm{E}-02 \\
6.1 \mathrm{E}-02 \\
4.8 \mathrm{E}-01 \\
7.5 \mathrm{E}-06 \\
1.5 \mathrm{E}-05\end{array}$ & $\begin{array}{l}2.7 \mathrm{E}-02 \\
7.5 \mathrm{E}-03 \\
2.6 \mathrm{E}-02 \\
7.8 \mathrm{E}-02 \\
1.1 \mathrm{E}-01 \\
7.0 \mathrm{E}-01 \\
2.0 \mathrm{E}-01 \\
1.1 \mathrm{E}-01 \\
2.5 \mathrm{E}-05 \\
2.5 \mathrm{E}-04 \\
1.3 \mathrm{E}-01 \\
1.3 \mathrm{E}-01 \\
5.5 \mathrm{E}-01 \\
1.4 \mathrm{E}-03 \\
2.8 \mathrm{E}-03\end{array}$ & $\begin{array}{l}1.8 \mathrm{E}-01 \\
2.8 \mathrm{E}-01 \\
7.5 \mathrm{E}-01 \\
2.2 \mathrm{E}-01 \\
3.1 \mathrm{E}-01 \\
1.6 \mathrm{E}+00 \\
7.9 \mathrm{E}-01 \\
2.2 \mathrm{E}-01 \\
1.4 \mathrm{E}-03 \\
1.5 \mathrm{E}-02 \\
9.7 \mathrm{E}-01 \\
9.7 \mathrm{E}-01 \\
1.0 \mathrm{E}+00 \\
3.8 \mathrm{E}-02 \\
6.6 \mathrm{E}-02\end{array}$ & $\begin{array}{l}4.8 E+01 \\
7.6 E+01 \\
2.0 E+02 \\
5.9 E+01 \\
8.4 E+01 \\
4.3 E+02 \\
2.1 E+02 \\
5.8 E+01 \\
3.9 E-01 \\
3.9 E+00 \\
2.6 E+02 \\
2.6 E+02 \\
2.8 E+02 \\
1.0 E+01 \\
1.8 E+01\end{array}$ \\
\hline $\begin{array}{l}{ }^{231} \mathrm{~Pa}+\mathrm{D}(\mathrm{c}) \\
{ }^{231} \mathrm{~Pa} \\
{ }^{233} \mathrm{~Pa} \\
{ }^{234} \mathrm{~Pa}\end{array}$ & $\begin{array}{l}1.3 \mathrm{E}-01 \\
9.1 \mathrm{E}-02 \\
6.9 \mathrm{E}-01 \\
6.2 \mathrm{E}+00\end{array}$ & $\begin{array}{l}1.0 \mathrm{E}-01 \\
3.7 \mathrm{E}-01 \\
4.0 \mathrm{E}-06 \\
3.6 \mathrm{E}-07\end{array}$ & $\begin{array}{l}6.4 E-01 \\
4.8 E+00 \\
1.5 E-03 \\
9.3 E-04\end{array}$ & $\begin{array}{l}8.7 E-01 \\
5.2 E+00 \\
6.9 E-01 \\
6.2 E+00\end{array}$ & $\begin{array}{l}2.3 E+02 \\
1.4 E+03 \\
1.9 E+02 \\
1.7 E+03\end{array}$ \\
\hline $\begin{array}{l}{ }^{232} U+D \\
{ }^{232} U \\
{ }^{233} U+D \\
{ }^{233} U \\
{ }^{234} U \\
{ }^{235} U+D \\
{ }^{236} U \\
{ }^{236} U \\
{ }^{237} U \\
{ }^{238} U+D \\
{ }^{238} U \\
{ }^{248} U-N a t(c) \\
246_{U+D}(c) \\
(d)\end{array}$ & $\begin{array}{l}4.2 \mathrm{E}-05 \\
3.0 \mathrm{E}-04 \\
9.9 \mathrm{E}-02 \\
4.7 \mathrm{E}-04 \\
1.8 \mathrm{E}-04 \\
1.2 \mathrm{E}-01 \\
2.3 \mathrm{E}-01 \\
9.6 \mathrm{E}-05 \\
3.0 \mathrm{E}-01 \\
2.4 \mathrm{E}-02 \\
5.4 \mathrm{E}-03 \\
8.0 \mathrm{E}-03 \\
3.8 \mathrm{E}-01 \\
1.2 \mathrm{E}+00\end{array}$ & $\begin{array}{l}3.8 \mathrm{E}-02 \\
2.6 \mathrm{E}-01 \\
8.8 \mathrm{E}-02 \\
5.7 \mathrm{E}-02 \\
5.7 \mathrm{E}-02 \\
2.6 \mathrm{E}-02 \\
5.3 \mathrm{E}-02 \\
5.3 \mathrm{E}-02 \\
1.5 \mathrm{E}-06 \\
1.6 \mathrm{E}-02 \\
5.3 \mathrm{E}-02 \\
5.5 \mathrm{E}-02 \\
3.2 \mathrm{E}-07 \\
9.7 \mathrm{E}-07\end{array}$ & $\begin{array}{l}1.2 \mathrm{E}-03 \\
8.3 \mathrm{E}-03 \\
9.5 \mathrm{E}-02 \\
3.3 \mathrm{E}-03 \\
3.3 \mathrm{E}-03 \\
1.8 \mathrm{E}-03 \\
3.3 \mathrm{E}-03 \\
3.0 \mathrm{E}-03 \\
3.8 \mathrm{E}-04 \\
1.8 \mathrm{E}-03 \\
3.0 \mathrm{E}-03 \\
3.1 \mathrm{E}-03 \\
1.8 \mathrm{E}-04 \\
5.5 \mathrm{E}-04\end{array}$ & $\begin{array}{l}3.9 \mathrm{E}-02 \\
2.7 \mathrm{E}-01 \\
2.8 \mathrm{E}-01 \\
6.1 \mathrm{E}-02 \\
6.1 \mathrm{E}-02 \\
1.5 \mathrm{E}-01 \\
2.9 \mathrm{E}-01 \\
5.6 \mathrm{E}-02 \\
3.0 \mathrm{E}-01 \\
4.2 \mathrm{E}-02 \\
6.1 \mathrm{E}-02 \\
6.6 \mathrm{E}-02 \\
3.8 \mathrm{E}-01 \\
1.2 \mathrm{E}+00\end{array}$ & $\begin{array}{l}1.0 E+01 \\
7.3 E+01 \\
7.6 E+01 \\
1.6 E+01 \\
1.6 E+01 \\
4.1 E+01 \\
7.8 E+01 \\
1.5 E+01 \\
8.1 E+01 \\
1.1 E+01 \\
1.6 E+01 \\
1.8 E+01 \\
1.0 E+02 \\
3.2 E+02\end{array}$ \\
\hline
\end{tabular}


TABLE 3.3. Annual Total Effective Dose Equivalent Factors for the Residential Use (Surface Soil) Scenario(a) (contd)

\begin{tabular}{|c|c|c|c|c|c|}
\hline Radionuclide & $\begin{array}{c}\text { External } \\
\text { (mrem) } \\
\end{array}$ & $\begin{array}{c}\text { Inhalation } \\
\text { (mrem) }\end{array}$ & $\begin{array}{c}\text { Food }(b) \\
\text { Ingestion } \\
\text { (mrem) }\end{array}$ & $\begin{array}{c}\text { TEDE } \\
\text { (mrem) }\end{array}$ & $\begin{array}{l}\text { TEDE } \\
(\mu S v)\end{array}$ \\
\hline $\begin{array}{l}{ }^{237} \mathrm{~Np}+\mathrm{D}(c) \\
{ }^{237} \mathrm{~Np} \\
{ }^{238} \mathrm{Np+D} \\
{ }^{238} \mathrm{~Np} \\
{ }^{239} \mathrm{~Np} \\
{ }^{239} \mathrm{~Np}(\mathrm{c})\end{array}$ & $\begin{array}{l}3.4 \mathrm{E}-01 \\
2.9 \mathrm{E}-02 \\
8.9 \mathrm{E}-01 \\
1.8 \mathrm{E}+00 \\
1.9 \mathrm{E}-01 \\
3.9 \mathrm{E}-01\end{array}$ & $\begin{array}{l}1.3 \mathrm{E}-01 \\
2.7 \mathrm{E}-01 \\
6.1 \mathrm{E}-02 \\
1.8 \mathrm{E}-05 \\
5.3 \mathrm{E}-07 \\
1.1 \mathrm{E}-06\end{array}$ & $\begin{array}{l}3.0 \mathrm{E}-01 \\
6.0 \mathrm{E}-01 \\
4.7 \mathrm{E}-03 \\
4.8 \mathrm{E}-04 \\
1.9 \mathrm{E}-04 \\
3.8 \mathrm{E}-04\end{array}$ & $\begin{array}{l}7.7 \mathrm{E}-01 \\
9.0 \mathrm{E}-01 \\
9.5 \mathrm{E}-01 \\
1.8 \mathrm{E}+00 \\
1.9 \mathrm{E}-01 \\
3.9 \mathrm{E}-01\end{array}$ & $\begin{array}{l}2.1 E+02 \\
2.4 E+02 \\
2.6 E+02 \\
4.9 E+02 \\
5.2 E+01 \\
1.1 E+02\end{array}$ \\
\hline $\begin{array}{l}{ }_{236}^{23} \mathrm{Pu} \\
{ }_{238} \mathrm{Pu} \\
{ }_{238} \mathrm{Pu} \\
{ }_{239} \mathrm{Pu} \\
{ }_{246} \mathrm{Pu} \\
{ }_{241} \mathrm{Pu}+\mathrm{D}(\mathrm{c}) \\
{ }_{241} \mathrm{Pu} \\
{ }_{242} \mathrm{Pu} \\
{ }_{243} \mathrm{Pu}+\mathrm{D}(\mathrm{c}) \\
{ }_{243} \mathrm{Pu} \\
{ }_{244} \mathrm{Pu}+\mathrm{D}(\mathrm{c}) \\
{ }_{244} \mathrm{Pu}\end{array}$ & $\begin{array}{l}9.2 \mathrm{E}-05 \\
9.3 \mathrm{E}-02 \\
6.1 \mathrm{E}-05 \\
1.4 \mathrm{E}-04 \\
6.1 \mathrm{E}-05 \\
6.8 \mathrm{E}-03 \\
1.1 \mathrm{E}-10 \\
5.1 \mathrm{E}-05 \\
1.4 \mathrm{E}-01 \\
3.2 \mathrm{E}-02 \\
2.9 \mathrm{E}-01 \\
3.6 \mathrm{E}-05\end{array}$ & $\begin{array}{l}3.2 \mathrm{E}-02 \\
7.9 \mathrm{E}-07 \\
1.2 \mathrm{E}-01 \\
1.3 \mathrm{E}-01 \\
1.3 \mathrm{E}-01 \\
9.4 \mathrm{E}-02 \\
2.1 \mathrm{E}-03 \\
1.2 \mathrm{E}-01 \\
3.1 \mathrm{E}-08 \\
6.1 \mathrm{E}-08 \\
2.9 \mathrm{E}-02 \\
1.1 \mathrm{E}-01\end{array}$ & $\begin{array}{l}1.7 \mathrm{E}-03 \\
1.1 \mathrm{E}-05 \\
8.8 \mathrm{E}-03 \\
9.8 \mathrm{E}-03 \\
9.6 \mathrm{E}-03 \\
5.1 \mathrm{E}-01 \\
1.8 \mathrm{E}-04 \\
9.1 \mathrm{E}-03 \\
4.1 \mathrm{E}-06 \\
8.3 \mathrm{E}-06 \\
2.5 \mathrm{E}-03 \\
9.3 \mathrm{E}-03\end{array}$ & $\begin{array}{l}3.3 E-02 \\
9.3 E-02 \\
1.3 E-01 \\
1.4 E-01 \\
1.4 E-01 \\
6.1 E-01 \\
2.2 E-03 \\
1.3 E-01 \\
1.4 E-01 \\
3.2 E-02 \\
3.2 E-01 \\
1.2 E-01\end{array}$ & $\begin{array}{l}9.0 \mathrm{E}+00 \\
2.5 \mathrm{E}+01 \\
3.6 \mathrm{E}+01 \\
3.7 \mathrm{E}+01 \\
3.7 \mathrm{E}+01 \\
1.7 \mathrm{E}+02 \\
6.1 \mathrm{E}-01 \\
3.4 \mathrm{E}+01 \\
3.8 \mathrm{E}+01 \\
8.7 \mathrm{E}+00 \\
8.6 \mathrm{E}+01 \\
3.3 \mathrm{E}+01\end{array}$ \\
\hline $\begin{array}{l}{ }^{241} \mathrm{Am} \\
{ }^{242 m} \mathrm{Am}+\mathrm{D} \\
{ }^{242 m} \mathrm{Am} \\
{ }^{242} \mathrm{Am}+\mathrm{D}(\mathrm{c}) \\
{ }^{242} \mathrm{Am} \\
{ }^{243} \mathrm{Am}+\mathrm{D} \\
{ }^{243} \mathrm{Am}\end{array}$ & $\begin{array}{l}1.4 \mathrm{E}-02 \\
7.1 \mathrm{E}-03 \\
5.7 \mathrm{E}-04 \\
9.4 \mathrm{E}-03 \\
2.7 \mathrm{E}-02 \\
1.5 \mathrm{E}-01 \\
5.1 \mathrm{E}-02\end{array}$ & $\begin{array}{l}1.9 \mathrm{E}-01 \\
7.2 \mathrm{E}-02 \\
1.7 \mathrm{E}-01 \\
3.7 \mathrm{E}-02 \\
2.3 \mathrm{E}-05 \\
6.0 \mathrm{E}-02 \\
1.8 \mathrm{E}-01\end{array}$ & $\begin{array}{l}1.0 \mathrm{E}+00 \\
2.6 \mathrm{E}-01 \\
9.8 \mathrm{E}-01 \\
6.7 \mathrm{E}-03 \\
3.5 \mathrm{E}-04 \\
3.4 \mathrm{E}-01 \\
1.0 \mathrm{E}+00\end{array}$ & $\begin{array}{l}1.2 \mathrm{E}+00 \\
3.4 \mathrm{E}-01 \\
1.1 \mathrm{E}+00 \\
5.3 \mathrm{E}-02 \\
2.7 \mathrm{E}-02 \\
5.5 \mathrm{E}-01 \\
1.3 \mathrm{E}+00\end{array}$ & $\begin{array}{l}3.3 \mathrm{E}+02 \\
9.2 \mathrm{E}+01 \\
3.1 \mathrm{E}+02 \\
1.4 \mathrm{E}+01 \\
7.4 \mathrm{E}+00 \\
1.5 \mathrm{E}+02 \\
3.4 \mathrm{E}+02\end{array}$ \\
\hline $\begin{array}{l}{ }^{242} \mathrm{Cm}+\mathrm{D}(\mathrm{c}) \\
{ }^{242} \mathrm{Cm} \\
{ }^{243} \mathrm{Cm} \\
{ }^{244} \mathrm{Cm}+\mathrm{D} \\
{ }^{244} \mathrm{Cm} \\
\left.{ }^{245} \mathrm{Cm}+\mathrm{C}\right) \\
{ }^{245} \mathrm{Cm} \\
{ }^{246} \mathrm{Cm} \\
{ }^{247} \mathrm{Cm} \text { Cm+D }(\mathrm{c}) \\
{ }^{247} \mathrm{Cm} \\
{ }^{248} \mathrm{Cm}\end{array}$ & $\begin{array}{l}6.3 \mathrm{E}-05 \\
5.8 \mathrm{E}-05 \\
2.9 \mathrm{E}-01 \\
2.4 \mathrm{E}-05 \\
4.9 \mathrm{E}-05 \\
4.7 \mathrm{E}-02 \\
1.3 \mathrm{E}-01 \\
4.1 \mathrm{E}-05 \\
7.8 \mathrm{E}-01 \\
1.3 \mathrm{E}+00 \\
3.7 \mathrm{E}-05\end{array}$ & $\begin{array}{l}7.0 \mathrm{E}-02 \\
6.6 \mathrm{E}-03 \\
2.5 \mathrm{E}-01 \\
5.0 \mathrm{E}-02 \\
1.0 \mathrm{E}-01 \\
1.2 \mathrm{E}-01 \\
1.8 \mathrm{E}-01 \\
1.8 \mathrm{E}-01 \\
8.6 \mathrm{E}-02 \\
1.7 \mathrm{E}-01 \\
6.6 \mathrm{E}-01\end{array}$ & $\begin{array}{l}1.2 \mathrm{E}-02 \\
1.3 \mathrm{E}-02 \\
3.0 \mathrm{E}-01 \\
1.2 \mathrm{E}-01 \\
2.3 \mathrm{E}-01 \\
4.8 \mathrm{E}-01 \\
4.3 \mathrm{E}-01 \\
4.3 \mathrm{E}-01 \\
2.0 \mathrm{E}-01 \\
4.0 \mathrm{E}-01 \\
1.6 \mathrm{E}+00\end{array}$ & $\begin{array}{l}8.2 \mathrm{E}-02 \\
1.9 \mathrm{E}-02 \\
8.4 \mathrm{E}-01 \\
1.7 \mathrm{E}-01 \\
3.3 \mathrm{E}-01 \\
6.6 \mathrm{E}-01 \\
7.4 \mathrm{E}-01 \\
6.1 \mathrm{E}-01 \\
1.1 \mathrm{E}+00 \\
1.9 \mathrm{E}+00 \\
2.2 \mathrm{E}+00\end{array}$ & $\begin{array}{l}2.2 E+01 \\
5.2 E+00 \\
2.3 E+02 \\
4.5 E+01 \\
9.0 E+01 \\
1.8 E+02 \\
2.0 E+02 \\
1.6 E+02 \\
2.9 E+02 \\
5.0 E+02 \\
6.0 E+02\end{array}$ \\
\hline${ }^{252} \mathrm{Cf}$ & 4.1E-05 & $5.7 \mathrm{E}-02$ & $2.8 \mathrm{E}-02$ & $8.5 E-02$ & $2.3 E+01$ \\
\hline
\end{tabular}

(a) Based on a surface soil activity level of $1 \mathrm{pCi} / \mathrm{g}(1 \mathrm{~Bq} / \mathrm{g})$. 
TABLE 3.3. Annual Total Effective Dose Equivalent Factors

for the Residual Use (Surface Soil) Scenario(a) (contd)

(b) Based on the total air deposition and root uptake contributions using an air concentration of $5 \times 10^{-5} \mathrm{pCi} / \mathrm{m}^{3}$ and root uptake factors as described in Appendix B.

(c) Where $+D$ means "plus daughters in equilibrium" referring to explicit daughters, or daughters with half-lives greater than 10 minutes. Separate values are also included for parents and daughters so that combined factors for non-equilibrium cases can be determined. Note: in some cases $+D$ also includes implicit daughters (i.e., uranium and thorium decay chains).

(d) Where +I means "plus implicit daughters in equilibrium" referring to daughters with half-lives less than 10 minutes. The decay energies associated with these daughters have been assigned to the parent, and the appropriate branching ratios (described in Appendix $D$ ) have been considered.

(e) Where Th-Nat includes an equilibrium mixture of ${ }^{232} \mathrm{Th}$ plus 10 daughters in the decay chain, as discussed in Appendix $D$.

(f) Where $1 \mathrm{Ci} \mathrm{U}$-Nat equals $48.9 \%{ }^{238} \mathrm{U}$ plus $48.9 \%{ }^{234} \mathrm{U}$ plus $2.25 \%{ }^{235} \mathrm{U}$. 


\subsubsection{Drinking Water Use Scenario}

To account for potential contamination of ground water used as a drinking water source, a drinking water scenario is defined (see the discussion of the ground-water resource model in Appendix B). This scenario is intended to account for the potential impacts on drinking water sources of residual radioactivity in soil and in structures that may be demolished and left onsite as buried rubble. For this scenario, an individual is assumed to ingest $730 \mathrm{~L} / \mathrm{yr}(2 \mathrm{~L} / \mathrm{d})$ of drinking water, obtained from an aquifer with a concentration calculated using the ground-water resource model discussed in Appendix B. The aquifer concentration is derived in a generic and somewhat conservative manner; thus, the magnitude of the annual TEDE factors for a given inventory may provide only an indication of when additional site specific data or modeling sophistication are warranted. For example, site specific considerations may include measured parameters such as leach rate and retention. The calculated annual TEDE factors for this scenario are shown in Table 3.4. The values shown are in units of mrem (and $\mu \mathrm{Sv}$ ) for a soil inventory of $1 \mathrm{pCi}(1 \mathrm{~Bq})$ for a listing of over 200 radionuclides. The + I (implicit daughter) and $+D$ (explicit daughter) notation has been used to provide maximum flexibility for consideration of all parent-daughter and decay chain situations. Further description of the parent-daughter and chain decay relationships used in this study is provided in Appendix D. Also included in Table 3.4 are listings by radionuclide of the maximum organ dose in $\mathrm{mrem} / \mathrm{yr}(\mu \mathrm{Sv} / \mathrm{yr})$ and the organ of concern. Again, the maximum organ dose rates shown in Table 3.4 are based on the dosimetry models, metabolic data, and recommendations of the ICRP found in Publication 30 (1979-1988). Because of changes in the metabolic data and the modeling approach, the maximum organ dose listings based on ICRP 30 do not always agree with information from ICRP 2 (1959).

Hand calculations were performed to verify the computer calculations for selected radionuclides using the pathway factors from Section 2.0 and the drinking water scenario assumptions. The results of the calculations indicated that drinking water concentrations and the resulting annual TEDE values in Table 3.4 were calculated correctly. An additional evaluation of the determination of the maximum organ dose was conducted. This is not always an easy evaluation, given the complexity of the ICRP 30 dosimetry system. For example, at first glance it may seem that a soluble element like Cs should produce a rather uniform dose to internal organs. However, the non-weighted target organ dose shows a maximum for either the small intestine wall or the stomach wall, depending on the isotope considered. Although somewhat of a calculational artifact, this result was verified by comparison with the information in Federal Guidance Report No. 11 (Eckerman, Wolbarst, and Richardson 1988). 
IABLE 3.4. Drinking Water Scenario Annual Committed Effective Dose Equivalent Factors for Exposure to Residual Radioactive Materials (a)

\begin{tabular}{|c|c|c|c|c|c|}
\hline \multirow[b]{2}{*}{ Radionuclide } & \multirow{2}{*}{$\begin{array}{c}\text { TEDE } \\
\text { (mrem) }\end{array}$} & \multirow{2}{*}{$\begin{array}{l}\text { TEDE } \\
(\mu S V) \\
\end{array}$} & \multicolumn{3}{|c|}{ Maximum Organ Dose } \\
\hline & & & (mrem) & $(\mu \mathrm{Sv})$ & $\operatorname{Organ}(b)$ \\
\hline${ }^{3} \mathrm{H}$ & $2.0 \mathrm{E}-12$ & $5.5 \mathrm{E}-10$ & $2.4 E-12$ & $6.5 E-10$ & SI Wall \\
\hline${ }^{10} \mathrm{Be}$ & $1.5 \mathrm{E}-11$ & $4.0 E-09$ & $1.6 \mathrm{E}-10$ & $4.2 E-08$ & LLI Wall \\
\hline${ }^{14} \mathrm{C}$ & $7.1 \mathrm{E}-12$ & $1.9 \mathrm{E}-09$ & $8.6 E-12$ & $2.3 E-09$ & LLI Wall \\
\hline${ }^{18} \mathrm{~F}$ & $7.4 \mathrm{E}-20$ & $2.0 \mathrm{E}-17$ & $7.4 \mathrm{E}-19$ & $2.0 \mathrm{E}-16$ & Stomach Wall \\
\hline $\begin{array}{l}{ }^{22} \mathrm{Na} \\
{ }^{24} \mathrm{Na}\end{array}$ & $\begin{array}{l}6.1 \mathrm{E}-14 \\
8.9 \mathrm{E}-20\end{array}$ & $\begin{array}{l}\text { 1. } 7 \mathrm{E}-11 \\
2.4 \mathrm{E}-17\end{array}$ & $\begin{array}{l}1.0 \mathrm{E}-13 \\
2.8 \mathrm{E}-19\end{array}$ & $\begin{array}{l}2.7 \mathrm{E}-11 \\
7.6 \mathrm{E}-17\end{array}$ & $\begin{array}{l}\text { Bone Surface } \\
\text { Stomach Wall }\end{array}$ \\
\hline $\begin{array}{l}{ }^{32} \mathrm{p} \\
{ }^{33} \mathrm{p}\end{array}$ & $\begin{array}{l}\text { 2.2E-15 } \\
7.2 \mathrm{E}-16\end{array}$ & $\begin{array}{l}6.0 \mathrm{E}-13 \\
1.9 \mathrm{E}-13\end{array}$ & $\begin{array}{l}8.1 \mathrm{E}-15 \\
3.9 \mathrm{E}-15\end{array}$ & $\begin{array}{l}2.2 \mathrm{E}-12 \\
1.1 \mathrm{E}-12\end{array}$ & $\begin{array}{l}\text { Red Marrow } \\
\text { Bone Surface }\end{array}$ \\
\hline${ }^{35} \mathrm{~S}$ & $2.9 \mathrm{E}-14$ & $7.9 \mathrm{E}-12$ & $1.5 E-13$ & $3.9 \mathrm{E}-11$ & LLI Wall \\
\hline${ }^{36} \mathrm{Cl}$ & $1.0 \mathrm{E}-11$ & $2.8 \mathrm{E}-09$ & $1.2 \mathrm{E}-11$ & $3.2 E-09$ & SI Wall \\
\hline${ }^{46} \mathrm{~K}$ & $8.2 E-14$ & $2.2 E-11$ & $9.7 E-14$ & $2.6 \mathrm{E}-11$ & SI Wall \\
\hline${ }^{41} \mathrm{Ca}$ & $\begin{array}{l}3.8 \mathrm{E}-13 \\
3.8 \mathrm{E}-13\end{array}$ & $\begin{array}{l}1.0 \mathrm{E}-10 \\
1.0 \mathrm{E}-10\end{array}$ & $\begin{array}{l}4.8 \mathrm{E}-12 \\
2.3 \mathrm{E}-12\end{array}$ & $\begin{array}{l}1.3 \mathrm{E}-09 \\
6.3 \mathrm{E}-10\end{array}$ & $\begin{array}{l}\text { Bone Surface } \\
\text { Bone Surface }\end{array}$ \\
\hline${ }^{46} \mathrm{SC}$ & $3.9 E-15$ & $1.1 E-12$ & $2.3 E-14$ & $6.3 E-12$ & LLI Wall \\
\hline${ }^{51} \mathrm{Cr}$ & $1.4 \mathrm{E}-16$ & $3.7 \mathrm{E}-14$ & $9.0 \mathrm{E}-16$ & $2.4 \mathrm{E}-13$ & LLI Wall \\
\hline $\begin{array}{l}{ }^{54} \mathrm{Mn} \\
{ }^{60} \mathrm{Mn}\end{array}$ & $\begin{array}{l}6.8 \mathrm{E}-14 \\
1.5 \mathrm{E}-20\end{array}$ & $\begin{array}{l}1.8 \mathrm{E}-11 \\
3.9 \mathrm{E}-18\end{array}$ & $\begin{array}{l}2.1 \mathrm{E}-13 \\
7.6 \mathrm{E}-20\end{array}$ & $\begin{array}{l}5.6 \mathrm{E}-11 \\
2.1 \mathrm{E}-17\end{array}$ & $\begin{array}{l}\text { LLI Wall } \\
\text { ULI Wall }\end{array}$ \\
\hline $\begin{array}{l}{ }^{55} \mathrm{Fe} \\
{ }^{59} \mathrm{Fe}\end{array}$ & $\begin{array}{l}2.3 \mathrm{E}-13 \\
1.9 \mathrm{E}-13\end{array}$ & $\begin{array}{l}6.3 \mathrm{E}-11 \\
5.1 \mathrm{E}-11\end{array}$ & $\begin{array}{l}8.5 \mathrm{E}-13 \\
9.0 \mathrm{E}-13\end{array}$ & $\begin{array}{l}2.3 \mathrm{E}-10 \\
2.4 \mathrm{E}-10\end{array}$ & $\begin{array}{l}\text { Spleen } \\
\text { LLI Wall }\end{array}$ \\
\hline $\begin{array}{l}{ }^{57} \mathrm{Co} \\
{ }^{58} \mathrm{Co} \\
{ }^{88} \mathrm{Co}\end{array}$ & $\begin{array}{l}2.0 \mathrm{E}-13 \\
1.9 \mathrm{E}-13 \\
4.4 \mathrm{E}-12\end{array}$ & $\begin{array}{l}5.3 E-11 \\
5.1 E-11 \\
1.2 E-09\end{array}$ & $\begin{array}{l}1.3 \mathrm{E}-12 \\
9.2 \mathrm{E}-13 \\
1.9 \mathrm{E}-11\end{array}$ & $\begin{array}{l}3.4 \mathrm{E}-10 \\
2.5 \mathrm{E}-10 \\
5.1 \mathrm{E}-09\end{array}$ & $\begin{array}{l}\text { LLI Wall } \\
\text { LLI Wall } \\
\text { LLI Wall }\end{array}$ \\
\hline $\begin{array}{l}{ }^{69} \mathrm{Ni} \\
{ }^{83} \mathrm{Ni} \\
{ }^{85} \mathrm{Ni}\end{array}$ & $\begin{array}{l}9.1 \mathrm{E}-14 \\
2.4 \mathrm{E}-13 \\
1.1 \mathrm{E}-19\end{array}$ & $\begin{array}{l}2.5 \mathrm{E}-11 \\
6.6 \mathrm{E}-11 \\
2.9 \mathrm{E}-17\end{array}$ & $\begin{array}{l}4.7 \mathrm{E}-13 \\
1.5 \mathrm{E}-12 \\
5.9 \mathrm{E}-19\end{array}$ & $\begin{array}{l}1.3 \mathrm{E}-10 \\
4.1 \mathrm{E}-10 \\
1.6 \mathrm{E}-16\end{array}$ & $\begin{array}{l}\text { LLI Wall } \\
\text { LLI Wall } \\
\text { ULI Wall }\end{array}$ \\
\hline${ }^{84} \mathrm{Cu}$ & $1.4 \mathrm{E}-17$ & $3.7 \mathrm{E}-15$ & $8.2 E-17$ & $2.2 \mathrm{E}-14$ & LLI Wall \\
\hline${ }^{60} \mathrm{Zn} Z \mathrm{Zn+D}$ & $\begin{array}{l}3.2 \mathrm{E}-12 \\
3.4 \mathrm{E}-18\end{array}$ & $\begin{array}{l}8.7 \mathrm{E}-10 \\
9.3 \mathrm{E}-16\end{array}$ & $\begin{array}{l}4.2 \mathrm{E}-12 \\
2.2 \mathrm{E}-17\end{array}$ & $\begin{array}{l}1.1 \mathrm{E}-09 \\
5.9 \mathrm{E}-15\end{array}$ & $\begin{array}{l}\text { LLI Wall } \\
\text { LLI Wall }\end{array}$ \\
\hline
\end{tabular}


TABLE 3.4. Drinking Water Scenario Annual Committed Effective Dose Equivalent Factors for Exposure to Residual Radioactive Materials (a) (contd)

\begin{tabular}{|c|c|c|c|c|c|}
\hline \multirow{2}{*}{ Radionuclide } & TEDE & TEDE & \multicolumn{3}{|c|}{ Maximum Organ Dose } \\
\hline & (mrem) & $(\mu S v)$ & (mrem) & $(\mu \mathrm{Sv})$ & $\operatorname{Organ}(b)$ \\
\hline${ }^{69} \mathrm{Zn}$ & $\begin{array}{l}6.4 \mathrm{E}-18 \\
2.0 \mathrm{E}-21\end{array}$ & $\begin{array}{l}1.7 \mathrm{E}-15 \\
5.4 \mathrm{E}-19\end{array}$ & $\begin{array}{l}4.4 \mathrm{E}-17 \\
1.7 \mathrm{E}-20\end{array}$ & $\begin{array}{l}1.2 \mathrm{E}-14 \\
4.7 \mathrm{E}-18\end{array}$ & $\begin{array}{l}\text { LLI Wall } \\
\text { Stomach Wall }\end{array}$ \\
\hline${ }^{76}$ As & $6.5 E-16$ & $1.8 \mathrm{E}-13$ & $5.4 E-15$ & 1. $5 E-12$ & LLI Wall \\
\hline${ }^{79} \mathrm{Se}$ & $\begin{array}{l}9.7 E-13 \\
3.1 E-12\end{array}$ & $\begin{array}{l}2.6 \mathrm{E}-10 \\
8.4 \mathrm{E}-10\end{array}$ & $\begin{array}{l}2.8 E-12 \\
1.9 E-11\end{array}$ & $\begin{array}{l}7.6 \mathrm{E}-10 \\
5.0 \mathrm{E}-09\end{array}$ & $\begin{array}{l}\text { Kidneys } \\
\text { Kidneys }\end{array}$ \\
\hline $\begin{array}{l}{ }^{82} \mathrm{Br} \\
{ }_{83}^{83} \mathrm{Br}+\mathrm{D} \\
{ }^{83} \mathrm{Br} \\
{ }^{84} \mathrm{Br}\end{array}$ & $\begin{array}{l}4.0 \mathrm{E}-16 \\
5.4 \mathrm{E}-20 \\
1.1 \mathrm{E}-19 \\
1.0 \mathrm{E}-20\end{array}$ & $\begin{array}{l}1.1 \mathrm{E}-13 \\
1.5 \mathrm{E}-17 \\
2.9 \mathrm{E}-17 \\
2.8 \mathrm{E}-18\end{array}$ & $\begin{array}{l}7.5 \mathrm{E}-16 \\
6.4 \mathrm{E}-19 \\
1.3 \mathrm{E}-18 \\
1.5 \mathrm{E}-19\end{array}$ & $\begin{array}{l}2.0 \mathrm{E}-13 \\
1.7 \mathrm{E}-16 \\
3.4 \mathrm{E}-16 \\
4.0 \mathrm{E}-17\end{array}$ & $\begin{array}{l}\text { Stomach Wall } \\
\text { Stomach Wall } \\
\text { Stomach Wall } \\
\text { Stomach Wall }\end{array}$ \\
\hline $\begin{array}{l}{ }^{86} \mathrm{Rb} \\
{ }^{88} \mathrm{Rb} \\
{ }^{89} \mathrm{Rb}+\mathrm{D} \\
{ }^{89} \mathrm{Rb}\end{array}$ & $\begin{array}{l}5.2 \mathrm{E}-16 \\
4.5 \mathrm{E}-24 \\
8.3 \mathrm{E}-23 \\
1.8 \mathrm{E}-24\end{array}$ & $\begin{array}{l}1.4 \mathrm{E}-13 \\
1.2 \mathrm{E}-21 \\
2.2 \mathrm{E}-20 \\
4.8 \mathrm{E}-22\end{array}$ & $\begin{array}{l}1.5 \mathrm{E}-15 \\
7.1 \mathrm{E}-23 \\
7.3 \mathrm{E}-22 \\
2.6 \mathrm{E}-23\end{array}$ & $\begin{array}{l}4.2 \mathrm{E}-13 \\
1.9 \mathrm{E}-20 \\
2.0 \mathrm{E}-19 \\
7.1 \mathrm{E}-21\end{array}$ & $\begin{array}{l}\text { Bone Surface } \\
\text { Stomach Wall } \\
\text { LLI Wall } \\
\text { Stomach Wall }\end{array}$ \\
\hline $\begin{array}{l}{ }^{85} \mathrm{Sr} \\
{ }^{89} \mathrm{Sr} \\
{ }^{90} \mathrm{Sr+D}(\mathrm{C}) \\
{ }^{96} \mathrm{Sr} \\
{ }^{91} \mathrm{Sr+D}(\mathrm{c}) \\
{ }^{91} \mathrm{Sr} \\
{ }^{92} \mathrm{Sr+D}(\mathrm{c}) \\
{ }^{92} \mathrm{Sr}\end{array}$ & $\begin{array}{l}6.5 \mathrm{E}-14 \\
2.1 \mathrm{E}-13 \\
2.0 \mathrm{E}-11 \\
3.8 \mathrm{E}-11 \\
7.3 \mathrm{E}-18 \\
3.8 \mathrm{E}-18 \\
2.3 \mathrm{E}-19 \\
2.0 \mathrm{E}-19\end{array}$ & $\begin{array}{l}1.7 \mathrm{E}-11 \\
5.8 \mathrm{E}-11 \\
5.5 \mathrm{E}-09 \\
1.0 \mathrm{E}-08 \\
2.0 \mathrm{E}-15 \\
1.0 \mathrm{E}-15 \\
6.2 \mathrm{E}-17 \\
5.5 \mathrm{E}-17\end{array}$ & $\begin{array}{l}2.0 \mathrm{E}-13 \\
1.9 \mathrm{E}-12 \\
2.2 \mathrm{E}-10 \\
4.4 \mathrm{E}-10 \\
7.9 \mathrm{E}-17 \\
2.2 \mathrm{E}-17 \\
1.5 \mathrm{E}-18 \\
1.4 \mathrm{E}-18\end{array}$ & $\begin{array}{l}5.3 \mathrm{E}-11 \\
5.2 \mathrm{E}-10 \\
5.9 \mathrm{E}-08 \\
1.2 \mathrm{E}-07 \\
2.1 \mathrm{E}-14 \\
5.9 \mathrm{E}-15 \\
4.1 \mathrm{E}-16 \\
3.8 \mathrm{E}-16\end{array}$ & $\begin{array}{l}\text { LLI Wall } \\
\text { LLI Wall } \\
\text { Bone Surface } \\
\text { Bone Surface } \\
\text { LLI Wal1 } \\
\text { LLI Wall } \\
\text { ULI Wall } \\
\text { ULI Wall }\end{array}$ \\
\hline $\begin{array}{l}{ }^{96} Y \\
{ }^{91} m_{Y} \\
{ }^{91 m_{Y}}(\mathrm{C}) \\
{ }^{91} Y \\
{ }^{92} Y \\
{ }^{93} Y\end{array}$ & $\begin{array}{l}7.9 \mathrm{E}-15 \\
5.7 \mathrm{E}-19 \\
4.9 \mathrm{E}-21 \\
3.0 \mathrm{E}-12 \\
4.4 \mathrm{E}-18 \\
8.6 \mathrm{E}-17\end{array}$ & $\begin{array}{l}2.1 \mathrm{E}-12 \\
1.5 \mathrm{E}-16 \\
1.3 \mathrm{E}-18 \\
8.2 \mathrm{E}-10 \\
1.2 \mathrm{E}-15 \\
2.3 \mathrm{E}-14\end{array}$ & $\begin{array}{l}8.7 E-14 \\
6.6 E-18 \\
2.3 E-20 \\
3.5 E-11 \\
2.9 E-17 \\
6.3 E-16\end{array}$ & $\begin{array}{l}2.3 \mathrm{E}-11 \\
1.8 \mathrm{E}-15 \\
6.2 \mathrm{E}-18 \\
9.6 \mathrm{E}-09 \\
7.8 \mathrm{E}-15 \\
1.7 \mathrm{E}-13\end{array}$ & $\begin{array}{l}\text { LLI Wall } \\
\text { LLI Wal1 } \\
\text { Stomach Wall } \\
\text { LLI Wal1 } \\
\text { ULI Wal1 } \\
\text { LLI Wal1 }\end{array}$ \\
\hline $\begin{array}{l}{ }^{93} \mathrm{Zr+D}(\mathrm{c}) \\
{ }^{93} \mathrm{Zr} \\
{ }^{95} \mathrm{Zr+D}(\mathrm{c}) \\
{ }^{95} \mathrm{Zr} \\
{ }^{97} \mathrm{Zr+D}(\mathrm{c}) \\
{ }^{97} \mathrm{Zr}\end{array}$ & $\begin{array}{l}3.4 \mathrm{E}-13 \\
5.1 \mathrm{E}-13 \\
1.2 \mathrm{E}-13 \\
1.4 \mathrm{E}-13 \\
1.4 \mathrm{E}-17 \\
4.2 \mathrm{E}-17\end{array}$ & $\begin{array}{l}9.1 \mathrm{E}-11 \\
1.4 \mathrm{E}-10 \\
3.1 \mathrm{E}-11 \\
3.7 \mathrm{E}-11 \\
3.9 \mathrm{E}-15 \\
1.1 \mathrm{E}-14\end{array}$ & $\begin{array}{l}5.3 \mathrm{E}-12 \\
1.1 \mathrm{E}-11 \\
8.1 \mathrm{E}-13 \\
1.1 \mathrm{E}-12 \\
1.1 \mathrm{E}-16 \\
3.3 \mathrm{E}-16\end{array}$ & $\begin{array}{l}1.4 \mathrm{E}-09 \\
2.8 \mathrm{E}-09 \\
2.2 \mathrm{E}-10 \\
2.9 \mathrm{E}-10 \\
3.0 \mathrm{E}-14 \\
9.0 \mathrm{E}-14\end{array}$ & $\begin{array}{l}\text { Bone Surface } \\
\text { Bone Surface } \\
\text { LLI Wall } \\
\text { LLI Wall } \\
\text { LLI Wall } \\
\text { LLI Wall }\end{array}$ \\
\hline${ }_{{ }^{95}{ }^{94} \mathrm{Nb}}^{\mathrm{95}} \mathrm{Nb}+\mathrm{D}$ & $\begin{array}{l}1.7 \mathrm{E}-13 \\
2.6 \mathrm{E}-12 \\
3.5 \mathrm{E}-16 \\
3.4 \mathrm{E}-16\end{array}$ & $\begin{array}{l}4.7 \mathrm{E}-11 \\
7.0 \mathrm{E}-10 \\
9.6 \mathrm{E}-14 \\
9.1 \mathrm{E}-14\end{array}$ & $\begin{array}{l}2.0 \mathrm{E}-12 \\
1.7 \mathrm{E}-11 \\
2.9 \mathrm{E}-15 \\
3.5 \mathrm{E}-15\end{array}$ & $\begin{array}{l}5.3 E-10 \\
4.7 E-09 \\
7.7 E-13 \\
9.5 E-13\end{array}$ & $\begin{array}{l}\text { LLI Wall } \\
\text { LLI Wall } \\
\text { LLI Wall } \\
\text { LLI Wall }\end{array}$ \\
\hline
\end{tabular}


TABLE 3.4. Drinking Water Scenario Annual Committed Effective Dose Equivalent Factors for Exposure to Residual Radioactive Materials (a) (contd)

\begin{tabular}{|c|c|c|c|c|c|}
\hline \multirow{2}{*}{ Radionuclide } & TEDE & TEDE & \multicolumn{3}{|c|}{ Maximum Organ Dose } \\
\hline & (mrem) & $(\mu S v)$ & (mrem) & $(\mu \mathrm{Sv})$ & Organ (b) \\
\hline $\begin{array}{l}{ }^{96} \mathrm{Nb} \\
{ }_{97}^{97} \mathrm{mb}+\mathrm{D} \\
\mathrm{Nb} \\
{ }^{87} \mathrm{Nb}\end{array}$ & $\begin{array}{l}3.4 E-14 \\
6.5 E-25 \\
2.1 E-26 \\
6.8 E-21\end{array}$ & $\begin{array}{l}9.3 \mathrm{E}-12 \\
1.8 \mathrm{E}-22 \\
5.7 \mathrm{E}-24 \\
1.8 \mathrm{E}-18\end{array}$ & $\begin{array}{l}2.1 \mathrm{E}-13 \\
3.8 \mathrm{E}-24 \\
1.5 \mathrm{E}-25 \\
3.9 \mathrm{E}-20\end{array}$ & $\begin{array}{l}5.6 \mathrm{E}-11 \\
1.0 \mathrm{E}-21 \\
3.9 \mathrm{E}-23 \\
1.1 \mathrm{E}-17\end{array}$ & $\begin{array}{l}\text { LLI Wall } \\
\text { Stomach Wall } \\
\text { Stomach Wall } \\
\text { Stomach Wall }\end{array}$ \\
\hline $\begin{array}{l}{ }^{93} \mathrm{Mo}+\mathrm{D} \\
{ }_{93}{ }_{\mathrm{Mo}}(\mathrm{c}) \\
{ }^{99} \mathrm{Mo}+\mathrm{D} \\
{ }^{99} \mathrm{Mo}\end{array}$ & $\begin{array}{l}3.4 \mathrm{E}-12 \\
5.2 \mathrm{E}-12 \\
1.4 \mathrm{E}-15 \\
2.7 \mathrm{E}-15\end{array}$ & $\begin{array}{l}9.1 \mathrm{E}-10 \\
1.4 \mathrm{E}-09 \\
3.9 \mathrm{E}-13 \\
7.4 \mathrm{E}-13\end{array}$ & $\begin{array}{l}1.2 E-11 \\
2.5 E-11 \\
5.6 E-15 \\
9.7 E-15\end{array}$ & $\begin{array}{l}3.4 E-09 \\
6.7 E-09 \\
1.5 E-12 \\
2.6 E-12\end{array}$ & $\begin{array}{l}\text { Liver } \\
\text { Liver } \\
\text { LLI Wall } \\
\text { LLI Wall }\end{array}$ \\
\hline $\begin{array}{l}{ }^{99 \mathrm{~m}} \mathrm{TC} \\
{ }^{99} \mathrm{TC} \\
{ }^{101} \mathrm{TC}\end{array}$ & $\begin{array}{l}6.1 E-19 \\
1.2 E-11 \\
8.5 E-22\end{array}$ & $\begin{array}{l}1.6 E-16 \\
3.1 E-09 \\
2.3 E-19\end{array}$ & $\begin{array}{l}3.0 \mathrm{E}-18 \\
1.0 \mathrm{E}-10 \\
6.5 \mathrm{E}-21\end{array}$ & $\begin{array}{l}8.1 E-16 \\
2.7 E-08 \\
1.7 E-18\end{array}$ & $\begin{array}{l}\text { ULI Wall } \\
\text { Thyroid } \\
\text { ULI Wall }\end{array}$ \\
\hline $\begin{array}{l}{ }_{{ }^{103} \mathrm{Ru} \mathrm{D}(\mathrm{c})} \\
{ }^{105} \mathrm{Ru} \\
{ }^{165} \mathrm{Ru+D} \text { (c) } \\
{ }^{106} \mathrm{Ru+I} \mathrm{Ru+I}(\mathrm{d})\end{array}$ & $\begin{array}{l}2.7 \mathrm{E}-13 \\
5.4 \mathrm{E}-13 \\
3.6 \mathrm{E}-18 \\
5.2 \mathrm{E}-18 \\
3.1 \mathrm{E}-11\end{array}$ & $\begin{array}{l}7.2 \mathrm{E}-11 \\
1.4 \mathrm{E}-10 \\
9.7 \mathrm{E}-16 \\
1.4 \mathrm{E}-15 \\
8.4 \mathrm{E}-09\end{array}$ & $\begin{array}{l}2.1 E-12 \\
4.3 E-12 \\
2.7 E-17 \\
2.8 E-17 \\
3.1 E-10\end{array}$ & $\begin{array}{l}5.8 E-10 \\
1.2 E-09 \\
7.2 E-15 \\
7.5 E-15 \\
8.4 E-08\end{array}$ & $\begin{array}{l}\text { LLI } \text { Wall } \\
\text { LLI } \text { Wall } \\
\text { LLI Wall } \\
\text { ULI } \text { Wall } \\
\text { LLI Wall }\end{array}$ \\
\hline $\begin{aligned}{ }^{103 m} \mathrm{Rh} \\
{ }^{106} \mathrm{Rh}\end{aligned}$ & $\begin{array}{l}1.8 E-21 \\
3.3 E-16\end{array}$ & $\begin{array}{l}5.0 E-19 \\
8.9 E-14\end{array}$ & $\begin{array}{l}1.5 \mathrm{E}-20 \\
3.1 \mathrm{E}-15\end{array}$ & $\begin{array}{l}4.0 E-18 \\
8.3 E-13\end{array}$ & $\begin{array}{l}\text { Stomach Wall } \\
\text { LLI Wall }\end{array}$ \\
\hline $\begin{array}{l}{ }^{163} \mathrm{Pd}+\mathrm{D} \\
{ }^{163} \mathrm{Pd} \\
{ }^{167} \mathrm{Pd} \\
{ }^{169} \mathrm{Pd}+\mathrm{I}\end{array}$ & $\begin{array}{l}1.2 \mathrm{E}-14 \\
2.3 \mathrm{E}-14 \\
4.9 \mathrm{E}-13 \\
4.1 \mathrm{E}-17\end{array}$ & $\begin{array}{l}3.1 \mathrm{E}-12 \\
6.2 \mathrm{E}-12 \\
1.3 \mathrm{E}-10 \\
1.1 \mathrm{E}-14\end{array}$ & $\begin{array}{l}1.2 \mathrm{E}-13 \\
2.5 \mathrm{E}-13 \\
5.5 \mathrm{E}-12 \\
3.3 \mathrm{E}-16\end{array}$ & $\begin{array}{l}3.4 \mathrm{E}-11 \\
6.7 \mathrm{E}-11 \\
1.5 \mathrm{E}-09 \\
9.0 \mathrm{E}-14\end{array}$ & $\begin{array}{l}\text { LLI } \text { Wall } \\
\text { LLI } \text { Wall } \\
\text { LLI } \text { Wall } \\
\text { LLI Wall }\end{array}$ \\
\hline${ }^{116 m} \mathrm{Ag}+\mathrm{I}(\mathrm{d})$ & $\begin{array}{l}4.6 E-14 \\
4.7 E-17\end{array}$ & $\begin{array}{l}1.2 \mathrm{E}-11 \\
1.3 \mathrm{E}-14\end{array}$ & $\begin{array}{l}3.5 E-13 \\
5.0 E-16\end{array}$ & $\begin{array}{l}9.4 \mathrm{E}-11 \\
1.4 \mathrm{E}-13\end{array}$ & $\begin{array}{l}\text { LLI Wall } \\
\text { LLI Wall }\end{array}$ \\
\hline $\begin{array}{l}{ }^{109} \mathrm{Cd} \\
{ }_{113{ }^{m}} \mathrm{Cd} \\
{ }_{115} \mathrm{Cd}(\mathrm{C}) \\
{ }^{115{ }^{m}} \mathrm{Cd}+\mathrm{C} \\
{ }^{116} \mathrm{Cd}\end{array}$ & $\begin{array}{l}4.4 E-12 \\
7.4 E-11 \\
2.3 E-13 \\
4.7 E-13 \\
4.5 E-16\end{array}$ & $\begin{array}{l}1.2 E-09 \\
2.0 E-08 \\
6.3 E-11 \\
1.3 E-10 \\
1.2 E-13\end{array}$ & $\begin{array}{l}5.0 \mathrm{E}-11 \\
9.7 \mathrm{E}-10 \\
1.5 \mathrm{E}-12 \\
2.9 \mathrm{E}-12 \\
4.2 \mathrm{E}-15\end{array}$ & $\begin{array}{l}1.4 E-08 \\
2.6 E-07 \\
3.9 E-10 \\
7.9 E-10 \\
1.1 E-12\end{array}$ & $\begin{array}{l}\text { Kidneys } \\
\text { Kidneys } \\
\text { LLI Wall } \\
\text { LLI Wall } \\
\text { LLI Wall }\end{array}$ \\
\hline${ }_{115 m}^{1111} \operatorname{In} \operatorname{In}_{I n}(d)$ & $\begin{array}{l}1.0 \mathrm{E}-15 \\
1.9 \mathrm{E}-12 \\
1.2 \mathrm{E}-18\end{array}$ & $\begin{array}{l}2.8 E-13 \\
5.0 E-10 \\
3.2 E-16\end{array}$ & $\begin{array}{l}5.9 E-15 \\
2.0 E-11 \\
7.0 E-18\end{array}$ & $\begin{array}{l}1.6 E-12 \\
5.4 E-09 \\
1.9 E-15\end{array}$ & $\begin{array}{l}\text { LLI } \text { Wall } \\
\text { LLI } \text { Wall } \\
\text { ULI } \text { Wall }\end{array}$ \\
\hline $\begin{array}{l}{ }_{117 m}^{119 m} \mathrm{Sn} \\
{ }_{121}^{121 m} \mathrm{Sn} \\
\mathrm{Sn} \\
\mathrm{Sn}\end{array}$ & $\begin{array}{l}9.3 \mathrm{E}-17 \\
4.2 \mathrm{E}-15 \\
6.4 \mathrm{E}-15 \\
7.8 \mathrm{E}-15\end{array}$ & $\begin{array}{l}2.5 \mathrm{E}-14 \\
1.1 \mathrm{E}-12 \\
1.7 \mathrm{E}-12 \\
2.1 \mathrm{E}-12\end{array}$ & $\begin{array}{l}9.6 \mathrm{E}-16 \\
4.5 \mathrm{E}-14 \\
6.7 \mathrm{E}-14 \\
8.3 \mathrm{E}-14\end{array}$ & $\begin{array}{l}2.6 \mathrm{E}-13 \\
1.2 \mathrm{E}-11 \\
1.8 \mathrm{E}-11 \\
2.2 \mathrm{E}-11\end{array}$ & $\begin{array}{l}\text { LLI } \text { Wall } \\
\text { LLI } \text { Wall } \\
\text { LLI } \text { Wall } \\
\text { LLI } \text { Wall }\end{array}$ \\
\hline
\end{tabular}


TABLE 3.4. Drinking Water Scenario Annual Committed Effective Dose Equivalent Factors for Exposure to Residual Radioactive Materials (a) (contd)

\begin{tabular}{|c|c|c|c|c|c|}
\hline & TEDE & TEDE & \multicolumn{3}{|c|}{ Maximum Organ Dose } \\
\hline Radionuclide & (mrem) & $(\mu S v)$ & (mrem) & $(\mu \mathrm{Sv})$ & $\operatorname{Organ}(\mathrm{b})$ \\
\hline $\begin{array}{l}{ }^{121} \mathrm{Sn} \\
{ }^{123} \mathrm{Sn} \\
{ }^{125} \mathrm{Sn+D}(\mathrm{c}) \\
{ }^{126} \mathrm{Sn} \\
{ }^{126} \mathrm{Sn+D}(\mathrm{c}) \\
{ }^{126} \mathrm{Sn}\end{array}$ & $\begin{array}{l}1.9 \mathrm{E}-19 \\
1.3 \mathrm{E}-14 \\
1.1 \mathrm{E}-16 \\
1.8 \mathrm{E}-16 \\
5.2 \mathrm{E}-14 \\
1.0 \mathrm{E}-13\end{array}$ & $\begin{array}{l}5.1 \mathrm{E}-17 \\
3.6 \mathrm{E}-12 \\
3.0 \mathrm{E}-14 \\
4.9 \mathrm{E}-14 \\
1.4 \mathrm{E}-11 \\
2.7 \mathrm{E}-11\end{array}$ & $\begin{array}{l}1.9 \mathrm{E}-18 \\
1.5 \mathrm{E}-13 \\
1.1 \mathrm{E}-15 \\
2.0 \mathrm{E}-15 \\
4.2 \mathrm{E}-13 \\
8.4 \mathrm{E}-13\end{array}$ & $\begin{array}{l}5.0 \mathrm{E}-16 \\
4.1 \mathrm{E}-11 \\
3.0 \mathrm{E}-13 \\
5.4 \mathrm{E}-13 \\
1.1 \mathrm{E}-10 \\
2.3 \mathrm{E}-10\end{array}$ & $\begin{array}{l}\text { LLI Wall } \\
\text { LLI Wal1 } \\
\text { LLI Wal1 } \\
\text { LLI Wall } \\
\text { LLI Wall } \\
\text { LLI Wall }\end{array}$ \\
\hline 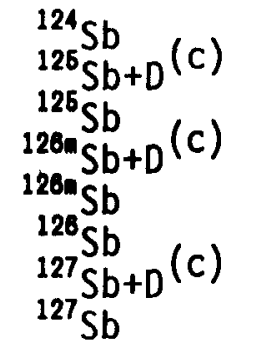 & $\begin{array}{l}5.4 \mathrm{E}-15 \\
1.3 \mathrm{E}-14 \\
1.2 \mathrm{E}-14 \\
3.8 \mathrm{E}-23 \\
2.6 \mathrm{E}-24 \\
2.5 \mathrm{E}-16 \\
1.0 \mathrm{E}-17 \\
1.8 \mathrm{E}-17\end{array}$ & $\begin{array}{l}1.5 \mathrm{E}-12 \\
3.5 \mathrm{E}-12 \\
3.4 \mathrm{E}-12 \\
1.0 \mathrm{E}-20 \\
7.0 \mathrm{E}-22 \\
6.8 \mathrm{E}-14 \\
2.8 \mathrm{E}-15 \\
4.7 \mathrm{E}-15\end{array}$ & $\begin{array}{l}4.7 \mathrm{E}-14 \\
1.0 \mathrm{E}-13 \\
1.1 \mathrm{E}-13 \\
2.5 \mathrm{E}-22 \\
2.7 \mathrm{E}-23 \\
1.8 \mathrm{E}-15 \\
9.7 \mathrm{E}-17 \\
1.8 \mathrm{E}-16\end{array}$ & $\begin{array}{l}1.3 \mathrm{E}-11 \\
2.7 \mathrm{E}-11 \\
2.9 \mathrm{E}-11 \\
6.7 \mathrm{E}-20 \\
7.4 \mathrm{E}-21 \\
4.8 \mathrm{E}-13 \\
2.6 \mathrm{E}-14 \\
4.9 \mathrm{E}-14\end{array}$ & $\begin{array}{l}\text { LLI Wal1 } \\
\text { LLI Wal1 } \\
\text { LLI Wall } \\
\text { LLI Wall } \\
\text { Stomach Wall } \\
\text { LLI Wal1 } \\
\text { LLI Wal1 } \\
\text { LLI Wall }\end{array}$ \\
\hline 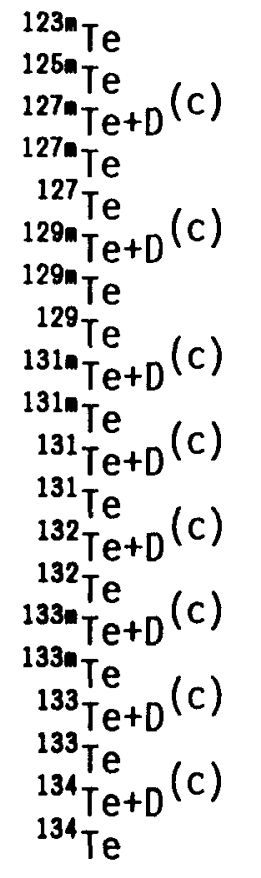 & $\begin{array}{l}5.2 \mathrm{E}-12 \\
1.2 \mathrm{E}-12 \\
3.6 \mathrm{E}-12 \\
6.6 \mathrm{E}-12 \\
1.1 \mathrm{E}-17 \\
3.8 \mathrm{E}-11 \\
6.1 \mathrm{E}-11 \\
6.1 \mathrm{E}-20 \\
4.7 \mathrm{E}-15 \\
2.7 \mathrm{E}-15 \\
7.6 \mathrm{E}-19 \\
6.4 \mathrm{E}-21 \\
8.1 \mathrm{E}-15 \\
1.6 \mathrm{E}-14 \\
5.2 \mathrm{E}-19 \\
1.2 \mathrm{E}-19 \\
2.5 \mathrm{E}-20 \\
1.2 \mathrm{E}-21 \\
1.9 \mathrm{E}-20 \\
1.6 \mathrm{E}-20\end{array}$ & $\begin{array}{l}1.4 \mathrm{E}-09 \\
3.2 \mathrm{E}-10 \\
9.8 \mathrm{E}-10 \\
1.8 \mathrm{E}-09 \\
3.0 \mathrm{E}-15 \\
1.0 \mathrm{E}-08 \\
1.7 \mathrm{E}-08 \\
1.7 \mathrm{E}-17 \\
1.3 \mathrm{E}-12 \\
7.3 \mathrm{E}-13 \\
2.1 \mathrm{E}-16 \\
1.7 \mathrm{E}-18 \\
2.2 \mathrm{E}-12 \\
4.4 \mathrm{E}-12 \\
1.4 \mathrm{E}-16 \\
3.2 \mathrm{E}-17 \\
6.7 \mathrm{E}-18 \\
3.3 \mathrm{E}-19 \\
5.2 \mathrm{E}-18 \\
4.4 \mathrm{E}-18\end{array}$ & $\begin{array}{l}8.3 \mathrm{E}-11 \\
1.6 \mathrm{E}-11 \\
3.2 \mathrm{E}-11 \\
6.3 \mathrm{E}-11 \\
7.4 \mathrm{E}-17 \\
1.5 \mathrm{E}-10 \\
2.4 \mathrm{E}-10 \\
3.5 \mathrm{E}-19 \\
1.3 \mathrm{E}-13 \\
2.2 \mathrm{E}-14 \\
2.4 \mathrm{E}-17 \\
1.0 \mathrm{E}-19 \\
8.6 \mathrm{E}-14 \\
1.6 \mathrm{E}-13 \\
1.5 \mathrm{E}-17 \\
2.0 \mathrm{E}-18 \\
7.5 \mathrm{E}-19 \\
2.3 \mathrm{E}-20 \\
1.6 \mathrm{E}-19 \\
1.8 \mathrm{E}-19\end{array}$ & $\begin{array}{l}2.3 \mathrm{E}-08 \\
4.2 \mathrm{E}-09 \\
8.6 \mathrm{E}-09 \\
1.7 \mathrm{E}-08 \\
2.0 \mathrm{E}-14 \\
4.0 \mathrm{E}-08 \\
6.5 \mathrm{E}-08 \\
9.6 \mathrm{E}-17 \\
3.4 \mathrm{E}-11 \\
6.0 \mathrm{E}-12 \\
6.6 \mathrm{E}-15 \\
2.7 \mathrm{E}-17 \\
2.3 \mathrm{E}-11 \\
4.4 \mathrm{E}-11 \\
4.0 \mathrm{E}-15 \\
5.3 \mathrm{E}-16 \\
2.0 \mathrm{E}-16 \\
6.1 \mathrm{E}-18 \\
4.4 \mathrm{E}-17 \\
4.8 \mathrm{E}-17\end{array}$ & $\begin{array}{l}\text { Bone Surface } \\
\text { Bone Surface } \\
\text { Bone Surface } \\
\text { Bone Surface } \\
\text { LLI Wall } \\
\text { Testes } \\
\text { Testes } \\
\text { Stomach wall } \\
\text { Thyroid } \\
\text { Thyroid } \\
\text { Thyroid } \\
\text { Thyroid } \\
\text { Thyroid } \\
\text { Thyroid } \\
\text { Thyroid } \\
\text { Thyroid } \\
\text { Thyroid } \\
\text { Thyroid } \\
\text { Thyroid } \\
\text { Thyroid }\end{array}$ \\
\hline $\begin{array}{l}{ }^{125} \mathrm{I} \\
{ }^{129} \mathrm{I} \\
{ }^{130} \mathrm{I} \\
{ }^{131} \mathrm{I}+\mathrm{D}(\mathrm{C}) \\
131 \mathrm{I} \\
{ }^{132} \mathrm{I} \\
{ }^{133} \mathrm{I}+\mathrm{D}(\mathrm{C})\end{array}$ & $\begin{array}{l}1.4 \mathrm{E}-11 \\
9.3 \mathrm{E}-10 \\
1.4 \mathrm{E}-16 \\
3.7 \mathrm{E}-13 \\
3.8 \mathrm{E}-13 \\
7.4 \mathrm{E}-19 \\
4.2 \mathrm{E}-16 \\
8.5 \mathrm{E}-16\end{array}$ & $\begin{array}{l}3.8 \mathrm{E}-09 \\
2.5 \mathrm{E}-07 \\
3.7 \mathrm{E}-14 \\
1.0 \mathrm{E}-10 \\
1.0 \mathrm{E}-10 \\
2.0 \mathrm{E}-16 \\
1.1 \mathrm{E}-13 \\
2.3 \mathrm{E}-13\end{array}$ & $\begin{array}{l}4.7 E-10 \\
3.1 E-08 \\
3.7 E-15 \\
1.2 E-11 \\
1.2 E-11 \\
1.1 E-17 \\
1.3 E-14 \\
2.6 E-14\end{array}$ & $\begin{array}{l}1.3 \mathrm{E}-07 \\
8.3 \mathrm{E}-06 \\
1.0 \mathrm{E}-12 \\
3.2 \mathrm{E}-09 \\
3.3 \mathrm{E}-09 \\
2.9 \mathrm{E}-15 \\
3.5 \mathrm{E}-12 \\
7.1 \mathrm{E}-12\end{array}$ & $\begin{array}{l}\text { Thyroid } \\
\text { Thyroid } \\
\text { Thyroid } \\
\text { Thyroid } \\
\text { Thyroid } \\
\text { Thyroid } \\
\text { Thyroid } \\
\text { Thyroid }\end{array}$ \\
\hline
\end{tabular}


TABLE 3.4. Drinking Water Scenario Annual Committed Effective Dose Equivalent Factors for Exposure to Residual Radioactive Materials(a) (contd)

\begin{tabular}{|c|c|c|c|c|c|}
\hline \multirow[b]{2}{*}{ Radionuclide } & \multirow{2}{*}{$\begin{array}{l}\text { TEDE } \\
\text { (mrem) }\end{array}$} & \multirow{2}{*}{$\begin{array}{l}\text { TEDE } \\
(\mu S v)\end{array}$} & \multicolumn{3}{|c|}{ Maximum Organ Dose } \\
\hline & & & (mrem) & $(\mu \mathrm{Sv})$ & Organ (b) \\
\hline${ }_{135}^{134} I_{I}^{13}+D(C)$ & $\begin{array}{l}4.0 E-20 \\
8.5 E-18 \\
1.8 E-17\end{array}$ & $\begin{array}{l}1.1 E-17 \\
2.3 E-15 \\
5.0 E-15\end{array}$ & $\begin{array}{l}3.3 E-19 \\
2.1 E-16 \\
4.6 E-16\end{array}$ & $\begin{array}{l}8.8 E-17 \\
5.7 E-14 \\
1.2 E-13\end{array}$ & $\begin{array}{l}\text { Stomach wall } \\
\text { Thyroid } \\
\text { Thyroid }\end{array}$ \\
\hline $\begin{array}{l}{ }^{134 m} \mathrm{Cs}+\mathrm{D}^{(\mathrm{C})} \\
{ }_{134 \mathrm{~m}} \mathrm{Cs} \\
{ }^{134} \mathrm{Cs} \\
{ }_{135} \mathrm{Cs} \\
{ }^{136} \mathrm{Cs} \quad(\mathrm{d}) \\
{ }^{137} \mathrm{Cs}+\mathrm{I} \\
{ }^{138} \mathrm{Cs}\end{array}$ & $\begin{array}{l}4.3 \mathrm{E}-23 \\
8.6 \mathrm{E}-23 \\
2.8 \mathrm{E}-13 \\
3.4 \mathrm{E}-14 \\
2.9 \mathrm{E}-16 \\
1.2 \mathrm{E}-13 \\
1.6 \mathrm{E}-23\end{array}$ & $\begin{array}{l}1.2 \mathrm{E}-20 \\
2.3 \mathrm{E}-20 \\
7.6 \mathrm{E}-11 \\
9.1 \mathrm{E}-12 \\
7.7 \mathrm{E}-14 \\
3.3 \mathrm{E}-11 \\
4.2 \mathrm{E}-21\end{array}$ & $\begin{array}{l}4.9 \mathrm{E}-22 \\
9.8 \mathrm{E}-22 \\
3.7 \mathrm{E}-13 \\
4.1 \mathrm{E}-14 \\
4.0 \mathrm{E}-16 \\
1.5 \mathrm{E}-13 \\
2.2 \mathrm{E}-22\end{array}$ & $\begin{array}{l}1.3 \mathrm{E}-19 \\
2.7 \mathrm{E}-19 \\
1.0 \mathrm{E}-10 \\
1.1 \mathrm{E}-11 \\
1.1 \mathrm{E}-13 \\
4.1 \mathrm{E}-11 \\
6.0 \mathrm{E}-20\end{array}$ & $\begin{array}{l}\text { Stomach Wall } \\
\text { Stomach Wall } \\
\text { SI Wall } \\
\text { SI Wall } \\
\text { SI Wall } \\
\text { SI Wall } \\
\text { Stomach Wall }\end{array}$ \\
\hline $\begin{array}{l}{ }^{139} \mathrm{Ba} \\
{ }^{146} \mathrm{Ba}+\mathrm{D} \\
{ }^{140} \mathrm{Ba} \\
{ }^{141} \mathrm{Ba}+\mathrm{D} \\
{ }^{141} \mathrm{Ba} \\
\left.{ }^{142} \mathrm{Ba}+\mathrm{C}\right) \\
{ }^{142} \mathrm{Ba}\end{array}$ & $\begin{array}{l}1.5 \mathrm{E}-22 \\
1.6 \mathrm{E}-16 \\
1.7 \mathrm{E}-16 \\
2.8 \mathrm{E}-23 \\
3.8 \mathrm{E}-24 \\
2.5 \mathrm{E}-24 \\
6.8 \mathrm{E}-25\end{array}$ & $\begin{array}{l}4.1 \mathrm{E}-20 \\
4.2 \mathrm{E}-14 \\
4.5 \mathrm{E}-14 \\
7.5 \mathrm{E}-21 \\
1.0 \mathrm{E}-21 \\
6.8 \mathrm{E}-22 \\
1.8 \mathrm{E}-22\end{array}$ & $\begin{array}{l}9.7 \mathrm{E}-22 \\
1.5 \mathrm{E}-15 \\
1.8 \mathrm{E}-15 \\
2.4 \mathrm{E}-22 \\
2.5 \mathrm{E}-23 \\
1.2 \mathrm{E}-23 \\
4.6 \mathrm{E}-24\end{array}$ & $\begin{array}{l}2.6 \mathrm{E}-19 \\
4.0 \mathrm{E}-13 \\
4.8 \mathrm{E}-13 \\
6.4 \mathrm{E}-20 \\
6.9 \mathrm{E}-21 \\
3.4 \mathrm{E}-21 \\
1.2 \mathrm{E}-21\end{array}$ & $\begin{array}{l}\text { Stomach Wall } \\
\text { LLI Wall } \\
\text { LLI Wall } \\
\text { LLI Wall } \\
\text { Stomach Wall } \\
\text { Stomach Wall } \\
\text { Stomach Wall }\end{array}$ \\
\hline $\begin{array}{l}{ }^{148} \mathrm{La} \\
141 \mathrm{La}+\mathrm{D}(\mathrm{c}) \\
{ }_{111} \mathrm{La} \\
{ }^{142} \mathrm{La}\end{array}$ & $\begin{array}{l}2.6 \mathrm{E}-18 \\
6.7 \mathrm{E}-21 \\
4.5 \mathrm{E}-21 \\
3.5 \mathrm{E}-22\end{array}$ & $\begin{array}{l}7.1 \mathrm{E}-16 \\
1.8 \mathrm{E}-18 \\
1.2 \mathrm{E}-18 \\
9.3 \mathrm{E}-20\end{array}$ & $\begin{array}{l}2.0 \mathrm{E}-17 \\
5.7 \mathrm{E}-20 \\
2.9 \mathrm{E}-20 \\
1.6 \mathrm{E}-21\end{array}$ & $\begin{array}{l}5.4 \mathrm{E}-15 \\
1.6 \mathrm{E}-17 \\
7.8 \mathrm{E}-18 \\
4.4 \mathrm{E}-19\end{array}$ & $\begin{array}{l}\text { LLI Wall } \\
\text { LLI Wall } \\
\text { ULI Wall } \\
\text { Stomach Wall }\end{array}$ \\
\hline $\begin{array}{l}{ }^{141} \mathrm{Ce} \\
{ }_{143} \mathrm{Ce}+\mathrm{D} \\
{ }_{143} \mathrm{Ce} \\
\left.{ }_{144} \mathrm{Ce} \text { ( }\right) \\
{ }^{144} \mathrm{Ce}\end{array}$ & $\begin{array}{l}1.4 \mathrm{E}-15 \\
4.2 \mathrm{E}-18 \\
4.1 \mathrm{E}-18 \\
8.9 \mathrm{E}-14 \\
1.8 \mathrm{E}-13\end{array}$ & $\begin{array}{l}3.9 \mathrm{E}-13 \\
1.1 \mathrm{E}-15 \\
1.1 \mathrm{E}-15 \\
2.4 \mathrm{E}-11 \\
4.8 \mathrm{E}-11\end{array}$ & $\begin{array}{l}1.6 \mathrm{E}-14 \\
4.5 \mathrm{E}-17 \\
4.0 \mathrm{E}-17 \\
1.1 \mathrm{E}-12 \\
2.1 \mathrm{E}-12\end{array}$ & $\begin{array}{l}4.3 \mathrm{E}-12 \\
1.2 \mathrm{E}-14 \\
1.1 \mathrm{E}-14 \\
2.9 \mathrm{E}-10 \\
5.8 \mathrm{E}-10\end{array}$ & $\begin{array}{l}\text { LLI Wall } \\
\text { LLI Wal1 } \\
\text { LLI Wall } \\
\text { LLI Wall } \\
\text { LLI Wal1 }\end{array}$ \\
\hline $\begin{array}{l}{ }_{143}^{144} \mathrm{Pr} \\
{ }^{144} \mathrm{Pr}+\mathrm{D} \\
{ }^{144} \mathrm{Pr} \\
{ }^{140} \mathrm{Pr}\end{array}$ & $\begin{array}{l}8.8 \mathrm{E}-14 \\
2.2 \mathrm{E}-22 \\
1.2 \mathrm{E}-22 \\
1.7 \mathrm{E}-21\end{array}$ & $\begin{array}{l}2.4 \mathrm{E}-11 \\
5.8 \mathrm{E}-20 \\
3.3 \mathrm{E}-20 \\
4.7 \mathrm{E}-19\end{array}$ & $\begin{array}{l}1.0 \mathrm{E}-12 \\
2.7 \mathrm{E}-21 \\
1.4 \mathrm{E}-21 \\
2.3 \mathrm{E}-20\end{array}$ & $\begin{array}{l}2.8 E-10 \\
7.2 E-19 \\
3.8 E-19 \\
6.3 E-18\end{array}$ & $\begin{array}{l}\text { LLI Wall } \\
\text { Stomach Wall } \\
\text { Stomach Wall } \\
\text { Stomach Wall }\end{array}$ \\
\hline $\begin{array}{l}{ }^{147} \mathrm{Nd}+\mathrm{D} \\
{ }^{147} \mathrm{Nd}\end{array}$ & $\begin{array}{l}3.2 E-14 \\
5.1 E-14\end{array}$ & $\begin{array}{l}8.6 \mathrm{E}-12 \\
1.4 \mathrm{E}-11\end{array}$ & $\begin{array}{l}3.4 E-13 \\
5.4 E-13\end{array}$ & $\begin{array}{l}9.3 \mathrm{E}-11 \\
1.5 \mathrm{E}-10\end{array}$ & $\begin{array}{l}\text { LLI Wall } \\
\text { LLI Wall }\end{array}$ \\
\hline $\begin{array}{l}{ }^{147} \mathrm{Pm} \\
{ }^{148 \mathrm{~m}} \mathrm{Pm}+\mathrm{D} \\
{ }_{148 \mathrm{~m}} \mathrm{Pm} \\
{ }_{148}^{149} \mathrm{Pm} \\
{ }^{161} \mathrm{Pm} \\
{ }^{14 m}\end{array}$ & $\begin{array}{l}2.7 \mathrm{E}-12 \\
1.3 \mathrm{E}-12 \\
1.3 \mathrm{E}-12 \\
3.2 \mathrm{E}-14 \\
2.0 \mathrm{E}-15 \\
4.2 \mathrm{E}-16\end{array}$ & $\begin{array}{l}7.3 \mathrm{E}-10 \\
3.6 \mathrm{E}-10 \\
3.5 \mathrm{E}-10 \\
8.7 \mathrm{E}-12 \\
5.5 \mathrm{E}-13 \\
1.1 \mathrm{E}-13\end{array}$ & $\begin{array}{l}3.3 \mathrm{E}-11 \\
9.3 \mathrm{E}-12 \\
8.8 \mathrm{E}-12 \\
3.5 \mathrm{E}-13 \\
2.2 \mathrm{E}-14 \\
3.8 \mathrm{E}-15\end{array}$ & $\begin{array}{l}8.8 \mathrm{E}-09 \\
2.5 \mathrm{E}-09 \\
2.4 \mathrm{E}-09 \\
9.5 \mathrm{E}-11 \\
5.9 \mathrm{E}-12 \\
1.0 \mathrm{E}-12\end{array}$ & $\begin{array}{l}\text { LLI Wall } \\
\text { LLI Wal1 } \\
\text { LLI Wall } \\
\text { LLI Wall } \\
\text { LLI Wal1 } \\
\text { LLI Wall }\end{array}$ \\
\hline
\end{tabular}


TABLE 3.4. Drinking Water Scenario Annual Committed Effective Dose Equivalent Factors for Exposure to Residual Radioactive Materials (a) (contd)

\begin{tabular}{|c|c|c|c|c|c|}
\hline & TEDE & TEDE & \multicolumn{3}{|c|}{ Maximum Organ Dose } \\
\hline Radionuclide & (mrem) & $(\mu \mathrm{Sv})$ & (mrem) & $(\mu \mathrm{Sv})$ & $\operatorname{Organ}(b)$ \\
\hline $\begin{array}{l}{ }^{147} \mathrm{Sm} \\
{ }^{151} \mathrm{Sm} \\
{ }^{153}\end{array}$ & $\begin{array}{l}6.2 \mathrm{E}-10 \\
1.2 \mathrm{E}-12 \\
1.2 \mathrm{E}-15\end{array}$ & $\begin{array}{l}1.7 E-07 \\
3.3 E-10 \\
3.1 E-13\end{array}$ & $\begin{array}{l}1.0 E-08 \\
1.2 E-11 \\
1.2 E-14\end{array}$ & $\begin{array}{l}2.8 \mathrm{E}-06 \\
3.2 \mathrm{E}-09 \\
3.1 \mathrm{E}-12\end{array}$ & $\begin{array}{l}\text { Bone Surface } \\
\text { LLI Wall } \\
\text { LLI Wall }\end{array}$ \\
\hline $\begin{array}{l}{ }^{152} \mathrm{Eu} \\
{ }^{154} \mathrm{Eu} \\
{ }^{156} \mathrm{Eu} \\
{ }^{158} \mathrm{Eu}\end{array}$ & $\begin{array}{l}2.2 \mathrm{E}-14 \\
3.1 \mathrm{E}-14 \\
4.9 \mathrm{E}-15 \\
2.4 \mathrm{E}-16\end{array}$ & $\begin{array}{l}5.9 \mathrm{E}-12 \\
8.5 \mathrm{E}-12 \\
1.3 \mathrm{E}-12 \\
6.4 \mathrm{E}-14\end{array}$ & $\begin{array}{l}1.3 E-13 \\
2.3 E-13 \\
4.3 E-14 \\
2.2 E-15\end{array}$ & $\begin{array}{l}3.5 \mathrm{E}-11 \\
6.2 \mathrm{E}-11 \\
1.2 \mathrm{E}-11 \\
6.0 \mathrm{E}-13\end{array}$ & $\begin{array}{l}\text { LLI Wall } \\
\text { LLI Wall } \\
\text { LLI Wall } \\
\text { LLI Wall }\end{array}$ \\
\hline${ }^{163} \mathrm{Gd}$ & $2.0 \mathrm{E}-12$ & $5.4 E-10$ & $1.7 \mathrm{E}-11$ & $4.5 E-09$ & LLI Wall \\
\hline${ }^{180} \mathrm{~Tb}$ & $3.0 \mathrm{E}-12$ & $8.1 E-10$ & $2.5 \mathrm{E}-11$ & $6.8 \mathrm{E}-09$ & LLI Wall \\
\hline${ }^{188 m_{n}} \mathrm{Ho}$ & $2.5 \mathrm{E}-11$ & $6.7 E-09$ & $1.3 E-10$ & $3.6 \mathrm{E}-08$ & LLI Wall \\
\hline $\begin{array}{l}{ }^{181} \mathrm{~W} \\
185 \mathrm{~W} \\
187 \mathrm{~W}\end{array}$ & $\begin{array}{l}2.7 \mathrm{E}-13 \\
7.8 \mathrm{E}-13 \\
2.1 \mathrm{E}-16\end{array}$ & $\begin{array}{l}7.2 \mathrm{E}-11 \\
2.1 \mathrm{E}-10 \\
5.7 \mathrm{E}-14\end{array}$ & $\begin{array}{l}1.7 \mathrm{E}-12 \\
8.2 \mathrm{E}-12 \\
1.7 \mathrm{E}-15\end{array}$ & $\begin{array}{l}4.7 E-10 \\
2.2 E-09 \\
4.6 E-13\end{array}$ & $\begin{array}{l}\text { LLI Wall } \\
\text { LLI Wall } \\
\text { LLI Wall }\end{array}$ \\
\hline${ }^{187} \mathrm{Re}$ & $4.5 E-14$ & $1.2 \mathrm{E}-11$ & $2.9 \mathrm{E}-13$ & $7.9 \mathrm{E}-11$ & ULI Wall \\
\hline $\begin{array}{l}{ }^{185} \text { Os } \\
{ }^{191} \text { Os }\end{array}$ & $\begin{array}{l}1.5 \mathrm{E}-12 \\
5.2 \mathrm{E}-14\end{array}$ & $\begin{array}{l}4.0 E-10 \\
1.4 E-11\end{array}$ & $\begin{array}{l}6.7 E-12 \\
5.5 E-13\end{array}$ & $\begin{array}{l}1.8 \mathrm{E}-09 \\
1.5 \mathrm{E}-10\end{array}$ & $\begin{array}{l}\text { LLI Wall } \\
\text { LLI Wall }\end{array}$ \\
\hline${ }^{192} \mathrm{Ir}$ & $2.7 \mathrm{E}-12$ & $7.3 E-10$ & $2.3 \mathrm{E}-11$ & $6.1 E-09$ & LLI Wall \\
\hline${ }^{203} \mathrm{Hg}$ & $2.4 \mathrm{E}-12$ & $6.3 E-10$ & $1.5 \mathrm{E}-11$ & $4.0 E-09$ & Kidneys \\
\hline $\begin{array}{l}{ }^{210} \mathrm{~Pb}+\mathrm{D} \\
{ }^{210} \mathrm{~Pb} \\
{ }^{212} \mathrm{~Pb}+\mathrm{C}(\mathrm{c}) \\
{ }^{212} \mathrm{~Pb}\end{array}$ & $\begin{array}{l}3.2 \mathrm{E}-10 \\
7.0 \mathrm{E}-10 \\
1.4 \mathrm{E}-17 \\
4.0 \mathrm{E}-17\end{array}$ & $\begin{array}{l}8.6 \mathrm{E}-08 \\
1.9 \mathrm{E}-07 \\
3.7 \mathrm{E}-15 \\
1.1 \mathrm{E}-14\end{array}$ & $\begin{array}{l}3.5 \mathrm{E}-09 \\
1.0 \mathrm{E}-08 \\
1.8 \mathrm{E}-16 \\
5.5 \mathrm{E}-16\end{array}$ & $\begin{array}{l}9.4 \mathrm{E}-07 \\
2.8 \mathrm{E}-06 \\
4.9 \mathrm{E}-14 \\
1.5 \mathrm{E}-13\end{array}$ & $\begin{array}{l}\text { Bone Surface } \\
\text { Bone Surface } \\
\text { Bone Surface } \\
\text { Bone Surface }\end{array}$ \\
\hline $\begin{array}{l}{ }^{210} \mathrm{Bi}+\mathrm{D} \\
{ }^{210} \mathrm{Bi} \\
{ }^{212} \mathrm{Bi}+\mathrm{C}(\mathrm{d})\end{array}$ & $\begin{array}{l}2.4 \mathrm{E}-12 \\
1.6 \mathrm{E}-14 \\
9.8 \mathrm{E}-20\end{array}$ & $\begin{array}{l}6.5 \mathrm{E}-10 \\
4.3 \mathrm{E}-12 \\
2.7 \mathrm{E}-17\end{array}$ & $\begin{array}{l}2.0 \mathrm{E}-11 \\
1.4 \mathrm{E}-13 \\
5.3 \mathrm{E}-19\end{array}$ & $\begin{array}{l}5.5 \mathrm{E}-09 \\
3.8 \mathrm{E}-11 \\
1.4 \mathrm{E}-16\end{array}$ & $\begin{array}{l}\text { Spleen } \\
\text { LLI Wall } \\
\text { Stomach Wall }\end{array}$ \\
\hline${ }^{216} \mathrm{Po}$ & $8.2 E-11$ & $2.2 E-08$ & $6.9 \mathrm{E}-10$ & $1.9 \mathrm{E}-07$ & Spleen \\
\hline $\begin{array}{l}{ }^{223} \mathrm{Ra}+\mathrm{I}(\mathrm{d}) \\
{ }^{224} \mathrm{Ra}+\mathrm{D}(\mathrm{c}) \\
{ }^{224} \mathrm{Ra}+\mathrm{I}(\mathrm{d}) \\
{ }^{225} \mathrm{Ra}+\mathrm{D}(\mathrm{c}) \\
{ }^{225} \mathrm{Ra} \\
{ }^{228} \mathrm{Ra}+\mathrm{D}\end{array}$ & $\begin{array}{l}2.4 \mathrm{E}-14 \\
1.6 \mathrm{E}-15 \\
7.1 \mathrm{E}-15 \\
4.3 \mathrm{E}-14 \\
2.0 \mathrm{E}-13 \\
1.2 \mathrm{E}-10\end{array}$ & $\begin{array}{l}6.6 \mathrm{E}-12 \\
4.4 \mathrm{E}-13 \\
1.9 \mathrm{E}-12 \\
1.2 \mathrm{E}-11 \\
5.4 \mathrm{E}-11 \\
3.3 \mathrm{E}-08\end{array}$ & $\begin{array}{l}3.7 \mathrm{E}-13 \\
1.7 \mathrm{E}-14 \\
6.5 \mathrm{E}-14 \\
7.7 \mathrm{E}-13 \\
5.1 \mathrm{E}-12 \\
1.5 \mathrm{E}-09\end{array}$ & $\begin{array}{l}1.0 \mathrm{E}-10 \\
4.7 \mathrm{E}-12 \\
1.8 \mathrm{E}-11 \\
2.1 \mathrm{E}-10 \\
1.4 \mathrm{E}-09 \\
4.0 \mathrm{E}-07\end{array}$ & $\begin{array}{l}\text { Bone Surface } \\
\text { Bone Surface } \\
\text { Bone Surface } \\
\text { Bone Surface } \\
\text { Bone Surface } \\
\text { Bone Surface }\end{array}$ \\
\hline
\end{tabular}


TABLE 3.4. Drinking Water Scenario Annual Committed Effective Dose Equivalent Factors for Exposure to Residual Radioactive Materials(a) (contd)

\begin{tabular}{|c|c|c|c|c|c|}
\hline & TEDE & TEDE & \multicolumn{3}{|c|}{ Maximum Organ Dose } \\
\hline Radionuclide & (mrem) & $(\mu S v)$ & (mrem) & $(\mu \mathrm{S} v)$ & $\operatorname{Organ}(\mathrm{b})$ \\
\hline $\begin{array}{l}{ }^{228}{ }_{\mathrm{Ra}}^{228} \\
{ }_{\mathrm{Ra}+\mathrm{D}}(\mathrm{c}) \\
{ }^{228} \mathrm{Ra}\end{array}$ & $\begin{array}{l}1.3 \mathrm{E}-10 \\
1.9 \mathrm{E}-11 \\
1.0 \mathrm{E}-10\end{array}$ & $\begin{array}{l}3.4 \mathrm{E}-08 \\
5.2 \mathrm{E}-09 \\
2.8 \mathrm{E}-08\end{array}$ & $\begin{array}{l}3.1 \mathrm{E}-09 \\
4.1 \mathrm{E}-10 \\
2.5 \mathrm{E}-09\end{array}$ & $\begin{array}{l}8.3 E-07 \\
1.1 E-07 \\
6.6 E-07\end{array}$ & $\begin{array}{l}\text { Bone Surface } \\
\text { Bone Surface } \\
\text { Bone Surface }\end{array}$ \\
\hline $\begin{array}{l}{ }^{225} \mathrm{AC}+1(\mathrm{~d}) \\
{ }^{227} \mathrm{AC}+\mathrm{D}(\mathrm{c}) \\
{ }^{2227} \mathrm{AC} \\
{ }^{228} \mathrm{AC}+\mathrm{D} \\
\mathrm{AC}\end{array}$ & $\begin{array}{l}7.6 \mathrm{E}-15 \\
2.3 \mathrm{E}-10 \\
1.8 \mathrm{E}-09 \\
2.0 \mathrm{E}-17 \\
5.7 \mathrm{E}-19\end{array}$ & $\begin{array}{l}2.1 \mathrm{E}-12 \\
6.2 \mathrm{E}-08 \\
4.9 \mathrm{E}-07 \\
5.3 \mathrm{E}-15 \\
1.5 \mathrm{E}-16\end{array}$ & $\begin{array}{l}6.9 E-14 \\
4.1 E-09 \\
3.3 E-08 \\
3.7 E-16 \\
3.0 E-18\end{array}$ & $\begin{array}{l}1.9 \mathrm{E}-11 \\
1.1 \mathrm{E}-06 \\
8.8 \mathrm{E}-06 \\
1.0 \mathrm{E}-13 \\
8.0 \mathrm{E}-16\end{array}$ & $\begin{array}{l}\text { LLI Wall } \\
\text { Bone Surface } \\
\text { Bone Surface } \\
\text { Bone Surface } \\
\text { Bone Surface }\end{array}$ \\
\hline $\begin{array}{l}{ }^{227} \mathrm{Th}+\mathrm{D}(\mathrm{c}) \\
{ }^{227} \mathrm{Th} \\
{ }^{228} \mathrm{Th}+\mathrm{D}(\mathrm{c}) \\
{ }^{228} \mathrm{Th} \\
{ }^{229} \mathrm{Th}+\mathrm{D}(\mathrm{c}) \\
{ }^{229} \mathrm{Th} \\
{ }^{236} \mathrm{Th}+\mathrm{D}(\mathrm{c}) \\
{ }^{238} \mathrm{Th} \\
{ }^{231} \mathrm{Th}+\mathrm{D} \\
{ }^{231} \mathrm{Th} \\
{ }^{232} \mathrm{Th}+\mathrm{D}(\mathrm{c}) \\
{ }^{232} \mathrm{Th}-\mathrm{Nat} \\
{ }^{234} \mathrm{Th} \\
{ }^{234} \mathrm{Th}+\mathrm{D} \\
{ }^{234} \mathrm{Th}+\mathrm{C} \\
\text { (d) }\end{array}$ & $\begin{array}{l}6.4 \mathrm{E}-14 \\
5.5 \mathrm{E}-14 \\
8.5 \mathrm{E}-12 \\
4.2 \mathrm{E}-11 \\
6.3 \mathrm{E}-11 \\
4.7 \mathrm{E}-10 \\
1.2 \mathrm{E}-10 \\
7.2 \mathrm{E}-11 \\
6.1 \mathrm{E}-19 \\
6.1 \mathrm{E}-18 \\
5.4 \mathrm{E}-11 \\
5.4 \mathrm{E}-11 \\
3.6 \mathrm{E}-10 \\
1.6 \mathrm{E}-14 \\
3.1 \mathrm{E}-14\end{array}$ & $\begin{array}{l}1.7 \mathrm{E}-11 \\
1.5 \mathrm{E}-11 \\
2.3 \mathrm{E}-09 \\
1.1 \mathrm{E}-08 \\
1.7 \mathrm{E}-08 \\
1.3 \mathrm{E}-07 \\
3.2 \mathrm{E}-08 \\
1.9 \mathrm{E}-08 \\
1.6 \mathrm{E}-16 \\
1.6 \mathrm{E}-15 \\
1.5 \mathrm{E}-08 \\
1.5 \mathrm{E}-08 \\
9.8 \mathrm{E}-08 \\
4.2 \mathrm{E}-12 \\
8.5 \mathrm{E}-12\end{array}$ & $\begin{array}{l}8.9 \mathrm{E}-13 \\
4.9 \mathrm{E}-13 \\
1.6 \mathrm{E}-10 \\
9.3 \mathrm{E}-10 \\
1.6 \mathrm{E}-09 \\
1.2 \mathrm{E}-08 \\
1.6 \mathrm{E}-09 \\
1.7 \mathrm{E}-09 \\
6.1 \mathrm{E}-18 \\
6.1 \mathrm{E}-17 \\
1.3 \mathrm{E}-09 \\
1.3 \mathrm{E}-09 \\
8.9 \mathrm{E}-09 \\
1.9 \mathrm{E}-13 \\
3.9 \mathrm{E}-13\end{array}$ & $\begin{array}{l}2.4 \mathrm{E}-10 \\
1.3 \mathrm{E}-10 \\
4.5 \mathrm{E}-08 \\
2.5 \mathrm{E}-07 \\
4.2 \mathrm{E}-07 \\
3.2 \mathrm{E}-06 \\
4.3 \mathrm{E}-07 \\
4.7 \mathrm{E}-07 \\
1.6 \mathrm{E}-15 \\
1.6 \mathrm{E}-14 \\
3.5 \mathrm{E}-07 \\
3.5 \mathrm{E}-07 \\
2.4 \mathrm{E}-06 \\
5.2 \mathrm{E}-11 \\
1.0 \mathrm{E}-10\end{array}$ & $\begin{array}{l}\text { Bone Surface } \\
\text { LLI Wall } \\
\text { Bone Surface } \\
\text { Bone Surface } \\
\text { Bone Surface } \\
\text { Bone Surface } \\
\text { Bone Surface } \\
\text { Bone Surface } \\
\text { LLI Wall } \\
\text { LLI Wall } \\
\text { Bone Surface } \\
\text { Bone Surface } \\
\text { Bone Surface } \\
\text { LLI Wall } \\
\text { LLI Wall }\end{array}$ \\
\hline $\begin{array}{l}{ }^{231} \mathrm{~Pa}+\mathrm{D} \\
{ }^{231} \mathrm{~Pa} \\
{ }^{233} \mathrm{Ca} \\
{ }^{234} \mathrm{~Pa}\end{array}$ & $\begin{array}{l}3.7 \mathrm{E}-10 \\
1.5 \mathrm{E}-09 \\
1.1 \mathrm{E}-14 \\
7.1 \mathrm{E}-19\end{array}$ & $\begin{array}{l}1.0 \mathrm{E}-07 \\
4.0 \mathrm{E}-07 \\
2.9 \mathrm{E}-12 \\
1.9 \mathrm{E}-16\end{array}$ & $\begin{array}{l}7.7 \mathrm{E}-09 \\
3.6 \mathrm{E}-08 \\
1.1 \mathrm{E}-13 \\
3.9 \mathrm{E}-18\end{array}$ & $\begin{array}{l}2.1 \mathrm{E}-06 \\
9.7 \mathrm{E}-06 \\
3.0 \mathrm{E}-11 \\
1.0 \mathrm{E}-15\end{array}$ & $\begin{array}{l}\text { Bone Surface } \\
\text { Bone Surface } \\
\text { LLI Wall } \\
\text { ULI Wall }\end{array}$ \\
\hline $\begin{array}{l}{ }^{232} U+D(c) \\
{ }^{232} U \\
{ }^{233} U+D \\
{ }^{233} U \\
{ }^{234} U \\
{ }^{235} U+D(c) \\
{ }^{235} U \\
{ }^{236} U \\
{ }^{237} U \\
{ }^{238} U+D(c) \\
{ }^{238} U \\
U-N a t(f) \\
{ }^{248} U+D(c) \\
{ }^{246} U+I \\
(d)\end{array}$ & $\begin{array}{l}3.9 \mathrm{E}-14 \\
2.7 \mathrm{E}-13 \\
1.7 \mathrm{E}-12 \\
1.0 \mathrm{E}-13 \\
1.0 \mathrm{E}-13 \\
5.9 \mathrm{E}-14 \\
1.1 \mathrm{E}-13 \\
9.6 \mathrm{E}-14 \\
1.8 \mathrm{E}-17 \\
7.2 \mathrm{E}-14 \\
1.5 \mathrm{E}-13 \\
1.3 \mathrm{E}-13 \\
6.8 \mathrm{E}-20 \\
2.0 \mathrm{E}-19\end{array}$ & $\begin{array}{l}1.0 \mathrm{E}-11 \\
7.3 \mathrm{E}-11 \\
4.7 \mathrm{E}-10 \\
2.8 \mathrm{E}-11 \\
2.8 \mathrm{E}-11 \\
1.6 \mathrm{E}-11 \\
3.0 \mathrm{E}-11 \\
2.6 \mathrm{E}-11 \\
4.9 \mathrm{E}-15 \\
2.0 \mathrm{E}-11 \\
4.0 \mathrm{E}-11 \\
3.4 \mathrm{E}-11 \\
1.8 \mathrm{E}-17 \\
5.5 \mathrm{E}-17\end{array}$ & $\begin{array}{l}5.5 \mathrm{E}-13 \\
3.9 \mathrm{E}-12 \\
4.2 \mathrm{E}-11 \\
7.6 \mathrm{E}-13 \\
7.6 \mathrm{E}-13 \\
4.4 \mathrm{E}-13 \\
8.4 \mathrm{E}-13 \\
6.8 \mathrm{E}-13 \\
1.9 \mathrm{E}-16 \\
6.5 \mathrm{E}-13 \\
1.1 \mathrm{E}-12 \\
9.1 \mathrm{E}-13 \\
5.4 \mathrm{E}-19 \\
1.6 \mathrm{E}-18\end{array}$ & $\begin{array}{l}1.5 \mathrm{E}-10 \\
1.0 \mathrm{E}-09 \\
1.1 \mathrm{E}-08 \\
2.1 \mathrm{E}-10 \\
2.1 \mathrm{E}-10 \\
1.2 \mathrm{E}-10 \\
2.3 \mathrm{E}-10 \\
1.8 \mathrm{E}-10 \\
5.1 \mathrm{E}-14 \\
1.7 \mathrm{E}-10 \\
2.9 \mathrm{E}-10 \\
2.5 \mathrm{E}-10 \\
1.4 \mathrm{E}-16 \\
4.3 \mathrm{E}-16\end{array}$ & $\begin{array}{l}\text { Bone Surface } \\
\text { Bone Surface } \\
\text { Bone Surface } \\
\text { LLI Wall } \\
\text { LLI Wall } \\
\text { LLI Wall } \\
\text { LLI Wall } \\
\text { LLI Wall } \\
\text { LLI Wall } \\
\text { LLI Wall } \\
\text { LLI Wall } \\
\text { LLI Wall } \\
\text { LLI Wall } \\
\text { LLI Wall }\end{array}$ \\
\hline
\end{tabular}


IABLE 3.4. Drinking Water Scenario Annual Committed Effective Dose Equivalent Factors for Exposure to Residual Radioactive Materials (a) (contd)

\begin{tabular}{|c|c|c|c|c|c|}
\hline \multirow[b]{2}{*}{ Radionuclide } & \multirow{2}{*}{$\begin{array}{c}\text { TEDE } \\
\text { (mrem) }\end{array}$} & \multirow{2}{*}{$\begin{array}{l}\text { TEDE } \\
(\mu \mathrm{S} v)\end{array}$} & \multicolumn{3}{|c|}{ Maximum Organ Dose } \\
\hline & & & (mrem) & $(\mu \mathrm{Sv})$ & $\operatorname{Organ}(b)$ \\
\hline $\begin{array}{l}{ }^{237} \mathrm{~Np}+\mathrm{D}(\mathrm{c}) \\
{ }^{237} \mathrm{~Np} \\
{ }^{238} \mathrm{~Np}+\mathrm{D} \\
{ }^{238} \mathrm{~Np} \\
{ }^{239} \mathrm{~Np}+\mathrm{C} \\
{ }^{239} \mathrm{~Np}\end{array}$ & $\begin{array}{l}3.9 \mathrm{E}-11 \\
7.9 \mathrm{E}-11 \\
3.6 \mathrm{E}-16 \\
8.5 \mathrm{E}-18 \\
4.3 \mathrm{E}-18 \\
8.7 \mathrm{E}-18\end{array}$ & $\begin{array}{l}1.1 \mathrm{E}-08 \\
2.1 \mathrm{E}-08 \\
9.8 \mathrm{E}-14 \\
2.3 \mathrm{E}-15 \\
1.2 \mathrm{E}-15 \\
2.3 \mathrm{E}-15\end{array}$ & $\begin{array}{l}9.1 \mathrm{E}-10 \\
1.8 \mathrm{E}-09 \\
6.3 \mathrm{E}-15 \\
7.2 \mathrm{E}-17 \\
4.3 \mathrm{E}-17 \\
8.7 \mathrm{E}-17\end{array}$ & $\begin{array}{l}2.5 \mathrm{E}-07 \\
4.9 \mathrm{E}-07 \\
1.7 \mathrm{E}-12 \\
2.0 \mathrm{E}-14 \\
1.2 \mathrm{E}-14 \\
2.3 \mathrm{E}-14\end{array}$ & $\begin{array}{l}\text { Bone Surface } \\
\text { Bone Surface } \\
\text { Bone Surface } \\
\text { LLI Wall } \\
\text { LLI Wall } \\
\text { LLI Wall }\end{array}$ \\
\hline $\begin{array}{l}{ }^{236} \mathrm{Pu} \\
{ }^{237} \mathrm{Pu} \\
{ }^{238} \mathrm{Pu} \\
{ }^{239} \mathrm{Pu} \\
{ }^{246} \mathrm{Pu} \\
{ }^{241} \mathrm{Pu}+\mathrm{D}(\mathrm{c}) \\
{ }^{241} \mathrm{Pu} \\
{ }^{242} \mathrm{Pu} \\
{ }^{243} \mathrm{Pu}+\mathrm{D}(\mathrm{C}) \\
{ }^{243} \mathrm{Pu} \\
{ }^{244} \mathrm{Pu}+\mathrm{D} \\
{ }^{244} \mathrm{Pu}\end{array}$ & $\begin{array}{l}8.1 \mathrm{E}-13 \\
4.1 \mathrm{E}-16 \\
5.1 \mathrm{E}-12 \\
5.6 \mathrm{E}-12 \\
5.6 \mathrm{E}-12 \\
2.6 \mathrm{E}-11 \\
1.0 \mathrm{E}-13 \\
5.1 \mathrm{E}-12 \\
2.8 \mathrm{E}-21 \\
5.6 \mathrm{E}-21 \\
1.4 \mathrm{E}-12 \\
5.3 \mathrm{E}-12\end{array}$ & $\begin{array}{l}2.2 \mathrm{E}-10 \\
1.1 \mathrm{E}-13 \\
1.4 \mathrm{E}-09 \\
1.5 \mathrm{E}-09 \\
1.5 \mathrm{E}-09 \\
7.1 \mathrm{E}-09 \\
2.7 \mathrm{E}-11 \\
1.4 \mathrm{E}-09 \\
7.5 \mathrm{E}-19 \\
1.5 \mathrm{E}-18 \\
3.7 \mathrm{E}-10 \\
1.4 \mathrm{E}-09\end{array}$ & $\begin{array}{l}1.0 \mathrm{E}-11 \\
3.5 \mathrm{E}-15 \\
8.9 \mathrm{E}-11 \\
1.0 \mathrm{E}-10 \\
1.0 \mathrm{E}-10 \\
5.0 \mathrm{E}-10 \\
1.9 \mathrm{E}-12 \\
9.2 \mathrm{E}-11 \\
1.8 \mathrm{E}-20 \\
3.7 \mathrm{E}-20 \\
2.3 \mathrm{E}-11 \\
9.3 \mathrm{E}-11\end{array}$ & $\begin{array}{l}2.7 \mathrm{E}-09 \\
9.5 \mathrm{E}-13 \\
2.4 \mathrm{E}-08 \\
2.7 \mathrm{E}-08 \\
2.7 \mathrm{E}-08 \\
1.3 \mathrm{E}-07 \\
5.1 \mathrm{E}-10 \\
2.5 \mathrm{E}-08 \\
4.9 \mathrm{E}-18 \\
9.9 \mathrm{E}-18 \\
6.2 \mathrm{E}-09 \\
2.5 \mathrm{E}-08\end{array}$ & $\begin{array}{l}\text { Bone Surface } \\
\text { LLI Wall } \\
\text { Bone Surface } \\
\text { Bone Surface } \\
\text { Bone Surface } \\
\text { Bone Surface } \\
\text { Bone Surface } \\
\text { Bone Surface } \\
\text { ULI Wall } \\
\text { ULI Wall } \\
\text { Bone Surface } \\
\text { Bone Surface }\end{array}$ \\
\hline $\begin{array}{l}{ }^{241} \mathrm{Am} \\
{ }^{242 m} \mathrm{Am}+\mathrm{D} \\
242 \mathrm{Am} \\
{ }^{242} \mathrm{Am} \\
{ }^{242} \mathrm{Am}+\mathrm{D} \\
{ }^{243} \mathrm{Am}(\mathrm{c}) \\
{ }^{243} \mathrm{Am}+\mathrm{Dm}\end{array}$ & $\begin{array}{l}4.8 E-10 \\
1.3 E-10 \\
4.6 E-10 \\
2.4 E-16 \\
2.6 E-18 \\
1.6 E-10 \\
4.8 E-10\end{array}$ & $\begin{array}{l}1.3 \mathrm{E}-07 \\
3.6 \mathrm{E}-08 \\
1.3 \mathrm{E}-07 \\
6.6 \mathrm{E}-14 \\
7.0 \mathrm{E}-16 \\
4.3 \mathrm{E}-08 \\
1.3 \mathrm{E}-07\end{array}$ & $\begin{array}{l}8.9 \mathrm{E}-09 \\
2.5 \mathrm{E}-09 \\
8.5 \mathrm{E}-09 \\
4.1 \mathrm{E}-15 \\
1.8 \mathrm{E}-17 \\
3.0 \mathrm{E}-09 \\
8.9 \mathrm{E}-09\end{array}$ & $\begin{array}{l}2.4 \mathrm{E}-06 \\
6.7 \mathrm{E}-07 \\
2.3 \mathrm{E}-06 \\
1.1 \mathrm{E}-12 \\
4.8 \mathrm{E}-15 \\
8.0 \mathrm{E}-07 \\
2.4 \mathrm{E}-06\end{array}$ & $\begin{array}{l}\text { Bone Surface } \\
\text { Bone Surface } \\
\text { Bone Surface } \\
\text { Bone Surface } \\
\text { LLI Wall } \\
\text { Bone Surface } \\
\text { Bone Surface }\end{array}$ \\
\hline $\begin{array}{l}{ }^{242} \mathrm{Cm}+\mathrm{D}(\mathrm{c}) \\
{ }^{242} \mathrm{Cm} \\
{ }^{243} \mathrm{Cm} \\
{ }^{244} \mathrm{Cm}+\mathrm{D}(\mathrm{c}) \\
{ }^{244} \mathrm{Cm} \\
{ }^{245} \mathrm{Cm}+\mathrm{D}(\mathrm{c}) \\
{ }^{245} \mathrm{Cm} \\
{ }^{246} \mathrm{Cm} \\
{ }^{247} \mathrm{Cm}+\mathrm{D}(\mathrm{c}) \\
{ }^{247} \mathrm{Cm} \\
{ }^{248} \mathrm{Cm}\end{array}$ & $\begin{array}{l}1.4 \mathrm{E}-12 \\
6.3 \mathrm{E}-13 \\
4.3 \mathrm{E}-11 \\
1.5 \mathrm{E}-11 \\
3.0 \mathrm{E}-11 \\
3.7 \mathrm{E}-11 \\
5.6 \mathrm{E}-11 \\
5.6 \mathrm{E}-11 \\
2.6 \mathrm{E}-11 \\
5.1 \mathrm{E}-11 \\
2.0 \mathrm{E}-10\end{array}$ & $\begin{array}{l}3.8 \mathrm{E}-10 \\
1.7 \mathrm{E}-10 \\
1.2 \mathrm{E}-08 \\
4.0 \mathrm{E}-09 \\
8.0 \mathrm{E}-09 \\
9.9 \mathrm{E}-09 \\
1.5 \mathrm{E}-08 \\
1.5 \mathrm{E}-08 \\
6.9 \mathrm{E}-09 \\
1.4 \mathrm{E}-08 \\
5.3 \mathrm{E}-08\end{array}$ & $\begin{array}{l}2.4 \mathrm{E}-11 \\
9.2 \mathrm{E}-12 \\
7.7 \mathrm{E}-10 \\
2.7 \mathrm{E}-10 \\
5.3 \mathrm{E}-10 \\
7.1 \mathrm{E}-10 \\
1.0 \mathrm{E}-09 \\
1.1 \mathrm{E}-09 \\
4.8 \mathrm{E}-10 \\
9.7 \mathrm{E}-10 \\
3.8 \mathrm{E}-09\end{array}$ & $\begin{array}{l}6.4 \mathrm{E}-09 \\
2.5 \mathrm{E}-09 \\
2.1 \mathrm{E}-07 \\
7.2 \mathrm{E}-08 \\
1.4 \mathrm{E}-07 \\
1.9 \mathrm{E}-07 \\
2.8 \mathrm{E}-07 \\
2.9 \mathrm{E}-07 \\
1.3 \mathrm{E}-07 \\
2.6 \mathrm{E}-07 \\
1.0 \mathrm{E}-06\end{array}$ & $\begin{array}{l}\text { Bone Surface } \\
\text { Bone Surface } \\
\text { Bone Surface } \\
\text { Bone Surface } \\
\text { Bone Surface } \\
\text { Bone Surface } \\
\text { Bone Surface } \\
\text { Bone Surface } \\
\text { Bone Surface } \\
\text { Bone Surface } \\
\text { Bone Surface }\end{array}$ \\
\hline${ }^{252} \mathrm{Cf}$ & $1.2 \mathrm{E}-10$ & $3.3 E-08$ & $2.5 \mathrm{E}-09$ & $6.7 E-07$ & Bone Surface \\
\hline
\end{tabular}

(a) Based on ingestion of $730 \mathrm{~L}$ of drinking water from a ground-water source and an inventory of $1 \mathrm{pCi}(1 \mathrm{~Bq})$ of activity, per radionuclide in soil. 
TABLE 3.4. Drinking Water Scenario Annual Committed Effective Dose Equivalent Factors for Exposure to Residual Radioactive Materials(a) (contd)

(b) Where SI is Small Intestine, ULI is Upper Large Intestine, and LLI is Lower Large Intestine.

(c) Where $+D$ means "plus daughters in equilibrium" referring to explicit daughters, or daughters with half-lives greater than 10 minutes. Separate values are also included for parents and daughters so that combined factors for non-equilibrium cases can be determined. Note: in some cases $+D$ also includes implicit daughters (i.e., uranium and thorium decay chains).

(d) Where +I means "plus implicit daughters in equilibrium" referring to daughters with half-lives less than 10 minutes. The decay energies associated with these daughters have been assigned to the parent, and the appropriate branching ratios (described in Appendix D) have been considered.

(e) Where Th-Nat includes an equilibrium mixture of ${ }^{232} \mathrm{Th}$ plus 10 daughters in the decay chain, as discussed in Appendix $D$.

(f) Where $1 \mathrm{Ci} \mathrm{U}$-Nat equals $48.9 \%{ }^{238} \mathrm{U}$ plus $48.9 \%{ }^{234} \mathrm{U}$ plus $2.25 \%{ }^{235} \mathrm{U}$. 


\subsection{SCENARIO PARAMETER SELECTIONS}

The input parameters and assumptions for each exposure pathway and scenario are selected to provide a reasonable estimate of the likely radiation dose to an average individual in a population. The parameters anc assumptions were not selected to be overly conservative; however, they still should overestimate the average potential radiation dose received. In general, default parameter values were selected to match literature values (such as those published by the ICRP), selected from values given in Regulatory Guide 1.109 (NRC 1977) or Oztunali et al. (1981), or are considered standard default values that are referenced by various national ( international authorities. A specific listing of the major parameters or assumptions for the scenarios identified for unrestricted release of buildings and land, their expected ranges, and a listing of the values selected for this study is given in Table 3.5. A discussion of the additional parameters and assumptions used in the analysis, listed by exposure pathway, is included in Appendix B. In every case, an attempt has been made to select values within the expected range--not at the extremes 0 the expected range. For example, the dietary intake rate was assumed to be $25 \%$ of the total diet defined in Regulatory Guide 1.109 (NRC 1977). Of additional concern is the use of potential dilution factors that may reduce the concentrations of material considered for each pathway and scenario. However, for this analysis, no dilution was considered and an initial concentration of $1 \mathrm{pCi} / \mathrm{g}(1 \mathrm{~Bq} / \mathrm{g})$ or $1 \mathrm{dpm} / 100 \mathrm{~cm}^{2}(1 \mathrm{~Bq} / 100 \mathrm{~cm} 2)$ was used.

Modified unit concentration TEDE factors can be obtained by multiplyin! the pathway dose conversion factors, discussed in Section 2.0, by appropria: modification factors, as described in Appendix B. In addition to dilution, the modification factors can account for changes in exposure duration, radionuclide concentrations in various media, and other pathway-specific parameters. For example, to change the exposure duration for the facilityinventory scenario for $2000 \mathrm{~h} / \mathrm{yr}$ to $4000 \mathrm{~h} / \mathrm{yr}$ the unit concentration TEDE factors for a given scenario are simply multiplied by 2 . In general, a simple linear multiplication should be used because linear chain models havi been used. 
Table 3.5. Expected Ranges for Major Scenario or Pathway

Parameters and the Selected Values Used in This Study

Scenario or Pathway

Parameter

Building Renovation

or Occupancy

- Inhalation

- Duration

0 to $8760 \mathrm{~h}$

- Breathing rate 125 to $333 \mathrm{~cm}^{3} / \mathrm{sec}$

- Concentration $10-6$ to $10-3 \mathrm{~g} / \mathrm{m}^{3}$

- Particle size

0.1 to $10 \mu \mathrm{m}$ AMAD

$1.0 \mu \mathrm{m} \operatorname{AMAD}(\mathrm{a})$

$500 \mathrm{~h}$ or $2000 \mathrm{~h}$

$270 \mathrm{~cm}^{3} / \mathrm{sec}$

$10^{-4} \mathrm{~g} / \mathrm{m}^{3}$

Dusty workplace (as discussed in Appendix B)

ICRP recommendations for standard man (1975)

ICRP generic value from ICRP 30 (19791988)

- Secondary Ingestion

- Ingestion rate 0 to $0.05 \mathrm{~g} / \mathrm{h}$

0.01 or $0.001 \mathrm{~g} / \mathrm{h}$

Literature value (as discussed in Appendix B)

- Duration

0 to $8760 \mathrm{~h}$

$500 \mathrm{~h}$ or $2000 \mathrm{~h}$

Renovation work;

Normal work year (50 work-weeks

- External

- Duration

0 to $8760 \mathrm{~h}$

$500 \mathrm{~h}$ or $2000 \mathrm{~h}$

Renovation work;

Normal work year

Residential Use

Surface Soil Scenario

- Inhalation

- Duration

0 to $8760 \mathrm{~h}$

$100 \mathrm{~h}$

Gardening;

$1700 \mathrm{~h}$ Outdoors;

$4380 \mathrm{~h}$ Indoors

- Breathing rate 125 to $333 \mathrm{~cm}^{3} / \mathrm{sec}$

$270 \mathrm{~cm} 3 / \mathrm{sec}$

ICRP recommendations

for standard man (1975) 
Table 3.5. Expected Ranges for Major Scenario or Pathway Parameters and the Selected Values Used in This Study (contd)

Scenario or Pathway

Parameter

Expected Range Selected Value

Comments

Residential Use

Surface Soil Scenario

(contd)

- Concentration $10-6$ to $10-3 \mathrm{~g} / \mathrm{m}^{3}$

$5 \times 10^{-4} \mathrm{~g} / \mathrm{m}^{3}$ Gardening dust;

$1 \times 10-5 \mathrm{~g} / \mathrm{m}^{3}$ Yardwork dust;

$5 \times 10^{-5} \mathrm{~g} / \mathrm{m}^{3} \quad$ EPA standard (indoors)

- Particle size 0.1 to $10 \mu \mathrm{m}$ AMAD $1.0 \mu \mathrm{m}$ AMAD

ICRP generic value from ICRP 30 (19791988)

- External

- Duration

0 to $8760 \mathrm{~h}$

$1800 \mathrm{~h}$

Outdoors;

$4380 \mathrm{~h}$ Indoors

work-weeks)

- Shielding factor 0 to 1.0

0.33

House shielding factor

- Ingestion

- Vegetables

0 to $190 \mathrm{~kg} / \mathrm{yr}$

$47.5 \mathrm{~kg} / \mathrm{yr}$

$25 \%$ of diet

- Milk

0 to $110 \mathrm{~L} / \mathrm{yr}$

$27.5 \mathrm{~L} / \mathrm{yr}$

$25 \%$ of diet

- Meat

0 to $76.5 \mathrm{~kg} / \mathrm{yr}$

$19.2 \mathrm{~kg} / \mathrm{yr}$

$25 \%$ of diet

- Eggs

0 to $19 \mathrm{~kg} / \mathrm{yr}$

$4.8 \mathrm{~kg} / \mathrm{yr}$

$25 \%$ of diet

- Air conc$10-6$ to $10^{-3} \mathrm{~g} / \mathrm{m}^{3}$

$5 \times 10-5$

EPA standard entration for (annual average) leaf deposition

Drinking Water Scenario

- Ingestion rate 0 to $4 \mathrm{~L} / \mathrm{d}$

$2 \mathrm{~L} / \mathrm{d}$

NRC (1977)

(a) Where AMAD means the activity mean aerodynamic diameter of the airborne particulate material. 


\subsection{EXAMPLE DOSE CALCULATIONS FOR REFERENCE BUILDINGS AND LAND}

This section describes example calculations performed to estimate the potential annual TEDE for a specified year of exposure after decommissioning, using the unit concentration TEDE factors discussed in Section 3.0. Example calculations are performed for illustration purposes only and are not intended to represent the exact mixtures or contamination levels found at any existing facility. The calculations in this section are performed using a radionuclide mixture for deposited surface contamination at a reference light-water power reactor, 30 years after shutdown. Additional example calculations for mixtures of radionuclides that potentially may be found at uranium facilities and radiopharmaceutical/industrial-source manufacturing plants are included in Appendix C. The calculated annual TEDEs are presented for each of the four major scenarios discussed in Section 3.0, and for exposure to residual radioactive materials during building renovation, normal building occupancy, residential use of surface soil, and drinking water from ground water sources.

\subsection{REFERENCE RADIONUCLIDE MIXTURE}

To illustrate the use of the unit concentration annual TEDE factors, a reference radionuclide mixture is defined for deposited surface contamination at light-water power reactor stations (Smith, Konzek, and Kennedy 1978; 0ak et al. 1980). This mixture and the descriptions of the reference light-water reactor are discussed in detail in the referenced documents. The mixture of radionuclides for power reactors and the additional mixtures discussed in Appendix $C$ are provided in terms of the relative activity (normalized to unity for the total mixture for each decay period), by radionuclide. No consideration is given to the total quantity present for the example calculations because actual contamination levels will vary depending on the success of decontamination procedures. For the example mixture calculations, concentrations of $100 \mathrm{pCi} / \mathrm{g}$ for volume sources (building material and soils) and $10,000 \mathrm{dpm} / 100 \mathrm{~cm} 2$ for building surface source levels are assumed. It is doubtful that any real facility would actually have radionuclide mixtures consisting of the exact relative activities or assumed concentrations of the radionuclides shown in the reference lists; they are intended to serve only as a basis for the example calculations.

For the reference light-water reactor, information from both a pressurized-water reactor (Smith, Konzek, and Kennedy 1978) and a boilingwater reactor (0ak et al. 1980) was reviewed. The mixture of radionuclides is anticipated to vary as a function of the operating history of the reactor, the plant design, the location within the facility, and the success of decontamination operations. In addition, potentially there will be mixtures resulting from neutron activation and mixtures containing fission products from the fuel. Thus, it is difficult to develop generic radionuclide mixtures that are truly representative of overall plant conditions. For illustrative purposes, a mixture associated with general surface contamination is defined, as shown in Table 4.1. This mixture contains both key activation and fission products that may be present during plant 
TABLE 4.1. Estimated Relative Activity of Radionuclides in Deposited Surface Contamination at the Reference Power Reactor(a)

\begin{tabular}{|c|c|c|c|}
\hline \multirow[b]{2}{*}{$\begin{array}{c}\text { Radio- } \\
\text { nuclides }\end{array}$} & \multirow[b]{2}{*}{ Half-Life $(y r)$} & \multicolumn{2}{|c|}{ Relative Activity } \\
\hline & & $\begin{array}{c}10 \text { Years } \\
\text { After Shutdown }\end{array}$ & $\begin{array}{c}30 \text { Years } \\
\text { After Shutdown }\end{array}$ \\
\hline $\begin{array}{l}54 \mathrm{Mn} \\
55 \mathrm{Fe} \\
60 \mathrm{Co}\end{array}$ & $\begin{array}{l}8.2 E-01 \\
2.7 E+00 \\
5.3 E+00\end{array}$ & $\begin{array}{l}8.5 E-07 \\
9.2 E-02 \\
2.9 E-01\end{array}$ & $\begin{array}{c}-(b) \\
1.1 E-03 \\
5.1 E-02\end{array}$ \\
\hline $\begin{array}{l}63 \mathrm{Ni} \\
65 \mathrm{Zn} \\
90 \mathrm{Sr+D}(\mathrm{c})\end{array}$ & $\begin{array}{l}1.0 E+02 \\
6.7 E-01 \\
2.8 E+01\end{array}$ & $\begin{array}{l}1.2 \mathrm{E}-02 \\
1.7 \mathrm{E}-06 \\
8.8 \mathrm{E}-02\end{array}$ & $\begin{array}{c}2.5 \mathrm{E}-02 \\
- \\
1.3 \mathrm{E}-01\end{array}$ \\
\hline $\begin{array}{l}110 \mathrm{mAg} \\
134 \mathrm{Cs} \\
137 \mathrm{Cs}+\mathrm{I}(\mathrm{d}) \\
144 \mathrm{Ce}\end{array}$ & $\begin{array}{l}6.8 \mathrm{E}-01 \\
2.1 \mathrm{E}+00 \\
3.0 \mathrm{E}+01 \\
7.8 \mathrm{E}-01\end{array}$ & $\begin{array}{l}1.2 \mathrm{E}-09 \\
1.1 \mathrm{E}-03 \\
5.2 \mathrm{E}-01 \\
1.1 \mathrm{E}-07\end{array}$ & $\begin{array}{l}-\overline{-} \\
3.4 \mathrm{E}-06 \\
8.2 \mathrm{E}-01 \\
-\end{array}$ \\
\hline Total & & 1.0 & 1.0 \\
\hline
\end{tabular}

(a) Based on information in Smith, Konzek, and Kennedy (1978) and Oak et al. (1980).

(b) A dash indicates a value less than 10-8.

(c) Where $+D$ means "plus explicit daughters in equilibrium" as discussed in Appendix D.

(d) Where +I means "plus implicit daughters in equilibrium" as discussed in Appendix D.

operations. Table 4.1 shows the variation in the relative activity of the mixture at 10 years and 30 years after plant shutdown; however, for purposes of the example calculations that follow, only the mixture after 30 years of radioactive decay is used. This decay period is chosen to be representative of decommissioning after a period of safe storage that allows for radioactive decay. After 30 years of decay, the level of 60 Co will have decayed to about $1 \%$ of its initial value, and about $82 \%$ of the activity for the mixture is $137 \mathrm{Cs}+\mathrm{I}$. About $13 \%$ of the mixture is $90 \mathrm{~S} r+\mathrm{D}$ and about $5 \%$ is $60 \mathrm{Co}$. For reactors where fission product releases from the fuel are minimal, the radionuclide mixture would be dominated by activation products, most notably $60 \mathrm{Co}$. However, the mixture of activation and fission products shown in Table 4.1 is used in this analys is to demonstrate the pathway contribution and relative importance of radionuclides from both sources.

\subsection{EXAMPLE DOSE CALCULATIONS FOR THE BUILDING RENOVATION SCENARIO}

As a demonstration of the application of the unit concentration annual TEDE factors for the building renovation scenario (described in Section 3.1.1) example calculations are next performed. The results shown in 
TABLE 4.2. Estimated Annual Total Effective Dose Equivalents for the Building Renovation (Volume Activity) Scenario Using the LightWater Power Reactor Surface Contamination Inventory(a)

\begin{tabular}{|c|c|c|c|c|c|}
\hline $\begin{array}{c}\text { Radio- } \\
\text { nuclide(b) }\end{array}$ & $\begin{array}{c}\text { External } \\
\text { (mrem) }\end{array}$ & $\begin{array}{c}\text { Inhalation } \\
\text { (mrem) }\end{array}$ & $\begin{array}{c}\text { Secondary } \\
\text { Ingestion } \\
\text { (mrem) }\end{array}$ & $\begin{array}{c}\text { TEDE } \\
\text { (mrem) }\end{array}$ & $\begin{array}{l}\text { TEDE } \\
(\mu \mathrm{SV})\end{array}$ \\
\hline $\begin{array}{l}{ }^{65} \mathrm{Fe} \\
{ }^{60} \mathrm{Co} \\
{ }^{63} \mathrm{Ni}\end{array}$ & $\begin{array}{l}1.8 E-06 \\
4.2 E-01 \\
2.5 E-08\end{array}$ & $\begin{array}{l}6.6 E-09 \\
4.8 E-05 \\
2.4 E-07\end{array}$ & $\begin{array}{l}3.2 E-07 \\
2.6 E-04 \\
6.3 E-06\end{array}$ & $\begin{array}{l}2.1 E-06 \\
4.2 E-01 \\
6.8 E-06\end{array}$ & $\begin{array}{l}2.1 E-05 \\
4.2 E+00 \\
6.8 E-05\end{array}$ \\
\hline $\begin{array}{l}{ }^{96} \mathrm{Sr}+\mathrm{D}(\mathrm{c}) \\
{ }_{134} \mathrm{Cs} \\
{ }^{137} \mathrm{Cs}+\mathrm{I}(\mathrm{d})\end{array}$ & $\begin{array}{l}1.4 \mathrm{E}-03 \\
1.9 \mathrm{E}-05 \\
1.6 \mathrm{E}+00 \\
\end{array}$ & $\begin{array}{l}6.7 \mathrm{E}-05 \\
6.8 \mathrm{E}-10 \\
5.5 \mathrm{E}-05 \\
\end{array}$ & $\begin{array}{l}4.2 \mathrm{E}-03 \\
1.1 \mathrm{E}-07 \\
9.8 \mathrm{E}-03 \\
\end{array}$ & $\begin{array}{l}5.7 \mathrm{E}-03 \\
1.9 \mathrm{E}-05 \\
1.6 \mathrm{E}+00 \\
\end{array}$ & $\begin{array}{l}5.7 \mathrm{E}-02 \\
1.9 \mathrm{E}-04 \\
1.6 \mathrm{E}+01 \\
\end{array}$ \\
\hline Total & $2.0 \mathrm{E}+00$ & $1.7 \mathrm{E}-04$ & $1.4 E-02$ & $2.0 E+00$ & $2.0 \mathrm{E}+01$ \\
\hline
\end{tabular}

(a) Using the radionuclide mixture shown in Table 4.1 with 30 years of radioactive decay and the annual TEDE factors from Table 3.1 .

(b) With an assumed contamination level of $100 \mathrm{pCi} / \mathrm{g}(3.7 \mathrm{~Bq} / \mathrm{g})$ of the radionuclide mixture.

(c) Where $+D$ means "plus explicit daughters in equilibrium" as discussed in Appendix $D$.

(d) Where +I means "plus implicit daughters in equilibrium" as discussed in Appendix D.

Table 4.2 are in terms of the dose by pathway and the annual TEDE from a year of exposure to an individual (mrem and $\mu \mathrm{Sv}$ ) for the light-water power reactor inventory decayed to 30 years after shutdown. The contamination level for volume sources in buildings was assumed to be $100 \mathrm{pCi} / \mathrm{g}(3.7 \mathrm{~Bq} / \mathrm{g})$. The results are the product of the annual TEDE factors in Table 3.1 (by radionuclide), the relative activity level for each radionuclide in the mixture, and the assumed contamination level for this example problem. The dominant radionuclide contributors to the dose in the mixture are $137 \mathrm{Cs}+\mathrm{I}$, $60 \mathrm{Co}$, and $90 \mathrm{~S} r+\mathrm{D}$. For this mixture and scenario, the most important exposure pathways are external exposure followed by secondary ingestion. The estimated annual TEDE resulting from a year of exposure to $100 \mathrm{pCi} / \mathrm{g}$ $(3.7 \mathrm{~Bq} / \mathrm{g})$ concentration of the radionuclide mixture is $2.0 \mathrm{mrem}$ $(20 \mu \mathrm{Sv})$.

\subsection{EXAMPLE DOSE CALCULATIONS FOR THE BUILDING OCCUPANCY SCENARIO}

Example calculations are next performed for the building occupancy scenario using the radionuclide mixture in Table 4.1, decayed to 30 years after shutdown. The assumed contamination level for the calculations is $10,000 \mathrm{dpm} / 100 \mathrm{~cm}^{2}\left(167 \mathrm{~Bq} / 100 \mathrm{~cm}^{2}\right)$ of the mixture. The results for an assumed year of exposure are shown in Table 4.3 in terms of the dose by exposure pathway and the annual TEDE to an individual (mrem and $\mu \mathrm{Sv}$ ). These 
TABLE 4.3. Estimated Annual Total Effective Dose Equivalents for the Building Occupancy (Surface Activity) Scenario Using the LightWater Power Reactor Surface Contamination Inventory(a)

\begin{tabular}{|c|c|c|c|c|c|}
\hline $\begin{array}{l}\text { Radio- } \\
\text { nuclide(b) }\end{array}$ & $\begin{array}{c}\text { External } \\
\text { (mrem) } \\
\end{array}$ & $\begin{array}{c}\text { Inhalation } \\
\text { (mrem) } \\
\end{array}$ & $\begin{array}{c}\text { Secondary } \\
\text { Ingestion } \\
\text { (mrem) }\end{array}$ & $\begin{array}{c}\text { TEDE } \\
\text { (mrem) }\end{array}$ & $\begin{array}{l}\text { TEDE } \\
(\mu \mathrm{SV}) \\
\end{array}$ \\
\hline $\begin{array}{l}{ }_{60}^{55} \mathrm{Fe} \\
{ }^{63} \mathrm{Co} \\
{ }^{63} \mathrm{Ni}\end{array}$ & $\begin{array}{l}1.6 \mathrm{E}-05 \\
4.3 \mathrm{E}-01 \\
2.0 \mathrm{E}-07\end{array}$ & $\begin{array}{l}2.6 \mathrm{E}-07 \\
1.9 \mathrm{E}-03 \\
9.5 \mathrm{E}-06\end{array}$ & $\begin{array}{l}1.3 \mathrm{E}-05 \\
1.0 \mathrm{E}-02 \\
2.5 \mathrm{E}-04\end{array}$ & $\begin{array}{l}2.9 E-05 \\
4.4 E-01 \\
2.5 E-04\end{array}$ & $\begin{array}{l}2.9 E-04 \\
4.4 E+00 \\
2.5 E-03\end{array}$ \\
\hline $\begin{array}{l}{ }^{96} \mathrm{Sr}+\mathrm{D}(\mathrm{c}) \\
{ }^{134} \mathrm{Cs} \\
{ }^{137} \mathrm{Cs}+\mathrm{I}(\mathrm{d})\end{array}$ & $\begin{array}{l}2.1 E-03 \\
2.0 E-05 \\
1.6 E+00 \\
\end{array}$ & $\begin{array}{l}2.7 E-03 \\
2.7 E-08 \\
2.2 E-03 \\
\end{array}$ & $\begin{array}{l}1.7 \mathrm{E}-01 \\
4.4 \mathrm{E}-06 \\
3.8 \mathrm{E}-01 \\
\end{array}$ & $\begin{array}{l}1.7 E-01 \\
2.4 E-05 \\
2.0 E+00 \\
\end{array}$ & $\begin{array}{l}1.7 E+00 \\
2.4 E-04 \\
2.0 E+01 \\
\end{array}$ \\
\hline Total & $2.0 \mathrm{E}+00$ & $6.8 E-03$ & $5.6 \mathrm{E}-01$ & $2.6 \mathrm{E}+00$ & $2.6 \mathrm{E}+01$ \\
\hline
\end{tabular}

(a) Using the radionuclide mixture shown in Table 4.1 with 30 years

of radioactive decay and the annual TEDE factors from Table 3.2 .

(b) With an assumed contamination level of $10,000 \mathrm{dpm} / 100 \mathrm{~cm}^{2}$ (167 $\mathrm{Bq} / 100 \mathrm{~cm} 2$ ) of the radionuclide mixture.

(c) Where $+D$ means "plus explicit daughters in equilibrium" as discussed in Appendix D.

(d) Where +I means "plus implicit daughters in equilibrium" as discussed in Appendix D.

results are the product of the unit concentration annual TEDE factors for this scenario from Table 3.2 by radionuclide, the relative activity level for each radionuclide in the mixture, and the assumed contamination level. The dominant radionuclide contributors to dose for this scenario are ${ }^{137} \mathrm{Cs}+\mathrm{I}$ and $60 \mathrm{Co}$, and the most important exposure pathway is external exposure. The estimated annual TEDE resulting from a $10000 \mathrm{dpm} / 100 \mathrm{~cm} 2(167 \mathrm{~Bq} / 100 \mathrm{~cm} 2)$ contamination level is 2.6 mrem (26 $\mu \mathrm{Sv})$.

\subsection{EXAMPLE DOSE CALCULATIONS FOR THE SURFACE SOIL ACTIVITY SCENARIO}

Example calculations for the surface soil activity scenario are conducted using an initial soil concentration of $100 \mathrm{pCi} / \mathrm{g}(3.7 \mathrm{~Bq} / \mathrm{g})$ of soil using the inventory in Table 4.1 , decayed to 30 years after plant shutdown. The results for one year of exposure are shown in Table 4.4 in terms of the dose by exposure pathway and the annual TEDE to an individual (mrem and $\mu \mathrm{Sv}$ ). The results are the product of the annual TEDE factors for this scenario by radionuclide from Table 3.3, the relative activity level for each radionuclide in the mixture, and the assumed contamination level.

The dominant radionuclide contributors to dose are $137 \mathrm{Cs}+1,60 \mathrm{Co}$, and $90 S r+D$ and the dominant exposure pathways are food ingestion and external 
TABLE 4.4. Estimated Annual Total Effective Dose Equivalents for the Residential Use (Surface Soil) Scenario Using the Light-Water Power Reactor Surface Contamination Inventory (a)

\begin{tabular}{|c|c|c|c|c|c|}
\hline $\begin{array}{c}\text { Radio- } \\
\text { nuclide (b) }\end{array}$ & $\begin{array}{c}\text { External } \\
\text { (mrem) }\end{array}$ & $\begin{array}{c}\text { Inhalation } \\
\text { (mrem) } \\
\end{array}$ & $\begin{array}{l}\text { Secondary } \\
\text { Ingestion } \\
\text { (mrem) }\end{array}$ & $\begin{array}{c}\text { TEDE } \\
\text { (mrem) }\end{array}$ & $\begin{array}{r}\text { TEDE } \\
(\mu S v) \\
\end{array}$ \\
\hline $\begin{array}{l}{ }^{55} \mathrm{Fe} \\
{ }^{68} \mathrm{Co} \\
{ }^{63} \mathrm{Ni}\end{array}$ & $\begin{array}{l}1.2 \mathrm{E}-05 \\
3.9 \mathrm{E}+01 \\
4.8 \mathrm{E}-07\end{array}$ & $\begin{array}{l}3.3 E-08 \\
4.2 E-04 \\
2.1 E-06\end{array}$ & $\begin{array}{l}3.8 \mathrm{E}-06 \\
3.1 \mathrm{E}-02 \\
3.2 \mathrm{E}-03\end{array}$ & $\begin{array}{l}1.5 E-05 \\
3.9 E+01 \\
3.2 E-03\end{array}$ & $\begin{array}{l}1.5 \mathrm{E}-04 \\
3.9 \mathrm{E}+02 \\
3.2 \mathrm{E}-02\end{array}$ \\
\hline $\begin{array}{l}{ }^{96} \mathrm{Sr}+\mathrm{D}(\mathrm{c}) \\
{ }_{134} \mathrm{Cs} \\
{ }^{137} \mathrm{Cs}+\mathrm{I}(\mathrm{d})\end{array}$ & $\begin{array}{l}1.2 \mathrm{E}-01 \\
1.8 \mathrm{E}-03 \\
1.6 \mathrm{E}+02 \\
\end{array}$ & $\begin{array}{l}6.0 \mathrm{E}-04 \\
6.1 \mathrm{E}-09 \\
4.8 \mathrm{E}-04 \\
\end{array}$ & $\begin{array}{l}1.2 \mathrm{E}+01 \\
1.7 \mathrm{E}-05 \\
2.5 \mathrm{E}-01 \\
\end{array}$ & $\begin{array}{l}1.2 E+01 \\
1.8 E-03 \\
1.6 E+02 \\
\end{array}$ & $\begin{array}{l}1.2 \mathrm{E}+02 \\
1.8 \mathrm{E}-02 \\
1.6 \mathrm{E}+03 \\
\end{array}$ \\
\hline Total & $2.0 \mathrm{E}+02$ & $1.5 \mathrm{E}-03$ & $1.2 \mathrm{E}+01$ & $2.1 \mathrm{E}+02$ & $2.1 E+03$ \\
\hline
\end{tabular}

(a) Using the radionuclide mixture shown in Table 4.1 with 30 years of radioactive decay and the annual TEDE factors from Table 3.3.

(b) With an assumed contamination level of $100 \mathrm{pCi} / \mathrm{g}(3.7 \mathrm{~Bq} / \mathrm{g})$.

(c) Where $+D$ means "plus explicit daughters in equilibrium" as discussed in Appendix D.

(d) Where +I means "plus implicit daughters in equilibrium" as discussed in Appendix D.

exposure. The estimated annual TEDE for the reference mixture with a concentration of $100 \mathrm{pCi} / \mathrm{g}(3.7 \mathrm{~Bq} / \mathrm{g})$ is $210 \mathrm{mrem}$, or $2100 \mu \mathrm{Sv}$.

\subsection{EXAMPLE DOSE CALCULATIONS FOR THE DRINKING WATER SCENARIO}

Example calculations for the drinking water scenario are conducted using an assumed contaminated area of $1000 \mathrm{~m}^{2}$, to a depth of $15 \mathrm{~cm}$, at a level of $100 \mathrm{pCi} / \mathrm{g}(3.7 \mathrm{~Bq} / \mathrm{g})$. Assuming a soil density of $1.6 \mathrm{~g} / \mathrm{cm}^{3}$, the total inventory is $24 \mathrm{mC} i(890 \mathrm{MBq})$. For the mixture of radionuclides shown in Table 4.1, decayed to 30 years after plant shutdown, the results for drinking water for 1 year are shown in Table 4.5 in terms of the annual TEDE to an individual (mrem and $\mu \mathrm{Sv}$ ) and the maximum organ dose rate by radionuclide. The results are the product of the annual TEDE factors by radionuclide from Table 3.4, the relative activity level for each radionuclide in the mixture, and the assumed contamination level for this example. The dominant contributors to dose for the mixture are $137 \mathrm{Cs}+\mathrm{I}$ and $90 \mathrm{sr}+\mathrm{D}$. The estimated annual TEDE for the mixture of radionuclides at the light-water power reactor is $0.073 \mathrm{mrem}(0.73 \mu \mathrm{Sv})$ and the maximum organ dose for the mixture is 0.69 mrem $(6.9 \mu \mathrm{Sv})$ to bone surface. The dominant contributor to organ dose for this mixture is $90 \mathrm{~S} r+D$. 
TABLE 4.5. Estimated Annual Total Effective Dose Equivalents for the Drinking Water Scenario Using the Light-Water Power Reactor Surface Contamination Inventory (a)

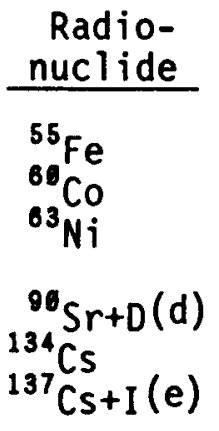

Total

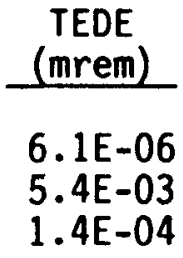

6. $2 \mathrm{E}-02$

2.3E-08

$2.4 \mathrm{E}-03$

$7.3 \mathrm{E}-02$

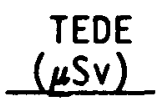

$6.1 E-05$

$5.4 \mathrm{E}-02$

$1.4 \mathrm{E}-03$

6.1E-01

2.3E-07

2.4E-02

7.3E-01

Maximum Organ Dose

\begin{tabular}{|c|c|c|}
\hline (mrem) & $(\mu S v)$ & $\operatorname{Organ}(c)$ \\
\hline $\begin{array}{l}2.2 E-05 \\
2.3 E-02 \\
9.0 E-04\end{array}$ & $\begin{array}{l}2.2 \mathrm{E}-04 \\
2.3 \mathrm{E}-01 \\
9.0 \mathrm{E}-03\end{array}$ & $\begin{array}{l}\text { Spleen } \\
\text { LLI Wall } \\
\text { LLI Wall }\end{array}$ \\
\hline $\begin{array}{l}6.9 E-01 \\
3.0 E-08 \\
3.0 E-03\end{array}$ & $\begin{array}{l}6.9 \mathrm{E}+00 \\
3.0 \mathrm{E}-07 \\
3.0 \mathrm{E}-02\end{array}$ & $\begin{array}{l}\text { Bone Surface } \\
\text { SI Wall } \\
\text { SI Wall }\end{array}$ \\
\hline
\end{tabular}

$6.9 \mathrm{E}-01$

$6.9 \mathrm{E}+00$

Bone Surface 


\subsection{DISCUSSION}

In support of the NRC's development of expanded criteria governing residual radioactive contamination for unrestricted use, a modeling evaluation was performed producing annual TEDE factors for anticipated radiation exposure pathways and scenarios. This section discusses the generic modeling approach, the generic scenarios and their limitations, and the relationship with existing standards.

\subsection{GENERIC MODELING APPROACH}

The generic modeling evaluation relies on a radiation exposure scenario analysis, including the major exposure pathways of direct exposure to penetrating radiation, inhalation, and ingestion. The modeling analysis is used to derive the annual TEDE to an average individual in a population exposed to residual radioactive material after decommissioning. The input parameters for each exposure pathway and scenario are selected to provide a reasonable estimate of the annual radiation dose. The parameters do not represent average conditions for all individuals exposed and were not selected to perform a worst case (overly conservative) analysis of the potential radiation dose to a maximum individual. Rather, the parameters were chosen from documented sources and previous analyses on the basis of the professional judgment of the study contributors. Although the dose estimates produced may be overestimations, in some cases, they are considered to be generally realistic as opposed to large overestimations (bounding cases). It is possible that for a few situations the parameters chosen could lead to an underestimate of the annual TEDE; however, the potential for underestimation is judged to be small, and the amount of the underestimation is unlikely to be large enough (or occur often enough) to warrant a more conservative approach. A more detailed consideration of the nuances of generic modeling follows.

Generic modeling evaluations, similar to the one described in this document, have become a rather common approach to setting risk-based radiation protection standards. They are useful in evaluating a wide variety of conditions; however, they often have limitations that need to be recognized. Models are intended to be an approximation of reality. Because of data limitations or lack of knowledge, sometimes generic modeling provides an oversimplification of actual conditions and may not account for important physical or chemical processes. When this occurs, or when it is suspected, attempts are made to use conservative assumptions and parameter selections to ensure that potential adverse consequences are overestimated. That is, where detailed knowledge is unavailable, there is an intentional error to provide greater safety. The results obtained by overestimating the adverse consequences may not be very useful because they may lead to prohibitive expenses for clean-up to meet regulatory requirements. However, for simple cases where trivial contamination exists, compliance reached by generic modeling is beneficial because it eliminates unwarranted regulation and expense while not significantly affecting public health and safety. 
For more complex situations, where a wide variation of contamination levels, mixtures, and physical/chemical properties exist, generic models and data sets may not produce a very convincing assurance of compliance. When complex conditions occur, there should be a reliance on site-specific data, if possible, and modifications to the generic approach should be made to account for the actual conditions. For example, direct measurements of radiation fields and their variation provide more meaningful information than generic attempts to model dose rates from contamination levels (i.e., actual measurements may obviate the need to model). A dilemma occurs when the costs of characterizing a site escalate beyond the basic demolition and waste disposal costs. The tendency in this situation may be to comply with a more restrictive generic limit than to attempt a full site characterization and compliance with a site-specific limit.

The pathway dose conversion factors and annual TEDE factors are presented for a listing of about 200 individual radionuclides. Key parentdaughter combinations and decay chains in equilibrium (i.e., for uranium and thorium) have been included. Daughters with half-lives less than 10 minutes are always assumed to be in equilibrium with their parent and are referred to as implicit daughters. Because the pathway-specific dose factors are based on unit activity, it is important to note that the unit activity includes contributions from both the parent and implicit daughters. To accomplish this, a +I notation for the radionuclides listings and an appropriate footnote are included with all dose factor or example problem result tables. Daughters with half-lives greater than 10 minutes that may not be in equilibrium with their parents are referred to as explicit daughters. These are shown in equilibrium conditions with the parent by a $+D$ notation with an appropriate footnote. Separate values for the parents and daughters are also included so that values for non-equilibrium cases can be determined. For cases where there is a short-lived parent with a long-lived daughter, separate listings for the parent and daughter are provided with no $+D$ listing. Finaliy, for decay chains, the listings include parents alone, parents with $+D$ and $+I$ notations (to include both implicit and explicit daughters) and daughters alone so that equilibrium and non-equilibrium cases can be considered. A further description of parent-daughter and decay chain situations is included in Appendix $D$.

\subsection{GENERIC SCENARIOS}

For residual contamination in buildings, this document provides derived annual TEDE factors that account for both potential building renovation (accounting for volume contamination sources) and routine building occupancy (accounting for surface contamination sources). These two scenarios were developed in an attempt to account for questions about the relative importance of volume versus surface activity and fixed versus removable contamination. For many alpha- and beta-emitting radionuclides, direct survey measurements may only account for surface activity because of self shielding. Surface measurement methods may not detect subsurface sources, and significant inventories of radioactive material may be missed. Surface contamination that is fixed (i.e., not easily removed by smearing) at the time of release may become removable with time. Loose surface contamination 
may result in additional radiation exposure pathways and higher estimated radiation doses, as described by scenarios and parameter values defined in this document. Because unrestricted release is considered, it cannot be assumed that subsurface sources or fixed contamination will remain that way after license termination. The two scenarios identified for buildings attempt to account for this potential situation. Because of modeling complexity, no attempt was made to model indoor radon concentrations that may result from residual uranium mixtures in buildings.

Residual radioactive soil contamination may be in a thin or thick soil layer, reside on the surface or be stabilized under a clean soil layer, cover a large or small area, and consist of many different radionuclides or mixtures of radionuclides. The derived annual TEDE factors provided in this document, in units of mrem per $\mathrm{pCi} / \mathrm{g}(\mu \mathrm{Sv}$ per $\mathrm{Bq} / \mathrm{g}$ ) of soil, relate to surface soils and are based on a scenario analysis that considers a combination of inhalation; external; contaminated drinking water ingestion; and agricultural food product ingestion exposure pathways. It is recognized that, for sites located in industrial locations, the potential for even light agricultural activities may be remote. Again, because of modeling complexity, no attempt was made to model indoor radon concentrations that could result from residual soil contamination.

Potential ingestion of contaminated drinking water required a simplified, yet conservative analysis. The model estimates the potential effect of the total radionuclide inventory on the ground-water resources. Perhaps this scenario and model are the most controversial aspects of the modeling analysis. The wide variability of physical and chemical conditions that potentially influence ground water, and the dependence on many parameters that may have a coupled dependency, make it difficult to model ground-water systems. In addition, a conceptual model of a ground-water system may not represent all the behavior of that system. Generic attempts at modeling ground-water systems generally encourage the use of overly conservative parameters, assumptions, and models. As a result, most generic modeling attempts have little meaning when compared with a real system. The existence of site-specific data may encourage a site-specific modeling effort, but such data are relatively costly to obtain, may be point values (both in time and location), and may not appropriately represent the actual system being modeled. The purpose of the generic modeling in this document was to derive an aquifer concentration from residual radioactive materials in soil (or in building materials that may ultimately be buried as rubble onsite) in a conservative manner that would indicate when additional site data or modeling sophistication are warranted. This approach is not intended for broader applications such as application to buried sources, disposed wastes, or sites with a history of spills contaminating soil deeper than $15 \mathrm{~cm}$.

The modeling approach considers leaching using leach-rate factors from previous NRC waste management literature (Oztunali et al. 1981) and simple dilution in a constant volume of ground water. No ground-water transport modeling is attempted. This approach was adopted to obtain results that are useful given the stated purpose of this document, especially in the recognition of cases with trivial contamination from a ground-water 
protection point of view. Because of the potential wide variation in parameter values, the selection of leach-rate factors associated with humid (high rainfall) sites from low-level radioactive waste literature is judged to be adequate for the generic analysis. In a similar manner, the infiltration rate and porosity values were chosen based on the high end of the range of infiltration rates and porosities determined for humid areas of the United States. The ground-water parameter values and concentration equations selected for this analysis are judged to provide a conservative analysis of potential ground-water concentrations.

As a demonstration of the application of the unit annual TEDE factors, example problems were developed. These example problems used mixtures of radionuclides that may be encountered for uranium facilities, light-water power reactors, and radiopharmaceutical/industrial source manufacturing facilities. For completeness, mixtures were defined for a uranium fuel fabrication facility and for depleted and enriched uranium materials. As shown in Appendix $C$, the results for all scenarios for the depleted and enriched uranium mixtures were within about $5 \%$. This similarity occurred because of the dominance of the inhalation pathway and the similarity of the inhalation committed dose equivalent factors for the major isotopes of uranium. When the uranium chains are present in equilibrium, the daughter products provide additional contributions to the dose analys is, as shown by the results for the mixture defined for the uranium fuel fabrication facility.

To ensure that the modeling results were produced correctly, hand calculations were performed to test the results of the computer analysis. In addition, the GENII computer software package has been extensively tested and reviewed in an effort to minimize computational errors. Separate hand calculations were performed during the development of the GENII program, and the results are included with the software documentation (Napier et al. 1988). Additional informal tests and comparisons with other modeling studies were made to determine if the generic modeling analys is was reasonable. Although some initial comparisons were made with existing regulations, these comparisons are not very useful, as described in the following section.

\subsection{RELATIONSHIP TO EXISTING STANDARDS}

The scenario-specific annual TEDE factors contained in this document were derived independently of existing standards or criteria concerning residual radioactive materials in buildings or soils. They are intended to provide a consistent basis for consideration of more than 200 radionuclides that potentially may be of interest at licensed facilities. Therefore, they may not compare with existing standards or regulations for specific facilities or mixtures of radionuclides. For example, current EPA regulations for cleanup of land and buildings contaminated with residual radioactive materials from inactive uranium processing sites (40 CFR 192, Subpart B) limit radium 226Ra concentrations in surface soils to $5 \mathrm{pCi} / \mathrm{g}$. Using the annual TEDE factors for 226Ra plus its daughters in equilibrium in surface soils (from Table 3.3) at a concentration of $5 \mathrm{pCi} / \mathrm{g}$, the result is an annual TEDE of approximately $1.3 \mathrm{mrem}(13 \mu \mathrm{Sv})$. Although this comparison 
may be interesting, it is not completely valid because the residential use (surface soil) scenario does not include an estimate of the indoor radon pathway, accounted for by the EPA standard. Similar difficulties (i.e., the use of different bases and assumptions) are encountered when attempting to compare the surface contamination limits with Regulatory Guide 1.86 or the soil limits with existing NRC guidance for uranium mill tailings. Thus, it is not practical to directly compare the values provided in this report with existing standards.

For the drinking water scenario, dose rate factors are included for determining the dose to the limiting organ. This was done to provide an approximate comparison with existing drinking water standards that limit the organ dose to $4 \mathrm{mrem} / \mathrm{yr}$ from concentrations of radionuclides in drinking water. The organ doses provided in this document were calculated using ICRP 30 methods, and the EPA regulations refer to ICRP 2 methods. The use of different dosimetry models and metabolic data means that the two values are not directly comparable for most radionuclides; however, they are useful in providing a rough estimate of the maximum organ dose. For most significant radionuclides, the ICRP 30 maximum organ dose rate times the weighting factor for the organ will not equal the annual TEDE because of the influence of the other organs in the analysis. An evaluation of the maximum organ dose determination was conducted as part of the review of the methods and results of this study. This was not an easy evaluation given the complexity of the ICRP 30 dosimetry system. For example, at first glance it may seem that a soluble element like cesium should produce a rather uniform dose to internal organs. However, the non-weighted target organ dose shows a maximum (by a few percent) for either the small intestine wall or the stomach wall, depending on the isotope considered. This occurs as somewhat of a calculational artifact (i.e., the computer program is able to recognize differences in the third significant figure and beyond to determine a maximum). Successful attempts were made to verify this result using information from the ICRP and other federal guidance. 



\subsection{REFERENCES}

10 CFR 20. 1986. U.S. Nuclear Regulatory Commission, "Standards for Protection Against Radiation." U.S. Code of Federal Regulations.

10 CFR 61. 1986. U.S. Nuclear Regulatory Commission, "Licensing Requirements for Land Disposal of Radioactive Waste." U.S. Code of Federal Regulations.

40 CFR 192. 1986. U.S. Environmental Protection Agency, "Health and Environmental Protection Standards for Uranium and Thorium Mill Tailings." U.S. Code of Federal Regulations.

46 FR 52061-3. October 23, 1981. "Disposal or Onsite Storage of Thorium or Uranium Wastes from Past Operations." Federal Register.

Aldrich, D. C., D. M. Ericson, Jr., and J. D. Johnson. 1978. Public Protection Strategies for Potential Nuclear Reactor Accidents: Sheltering Concepts with Existing Public and Private Structures. SAND77-1725. Sandia Laboratories, Albuquerque, New Mexico.

American Society of Mechanical Engineers (ASME). 1986. Quality Assurance Program Requirements for Nuclear Facilities. ANSI/ASME NQA-1, American National Standards Institute, New York.

Anspaugh, L. R., J. H. Shinn, P. L. Phelps, and N. C. Kennedy. 1974. "Resuspension and Redistribution of Plutonium in Soils." Health Phys. (29) $4: 571-582$.

Baker, D. A., G. R. Hoenes, and J. K. Soldat. 1976. F00D - An Interactive Code to Calculate Internal Radiation Doses from Contaminated Food Products. BNWL-SA-5523. Pacific Northwest Laboratory, Richland, Washington.

Bateman, H. 1910. "Solution of a System of Differential Equations Occurring in the Theory of Radio-active Transformations." Proc. Cambridge Phil. Soc. $15: 423-427$.

Codel1, R. B. 1984. Simplified Analys is for Liquid Pathway Studies. NUREG1054. U.S. Nuclear Regulatory Commission, Washington, D.C.

Codell, R. B., K. T. Key, and G. Whelan. 1982. A Collection of Mathematical Models for Dispersion in Surface Water. NUREG-0868. U.S. Nuclear Regulatory Commission, Washington, D.C.

Dunster, H. J. 1962. Maximum Permissible Levels of Skin Contamination. AHSB (RP)R28, United Kingdom Atomic Energy Authority, London. 
Eckerman, K. F., A. B. Wolbarst, and A. C. B. Richardson. 1988. Limiting Values of Radionuclide Intake and Air Concentration and Dose Conversion Factors for Inhalation, Submersion, and Ingestion. Federal Guidance Report No. 11, EPA-520/1-88-020. Prepared by Oak Ridge National Laboratory for the U.S. Environmental Protection Agency, Washington, D.C.

Elder, H. K., and D. E. Blahnik. 1980. Technology, Safety and Costs of Decommissioning a Reference Uranium Fabrication Plant. NUREG/CR-1266, Vols. 1 and 2, U.S. Nuclear Regulatory Commission, Washington, D.C.

Enge 1, R. L., J. Greenborg, and M. M. Hendrickson. 1966. ISOSHLD - A Computer Code for General Purpose Isotope Shielding Analysis. BNWL-236, Pacific Northwest Laboratory, Richland, Washington.

Freeze, R. A., and J. A. Cherry. 1979. Groundwater. Prentice-Hall, Inc., Englewood Cliffs, New Jersey.

Gallacher, J. E. J., P. C. Elwood, K. M. Phillips, B. E. Davies, and D. T. Jones. 1984. "Relation Between Pica and Blood Lead in Areas of Differing Lead Exposure." In Archives of Disease in Childhood, vol. 59, pp. 40-44, New York.

Gibson, J. A. B., and A. D. Wrixon. 1979. "Methods for the Calculation of Derived Working Limits for Surface Contamination by Low-Toxicity

Radionuclides." Health Phys. (36) 3:311-321.

Goode, D. J., S. M. Neuder, R. A. Pennifill, and T. Ginn. 1986. Onsite Disposal of Radioactive Waste. NUREG-1101, vol. 3. U.S. Nuclear Regulatory Commission, Washington, D.C.

Healy, J. W., and J. J. Fuquay. 1959. "Wind Pickup of Radioactive Particles from the Ground." Progress in Nuclear Energy. Series XII, Health Physics Vol. I, Pergamon Press, New York.

Healy J.W. 1971. Surface Contamination: Decision Levels. LA-4558-MS, Los Alamos Scientific Laboratory, Los Alamos, New Mexico.

Healy, J. W., J. C. Rodgers, and C. L. Wienke. 1979. Interim Soil Limits for D\&D Projects. LA-UR-79-1865. LOS Alamos Scientific Laboratory, LoS Alamos, New Mexico.

International Commission on Radiological Protection (ICRP). 1959.

Recommendations of the International Commission on Radiological Protection. ICRP Publication 2, Pergamon Press, New York.

International Commission on Radiological Protection (ICRP). 1975. Report of the Task Group on Reference Man. ICRP Publication 23, Pergamon Press, New York.

International Commission on Radiological Protection (ICRP). 1977. Recommendations of the International Commission on Radiological Protection. ICRP Publication 26, Pergamon Press, New York. 
International Commission on Radiological Protection (ICRP). 1979-1988. Limits for Intakes of Radionuclides by Workers. ICRP Publication 30, Parts 1-4 (and supplements), Vol. 2, No. $3 / 4$ through Vol. 19, No. 4, Pergamon Press, New York.

Isherwood, D. 1981. Geosciences Data Base Handbook for Modeling a Nuclear Waste Repository. NUREG/CR-0912, UCRL-52719. Prepared by Lawrence Livermore Laboratory for the U.S. Nuclear Regulatory Commission, Washington, D.C.

Jensen, P. H. 1985. "Shielding Factors for Gamma Radiation From Activity Deposited on Structures and Ground Surfaces." Nuc. Tech. 68:29-39

Kennedy, W. E., Jr., R. A. Peloquin, B. A. Napier, and S. M. Neuder. 1987. Intruder Dose Pathway Analysis for the Onsite Disposal of Radioactive Wastes: The ONSITE/MAXI1 Computer Program. NUREG/CR-3620 (Supplement 2), PNL-4054, Prepared by Pacific Northwest Laboratory for the U.S. Nuclear Regulatory Commission, Washington, D.C.

Kennedy, W. E., Jr., E. C. Watson, D. W. Murphy, B. J. Harrer, R. Harty, and J. M. Aldrich. 1981. A Review of Removable Surface Contamination on Radioactive Materials Transportation Containers. NUREG/CR-1859, PNL-3666, Prepared by Pacific Northwest Laboratory for the U.S. Nuclear Regulatory Commission, Washington, D.C.

Kocher, D. C. 1978. Effects of Man's Residence Inside Building Structures on Radiation Doses from Routine Releases of Radionuclides to the Atmosphere. ORNL/TM-6526. Oak Ridge National Laboratory, Oak Ridge, Tennessee.

Kocher, D. C. 1981a. Dose-Rate Conversion Factors for External Exposure to Photons and Electrons. NUREG/CR-1918, Prepared by Oak Ridge National Laboratory for the U.S. Nuclear Regulatory Commission, Washington, D.C.

Kocher, D. C. 1981b. Radioactive Decay Data Tables: A Handbook of Decay Data for Application to Radiation Dosimetry and Radiological Assessments. DOE/TIC-11026. Prepared by Oak Ridge National Laboratory for the U.S. Department of Energy, Washington, D.C.

Konikow, L. F., and J. D. Bredehoeft. 1978. "Computer Model of TwoDimensional Solute Transport and Dispersion in Ground-Water." Book 7, Chapter C2 in U.S. Geological Survey Techniques of Water Resources Investigations. U.S. Geological Survey, Washington, D.C.

LaGoy, P. K. 1987. "Estimated Soil Ingestion Rates for Use in Risk Assessment," Risk Analysis. 7(3):355-359, Plenum Press, New York.

Lepow, M. L., L. Bruckman, M. Gillette, S. Varkowitx, R. Robino, and J. Kapish. 1975. "Investigations into Sources of Lead in the Environment of Urban Children." Environ. Res. 10:414-426. 
Milham, R. C., J. F. Schubert, J. R. Watts, A. L. Boni, and J. C. Corey. 1975. "Measured Plutonium Resuspension and Resulting Dose from Agricultural Operations on an 01d Field at the Savannah River Plant in the Southeastern United States." IAEA/USERDA International Symposium on Transuranic Nuclides in the Environment. IAEA-SM-199/83, International Atomic Energy Agency, Vienna.

Miller, D. W., ed. 1980. Waste Disposal Effects on Ground Water. Premier Press, Berkeley, California.

Mishima, J., and L. C. Schwendiman. 1972. "Airborne Release of Plutonium and its Compounds During Overheating Accidents." Pacific Northwest Laboratory Annual Report for the DOE Assistant Secretary for the Environment, part I, Biology and Medicine. BNWL-1651, Pacific Northwest Laboratory, Richland, Washington.

Napier, B. A., R. A. Peloquin, D. L. Strenge, and J. V. Ramsdell. 1988. Hanford Environmental Dosimetry Upgrade Project, GENII - the Hanford Environmental Radiation Dosimetry Software Package. PNL-6584, vols. 1-3. Pacific Northwest Laboratory, Richland, Washington.

Oak, G. D., G. M. Holter, W. E. Kennedy, Jr., and G. J. Konzek. 1980. Technology, Safety and Costs of Decommissioning a Reference Boiling-Water Reactor Power Station. NUREG/CR-0672. Prepared by Pacific Northwest Laboratory for the U.S. Nuclear Regulatory Commission, Washington, D.C.

Oak Ridge National Laboratory (ORNL). 1981. RSIC Data Library Collection DRALIST, Radioactive Decay Data for Application to Radiation Dosimetry and Radiological Assessments. DLC-80, 0ak Ridge, Tennessee.

Oblath, S. B. 1989. "Leaching from Solidified Waste Forms under Saturated and Unsaturated Conditions." Environ. Sci. Technol. 23(9):1098-1102.

Oztunali, 0. I., G. C. Re, P. M. Moskowitz, E. D. Picazo, and C. J. Pitt. 1981. Data Base for Radioactive Waste Management: Impacts Analyses Methodology Report. NUREG.CR-1759, vol. 3. Prepared by Dames and Moore, Inc. for the U.S. NucTear Regulatory Commission, Washington, D.C.

Oztunali, 0. I., and G. W. Roles. 1986. Update of Part 61 Impacts Analysis Methodology. NUREG/CR-4370, vol. 1. Prepared by Dames and Moore, Inc. for the U.S. Nuclear Regulatory Commission, Washington, D.C.

Sayre, J. W., E. Charney, J. Vostal, and I. B. Pless. 1974. "House and Hand Dust as a Potential Source of Childhood Lead Exposure." Am J of Dis Chil. $127: 167-170$.

Sehmel, G. A. 1974. "Particle Resuspension from an Asphalt Road." Atmosphere-Surface Exchange of Particulate and Gaseous Pollutants. Conf740921, U.S. Atomic Energy Agency, Washington, D.C. 
Sehme1, G. A. 1976. "Particle Resuspension from Truck Traffic in a Cheat Grass Area." Pacific Northwest Laboratory Annual Report for 1975 to the DOE Assistant Secretary for the Environment, Part 3, Atmospheric Sciences. BNWL2000, Pacific Northwest Laboratory, Richland, Washington.

Sehme1, G. A., and F. D. Lloyd. 1976. "Resuspension Rates from a Circular Field Source." Pacific Northwest Laboratory Annual Report for 1975 to the DOE Assistant Secretary for the Environment, Part 3, Atmospheric Sciences. BNWL-2000, Pacific Northwest Laboratory, Richland, Washington.

Smith, R. I., G. J. Konzek, and W. E. Kennedy, Jr. 1978. Technology, Safety and Costs of Decommissioning a Reference Pressurized Water Reactor Power Station. NUREG/CR-0130, vol. 1-2. Prepared by Pacific Northwest Laboratory for the U.S. Nuclear Regulatory Commission, Washington, D.C.

Soldat, J. K., and R. D. Harr. 1971. "Radiation Dose Model" in HERMES - A Digital Computer Code for Estimating Regional Radiological Effects from the Nuclear Power Industry (J. F. Fletcher and W. L. Dotson, compllers). HEDLTME-71-68. Hanford Engineering and Development Laboratory, Richland, Washington.

Soldat, J. K., N. M. Robinson, and D. A Baker. 1974. Models and Computer Codes for Evaluating Environmental Radiation Doses. BNWL-1754, Pacific Northwest Laboratory, Richland, Washington.

Stewart, K. 1964. "The Resuspension of Particulate Material from Surfaces." Proceedings of the Surface Contamination Symposium. Pergamon Press, New York.

Tracy, J. V. 1982. Users Guide and Documentation for Adsorption and Decay Modifications to the USGS Solute Transport Model. NUREG/CR-2502. U.S. Nuclear Regulatory Commission, Washington, D.C.

U.S. Nucilear Regulatory Commission (NRC). 1981. Draft Environmental Impact Statement on 10 CFR 61 , "Licensing Requirements for Land Disposal of Radioactive Waste." NUREG-0782, Vols. 1-4, U.S. Nuclear Regulatory Commission, Washington, D.C.

U.S. Nuclear Regulatory Commission (NRC). 1977. Calculation of Annual Doses to Man From Routine Releases of Reactor Effluents for the Purpose of Evaluating Compliance with 10 CFR Part 50, Appendix I. Regulatory Guide 1.109, Rev. 1, U.S. Nuclear Regulatory Commission, Washington, D.C.

U.S. Nuclear Regulatory Commission (NRC). 1974. Termination of Operating Licenses for Nuclear Reactors. Regulatory Guide 1.86, U.S. Nuclear Regulatory Commission, Washington, D.C.

Walter, S. D., A. J. Yankel, and I. H. Von Lindern. 1980. "Age-Specific Risk Factors for Lead Absorption in Children." Archives of Environ. Health. $53(1): 53-58$. 
Wilson, J. L., and P. J. Miller. 1979. "Two-Dimensional Plume in Uniform Groundwater Flow, Closure." J. Hydraulics Division, ASCE, 105(HY12):15671570 .

Witherspoon, J. P. 1982. Technology and Cost of Termination Surveys Associated With Decommissioning of Nuclear Facilities. NUREG/CR-32241, ORNL/HASRD-121. Prepared by Oak Ridge National Laboratory, Oak Ridge, Tennessee. 
APPENDIX A

EXTERNAL EXPOSURE MODEL SENSITIVITY STUDIES 

APPENDIX A

\section{EXTERNAL EXPOSURE MODELING SENSITIVITY STUDIES}

Several potentially important conditions exist that may affect the selection of modeling methods used in the external exposure pathway analysis. Significant conditions include: 1) the relationship between external dose rate and source area for various source energies; 2) the relationship between external dose rate and room volume (with various surface contamination conditions); 3 ) the external dose rate for both surface and volume sources, as a function of room size; and 4) the dose rate versus fractional hectare of contaminated soil source area for both shielded and non-shielded conditions.

To determine how to best model these situations for the purposes of this study, a series of sensitivity studies were conducted using a generic modeling approach. The results of these sensitivity studies should also serve as useful illustrations of the determination of the important conditions that may contribute external exposure. When conducting the dose calculations for determining the unit concentration dose conversion factors for the external exposure pathway, described in Section 2.2.1, dose factors generated by the GENII software package were used. These factors are in terms of the effective dose equivalent and account for the sum of weighted organ doses (Kocher 1981).

For each situation considered in the sensitivity studies, only the equivalent penetrating gamma component of the radiation was modeled. That is, for each situation the exposure in air (mR/h) was converted to approximate dose equivalent rate $(\mathrm{mrem} / \mathrm{h})$ by calculating the dose at the center of a sphere of tissue with a radius of $5 \mathrm{~cm}$. The $5-\mathrm{cm}$ tissue depth was used only in the sensitivity studies and not as the basis for the external exposure pathway dose rate conversion factors developed and described in Section 2.2.1. This procedure eliminated the surface effects from low energy photons, but enabled a determination of the appropriate modeling approach for external exposure in buildings and on land surfaces. The results of the sensitivity studies for each of the modeling considerations for the external exposure pathway are described in the following sections.

\section{A.1 WHOLE BODY DOSE RATE VERSUS SOURCE AREA}

As a first step in evaluating external whole body dose rate relationships, a series of calculations for specific gamma-emitters from Kennedy et al. (1987) was considered to determine the influence of source size on the approximate whole-body dose rate. The dose rates for the sensitivity study were estimated using a version of the ISOSHLD computer program (Engel et al. 1966) that employed a single disk source geometry. The dose rates were calculated for selected radionuclides at a 5 -cm tissue depth located $1 \mathrm{~m}$ in air above the source. The results of these calculations are shown in Figure A.1 (Kennedy et al. 1987) in units of mrem/h. Because the curves in Figure A.1 are parallel, the correction factor for a reduced source area is uniform over a large range of gamma energies. This result occurs

A.1 


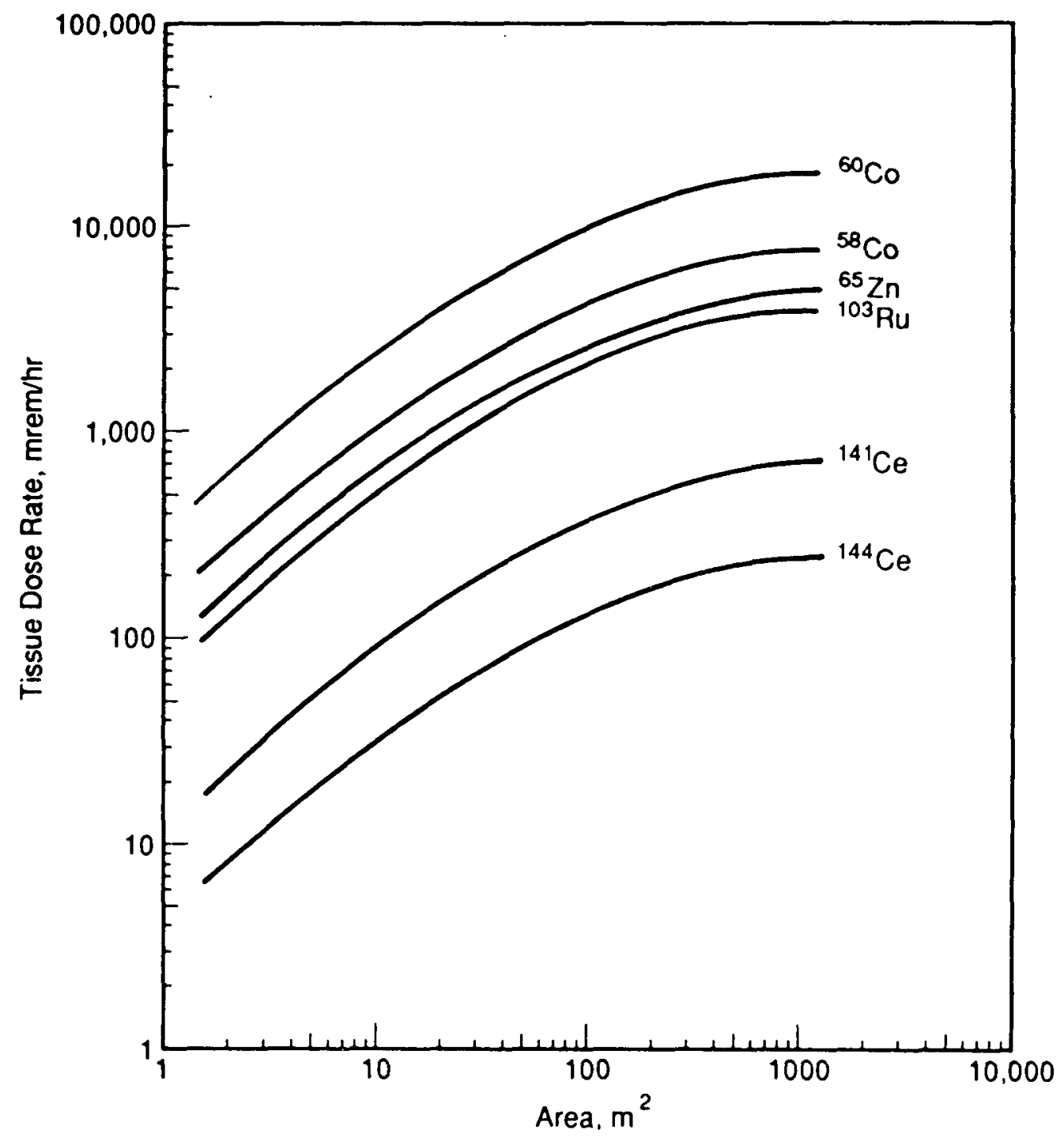

FIGURE A.1. Estimated Tissue Dose Rate Versus Source Area

because the penetrating gamma component (through $5 \mathrm{~cm}$ of body tissue) was calculated, eliminating the low-energy photon effects. This result indicates that there is a large, but uniform, variation of the predicted external dose rate versus source size.

\section{A.2 EXTERNAL DOSE RATE VERSUS ROOM VOLUME}

The ISOSHLD (Engel et al. 1966) computer program was next used to estimate the relationships between the external dose rate and the size of contaminated room, for both uniform and non-uniform contamination conditions. 
It is important to determine this relationship so that an adequate modeling basis for a generic room can be established. The results of the calculations are shown in Figure A.2 for a range of room volumes, assuming a room height of $3 \mathrm{~m}$, and for both uniform and non-uniform contamination conditions

(0ak et al. 1980). The curves in Figure A.2 were calculated for the gamma energies of $60 \mathrm{Co}$; however, this basic relationship is valid over a wide range of gamma energies, and parallel curves could be developed as discussed in Section A.1. As in the previous sensitivity study, the approximate dose rates $(\mathrm{mrem} / \mathrm{h})$ are estimated at a depth of $5 \mathrm{~cm}$ in tissue.

The dose rates were calculated for 60 Co alone, assuming a contamination level of $1 \mathrm{Ci} / \mathrm{m}^{2}$ on the floor. Because there is a definite relationship between dose rate and source energy, the shape of the curves in Figure A.2 should be similar for the source energies considered in Figure A.1, even though the magnitude of the dose rate will vary. Figure A.2 graphically illustrates the difference between an evenly contaminated room and one whose walls and ceiling contain $50 \%$ and $10 \%$, respectively, of the floor contamination level. For reference, the straight line represents the dose rate from an infinite flat-plane source of evenly distributed $60 \mathrm{Co}$ contamination with a concentration of $1 \mathrm{Ci} / \mathrm{m}^{2}$. It can be seen that the dose rate for a large, non-uniformly contaminated room may slightly exceed that of a uniformly contaminated infinite plane because of contributions to the dose

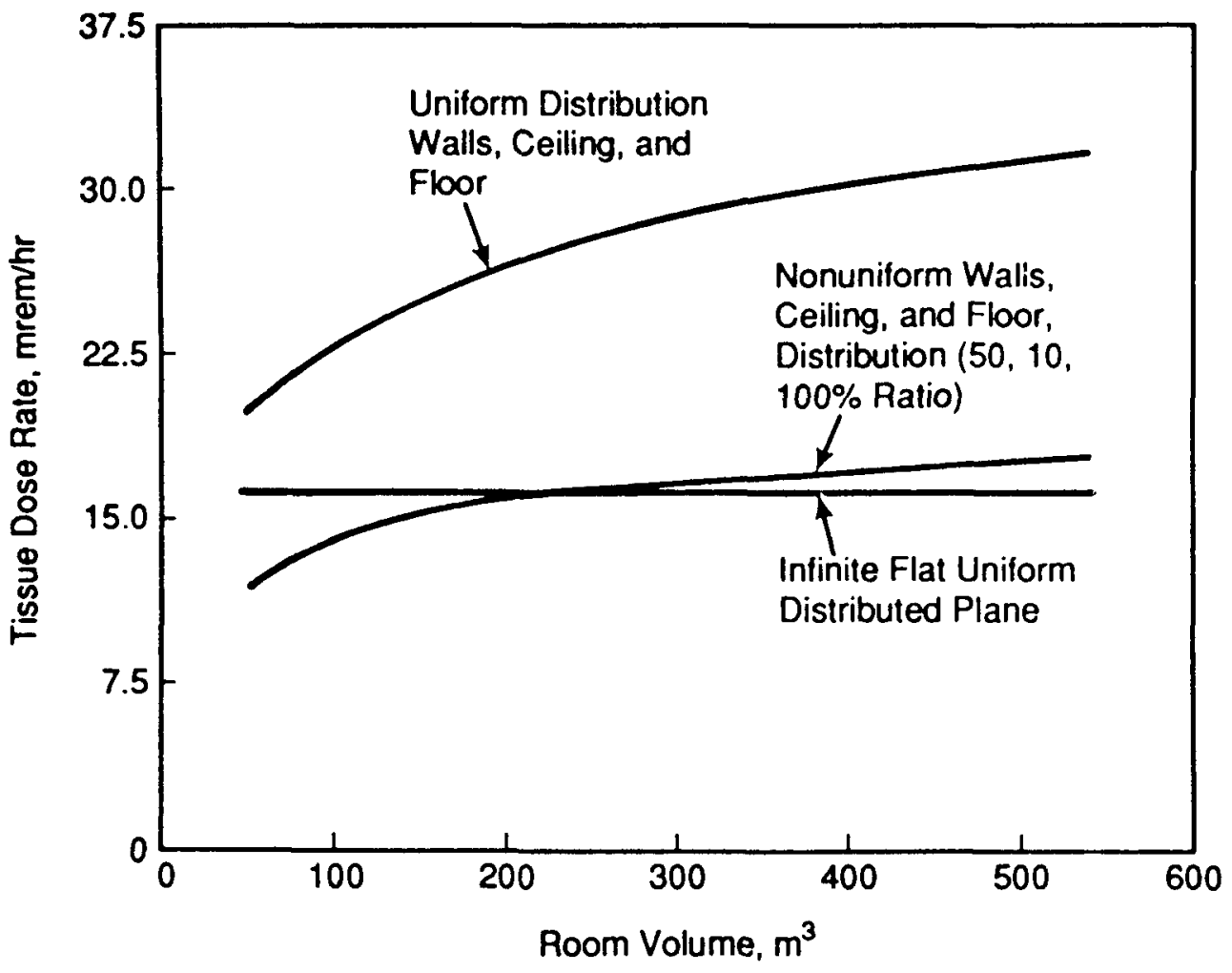

FIGURE A.2. Estimated Tissue Dose Rate at $1 \mathrm{~m}$ Above a Floor Having $1 \mathrm{Ci} / \mathrm{m}^{2}$ as a Function of Room Volume 
rate from the ceiling and walls. However, for most structures where the contamination levels are predicted to be lower on the walls and ceiling, dose rates calculated for an infinite flat plane provide a close approximation to the more complicated model that accounts for contaminated walls and ceiling. For situations where there may be a significant contribution from walls or ceilings, a modified approach to estimating dose rates in rooms should be adopted.

\section{A.3 EXTERNAL DOSE RATE VERSUS SOURCE CONFIGURATION}

Residual contamination on various surfaces in contaminated rooms will vary between surface and volume contamination conditions, depending on the type of surface and the mixture of radionuclides encountered. The potential effect of such variations on the external whole body dose rate was investigated for the mixture of radionuclides found in $3 \%$ enriched (unirradiated) uranium after 10 years of radioactive decay (Elder and Blahnik 1980). This mixture is shown in Table 4.1 in Section 4.1. For this mixture, the dose rate in $(\mathrm{mrem} / \mathrm{h})$ for selected source conditions as a function of room volume are shown in Figure A.3. All curves shown are based on the nonuniform distribution of contamination shown in Figure A.2 (i.e., the walls and ceiling have $50 \%$ and $10 \%$, respectively, of the contamination level found on the floor).

As a reference, the external whole-body dose rate expected from ${ }^{60} \mathrm{Co}$ surface contamination (from Figure A.2) is also shown in Figure A.3. For this mixture of radionuclides, three source configurations on (or in) concrete are plotted: 1) surface contamination (representing non-porous surfaces); 2) a volume source consisting of $90 \%$ of the activity in the first centimeter, with the remaining $10 \%$ of the activity uniformly distributed to a depth of $6 \mathrm{~cm}$; and 3) a volume source uniformly distributed to a depth of 6 $\mathrm{cm}$. For all curves shown in Figure A.3, the units have been normalized to mrem/h per $\mathrm{Ci} / \mathrm{m}^{2}$; that is, for the volume sources, $1 \mathrm{Ci}$ of the mixture of radionuclides is assumed to be diluted in each volume source (up to a maximum depth of $6 \mathrm{~cm}$ ) per square meter of area. For surface contamination, the concentration is $1 \mathrm{Ci} / \mathrm{m}^{2}$. Thus, the distribution of radionuclides in the volume sources is different than for the surface source, but the total $\mathrm{Ci} / \mathrm{m}^{2}$ in both stays the same. As shown by the curves in Figure A.3, the external effective dose rate for the mixture of radionuclides in the $3 \%$ enriched uranium inventory is quite small compared with the estimated external effective dose rate for a pure 60 Co source, at the same contamination level. This result is consistent with a comparison of the total gamma energy for the uranium mixture and $60 \mathrm{Co}$.

\section{A.4 EXTERNAL DOSE RATES FOR SOIL CONTAMINATION CONDITIONS}

The levels and mixtures of residual radioactivity in soil can vary widely depending on the type of facility considered and its operating history. A series of calculations were next performed to determine the potential variation of external dose rate as a function of source area, source volume, and depth of cover material. The results of sequential 


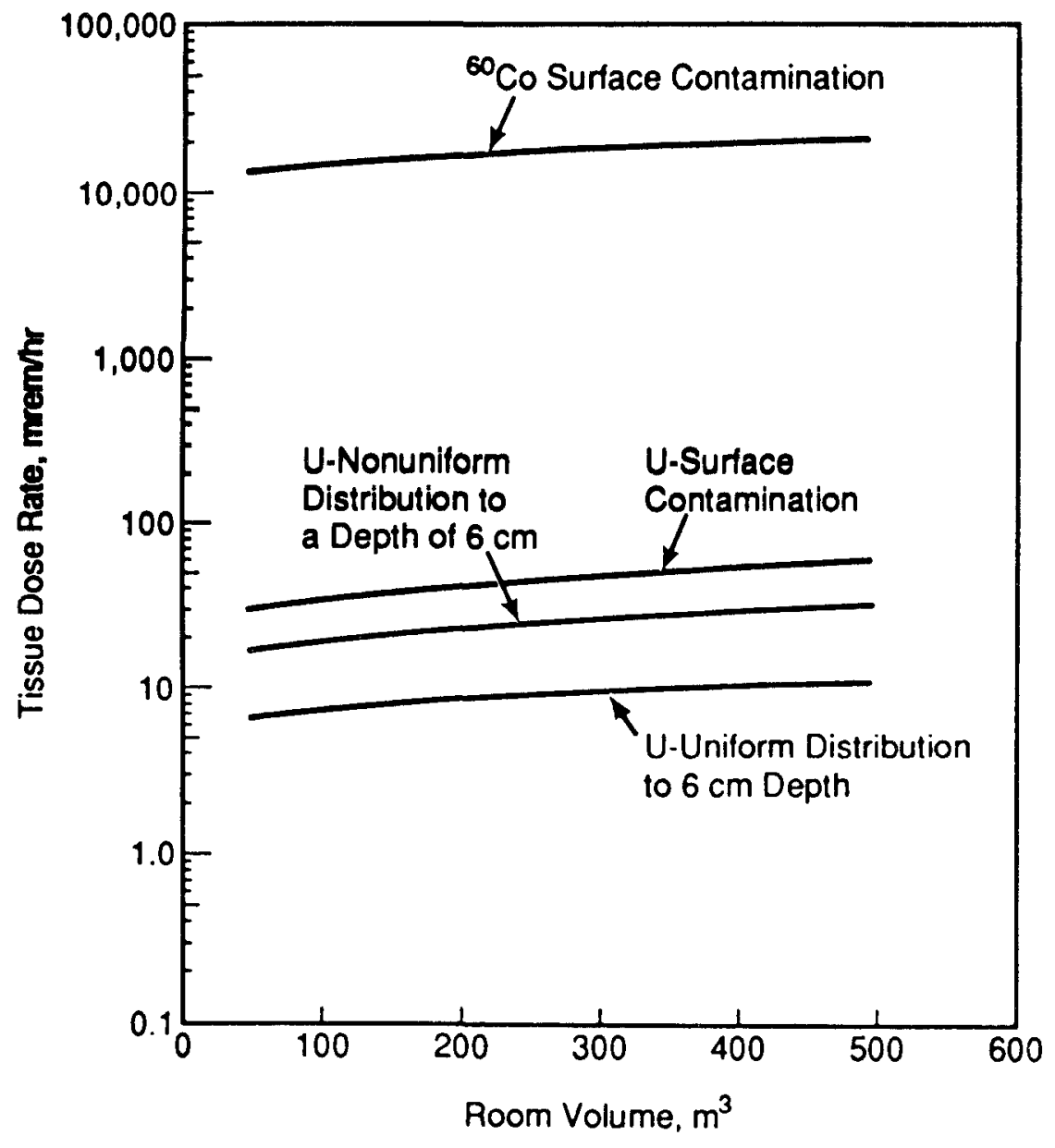

FIGURE A.3. Estimated Tissue Dose Rate for Selected Source Configurations Versus Room Volume for an Effective Contamination Level of $1 \mathrm{Ci} / \mathrm{m}^{2}$

calculations made for these different soil contamination conditions are shown as a family of curves in Figure A.4. The source areas were varied from about $1 \mathrm{~m}^{2}$ to 1 ha $\left(10,000 \mathrm{~m}^{2}\right)$. Three source thicknesses of 1,15 , and $100 \mathrm{~cm}$ were considered. In addition, calculations were performed for two thicknesses of overburden (clean soil as a shield in the calculations), with layers of 0.15 and $0.5 \mathrm{~m}$, to illustrate the effect of soil shielding on the estimated dose rate. As for the calculations made supporting Figure A.3, the units in Figure A.4 have been normalized to $\mathrm{mrem} / \mathrm{h}$ per $\mathrm{Ci} / \mathrm{m}^{2}$; that is, for the volume sources, $1 \mathrm{Ci}$ of the mixture of radionuclides for the reference uranium fuel fabrication facility was assumed to be diluted in slabs of 1, 15, and $100 \mathrm{~cm}$ thickness for each square meter of surface area. As a result, the concentration in $\mathrm{Ci} / \mathrm{m}^{3}$ of soil varies, but the total activity per unit area 
remains constant. As a comparison, the results for a $1-m-t h i c k$ slab of soil containing ${ }^{60} \mathrm{Co}$ are also shown in Figure A.4. These results show that the external whole body dose rate decreases with increasing overburden and

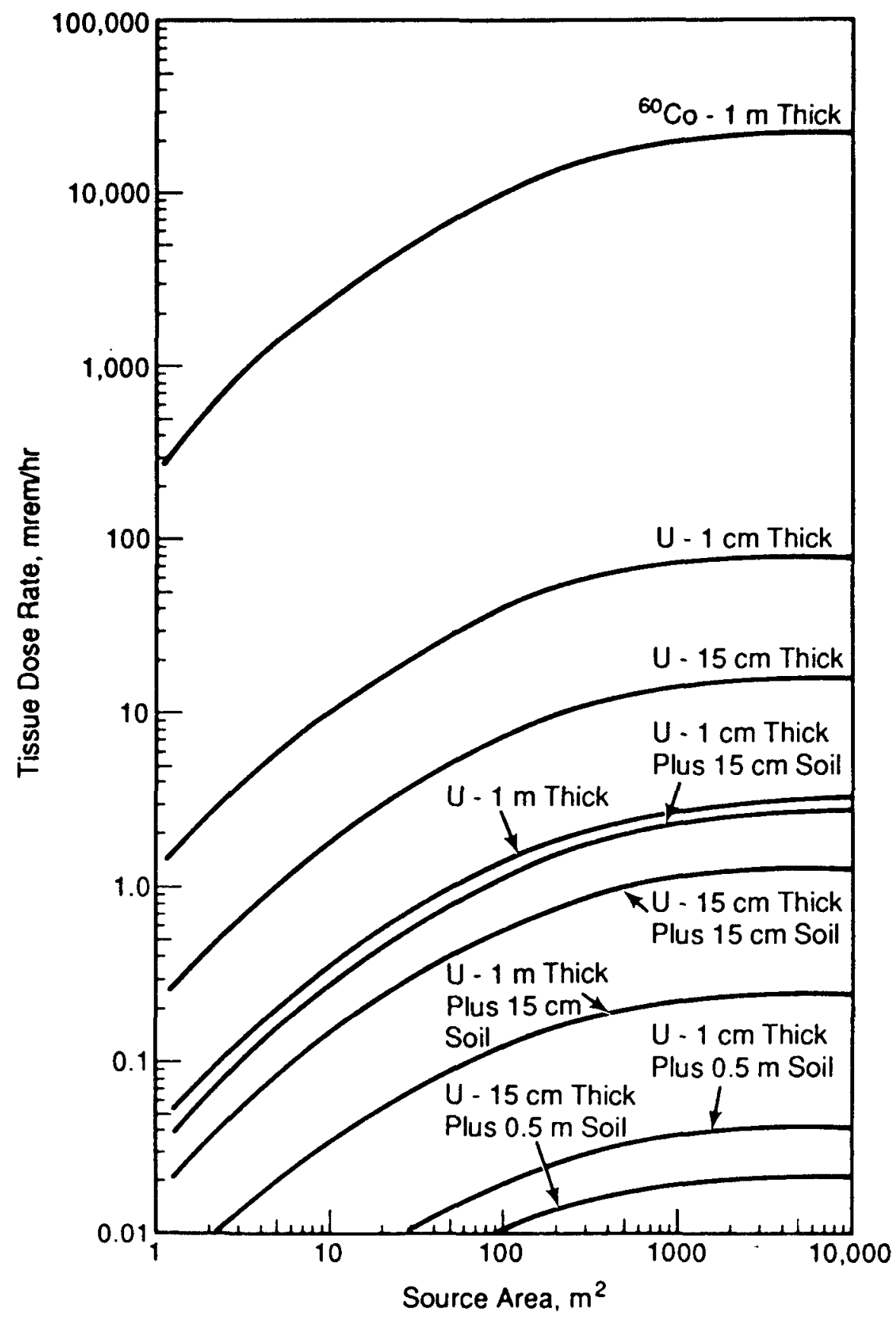

FIGURE A.4. Estimated Tissue Dose Rate for Selected Conditions Versus Area of Soil Contamination for an Effective Concentration of $1 \mathrm{Ci} / \mathrm{m}^{2}$ 
decreases with increasing source thickness as the concentration decreases. The calculated results shown in Figure A.4 agree with what should be expected for these source/shield configurations.

\section{A.5 EXTERNAL DOSE RATE MODELING APPROACH}

The external exposure dose rate conversion factors for the study were generated using the GENII software package. As part of this package, the EXTDF module allows easy and uniform calculation of plane or slab sources of contamination. The EXTDF code is a modification and enhancement of the wellknown shielding code ISOSHLD (Engel, Greenborg, and Hendrickson 1966). The data libraries of radionuclide decay information and gamma and beta yields are based on the original DRALIST compilation of Kocher (1981b) as updated by Oak Ridge National Laboratory (ORNL 1981). External exposure rates for unit quantities of individual radionuclides are calculated considering contributions from electromagnetic radiation from gamma- and $x$-rays; bremsstralung from the slowing-down of beta particles, auger electrons, and conversion electrons; and annihilation radiation from positrons. Alpha particles are not calculated to contribute to the exposure rates. The source of the radionuclide data is the DRALIST file (Oak Ridge National Laboratory 1981), which was adapted for GENII by including implicit daughter energies with their parents. This is an extremely comprehensive list, containing most known radiations, including many with very low frequencies of occurrence (e.g., a $0.8 \mathrm{MeV}$ gamma ray from Po-210 which only occurs once in every 105 decays).

Some radionuclides are pure alpha emitters (e.g. Sm-147). These nuclides will have a calculated exposure rate of zero. Some radionuclides are considered to have "no" emissions, but in actuality have very infrequent or very low-energy radiations. Examples of these types are $\mathrm{Fe}-55$ or $\mathrm{Ni}-59$, which usually decay via internal conversion. Each of these two radionuclides actually has a number of low-energy auger or internal conversion electrons, which may lead to low-energy bremsstralung, and also have a few very-lowenergy gamma or $x$-rays associated with their decays.

Bremsstralung is calculated as a function of the energy of the electron emitted, and the atomic number of the source matrix, using the procedure of the ISOSHLD shielding code (Engel, Greenborg, and Hendrickson 1966). This technique assigns a number and energy of emitted photons as a function of the electron energy. Energies less than $5 \mathrm{keV}$ do not result in emitted photons. An example radionuclide with a beta particle emitted $100 \%$ of the time, but at a very low energy, is Re-187. The maximum beta energy per decay of $\operatorname{Re}-187$ is $0.0026 \mathrm{keV}$, an thus no contribution from bremsstralung is included. For radionuclides which emit positrons, the energy of the annihilation gamma is treated as if the gamma were from any other source.

The ISOSHLD code uses a point kernel integration technique (i.e., the dose at the exposure point is the contribution from a large number of individual point sources). A numerical integration is carried out over the source volume to obtain the total dose. The geometries available in the EXTDF code are the standard 14 available in ISOSHLD. 
The ISOSHLD model works on discrete energy groups, rather than individual energies. Twenty-five energy groups are included in the model. The lowest energy group is from 0.01 to $0.02 \mathrm{Mev}$ (10 to $20 \mathrm{keV}$ ). Any photon radiations of energies lower than this value are lumped into the 0.01 to 0.02 $\mathrm{MeV}$ group. This tends to somewhat overestimate the resultant exposure rate, because the average energy of this group, $0.015 \mathrm{MeV}$, may over-represent the actual energy value for a given radionuclide. This is done to provide a conservative value of the dose response. However, for such radionuclides, even the overestimation is a very small number, relative to those radionuclides with significant energy photon emissions.

Conversion of the calculated exposure rates to effective dose equivalent rates is done via an energy-dependent external-exposure-to-effective-dose conversion factor. It includes attenuation in the body to the depth of each exposed organ. This factor is applied to each of the ISOSHLD energy groups. As for the shielding calculation, doses for photons of energy less than 0.01 $\mathrm{MeV}$ are lumped into the 0.01 to $0.02 \mathrm{MeV}$ group. This has the effect of somewhat overestimating doses from very-low-energy photons. However, for such radionuclides, even the overestimation will be a very small number, relative to those radionuclides with significant emissions.

\section{A.6 SUMMARY}

To best determine how to model a wide variety of external exposure conditions for buildings and soils for this study, a series of sensitivity studies were conducted. The results of these studies were used to determine the source geometry configurations described in Section 2.1.2. In summary:

- For buildings with non-homogeneous distributions of source materials (i.e., higher contamination concentrations on the floor than on the walls or ceiling), dose rate factors for an infinite flat plane provide an appropriate solution over a wide variation of room volumes (as shown in Figure A.2). For situations where the ceiling may be a significant source, the infinite flat plane conversion factors may be doubled to account for two-plane sources.

- For surface soils, the use of a large cylinder source solution, with an assumed radius of $5 \mathrm{~m}$ and a contamination depth of $15 \mathrm{~cm}$ (the assumed plow layer) will provide an adequate modeling bas is for source areas greater than about $80 \mathrm{~m}^{2}$ (as shown in Figure A.4). Although subsurface sources may be important, for this study the external exposure pathway dose factors represent a surface soil, volume source. 
APPENDIX B

PATHWAY ANALYSIS PARAMETERS, ASSUMPTIONS, AND MODIFICATIONS 
APPENDIX B

\section{PATHWAY ANALYSIS PARAMETERS, ASSUMPTIONS, AND MODIFICATIONS}

This appendix contains supporting information to the radiation exposure pathway and scenario analysis conducted for residual radioactive materials in buildings and on land. The support information includes discussions of the derivation of the concentrations in specific media (air, food products, and ground water); describes the key model parameters and assumptions used in the analysis; and describes modifications that can be applied to create sitespecific scenarios and annual TEDE factors.

\section{B.1 EXTERNAL EXPOSURE}

The equation used to model external exposure is shown in Equation 2.1 of Section 2.2.1 of this document. In this equation, the radiation dose equivalent from external sources is the product of the concentration of radionuclides $\left(C_{i p}\right)$, the usage factor or exposure duration $\left(U_{p}\right)$, and a dose rate equivalent factor $\left(D_{i p}\right)$. Details concerning the sensitivity studies conducted to determine the best modeling approach for the external exposure dose equivalent factor $\left(D_{i p}\right)$ for the scenarios considered in this study are described in Appendix $A$. The assumed concentration used in the scenariospecific annual TEDE factors shown in Section 3.0 were either $1 \mathrm{pCi} / \mathrm{g}$ (1 $\mathrm{Bq} / \mathrm{g})$ for volume sources, $1 \mathrm{dpm} / 100 \mathrm{~cm} 2\left(1 \mathrm{Bp} / 100 \mathrm{~cm}^{2}\right)$ for surface sources, or an inventory of $1 \mathrm{pCi}(1 \mathrm{~Bq})$ for estimating concentrations in ground water sources. The assumed exposure duration varied for each scenario, as described in Section 3.0.

Simple modifications can be made to the external exposure pathway and annual TEDE factors in this document by a ratio multiplication. To account for a different exposure duration, the external dose conversion factors must be multiplied by the desired exposure duration and divided by the assumed exposure duration. For example, if an external exposure duration of 4000 $\mathrm{h} / \mathrm{yr}$ is desired for the building occupancy (surface activity) scenario instead of the assumed $2000 \mathrm{~h} / \mathrm{yr}$ duration, the external exposure pathway factors in Table 3.2 must be modified by multiplying by 2 (i.e., 4000/2000). Then, the modified annual TEDE is obtained by adding the modified external exposure factors to the other pathway factors. If inhalation and secondary ingestion parameters are also modified, the respective dose rates should be multiplied by the ratio of the parameters. Then the modified pathway dose rates should be summed to get the modified annual TEDE factor.

For example, to account for a concentration $\left(C_{i p}\right)$ different than the assumed unit levels, the unit concentration annual TEDE factors should be multiplied by the desired concentration. If the modified concentration of radionuclide $i$ for the building renovation (volume activity) scenario is $5 \mathrm{pCi} / \mathrm{g}$ instead of $1 \mathrm{pCi} / \mathrm{g}$, the individual pathway dose rates, and consequently the annual TEDE value shown in Table 3.1 , should be multiplied by 5 . 
More significant changes to the basic dose equivalent factors, accounting for different geometry conditions (radionuclides in smaller source areas or mixed to different depths) or different types of materials (perhaps wood instead of concrete surfaces) require the generation of modified external exposure files using the GENII software package. Details on how to accomplish these changes are found in the documentation of the GENII software (Napier et al. 1988). Appendix D shows the input to the GENII code prepared for developing the external exposure factors used in this analysis and the report generators used to develop the tables of dose rate, committed effective dose rate and annual TEDE factor tables found in Sections 2.0 and 3.0.

\section{B.2 INHALATION}

For inhalation, the model used to describe inhalation exposures is shown in Equation 2.2 of Section 2.2.2. In this equation, the committed effective dose equivalent is found as the product of the ventilation (breathing) rate $(v)$, the exposure duration $(t)$, the concentration of respirable dust in the air $\left(C_{d}\right)$, the concentration of radioactive material in the dust $\left(C_{w}, i\right)$, and the inhalation dose conversion factor $(D F I N H, i)$. As previously described for external exposure, simple modifications can be made to the inhalation pathway and annual TEDE factors accounting for a modified exposure duration or radionuclide concentration. In a similar way, the pathway factors can be modified accounting for a modified ventilation rate by multiplying by the desired value and dividing by the assumed value.

The concentration of respirable dust $\left(C_{d}\right)$ in the air will vary depending upon a variety of factors, including the physical condition (such as the particle size) of the material being handled, the quantity of the material present, and the building ventilation or wind conditions. There are three generic modeling approaches for estimating the concentrations of respirable dust in the air: the use of resuspension factors, resuspension rates, and mass loading factors. A summary of reported resuspension information in shown in Table B.1.

For the resuspension factor method, the average airborne concentration is a function of a resuspension factor and the surface contamination level as shown in Equation B.1.

$$
\chi=S_{f} S_{A}
$$

where $\chi=$ average airborne concentration, $\mathrm{pCi} / \mathrm{m}^{3}\left(\mathrm{~Bq} / \mathrm{m}^{3}\right)$

$S_{f}=$ resuspension factor, $m^{-1}$

$S_{A}=$ surface radioactivity, $\mathrm{pCi} / \mathrm{m}^{2}\left(\mathrm{~Bq} / \mathrm{m}^{2}\right)$.

The typical ranges for reported resuspension factors from Table B.1 is from about $10^{-11}$ to $10^{-5} \mathrm{~m}^{-1}$. To apply a resuspension factor analysis, there 
TABLE B.1. Reported Resuspension Information

Condition, Author and Reference

Wind Stress on:

\section{Bare Soil}

(Stewart 1964)

(Stewart 1964)

(Stewart 1964)

(Mishima and

Schwendiman

1972)

Vegetation:

(Healy and Fuquay 1959)

(Mishima and

Schwendiman

1972)

(Sehmel and

Lloyd 1976)

(Stewart 1964)

(Sehmel 1974)

(Anspaugh 1974)

Mechanical Mixing:

(Stewart 1964)

(Sehmel 1976)

(Sehmel 1974)

(Milham et al. 1975)

(Healy 1971)
Resuspended

Contaminant and Form

Factor or Rate Range

91Y aqueous chloride

210 po as oxide

$\mathrm{U}_{3} \mathrm{O}_{8}$

U02 powder

$1.2 E-4$ to $1.6 E-4 h-1$

$2 E-11$ to $8 E-9 m-1$

$9 E-8$ to $1 E-7 m^{-1}$

$9 E-8$ to $5 E-7 m-1$

Flourescent powder

$2.9 \mathrm{E}-8$ to $6 \mathrm{E}-7 \mathrm{~m}-1$

$\mathrm{UO}_{2}$ powder

$7 E-7$ to $5.9 E-5 h-1$

Calcium molybdate

$1 E-10$ to $1 E-8$ sec-1

$\mathrm{U}_{3} \mathrm{O}_{8}$

$3 E-8$ to $5 E-5 m-1$

ZnS

$3.4 \mathrm{E}-8 \mathrm{sec}^{-1}$

$\mathrm{Pu}$

2.7E-12 to $4.8 \mathrm{E}-10 \mathrm{sec}^{-1}$ 
must be a reasonably uniform level of removable surface contamination, as would be found for soil contamination conditions. Inside buildings after decontamination operations, it is unlikely that significant removable surface contamination would be present.

Alternatively, the resuspension rate method is often used to predict air concentrations within buildings. The average air concentration can be expressed as a function of the resuspension rate and the room ventilation rate by (Healy 1971):

$$
\chi=\frac{\mathrm{fAS} A}{\mathrm{Vn}_{\mathrm{n}}}
$$

where $f=$ the resuspension rate, $h-1$

$A=$ the surface area from which resuspension occurs, $\mathrm{m}^{2}$

$V=$ the volume of air in the room, $\mathrm{m}^{3}$

$n=$ the rate of room air exchange, $h-1$

and where $\chi$ and $S_{A}$ are as previously defined.

(NOTE: $\chi / S_{A}=S_{f}$, the resuspension factor, $m^{-1}$ ).

The typical range of reported resuspension rates from Table $B .1$ is $10-6$ to $10-3 h-1$.

Perhaps the simplest method of estimating air concentrations is to use mass loading factors. For this method, the average air concentration is assumed in terms of $\mathrm{g} / \mathrm{m}^{3}$ of air. This concentration is converted to units of activity using the concentration of the source material. For this analysis, air concentrations have been assumed in the range of $10^{-5}$ to $10^{-3} \mathrm{~g} / \mathrm{m} 3$. This range is roughly consistent with the reported resuspension factor and rate ranges, for selected conditions, and includes the EPA standard for long-term dust loading, namely $5 \times 10^{-5} \mathrm{~g} / \mathrm{m}^{3}$. Local annual averaged dust loadings may be higher or lower than this reference value, depending on soil and wind conditions. Modifications to the assumed air concentrations for each scenario can be made by dividing the inhalation pathway factors by the assumed air concentration and multiplying by the modified concentration in a manner similar to the corrections that can be made for modified exposure durations or ventilation rates. Because of the linear form of Equation 2.2, doubling the air concentration will have the effect of doubling the committed effective dose equivalent from inhalation.

More significant changes to the basic dose equivalent factors, accounting for different solubility classes or particle sizes, require the generation of modified inhalation exposure pathway files using the GENII software package. Details on how to modify the inhalation pathway factors can be found in the recommendations of the ICRP in Publication 30 (1979-1988) and in the documentation of the GENII software (Napier et al. 1988). For 
this analysis, the inhalation committed effective dose equivalent factors were calculated using an assumed particle size distribution of $1 \mu \mathrm{m}$ AMAD. $A$ listing of the assumed solubility classes, by element, used for this analys is is shown in Table B.2. These solubility classes were selected to maximize the potential inhalation dose as calculated using the ICRP lung model.

\section{B.3 SECONDARY INGESTION}

For secondary ingestion, the model used to describe the committed effective dose equivalent is shown in Equation 2.3 of Section 2.2.3. In this equation, the committed effective dose equivalent is found as the product of the intake rate (I), the exposure duration $(t)$, the concentration in the removable surface contamination ( $\left.C_{I N G}, j\right)$, and the ingestion dose conversion factor (DFING, $i$ ). In general, the most soluble form of each element was assumed to maximize the calculated ingestion dose. As previously described for external exposure, simple modifications to the pathway dose conversion factors can be made for the exposure duration and concentration of removable surface contamination by multiplying by the desired parameter value and dividing by the assumed value.

To estimate the ingestion or intake rate, a literature survey was conducted. Previous dose evaluations of the ingestion of surface contamination have been directed toward chronic occupational exposure situations (Dunster 1962; Gibson and Wrixon 1979; Healy 1971; Kennedy et al. 1981). Additional studies have been conducted in estimating the quantities of lead contamination that could be ingested by different age groups (Sayre et al. 1974; Lepow et al. 1975; Walter, Yankel, and Von Lindern 1980; Gallacher et al. 1984; LaGoy 1987). A summary of the referenced surface contamination ingestion data is given in Table B.3. A review of previous work on secondary ingestion pathways indicates that no quantitative data for radioactive materials are available. Because of this lack of data, previous dose estimates for secondary ingestion have relied on assumed ingestion rates. Quantitative data for ingestion of lead by children indicate that they may ingest 11 to $50 \mathrm{mg}$ of lead from hand surfaces with a frequency of up to 10 times per day (Sayre et al. 1974; Lepow et al. 1975; Walter, Yankel, and Von Lindern 1980; Gallacher et al. 1984; LaGoy 1987). The total quantity ingested per day by children may range upward from about $0.1 \mathrm{~g}$.

For our analysis, adult workers are assumed to ingest less removable contamination than children, and renovation workers are assumed to ingest at a higher rate than workers who have routine occupancy. To estimate the potential radiation doses resulting from secondary ingestion, adult renovation workers are assumed to ingest $10 \mathrm{mg}$ of contamination per hour of exposure. Workers during routine building occupancy are assumed to ingest surface contamination at a rate of $1 \mathrm{mg}$ of contamination per hour of exposure.

Modifications to the pathway dose rate factors and annual TEDE factors to account for a modified secondary ingestion rate can be made by a simple ratio multiplication, as previously described. Again, because of the linear form of Equation 2.3, doubling the secondary ingestion rate will have the 
TABLE B.2. Inhalation Solubility Class Information

\begin{tabular}{|c|c|c|c|}
\hline Element & $\begin{array}{l}\text { Inhalation } \\
\text { Solubility } \\
\text { Class(a) } \\
\end{array}$ & Element & $\begin{array}{l}\text { Inhalation } \\
\text { Solubility } \\
\text { Class (a) } \\
\end{array}$ \\
\hline$H$ & D & $S b$ & W \\
\hline $\mathrm{Be}$ & $Y$ & $\mathrm{Te}$ & W \\
\hline C & D & I & $D$ \\
\hline$N$ & 0 & Cs & D \\
\hline $\mathrm{F}$ & D & $\mathrm{Ba}$ & D \\
\hline $\mathrm{Na}$ & D & La & $D$ \\
\hline$P$ & D & $\mathrm{Ce}$ & $Y$ \\
\hline$S$ & $W$ & $\mathrm{Pr}$ & $Y$ \\
\hline $\mathrm{Cl}$ & D & $\mathrm{Nd}$ & $Y$ \\
\hline$K$ & D & $\mathrm{Pm}$ & $Y$ \\
\hline $\mathrm{Ca}$ & W & $\mathrm{Sm}$ & $W$ \\
\hline Sc & $Y$ & Eu & W \\
\hline $\mathrm{Cr}$ & $Y$ & $\mathrm{Gd}$ & D \\
\hline $\mathrm{Mn}$ & $W$ & Tb & $W$ \\
\hline $\mathrm{Fe}$ & $W$ & Ho & W \\
\hline Co & Y & W & $D$ \\
\hline $\mathrm{Ni}$ & $W$ & $\operatorname{Re}$ & $W$ \\
\hline $\mathrm{Cu}$ & $D$ & Os & D \\
\hline $\mathrm{Zn}$ & $Y$ & Ir & $Y$ \\
\hline As & $W$ & $\mathrm{Hg}$ & D \\
\hline $\mathrm{Se}$ & W & $\mathrm{Pb}$ & D \\
\hline $\mathrm{Br}$ & D & $\mathrm{Bi}$ & $W$ \\
\hline $\mathrm{Rb}$ & D & Po & $W$ \\
\hline $\mathrm{Sr}$ & $D$ & $\mathrm{Fr}$ & D \\
\hline$Y$ & $Y$ & $\mathrm{Ra}$ & $W$ \\
\hline $\mathrm{Zr}$ & $W$ & $A C$ & $Y$ \\
\hline $\mathrm{Nb}$ & $Y$ & Th & $Y$ \\
\hline Mo & D & $\mathrm{Pa}$ & $Y$ \\
\hline Tc & $W$ & U & Y \\
\hline Ru & $Y$ & $N p$ & $W$ \\
\hline $\mathrm{Rh}$ & Y & $\mathrm{Pu}$ & $Y$ \\
\hline $\mathrm{Pd}$ & $Y$ & Am & $W$ \\
\hline $\mathrm{Ag}$ & D & $\mathrm{Cm}$ & W \\
\hline $\mathrm{Cd}$ & D & $\mathrm{Cf}$ & W \\
\hline In & D & & \\
\hline Sn & W & & \\
\hline
\end{tabular}

(a) Where $D, W$, and $Y$ correspond to the ICRP definitions and roughly relate to lung clearance times of: $D=$ days, $W=$ weeks, and $Y=$ years.

B.6 
TABLE B.3. Referenced Surface-Contamination Ingestion Data

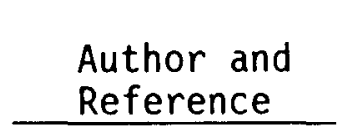

Dunster (1962)

Gibson and

Wrixon (1979)

Healy (1971)

Kennedy et al.

(1981)

Sayre et al. (1974)

Lepow et al.

(1975)

$0.1 \mathrm{~g} / \mathrm{day}$

(children)

$0.018 \mathrm{~m}^{2}$ of dust on hands (children)

Water, Yankel, and Von Lindern (1980)

Gallacher et al. (1984)
Reported Ingestion Rate or Other Value

$10-3 \mathrm{~m}^{2} /$ day

$10^{-3} \mathrm{~m}^{2} /$ day

$10^{-4} \mathrm{~m}^{2} / \mathrm{h}$

$10^{-4} \mathrm{~m}^{2} / \mathrm{h}$
Age dependent

Comments

Chronic ingestion of $M P C_{W}$ values of $226 \mathrm{Ra}, 90 \mathrm{Sr}$, and $210 \mathrm{~Pb}$ to derive permissible levels of skin contamination.

Chronic ingestion. No data available to improve Dunster's model (MPCW analysis)

Chronic ingestion during $8 \mathrm{~h}$ for workers, $24 \mathrm{~h}$ for members of the public. These are arbitrary assumptions in an effort to account for presumed higher intake by children--2.4 E-3m²/day.

Chronic ingestion of removable surface contamination on transportation containers. Dose estimates for both workers and members of the public. (2000 h/yr exposure).

Dirt-hand-mouth ingestion route by children for intake of lead dust. Measurement of the amount of lead dust on children's hands compared with the level present on surfaces in a house.

"Hands-in-mouth" exposure route is the principal cause of excessive lead ingestion. Mean measured weight on children's hands was $11 \mathrm{mg}$. Assuming a mouthing frequency of 10 times/d for small children yields an estimate of $0.1 \mathrm{~g}$ of dirt ingested/d.

Secondary risk factors for lead ingestion were found to be age dependent. Household dustiness is a factor for ages 2 years and under; soil lead is a factor for ages 2 to 7 years.

Data comparing environmental and hand contamination of lead on children's hands was equivalent to 20 to $50 \mathrm{mg}$. This level was estimated to equal about $1 \mathrm{E}-3$ to $3 \mathrm{E}-3 \mathrm{~m}^{2}$ at the level present in outdoor areas. 
effect of doubling the committed effective dose equivalent rate from secondary ingestion.

\section{B.4 INGESTION OF AGRICULTURAL FOOD PRODUCTS}

For ingestion of agricultural food products, the model used to describe the committed effective dose equivalent is shown in Equation 2.4 of Section 2.2.4. This equation contains many parameters describing the behavior of radionuclides in soil and in various food products. The first term in the brackets of Equation 2.4 relates to the concentration resulting from direct deposition of airborne and irrigation-borne material on foliage during the growing season. The airborne material considered is resuspended material from the soil in which the plants are growing. The second term relates to the plant uptake from the soil. The third and fourth terms account for uptake of radionuclides contained in the plow layer $(0.15 \mathrm{~m})$ and material below the plow layer (below a depth of $0.15 \mathrm{~m}$ ).

Although the form of Equation 2.4 is complicated and contains many terms, simple modifications to selected parameters can be made using the ratio methods previously discussed. These modifications include changes to the soil concentration, total assumed diet, and air concentration from resuspension (for the air deposition values in Table 2.4). Again, the modification procedure is to multiply the annual TEDE shown in Table 3.3 by the desired parameter value and divide by the previously assumed value.

Additional details concerning the GENII input parameters and implicit assumptions are contained in Appendix $D$. A computer-readable listing of the food transfer coefficients, by element, is given in Appendix D. More detailed information concerning the default data for the agriculture food product ingestion pathway is provided in Section B.6.

\section{B.5 DRINKING WATER INGESTION}

Residual radioactive contamination in soil has the potential to contaminate ground water in either the saturated or unsaturated zones. The primary mechanisms controlling potential ground-water contamination include infiltration and leaching, transport through the unsaturated zone, and transport through the saturated zone. Many additional processes influence these mechanisms, including precipitation rates, land surface properties, soil properties, the chemical, nature of the radioactive contamination, spatial distributions of the contamination, and advection/retardation in the aquifer. More complete discussions of ground water can be found in Freeze and Cherry (1979), Isherwood (1981), and Wilson and Miller (1979). Previous efforts by the NRC have established a family of models that have fairly broad application to matters related to waste management. These models include those in references by Goode et al. (1986); Konikow and Bredehoeft (1978); Tracy (1982); Codell, Key, and Whelan (1982); and Codell (1984).

The wide variability of physical and chemical conditions that potentially influence ground water, and the dependence on many parameteis that may have a coupled dependency, makes it difficult to model ground-water systems. In addition, a conceptual model of a ground-water system is an 
approximation of reality and may not represent all of the behavior of that system. The cost of site characterization, model selection, and model validation may be prohibitive if a trivial source of contamination exists. As a result of the potential system variability, modeling uncertainty, and data collection/modeling costs, generic modeling attempts generally encourage the use of worst-case (overly conservative) parameters, assumptions, and models to avoid under-prediction of a ground-water system response. The existence of site data may allow the use of more realistic and sophisticated models, but the data may be point values (in both location and time) and may still not appropriately represent the actual system being modeled.

A middle ground between costly site characterization and overly conservative generic modeling may be the use of simple generic models for estimating drinking water concentrations with the use of data and assumptions that should be readily available for any site. The purpose of generic modeling conducted for this document is to derive an aquifer concentration from residual radioactive materials in soil in a conservative manner that will indicate when additional site data or modeling sophistication are warranted. It is recognized that the results may be prohibitive for all but trivial cases. However, it is also recognized that the types of data and assumptions used in the generic approach should be easily obtained for any given site so that some site-specific modifications are possible.

The modeling approach developed for the onsite disposal of radioactive wastes (Goode et al. 1986) is potentially applicable to residual radioactive soil contamination. Goode et al. (1986) provide a discussion of a methodology for estimating the potential contamination of ground water by materials disposed in soils by licensees. Their methodology includes the formulation of a conceptual model, representation of the conceptual model mathematically, estimation of conservative parameters, and prediction of receptor concentrations. Conservative models, assumptions, and parameter selections (i.e., those that are likely to overestimate the receptor concentration) are used for their methodology because of the need to ensure that underestimates of the potential consequences do not occur. When valid site data exist, they recommend that more realistic parameters and models should be used to refine the predictions. In an appendix, Goode et al. (1986) provide an overview of the types of mathematical models that should be considered when developing a detailed evaluation of potential waste disposal impacts on ground-water resources.

Goode et al. (1986) provide a methodology for predicting the well concentrations for an individual who drinks water from an onsite well. The simple conceptual model considers immediate release of materials to the soil. The total radionuclide inventory is assumed to be dispersed in the annual flow rate in the aquifer, $F$, taken to be $91,250 \mathrm{~L}$ of ground water. This volume of water represents the annual volume of water from a domestic well, or $250 \mathrm{~L} /$ day (Miller 1980), used by one person for all purposes, of which $2 \mathrm{~L} /$ day are assumed to be consumed as drinking water. The concentration of each radionuclide in the water is simply the total inventory divided by 91,250 , corrected for radioactive decay. This concentration is described mathematically, Equation B.3, (Goode et al. 1986) as: 


$$
C_{a}(t)=\frac{M_{0}}{(1 y r)(F)} \int_{0}^{1} e^{(-\lambda R t)} d t
$$

where $C_{a}=$ the concentration of each radionuclide in the aquifer at time $t$, $\mathrm{PCi} / \mathrm{L}(\mathrm{Bq} / \mathrm{L})$

$M_{0}=$ the total radioactivity at the time of site release, $\mathrm{pCi}(\mathrm{Bq})$ $\lambda_{R}=t_{y r^{-1}}$ radioactive decay constant for each radionuclide considered,

$\mathrm{F}=$ the dilution flow, $91,250 \mathrm{~L} / \mathrm{yr}$ for an individual

$t=$ an averaging time of 1 year.

Equation B.3 does not account for the leach rate of the radionuclide inventory in soils. A conservative method of estimating the concentration of radionuclides in a ground-water aquifer is to use a simple leach-rate model. Leach rates are dependent on the chemical properties of the radionuclides and soil, and the rate of local water movement. For this model, it is assumed that radionuclides are transferred to the ground water because of contact with infiltrating water (i.e., as a function of the solubility of material in water with no retardation in soils). For most sites, saturation conditions occur only during part of the year; however for this analysis, continuous saturated conditions are assumed. This condition is unlikely for surface soils, as considered for the residential use scenario and should define a conservative case.

To account for leach rate conditions, Equation B. 3 can be modified through the following procedure. Figure B.1 shows a representation of the movement of material from a simple two-box leach model. Box 1 in the figure represents the initial inventory, with removal of material by either radioactive decay or leaching into Box 2. The initial quantity of material in Box $1, Q_{1}(0)$, equals Mo. The initial quantity of material in Box 2, Q2 $(0)$, equals zero. The material in Box 2 serves as the material used to estimate the annual average concentration in the ground-water system.

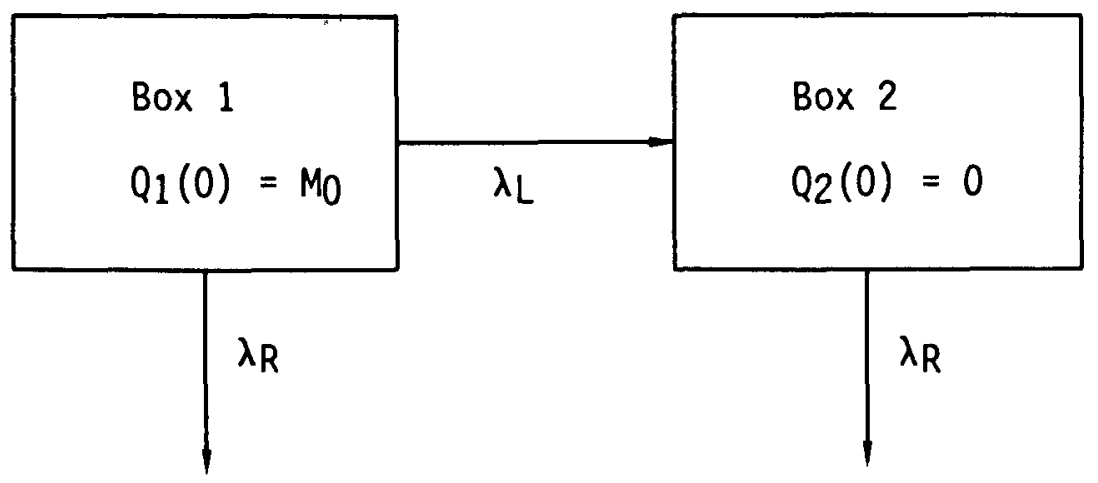

FIGURE B.1. Two-Box Representation of a Simple Leach-Rate System 
The rate of removal of material from Box 1 is described by:

$$
\frac{d Q_{1}}{d t}=-\left(\lambda_{R}+\lambda_{L}\right) Q_{1}
$$

and the change of material from Box 2 is described by:

$$
\frac{d Q_{2}}{d t}=\lambda_{L} Q_{1}-\lambda_{R} Q_{2}
$$

where $\lambda_{L}=$ the leach rate constant, $y r^{-1}$

$Q_{1}=$ the quantity of material in the initial inventory, $\mathrm{pCi}(\mathrm{Bq})$

$Q_{2}=$ the quantity of material leached from the initial inventory, $\mathrm{pCi}$ $(\mathrm{Bq})$

$\lambda_{R}=$ the radioactive decay constant for each radionuclide considered, $\mathrm{yr}^{-1}$.

The quantity in Box 1 equals:

$$
Q_{1}=M_{0} e^{-\left(\lambda_{R}+\lambda_{L}\right) t}
$$

where the parameters are as previously defined.

Solving for the quantity in Box 2, results in:

$$
Q_{2}=M_{0} e^{-\lambda_{R} t}\left(1-e^{-\left(\lambda_{L}\right) t}\right)
$$

where the parameters are as previously defined.

The average concentration in the aquifer is defined by:

$$
\bar{W}=\frac{\int_{0}^{t} Q_{2} d t}{\int_{0}^{t} F d t}
$$


The average concentration in the aquifer, $\bar{W}$, is the ratio of integrals defined in Equation B.8. Solving these integrals, $\bar{W}$ equals:

$$
\bar{W}=\frac{M_{0}}{F t}\left[\frac{\left[1-e^{-\lambda_{R} t}\right]}{\lambda_{R}}-\left(\frac{1-e^{-\left(\lambda_{R}+\lambda_{L}\right) t}}{\left(\lambda_{R}+\lambda_{L}\right)}\right)\right]
$$

The dilution flow in the aquifer is assumed to be $9.1 \times 10^{4} \mathrm{~L} / \mathrm{yr}$, or enough water to supply an average person. The leach rate, $\lambda L$, is defined as follows:

$$
\lambda_{L}=k I(H n)
$$

where $k$ = the partition coefficient, listed by element in Table B.4, from Oztunali et al. (1981)

$I=$ the infiltration rate, chosen to be $0.18 \mathrm{~m} / \mathrm{yr}$ based on the high end of the range of infiltration rated determined for humid areas of the United States (Oztunali, et al. 1981)

$H=$ the assumed thickness of the soil layer containing the residual radioactive material, chosen to be $15 \mathrm{~cm}$ (consistent with previous plow layer assumptions for the agricultural food pathway)

$\mathrm{n}=$ the porosity of the waste/soil mixture, chosen to be 0.3 , associated with only partially compacted soils, as described in Oztunali et. al. (1981).

Again, the use of partition coefficients assumes movement with the saturated water and does not account for further retardation by the soils. The data from 0ztunali et al. (1981) are representative of Maxey Flats and West Valley low-level waste disposal trenches. Because of the presence of organic chemicals and chelating agents at these sites, and because of the continuous full saturation conditions that exist there, these data represent extreme leachability conditions (0ztunali et al. 1981). No attempt was made to reduce the leach rate conditions to account for periods when full saturation does not occur. This approach is not consistent with previous assumptions for low-level waste disposal (0ztunali et al. 1981) where wastesoil leach rates were assumed to be a linear function of the degree of saturation. However, it is consistent with recent literature reports (0blath 1989).

Selection of $0.18 \mathrm{~m} / \mathrm{yr}$ as the infiltration rate determined for humid areas should provide a bounding assumption because many sites are located in areas of lower annual rainfall. Also, selection of 0.3 as the porosity of the soil was intended to provide a bounding analysis. However, these parameters should be readily available and site-specific modifications to this generic model should be possible. 
TABLE B.4. Ground Water Partition Coefficient Information

\begin{tabular}{|c|c|c|c|}
\hline Element & $\begin{array}{c}\text { Ground Water } \\
\text { Partition } \\
\text { Coefficient (a) } \\
\end{array}$ & Element & $\begin{array}{c}\text { Ground Water } \\
\text { Partition } \\
\text { Coefficient }(a) \\
\end{array}$ \\
\hline $\begin{array}{l}\mathrm{H} \\
\mathrm{Be} \\
\mathrm{C}\end{array}$ & $\begin{array}{l}1.15 \\
0.1 \\
1.15 E-1\end{array}$ & $\begin{array}{l}\mathrm{Sb} \\
\mathrm{Te} \\
\mathrm{I}\end{array}$ & $\begin{array}{l}1.62 \mathrm{E}-4 \\
0.1 \\
1.15 \mathrm{E}-1\end{array}$ \\
\hline $\begin{array}{l}\mathrm{N} \\
\mathrm{F} \\
\mathrm{Na}\end{array}$ & $\begin{array}{l}0.1 \\
1.15 \mathrm{E}-1 \\
1.61 \mathrm{E}-4\end{array}$ & $\begin{array}{l}\text { Cs } \\
\text { Ba } \\
\text { La }\end{array}$ & $\begin{array}{l}1.62 \mathrm{E}-4 \\
1.11 \mathrm{E}-4 \\
1.11 \mathrm{E}-4\end{array}$ \\
\hline $\begin{array}{l}\mathrm{P} \\
\mathrm{S} \\
\mathrm{Cl}\end{array}$ & $\begin{array}{l}1.26 \mathrm{E}-3 \\
1.20 \mathrm{E}-2 \\
1.15 \mathrm{E}-1\end{array}$ & $\begin{array}{l}\mathrm{Ce} \\
\mathrm{Pr} \\
\mathrm{Nd}\end{array}$ & $\begin{array}{l}4.67 \mathrm{E}-4 \\
0.1 \\
0.1\end{array}$ \\
\hline $\begin{array}{l}\mathrm{K} \\
\mathrm{Ca} \\
\mathrm{SC}\end{array}$ & $\begin{array}{l}1.62 \mathrm{E}-4 \\
9.86 \mathrm{E}-3 \\
1.11 \mathrm{E}-4\end{array}$ & $\begin{array}{l}\text { Pm } \\
\text { Sm } \\
\text { Eu }\end{array}$ & $\begin{array}{l}0.1 \\
0.1 \\
1.11 \mathrm{E}-4\end{array}$ \\
\hline $\begin{array}{l}\mathrm{Cr} \\
\mathrm{Mn} \\
\mathrm{Fe}\end{array}$ & $\begin{array}{l}1.26 \mathrm{E}-3 \\
1.26 \mathrm{E}-3 \\
1.48 \mathrm{E}-2\end{array}$ & $\begin{array}{l}\text { Gd } \\
\text { Tb } \\
\text { Ho }\end{array}$ & $\begin{array}{l}0.1 \\
0.1 \\
0.1\end{array}$ \\
\hline $\begin{array}{l}\mathrm{Co} \\
\mathrm{Ni} \\
\mathrm{Cu}\end{array}$ & $\begin{array}{l}1.48 \mathrm{E}-2 \\
1.48 \mathrm{E}-2 \\
0.1\end{array}$ & $\begin{array}{l}W \\
\operatorname{Re} \\
\text { Os }\end{array}$ & $\begin{array}{l}0.1 \\
0.1 \\
0.1\end{array}$ \\
\hline $\begin{array}{l}\text { Zn } \\
\text { As } \\
\text { Se }\end{array}$ & $\begin{array}{l}1.48 \mathrm{E}-2 \\
0.1 \\
1.20 \mathrm{E}-2\end{array}$ & $\begin{array}{l}\mathrm{Ir} \\
\mathrm{Hg} \\
\mathrm{Pb}\end{array}$ & $\begin{array}{l}0.1 \\
0.1 \\
4.11 E-3\end{array}$ \\
\hline $\begin{array}{l}\mathrm{Br} \\
\mathrm{Rb} \\
\mathrm{Sr}\end{array}$ & $\begin{array}{l}1.15 \mathrm{E}-1 \\
1.62 \mathrm{E}-4 \\
9.86 \mathrm{E}-3\end{array}$ & $\begin{array}{l}\mathrm{Bi} \\
\mathrm{Po} \\
\mathrm{Fr}\end{array}$ & $\begin{array}{l}0.1 \\
4.11 E-3 \\
0.1\end{array}$ \\
\hline $\begin{array}{l}Y \\
Z r \\
N b\end{array}$ & $\begin{array}{l}0.1 \\
9.86 \mathrm{E}-3 \\
1.11 \mathrm{E}-2\end{array}$ & $\begin{array}{l}\text { Ra } \\
\text { Ac } \\
\text { Th }\end{array}$ & $\begin{array}{l}4.11 \mathrm{E}-3 \\
4.11 \mathrm{E}-3 \\
4.11 \mathrm{E}-3\end{array}$ \\
\hline $\begin{array}{l}\text { Mo } \\
\text { Tc } \\
\text { Ru }\end{array}$ & $\begin{array}{l}1.15 \mathrm{E}-1 \\
1.15 \mathrm{E}-1 \\
1.15 \mathrm{E}-1\end{array}$ & $\begin{array}{l}\mathrm{Pa} \\
U_{\mathrm{Np}}\end{array}$ & $\begin{array}{l}4.11 \mathrm{E}-3 \\
1.24 \mathrm{E}-4 \\
4.67 \mathrm{E}-4\end{array}$ \\
\hline $\begin{array}{l}\mathrm{Rh} \\
\mathrm{Pd} \\
\mathrm{Ag}\end{array}$ & $\begin{array}{l}0.1 \\
0.1 \\
1.62 \mathrm{E}-4\end{array}$ & $\begin{array}{l}\mathrm{Pu} \\
\mathrm{Am} \\
\mathrm{Cm}\end{array}$ & $\begin{array}{l}4.67 \mathrm{E}-4 \\
4.11 \mathrm{E}-3 \\
4.67 \mathrm{E}-4\end{array}$ \\
\hline $\begin{array}{l}\text { Cd } \\
\text { In } \\
\text { Sn }\end{array}$ & $\begin{array}{l}1.48 \mathrm{E}-2 \\
0.1 \\
1.62 \mathrm{E}-4\end{array}$ & $\mathrm{Cf}$ & $4.11 E-3$ \\
\hline
\end{tabular}

(a) Based on information in the data base supporting 10 CFR 61 (Oztunali et al. 1981). 
As stated before, this method does not include any correction for retardation; that is, materials are assumed to move with water. For shortlived radionuclides, this method is conservative because retardation would provide a hold-up period of several years to several decades during groundwater transport. For longer lived radionuclides, this method may also be quite conservative because the expected ground-water plume for long-lived materials may be spread out over a long period of time, at a lower concentration. Alternative parameter values could be obtained for the partition coefficients based on alternative referenced data. Although the data selected for the partition coefficient are from a reference concerning low-level radioactive waste disposal, the values used in this study are felt to be generic, conservative, and adequate for the level of analys is considered in this document. As described in Section 3.0, these ground-water equations are used to estimate drinking water concentrations resulting from residual radioactive material inventories in surface soils. These drinking water concentrations allow for the calculation of annual TEDE factors through a drinking water scenario. Again, the results of the analysis are intended to provide an indication of when additional site data or modeling sophistication are warranted.

Modifications can be made to the derived annual TEDE factors to account for modified assumptions concerning the quantity of drinking water ingested and the concentrations of radionuclides in the water supply. To obtain modified annual TEDE factors, simply multiply the TEDE factor in Table 3.4 by the modified parameter value and divide by the previously assumed parameter value.

\section{B.6 INTERNAL PATHWAY PARAMETERS USED IN THE GENI I COMPUTER PROGRAM}

Additional parameter values have been used in the calculation of pathway dose conversion factors using the GENII computer program. Listings of the major parameters, the values assumed, and notes concerning the reference used for the values are found in Table B.5. Table B.5 provides information for each of the major pathways of exposure and identifies the parameter name used by the GENII program. Additional details about the input for the GENII program, with computer-readable files for the generation of the pathway dose conversion factors and the annual TEDE factors, are found in Appendix D. 
TABLE B.5. Parameter Values Used by the GENII Computer Program for Calculation of Internal Dose Conversion Factors

Pathway/

GENII

Parameter

Name

Value

Definition/Notes

Inhalation Pathway

INHCON

RINH

HRINH

$1 \mathrm{hr} / \mathrm{yr}$

Secondary Ingestion Pathway

DWCON

$1 \mathrm{pCi} / \mathrm{L}$

DWUSAG

$1 \mathrm{~L} / \mathrm{yr}$

Ingestion Pathway - Air Contribution

ARFCON

$1 \mathrm{pCi} / \mathrm{m}^{3}$

DPVL

$8.0 \mathrm{E}-4 \mathrm{~m} / \mathrm{sec}(\mathrm{a})$

DEPFR1

WTIM

$14 d$
Air concentration for inhalation.

Inhalation rate (ICRP 1975; Napier et al. 1988).

Inhalation exposure duration.

Unit concentration as a basis.

Unit consumption rate as a basis.

Air concentration for plant uptake.

Deposition velocity from Oztunali et a). (1981). (a)

Air interception fraction based on standing biomass and dry/wet weight of food type as discussed in Section 4.7.4, Vol. 1 of Napier et al. (1988). Values used for standing biomass and dry-to-wet ratios were taken from Table 4.25, Vol. 1 of Napier et al. (1988).

Weathering half-time (Oztunali et al. 1981, Napier et a1. 1988).

(a) Exceptions: For iodine a value of $1.0 \mathrm{E}-2 \mathrm{~m} / \mathrm{sec}$ and for noble gases a value of $0.0 \mathrm{~m} / \mathrm{sec}$ were used. 
IABLE B.5. Parameter Values Used by the GENII Computer Program for Calculation of Internal Dose Conversion Factors (contd)

\begin{tabular}{|c|c|}
\hline $\begin{array}{l}\text { Pathway/ } \\
\text { GENII } \\
\text { Parameter } \\
\text { Name } \\
\end{array}$ & Value \\
\hline Ingestion & Pathway - Soil Contribution \\
\hline SOLCON & $1 \mathrm{pCi} / \mathrm{g}$ \\
\hline BVI & (a) \\
\hline Ingestion & Pathway - Both Air and Soil \\
\hline GRWP & $60 d(b)$ \\
\hline YELD & $2 \mathrm{~kg} / \mathrm{m}^{2}(\mathrm{c})$ \\
\hline CONSUM & $50 \mathrm{~kg} / \mathrm{d}$ \\
\hline
\end{tabular}

FMI

(d)

Soil concentration for plant uptake.

Soil-to-plant transfer factor by radionuclide and $p 1$ ant type. Values from Oztunali et al. (1981) were used when available. Additional values from NRC (1977) and Napier et al. (1988) were used, when necessary.

Definition/Notes

Growing period for plants (NRC 1977).

Average crop yield (NRC 1977).

Animal consumption rate of feed and forage (Oztunali et al. 1981; NRC 1977).

Transfer coefficient that relates daily intake rate by an animal to the concentration in an edible animal product. Values from 0ztunali et al. (1981) were used when available.

Additional values from NRC (1977) and Napier et al. (1988) were used, when necessary.

(a) Dry plant/dry soil. Values in NRC (1977) and Oztunali et al. (1981) converted to dry plant weight using dry-to-wet ratios from Table 4.25, Vol. 1 of Napier et al. (1988), by radionuclide. See Table D.5 in Appendix D.

(b) With the exception of $30 \mathrm{~d}$ for fresh forage eaten by milk cow.

(c) With exception of fresh forage where a value of $0.7 \mathrm{~kg} / \mathrm{m} 2$ was indicated.

(d) Concentrations in $\mathrm{pCi} / \mathrm{kg}$ in meat or milk per $\mathrm{pCi} / \mathrm{d}$ ingested by animal. Radionuclide-specific. See columns 6 (beef) and 8 (milk cow) of Table D.5 in Appendix D. 
TABLE B.5. Parameter Values Used by the GENII Computer Program for Calculation of Internal Dose Conversion Factors (contd)

\section{Pathway/ \\ GENI I \\ Parameter \\ Name \\ DIETFR}

STORTM

$\begin{array}{rl}90 & d \\ 0 & d\end{array}$

HOLDUP

$\begin{array}{rl}1 & d \\ 14 & d \\ 20 & d \\ 2 & d\end{array}$

CONS

$$
\begin{array}{r}
9.8 \mathrm{~kg} / \mathrm{yr} \\
91 \mathrm{~kg} / \mathrm{yr} \\
42 \mathrm{~kg} / \mathrm{yr} \\
47 \mathrm{~kg} / \mathrm{yr}
\end{array}
$$

CONSA
Definition/Notes

Animal diet fraction for feed and fresh forage. Beef cattle are assumed to eat stored feed exclusively and milk cows are assumed to eat fresh forage exclusively based on an implicit assumption in NRC (1977)

Storage time between harvest of animal feed and animal consumption (NRC 1977) :

Beef

Milk

Holdup time between harvest or slaughter and human consumption of food (NRC 1977):

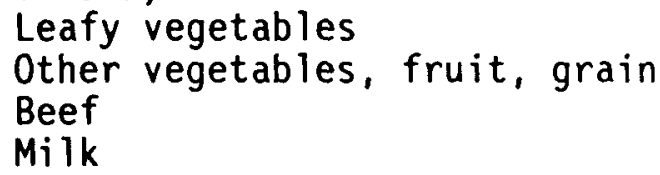
Beef

Milk

Human consumption rate of food (NRC

1977, Oztunali et al. 1981):

Leafy vegetables

Other vegetables

Fruit

Grains and cereal

Values based on a total of $190 \mathrm{~kg} / \mathrm{yr}$ in NRC (1977) and Oztunali et al. (1981), pro-rated by food type based of food type distribution for the average individual in Napier et al. (1988).

Human consumption of animal products

(NRC 1977; Oztunali et al. 1981): Beef $\mathrm{Milk}$ 

APPENDIX C

EXAMPLE DOSE CALCULATIONS FOR REFERENCE BUILDINGS AND LAND 



\section{APPENDIX C \\ EXAMPLE DOSE CALCULATIONS FOR REFERENCE BUILDINGS AND LAND}

This appendix contains additional example calculations performed to estimate the potential annual TEDE from mixtures of radionuclides associated with reference facilities after decommissioning. This appendix is an extension to the discussion on example calculations presented in Section 4.0. The calculations are performed for illustrative purposes using the unit concentration annual TEDE factors from Section 3.0 of this report and reference radionuclide mixtures for example facilities obtained from literature sources. The reference mixtures represent the types of radionuclides and relative activity levels that potentially may be found at uranium facilities, light-water power reactors, and radiopharmaceutical/ industrial-source manufacturing plants. The mixtures and assumed levels are not intended to represent the exact mixtures or levels found at any existing facility. The example calculations consider both residual radioactive contamination in buildings and on land. The results are presented in terms of the calculated annual TEDE for exposure during building renovation and routine occupancy, and during residential use of surface soil and drinking water from ground-water sources.

\section{C.1 REFERENCE RADIONUCLIDE MIXTURES}

As an illustration of the use of the unit concentration annual TEDE factors, reference radionuclide mixtures are defined that are representative of those expected to be found at a uranium facilities (Elder and Blahnik 1980; 10 CFR 20, App. B), light-water power reactor stations (Smith, Konzek, and Kennedy 1978; Oak et al. 1980), and a plant that manufactures a variety of radiopharmaceutical/industrial sources (Kennedy et al. 1981). These mixtures and the descriptions of the reference facilities are discussed in detail in the referenced documents. Each of the mixtures is provided in terms of the relative activity (normalized to unity for the total mixture), by radionuclide, in the mixture without consideration of the total quantity present. For the example mixture calculations, assumed concentrations of 100 $\mathrm{pCi} / \mathrm{g}$ for volume sources (building material and soils) and $10,000 \mathrm{dpm} / 100 \mathrm{~cm}^{2}$ for surface source levels are assumed. It is doubtful that any real facility would actually have radionuclide mixtures consisting of the exact relative activities or assumed concentrations of the radionuclides shown in the reference mixtures. They are intended to serve only as a generic basis for the example calculations.

The mixture of radionuclides found 10 years after plant shutdown at a reference uranium fuel fabrication facility are listed in Table C.1 (Elder and Blahnik 1980). The facility was assumed to operate for a plant life of 40 years allowing the buildup of a residual inventory of material within the facility and on the surrounding land. Because of the long half-lives of the uranium isotopes, and because the uranium has been chemically separated from its full decay chain, only the primary decay products through thorium have been included. Because of the long half-lives of most of the radionuclides shown, the relative activity of the radionuclides is fairly constant over time. Thus, the relative activity is shown only for a decay period of 10 
years after plant shutdown. For this mixture, approximately $51 \%$ of the activity is from $234 \mathrm{U}, 16 \%$ from each of $234 \mathrm{Th}$ and $234 \mathrm{~Pa}$, and $12 \%$ from $238 \mathrm{U}$.

Other types of uranium facilities may handle either depleted or enriched mixtures of uranium isotopes. Estimates of the activity by major

radionuclide are obtained from the footnotes to 10 CFR 20, Appendix B. For depleted uranium, the total specific activity is defined as $3.6 \times 10^{-7} \mathrm{Ci} / \mathrm{g}$ consisting of equal parts of $238 \mathrm{U}$ and $234 \mathrm{U}$. For enriched uranium, the footnotes to Appendix $B$ of 10 CFR 20 provide a formula for estimating specific activity:

$$
S A=\left(0.4+0.38 E+0.0034 E^{2}\right) \times 10-6, \quad E \geq 0.72
$$

where $S A=$ the specific activity of the uranium mixture $(\mathrm{C} / \mathrm{g}$ of uranium activity per gram of uranium, and

$E=$ the percentage, by weight, of $235 \mathrm{U}$.

Mixtures of radionuclides for depleted and enriched uranium that may be encountered at various facilities are shown in Table C.2.

For the reference light-water reactor, information from both a pressurized-water reactor (Smith, Konzek, and Kennedy 1978) and a boilingwater reactor (0ak et al. 1980) was reviewed. The mixture of radionuclides is anticipated to vary as a function of the operating history of the reactor, the plant design, the location within the facility, and the success of decontamination operations. In addition to the surface contamination mixture discussed in Section 4.1, a mixture representing neutron-activated concrete is shown in Table C.3. The mixture of radionuclides for activated concrete will vary depending on the nature of the concrete and its neutron-activation history; the mixture shown is felt to be an adequate bas is for the example dose calculations that follow. Table $C .3$ shows the variation in the relative activity of the mixture at 10 years and 30 years after plant shutdown; however, for purposes of the example calculations that follow, only the mixture after 30 years of decay is used. For this mixture, about $89 \%$ of the relative activity is from $63 \mathrm{Ni}$, and about $8.5 \%$ is from $60 \mathrm{Co}$. For periods of less decay (i.e., closer to plant shutdown), the mixture is dominated by 60 Co instead of the longer lived $63 \mathrm{Ni}$, and the activity level is much greater. For purposes of this example, only the mixture after 30 years of decay is used because it is representative of the potential safe storage period before dismantlement occurs. The 30-year decay period is intended to allow overall dose rates and inventories of short-lived activation products to decay so that occupational doses during dismantlement will be optimized.

There are a large number of radionuclides that are used to make radiopharmaceuticals or as industrial sources. For this study, we have assumed that the representative mixture shown in Table $C .4$ is handled at a facility that supplies both radiopharmaceuticals and industrial sources. The relative contamination level of each radionuclide in the mixture is assumed at plant shutdown with a fractional activity of either 0.1 or 0.05 . The effect of radioactive decay on this mixture is shown for a 5-year decay 
TABLE C.1. Estimated Relative Activity of Radionuclides at a Reference Uranium Fuel Fabrication Facility 10 Years After Shutdown(a)

Radionuclide

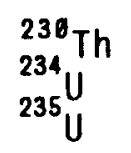

${ }^{231} \mathrm{Th}$

${ }^{234} \mathrm{Th}+\mathrm{I}$

Total
Half-Life (yr)

$7.7 \mathrm{E}+04$

$2.4 \mathrm{E}+05$

$7.0 E+08$

2.9E-03

$3.3 \mathrm{E}+04$

$4.5 E+09$

$6.6 \mathrm{E}-02$

7.6E-04
Relative Activity

$1.6 \mathrm{E}-04$

5. $1 \mathrm{E}-01$

$2.3 \mathrm{E}-02$

2.3E-02

$1.5 \mathrm{E}-05$

$1.2 \mathrm{E}-01$

2.3E-01

$1.2 \mathrm{E}-04$

(a) Based on information in Table 7.1-1 of Elder and Blahnik (1980) and Table 4.1 of Witherspoon (1982).

TABLE C.2. Estimated Relative Activity of Radionuclides in Depleted and Enriched Uranium Mixtures(a)

Radionuclide

Half-Life (yr)

Relative Activity

Depleted

$\begin{array}{ccc}{ }^{234} \mathrm{U} & 2.4 \mathrm{E}+05 & 8.4 \mathrm{E}-02 \\ { }^{235} \mathrm{U} & 7.0 \mathrm{E}+08 & 1.5 \mathrm{E}-02 \\ { }^{238} \mathrm{U} & 4.5 \mathrm{E}+09 & 9.0 \mathrm{E}-01 \\ & & \\ \text { Total } & & 1.0\end{array}$

Enriched $(4 \%)$

$\begin{array}{lll}{ }^{234} \mathrm{U} & 6.6 \mathrm{E}-02 & 7.7 \mathrm{E}-01 \\ { }^{235} \mathrm{U} & 7.0 \mathrm{E}+08 & 2.8 \mathrm{E}-02 \\ 238 \mathrm{U} & 4.5 \mathrm{E}+09 & 2.0 \mathrm{E}-01 \\ & & 1.0 \\ \text { Total } & & \end{array}$

(a) Based on information in the footnotes to Appendix $B$ of 10 CFR 20 using the activities of the uranium isotopes only. 
TABLE C.3. Estimated Relative Activity of Radionuclides in NeutronActivated Concrete at the Reference Power Reactor(a)

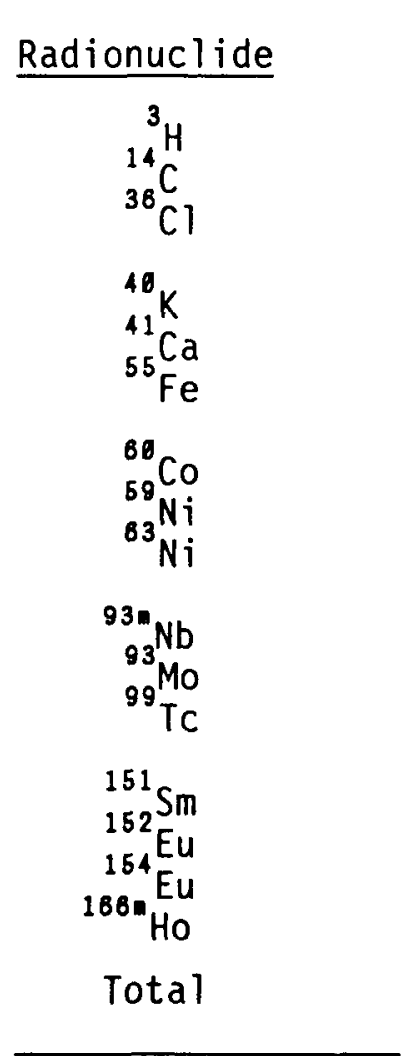

Half-Life (yr)

$1.2 \mathrm{E}+01$

$5.7 E+03$

$3.1 \mathrm{E}+05$

$1.3 \mathrm{E}+09$

$8.0 \mathrm{E}+04$

$2.7 \mathrm{E}+00$

$5.3 E+00$

$8.0 \mathrm{E}+04$

$1.0 E+02$

$1.4 \mathrm{E}+01$

$3.5 E+03$

2. $1 E+05$

9. $0 \mathrm{E}+01$

1. $4 E+01$

$8.8 \mathrm{E}+00$

1. $2 \mathrm{E}+03$
Relative Activity

\begin{tabular}{cc}
10 Years & 30 Years \\
After Shutdown & After Shutdown \\
\hline
\end{tabular}

4. $6 \mathrm{E}-06$

4. $4 \mathrm{E}-06$

4. $3 E-04$

1. $3 \mathrm{E}-03$

3. $9 \mathrm{E}-06$

1. $2 \mathrm{E}-05$

1.2E-04

2.6E-03

3.6E-04

$7.8 \mathrm{E}-03$

2.7E-01

4. $1 \mathrm{E}-03$

3. $8 \mathrm{E}-01$

8.5E-02

2.7E-03

8.1E-03

3. $4 \mathrm{E}-01$

8. $9 \mathrm{E}-01$

6.5E-06

2.0E-05

1.3E-06

4.1E-06

1.5E-07

4. $4 \mathrm{E}-07$

8. $4 \mathrm{E}-08$

2. $2 \mathrm{E}-07$

1. $5 \mathrm{E}-03$

$5.5 \mathrm{E}-04$

1. $4 \mathrm{E}-04$

1.1E-05

3. 3E-06

1.0E-04

1.0

1.0

(a) Based on information in Smith, Konzek, and Kennedy (1978) and Oak et al. (1980). 
TABLE C.4. Estimated Relative Activity of Radionuclides at the Reference Radiopharmaceutical/Industrial-Source Facility (a)

Relative Activity

\section{Radionuclide}

${ }_{2}^{14}{ }_{2}^{3} \mathrm{Ca}$

${ }_{57}^{59} \mathrm{Fe}$

${ }^{6} \mathrm{Co}$

${ }_{125}^{99} \mathrm{TC}$

$131 \mathrm{I}$

${ }_{137}^{134} \mathrm{Cs}$

${ }_{192}^{137} \mathrm{CS}+\mathrm{I}(\mathrm{C})$

${ }_{210}^{210} \mathrm{~Pb}$
${ }_{228} \mathrm{Po}$
$\mathrm{Ra}$

Total
Half-Life (yr)

1. $2 \mathrm{E}+01$

5.7E+03

2. $6 \mathrm{E}+00$

1. $2 \mathrm{E}-01$

7. $4 \mathrm{E}-01$

$5.3 \mathrm{E}+00$

6.9E-04

1. $6 \mathrm{E}-01$

2. 2E-02

2. $1 \mathrm{E}+02$

3. $0 \mathrm{E}+01$

2. OE-01

2. $2 E+01$

3. $8 \mathrm{E}-01$

1. $6 \mathrm{E}+03$
At Shutdown

1. $0 E-01$

5. OE-02

1. $0 \mathrm{E}-01$

5. OE-02

5. $0 \mathrm{E}-02$

1. $0 \mathrm{E}-01$

5. $0 \mathrm{E}-02$

5. $0 \mathrm{E}-02$

5. OE-02

5. $0 \mathrm{E}-02$

1. $0 \mathrm{E}-01$

5.0E-02

5. $0 \mathrm{E}-02$

5. OE-02

1. $0 \mathrm{E}-01$

1.0
5 Years

After Shutdown

1. $4 \mathrm{E}-01$

9. $6 \mathrm{E}-02$

5.2E-02

9.2E-04

1. $0 \mathrm{E}-01$

1. $8 \mathrm{E}-02$

1. $7 \mathrm{E}-01$

1. $9 E-01$

1. $1 E-01$

$1.1 E-01$

1.0

(a) Based on information in Kennedy et al. (1981).

(b) A dash indicates a value less than 10-8.

(c) Where +I means "plus implicit daughters in equilibrium" as discussed in Appendix $D$ 
period after shutdown. Because many of the radionuclides commonly used as radiopharmaceuticals or as industrial sources have short half-lives, five of the radionuclides included at plant shutdown have decayed to trivial levels. Only, $3 \mathrm{H}, 60 \mathrm{Co}, 210 \mathrm{~Pb}, 210 \mathrm{Po}$, and $226 \mathrm{Ra}$ have activity levels greater or equal to $10 \%$ of the total for the mixture. For the example problems that follow, only the mixture after 5 years of decay is considered.

\section{C.2 EXAMPLE DOSE CALCULATIONS FOR THE BUILDING RENOVATION SCENARIO}

Example calculations are next performed for the reference mixtures and assumed levels of radionuclides discussed in the previous section for the building renovation (volume activity) scenario. The results are presented in Tables C.5 through $C .8$ as the estimated rate by exposure pathway and the annual TEDE to an individual (in mrem and $\mu S v$ of exposure). The initial contamination level was assumed to be $100 \mathrm{pCi} / \mathrm{g}$ for each mixture. As shown in these tables, the estimated dose varies depending on the mixture.

Because of the long half-lives of the uranium decay chain, the example calculations for the uranium fuel fabrication facility are performed for only one decay period--10 years after plant shutdown. The reference mixture of radionuclides is shown in Table C.1. The resulting estimated annual TEDE for the building renovation scenario for the uranium fuel fabrication facility are shown in Table C.5. The annual TEDE is $0.46 \mathrm{mrem}(4.6 \mu \mathrm{Sv})$. The dominant contributor to the dose is $234 \mathrm{U}$ through the inhalation pathway.

For the depleted and enriched uranium mixtures shown in Table C.2, the resulting estimated annual TEDEs for the building renovation scenario are shown in Table C.6. The annual TEDE from depleted uranium is 0.62 mrem $(6.2$ $\mu \mathrm{Sv})$. The dominant exposure pathway for depleted uranium is inhalation and the dominant radionuclide is $238 \mathrm{U}$. The annual TEDE from enriched uranium is $0.66 \mathrm{mrem}(6.6 \mu \mathrm{Sv})$. The dominant exposure pathway is inhalation and the dominant radionuclide in the mixture is $234 \mathrm{U}$.

For the neutron-activated concrete mixture at the light-water power reactor, the calculations were performed for the mixture decayed to 30 years after plant shutdown. The reference mixture of radionuclides is shown in Table C.3 and the resulting annual TEDEs are shown in Table C.7. The estimated annual TEDE for this mixture is $0.71 \mathrm{mrem}(7.1 \mu \mathrm{Sv})$, dominated by 60 Co and the external exposure pathway.

Example annual TEDEs for the radiopharmaceutical/industrial source mixture with 5 years of radioactive decay, shown in Table C.4, for the building renovation scenario are shown in Table C.8. The estimated annual TEDE for this mixture and exposure scenario is 2.3 mrem ( $23 \mu \mathrm{Sv}$ ). The dominant radionuclide contributors to the dose in the mixture are $60 \mathrm{Co}$, $137 \mathrm{Cs}$, and $22 \mathrm{Na}$. The dominant exposure pathway is external exposure.

\section{C.3 EXAMPLE DOSE CALCULATIONS FOR THE BUILDING OCCUPANCY SCENARIO}

Example calculations are next performed that consider the reference mixtures and assumed levels of radionuclides discussed in the previous section for the building occupancy (surface activity) scenario. The results 
are presented in Tables C.9 through C.12 in terms of the estimated dose by exposure pathway and the annual TEDE to an individual (in mrem and $\mu S v$ ). The initial contamination level for this scenario was assumed to be $10000 \mathrm{dpm} / 100$ $\mathrm{cm}^{2}\left(167 \mathrm{~Bq} / 100 \mathrm{~cm}^{2}\right)$ for each mixture. As shown in these tables, the estimated dose varies depending on the mixture and exposure pathway.

The example calculations for the uranium fuel fabrication facility were performed for the mixture shown in Table C.1 with 10 years of decay after plant shutdown. The resulting estimated annual TEDE for the building occupancy scenario for the uranium fuel fabrication facility are shown in Table C.9. The annual TEDE from surface sources is 34 mrem $(340 \mu \mathrm{Sv})$. The dominant contributor to the dose is $234 \mathrm{U}$ through the inhalation pathway.

For the depleted and enriched uranium mixtures shown in Table C.2, the resulting estimated annual TEDEs for the building occupancy scenario are shown in Table C.10. The annual TEDE from depleted uranium is 25 mrem (250 $\mu \mathrm{Sv})$. Inhalation is the dominant pathway and $238 \mathrm{U}$ is the dominant radionuclide contributor to dose. For enriched uranium, the annual TEDE is $27 \mathrm{mrem}(270 \mu \mathrm{Sv})$. The dominant contributor to dose is $234 \mathrm{U}$ through the inhalation pathway.

For the neutron-activated concrete mixture at the light-water power reactor, the calculations were performed for the mixture decayed to 30 years after plant shutdown. The results are shown in Table C.11. The estimated annual TEDE for this mixture and scenario is $0.74 \mathrm{mrem}(7.4 \mu \mathrm{Sv})$, dominated by 60 Co and the external exposure pathway.

Example annual TEDEs for the radiopharmaceutical/industrial-source mixture with 5 years of radioactive decay for the building surface activity scenario are shown in Table C.12. The estimated annual TEDE for this mixture and exposure scenario is $29 \mathrm{mrem} / \mathrm{yr}(290 \mu \mathrm{Sv} / \mathrm{yr})$. The dominant radionuclide contributors to the dose in the mixture are $210 \mathrm{~Pb}, 210 \mathrm{Po}$, and $226 \mathrm{Ra}$. The dominant exposure pathway is secondary ingestion. 
TABLE C.5. Estimated Annual Total Effective Dose Equivalents for the Building Renovation (Volume Activity) Scenario Using the Uranium Fuel Fabrication Facility Inventory 10 Years After Shutdown(a)

\begin{tabular}{|c|c|c|c|c|c|}
\hline $\begin{array}{c}\text { Radio- } \\
\text { nuclide (b) }\end{array}$ & $\begin{array}{c}\text { External } \\
\text { (mrem) }\end{array}$ & $\begin{array}{c}\text { Inhalation } \\
\text { (mrem) }\end{array}$ & $\begin{array}{c}\text { Secondary } \\
\text { Ingestion } \\
\text { (mrem) }\end{array}$ & $\begin{array}{c}\text { TEDE } \\
\text { (mrem) }\end{array}$ & $\begin{array}{r}\text { TEDE } \\
(\mu S V)\end{array}$ \\
\hline $\begin{array}{l}{ }^{236} \mathrm{Th} \\
{ }^{231} \mathrm{Th} \\
{ }^{234} \mathrm{Th}+\mathrm{I}(\mathrm{c})\end{array}$ & $\begin{array}{l}1.8 \mathrm{E}-07 \\
6.2 \mathrm{E}-04 \\
1.7 \mathrm{E}-02\end{array}$ & $\begin{array}{l}2.2 \mathrm{E}-04 \\
9.4 \mathrm{E}-08 \\
3.9 \mathrm{E}-05\end{array}$ & $\begin{array}{l}7.0 E-04 \\
1.5 E-05 \\
1.5 E-03\end{array}$ & $\begin{array}{l}9.3 \mathrm{E}-04 \\
6.4 \mathrm{E}-04 \\
1.9 \mathrm{E}-03\end{array}$ & $\begin{array}{l}9.3 \mathrm{E}-03 \\
6.4 \mathrm{E}-03 \\
1.9 \mathrm{E}-01\end{array}$ \\
\hline${ }^{234} \mathrm{~Pa}$ & $\begin{array}{l}7.9 \mathrm{E}-04 \\
4.2 \mathrm{E}-04\end{array}$ & $\begin{array}{l}\text { 4. } 9 \mathrm{E}-10 \\
3.3 \mathrm{E}-01\end{array}$ & $\begin{array}{l}1.3 \mathrm{E}-07 \\
6.6 \mathrm{E}-03\end{array}$ & $\begin{array}{l}7.9 \mathrm{E}-04 \\
3.4 \mathrm{E}-01\end{array}$ & $\begin{array}{l}7.9 E-03 \\
3.4 E+00\end{array}$ \\
\hline${ }^{235} U$ & $\begin{array}{l}7.4 \mathrm{E}-03 \\
9.4 \mathrm{E}-04\end{array}$ & $\begin{array}{l}1.4 \mathrm{E}-02 \\
7.2 \mathrm{E}-02\end{array}$ & $\begin{array}{l}3.0 \mathrm{E}-04 \\
1.6 \mathrm{E}-03\end{array}$ & $\begin{array}{l}2.1 \mathrm{E}-02 \\
7.4 \mathrm{E}-02\end{array}$ & $\begin{array}{l}2.1 \mathrm{E}-01 \\
7.4 \mathrm{E}-01\end{array}$ \\
\hline Total & $\overline{2.7 \mathrm{E}-02}$ & $\overline{4.2 \mathrm{E}-01}$ & $\overline{1.1 \mathrm{E}-02}$ & $\overline{4.6 \mathrm{E}-01}$ & $\overline{4.6 E+00}$ \\
\hline
\end{tabular}

(a) Using the radionuclide mixture shown in Table C.1 with 10 years of radioactive decay and the annual TEDE factors from Table 3.1.

(b) With an assumed contamination level of $100 \mathrm{pCi} / \mathrm{g}(3.7 \mathrm{~Bq} / \mathrm{g})$ of the radionuclide mixture.

(c) Where +I means "plus implicit daughters in equilibrium" as discussed in Appendix D.

TABLE C.6. Estimated Annual Total Effective Dose Equivalents for the Building Renovation (Volume Activity) Scenario Using the Depleted and Enriched Uranium Mixture(a)

\begin{tabular}{|c|c|c|c|c|c|}
\hline $\begin{array}{l}\text { Radio- } \\
\text { nuclide(b) }\end{array}$ & $\begin{array}{c}\text { External } \\
\text { (mrem) }\end{array}$ & $\begin{array}{c}\text { Inhalation } \\
\text { (mrem) }\end{array}$ & $\begin{array}{l}\text { Secondary } \\
\text { Ingestion } \\
\text { (mrem) }\end{array}$ & $\begin{array}{c}\text { TEDE } \\
\text { (mrem) }\end{array}$ & $\begin{array}{r}\text { TEDE } \\
(\mu S v) \\
\end{array}$ \\
\hline \multicolumn{6}{|l|}{ Depleted } \\
\hline $\begin{array}{l}{ }^{234} \mathrm{U} \\
235 \mathrm{U} \\
238 \mathrm{U}\end{array}$ & $\begin{array}{l}6.9 \mathrm{E}-05 \\
4.8 \mathrm{E}-03 \\
7.2 \mathrm{E}-03\end{array}$ & $\begin{array}{l}5.5 \mathrm{E}-02 \\
9.0 \mathrm{E}-03 \\
5.4 \mathrm{E}-01\end{array}$ & $\begin{array}{l}1.1 \mathrm{E}-03 \\
2.0 \mathrm{E}-04 \\
1.2 \mathrm{E}-02\end{array}$ & $\begin{array}{l}5.5 \mathrm{E}-02 \\
1.4 \mathrm{E}-02 \\
5.6 \mathrm{E}-01\end{array}$ & $\begin{array}{l}5.5 \mathrm{E}-01 \\
1.4 \mathrm{E}-01 \\
5.6 \mathrm{E}+00\end{array}$ \\
\hline Total & $\overline{1.2 \mathrm{E}-02}$ & $\overline{6.0 \mathrm{E}-01}$ & $\overline{1.3 \mathrm{E}-02}$ & $\overline{6.2 E-01}$ & $\overline{6.2 E+00}$ \\
\hline
\end{tabular}

Enriched $(4 \%)$

$\begin{array}{cccccc}{ }^{234} \mathrm{U} & 6.6 \mathrm{E}-04 & 5.0 \mathrm{E}-01 & 1.0 \mathrm{E}-02 & 5.1 \mathrm{E}-01 & 5.1 \mathrm{E}+00 \\ 235 \mathrm{U} & 9.0 \mathrm{E}-03 & 1.7 \mathrm{E}-02 & 3.6 \mathrm{E}-04 & 2.6 \mathrm{E}-02 & 2.6 \mathrm{E}-01 \\ 238 \mathrm{U} & 4.1 \mathrm{E}-05 & 1.2 \mathrm{E}-01 & 2.6 \mathrm{E}-03 & 1.2 \mathrm{E}-01 & 1.2 \mathrm{E}+00 \\ \text { Total } & \overline{9.7 \mathrm{E}-03} & \overline{6.4 \mathrm{E}-01} & \overline{1.3 \mathrm{E}-02} & \overline{6.6 \mathrm{E}-01} & \overline{6.6 \mathrm{E}+00}\end{array}$

(a) Using the uranium isotope mixture shown in Table C.2 and the annual TEDE factors from Table 3.1.

(b) With an assumed contamination level of $100 \mathrm{pCi} / \mathrm{g}(3.7 \mathrm{~Bq} / \mathrm{g})$ of the radionuclide mixture. 
TABLE C.7. Estimated Annual Total Effective Dose Equivalents for the Building Renovation (Volume Activity) Scenario Using the Neutron-Activated Power Reactor Concrete Inventory 30 Years After Shutdown (a)

\begin{tabular}{|c|c|c|c|c|c|}
\hline $\begin{array}{l}\text { Radio- } \\
\text { nuclide(b) }\end{array}$ & $\begin{array}{c}\text { External } \\
\text { (mrem) } \\
\end{array}$ & $\begin{array}{c}\text { Inhalation } \\
\text { (mrem) }\end{array}$ & $\begin{array}{c}\text { Secondary } \\
\text { Ingestion } \\
\text { (mrem) }\end{array}$ & $\begin{array}{c}\text { TEDE } \\
\text { (mrem) }\end{array}$ & $\begin{array}{r}\text { TEDE } \\
(\mu S v) \\
\end{array}$ \\
\hline${ }^{{ }^{14} \mathrm{C}} \mathrm{C}$ & $\begin{array}{l}2.2 E-15 \\
2.7 E-08 \\
1.6 E-08\end{array}$ & $\begin{array}{l}1.8 \mathrm{E}-12 \\
1.2 \mathrm{E}-08 \\
1.1 \mathrm{E}-10\end{array}$ & $\begin{array}{l}1.2 E-10 \\
1.2 E-06 \\
1.7 E-08\end{array}$ & $\begin{array}{l}1.3 E-10 \\
1.3 E-06 \\
3.2 E-08\end{array}$ & $\begin{array}{l}1.3 E-09 \\
1.3 E-06 \\
3.2 E-07\end{array}$ \\
\hline $\begin{array}{l}{ }^{40} \mathrm{~K} \\
{ }^{41} \mathrm{Ca} \\
{ }^{55} \mathrm{Fe}\end{array}$ & $\begin{array}{l}1.9 E-04 \\
5.4 E-06 \\
6.6 E-06\end{array}$ & $\begin{array}{l}1.8 E-08 \\
4.7 E-08 \\
2.5 E-08\end{array}$ & $\begin{array}{l}3.1 \mathrm{E}-06 \\
4.7 \mathrm{E}-06 \\
1.2 \mathrm{E}-06\end{array}$ & $\begin{array}{l}2.0 E-04 \\
1.0 E-05 \\
7.8 E-06\end{array}$ & $\begin{array}{l}2.0 E-03 \\
1.0 E-04 \\
7.8 E-05\end{array}$ \\
\hline $\begin{array}{l}{ }^{66} \mathrm{Co} \\
{ }^{69} \mathrm{Ni} \\
{ }^{63} \mathrm{Ni}\end{array}$ & $\begin{array}{l}7.1 E-01 \\
1.5 E-05 \\
8.8 E-07\end{array}$ & $\begin{array}{l}8.1 E-05 \\
3.2 E-08 \\
8.5 E-06\end{array}$ & $\begin{array}{l}4.2 E-04 \\
7.7 E-07 \\
2.2 E-04\end{array}$ & $\begin{array}{l}7.1 E-01 \\
1.6 E-05 \\
2.4 E-04\end{array}$ & $\begin{array}{l}7.1 E+00 \\
1.6 E-04 \\
2.4 E-03\end{array}$ \\
\hline $\begin{array}{l}{ }_{93 m}^{9 m b} \\
{ }_{93} \mathrm{Mo} \\
{ }^{99} \mathrm{TC}\end{array}$ & $\begin{array}{l}1.5 E-08 \\
1.5 E-08 \\
4.8 E-11\end{array}$ & $\begin{array}{l}3.0 E-09 \\
1.9 E-11 \\
1.9 E-11\end{array}$ & $\begin{array}{l}5.0 E-09 \\
2.9 E-09 \\
6.6 E-10\end{array}$ & $\begin{array}{l}2.4 E-08 \\
1.8 E-08 \\
7.5 E-10\end{array}$ & $\begin{array}{l}2.4 E-07 \\
1.8 E-07 \\
7.5 E-09\end{array}$ \\
\hline $\begin{array}{l}{ }^{151} \mathrm{Sm} \\
152 \mathrm{Eu} \\
154 \mathrm{Eu} \\
166 \mathrm{~m} \mathrm{Ho}\end{array}$ & $\begin{array}{l}2.6 \mathrm{E}-12 \\
2.2 \mathrm{E}-03 \\
4.5 \mathrm{E}-05 \\
5.1 \mathrm{E}-04\end{array}$ & $\begin{array}{l}3.3 \mathrm{E}-11 \\
5.5 \mathrm{E}-07 \\
1.4 \mathrm{E}-08 \\
3.6 \mathrm{E}-07\end{array}$ & $\begin{array}{l}4.2 E-11 \\
1.7 E-06 \\
5.1 E-08 \\
3.8 E-07\end{array}$ & $\begin{array}{l}7.7 \mathrm{E}-11 \\
2.2 \mathrm{E}-03 \\
4.5 \mathrm{E}-05 \\
5.1 \mathrm{E}-04\end{array}$ & $\begin{array}{l}7.7 \mathrm{E}-10 \\
2.2 \mathrm{E}-02 \\
4.5 \mathrm{E}-04 \\
5.1 \mathrm{E}-03\end{array}$ \\
\hline Total & $\overline{7.1 \mathrm{E}-01}$ & $\overline{9.1 E-05}$ & $\overline{6.5 E-04}$ & $\overline{7.1 E-01}$ & $\overline{7.1 E+00}$ \\
\hline
\end{tabular}

(a) Using the radionuclide mixture shown in Table C.3 with 30 years of radioactive decay and the annual TEDE factors from Table 3.1.

(b) With an assumed contamination level of $100 \mathrm{pCi} / \mathrm{g}(3.7 \mathrm{~Bq} / \mathrm{g})$ of the radionuclide mixture. 
IABLE C.8. Estimated Annual Total Effective Dose Equivalents for the Building Renovation (Volume Activity) Scenario Using the Reference Radiopharmaceutical/Industrial-Source Inventory 5 Years After Shutdown(a)

\begin{tabular}{|c|c|c|c|c|c|}
\hline $\begin{array}{c}\text { Radio- } \\
\text { nuclide(b) }\end{array}$ & $\begin{array}{c}\text { External } \\
\text { (mrem) }\end{array}$ & $\begin{array}{c}\text { Inhalation } \\
\text { (mrem) }\end{array}$ & $\begin{array}{l}\text { Secondary } \\
\text { Ingestion } \\
\text { (mrem) }\end{array}$ & $\begin{array}{c}\text { TEDE } \\
\text { (mrem) }\end{array}$ & $\begin{array}{r}\text { TEDE } \\
(\mu \mathrm{SV}) \\
\end{array}$ \\
\hline $\begin{array}{l}{ }^{3} \mathrm{H} \\
{ }^{14} \mathrm{C} \\
{ }^{22} \mathrm{Na}\end{array}$ & $\begin{array}{l}7.1 \mathrm{E}-11 \\
2.0 \mathrm{E}-06 \\
3.6 \mathrm{E}-01\end{array}$ & $\begin{array}{l}5.7 \mathrm{E}-08 \\
9.1 \mathrm{E}-07 \\
2.2 \mathrm{E}-06\end{array}$ & $\begin{array}{l}3.9 \mathrm{E}-06 \\
9.1 \mathrm{E}-05 \\
3.6 \mathrm{E}-04\end{array}$ & $\begin{array}{l}4.0 \mathrm{E}-06 \\
9.4 \mathrm{E}-05 \\
3.4 \mathrm{E}-01\end{array}$ & $\begin{array}{l}4.0 \mathrm{E}-05 \\
9.4 \mathrm{E}-04 \\
3.4 \mathrm{E}+00\end{array}$ \\
\hline $\begin{array}{l}{ }^{57} \mathrm{Co} \\
{ }^{68} \mathrm{Co} \\
{ }^{134} \mathrm{Cs}\end{array}$ & $\begin{array}{l}3.1 \mathrm{E}-04 \\
8.3 \mathrm{E}-01 \\
1.0 \mathrm{E}-01\end{array}$ & $\begin{array}{l}3.8 \mathrm{E}-08 \\
9.5 \mathrm{E}-05 \\
3.6 \mathrm{E}-06\end{array}$ & $\begin{array}{l}3.5 \mathrm{E}-07 \\
5.0 \mathrm{E}-04 \\
5.9 \mathrm{E}-04\end{array}$ & $\begin{array}{l}3.1 \mathrm{E}-04 \\
8.3 \mathrm{E}-01 \\
1.0 \mathrm{E}-01\end{array}$ & $\begin{array}{l}3.1 \mathrm{E}-03 \\
8.3 \mathrm{E}+00 \\
1.0 \mathrm{E}+00\end{array}$ \\
\hline $\begin{array}{l}{ }^{137} \mathrm{CS}+\mathrm{I}(\mathrm{c}) \\
{ }^{216} \mathrm{~Pb} \\
{ }^{116} \mathrm{~Pb} \\
{ }^{226} \mathrm{~Pa}\end{array}$ & $\begin{array}{l}3.2 \mathrm{E}-01 \\
6.8 \mathrm{E}-04 \\
3.3 \mathrm{E}-06 \\
1.3 \mathrm{E}-03\end{array}$ & $\begin{array}{l}1.1 E-05 \\
1.2 E-02 \\
4.6 E-03 \\
4.4 E-03\end{array}$ & $\begin{array}{l}2.0 E-03 \\
5.1 E-01 \\
1.0 E-01 \\
5.2 E-02\end{array}$ & $\begin{array}{l}3.2 \mathrm{E}-01 \\
5.3 \mathrm{E}-01 \\
1.1 \mathrm{E}-01 \\
5.8 \mathrm{E}-02\end{array}$ & $\begin{array}{l}3.2 E+00 \\
5.3 E+00 \\
1.1 E+00 \\
5.8 E-01\end{array}$ \\
\hline Total & $\overline{1.6 \mathrm{E}+00}$ & $\overline{2.1 \mathrm{E}-02}$ & $\overline{6.6 E-01}$ & $\overline{2.3 E+00}$ & $\overline{2.3 E+01}$ \\
\hline
\end{tabular}

(a) Using the radionuclide mixture shown in Table C.4 with 5 years of radioactive decay and the annual TEDE factors from Table 3.1 .

(b) With an assumed contamination level of $100 \mathrm{pCi} / \mathrm{g}(3.7 \mathrm{~Bq} / \mathrm{g})$ of the radionuclide mixture.

(c) Where +I means "plus implicit daughters in equilibrium" as discussed in Appendix D. 
TABLE C.9. Estimated Annual Total Effective Dose Equivalents for the Building Occupancy (Surface Activity) Scenario Using the Uranium Fuel Fabrication Facility Inventory 10 Years After Shutdown(a)

\begin{tabular}{|c|c|c|c|c|c|}
\hline $\begin{array}{l}\text { Radio- } \\
\text { nuclide(b) }\end{array}$ & $\begin{array}{c}\text { External } \\
\text { (mrem) } \\
\end{array}$ & $\begin{array}{c}\text { Inhalation } \\
\text { (mrem) } \\
\end{array}$ & $\begin{array}{c}\text { Secondary } \\
\text { Ingestion } \\
\text { (mrem) }\end{array}$ & $\begin{array}{l}\text { TEDE } \\
\text { (mrem) }\end{array}$ & $\begin{array}{l}\text { TEDE } \\
(\mu S v)\end{array}$ \\
\hline $\begin{array}{l}{ }^{236} \mathrm{Th} \\
{ }^{231} \mathrm{Th} \\
{ }^{234} \mathrm{Th}+\mathrm{I}(\mathrm{c})\end{array}$ & $\begin{array}{l}8.6 E-07 \\
2.0 E-03 \\
2.2 E-02\end{array}$ & $\begin{array}{l}9.0 E-03 \\
3.7 E-06 \\
1.6 E-03\end{array}$ & $\begin{array}{l}2.9 E-02 \\
6.0 E-04 \\
6.0 E-02\end{array}$ & $\begin{array}{l}3.7 E-02 \\
2.5 E-03 \\
8.3 E-02\end{array}$ & $\begin{array}{l}3.7 E-01 \\
2.5 E-02 \\
8.3 E-01\end{array}$ \\
\hline $\begin{array}{l}{ }_{234}^{234} \mathrm{~Pa} \\
{ }^{23}\end{array}$ & $\begin{array}{l}8.3 E-04 \\
6.5 E-03\end{array}$ & $\begin{array}{l}1.9 E-08 \\
2.9 E+01\end{array}$ & $\begin{array}{l}5.3 E-06 \\
5.7 E-01\end{array}$ & $\begin{array}{l}8.4 E-04 \\
3.0 E+01\end{array}$ & $\begin{array}{l}8.4 E-03 \\
3.0 E+02\end{array}$ \\
\hline${ }^{235} \mathrm{U}$ & $\begin{array}{l}9.7 E-03 \\
1.8 E-03\end{array}$ & $\begin{array}{l}5.5 E-01 \\
2.9 E+00\end{array}$ & $\begin{array}{l}1.2 E-02 \\
6.0 E-02\end{array}$ & $\begin{array}{l}5.8 E-01 \\
3.0 E+00\end{array}$ & $\begin{array}{l}5.8 E+00 \\
3.0 E+01\end{array}$ \\
\hline Total & $\overline{4.3 E-02}$ & $\overline{3.2 E+01}$ & $\overline{7.3 E-01}$ & $\overline{3.4 E+01}$ & $\overline{3.4 E+02}$ \\
\hline
\end{tabular}

(a) Using the radionuclide mixture shown in Table C.1 with 10 years of radioactive decay and the annual TEDE factors from Table 3.2.

(b) With an assumed contamination level of $10,000 \mathrm{dpm} / \mathrm{cm}^{2}(167 \mathrm{~Bq} / 100 \mathrm{~cm} 2)$ of the radionuclide mixture.

(c) Where +I means "plus implicit daughters in equilibrium" as discussed in Appendix D.

IABLE C.10. Estimated Annual Total Effective Dose Equivalents for the Building Occupancy (Surface Activity) Scenario Using the Depleted and Enriched Uranium Mixture(a)

\begin{tabular}{|c|c|c|c|c|c|}
\hline $\begin{array}{l}\text { Radio- } \\
\text { nuclide(b) }\end{array}$ & $\begin{array}{c}\text { External } \\
\text { (mrem) }\end{array}$ & $\begin{array}{c}\text { Inhalation } \\
\text { (mrem) } \\
\end{array}$ & $\begin{array}{c}\text { Secondary } \\
\text { Ingestion } \\
\text { (mrem) }\end{array}$ & $\begin{array}{c}\text { TEDE } \\
\text { (mrem) }\end{array}$ & $\begin{array}{l}\text { TEDE } \\
(\mu S V)\end{array}$ \\
\hline \multicolumn{6}{|l|}{ Depleted } \\
\hline $\begin{array}{l}{ }^{234} U \\
{ }^{235} U\end{array}$ & $\begin{array}{l}5.0 E-04 \\
6.3 E-03 \\
1.4 E-02\end{array}$ & $\begin{array}{l}2.2 E+00 \\
3.6 E-01 \\
2.2 E+01\end{array}$ & $\begin{array}{l}4.4 E-02 \\
7.8 E-03 \\
4.5 E-01\end{array}$ & $\begin{array}{l}2.3 E+00 \\
3.8 E-01 \\
2.2 E+01\end{array}$ & $\begin{array}{l}2.2 E+01 \\
3.8 E+00 \\
2.2 E+02\end{array}$ \\
\hline Total & $\overline{1.8 \mathrm{E}-02}$ & $\overline{2.5 E+01}$ & $\overline{5.0 \mathrm{E}-01}$ & $\overline{2.5 E+01}$ & $2.5 E+02$ \\
\hline
\end{tabular}

Enriched $(4 \%)$

$\begin{array}{cccccc}{ }^{234} \mathrm{U} & 4.5 \mathrm{E}-03 & 2.0 \mathrm{E}+01 & 4.0 \mathrm{E}-01 & 2.1 \mathrm{E}+01 & 2.1 \mathrm{E}+02 \\ { }^{235} \mathrm{U} & 1.2 \mathrm{E}-02 & 6.7 \mathrm{E}-01 & 1.5 \mathrm{E}-02 & 7.0 \mathrm{E}-01 & 7.0 \mathrm{E}+00 \\ { }^{238} \mathrm{U} & 3.0 \mathrm{E}-03 & 4.8 \mathrm{E}+00 & 1.0 \mathrm{E}-01 & 5.0 \mathrm{E}+00 & 5.0 \mathrm{E}+01 \\ \text { Total } & \overline{2.0 E-03} & \overline{2.6 \mathrm{E}+01} & \overline{5.2 E-01} & \overline{2.7 E+01} & \overline{2.7 E+02}\end{array}$

(a) Using the uranium isotope mixture shown in Table C.2 and the annual TEDE factors from Table 3.2.

(b) With an assumed contamination level of $10,000 \mathrm{dpm} / \mathrm{cm}^{2}(167 \mathrm{~Bq} / 100 \mathrm{~cm} 2)$ of the radionuclide mixture. 
TABLE C.11. Estimated Annual Total Effective Dose Equivalents for the Building Occupancy (Surface Activity) Scenario Using the Neutron-Activated Power Reactor Concrete Inventory 30 Years After Shutdown (a)

\begin{tabular}{|c|c|c|c|c|c|}
\hline $\begin{array}{l}\text { Radio- } \\
\text { nuclide(b) }\end{array}$ & $\begin{array}{c}\text { External } \\
\text { (mrem) } \\
\end{array}$ & $\begin{array}{c}\text { Inhalation } \\
\text { (mrem) }\end{array}$ & $\begin{array}{l}\text { Ingest ion } \\
\text { (mrem) }\end{array}$ & $\begin{array}{c}\text { TEDE } \\
\text { (mrem) }\end{array}$ & $\begin{array}{r}\text { TEDE } \\
(\mu S V) \\
\end{array}$ \\
\hline $\begin{array}{l}{ }^{3} \mathrm{H} \\
{ }^{14} \mathrm{C} \\
{ }^{38} \mathrm{Cl}\end{array}$ & $\begin{array}{l}2.1 \mathrm{E}-14 \\
1.3 \mathrm{E}-07 \\
3.1 \mathrm{E}-08\end{array}$ & $\begin{array}{l}7.0 \mathrm{E}-11 \\
4.9 \mathrm{E}-07 \\
4.3 \mathrm{E}-09\end{array}$ & $\begin{array}{l}4.8 \mathrm{E}-09 \\
4.9 \mathrm{E}-05 \\
6.7 \mathrm{E}-07\end{array}$ & $\begin{array}{l}4.8 E-09 \\
4.9 E-05 \\
7.1 E-07\end{array}$ & $\begin{array}{l}4.8 \mathrm{E}-08 \\
4.9 \mathrm{E}-04 \\
7.1 \mathrm{E}-06\end{array}$ \\
\hline $\begin{array}{l}{ }^{48} \mathrm{~K} \\
{ }^{41} \mathrm{Ca} \\
{ }^{55} \mathrm{Fe}\end{array}$ & $\begin{array}{l}1.9 E-04 \\
5.1 E-06 \\
6.2 E-05\end{array}$ & $\begin{array}{l}7.2 \mathrm{E}-07 \\
1.9 \mathrm{E}-06 \\
9.8 \mathrm{E}-07\end{array}$ & $\begin{array}{l}1.2 \mathrm{E}-04 \\
1.9 \mathrm{E}-04 \\
4.9 \mathrm{E}-05\end{array}$ & $\begin{array}{l}3.2 E-04 \\
4.2 E-04 \\
1.1 E-04\end{array}$ & $\begin{array}{l}3.2 E-03 \\
4.2 E-03 \\
1.1 E-03\end{array}$ \\
\hline $\begin{array}{l}{ }^{80} \mathrm{Co} \\
{ }^{59} \mathrm{Ni} \\
{ }^{83} \mathrm{Ni}\end{array}$ & $\begin{array}{l}7.1 \mathrm{E}-01 \\
1.5 \mathrm{E}-04 \\
7.2 \mathrm{E}-06\end{array}$ & $\begin{array}{l}3.2 \mathrm{E}-03 \\
1.3 \mathrm{E}-06 \\
3.4 \mathrm{E}-04\end{array}$ & $\begin{array}{l}1.7 \mathrm{E}-02 \\
3.1 \mathrm{E}-05 \\
8.9 \mathrm{E}-03\end{array}$ & $\begin{array}{l}7.3 \mathrm{E}-01 \\
1.8 \mathrm{E}-04 \\
9.8 \mathrm{E}-03\end{array}$ & $\begin{array}{l}7.3 \mathrm{E}+00 \\
1.8 \mathrm{E}-03 \\
9.8 \mathrm{E}-02\end{array}$ \\
\hline $\begin{array}{l}{ }^{931} \mathrm{Nb} \\
{ }^{93} \mathrm{Mb} \\
{ }^{99} \mathrm{Mc} \\
{ }_{\mathrm{TC}}\end{array}$ & $\begin{array}{l}1.4 \mathrm{E}-07 \\
1.4 \mathrm{E}-07 \\
1.7 \mathrm{E}-10\end{array}$ & $\begin{array}{l}1.2 \mathrm{E}-07 \\
7.8 \mathrm{E}-10 \\
7.9 \mathrm{E}-10\end{array}$ & $\begin{array}{l}2.0 \mathrm{E}-07 \\
1.2 \mathrm{E}-07 \\
2.7 \mathrm{E}-08\end{array}$ & $\begin{array}{l}4.6 \mathrm{E}-07 \\
2.6 \mathrm{E}-07 \\
2.8 \mathrm{E}-08\end{array}$ & $\begin{array}{l}4.6 \mathrm{E}-06 \\
2.6 \mathrm{E}-06 \\
2.8 \mathrm{E}-07\end{array}$ \\
\hline $\begin{array}{c}{ }^{151} \mathrm{Sm} \\
{ }_{152} \mathrm{Eu} \\
{ }_{154} \mathrm{Eu} \\
{ }^{168 \mathrm{~m}_{\mathrm{Ho}}}\end{array}$ & $\begin{array}{l}2.4 E-11 \\
2.3 E-03 \\
4.7 E-05 \\
5.5 E-04\end{array}$ & $\begin{array}{l}1.3 \mathrm{E}-09 \\
2.2 \mathrm{E}-05 \\
5.7 \mathrm{E}-07 \\
1.4 \mathrm{E}-05\end{array}$ & $\begin{array}{l}1.7 \mathrm{E}-09 \\
7.2 \mathrm{E}-05 \\
2.0 \mathrm{E}-06 \\
1.5 \mathrm{E}-05\end{array}$ & $\begin{array}{l}3.1 E-09 \\
2.4 E-03 \\
4.9 E-05 \\
5.8 E-04\end{array}$ & $\begin{array}{l}3.1 \mathrm{E}-08 \\
2.4 \mathrm{E}-02 \\
4.9 \mathrm{E}-04 \\
5.8 \mathrm{E}-03\end{array}$ \\
\hline Total & $\overline{7.1 \mathrm{E}-01}$ & $\overline{3.6 \mathrm{E}-03}$ & $\overline{2.6 \mathrm{E}-02}$ & $\overline{7.4 E-01}$ & $\overline{7.4 E+00}$ \\
\hline
\end{tabular}

(a) Using the radionuclide mixture shown in Table C.3 with 30 years of radioactive decay and the annual TEDE factors from Table 3.2.

(b) With an assumed contamination level of $10,000 \mathrm{dpm} / 100 \mathrm{~cm} 2$ $\left(167 \mathrm{~Bq} / 100 \mathrm{~cm}^{2}\right)$ of the radionuclide mixture. 
TABLE C.12 Estimated Annual Total Effective Dose Equivalents for the Building Occupancy (Surface Activity) Scenario Using the Reference Radiopharmaceutical/Industrial Source Inventory 5 Years After Shutdown(a)

\begin{tabular}{|c|c|c|c|c|c|}
\hline $\begin{array}{l}\text { Radio- } \\
\text { nuclide(b) }\end{array}$ & $\begin{array}{c}\text { External } \\
\text { (mrem) }\end{array}$ & $\begin{array}{c}\text { Inhalation } \\
\text { (mrem) }\end{array}$ & $\begin{array}{l}\text { Secondary } \\
\text { Ingestion } \\
\text { (mrem) }\end{array}$ & $\begin{array}{c}\text { TEDE } \\
\text { (mrem) }\end{array}$ & $\begin{array}{r}\text { TEDE } \\
(\mu S V) \\
\end{array}$ \\
\hline $\begin{array}{l}{ }^{3} \mathrm{H} \\
{ }^{14} \mathrm{C} \\
{ }^{22} \mathrm{Na}\end{array}$ & $\begin{array}{l}6.7 \mathrm{E}-10 \\
9.6 \mathrm{E}-06 \\
3.7 \mathrm{E}-01\end{array}$ & $\begin{array}{l}2.2 E-06 \\
3.6 E-05 \\
8.8 E-05\end{array}$ & $\begin{array}{l}1.5 \mathrm{E}-04 \\
3.6 \mathrm{E}-03 \\
1.5 \mathrm{E}-02\end{array}$ & $\begin{array}{l}1.5 E-04 \\
3.6 E-03 \\
3.9 E-01\end{array}$ & $\begin{array}{l}1.5 E-03 \\
3.6 E-02 \\
3.9 E+00\end{array}$ \\
\hline $\begin{array}{l}{ }^{57} \mathrm{Co} \\
{ }^{88} \mathrm{Co} \\
{ }^{134} \mathrm{Cs}\end{array}$ & $\begin{array}{l}4.3 E-04 \\
8.4 \mathrm{E}-01 \\
1.1 \mathrm{E}-01\end{array}$ & $\begin{array}{l}1.6 \mathrm{E}-06 \\
3.8 \mathrm{E}-03 \\
1.4 \mathrm{E}-04\end{array}$ & $\begin{array}{l}1.4 \mathrm{E}-05 \\
2.0 \mathrm{E}-02 \\
2.3 \mathrm{E}-02\end{array}$ & $\begin{array}{l}4.5 E-04 \\
8.6 E-01 \\
1.3 E-01\end{array}$ & $\begin{array}{l}4.5 \mathrm{E}-03 \\
8.6 \mathrm{E}+00 \\
1.3 \mathrm{E}+00\end{array}$ \\
\hline $\begin{array}{l}{ }^{137} \mathrm{Cs}+\mathrm{I}(\mathrm{c}) \\
{ }^{216} \mathrm{~Pb} \\
216 \mathrm{Po} \\
{ }^{228} \mathrm{Ra}\end{array}$ & $\begin{array}{l}3.4 E-01 \\
3.6 E-03 \\
3.4 E-06 \\
1.9 E-03\end{array}$ & $\begin{array}{l}4.6 \mathrm{E}-04 \\
4.9 \mathrm{E}-01 \\
1.9 \mathrm{E}-01 \\
1.8 \mathrm{E}-01\end{array}$ & $\begin{array}{l}8.0 E-02 \\
2.1 E+01 \\
4.2 E+00 \\
2.1 E+00\end{array}$ & $\begin{array}{l}4.2 E-01 \\
2.1 E+01 \\
4.4 E+00 \\
2.3 E+00\end{array}$ & $\begin{array}{l}4.2 E+00 \\
2.1 E+02 \\
4.4 E+01 \\
2.3 E+01\end{array}$ \\
\hline Total & $\overline{1.7 E+00}$ & $\overline{8.6 \mathrm{E}-01}$ & $\overline{2.7 E+01}$ & $\overline{2.9 E+01}$ & $\overline{2.9 E+02}$ \\
\hline
\end{tabular}

(a) Using the radionuclide mixture shown in Table C.4 with 5 years of radioactive decay and the annual TEDE factors from Table 3.2.

(b) With an assumed contamination level of $10,000 \mathrm{dpm} / 100 \mathrm{~cm} 2$ (167 Bq/100 $\mathrm{cm}^{2}$ ) of the radionuclide mixture.

(c) Where +I means "plus implicit daughters in equilibrium" as discussed in Appendix D. 


\section{C.4 EXAMPLE DOSE CALCULATIONS FOR THE RESIDENTIAL USE (SURFACE SOIL) SCENARIO}

Example calculations that consider the reference mixtures and assumed levels of radionuclides discussed in Section C.I for the residential use (surface soil) activity scenario follow. The results are presented in Tables C.13 through C.16 in terms of the estimated dose by exposure pathway and the annual TEDE to an individual (in mrem and $\mu \mathrm{Sv}$ ). The initial contamination level for this scenario was assumed to be $100 \mathrm{pCi} / \mathrm{g}$ in soil for each mixture.

The example calculations for the uranium fuel fabrication facility are performed for the inventory shown in Table C.1 for 10 years after plant shutdown. The resulting estimated annual TEDEs for the surface soil activity scenario for the uranium fuel fabrication facility are shown in Table C.13. The annual TEDE from 1 year of residential use of surface soil is $6.1 \mathrm{mrem}$ $(61 \mu \mathrm{Sv})$. The dominant contributor to the dose is $234 \mathrm{U}$ through the inhalation pathway.

For the depleted and enriched uranium mixtures shown in Table C.2, the resulting estimated annual TEDEs for the residential use scenario are shown in Table C.14. The annual TEDE from depleted uranium is 6.4 mrem (64 $\mu \mathrm{Sv})$. The dominant exposure pathway for depleted uranium is inhalation and the dominant contributor to dose is $238 \mathrm{U}$. For enriched uranium, the annual TEDE is $6.7 \mathrm{mrem}(67 \mu \mathrm{SV})$, dominated by inhalation of $234 \mathrm{U}$.

For the neutron-activated concrete mixture at the light-water power reactor, the calculations were performed for the mixture shown in Table C.2 decayed to 30 years after plant shutdown. The results are shown in Table C.15. The estimated annual TEDE for this mixture is $66 \mathrm{mrem}(660 \mu \mathrm{Sv}$, dominated by ${ }^{60} \mathrm{Co}$ and the external exposure pathway.

Example annual TEDEs for the radiopharmaceutical/industrial source mixture shown in Table C.3 with 5 years of radioactive decay for the surface soil activity scenario are shown in Table C.16. The estimated annual TEDE for this mixture and exposure scenario is $180 \mathrm{mrem}(1800 \mu \mathrm{Sv})$. The dominant radionuclide contributors to the dose in the mixture are $60 \mathrm{Co},{ }^{137} \mathrm{Cs}+\mathrm{I}$, and $22 \mathrm{Na}$. The dominant exposure pathway is external exposure. 
TABLE C.13. Estimated Annual Total Effective Dose Equivalents for the Residential Use (Surface Soil) Scenario Using the Uranium Fuel Fabrication Facility Inventory 10 Years After Shutdown(a)

\begin{tabular}{|c|c|c|c|c|c|}
\hline $\begin{array}{c}\text { Radio- } \\
\text { nuclide(b) }\end{array}$ & $\begin{array}{c}\text { External } \\
\text { (mrem) }\end{array}$ & $\begin{array}{c}\text { Inhalation } \\
\text { (mrem) }\end{array}$ & $\begin{array}{c}\text { Food } \\
\text { Ingestion } \\
\text { (mrem) }\end{array}$ & $\begin{array}{c}\text { TEDE } \\
\text { (mrem) }\end{array}$ & $\begin{array}{r}\text { TEDE } \\
(\mu \mathrm{SV}) \\
\end{array}$ \\
\hline $\begin{array}{l}{ }^{238} \mathrm{Th} \\
{ }^{231} \mathrm{Th} \\
{ }^{234} \mathrm{Th}+\mathrm{I}(\mathrm{c})\end{array}$ & $\begin{array}{l}6.4 \mathrm{E}-06 \\
3.2 \mathrm{E}-02 \\
1.5 \mathrm{E}+00\end{array}$ & $\begin{array}{l}1.9 \mathrm{E}-03 \\
8.3 \mathrm{E}-07 \\
3.4 \mathrm{E}-04\end{array}$ & $\begin{array}{l}3.2 E-02 \\
5.8 E-04 \\
6.4 E-02\end{array}$ & $\begin{array}{l}3.4 \mathrm{E}-02 \\
3.4 \mathrm{E}-02 \\
1.5 \mathrm{E}+00\end{array}$ & $\begin{array}{l}3.4 E-01 \\
3.4 E-01 \\
1.5 E+01\end{array}$ \\
\hline $\begin{array}{l}{ }_{234}^{234} \mathrm{~Pa} \\
{ }^{23} \mathrm{U}\end{array}$ & $\begin{array}{l}7.4 \mathrm{E}-02 \\
9.1 \mathrm{E}-03\end{array}$ & $\begin{array}{l}4.3 E-09 \\
2.9 E+00\end{array}$ & $\begin{array}{l}1.1 \mathrm{E}-05 \\
1.7 \mathrm{E}-01\end{array}$ & $\begin{array}{l}7.4 \mathrm{E}-02 \\
3.1 \mathrm{E}+00\end{array}$ & $\begin{array}{l}7.4 E-01 \\
3.1 E+01\end{array}$ \\
\hline${ }^{235} \mathrm{U}$ & $\begin{array}{l}5.3 \mathrm{E}-01 \\
6.5 \mathrm{E}-02\end{array}$ & $\begin{array}{l}1.2 \mathrm{E}-01 \\
6.4 \mathrm{E}-01\end{array}$ & $\begin{array}{l}7.6 \mathrm{E}-03 \\
3.6 \mathrm{E}-02\end{array}$ & $\begin{array}{l}6.6 \mathrm{E}-01 \\
7.3 \mathrm{E}-01\end{array}$ & $\begin{array}{l}6.6 E+00 \\
7.3 E+00\end{array}$ \\
\hline Total & $\overline{2.2 E+00}$ & $\overline{3.7 E+00}$ & $\overline{3.0 E-01}$ & $\overline{6.1 E+00}$ & $\overline{6.1 E+01}$ \\
\hline
\end{tabular}

(a) Using the radionuclide mixture shown in Table C.1 with 10 years of radioactive decay and the annual TEDE factors from Table 3.3.

(b) With an assumed contamination level of $100 \mathrm{pCi} / \mathrm{g}(3.7 \mathrm{~Bq} / \mathrm{g})$ of the radionuclide mixture.

(c) Where $+I$ means "plus implicit daughters in equilibrium" as discussed in Appendix D.

TABLE C.14. Estimated Annual Total Effective Dose Equivalents for Residential Use (Surface Soil) Scenario Using the Depleted and Enriched Uranium Mixture(a)

\begin{tabular}{|c|c|c|c|c|c|}
\hline $\begin{array}{l}\text { Radio- } \\
\text { nuclide(b) }\end{array}$ & $\begin{array}{c}\text { External } \\
\text { (mrem) } \\
\end{array}$ & $\begin{array}{c}\text { Inhalation } \\
\text { (mrem) }\end{array}$ & $\begin{array}{c}\text { Food } \\
\text { Ingestion } \\
\text { (mrem) }\end{array}$ & $\begin{array}{c}\text { TEDE } \\
\text { (mrem) }\end{array}$ & $\begin{array}{r}\text { TEDE } \\
(\mu \mathrm{SV}) \\
\end{array}$ \\
\hline \multicolumn{6}{|l|}{ Depleted } \\
\hline $\begin{array}{l}{ }^{234} \mathrm{U} \\
{ }^{235} \mathrm{U} \\
{ }^{238} \mathrm{U}\end{array}$ & $\begin{array}{l}1.5 \mathrm{E}-03 \\
3.4 \mathrm{E}-01 \\
4.9 \mathrm{E}-01\end{array}$ & $\begin{array}{l}4.8 \mathrm{E}-01 \\
8.0 \mathrm{E}-02 \\
4.8 \mathrm{E}+00\end{array}$ & $\begin{array}{l}2.8 \mathrm{E}-02 \\
5.0 \mathrm{E}-03 \\
2.7 \mathrm{E}-01\end{array}$ & $\begin{array}{l}5.1 E-01 \\
4.4 E-01 \\
5.5 E+00\end{array}$ & $\begin{array}{l}5.1 E+00 \\
4.4 E+00 \\
5.5 E+01\end{array}$ \\
\hline Total & $\overline{8.3 E-01}$ & $\overline{5.4 E+00}$ & $\overline{3.0 \mathrm{E}-01}$ & $\overline{6.4 E+00}$ & $\overline{6.4 E+01}$ \\
\hline
\end{tabular}

Enriched (4\%)

$\begin{array}{rlllll}{ }^{234} \mathrm{U} & 1.4 \mathrm{E}-02 & 4.4 \mathrm{E}+00 & 2.5 \mathrm{E}-01 & 4.7 \mathrm{E}+00 & 4.7 \mathrm{E}+01 \\ { }^{235} \mathrm{U} & 6.4 \mathrm{E}-01 & 1.5 \mathrm{E}-01 & 9.2 \mathrm{E}-03 & 8.1 \mathrm{E}-01 & 8.1 \mathrm{E}+00 \\ { }^{238} \mathrm{U} & 1.1 \mathrm{E}-01 & 1.1 \mathrm{E}+00 & 6.0 \mathrm{E}-02 & 1.2 \mathrm{E}+00 & 1.2 \mathrm{E}+01 \\ { }^{2} \mathrm{U} & \overline{7.6 \mathrm{E}-01} & \overline{5.6 \mathrm{E}+00} & \overline{3.2 \mathrm{E}-01} & \overline{6.7 \mathrm{E}+00} & \overline{6.7 \mathrm{E}+01}\end{array}$

(a) Using the uranium isotope mixture shown in Table C.2 with 10 years of radioactive decay and the annual TEDE factors from Table 3.3.

(b) With an assumed contamination level of $100 \mathrm{pCi} / \mathrm{g}(3.7 \mathrm{~Bq} / \mathrm{g})$ of the radionuclide mixture. 
TABLE C.15. Estimated Annual Total Effective Dose Equivalents for the Residential Use (Surface Soil) Scenario Using the Neutron-Activated Power Reactor Concrete Inventory 30 Years After Shutdown (a)

\begin{tabular}{|c|c|c|c|c|c|}
\hline $\begin{array}{c}\text { Radio- } \\
\text { nuclide(b) }\end{array}$ & $\begin{array}{c}\text { External } \\
\text { (mrem) }\end{array}$ & $\begin{array}{l}\text { Inhalation } \\
\text { (mrem) }\end{array}$ & $\begin{array}{c}\text { Food } \\
\text { Ingest ion } \\
\text { (mrem) }\end{array}$ & $\begin{array}{c}\text { TEDE } \\
\text { (mrem) }\end{array}$ & $\begin{array}{r}\text { TEDE } \\
(\mu S v) \\
\end{array}$ \\
\hline $\begin{array}{l}{ }^{3} \mathrm{H} \\
{ }^{14} \mathrm{C} \\
{ }^{36} \mathrm{Cl}\end{array}$ & $\begin{array}{l}1.5 \mathrm{E}-14 \\
9.5 \mathrm{E}-07 \\
1.0 \mathrm{E}-06\end{array}$ & $\begin{array}{l}1.5 \mathrm{E}-11 \\
1.1 \mathrm{E}-07 \\
9.5 \mathrm{E}-10\end{array}$ & $\begin{array}{l}1.3 \mathrm{E}-11 \\
1.2 \mathrm{E}-06 \\
3.4 \mathrm{E}-03\end{array}$ & $\begin{array}{l}2.9 \mathrm{E}-11 \\
2.2 \mathrm{E}-06 \\
3.4 \mathrm{E}-03\end{array}$ & $\begin{array}{l}2.9 E-10 \\
2.2 E-05 \\
3.4 E-02\end{array}$ \\
\hline $\begin{array}{l}{ }^{46} \mathrm{~K} \\
{ }^{41} \mathrm{Ca} \\
{ }^{55} \mathrm{Fe}\end{array}$ & $\begin{array}{l}1.8 \mathrm{E}-02 \\
3.6 \mathrm{E}-05 \\
4.5 \mathrm{E}-05\end{array}$ & $\begin{array}{l}1.6 \mathrm{E}-07 \\
4.1 \mathrm{E}-07 \\
2.2 \mathrm{E}-07\end{array}$ & $\begin{array}{l}2.9 \mathrm{E}-02 \\
4.5 \mathrm{E}-03 \\
1.4 \mathrm{E}-05\end{array}$ & $\begin{array}{l}4.7 \mathrm{E}-02 \\
4.5 \mathrm{E}-03 \\
5.7 \mathrm{E}-05\end{array}$ & $\begin{array}{l}4.7 \mathrm{E}-01 \\
4.5 \mathrm{E}-02 \\
5.7 \mathrm{E}-04\end{array}$ \\
\hline $\begin{array}{l}{ }^{86} \mathrm{Co} \\
{ }^{59} \mathrm{Ni} \\
{ }^{83} \mathrm{Ni}\end{array}$ & $\begin{array}{l}6.6 \mathrm{E}+01 \\
1.0 \mathrm{E}-04 \\
1.7 \mathrm{E}-05\end{array}$ & $\begin{array}{l}7.1 \mathrm{E}-04 \\
2.8 \mathrm{E}-07 \\
7.4 \mathrm{E}-05\end{array}$ & $\begin{array}{l}5.1 \mathrm{E}-02 \\
3.9 \mathrm{E}-04 \\
1.2 \mathrm{E}-01\end{array}$ & $\begin{array}{l}6.6 \mathrm{E}+01 \\
4.9 \mathrm{E}-04 \\
1.2 \mathrm{E}-01\end{array}$ & $\begin{array}{l}6.6 \mathrm{E}+02 \\
4.9 \mathrm{E}-03 \\
1.2 \mathrm{E}+00\end{array}$ \\
\hline $\begin{array}{l}{ }^{93 n_{\mathrm{Nb}}} \\
{ }^{93} \mathrm{Mo} \\
{ }^{99} \mathrm{TC}\end{array}$ & $\begin{array}{l}1.0 \mathrm{E}-07 \\
1.0 \mathrm{E}-07 \\
2.2 \mathrm{E}-09\end{array}$ & $\begin{array}{l}2.6 \mathrm{E}-08 \\
1.7 \mathrm{E}-10 \\
1.7 \mathrm{E}-10\end{array}$ & $\begin{array}{l}3.6 \mathrm{E}-06 \\
4.1 \mathrm{E}-06 \\
1.3 \mathrm{E}-05\end{array}$ & $\begin{array}{l}3.6 E-06 \\
4.2 E-06 \\
1.3 E-05\end{array}$ & $\begin{array}{l}3.6 \mathrm{E}-05 \\
4.2 \mathrm{E}-05 \\
1.3 \mathrm{E}-04\end{array}$ \\
\hline $\begin{array}{r}{ }^{151} \mathrm{Sm} \\
{ }^{152} \mathrm{Eu} \\
{ }^{154} \mathrm{Eu} \\
1681 \mathrm{Ho}\end{array}$ & $\begin{array}{l}4.2 \mathrm{E}-11 \\
2.0 \mathrm{E}-01 \\
4.2 \mathrm{E}-03 \\
4.8 \mathrm{E}-02\end{array}$ & $\begin{array}{l}2.9 \mathrm{E}-10 \\
4.8 \mathrm{E}-06 \\
1.2 \mathrm{E}-07 \\
3.4 \mathrm{E}-06\end{array}$ & $\begin{array}{l}8.8 \mathrm{E}-10 \\
4.6 \mathrm{E}-05 \\
1.3 \mathrm{E}-06 \\
1.1 \mathrm{E}-05\end{array}$ & $\begin{array}{l}1.2 \mathrm{E}-09 \\
2.0 \mathrm{E}-01 \\
4.2 \mathrm{E}-03 \\
4.8 \mathrm{E}-02\end{array}$ & $\begin{array}{l}1.2 \mathrm{E}-08 \\
2.0 \mathrm{E}+00 \\
4.2 \mathrm{E}-02 \\
4.8 \mathrm{E}-01\end{array}$ \\
\hline Total & $\overline{6.6 \mathrm{E}+01}$ & $\overline{7.8 \mathrm{E}-04}$ & $\overline{2.1 \mathrm{E}-01}$ & $\overline{6.6 E+01}$ & $\overline{6.6 E+02}$ \\
\hline
\end{tabular}

(a) Using the radionuclide mixture shown in Table C. 3 with 30 years of radioactive decay and the annual TEDE factors from Table 3.3.

(b) With an assumed contamination level of $100 \mathrm{pCi} / \mathrm{g}(3.7 \mathrm{~Bq} / \mathrm{g})$ of the radionuclide mixture. 
TABLE C.16. Estimated Annual Total Effective Dose Equivalents for the Residential Use (Surface Soil) Scenario Using the Reference Radiopharmaceutical/Industrial-Source Inventory 5 Years After Shutdown(a)

\begin{tabular}{|c|c|c|c|c|c|}
\hline $\begin{array}{l}\text { Radio- } \\
\text { nuclide (b) }\end{array}$ & $\begin{array}{c}\text { External } \\
\text { (mrem) } \\
\end{array}$ & $\begin{array}{c}\text { Inhalation } \\
\text { (mrem) } \\
\end{array}$ & $\begin{array}{c}\text { Food } \\
\text { Ingestion } \\
\text { (mrem) }\end{array}$ & $\begin{array}{c}\text { TEDE } \\
\text { (mrem) }\end{array}$ & $\begin{array}{l}\text { TEDE } \\
(\mu S v) \\
\end{array}$ \\
\hline $\begin{array}{l}{ }^{3} \mathrm{H} \\
{ }^{2} \mathrm{C} \\
{ }_{\mathrm{Na}}\end{array}$ & $\begin{array}{l}4.8 E-10 \\
7.0 E-05 \\
3.6 E+01\end{array}$ & $\begin{array}{l}5.0 E-07 \\
7.9 E-06 \\
2.0 E-05\end{array}$ & $\begin{array}{l}4.1 E-07 \\
8.6 E-05 \\
7.3 E-01\end{array}$ & $\begin{array}{l}9.1 \mathrm{E}-07 \\
1.6 \mathrm{E}-04 \\
3.7 \mathrm{E}+01\end{array}$ & $\begin{array}{l}9.1 E-06 \\
1.6 E-03 \\
3.7 E+02\end{array}$ \\
\hline $\begin{array}{l}{ }^{57} \mathrm{Co} \\
{ }^{80} \mathrm{Co} \\
{ }^{134} \mathrm{Cs}\end{array}$ & $\begin{array}{l}2.3 E-02 \\
7.7 E+01 \\
9.5 E+00\end{array}$ & $\begin{array}{l}3.3 E-07 \\
8.3 E-04 \\
3.2 E-05\end{array}$ & $\begin{array}{l}4.0 E-05 \\
6.0 E-02 \\
9.0 E-02\end{array}$ & $\begin{array}{l}2.3 E-02 \\
7.7 E+01 \\
9.7 E+00\end{array}$ & $\begin{array}{l}2.3 E-01 \\
7.7 E+02 \\
9.7 E+01\end{array}$ \\
\hline $\begin{array}{l}{ }^{137} \mathrm{Cs}+\mathrm{I}(\mathrm{c}) \\
210 \mathrm{~Pb} \\
210 \mathrm{Po} \\
{ }^{226} \mathrm{Ra}\end{array}$ & $\begin{array}{l}3.2 \mathrm{E}+01 \\
1.9 \mathrm{E}-02 \\
2.9 \mathrm{E}-04 \\
9.9 \mathrm{E}-02\end{array}$ & $\begin{array}{l}1.0 \mathrm{E}-04 \\
1.1 \mathrm{E}-01 \\
4.1 \mathrm{E}-02 \\
3.9 \mathrm{E}-02\end{array}$ & $\begin{array}{l}5.3 E-02 \\
1.9 E+01 \\
1.6 E+00 \\
7.5 E+00\end{array}$ & $\begin{array}{l}3.2 \mathrm{E}+01 \\
1.9 \mathrm{E}+01 \\
1.6 \mathrm{E}+00 \\
7.6 \mathrm{E}+00\end{array}$ & $\begin{array}{l}3.2 \mathrm{E}+02 \\
1.9 \mathrm{E}+02 \\
1.6 \mathrm{E}+01 \\
7.6 \mathrm{E}+01\end{array}$ \\
\hline Total & $\overline{1.6 \mathrm{E}+02}$ & $\overline{1.9 \mathrm{E}-01}$ & $\overline{2 . \overline{9 E+01}}$ & $\overline{1.8 \mathrm{E}+02}$ & $\overline{1.8 E+03}$ \\
\hline
\end{tabular}

(a) Using the radionuclide mixture shown in Table C.4 with 5 years of radioactive decay and the annual TEDE factors from Table 3.3.

(b) With an assumed contamination level of $100 \mathrm{pCi} / \mathrm{g}(3.7 \mathrm{~Bq} / \mathrm{g})$ of the radionuclide mixture.

(c) Where +I means "plus implicit daughters in equilibrium" as discussed in Appendix D. 


\section{C.5 EXAMPLE DOSE CALCULATIONS FOR THE DRINKING WATER SCENARIO}

Example calculations that consider the reference mixtures and assumed levels of radionuclides discussed in Section C.1 for the drinking water scenario follow. The results are presented in Tables $\mathrm{C} .17$ through $\mathrm{C} .20$ in terms of the estimated annual TEDE to an individual (in mrem and $\mu S v$ ) and the maximum organ dose. The initial contamination level for this scenario was assumed to be $100 \mathrm{pCi} / \mathrm{g}$ over a contaminated area of $1000 \mathrm{~m}^{2}$ to a depth of $0.15 \mathrm{~m}$ (the plow layer used for the surface soil scenario). Assuming a soil density of $1.6 \mathrm{~g} / \mathrm{cm}^{3}$, the total inventory for this example is $24 \mathrm{mCi}$ ( 890 $\mathrm{MBq}$ ). As shown in these tables, the estimated dose varies depending on the mixture.

The example calculations for the uranium fuel fabrication facility were performed for the radionuclide mixture shown in Table C.1 for 10 years of decay after plant shutdown. The resulting estimated annual TEDE for the drinking water scenario are shown in Table C.17. The annual TEDE from drinking water is $0.0064 \mathrm{mrem}(0.064 \mu \mathrm{Sv})$. The estimated maximum organ dose for this mixture is $0.061 \mathrm{mrem}(0.61 \mu \mathrm{Sv})$, and the critical organ is bone surface. The dominant radionuclide for the organ dose is $230 \mathrm{Th}$.

For the depleted and enriched uranium mixtures shown in Table C.2, the estimated TEDEs are shown for the drinking water scenario in Table C.18. The estimated annual TEDE for depleted uranium is $0.0035 \mathrm{mrem}(0.035 \mu \mathrm{Sv})$. The critical organ dose is 0.026 mrem $(0.26 \mu \mathrm{Sv})$ and the critical organ is lower large intestine wall. The dominant contributor to dose is $238 \mathrm{U}$. The estimated annual TEDE for enriched uranium is $0.0026 \mathrm{mrem}(0.026 \mu \mathrm{Sv})$. The critical organ dose is $0.02 \mathrm{mrem}(0.2 \mu \mathrm{Sv})$, and the critical organ is lower large intestine. The dominant radionuclide contributor to organ dose is $234 u$.

For the neutron-activated concrete mixture at the light-water power reactor, the calculations were performed for the mixture decayed to 30 years after plant shutdown. The results for the drinking water scenario are shown in Table C.19. The estimated annual TEDE for this mixture is $0.014 \mathrm{mrem}$ $(0.14 \mu \mathrm{Sv})$, dominated by $60 \mathrm{Co}$ and $63 \mathrm{Ni}$. The estimated maximum organ dose is $0.072 \mathrm{mrem}(0.72 \mathrm{\mu Sv})$, and the critical organ is lower large intestine wall. The dominant radionuclide contributor to organ dose is $60 \mathrm{Co}$.

Example annual TEDEs for the radiopharmaceutical/industrial-source mixture with 5 years of radioactive decay for the drinking water scenario are shown in Table C.20. The estimated annual TEDE for this mixture and exposure scenario is 3.8 mrem $(38 \mu \mathrm{Sv})$. The dominant radionuclide contributors to the dose in the mixture are $210 \mathrm{~Pb}$ and $226 \mathrm{Ra}$. The estimated maximum organ dose is 54 mrem $(540 \mu \mathrm{Sv})$, and the critical organ is bone surface. The dominant contributor to organ dose is $210 \mathrm{pb}$. 
TABLE C.17. Estimated Annual Total Effective Dose Equivalents for the Drinking Water Scenario Using the Uranium Fuel Fabrication Facility Inventory 10 Years After Shutdown (a)

\begin{tabular}{|c|c|c|c|c|c|}
\hline \multirow[b]{2}{*}{$\begin{array}{l}\text { Radio- } \\
\text { nuclide(b) }\end{array}$} & \multirow{2}{*}{$\begin{array}{c}\text { TEDE } \\
\text { (mrem) }\end{array}$} & \multirow[b]{2}{*}{$\begin{array}{l}\text { TEDE } \\
(\mu S v)\end{array}$} & \\
\hline & & & (mrem) & $(\mu S v)$ & $\operatorname{Organ}(\mathrm{c})$ \\
\hline $\begin{array}{l}230 \mathrm{Th} \\
231 \mathrm{Th} \\
234 \mathrm{Th}+\mathrm{I}(\mathrm{d})\end{array}$ & $\begin{array}{l}4.6 E-03 \\
3.4 E-09 \\
1.7 E-04\end{array}$ & $\begin{array}{l}4.6 E-02 \\
3.4 E-08 \\
1.7 E-03\end{array}$ & $\begin{array}{l}6.1 \mathrm{E}-02 \\
3.4 \mathrm{E}-08 \\
2.2 \mathrm{E}-03\end{array}$ & $\begin{array}{l}6.1 E-01 \\
3.4 E-07 \\
2.2 E-02\end{array}$ & $\begin{array}{l}\text { Bone Surface } \\
\text { LLI Wall } \\
\text { LLI Wall }\end{array}$ \\
\hline $\begin{array}{l}{ }^{234} \mathrm{~Pa} \\
{ }^{234}\end{array}$ & $\begin{array}{l}2.0 \mathrm{E}-12 \\
1.2 \mathrm{E}-03\end{array}$ & $\begin{array}{l}2.0 \mathrm{E}-11 \\
1.2 \mathrm{E}-02\end{array}$ & $\begin{array}{l}1.1 \mathrm{E}-11 \\
9.3 \mathrm{E}-03\end{array}$ & $\begin{array}{l}1.1 \mathrm{E}-10 \\
9.3 \mathrm{E}-02\end{array}$ & $\begin{array}{l}\text { ULI Wall } \\
\text { LLI Wall }\end{array}$ \\
\hline${ }^{238} \mathrm{U}$ & $\begin{array}{l}6.1 E-05 \\
4.3 E-04\end{array}$ & $\begin{array}{l}6.1 E-04 \\
4.3 E-03\end{array}$ & $\begin{array}{l}4.6 E-04 \\
3.2 E-03\end{array}$ & $\begin{array}{l}4.6 E-03 \\
3.2 E-02\end{array}$ & $\begin{array}{l}\text { LLI Wall } \\
\text { LLI Wall }\end{array}$ \\
\hline Total & $\overline{6.4 E-03}$ & $\overline{6.4 E-02}$ & $\overline{6.1 E-02}$ & $\overline{6.1 E-01}$ & $\overline{\text { Bone Surface }}$ \\
\hline
\end{tabular}

(a) Using the radionuclide mixture shown in Table C.1 with 10 years of radioactive decay and the annual TEDE factors from Table 3.4.

(b) Assuming a contaminated area of $1,000 \mathrm{~m}^{2}$, to a depth of $15 \mathrm{~cm}$, at a level of $100 \mathrm{pCi} / \mathrm{g}$. Assuming a soil density of $1.6 \mathrm{~g} / \mathrm{cm}^{3}$, the total inventory is $24 \mathrm{mCi}(890 \mathrm{MBq})$.

(c) Where LLI is lower large intestine and ULI is upper large intestine.

(d) Where +I means "plus implicit daughters in equilibrium" as discussed in Appendix $D$. 
TABLE C.18. Estimated Annual Total Effective Dose Equivalents for the Drinking Water Scenario Using the Depleted and Enriched Uranium Mixtures (a)

Radionuclide(b)

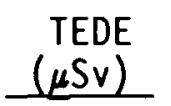

TEDE

(mrem)
Maximum Organ Dose

(mrem) $(\mu S v)$

Depleted

$\begin{array}{llllll}{ }^{234} \mathrm{U} & 2.2 \mathrm{E}-04 & 2.2 \mathrm{E}-03 & 1.5 \mathrm{E}-03 & 1.5 \mathrm{E}-02 & \text { LLI Wall } \\ 235 \mathrm{U} & 4.0 \mathrm{E}-05 & 4.0 \mathrm{E}-04 & 3.0 \mathrm{E}-04 & 3.0 \mathrm{E}-03 & \text { LLI Wall } \\ 238 \mathrm{U} & 3.2 \mathrm{E}-03 & 3.2 \mathrm{E}-02 & 2.4 \mathrm{E}-02 & 2.4 \mathrm{E}-01 & \text { LLI Wall } \\ \text { Total } & \overline{3.5 \mathrm{E}-03} & \overline{3.5 \mathrm{E}-02} & \overline{2.6 \mathrm{E}-02} & \overline{2.6 \mathrm{E}-01} & \text { LLI Wal1 }\end{array}$

Enriched

$\begin{array}{cccccc}{ }^{234} \mathrm{U} & 1.8 \mathrm{E}-03 & 1.8 \mathrm{E}-02 & 1.4 \mathrm{E}-02 & 1.4 \mathrm{E}-01 & \text { LLI Wall } \\ { }^{235} \mathrm{U} & 7.4 \mathrm{E}-05 & 7.4 \mathrm{E}-04 & 5.6 \mathrm{E}-04 & 5.6 \mathrm{E}-03 & \text { LLI Wall } \\ { }^{238} \mathrm{U} & 7.2 \mathrm{E}-04 & 7.2 \mathrm{E}-03 & 5.3 \mathrm{E}-03 & 5.3 \mathrm{E}-02 & \text { LLI Wall } \\ \text { Total } & \overline{2.6 \mathrm{E}-03} & \overline{2.6 \mathrm{E}-02} & \overline{2.0 \mathrm{E}-02} & \overline{2.0 \mathrm{E}-01} & \overline{\text { LLI Wall }}\end{array}$

(a) Using the uranium isotope mixture shown in Table C.2 and the annual TEDE factors from Table 3.4 .

(b) Assuming a contaminated area of $1,000 \mathrm{~m}^{2}$, to a depth of $15 \mathrm{~cm}$, at a level of $100 \mathrm{pCi} / \mathrm{g}$. Assuming a soil density of $1.6 \mathrm{~g} / \mathrm{cm}^{3}$, the total inventory is $24 \mathrm{mCi}(890 \mathrm{MBq})$.

(c) Where LLI is lower large intestine. 
TABLE C.19. Estimated Annual Total Effective Dose Equivalents for the Drinking Water Scenario Using the Neutron-Activated Power Reactor Concrete Inventory 30 Years After Shutdown(a)

\begin{tabular}{|c|c|c|c|c|c|}
\hline \multirow[b]{2}{*}{$\begin{array}{l}\text { Radio- } \\
\text { nuclide(b) } \\
\end{array}$} & \multirow[b]{2}{*}{$\begin{array}{c}\text { TEDE } \\
\text { (mrem) }\end{array}$} & \multirow[b]{2}{*}{$\begin{array}{r}\text { TEDE } \\
(\mu S V) \\
\end{array}$} & \multicolumn{3}{|c|}{ Maximum Organ Dose } \\
\hline & & & (mrem) & $(\mu S v)$ & $\operatorname{Organ}(c)$ \\
\hline${ }^{14}{ }^{36} \mathrm{C}$ & $\begin{array}{l}2.1 E-07 \\
2.2 E-04 \\
2.9 E-06\end{array}$ & $\begin{array}{l}2.1 \mathrm{E}-06 \\
2.2 \mathrm{E}-03 \\
2.9 \mathrm{E}-05\end{array}$ & $\begin{array}{l}2.5 E-07 \\
2.7 E-04 \\
3.5 E-06\end{array}$ & $\begin{array}{l}2.5 E-06 \\
2.7 E-03 \\
3.5 E-05\end{array}$ & $\begin{array}{l}\text { SI Wall } \\
\text { LLI Wall } \\
\text { SI Wall }\end{array}$ \\
\hline $\begin{array}{l}{ }^{40} \mathrm{~K} \\
{ }^{41} \mathrm{Ca} \\
{ }^{55} \mathrm{Fe}\end{array}$ & $\begin{array}{l}7.1 E-07 \\
7.1 E-05 \\
2.3 E-05\end{array}$ & $\begin{array}{l}7.1 E-06 \\
7.1 E-04 \\
2.3 E-04\end{array}$ & $\begin{array}{l}8.4 \mathrm{E}-07 \\
9.0 \mathrm{E}-04 \\
8.4 \mathrm{E}-05\end{array}$ & $\begin{array}{l}8.4 E-06 \\
9.0 E-03 \\
8.4 E-04\end{array}$ & $\begin{array}{l}\text { SI Wall } \\
\text { Bone Surface } \\
\text { Spleen }\end{array}$ \\
\hline $\begin{array}{l}{ }^{68} \mathrm{Co} \\
{ }^{59} \mathrm{Ni} \\
{ }^{83} \mathrm{Ni}\end{array}$ & $\begin{array}{l}9.0 E-03 \\
1.8 E-05 \\
5.1 E-03\end{array}$ & $\begin{array}{l}9.0 \mathrm{E}-02 \\
1.8 \mathrm{E}-04 \\
5.1 \mathrm{E}-02\end{array}$ & $\begin{array}{l}3.9 E-02 \\
9.1 E-05 \\
3.2 E-02\end{array}$ & $\begin{array}{l}3.9 E-01 \\
9.1 E-04 \\
3.2 E-01\end{array}$ & $\begin{array}{l}\text { LLI Wal1 } \\
\text { LLI Wal1 } \\
\text { LLI Wall }\end{array}$ \\
\hline $\begin{array}{l}93=\mathrm{Nb} \\
{ }^{93} \mathrm{Mo} \\
{ }^{99} \mathrm{TC}\end{array}$ & $\begin{array}{l}8.2 E-08 \\
5.1 E-07 \\
1.3 E-07\end{array}$ & $\begin{array}{l}8.2 E-07 \\
5.1 E-06 \\
1.3 E-06\end{array}$ & $\begin{array}{l}9.6 \mathrm{E}-07 \\
2.5 \mathrm{E}-06 \\
1.1 \mathrm{E}-06\end{array}$ & $\begin{array}{l}9.6 E-06 \\
2.5 E-05 \\
1.1 E-05\end{array}$ & $\begin{array}{l}\text { LLI Wal } 1 \\
\text { Liver } \\
\text { Thyroid }\end{array}$ \\
\hline $\begin{array}{l}{ }^{151} \mathrm{Sm} \\
{ }_{152} \mathrm{Eu} \\
{ }_{154} \mathrm{Eu} \\
166 \mathrm{mo}\end{array}$ & $\begin{array}{l}6.3 E-09 \\
2.9 E-07 \\
8.2 E-09 \\
6.0 E-05\end{array}$ & $\begin{array}{l}6.3 E-08 \\
2.9 E-06 \\
8.2 E-08 \\
6.0 E-04\end{array}$ & $\begin{array}{l}6.3 E-08 \\
1.7 E-06 \\
6.1 E-08 \\
3.1 E-04\end{array}$ & $\begin{array}{l}6.3 E-07 \\
1.7 E-05 \\
6.1 E-07 \\
3.1 E-03\end{array}$ & $\begin{array}{l}\text { LLI Wall } \\
\text { LLI Wall } \\
\text { LLI Wall } \\
\text { LLI Wall }\end{array}$ \\
\hline Total & $\overline{1.4 \mathrm{E}-02}$ & $\overline{1.4 E-01}$ & $\overline{7.2 \mathrm{E}-02}$ & $\overline{7.2 E-01}$ & LLI Wall \\
\hline
\end{tabular}

(a) Using the radionuclide mixture shown in Table C. 3 with 30 years of radioactive decay and the annual TEDE factors from Table 3.4.

(b) Assuming a contaminated area of $1000 \mathrm{~m}^{2}$ to a depth of $1.5 \mathrm{~cm}$, at a level of $100 \mathrm{pCi} / \mathrm{g}$. Assuming a soil density of $1.6 \mathrm{~g} / \mathrm{cm}^{3}$, the total inventory is $24 \mathrm{mCi}(890 \mathrm{MBq})$.

(c) Where SI is small intestine and LLI is lower large intestine. 
TABLE C.20. Estimated Annual Total Effective Dose Equivalents for the Drinking Water Scenario Using the Reference Radiopharmaceutical/ Industrial-Source Inventory 5 Years After Shutdown(a)

\begin{tabular}{|c|c|c|c|c|c|}
\hline \multirow[b]{2}{*}{$\begin{array}{l}\text { Radio- } \\
\text { nuclide(b) }\end{array}$} & \multirow[b]{2}{*}{$\begin{array}{c}\text { TEDE } \\
\text { (mrem) }\end{array}$} & \multirow[b]{2}{*}{$\begin{array}{l}\text { TEDE } \\
(\mu \mathrm{SV}) \\
\end{array}$} & \multicolumn{3}{|c|}{ Maximum Organ Dose } \\
\hline & & & (mrem) & $(\mu S v)$ & $\operatorname{Organ}(c)$ \\
\hline${ }^{2}{ }^{3} \mathrm{C} \mathrm{C}$ & $\begin{array}{l}6.7 \mathrm{E}-03 \\
1.6 \mathrm{E}-02 \\
7.6 \mathrm{E}-05\end{array}$ & $\begin{array}{l}6.7 E-02 \\
1.6 E-01 \\
7.6 E-04\end{array}$ & $\begin{array}{l}8.1 E-03 \\
2.0 E-02 \\
1.2 E-04\end{array}$ & $\begin{array}{l}8.1 E-02 \\
2.0 E-01 \\
1.2 E-03\end{array}$ & $\begin{array}{l}\text { SI Wall } \\
\text { LLI Wall } \\
\text { Bone Surface }\end{array}$ \\
\hline $\begin{array}{l}{ }^{87} \mathrm{Co} \\
{ }^{80} \mathrm{Co} \\
{ }^{134} \mathrm{Cs}\end{array}$ & $\begin{array}{l}4.4 \mathrm{E}-06 \\
1.1 \mathrm{E}-02 \\
1.2 \mathrm{E}-04\end{array}$ & $\begin{array}{l}4.4 E-05 \\
1.1 E-01 \\
1.2 E-03\end{array}$ & $\begin{array}{l}2.9 \mathrm{E}-05 \\
4.6 \mathrm{E}-02 \\
1.6 \mathrm{E}-04\end{array}$ & $\begin{array}{l}2.9 E-04 \\
4.6 E-01 \\
1.6 E-03\end{array}$ & $\begin{array}{l}\text { LLI Wall } \\
\text { LLI Wall } \\
\text { SI Wall }\end{array}$ \\
\hline $\begin{array}{l}{ }^{137} \mathrm{Cs}+\mathrm{I}(\mathrm{d}) \\
210 \mathrm{~Pb} \\
210 \mathrm{PO} \\
{ }^{226} \mathrm{Ra}\end{array}$ & $\begin{array}{l}4.9 \mathrm{E}-04 \\
3.2 \mathrm{E}+00 \\
2.0 \mathrm{E}-01 \\
3.4 \mathrm{E}-01\end{array}$ & $\begin{array}{l}4.9 \mathrm{E}-03 \\
3.2 \mathrm{E}+01 \\
2.0 \mathrm{E}+00 \\
3.4 \mathrm{E}+00\end{array}$ & $\begin{array}{l}6.1 E-04 \\
4.6 E+01 \\
1.7 E+00 \\
8.2 E+00\end{array}$ & $\begin{array}{l}6.1 E-03 \\
4.6 E+02 \\
1.7 E+01 \\
8.2 E+01\end{array}$ & $\begin{array}{l}\text { SI Wall } \\
\text { Bone Surface } \\
\text { Spleen } \\
\text { Bone Surface }\end{array}$ \\
\hline Total & $\overline{3.8 \bar{E}+00}$ & $\overline{3.8 E+01}$ & $\overline{5.4 E+01}$ & $\overline{5.4 E+02}$ & $\overline{\text { Bone Surface }}$ \\
\hline
\end{tabular}

(a) Using the radionuclide mixture shown in Table C.4 with 5 years of radioactive decay and the annual TEDE factors from Table 3.4.

(b) Assuming a contaminated area of $1000 \mathrm{~m}^{2}$ to a depth of $15 \mathrm{~cm}$, at a level of $100 \mathrm{pCi} / \mathrm{g}$. Assuming a soil density of $1.6 \mathrm{~g} / \mathrm{cm}^{3}$, the total inventory is $24 \mathrm{mCi}(890 \mathrm{MBq})$.

(c) Where SI is small intestine and LLI is lower large intestine.

(d) Where +I means "plus implicit daughters in equilibrium" as discussed in Appendix D. 
APPENDIX 0

COMPUTERIZED MODEL INPUT/OUTPUT 



\section{APPENDIX D}

\section{COMPUTERIZED MODEL INPUT/OUTPUT}

This appendix discusses radionuclide chain decay as applied in the generation of the dose rate or committed effective dose rate conversion factors (Tables 2.1 through 2.5) and the TEDE rate factors (Tables 3.1 through 3.4) and a description of the computerized approach used in the preparation of those tables. In addition, listings are included of the computer programs, data libraries, and associated data files used in the generation of the dose rate, committed effective dose rate conversion factors, and TEDE rate factors.

\section{D.1 Radioactive Chain Decay}

Radioactive nuclei sometimes decay through a number of unstable daughters before reaching a stable (or very-long-lived) end state. These decay chains are important in that the decay energies of the daughter products contribute to the total dose received from intake of the parent. Tables 2.1 through 2.5 and Tables 3.1 through 3.4 contain listings for both: 1) radionuclides with daughters in equilibrium with their parents and 2) as individual radionuclides without any daughter contributions. The dose rate factors for radionuclides with daughters in equilibrium are useful for cases where the equilibrium conditions are not known. The individual radionuclide entries allow the user to calculate dose rate factors for mixtures where radionuclide daughters may not be in equilibrium with their parents.

The data on radionuclide half-lives, decay chains, and various fractional branching ratios within chains used in GENII are largely taken from the DRALIST data of Kocher (1981b) for consistency with gamma energy data used elsewhere in the GENII code package. Radionuclides of half-life less than 10 minutes are of little importance in environmental calculations and in terms of internal dose, except where they are members of longer decay chains. Daughters of half-life less than 10 minutes are always assumed to be in equilibrium with their parent and are referred to here as implicit daughters. These daughters are given a + I notation in the dose factor tables with a footnote explaining the meaning. An example of a radionuclide with the $+I$ notation is $137 \mathrm{Cs}+\mathrm{I}$. In GENII, the decay energies associated with these short-lived daughters have been assigned to the parent. This assignment includes correction of the SEE factors in the dose calculations. The appropriate branching ratios have been considered.

Daughters with half-lives greater that 10 minutes that may not be in equilibrium with their parents are referred to here as explicit daughters. These daughters are given $a+D$ notation in the dose factor tables with a footnote explaining the meaning. An example of a radionuclide with the $+D$ notation is $90 \mathrm{~S} r+\mathrm{D}$. The GENII software tracks explicit daughters independently throughout all calculations. Radionuclide decay and daughter ingrowth chain organization, branching ratios, and explicit/implicit daughter 
classification used for the generation of Tables 2.1 through 2.5 and Tables 3.1 through 3.4 are presented in Table 0.1 .

For cases where there is a short-lived parent with a long-lived daughter, no attempt is made to combine the parent and daughter. Instead, listings for both parent and daughter are included separately so that the user may define the equilibrium condition. Finally, for decay chains, the listings include parents with $+D$ to include explicit daughters, as determined by the decay chain data. For example, $224 \mathrm{Ra}+\mathrm{D}$ is listed to include the implicit daughters $220 \mathrm{Ra}$ and $216 \mathrm{Po}$, plus the explicit daughters $212 \mathrm{~Pb}$ and $212 \mathrm{Bi}$ with its implicit daughters $21 \mathrm{P}_{\mathrm{O}}$ and $208 \mathrm{Tl}$, and $224 \mathrm{Ra}$ is listed separately to permit separate equilibrium calculations. Table D.1 contains listings of the data concerning the decay schemes, branching ratios, and explicit/implicit daughter classifications used in the decay chain calculations.

The computer programs within the GENII package include a generalized chain decay processor. This processor can give the activity of any member of a decay chain as a function of time from any initializing condition. The chain processor operates on a recursive application of the Bateman equations (Bateman 1910). The Bateman equations provide a simple means of solving the differential equations describing chain decay. The basic form of the equation is

$$
\begin{aligned}
& A_{n}=N_{10}\left[c_{1} e^{-\lambda_{1} t}+c_{2} e^{-\lambda_{2} t}+\ldots+c_{n} e^{-\lambda_{n} t}\right] \\
& c_{i}=\frac{\lambda_{1} \lambda_{2} \ldots \lambda_{i-1}}{n}\left(\lambda_{j}-\lambda_{i}\right) \\
& \substack{j=1 \\
j \neq i}
\end{aligned}
$$

where $A_{n}=$ the activity of the $n$th daughter

$N_{10}=$ the initial activity of the parent at time $t=0$

$\lambda_{i}=$ the decay constant for nuclide $i$ of the chain.

A variant in general decay schemes is branching decay. For this case the Bateman solution is applied in GENII by using partial decay constants (that is, the decay constant times the branching fraction) in the numerator of the constants $C_{j}$. If the decay chain branches and subsequently the two branches are rejoined (as in the natural radioactive series), the two branches are treated by this method as separate chains. The production of a common member beyond the branch point is the sum of the two paths.

An option can be activated in GENII where initial concentrations of daughters are brought to equilibrium with the parent identified on input. This feature was used to calculate dose rate factors (Tables 2.1 through 2.5) for radionuclides where daughters were assumed to be in equilibrium with their parents ( $+D$ designation in the tables). To calculate dose rate factors in GENII without contribution from explicit daughters, a special version of 
the radionuclide decay library was prepared where radionuclides were treated without progeny.

\section{D.2 Computerized Model Input/Output}

This section contains listings of the computer programs and data files used to produce the dose rate or committed effective dose rate conversion factors (Tables 2.1 through 2.5) and the TEDE rate factors (Tables 3.1 through 3.4). These computer programs are essentially report generators operating on the output from the GENII computer program to produce the tables in a usable form. The tables in Section 2.0 contain dose rate or committed effective dose rate conversion factors, by exposure pathway, for exposure to residual radioactive contamination. The tables in Section 3.0 contain TEDE rate factors for unit concentrations of radionuclides in building volume and surface materials, and in soil for the four identified radiation exposure scenarios.

The FORTRAN-language computer program PHASE8, listed in Table 0.2 was developed and used to generate the basic values shown in tables in Sections 2 and 3, complete with word processing commands. PHASE8 processed five input files: RMDLIBDA.SHT, EXTERNAL.DF, EXTDAU.DF, INTERNAL.DF, and INTNODAU.DF. The latter four files were created by programs in the GENII Software System (Versions $1.425,1.429$, and 1.430).

The file RMDLIBDA.SHT, shown as Table 0.3 , contains the control list of radionuclides to be included in the tables. This file is shown in its computer-readable format and also contains the radionuclide half-life in days, the radionuclide decay chain equilibrium multiplier, the default solubility classification for inhalation, and the partitioning coefficient for the leach-rate calculations included for the drinking water scenario.

The file EXTERNAL.DF, shown as Table 0.4, contains external dose factors for radionuclides considered individually. EXTERNAL. DF was created from output files of the program EXTDF of the GENII software system. This file is shown in computer-readable format. It contains dose factors for three configurations: 1) an infinite disk surface source of concrete; 2) a 1-cmthick, 5-m-diameter cylinder source of concrete; and 3) a 15-cm-thick infinite slab source of soil. Units are rem/h per $\mathrm{Ci} / \mathrm{cm}^{2}$ or $\mathrm{cm}^{3}$, depending on whether a surface or volume source is considered. The file EXTDAU.DF shown as Table $D .5$ contains external dose factors where daughters are in equilibrium with their parents (used for radionuclides with the $+D$ designation in Tables 2.1-2.5 and 3.1-3.4. This file is shown in computerreadable format. It contains dose factors for the three configurations considered in file EXTERNAL.DF as discussed above. For the surface source configuration, units are rem/h per $\mathrm{pCi}-\mathrm{m} / \mathrm{e}$ ffective $\mathrm{m}^{2}$. For the volume and

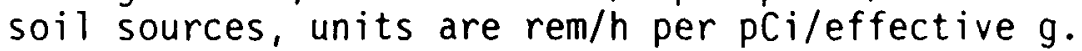

The files INTERNAL.DF, shown as Table 0.6, and INTNODAU.DF, shown as Table 0.7 , consist of inhalation committed effective dose rate equivalents, secondary ingestion committed effective dose rate equivalents, ingestion committed effective dose rate equivalents (air contribution and soil 
contribution from ingestion of food products), and drinking water maximum organ dose rate factors that were created from GENII DOSEQA.OUT files. The organ receiving the highest dose from the drinking water pathway is also identified. Tables $D .6$ and $D .7$ are shown in computer-readable format. The units considered are as follows: for inhalation, rem/h $\mathrm{per} \mathrm{pCi} / \mathrm{m}^{3}$; for secondary ingestion, rem per $\mathrm{pCi}$ ingested; for the air contribution to the ingestion pathway, rem per $\mathrm{pCi} / \mathrm{m}^{3}$ of air; for the soil contribution to the ingestion pathway, rem per $\mathrm{pCi} / \mathrm{g}$ of soil; and for drinking water ingestion, rem per $\mathrm{pC} i$. INTERNAL.DF (Table D.6) contains dose factors where daughters are in equilibrium with their parents (used for radionuclides with the $+D$ designation in Tables 2.1-2.5 and 3.1-3.4). INTNODAU.DF (Table D.7) contains dose factors for radionuclides considered individually.

The EXTDF output files used to generate EXTERNAL.DF for the three configurations are shown as Tables $0.8-0.10$. Table D.8 shows the results for an infinite disk surface source of concrete. Table D.9 shows the results for a 1-cm-thick, 5-m-diameter cylinder source of concrete. Table 0.10 shows the results for a 15-cm-thick infinite slab source of soil. The files are shown in computer-generated format. The file EXTERNAL.DF (Table D.4) was generated from the output files shown in Tables D.8 through D. 10 .

The file EXTDAU.DF (Table D.5) was constructed by extracting appropriate columns from three DOSEQA.OUT files generated from multiple runs of the GENII software. The GENII DOSEQA.OUT files allow tabulation and quality-control checking of multiple GENII runs. The organization of the DOSEQA.OUT file is described in Section 2.2.17, Volume 2 of the GENII documentation. Three BASIC-language programs were written to control GENII execution, each producing a separate DOSEQA.OUT file. EXTDAU.DF was then constructed manually from the DOSEQA.OUT files. Table D.11 lists the BASIC-language GENII-control program GENEXT1.BAS used to generate dose factors for an infinite disk source of concrete (first column of file EXTDAU.DF). The GENII-control program used to generate dose factors for the remaining two configurations are not shown because of their similarity to GENEXT1.BAS; only filenames differ. The GENII input file templates, EXTOPT.TPL and EXTPAR.TPL are shown in computer-readable format in Tables 0.12 and $D .13$, respectively. Unique versions of the GENII external dose factor file (GRDF.DAT) were used for each of the three configurations. These files were created by running EXTDF with the EXTDF input files shown in Tables $0.8-0.10$, modified so that the output is in GENII-compatible units. The output from EXTDF was substituted for the surface soil exposure dose rate factor column in three versions of the GRDF.DAT file used in the three GENII runs used to generate EXTDAU.DF.

The files INTERNAL.DF (Table D.6) and INTNODAU.DF were constructed by extracting appropriate columns from eight DOSEQA.OUT files generated from multiple runs of the GENII software. The GENII DOSEQA. OUT files allow tabulation and quality-control checking of multiple GENII runs. The organization of the DOSEQA.OUT is described in Section 2.2.17, Volume 2 of the GENII documentation. Eight BASIC-language control programs were written to control GENII executions, each producing a separate DOSEQA.OUT file. INTERNAL.DF (Table 0.6) and INTNODAU.DF (Table 0.7) were then constructed manually from the DOSEQA.OUT files. Four control programs were used to 
control pathway calculations factors for each of the internal dose factor files. Table D.14 summarizes program and file usage for generation of INTERNAL.DF (Table D.6) and INTNODAU.DF (Table D.7). Tables D.15 through D.18 list the BASIC-1 anguage GENII-control programs GENALL.BAS, GENALL2.BAS, GENALL3.BAS, and GENALL4.BAS, respectively. The programs GENALLN.BAS, GENALL2N.BAS, GENALL3N.BAS, and GENALL4N.BAS are not shown because of the ir similarity to the files shown in Table $D .15$ through $D .18$; only filenames differ. The GENII input file templates SOILOPT.TPL, SOILPAR.TPL, DRINKOPT.TPL, and DRINKPAR.TPL are shown in computer-readable format as Tables D.19 through D.22, respectively.

To create EXTDAU.DF (Table D.5) and INTERNAL.DF (Table D.6), the GENII radionuclide master library (RMDLIB.DAT), shown as Table 0.23 in computerreadable format, was used to control radionuclide chain decay. To generate INTNODAU.DF (Table 0.7), a special version of the radionuclide master library where all radionuclides are processed without daughters, shown as Table 0.24, was used. The GENII executions used a modified version of the food transfer coefficient library to track the data base for 10 CFR 61 and Regulatory Guide 1.109 (NRC 1977) values. Table D.25 contains the version of FTRANS.DAT (food transfer coefficient data), in computer-readable format, used by GENII to generate the food product ingestion TEDE factors from the soil contribution to ingestion. For the air contribution to ingestion, calculations were made using a version of this file with the soil-to-plant uptake factors set to 0.0 . Values used from this file are identified in Table B.4 of Appendix B. 
TABLE D.1.
Radionuclide Decay and Daughter Ingrowth Chain
Organization in the Radionuclide Master Library

\begin{tabular}{|c|c|c|c|c|c|c|c|}
\hline \multirow{2}{*}{$\begin{array}{l}\text { Chain } \\
\text { Position } \\
\text { Index (c) } \\
\begin{array}{c}1 \\
2\end{array}\end{array}$} & \multirow{2}{*}{$\begin{array}{l}\text { Radio- } \\
\text { nuclide(d) } \\
\text { ZN69M } \\
\text { ZN69 }\end{array}$} & & $\begin{array}{l}\text { xplicit } \\
\text { rimary } \\
\text { ecursor } \\
\text { anching } \\
\text { atio(e) }\end{array}$ & $\begin{array}{c}u g h \\
\mathrm{~A} \\
\mathrm{Pr} \\
\mathrm{Br} \\
\mathrm{F}\end{array}$ & $\begin{array}{l}\text { ters }(b) \\
\text { ternate } \\
\text { ecursor } \\
\text { anching } \\
\text { atio(f) }\end{array}$ & Implicit & Daughters (g) \\
\hline & & 1 & 1.0 & & & & \\
\hline $\begin{array}{l}1 \\
2\end{array}$ & $\begin{array}{l}\text { RB89 } \\
\text { SR89 }\end{array}$ & 1 & 1.0 & & & & \\
\hline$\frac{1}{2}$ & $\begin{array}{l}\text { SR90 } \\
Y 90\end{array}$ & 1 & 1.0 & & & & \\
\hline $\begin{array}{l}1 \\
2 \\
3\end{array}$ & $\begin{array}{l}\text { SR91 } \\
\text { Y } 91 \mathrm{M} \\
\text { Y } 91\end{array}$ & $\begin{array}{l}1 \\
2\end{array}$ & $\begin{array}{l}0.5740 \\
1.0\end{array}$ & 1 & 0.4260 & & \\
\hline $\begin{array}{l}1 \\
2\end{array}$ & $\begin{array}{l}\text { SR92 } \\
\text { Y } 92\end{array}$ & 1 & 1.0 & & & & \\
\hline $\begin{array}{l}1 \\
2 \\
3\end{array}$ & $\begin{array}{l}\text { M093 } \\
\text { ZR93 } \\
\text { NB93M }\end{array}$ & 1 & 1.0 & 2 & 1.0 & & \\
\hline $\begin{array}{l}1 \\
2 \\
3\end{array}$ & $\begin{array}{l}\text { ZR95 } \\
\text { NB95M } \\
\text { NB95 }\end{array}$ & $\begin{array}{l}1 \\
2\end{array}$ & $\begin{array}{l}0.0080 \\
0.9450\end{array}$ & 1 & 0.9922 & & \\
\hline $\begin{array}{l}1 \\
2 \\
3\end{array}$ & $\begin{array}{l}\text { ZR97 } \\
\text { NB97M } \\
\text { NB97 }\end{array}$ & $\begin{array}{l}1 \\
2\end{array}$ & $\begin{array}{l}0.9470 \\
1.0\end{array}$ & 1 & 0.0530 & & \\
\hline $\begin{array}{l}1 \\
2\end{array}$ & $\begin{array}{l}\text { M099 } \\
\text { TC99M }\end{array}$ & 1 & 0.8860 & & & & \\
\hline $\begin{array}{l}1 \\
2 \\
3\end{array}$ & $\begin{array}{l}\text { RU103 } \\
\text { PD103 } \\
\text { RH103M }\end{array}$ & 1 & 0.9974 & 2 & 0.9997 & & \\
\hline $\begin{array}{l}1 \\
2\end{array}$ & $\begin{array}{l}\text { RU105 } \\
\text { RH105 }\end{array}$ & 1 & 1.0 & & & RH105M & 1.000 \\
\hline 1 & RU106 & & & & & RH106 & 1.000 \\
\hline 1 & PD109 & & & & & AG109M & 1.000 \\
\hline 1 & AG110M & & & & & AG110 & 0.985 \\
\hline
\end{tabular}


TABLE D.1. Radionuclide Decay and Daughter Ingrpwth Chain Organization in the Radionuclide Master Library (contd)

Chain $\frac{\text { Explicit Daughters }(b)}{\text { Primary Alternate }}$ Position Radio- Branching Branching Index(c) nuclide(d) Ratio(e) Ratio(f) Implicit Daughters (g)

\begin{tabular}{|c|c|c|c|c|c|}
\hline $\begin{array}{l}1 \\
2\end{array}$ & $\begin{array}{l}\text { CD115M } \\
\text { CD115 } \\
\text { IN115M }\end{array}$ & 2 & 1.0 & & \\
\hline & IN114M & & & & \\
\hline & $\begin{array}{l}\text { SN113 } \\
\text { IN113M }\end{array}$ & 1 & 1.0 & & \\
\hline 2 & $\begin{array}{l}\text { SN125 } \\
\text { SB125 } \\
\text { TE } 125 M\end{array}$ & $\begin{array}{l}1 \\
2\end{array}$ & $\begin{array}{l}1.0 \\
0.2310\end{array}$ & & \\
\hline 1 & $\begin{array}{l}\text { SN126 } \\
\text { SB126M } \\
\text { SB126 }\end{array}$ & $\begin{array}{l}1 \\
2\end{array}$ & $\begin{array}{l}1.0 \\
0.1400\end{array}$ & & \\
\hline 1 & $\begin{array}{l}\text { SB127 } \\
\text { TE127M } \\
\text { TE127 }\end{array}$ & $\begin{array}{l}1 \\
2\end{array}$ & $\begin{array}{l}0.1690 \\
0.9820\end{array}$ & 1 & 0.8310 \\
\hline & $\begin{array}{l}\text { TE129M } \\
\text { TE129 }\end{array}$ & 1 & 0.6290 & & \\
\hline & $\begin{array}{l}\text { TE131M } \\
\text { TE131 } \\
\text { I } 131\end{array}$ & $\begin{array}{l}1 \\
2\end{array}$ & $\begin{array}{l}0.2220 \\
1.0\end{array}$ & 1 & 0.7780 \\
\hline & $\begin{array}{r}\text { TE132 } \\
\text { I } 132\end{array}$ & 1 & 1.0 & & \\
\hline & $\begin{array}{l}\text { TE133M } \\
\text { TE133 } \\
\text { I } 133\end{array}$ & $\begin{array}{l}1 \\
2\end{array}$ & $\begin{array}{l}0.1300 \\
1.0\end{array}$ & 1 & 0.8700 \\
\hline & $\begin{array}{r}\text { TE } 134 \\
\text { I } 134\end{array}$ & 1 & 1.0 & & \\
\hline
\end{tabular}


TABLE D.1. Radionuclide Decay and Daughter Ingrgwth Chain Organization in the Radionuclide Master Library ${ }^{2}$ (contd)

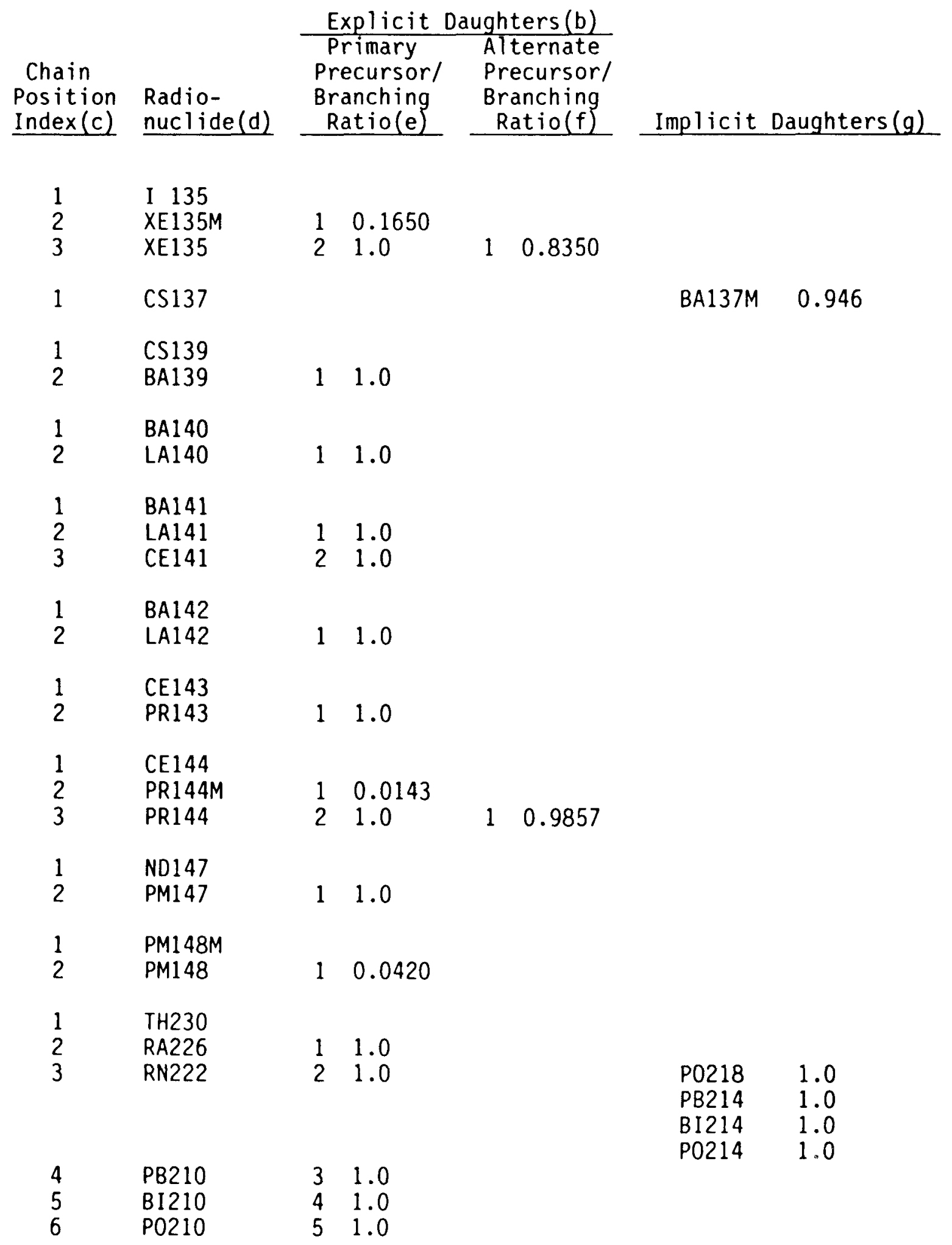


TABLE D.1. Radionuclide Decay and Daughter Ingrpwth Chain Organization in the Radionuclide Master Library (contd)

Chain

Position Radio-

Index(c) nuclide(d)

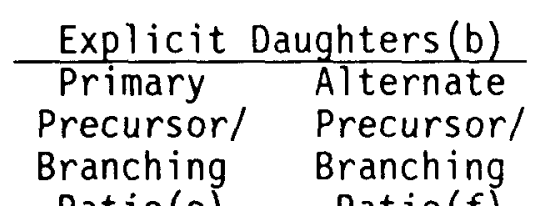

Ratio(e) Ratio(f)
Implicit Daughters $(g)$

$\begin{array}{llll}1 & \text { TH232 } & & \\ 2 & \text { RA228 } & 1 & 1.0 \\ 3 & \text { AC228 } & 2 & 1.0 \\ 4 & \text { TH228 } & 3 & 1.0 \\ 5 & \text { RA224 } & 4 & 1.0 \\ & & & \\ 6 & \text { PB212 } & 5 & 1.0 \\ 7 & \text { BI212 } & 6 & 1.0\end{array}$

RN220 $\quad 1.0$

P0216 1.0

P0212 0.6407

TL208 0.3593

$\begin{array}{llllllll}1 & \text { U 235 } & & & & & \\ 2 & \text { TH231 } & 1 & 1.0 & & & & \\ 1 & \text { PA231 } & & & & & \\ 2 & \text { AC227 } & 1 & 1.0 & & & & \\ 3 & \text { TH227 } & 2 & 0.9862 & & & & \\ 4 & \text { FR223 } & 2 & 0.0138 & & & & \\ 5 & \text { RA223 } & 3 & 1.0 & 4 & 1.0 & \text { RN219 } & 1.0 \\ & & & & & & \text { PO215 } & 1.0 \\ & & & & & & \text { PB211 } & 1.0 \\ & & & & & \text { BI211 } & 1.0 \\ & & & & & \text { TL207 } & 1.0\end{array}$

$\begin{array}{ll}1 & \text { NP237 } \\ 2 & \text { PA233 }\end{array}$

11.0

$1 \quad$ U 233

2 TH229

3 RA225

$4 \quad$ AC225

11.0

21.0

31.0

FR221 1.0

AT217 1.0

BI213 1.0

P0213 0.9784

TL209 0.0216

PB209 1.0

$\begin{array}{llll}1 & \text { U } 238 & & \\ 2 & \text { TH234 } & 1 & 1.0 \\ 3 & \text { PA234 } & 2 & 0.0016\end{array}$

PA234M 0.998 
TABLE D.1. Radionuclide Decay and Daughter Ingrgwth Chain Organization in the Radionuclide Master Library (contd)

\begin{tabular}{|c|c|c|c|c|c|c|}
\hline $\begin{array}{l}\text { Chain } \\
\text { Position } \\
\text { Index (c) } \\
\end{array}$ & $\begin{array}{l}\text { Radio- } \\
\text { nuclide(d) }\end{array}$ & & $\begin{array}{l}\text { xplicit } \\
\text { rimary } \\
\text { ecursor/ } \\
\text { anching } \\
\text { atio(e) }\end{array}$ & $\begin{array}{l}\text { ughters }(b) \\
\text { Alternate } \\
\text { Precursor } \\
\text { Branching } \\
\text { Ratio(f) }\end{array}$ & Implicit & Daughters $(g)$ \\
\hline $\begin{array}{l}1 \\
2 \\
3 \\
4\end{array}$ & $\begin{array}{l}\text { AM242M } \\
\text { AM242 } \\
\text { CM242 } \\
\text { PU238 }\end{array}$ & $\begin{array}{l}1 \\
2 \\
3\end{array}$ & $\begin{array}{l}1.0 \\
0.8270 \\
1.0\end{array}$ & & & \\
\hline $\begin{array}{l}1 \\
2\end{array}$ & $\begin{array}{l}\text { NP238 } \\
\text { PU238 }\end{array}$ & 1 & 1.0 & & & \\
\hline $\begin{array}{l}1 \\
2\end{array}$ & $\begin{array}{l}\text { PU244 } \\
\text { U } 240\end{array}$ & 1 & 0.9988 & & NP240M & 1.0 \\
\hline $\begin{array}{l}1 \\
2 \\
3\end{array}$ & $\begin{array}{l}\text { CM245 } \\
\text { PU241 } \\
\text { AM241 }\end{array}$ & $\begin{array}{l}1 \\
2\end{array}$ & $\begin{array}{l}1.0 \\
1.0\end{array}$ & & & \\
\hline $\begin{array}{l}1 \\
2\end{array}$ & $\begin{array}{l}\text { CM247 } \\
\text { PU243 }\end{array}$ & 1 & 1.0 & & & \\
\hline $\begin{array}{l}1 \\
2 \\
3 \\
4\end{array}$ & $\begin{array}{l}\text { CM243 } \\
\text { AM243 } \\
\text { NP239 } \\
\text { PU239 }\end{array}$ & $\begin{array}{l}1 \\
2 \\
2\end{array}$ & $\begin{array}{l}0.0024 \\
1.0 \\
0.9976\end{array}$ & & & \\
\hline
\end{tabular}

(a) The radionuclide master library (RMDLIB.DAT) contains all radiological decay and daughter ingrowth data used by the GENII Software System to calculate internal and external dose factors included in this document. In RMDLIB.DAT radionuclides are organized into chains controlled by integer indices.

(b) Daughters of half-life greater than 10 minutes that may not be in equilibrium with its parent. Explicit daughters are tracked independently throughout the system.

(c) Indicator of relative position in the decay chain (where 1 is highest position).

(d) Radionuclide name as specified in RMDLIB.DAT.

(e) Chain position index of primary precursor in decay chain (as defined in the first column of this table) followed by the branching ratio.

(f) Chain position index of alternate precursor in decay chain (as defined in the first column of this table) followed by the branching ratio. 
TABLE D.1. Radionuclide Decay and Daughter Ingrowth Chain
Organization in the Radionuclide Master Library (a) (contd)

(g) Daughters of half-life less than 10 minutes that are always assumed to be in equilibrium with their parent. The decay energies associated with these short-lived daughters has been assigned to the parent. This assignment includes correction of radioactive decay energies for both internal and external dose calculations. Listed in the table are the implicit daughters and their branching ratios included for the parent listed in the second column of the table. 
TABLE D.2. PHASE8 Computer Program Listing

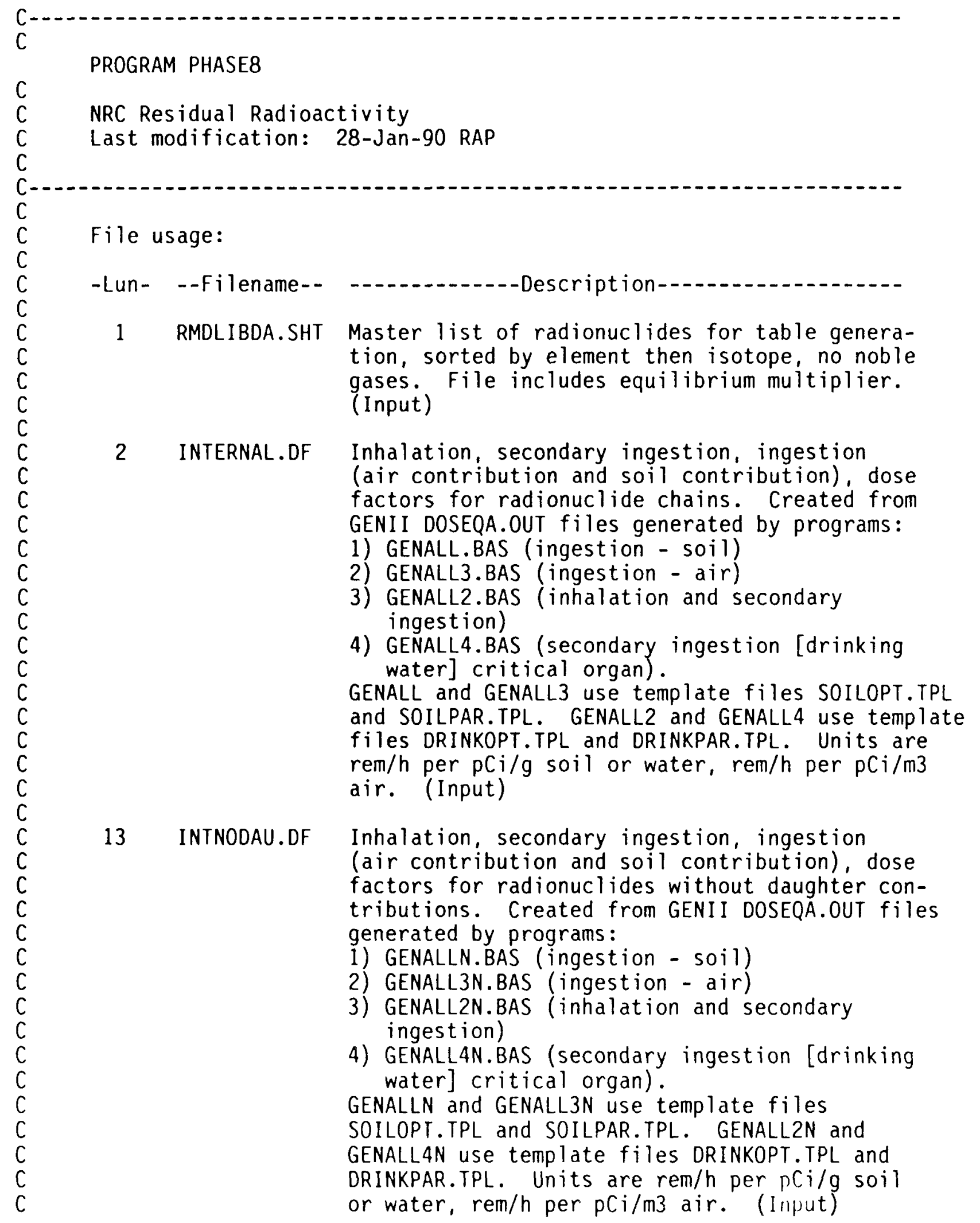


TABLE D.2. PHASE8 Computer Program Listing (contd)

External dose factors for radionuclides without daughters:

1) Infinite disk surface source, concrete

1) $1 \mathrm{~cm}$ thick disk source, $5 \mathrm{~m}$ source, concrete

3) $15 \mathrm{~cm}$ thick infinite slab source, soil. Created from EXTOF runs SURC, VOLC1, and VOLSOL15. Units are $\mathrm{rem} / \mathrm{h}$ per $\mathrm{Ci} / \mathrm{cm} 2$ or $\mathrm{cm} 3$. (Input)

4 TABLE2.1

External dose coversion factors for exposure to residual radioactive materials. (Output)

TABLE2.2

Inhalation committed effective dose conversion factors for exposure to residual radioactive materials. (Output)

TABLE2.3

Secondary ingestion committed effective dose conversion factors for exposure to residual radioactive materials. (Output)

7 TABLE2.5

Agricultural food product ingestion committed effective dose conversion factors for exposure to residual radioactive materials. (Output)

12 TABLE2.4

Maximum organ dose conversion factors for ingestion of drinking water. (Output)

7 TABLE2.5

Agricultural food product ingestion committed effective dose conversion factors for exposure to residual radioactive materials. (Output)

8 TABLE3.1

Total effective dose equivalents for the building volume activity scenario. (Output)

$9 \quad$ TABLE3.2

Total effective dose equivalents for the building surface activity scenario. (Output)

10 TABLE3. 3

Total effective dose equivalent for the surface soil scenario. (Output)

11 TABLE3.4 Drinking water commetted effective dose equivalents for exposure to residual radioactive materials. (Output)

14 TABLE.1

Allowable levels of contamination following decommissioning of nuclear facilities-building volume and surface sources. (Output)

15 TABLE.2

Allowable levels of contamination following 
IABLE D.2. PHASE8 Computer Program Listing (contd)

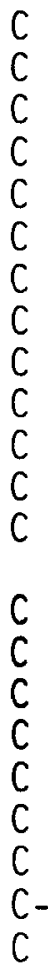

decommissioning of nuclear facilities-surface soil total inventory sources. (Output)

16 EXTDAU.DF External dose factors for radionuclides with daughters:

1) Infinite disk surface source, concrete

1) $1 \mathrm{~cm}$ thick disk source, $5 \mathrm{~m}$ source, concrete

3) $15 \mathrm{~cm}$ thick infinite slab source, soil. GRDF1.DAT, GRDF2.DAT, and GRDF3.DAT created from EXTDF runs EXT1, EXT2, and EXT3. BASIC programs GENEXT1, GENEXT2, and GENEXT3 were then run and this file was then created from the DOSEQA.OUT files. Units are rem/h per $\mathrm{pCi} / \mathrm{m} 2$. (Input)

17 CONTROL.1

18 CONTROL.2

TABLE 3.1 Internal controlling dose list TABLE 3.2 Internal controlling dose list 19 CONTROL.3 TABLE 3.3 Internal controlling dose list

20 PHASE8.QA

External dose factor QA file for verification

REAL REXT (3,300), REXTD (3,300),

$\operatorname{RINH}(300), \operatorname{RING}(2,300), \operatorname{RSING}(300), \operatorname{DWORG}(300)$,

$\operatorname{RINHN}(300), \operatorname{RINGN}(2,300), \operatorname{RSINGN}(300)$,

DWORGN (300)

REAL*8 RLAM, RLAML, REXP, REXP2, CONV1, CONV2

CHARACTER ELTM*2, ELT*2, AWM*6, AW*6, ORGAN*21(300), ORGNO*21(300),

$O R G \star 21, O R G 4 A \star 21, O R G 5 A * 21, O R G 8 A \star 21$, $O R G 4 B \star 21, O R G 5 B \star 21, O R G 8 B \star 21$,

$\operatorname{ELT} 1 * 2(300), A W 1 \star 6(300), D U M \star 5, E L T 2 * 2(300), A W 2 * 6(300)$, $A D V U P \star 1, A D V D W N * 1, N E X T A B * 1$,

LITFNT*6, BIGFNT*6,

RITNAM $* 29(3)$, UNAT $* 29(3)$, THNAT $* 29(3)$,

ELTOLD*2，TMPNAM*29，FOOT*3(6)，DESIG*2(2), CNTRL*10(2)

INTEGER IBETA, IMPDAU

LOGICAL U234，U235，U238，TH232

DATA ELTOLD /' '/

DATA FOOT /'(a)', ' (b) ', ' (c)', ' (d) ', '(e)' , ' (f)' '/

DATA DESIG $/{ }^{\prime}+D^{\prime}, '+I^{\prime} /$

DATA CNTRL /'Inhalation', 'Ingestion '/

$A L O G=0.6931$ 
TABLE D.2. PHASE8 Computer Program Listing (contd)
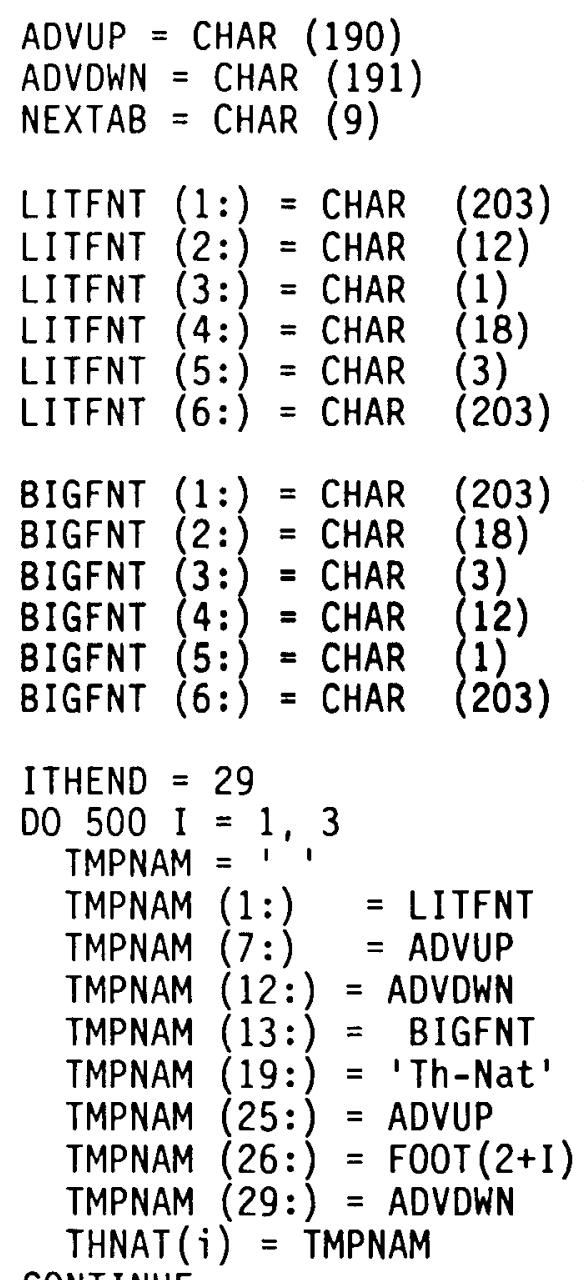

500 CONTINUE

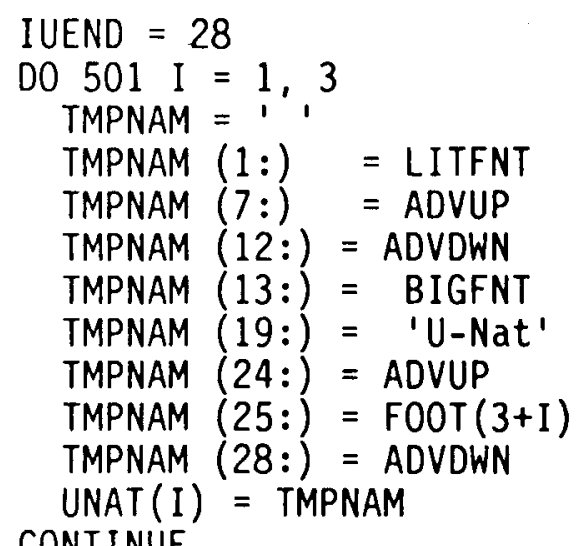

501 CONTINUE

C---- Open and read input files

OPEN (1, FILE ='rmd libda. sht', STATUS=' OLD' $)$

$\operatorname{READ}(1, '(A)$ ') DUM 
IABLE D.2. PHASE8 Computer Program Listing (contd)

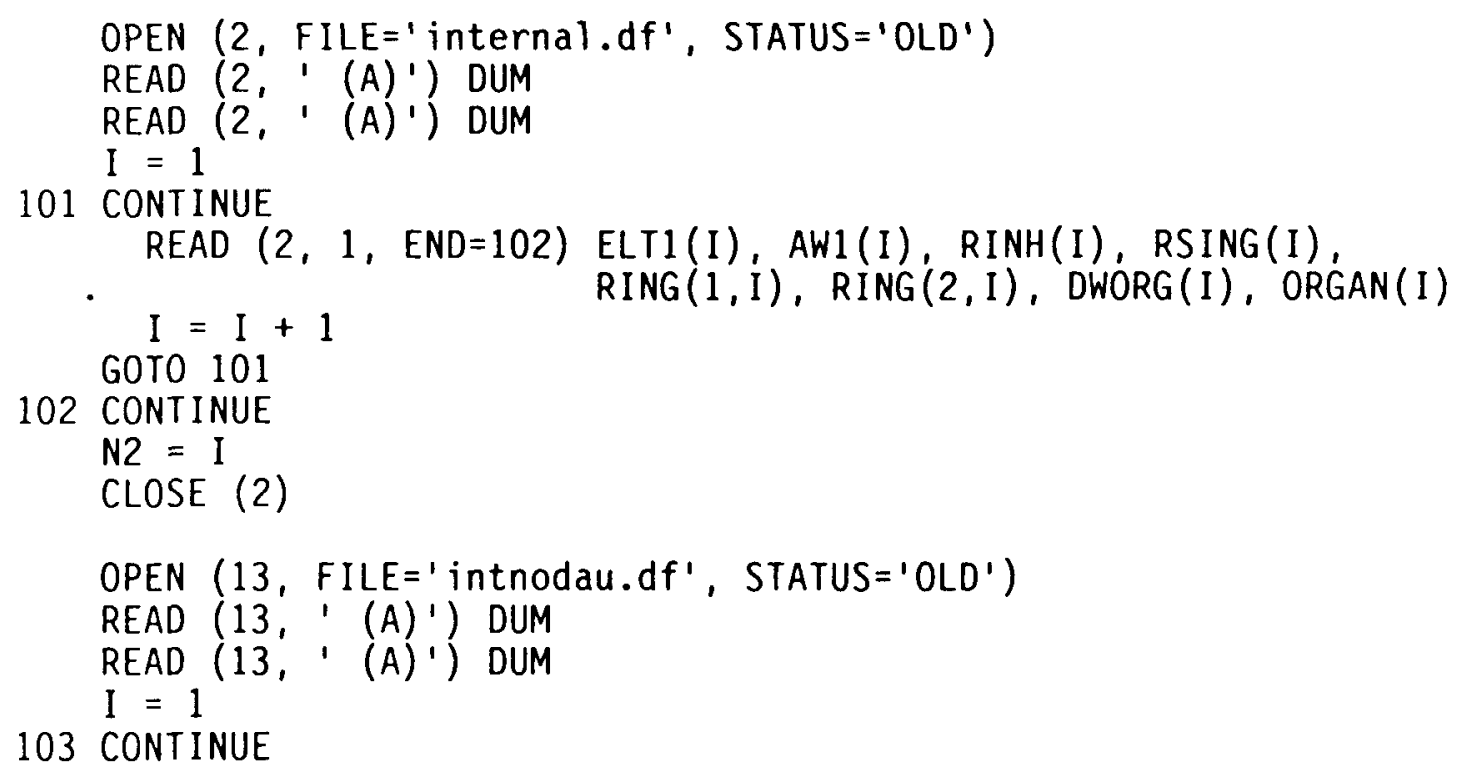


IABLE D.2. PHASE8 Computer Program Listing (contd)

$\operatorname{READ}(16, '$ ' (A)') DUM

$\operatorname{READ}\left(16, '(A)^{\prime}\right)$ DUM

$\mathrm{I}=1$

120 CONTINUE

$\operatorname{READ}(16,1, E N D=121)$ ELT, AW,

$\operatorname{REXTD}(1,1), \operatorname{REXTD}(2, I), \operatorname{REXTD}(3, I)$

C Write $(*, *) ' 1, \operatorname{ElT}, \operatorname{AW}, \operatorname{ReXtD}(1, I), \operatorname{ReXto}(2, I), \operatorname{ReXto}(3, I)$

IF (ELT .NE. ELT1(I) . AND. AW .NE. AW1(I)) THEN

WRITE $(*, *)$ ' Error with EXTDAU.DF order',

ENDIF

ELT, ELT1(I), AW, AW1(I), '\#'

$\mathrm{I}=\mathrm{I}+1$

GOTO 120

121 CONTINUE

CLOSE (16)

C-.-- Open output files and print headings

$\operatorname{OPEN}\left(4, F I L E={ }^{\prime}\right.$ table2.1', STATUS='OLD', ACCESS='APPEND' $)$ WRITE $\left(4,{ }^{\prime}(6 A)^{\prime}\right)$

- $\operatorname{CHAR}(199), \operatorname{CHAR}(0), \operatorname{CHAR}(1), \operatorname{CHAR}(0), \operatorname{CHAR}(6), \operatorname{CHAR}(199)$

C . 'TABLE2.1 External dose conversion factors for exposure',

C . ' to residual radioactive materials'

$\operatorname{OPEN}\left(5, F I L E={ }^{\prime}\right.$ table $2.2^{\prime}$, STATUS $={ }^{\prime} O L D^{\prime}$, ACCESS $={ }^{\prime}$ APPEND $\left.{ }^{\prime}\right)$ WRITE $(5, '(6 A)$ ')

CHAR(199), CHAR(0), $\operatorname{CHAR}(1), \operatorname{CHAR}(0), \operatorname{CHAR}(16), \operatorname{CHAR}(199)$

C . 'TABLE2.2 Inhalation committed effective dose conversion fac-',

C. ' tors for exposure to residual radioactive materials'

OPEN (6, FILE=' table2.3', STATUS='OLD', ACCESS='APPEND' ) WRITE $\left(6,{ }^{\prime}(6 A)^{\prime}\right)$

CHAR(199), CHAR(0), CHAR(1), CHAR(0), CHAR(25), CHAR(199)

C . 'TABLE2.3 Secondary ingestion committed effective dose conver-',

C .' sion factors for exposure to residual radioactive',

C.' materials.'

OPEN $\left(7, F I L E='\right.$ table $2.4 '$, STATUS $={ }^{\prime}$ OLD', ACCESS $={ }^{\prime}$ APPEND' $)$ WRITE $(7, '(6 A) ')$

CHAR(199), Char(0), $\operatorname{CHAR}(1), \operatorname{CHAR}(0), \operatorname{CHAR}(35), \operatorname{CHAR}(199)$

C . 'TABLE2.4 Maximum organ dose conversion factors for',

C . ' ingestion of drinking water'

OPEN $\left(12, F I L E='\right.$ table $2.5^{\prime}$, STATUS $='$ OLD', ACCESS $={ }^{\prime}$ APPEND' $)$ WRITE $\left(12,{ }^{\prime}(6 A)^{\prime}\right)$

CHAR(199), $\operatorname{CHAR}(0), \operatorname{CHAR}(1), \operatorname{CHAR}(0), \operatorname{CHAR}(47), \operatorname{CHAR}(199)$

C . 'TABLE2.5 Agricultural food product ingestion committed',

C . ' effective dose conversion factors for exposure',

C. ' to residual radioactive materials', 
TABLE 0.2. PHASE8 Computer Program Listing (contd)

C . ' ' (air and soil contributions)'

OPEN (8, FILE $={ }^{\prime}$ table $3.1 '$, STATUS='OLD', ACCESS $={ }^{\prime}$ APPEND') WRITE $(8, '(6 A) ')$

CHAR(199), CHAR(0), CHAR(1), ChaR(0), Char(4), ChAR(199)

C. 'TABLE3.1 Total effective dose equivalents for the',

C . ' building volume activity scenario'

$\operatorname{OPEN}\left(9, F I L E={ }^{\prime}\right.$ table $3.2^{\prime}$, STATUS $={ }^{\prime}$ OLD', ACCESS $={ }^{\prime}$ APPEND' $)$ WRITE $\left(9, '(6 A)^{\prime}\right)$

- $\operatorname{CHAR}(199), \operatorname{CHAR}(0), \operatorname{CHar}(1), \operatorname{CHar}(0), \operatorname{CHar}(14), \operatorname{CHar}(199)$

C. 'TABLE3.2 Total effective dose equivalents for the',

C . ' building surface activity scenario'

OPEN $\left(10, F I L E={ }^{\prime}\right.$ table $3.3^{\prime}$, STATUS $={ }^{\prime} O L D^{\prime}$, ACCESS $={ }^{\prime}$ APPEND $\left.{ }^{\prime}\right)$ WRITE $(10, '(6 A) ')$

- $\operatorname{CHAR}(199), \operatorname{CHAR}(0), \operatorname{CHAR}(1), \operatorname{CHAR}(0), \operatorname{CHAR}(25), \operatorname{CHAR}(199)$

C. 'TABLE3.3 Total effective dose equivalent for the surface',

C.' soil scenario'

OPEN $\left(11, F I L E={ }^{\prime}\right.$ table $3.4^{\prime}$, STATUS $={ }^{\prime}$ OLD', ACCESS $={ }^{\prime}$ APPEND' $)$ WRITE (11,' (6A)')

CHAR(199), Char(0), Char(1), $\operatorname{CHAR}(0), \operatorname{CHAR}(35), \operatorname{CHAR}(199)$

C . 'TABLE3.4 Drinking water committed effective dose equivalents',

C . ' for exposure to residual radioactive materials'

OPEN $\left(14, F I L E={ }^{\prime}\right.$ table $.1 '$, STATUS='OLD', ACCESS ='APPEND' $)$

WRITE $\left(14, '(6 A)^{\prime}\right)$

- $\operatorname{CHAR}(199), \operatorname{CHaR}(0), \operatorname{CHaR}(1), \operatorname{CHaR}(0), \operatorname{Char}(4), \operatorname{CHaR}(199)$

OPEN $\left(15, F I L E='\right.$ table. $2^{\prime}$, STATUS='OLD', ACCESS ='APPEND' $)$ WRITE $(15, '(6 A) ')$

- $\operatorname{CHar}(199), \operatorname{Char}(0), \operatorname{Char}(1), \operatorname{CHar}(0), \operatorname{Char}(14), \operatorname{CHar}(199)$

OPEN (17, FILE=' control.1', STATUS=' unknown' $)$

OPEN (18, FILE $=$ ' control.2', STATUS $=$ ' unknown' $)$

OPEN $(19$, FILE $=$ ' control.3', STATUS=' unknown' $)$

C OPEN (20, FILE='phase8.qa', STATUS='unknown')

C--.- Process master list

7 CONTINUE

U234 $=$.FALSE.
U235 $=$. FALSE.
U238 $=$. FALSE.
TH232 $=$.FALSE.

READ (1, 14, END=9) ELTM, AWM, HL, CHADJ, PC, IBETA, IMPDAU, 
IABLE D.2. PHASE8 Computer Program Listing (contd) CHADJI

DO 201 IINT $=1, \mathrm{~N} 2$

IF (ELTI(IINT) .EQ. ELTM .AND. AWI(IINT) .EQ. AWM) GOTO 202

201 CONTINUE

202 CONTINUE

D0 203 IEXT $=1, \mathrm{~N} 3$

203 CONTINUE

204 CONTINUE

C

TEMP $=$ CHADJ - CHADJI

IF (TEMP . NE. 1.0) THEN

Explicit daughter--

$\mathrm{NT}=2$

IFOOT $=1$

IDESIG $=1$

ELSEIF (IMPDAU .GT. 0.0) THEN

C Implicit daughter--

NT $=1$

IFOOT $=2$

IDESIG $=2$

ELSE

$$
\begin{aligned}
& \text { NT }=1 \\
& \text { IFOOT }=0 \\
& \text { IDESIG }=0
\end{aligned}
$$

ENDIF

IF (ELTM .EQ. ' $U$ ') THEN

IF (AWM .EQ. '234 1 ') U234 = .TRUE.

IF (AWM .EQ. '235 $\quad$ ') U235 = .TRUE.

IF (AWM .EQ. '238 ') U238 = . TRUE.

ELSEIF (ELTM .EQ. 'TH' .AND. AWM .EQ. '232 ') THEN

TH232 = . TRUE.

ENDIF

D0 400 IT $=1$, NT

C--.-- Construct name for printing

IF (IT .EQ. 2 .AND. IMPDAU .GT. 0) THEN

IDESIG $=2$

IFOOT $=2$

ENDIF

DO $401 K=1,3$
TMPNAM $=1,3$
IMPNAM $(1:)=$ LITFNT
TMPNAM $(7:) \quad=$ ADVUP

D. 19 
TABLE D.2. PHASE8 Computer Program Listing (contd)

8

DO $8 I=1,4$

IF (AWM (I:I) .NE. ' ' ') IPOS = I

CONTINUE

TMPNAM (12-IPOS:) = AWM

IPOS = IPOS + 1

C

Change $M$ to $\mathrm{m}-$ -

IF (TMPNAM(11:11).EQ. 'M') $\operatorname{TMPNAM}(11: 11)=' m$ '

$\operatorname{TMPNAM}(12:)=$ ADVDWN

$\operatorname{TMPNAM}(13:)=$ BIGFNT

$\operatorname{TMPNAM}(19:)=\operatorname{ELTM}(1: 1)$

IF (ELTM (2:2) .EQ. ' ' ) THEN

ISTART $=20$

ELSE

IASC = ICHAR (ELTM(2:2))

$I A S C=I A S C+32$

$\operatorname{TMPNAM}(20: 20)=$ CHAR (IASC)

ISTART $=21$

ENDIF

IEND = ISTART

IF ((IT .EQ. 1 .AND. IDESIG .GT. 0) .OR .

(IT .EQ. 2 .AND. IMPDAU .GT. 0)) THEN

TMPNAM (ISTART:) = DESIG (IDESIG)

TMPNAM (ISTART+2:) = ADVUP

TMPNAM (ISTART $+3:)=$ FOOT $($ IFOOT $+K-1)$

TMPNAM (ISTART+6:) = ADVDWN

\section{ENDIF}

IEND = ISTART + 6

$\operatorname{RITNAM}(K)=$ TMPNAM

401 CONTINUE

IF (ELTM .NE. ELTOLD .AND. IT .EQ. 1) THEN DO $301 K=4,12$,
WRITE $(K, *) 11$

301 CONTINUE

$\operatorname{WRITE}\left(14,{ }^{*}\right), 1$

ENDIF

WRITE $(15, *)$ ' '

C-.--- Table 2.1 - External

C No daughters:

C Surface: 
TABLE D.2. PHASE8 Computer Program Listing (contd)

Volume and soil:

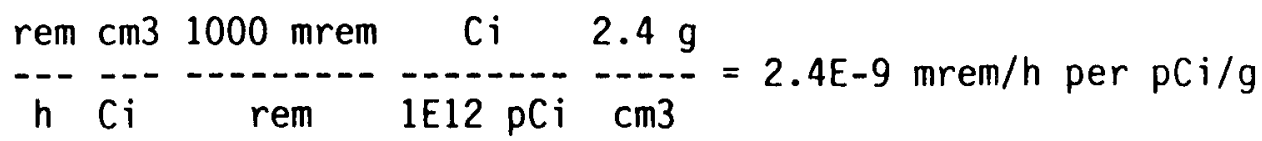

With daughters:

C

Surface - EXTDF conversion to GENII surface units:

\begin{tabular}{cccccc}
$\mathrm{rem}$ & $\mathrm{cm} 2$ & $\mathrm{~Sv}$ & $8766 \mathrm{~h}$ & $\mathrm{Ci}$ & $\mathrm{m} 2$ \\
\hdashline $\mathrm{h}$ & $\mathrm{Ci}$ & $100 \mathrm{rem}$ & yr & $3.7 \mathrm{E} 10 \mathrm{~Bq}$ & $1 . \mathrm{E} 4 \mathrm{~cm} 2$
\end{tabular}$=2.37 \mathrm{E}-13 \mathrm{~Sv} / \mathrm{h} \mathrm{per}$

Surface - in GENII:

Sv m2 $0.037 \mathrm{~Bq}$ yr 100 rem "m2"

yr Bq $\mathrm{pCi} \quad 8766 \mathrm{~h}$ Sv $0.15 \mathrm{m3}=2.8 \mathrm{E}-3 \mathrm{rem} / \mathrm{h} \mathrm{per}$

Surface:

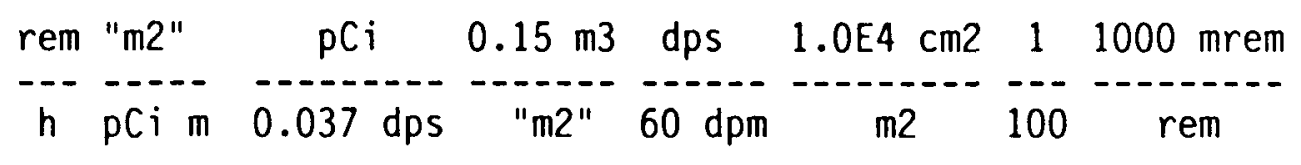

$=6.76 \mathrm{mrem} / \mathrm{h}$ per $\mathrm{dpm} / 100 \mathrm{~cm} 2$

Volume and soil - EXTDF conversion to GENII surface units:

\begin{tabular}{cccccc} 
rem $\mathrm{cm} 3$ & $\mathrm{SV}$ & $8766 \mathrm{~h}$ & $\mathrm{Ci}$ & $\mathrm{m} 3$ & \\
\hdashline $\mathrm{h} \quad \mathrm{Ci}$ & $100 \mathrm{rem}$ & yr & $3.7 \mathrm{E} 10 \mathrm{~Bq}$ & $1 . \mathrm{E} 6 \mathrm{~cm} 3$
\end{tabular}$=2.37 \mathrm{E}-15 \mathrm{~Sv} / \mathrm{h} \mathrm{per}$

Note: EXTDF was run with IEXTU $=6$ instead of IEXTU $=3$ for these runs (GENII soil default), consequently a unit conversion of 2.37E-13 was used instead of 2.37-15 (\# $\mathrm{cm} 2 \mathrm{vs} . \mathrm{cm} 3)$. A correction of 100.0 is applied in the final step. 
IABLE D.2. PHASE8 Computer Program Listing (contd)

C Volume and soil - in GENII:

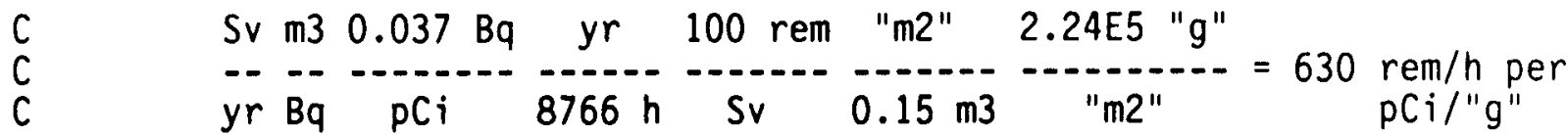

C Volume and soil:

$c$
$c$

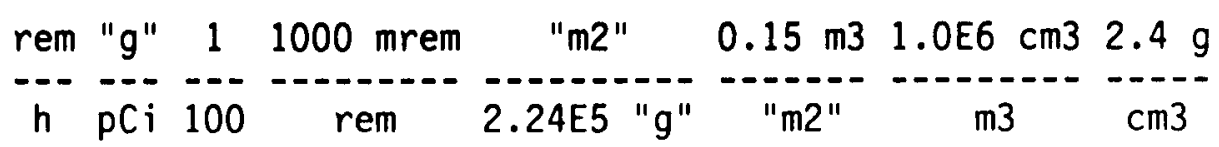

$=16.07 \mathrm{mrem} / \mathrm{h}$ per $\mathrm{pCi} / \mathrm{g}$

Si unit conversions:

C

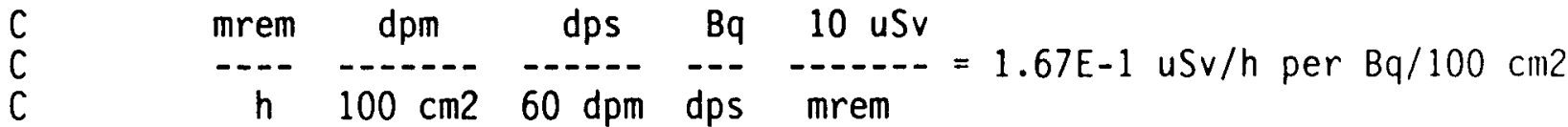

$\begin{array}{lcccc}\mathrm{C} & \mathrm{mrem} & \mathrm{g} & 10 \mathrm{uSv} & 27 \mathrm{pCi} \\ \mathrm{C} & \mathrm{h} & \mathrm{pCi} & \mathrm{mrem} & \mathrm{Bq}\end{array}$

IF (IT .EQ. 1 .AND. NT .GT. 1) THEN

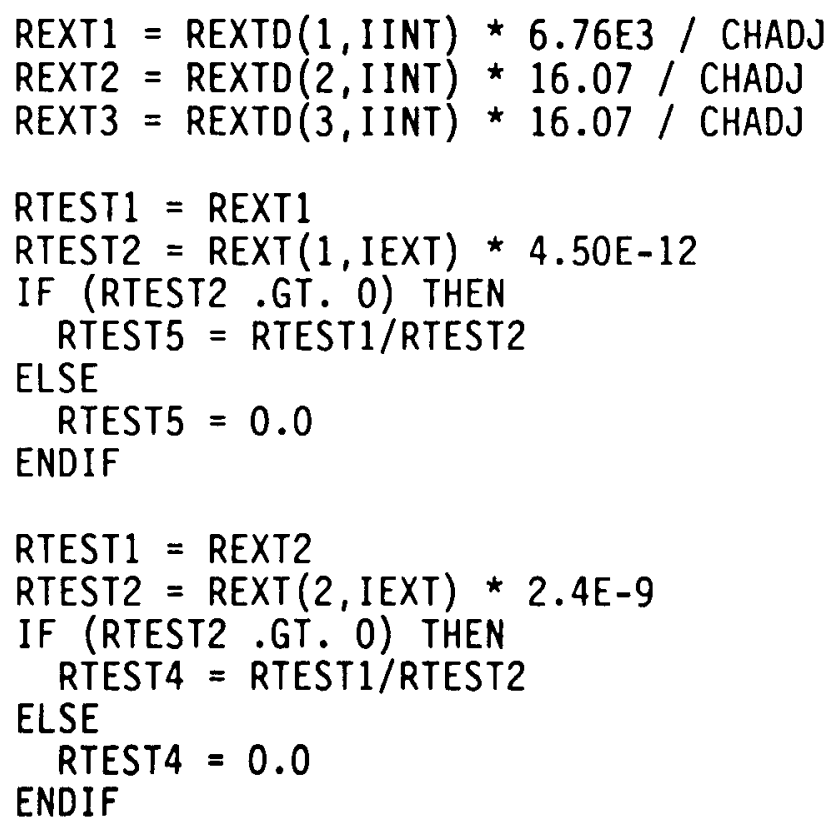


TABLE 0.2. PHASE8 Computer Program Listing (contd)

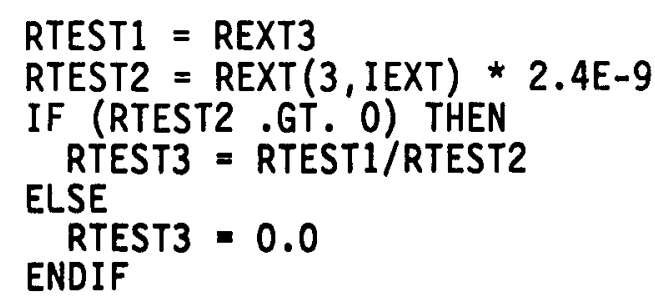

WRITE $(*$ 89) ELTM, AWM, CHADJ, RTEST5, RTEST4, RTEST3

C WRITE $(20,89)$ ELTM, AWM, CHADJ, RTEST5, RTEST4, RTEST3 FORMAT (' $, A 2, A 4,1 P, 4 G 10.2)$

ELSE

$$
\begin{aligned}
& \text { REXT1 }=\operatorname{REXT}(1, \text { IEXT) } \star 4.50 E-12 \\
& \text { REXT2 }=\operatorname{REXT}(2, \text { IEXT) } \star 2.4 E-9 \\
& \text { REXT3 }=\operatorname{REXT}(3, \text { IEXT) } \star 2.4 E-9
\end{aligned}
$$

ENDIF

$$
\begin{array}{ll}
\text { WRITE }(4,12) & \text { NEXTAB, RITNAM(1) }(1: \text { IEND), NEXTAB, } \\
& \text { REXT1, NEXTAB, REXT1 *1.67E-1, NEXTAB, } \\
& \text { REXT2, NEXTAB, REXT2 *270.0, NEXTAB, } \\
& \text { REXT3, NEXTAB, REXT3 } * 270.0
\end{array}
$$

C

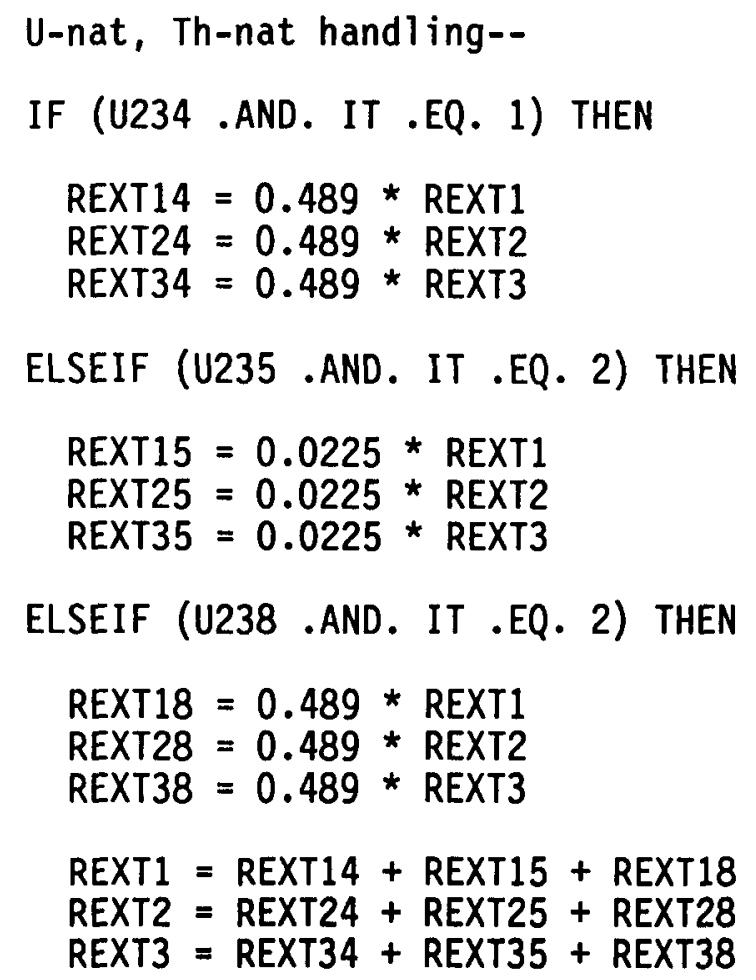


TABLE D.2. PHASE8 Computer Program Listing (contd)

WRITE $(4,12)$ NEXTAB, UNAT(1) (1:IUEND), NEXTAB, REXT1, NEXTAB, REXT1 *1.67E-1, NEXTAB, REXT2, NEXTAB, REXT2 *270.0, NEXTAB, REXT3，NEXTAB， REXT3 * 270.0

ELSEIF (TH232 .AND. IT .EQ. 1) THEN

WRITE $(4,12)$ NEXTAB, THNAT(1) (1:ITHEND), NEXTAB, REXT1, NEXTAB, REXT1 * 1.67E-1, NEXTAB, REXT2, NEXTAB, REXT2 *270.0, NEXTAB, REXT3, NEXTAB, REXT3 * 270.0

ENDIF

C---.- Table 2.2-2.5 - Ingestions

$c$
$c$

\begin{tabular}{ccc} 
rem & $\mathrm{m} 3$ & $1000 \mathrm{mrem}$ \\
\hdashline $\mathrm{h}$ & $\mathrm{pCi}$ & $\mathrm{rem}$
\end{tabular}$=1000 \mathrm{mrem} / \mathrm{h}$ per $\mathrm{pCi} / \mathrm{m} 3 \quad$ (air)

C

rem g 1000 mrem

h $\mathrm{pCi}_{\mathrm{rem}} \mathrm{rem}=1000 \mathrm{mrem} / \mathrm{h}$ per $\mathrm{pCi} / \mathrm{g}$ (soil)

rem $L \quad 1000$ mrem

h $\mathrm{pCi}$ rem $=1000 \mathrm{mrem} / \mathrm{h}$ per $\mathrm{pCi} / \mathrm{L} \quad$ (drinking water)

IF (IT .EQ. 1) THEN RINH1 $=$ RINH (IINT) * $1000.0 /$ CHADJ

RSINGI = RSING(IINT) *1000.0/ CHADJ

RING1 = RING(1,IINT) *1000.0/CHADJ

RING2 $=$ RING $(2$, IINT $) * 1000.0 /$ CHADJ

$D W=$ DWORG $($ IINT $) * 1000.0 /$ CHADJ

ELSE $O R G=$ ORGAN (IINT)

$$
\begin{aligned}
& \text { RINH1 }=\text { RINHN (IINT) } * 1000.0 \\
& \text { RSING1 }=\text { RSINGN (IINT) } * 1000.0 \\
& \text { RING1 }=\text { RINGN (IIINT) } * 1000.0 \\
& \text { RING2 }=\text { RINGN (2, IINT) } * 1000.0 \\
& \text { DW }=\text { DWORGN (IINT) } * 1000.0 \\
& \text { ORG }=\text { ORGNO(IINT) } \\
& \text { ENDIF }
\end{aligned}
$$

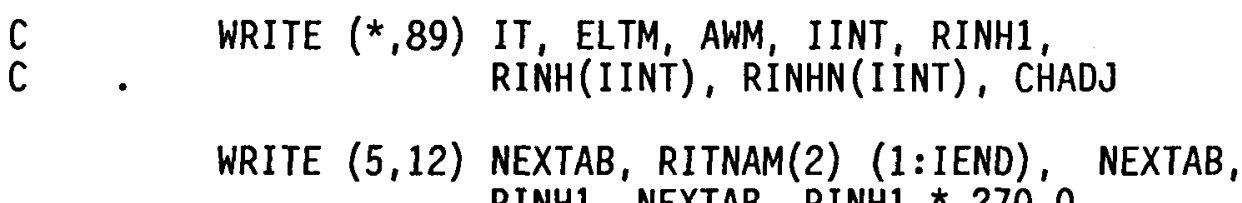
RINH1, NEXTAB， RINH1 * 270.0 
TABLE D.2. PHASE8 Computer Program Listing (contd)

WRITE $(6,12)$ NEXTAB, RITNAM(1) (1:IEND), NEXTAB, RSING1， NEXTAB， RSING1 * 270.0

WRITE $(7,13)$ NEXTAB, RITNAM(2) (1:IEND), NEXTAB, DW, NEXTAB, DW * 270.0, NEXTAB, ORG

WRITE $(12,12)$ NEXTAB, RITNAM(1) (1:IEND), NEXTAB, RING1, NEXTAB, RING1 *270.0, NEXTAB, RING2, NEXTAB， RING2 * 270.0

C U-nat, Th-nat handling--

IF (U234 .AND. IT .EQ. 1) THEN

$$
\begin{aligned}
& \text { AINH14 }=0.489 * \text { RINH1 } \\
& \text { ASING14 }=0.489 * \text { RSING1 } \\
& \text { AING14 }=0.489 * \text { RING1 } \\
& \text { AING24 }=0.489 * \text { RING2 } \\
& \text { AW4 }=0.489 * \text { DW } \\
& \text { ORG4A }=\text { ORG }
\end{aligned}
$$

C

$$
\text { WRITE }\left(*{ }^{*}\right) \text { 'U-234 set' }
$$

ELSEIF (U235 .AND. IT .EQ. 2) THEN

$$
\begin{aligned}
& \text { AINH15 }=0.0225 * \text { RINH1 } \\
& \text { ASING15 }=0.0225 * \text { RSING1 } \\
& \text { AING15 }=0.0225 * \text { RING1 } \\
& \text { AING25 }=0.0225 * \text { RING2 } \\
& \text { AW5 }=0.0225 * \text { DW } \\
& \text { ORG5A }=\text { ORG }
\end{aligned}
$$

ELSEIF (U238 .AND. IT .EQ. 2) THEN

$$
\begin{aligned}
& \text { AINH18 }=0.489 * \text { RINH1 } \\
& \text { ASING18 }=0.489 * \text { RSING1 } \\
& \text { AING18 }=0.489 * \text { RING1 } \\
& \text { AING28 }=0.489 * \text { RING2 } \\
& \text { AW8 }=0.489 * \text { DW } \\
& \text { ORG8A }=\text { ORG } \\
& \text { WRITE }(*, *) ' U-238 \text { set }-2 ', \text { ORG8A, IT } \\
& \text { RINH1 = AINH14 + AINH15 + AINH18 } \\
& \text { RSING1 = ASING14 + ASING15 + ASING18 }
\end{aligned}
$$


TABLE D.2. PHASE8 Computer Program Listing (contd)

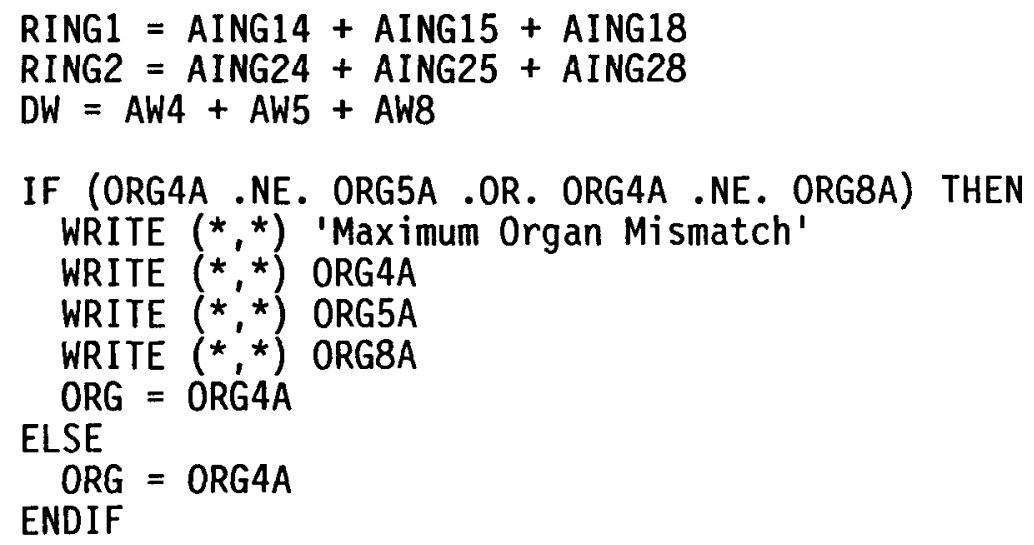

ENDIF

C...-.- Tabl scenario 
TABLE D.2. PHASE8 Computer Program Listing (contd)

ELSEIF (U238 .AND. IT .EQ. 2) THEN

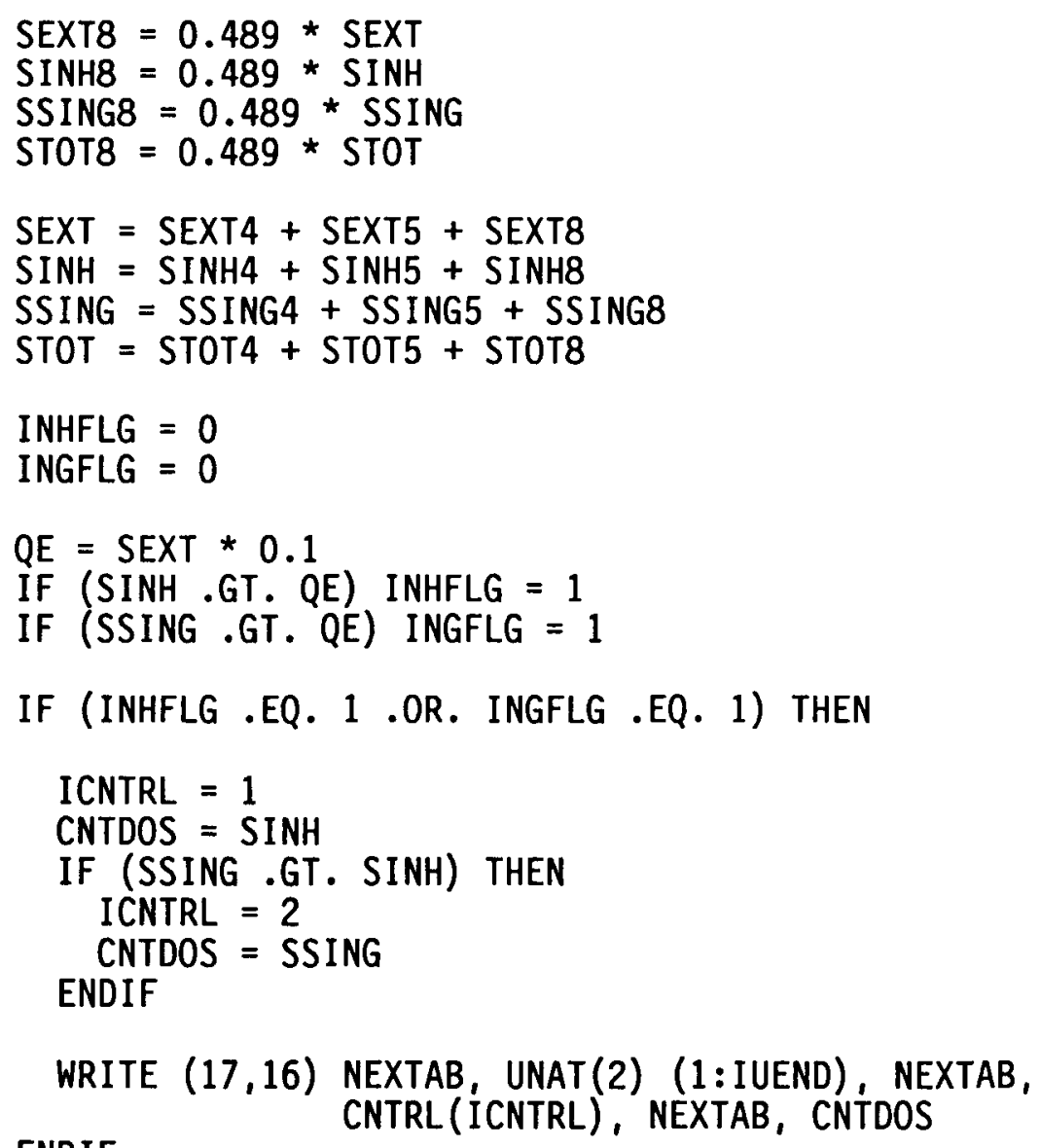

WRITE $(8,12)$ NEXTAB, UNAT(2) (1: IUEND), NEXTAB, SEXT, NEXTAB, SINH, NEXTAB, SSING, NEXTAB, STOT, NEXTAB, STOT * 270.0

ELSEIF (TH232 .AND. IT .EQ. 1) THEN

IF (INHFLG .EQ. 1 .OR. INGFLG .EQ. 1) THEN

WRITE $(17,16)$ NEXTAB, THNAT $(2)$ (1: ITHEND), NEXTAB, ENDIF CNTRL(ICNTRL), NEXTAB, CNTDOS

WRITE $(8,12)$ NEXTAB, THNAT(2) (1: ITHEND), NEXTAB, SEXT, NEXTAB, SINH, NEXTAB, SSING, NEXTAB, STOT, NEXTAB, STOT * 270.0

ENDIF 
TABLE D.2. PHASE8 Computer Program Listing (contd)

C-..-- Table 3.2 - Building surface activity scenario

C 2000 hours exposure

C $\quad 1.0 \mathrm{E}-5 \mathrm{pCi} / \mathrm{m} 3$ air concentration

C $\quad 0.001 \mathrm{pCi} / \mathrm{h} \operatorname{diet}$

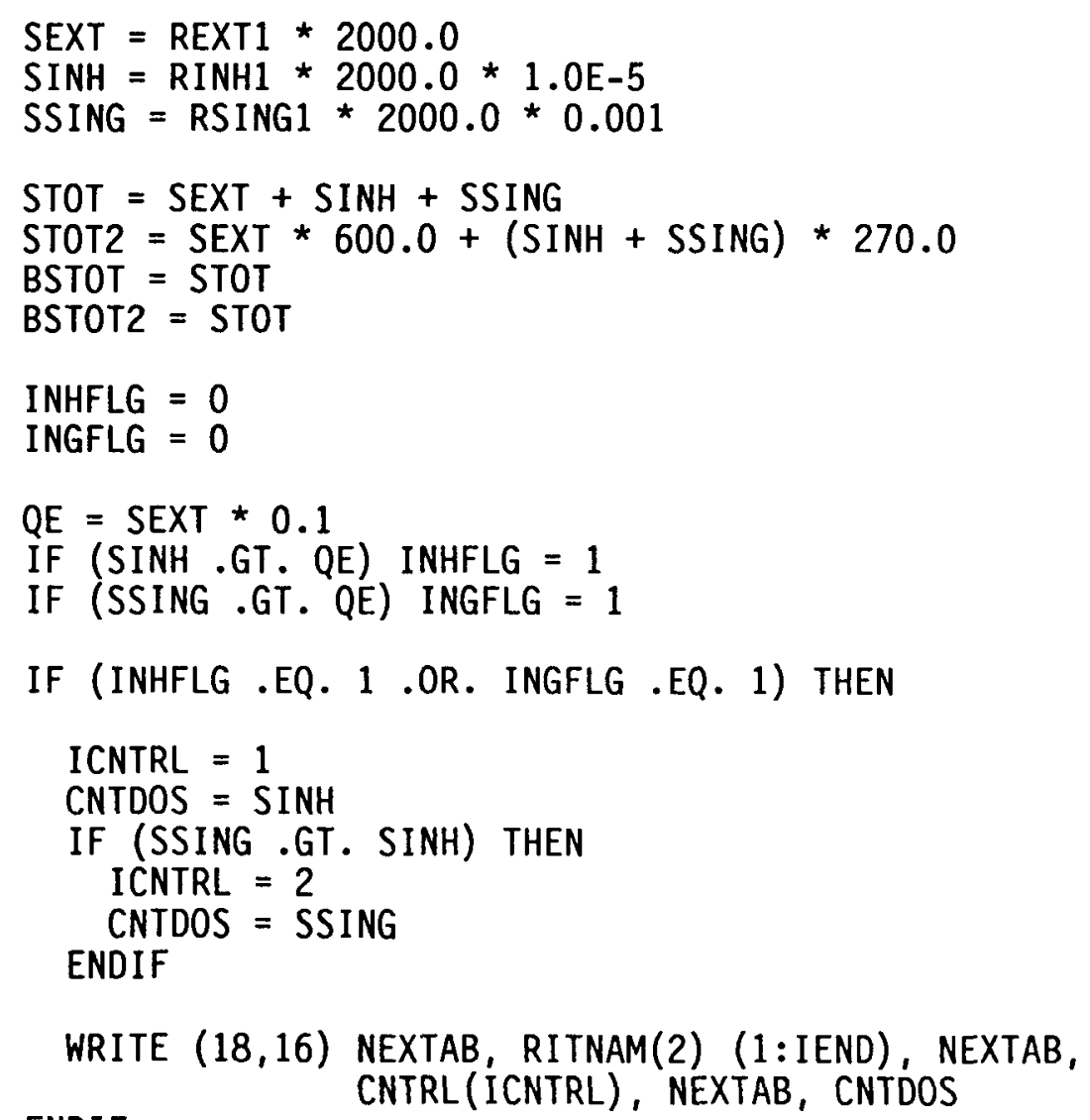

WRItE $(9,12)$ NEXTAB, RITNAM(2) (1: IEND)， NEXTAB, SEXT, NEXTAB, SINH, NEXTAB, SSING, NEXTAB, STOT, NEXTAB, STOT2

U-nat, Th-nat handling--

IF (U234 .AND. IT .EQ. 1) THEN

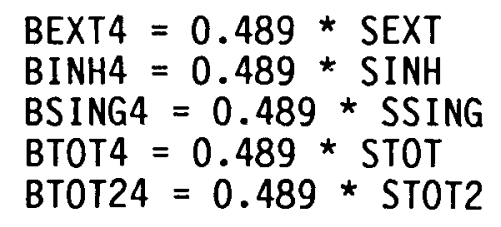


TABLE D.2. PHASE8 Computer Program Listing (contd)

ELSEIF (U235 .AND. IT .EQ. 2) THEN

$$
\begin{aligned}
& \text { BEXT5 }=0.0225 * \text { SEXT } \\
& \text { BINH5 }=0.0225 * \text { SINH } \\
& \text { BSING5 }=0.0225 * \text { SSING } \\
& \text { BTOT5 }=0.0225 * \text { STOT } \\
& \text { BTOT25 }=0.0225 * \text { STOT2 }
\end{aligned}
$$

ELSEIF (U238 .AND. IT .EQ. 2) THEN

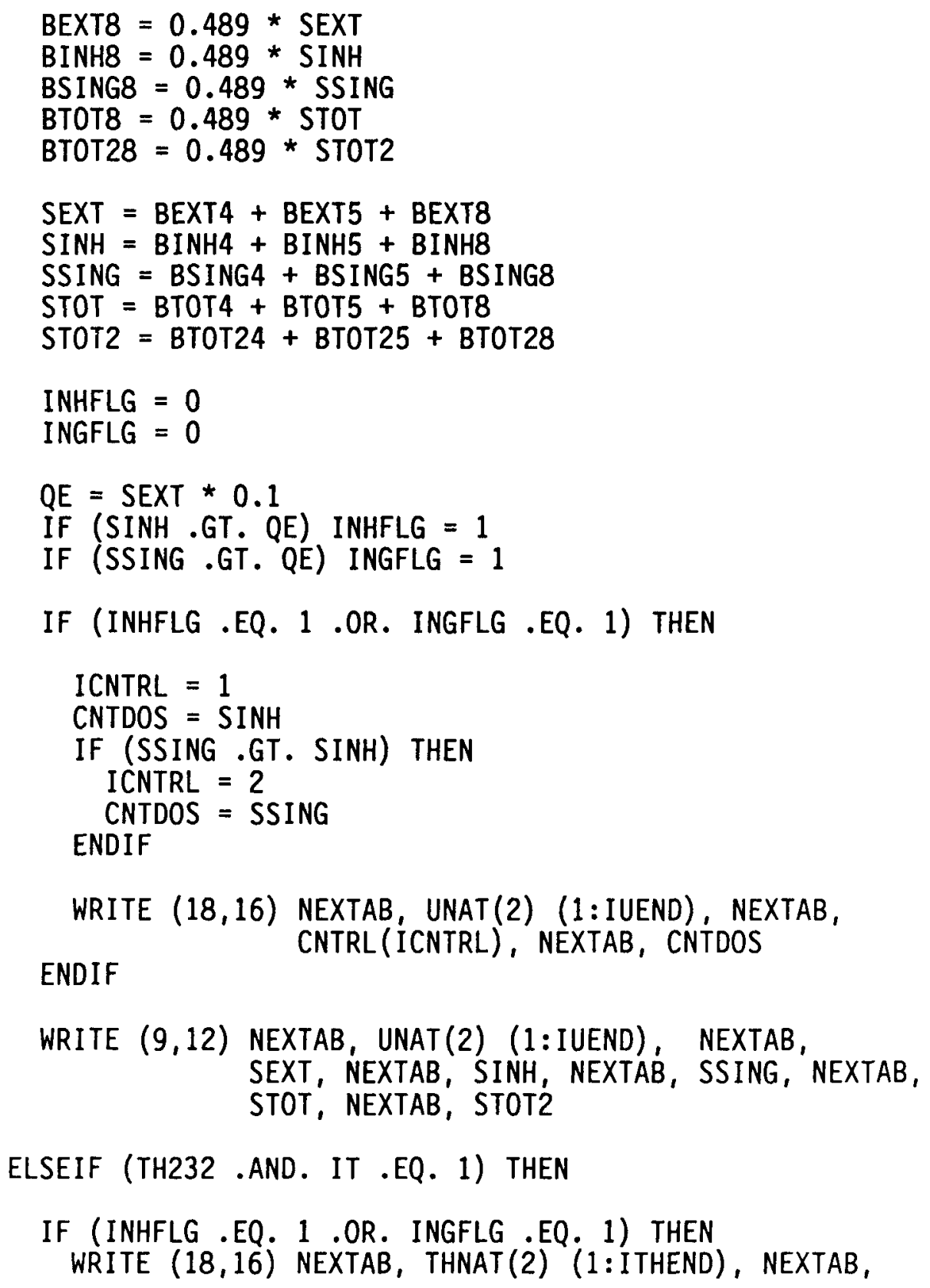


IABLE D.2. PHASE8 Computer Program Listing (contd)

ENDIF

CNTRL (ICNTRL)， NEXTAB， CNTDOS

WRITE $(9,12)$ NEXTAB, THNAT (2) (1:ITHEND), NEXTAB,
SEXT, NEXTAB, SINH, NEXTAB, SSING, NEXTAB,
STOT, NEXTAB, STOT2

ENDIF

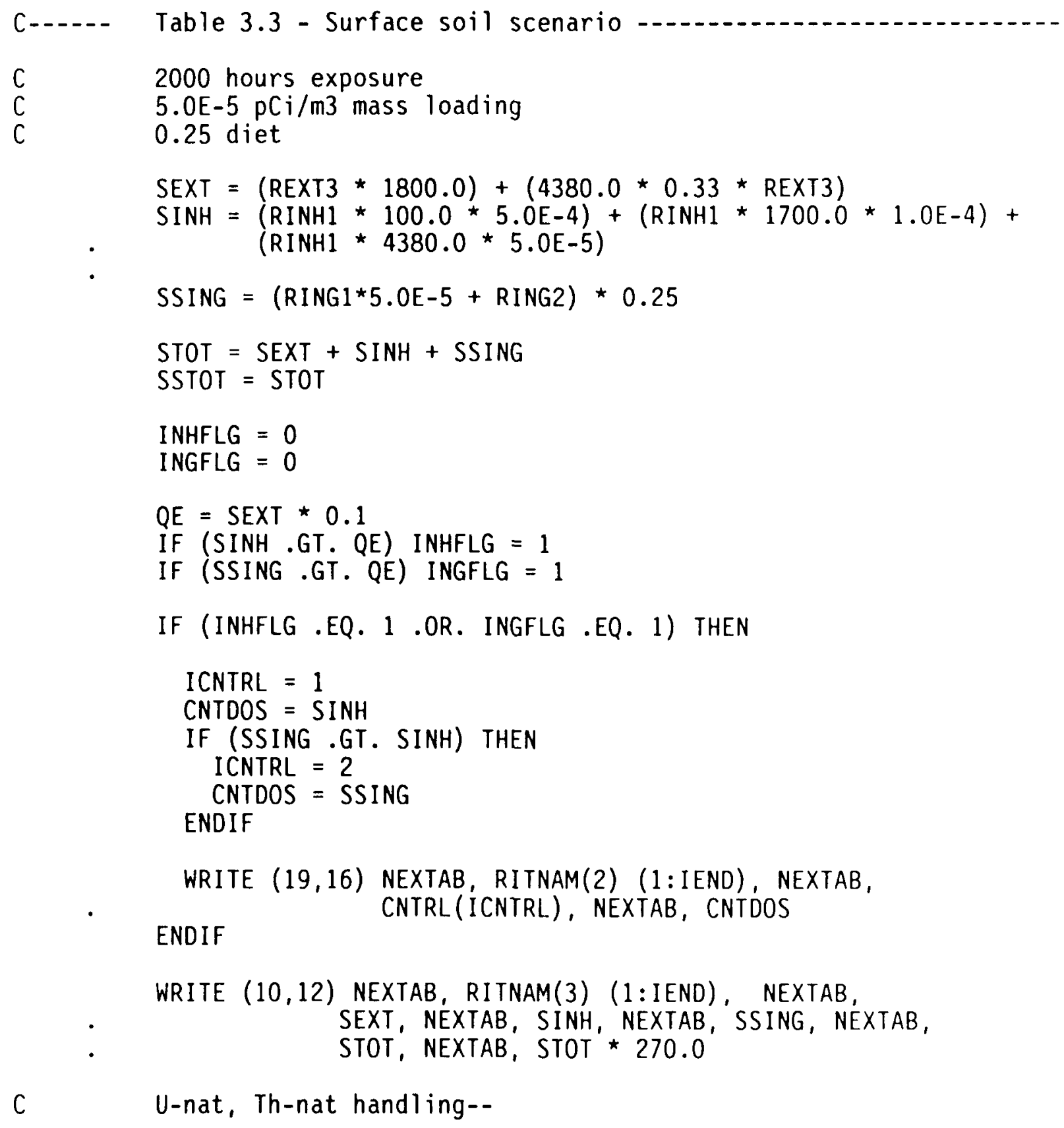


TABLE D.2. PHASE8 Computer Program Listing (contd)

IF (U234 .AND. IT .EQ. 1) THEN

CEXT4 $=0.489 *$ SEXT

CINH4 $=0.489 *$ SINH

CSING $4=0.489 *$ SSING

CTOT4 $=0.489 *$ STOT

ELSEIF (U235 .AND. IT .EQ. 2) THEN

CEXT5 $=0.0225 *$ SEXT

CINH5 $=0.0225 *$ SINH

CSING5 $=0.0225 *$ SSING

CTOT $5=0.0225 *$ STOT

ELSEIF (U238 .AND. IT .EQ. 2) THEN

CEXT8 $=0.489 *$ SEXT

CINH8 $=0.489 *$ SINH

CSING8 $=0.489 *$ SSING

CTOT $8=0.489 *$ STOT

SEXT = CEXT4 + CEXT5 + CEXT8

SINH $=$ CINH4 + CINH5 + CINH8

SSING $=$ CSING $4+$ CSING5 + CSING8

STOT = CTOT 4 + CTOT5 + CTOT8

INHFLG $=0$

INGFLG $=0$

$\mathrm{QE}=$ SEXT *0.1

IF (SINH .GT.QE) INHFLG $=1$

IF (SSING .GT. QE) INGFLG $=1$

IF (INHFLG .EQ. 1 .OR. INGFLG .EQ. 1) THEN

ICNTRL $=1$

CNTDOS = SINH

IF (SSING .GT. SINH) THEN

ICNTRL $=2$

CNTDOS = SSING

ENDIF

WRITE $(19,16)$ NEXTAB，UNAT(2) (1: IUEND), NEXTAB,

ENDIF

CNTRL (ICNTRL)， NEXTAB， CNTDOS

WRITE $(10,12)$ NEXTAB, UNAT(3) (1:IUEND), NEXTAB, SEXT, NEXTAB, SINH, NEXTAB, SSING, NEXTAB, STOT, NEXTAB， STOT * 270.0 
TABLE D.2. PHASE8 Computer Program Listing (contd)

ELSEIF (TH232 .AND. IT .EQ. 1) THEN

IF (INHFLG .EQ. 1 .OR. INGFLG .EQ. 1) THEN

WRITE $(19,16)$ NEXTAB, THNAT (2) (1:I ITHEND), NEXTAB,

ENDIF

CNTRL (ICNTRL), NEXTAB, CNTDOS

WRITE $(10,12)$ NEXTAB, THNAT(3) (1:ITHEND), NEXTAB,

SEXT, NEXTAB, SINH, NEXTAB, SSING, NEXTAB,

STOT, NEXTAB, STOT * 270.0

ENDIF

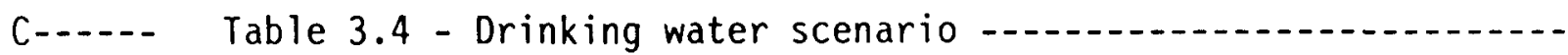

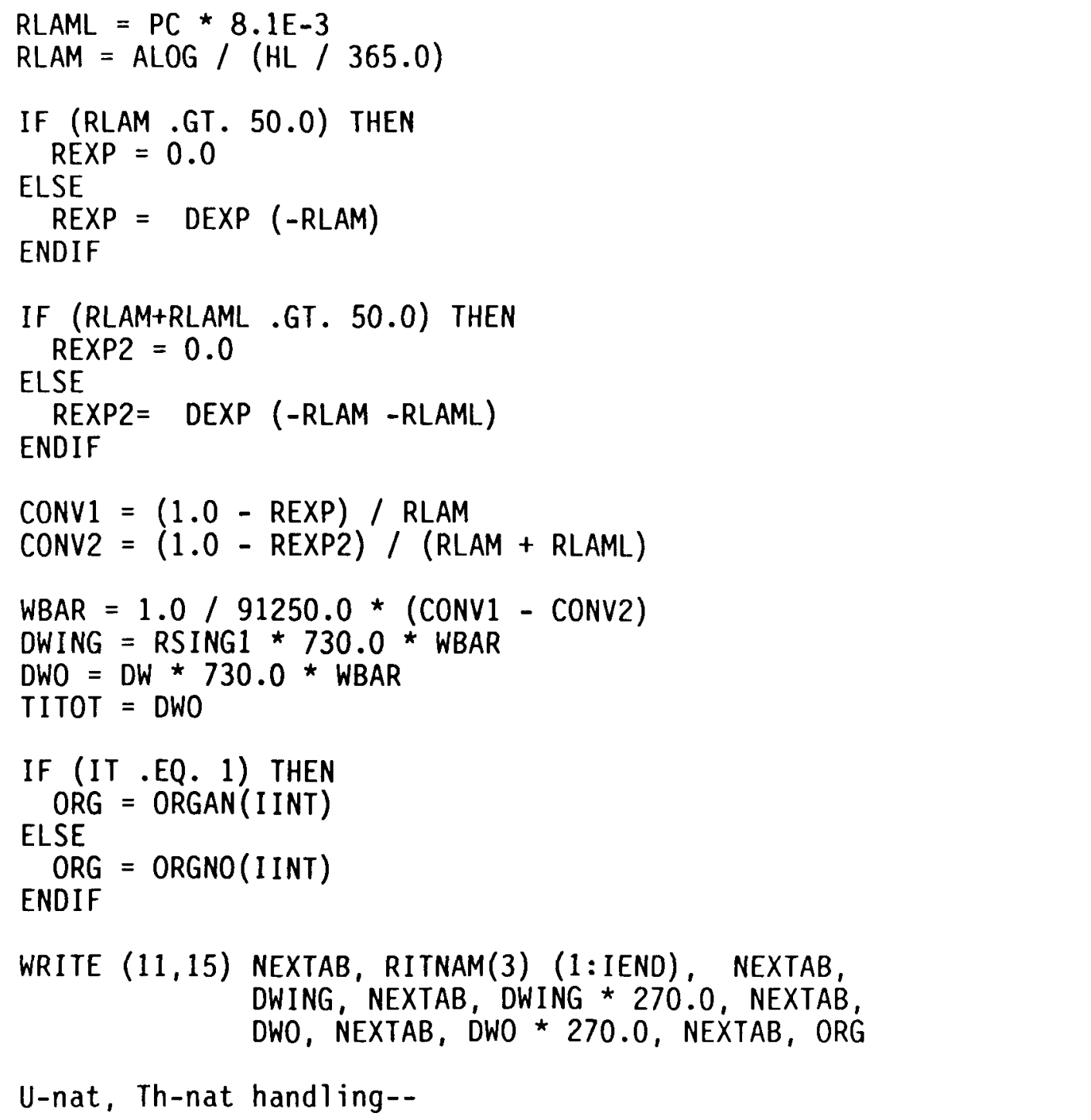


IABLE D.2. PHASE8 Computer Program Listing (contd)

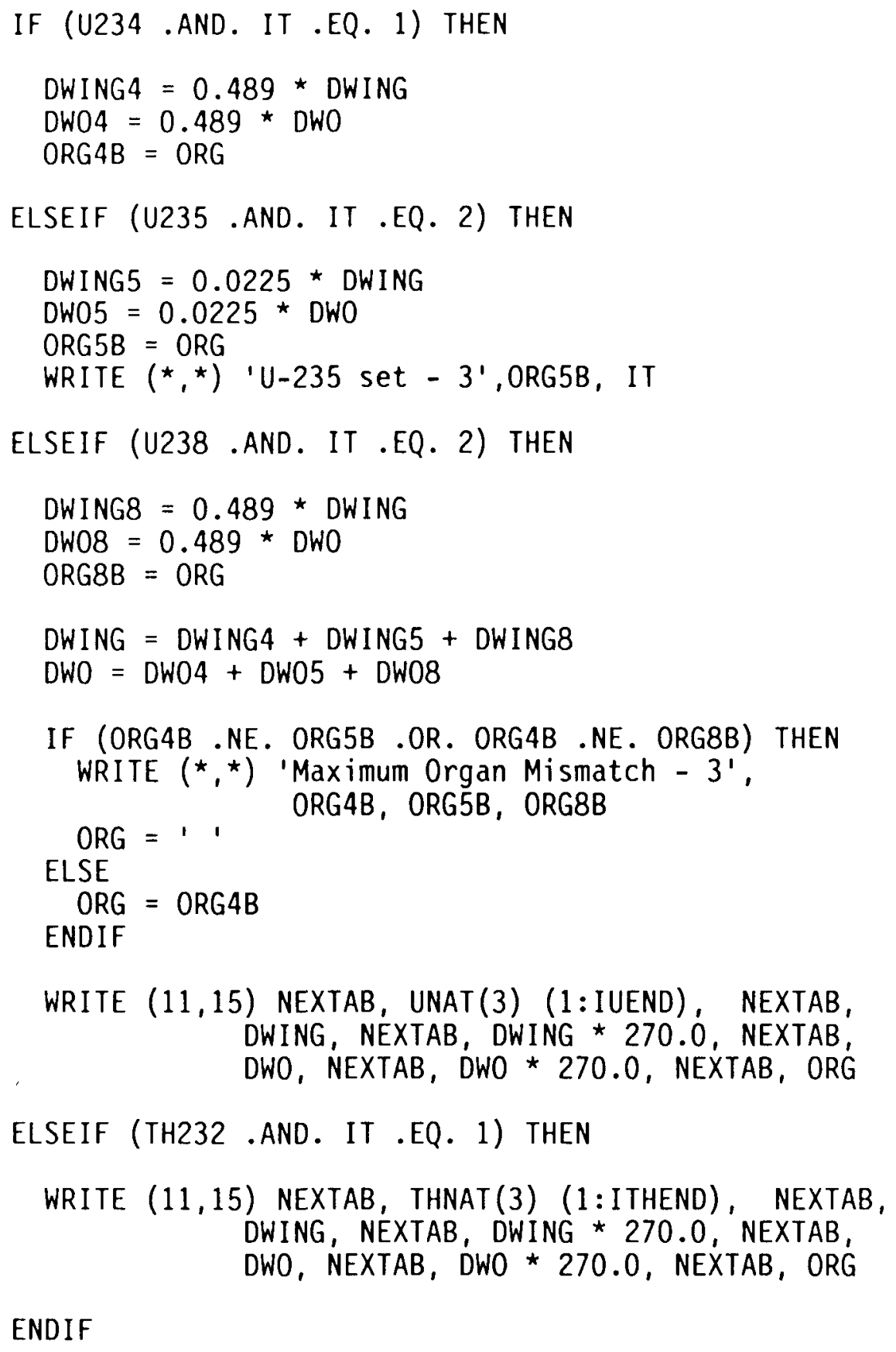

ENDIF

C-.--- Table 1 - Allowable limits for buildings

RLIMRM $=10.0$

RLIMSV $=100.0$

C Building volume-- 
IABLE D.2. PHASE8 Computer Program Listing (contd)

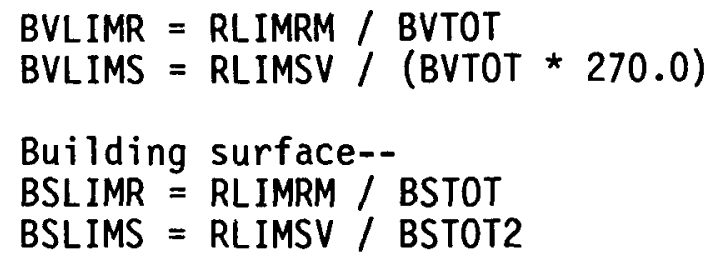

C

U-nat, Th-nat handling--

IF (U234 .AND. IT .EQ . 1) THEN

BVTOT4 $=0.489 *$ BVTOT

BSTOT4 $=0.489 *$ BSTOT

BST42 $=0.489 *$ BSTOT2

ELSEIF (U235 .AND. IT .EQ. 2) THEN BVTOT5 $=0.0225 *$ BVTOT

BSTOT5 $=0.0225 *$ BSTOT

BST52 $=0.0225 *$ BSTOT2

ELSEIF (U238 .AND. IT .EQ. 2) THEN BVTOT $8=0.489 *$ BVTOT

BSTOT8 $=0.489 *$ BSTOT

BST82 $=0.489 *$ BSTOT2

BVTOT = BVTOT4 + BVTOT5 + BVTOT8

BSTOT = BSTOT4 + BSTOT5 + BSTOT8

BSTOT2 $=$ BST42 + BST52 + BST82

C Building volume--

BVLIMR = RLIMRM / BVTOT

BVLIMS = RLIMSV / (BVTOT * 270.0)

C Building surface--

BSLIMR = RLIMRM / BSTOT

BSLIMS = RLIMSV / BSTOT2

WRITE $(14,12)$ NEXTAB, UNAT (2) (1: IUEND), NEXTAB, BVLIMR, NEXTAB, BVLIMS, NEXTAB, BSLIMR, NEXTAB, BSLIMS

ELSEIF (TH232 .AND. IT .EQ. 1) THEN WRITE $(14,12)$ NEXTAB, THNAT (2) (1: ITHEND), NEXTAB, BVLIMR, NEXTAB, BVLIMS, NEXTAB, BSLIMR， NEXTAB，BSLIMS 
TABLE D.2. PHASE8 Computer Program Listing (contd)

ENDIF

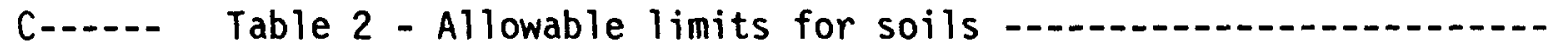

RLIMRM $=10.0$

RLIMSV $=100.0$

C Surface soil--

SSLIMR = RLIMRM / SSTOT

SSLIMS $=$ RLIMSV $/($ SSTOT $* 270.0)$

C Total inventory--

TILIMR = RLIMRM / TITOT

TILIMS $=$ RLIMSV / (TITOT * 270.0)

WRITE $(15,12)$ NEXTAB, RITNAM(2) (1:IEND), NEXTAB, SSLIMR, NEXTAB, SSLIMS, NEXTAB, TILIMS, NEXTAB, TILIMS

C

U-nat, Th-nat handling--

IF (U234 .AND. IT .EQ. 1) THEN

SSTOT4 $=0.489 *$ SSTOT

TITOT4 $=0.489 *$ TITOT

ELSEIF (U235 .AND. IT .EQ. 2) THEN

SSTOT5 $=0.0225 *$ SSTOT

TITOT5 $=0.0225 *$ TITOT

ELSEIF (U238 .AND. IT .EQ. 2) THEN

SSTOT $8=0.489 *$ SSTOT

TITOT8 $=0.489 *$ TITOT

SSTOT = SSTOT4 + SSTOT5 + SSTOT8

TITOT = TITOT4 + TITOT5 + TITOT8

C Surface soil--

SSLIMR = RLIMRM / SSTOT

SSLIMS $=$ RLIMSV $/($ SSTOT * 270.0)

C

Total inventory--

TILIMR = RLIMRM / TITOT

TILIMS $=$ RLIMSV $/($ TITOT * 270.0)

WRITE $(15,12)$ NEXTAB, UNAT(2) (1: IUEND), NEXTAB, SSLIMR, NEXTAB, SSLIMS, NEXTAB,

TILIMR， NEXTAB，TILIMS

ELSEIF (TH232 .AND. IT .EQ. 1) THEN 


\section{TABLE D.2. PHASE8 Computer Program Listing (contd)}

\section{WRITE $(15,12)$ NEXTAB, THNAT(2) (1:ITHEND), NEXTAB,}

SSLIMR, NEXTAB, SSLIMS, NEXTAB,

TILIMR, NEXTAB, TILIMS

\section{ENDIF}

400 CONTINUE

ELTOLD = ELTM

GOTO 7

C-..-.- End of loop

9 CONTINUE

DO $502 K=4,12$

502 CONTINUE

\section{CALL EXIT (1)}

C-.-- Format Statements

1 FORMAT (A2, A5, 5E8.0, A21)

2 FORMAT (A2, A6, 3(E8.0, 2X))

3 FORMAT $('$, , A)

12 FORMAT (A1, A, A1, 1P, 6(E7.1, A1))

13 FORMAT (A1, A, A1, 1P, 2(E7.1, A1), A21, A1)

14 FORMAT (A2, A6, 3X, E8.0, E9.0, 11X, E7.0, 1X, 2I2, E7.0)

15 FORMAT (A1, $A, A 1,1 P, 4(E 7.1, A 1), A 21, A 1$ )

16 FORMAT (A1, A, A1, A, A1, 1PE7.1, A1) 
TABLE D.3. Radionuclide Control List (RMDLIBDA.SHT)

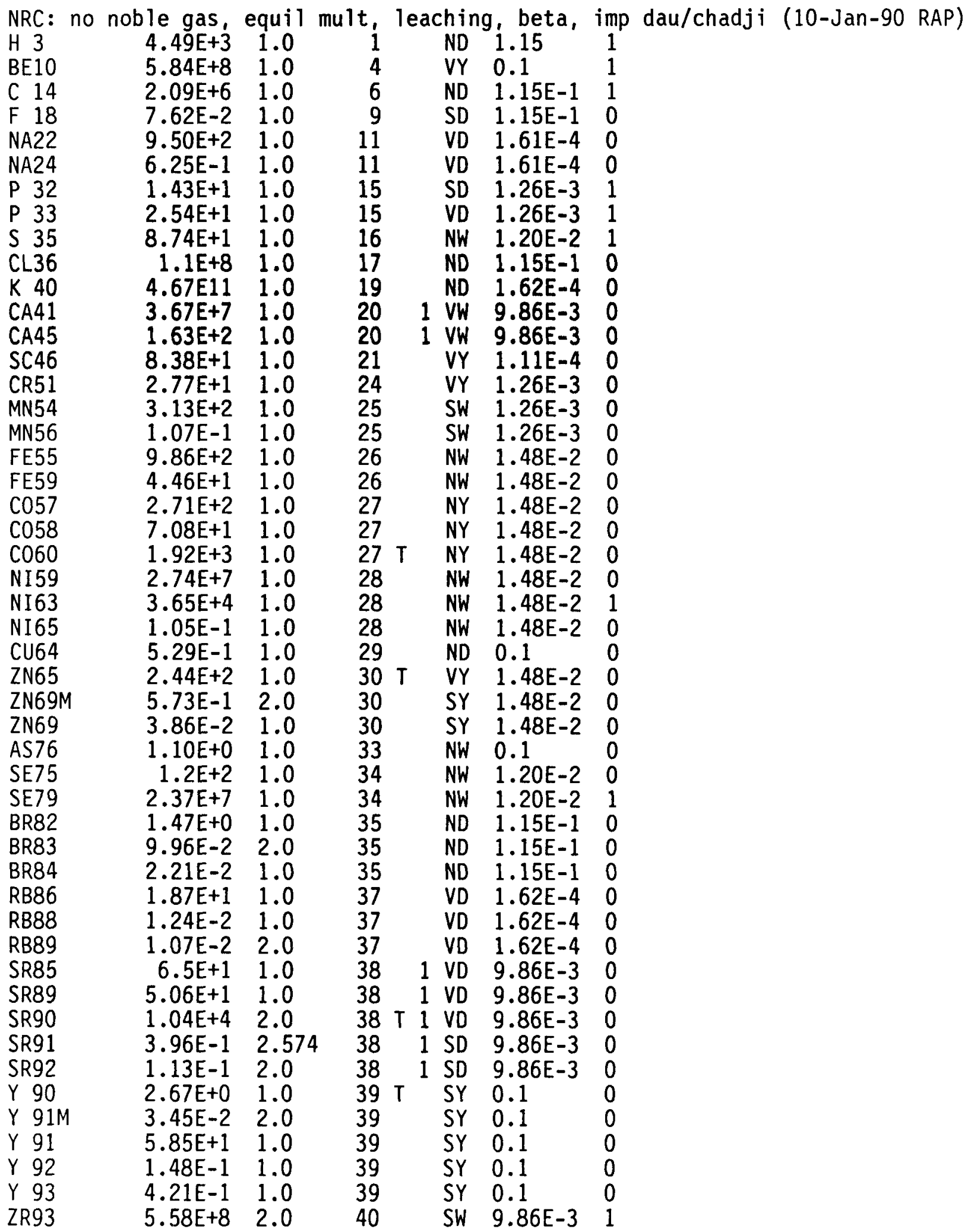


TABLE D.3. Radionuclide Control List (RMDLIBDA.SHT) (contd)

\begin{tabular}{|c|c|c|c|c|c|c|}
\hline $\begin{array}{l}\text { ZR95 } \\
\text { ZR97 } \\
\text { NB93M } \\
\text { NB94 } \\
\text { NB95M } \\
\text { NB95 } \\
\text { NB97M } \\
\text { NB97 } \\
\text { M093 } \\
\text { MO99 } \\
\text { TC99M } \\
\text { TC99 } \\
\text { TC101 } \\
\text { RU103 } \\
\text { RU105 } \\
\text { RU106 } \\
\text { RH103M } \\
\text { RH105 } \\
\text { PD103 } \\
\text { PD107 } \\
\text { PD109 } \\
\text { AG110M } \\
\text { AG111 } \\
\text { CD109 } \\
\text { CD113M } \\
\text { CD115M } \\
\text { CD115 } \\
\text { IN111 } \\
\text { IN114M } \\
\text { IN115M } \\
\text { SN117M } \\
\text { SN119M } \\
\text { SN121M } \\
\text { SN121 } \\
\text { SN123 } \\
\text { SN125 } \\
\text { SN126 } \\
\text { SB124 } \\
\text { SB125 } \\
\text { SB126M } \\
\text { SB126 } \\
\text { SB127 } \\
\text { TE123M } \\
\text { TE125M } \\
\text { TE127M } \\
\text { TE127 } \\
\text { TE129M } \\
\text { TE129 } \\
\text { TE131M } \\
\text { TE131 }\end{array}$ & $\begin{array}{l}6.40 \mathrm{E}+1 \\
7.04 \mathrm{E}-1 \\
5.33 \mathrm{E}+3 \\
7.41 \mathrm{E}+6 \\
3.61 \mathrm{E}+0 \\
3.51 \mathrm{E}+1 \\
6.94 \mathrm{E}-4 \\
5.01 \mathrm{E}-2 \\
1.28 \mathrm{E}+6 \\
2.75 \mathrm{E}+0 \\
2.51 \mathrm{E}-1 \\
7.77 \mathrm{E}+7 \\
9.86 \mathrm{E}-3 \\
3.94 \mathrm{E}+1 \\
1.85 \mathrm{E}-1 \\
3.68 \mathrm{E}+2 \\
3.90 \mathrm{E}-2 \\
1.47 \mathrm{E}+0 \\
1.70 \mathrm{E}+1 \\
2.37 \mathrm{E}+9 \\
5.61 \mathrm{E}-1 \\
2.50 \mathrm{E}+2 \\
7.46 \mathrm{E}+0 \\
4.64 \mathrm{E}+2 \\
5.00 \mathrm{E}+3 \\
4.46 \mathrm{E}+1 \\
2.23 \mathrm{E}+0 \\
2.8 \mathrm{E}+0 \\
5.00 \mathrm{E}+1 \\
1.82 \mathrm{E}-1 \\
1.40 \mathrm{E}+1 \\
2.93 \mathrm{E}+2 \\
2.01 \mathrm{E}+4 \\
1.13 \mathrm{E}+0 \\
1.29 \mathrm{E}+2 \\
9.64 \mathrm{E}+0 \\
3.65 \mathrm{E}+7 \\
6.02 \mathrm{E}+1 \\
1.01 \mathrm{E}+3 \\
1.32 \mathrm{E}-2 \\
1.24 \mathrm{E}+1 \\
3.85 \mathrm{E}+0 \\
1.20 \mathrm{E}+2 \\
5.80 \mathrm{E}+1 \\
1.09 \mathrm{E}+2 \\
3.90 \mathrm{E}-1 \\
3.36 \mathrm{E}+1 \\
4.83 \mathrm{E}-2 \\
1.25 \mathrm{E}+0 \\
1.74 \mathrm{E}-2\end{array}$ & $\begin{array}{l}2.007 \\
2.947 \\
1.0 \\
1.0 \\
1.945 \\
1.0 \\
2.0 \\
1.0 \\
2.0 \\
1.886 \\
1.0 \\
1.0 \\
1.0 \\
1.9974 \\
3.0 \\
2.0 \\
1.0 \\
1.0 \\
1.9997 \\
1.0 \\
2.0 \\
1.985 \\
1.0 \\
1.0 \\
1.0 \\
2.0 \\
1.0 \\
1.0 \\
1.957 \\
1.0 \\
1.0 \\
1.0 \\
1.776 \\
1.0 \\
1.0 \\
2.23 \\
2.14 \\
1.0 \\
1.231 \\
1.14 \\
1.0 \\
2.166 \\
1.0 \\
1.0 \\
1.982 \\
1.0 \\
1.629 \\
1.0 \\
2.233 \\
2.0109\end{array}$ & $\begin{array}{l}40 \\
40 \\
41 \\
41 \\
41 \\
41 \\
41 \\
41 \\
42 \\
42 \\
43 \\
43 \\
43 \\
44 \\
44 \\
44 \\
45 \\
45 \\
46 \\
46 \\
46 \\
47 \\
47 \\
48 \\
48 \\
48 \\
48 \\
49 \\
49 \\
49 \\
50 \\
50 \\
50 \\
50 \\
50 \\
50 \\
50 \\
51 \\
51 \\
51 \\
51 \\
51 \\
52 \\
52 \\
52 \\
52 \\
52 \\
52 \\
52 \\
52\end{array}$ & $\begin{array}{l}\text { SW } \\
\text { SW } \\
\text { VY } \\
\text { VY } \\
\text { SY } \\
\text { SY } \\
\text { SY } \\
\text { SY } \\
\text { SD } \\
\text { SD } \\
\text { NW } \\
\text { NW } \\
\text { NW } \\
\text { NY } \\
\text { NY } \\
\text { NY } \\
\text { NY } \\
N Y \\
\text { VY } \\
\text { VY } \\
\text { VY } \\
\text { ND } \\
\text { ND } \\
\text { ND } \\
\text { ND } \\
\text { ND } \\
N D \\
\text { VD } \\
\text { VD } \\
\text { VD } \\
\text { SW } \\
\text { VW } \\
\text { VW } \\
\text { VW } \\
\text { VW } \\
\text { SW } \\
\text { VW } \\
\text { SW } \\
\text { SW } \\
\text { SW } \\
\text { SW } \\
\text { SW } \\
\text { SW } \\
\text { SW } \\
\text { SW } \\
\text { SW } \\
\text { SW } \\
\text { SW } \\
\text { SW } \\
\text { SW }\end{array}$ & $\begin{array}{l}9.86 \mathrm{E}-3 \\
9.86 \mathrm{E}-3 \\
1.11 \mathrm{E}-2 \\
1.11 \mathrm{E}-2 \\
1.11 \mathrm{E}-2 \\
1.11 \mathrm{E}-2 \\
1.11 \mathrm{E}-2 \\
1.11 \mathrm{E}-2 \\
1.15 \mathrm{E}-1 \\
1.15 \mathrm{E}-1 \\
1.15 \mathrm{E}-1 \\
1.15 \mathrm{E}-1 \\
1.15 \mathrm{E}-1 \\
1.15 \mathrm{E}-1 \\
1.15 \mathrm{E}-1 \\
1.15 \mathrm{E}-1 \\
0.1 \\
0.1 \\
0.1 \\
0.1 \\
0.1 \\
1.62 \mathrm{E}-4 \\
1.62 \mathrm{E}-4 \\
1.48 \mathrm{E}-2 \\
1.48 \mathrm{E}-2 \\
1.48 \mathrm{E}-2 \\
1.48 \mathrm{E}-2 \\
0.1 \\
0.1 \\
0.1 \\
1.62 \mathrm{E}-4 \\
1.62 \mathrm{E}-4 \\
1.62 \mathrm{E}-4 \\
1.62 \mathrm{E}-4 \\
1.62 \mathrm{E}-4 \\
1.62 \mathrm{E}-4 \\
1.62 \mathrm{E}-4 \\
1.62 \mathrm{E}-4 \\
1.62 \mathrm{E}-4 \\
1.62 \mathrm{E}-4 \\
1.62 \mathrm{E}-4 \\
1.62 \mathrm{E}-4 \\
0.1 \\
0.1 \\
0.1 \\
0.1 \\
0.1 \\
0.1 \\
0.1 \\
0.1 \\
\end{array}$ & $\begin{array}{lll}0 & \\
0 & & \\
0 & & \\
0 & & \\
0 & & \\
0 & & \\
0 & & \\
0 & & \\
0 & & \\
0 & & \\
0 & & \\
0 & & \\
0 & & \\
0 & & \\
0 & 1 & 1.0 \\
1 & 1 & 1.0 \\
0 & & \\
0 & & \\
0 & & \\
1 & & \\
0 & 1 & 1.0 \\
0 & 1 & 0.985 \\
0 & & \\
0 & & \\
1 & & \\
0 & & \\
0 & & \\
0 & & \\
0 & 1 & 0.957 \\
0 & & \\
0 & & \\
0 & & \\
2 & & \\
1 & & \\
0 & & \\
0 & & \\
0 & & \\
0 & & \\
0 & & \\
0 & & \\
0 & & \\
0 & & \\
0 & & \\
0 & & \\
0 & & \\
0 & & \\
0 & & \\
0 & & \\
0 & & \\
0 & & \end{array}$ \\
\hline
\end{tabular}


TABLE D.3. Radionuclide Control List (RMDLIBDA.SHT) (contd)

\begin{tabular}{|c|c|c|c|c|c|c|}
\hline $\begin{array}{l}\text { TE132 } \\
\text { TE133M } \\
\text { TE133 } \\
\text { TE134 } \\
\text { I } 125 \\
\text { I } 129 \\
\text { I } 130 \\
\text { I } 131 \\
\text { I } 132 \\
\text { I } 133 \\
\text { I } 134 \\
\text { I } 135 \\
\text { CS134M } \\
\text { CS134 } \\
\text { CS135 } \\
\text { CS136 } \\
\text { CS137 } \\
\text { CS138 } \\
\text { BA139 } \\
\text { BA140 } \\
\text { BA141 } \\
\text { BA142 } \\
\text { LA140 } \\
\text { LA141 } \\
\text { LA142 } \\
\text { CE141 } \\
\text { CE143 } \\
\text { CE144 } \\
\text { PR143 } \\
\text { PR144M } \\
\text { PR144 } \\
\text { ND147 } \\
\text { PM147 } \\
\text { PM148M } \\
\text { PM148 } \\
\text { PM149 } \\
\text { PM151 } \\
\text { SM147 } \\
\text { SM151 } \\
\text { SM153 } \\
\text { EU152 } \\
\text { EU154 } \\
\text { EU155 } \\
\text { EU156 } \\
\text { GD153 } \\
\text { TB160 } \\
\text { HO166M } \\
\text { W } 181 \\
\text { W } 185 \\
\text { W } 187\end{array}$ & $\begin{array}{l}3.26 \mathrm{E}+0 \\
3.85 \mathrm{E}-2 \\
8.65 \mathrm{E}-3 \\
2.90 \mathrm{E}-2 \\
6.01 \mathrm{E}+1 \\
5.73 \mathrm{E}+9 \\
5.15 \mathrm{E}-1 \\
8.04 \mathrm{E}+0 \\
9.58 \mathrm{E}-2 \\
8.67 \mathrm{E}-1 \\
3.65 \mathrm{E}-2 \\
2.75 \mathrm{E}-1 \\
1.21 \mathrm{E}-1 \\
7.53 \mathrm{E}+2 \\
8.40 \mathrm{E}+8 \\
1.32 \mathrm{E}+1 \\
1.10 \mathrm{E}+4 \\
2.24 \mathrm{E}-2 \\
5.77 \mathrm{E}-2 \\
1.28 \mathrm{E}+1 \\
1.27 \mathrm{E}-2 \\
7.43 \mathrm{E}-3 \\
1.68 \mathrm{E}+0 \\
1.64 \mathrm{E}-1 \\
6.63 \mathrm{E}-2 \\
3.25 \mathrm{E}+1 \\
1.38 \mathrm{E}+0 \\
2.84 \mathrm{E}+2 \\
1.36 \mathrm{E}+1 \\
5.00 \mathrm{E}-3 \\
1.20 \mathrm{E}-2 \\
1.10 \mathrm{E}+1 \\
9.58 \mathrm{E}+2 \\
4.13 \mathrm{E}+1 \\
5.37 \mathrm{E}+0 \\
2.21 \mathrm{E}+0 \\
1.18 \mathrm{E}+0 \\
3.87 \mathrm{E} 13 \\
3.29 \mathrm{E}+4 \\
1.95 \mathrm{E}+0 \\
4.96 \mathrm{E}+3 \\
3.21 \mathrm{E}+3 \\
1.81 \mathrm{E}+3 \\
1.52 \mathrm{E}+1 \\
2.42 \mathrm{E}+2 \\
7.23 \mathrm{E}+1 \\
4.38 \mathrm{E}+5 \\
1.21 \mathrm{E}+2 \\
7.51 \mathrm{E}+1 \\
9.93 \mathrm{E}-1\end{array}$ & $\begin{array}{l}2.0 \\
3.1588 \\
3.0288 \\
2.0 \\
1.0 \\
1.0 \\
1.0 \\
1.0109 \\
1.0 \\
2.0288 \\
1.0 \\
2.165 \\
2.0 \\
1.0 \\
1.0 \\
1.0 \\
1.946 \\
1.0 \\
1.0 \\
2.0 \\
3.0 \\
2.0 \\
1.0 \\
2.0 \\
1.0 \\
1.0 \\
2.0 \\
2.0143 \\
1.0 \\
2.0 \\
1.0 \\
2.0 \\
1.0 \\
1.0420 \\
1.0 \\
1.0 \\
1.0 \\
1.0 \\
1.0 \\
1.0 \\
1.0 \\
1.0 \\
1.0 \\
1.0 \\
1.0 \\
1.0 \\
1.0 \\
1.0 \\
1.0 \\
1.0\end{array}$ & $\begin{array}{l}52 \\
52 \\
52 \\
52 \\
53 \\
53 \\
53 \\
53 \\
53 \\
53 \\
53 \\
53 \\
55 \\
55 \\
55 \\
55 \\
55 \\
55 \\
56 \\
56 \\
56 \\
56 \\
57 \\
57 \\
57 \\
58 \\
58 \\
58 \\
59 \\
59 \\
59 \\
60 \\
61 \\
61 \\
61 \\
61 \\
61 \\
62 \\
62 \\
62 \\
63 \\
63 \\
63 \\
63 \\
64 \\
65 \\
67 \\
74 \\
74 \\
74\end{array}$ & $\begin{array}{l}\text { SW } \\
\text { SW } \\
\text { SW } \\
\text { SW } \\
2 \text { ND } \\
2 \text { ND } \\
2 \text { ND } \\
2 \text { ND } \\
2 \text { ND } \\
2 \text { ND } \\
2 \text { ND } \\
2 \text { ND } \\
\text { ND } \\
\text { ND } \\
\text { ND } \\
\text { ND } \\
\text { ND } \\
\text { ND } \\
1 \text { SD } \\
1 \text { SD } \\
1 \text { SD } \\
1 \text { SD } \\
\text { SD } \\
\text { SD } \\
\text { SD } \\
\text { SY } \\
\text { SY } \\
\text { SY } \\
\text { SY } \\
\text { SY } \\
\text { SY } \\
\text { SY } \\
\text { SY } \\
\text { SY } \\
\text { SY } \\
\text { SY } \\
\text { SY } \\
\text { SW } \\
\text { SW } \\
\text { SW } \\
\text { SW } \\
\text { SW } \\
\text { SW } \\
\text { SW } \\
\text { SD } \\
\text { SW } \\
\text { SW } \\
\text { VD } \\
\text { VD } \\
\text { SD }\end{array}$ & $\begin{array}{l}0.1 \\
0.1 \\
0.1 \\
0.1 \\
1.15 \mathrm{E}-1 \\
1.15 \mathrm{E}-1 \\
1.15 \mathrm{E}-1 \\
1.15 \mathrm{E}-1 \\
1.15 \mathrm{E}-1 \\
1.15 \mathrm{E}-1 \\
1.15 \mathrm{E}-1 \\
1.15 \mathrm{E}-1 \\
1.62 \mathrm{E}-4 \\
1.62 \mathrm{E}-4 \\
1.62 \mathrm{E}-4 \\
1.62 \mathrm{E}-4 \\
1.62 \mathrm{E}-4 \\
1.62 \mathrm{E}-4 \\
1.11 \mathrm{E}-4 \\
1.11 \mathrm{E}-4 \\
1.11 \mathrm{E}-4 \\
1.11 \mathrm{E}-4 \\
1.11 \mathrm{E}-4 \\
1.11 \mathrm{E}-4 \\
1.11 \mathrm{E}-4 \\
4.67 \mathrm{E}-4 \\
4.67 \mathrm{E}-4 \\
4.67 \mathrm{E}-4 \\
0.1 \\
0.1 \\
0.1 \\
0.1 \\
0.1 \\
0.1 \\
0.1 \\
0.1 \\
0.1 \\
0.1 \\
0.1 \\
0.1 \\
1.11 \mathrm{E}-4 \\
1.11 \mathrm{E}-4 \\
1.11 \mathrm{E}-4 \\
1.11 \mathrm{E}-4 \\
0.1 \\
0.1 \\
0.1 \\
0.1 \\
0.1 \\
0.1 \\
\end{array}$ & $\begin{array}{l}0 \\
0 \\
0 \\
0 \\
0 \\
0 \\
0 \\
0 \\
0 \\
0 \\
0 \\
0 \\
0 \\
0 \\
1 \\
0 \\
1 \\
0 \\
0 \\
0 \\
0 \\
0 \\
0 \\
0 \\
0 \\
0 \\
0 \\
0 \\
0 \\
0 \\
0 \\
0 \\
0 \\
0 \\
0 \\
0 \\
0 \\
0 \\
0 \\
1 \\
0 \\
0 \\
0 \\
0 \\
0 \\
0 \\
0 \\
0 \\
0 \\
0 \\
0 \\
0 \\
\\
\\
\end{array}$ \\
\hline
\end{tabular}


TABLE D.3. Radionuclide Control List (RMDLIBDA.SHT) (contd)

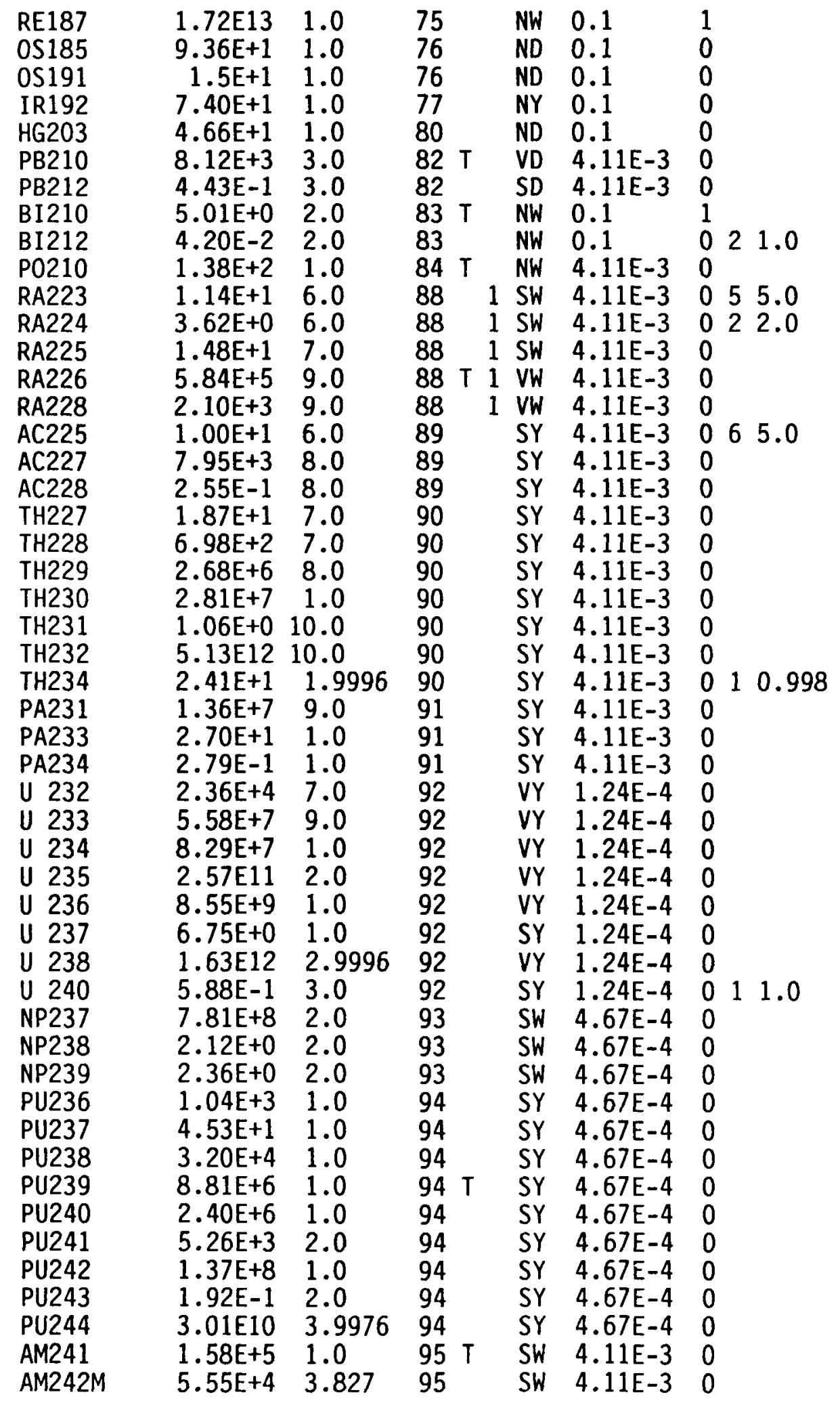


TABLE D.3. Radionuclide Control List (RMDLIBDA.SHT) (contd)

$\begin{array}{lllllll}\text { AM242 } & 6.68 E-1 & 2.827 & 95 & \text { SW } & 4.11 E-3 & 0 \\ \text { AM243 } & 2.69 E+6 & 3.0 & 95 & \text { SW } & 4.11 E-3 & 0 \\ \text { CM242 } & 1.63 E+2 & 1.8270 & 96 & \text { SW } & 4.67 E-4 & 0 \\ \text { CM243 } & 1.04 E+4 & 1.0 & 96 & \text { SW } & 4.67 E-4 & 0 \\ \text { CM244 } & 6.61 E+3 & 2.0 & 96 & \text { SW } & 4.67 E-4 & 0 \\ \text { CM245 } & 3.10 E+6 & 3.0 & 96 & \text { SW } & 4.67 E-4 & 0 \\ \text { CM246 } & 1.73 E+6 & 1.0 & 96 & \text { SW } & 4.67 E-4 & 0 \\ \text { CM247 } & 5.69 E+9 & 2.0 & 96 & \text { SW } & 4.67 E-4 & 0 \\ \text { CM248 } & 1.24 E+8 & 1.0 & 96 & \text { SW } & 4.67 E-4 & 0 \\ \text { CF252 } & 9.63 E+2 & 1.0 & 98 & \text { SW } & 4.11 E-3 & 0\end{array}$




\section{TABLE D.4. External Dose Rate Factors for Individual}

Radionuclides (EXTERNAL.DF)

NRC Residual Radiation External DFs (rem/h per Ci/cm2 or cm3) 21-Dec-89 RAP Col 1: Infinite disk source, concrete

Col 2: $1 \mathrm{~cm}$ thick, $5 \mathrm{~m}$ disk source, concrete

Col 3: $15 \mathrm{~cm}$ thick infinite slab source, soil

H $3 \quad 5.29 \mathrm{E}-05 \quad 4.23 \mathrm{E}-06 \quad 4.38 \mathrm{E}-06$

$\begin{array}{llll}\mathrm{BE} 10 & 1.89 \mathrm{E}+01 \quad 5.87 \mathrm{E}+00 \quad 5.48 \mathrm{E}+01\end{array}$

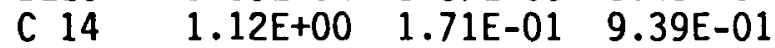

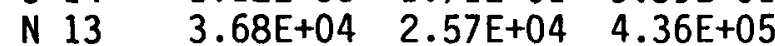

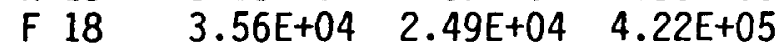

$\begin{array}{llll}\mathrm{NA} 22 & 7.98 \mathrm{E}+04 & 5.78 \mathrm{E}+04 & 8.89 \mathrm{E}+05\end{array}$

$\begin{array}{llll}\mathrm{NA} 24 & 1.48 \mathrm{E}+05 & 1.14 \mathrm{E}+05 & 1.72 \mathrm{E}+06\end{array}$

$\begin{array}{llll}\mathrm{SI} 31 & 1.63 \mathrm{E}+02 & 9.38 \mathrm{E}+01 & 1.22 \mathrm{E}+03\end{array}$

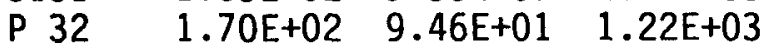

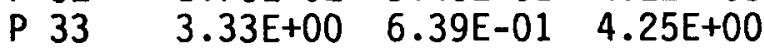

S $35 \quad 1.26 \mathrm{E}+00 \quad 1.99 \mathrm{E}-01 \quad 1.13 \mathrm{E}+00$

$\begin{array}{llll}\text { CL36 } & 2.91 E+01 & 1.06 E+01 & 1.09 E+02\end{array}$

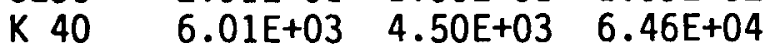

$\begin{array}{llll}\text { AR39 } & 1.88 E+01 & 5.89 E+00 & 5.52 E+01\end{array}$

AR41 4.64E+04 3.45E+04 4.93E+05

CA41 $7.19 E+01 \quad 5.75 E+00 \quad 5.95 E+00$

CA45 3.45E+00 6.71E-01 4.51E+00

$\begin{array}{llll}\text { SC46 } & 7.92 E+04 & 5.82 E+04 & 8.28 E+05\end{array}$

$\begin{array}{llll}\text { CR51 } & 1.48 \mathrm{E}+03 & 9.28 \mathrm{E}+02 & 1.46 \mathrm{E}+04\end{array}$

$\begin{array}{llll}\text { MN54 } & 3.27 \mathrm{E}+04 & 2.35 \mathrm{E}+04 & 3.31 \mathrm{E}+05\end{array}$

$\begin{array}{llll}\text { MN56 } & 6.49 E+04 & 4.84 E+04 & 7.14 E+05\end{array}$

$\begin{array}{llll}F E 55 & 1.64 E+02 & 1.31 E+01 & 1.36 E+01\end{array}$

FE59 4.23E+04 3.14E+04 4.57E+05

$\begin{array}{llll}\mathrm{C} 057 & 5.25 \mathrm{E}+03 \quad 2.85 \mathrm{E}+03 & 3.15 \mathrm{E}+04\end{array}$

$\begin{array}{lll}\mathrm{C} 058 & 3.86 \mathrm{E}+04 \quad 2.76 \mathrm{E}+04 \quad 4.01 \mathrm{E}+05\end{array}$

$\begin{array}{llll}\text { C060 } & 9.34 \mathrm{E}+04 & 6.95 \mathrm{E}+04 & 9.93 \mathrm{E}+05\end{array}$

NI59 2.00E+02 1.60E+01 1.65E+01

$\begin{array}{llll}\text { NI63 } & 8.94 E-02 & 8.23 E-03 & 2.39 E-02\end{array}$

$\begin{array}{llll}N I 65 & 2.15 \mathrm{E}+04 & 1.60 \mathrm{E}+04 & 2.31 \mathrm{E}+05\end{array}$

$\begin{array}{llll}\text { CU64 } & 6.92 \mathrm{E}+03 & 4.78 \mathrm{E}+03 & 8.05 \mathrm{E}+04\end{array}$

$\begin{array}{llll}\text { ZN65 } & 2.44 E+04 & 1.80 E+04 & 2.58 E+05\end{array}$

$\begin{array}{llll}\text { ZN69M } & 1.75 E+04 & 1.22 E+04 & 2.07 E+05\end{array}$

$\begin{array}{llll}\text { ZN69 } & 4.82 \mathrm{E}+01 & 1.93 \mathrm{E}+01 & 2.10 \mathrm{E}+02\end{array}$

$\begin{array}{llll}\text { GA72 } & 1.02 E+05 & 7.61 E+04 & 1.13 E+06\end{array}$

$\begin{array}{llll}\text { AS76 } & 1.91 E+04 & 1.36 E+04 & 2.04 E+05\end{array}$

SE75 $1.46 E+04 \quad 9.06 E+03 \quad 1.22 E+05$

SE79 8.66E-01 1.26E-01 6.70E-01

BR82 1.07E+05 7.71E+04 $1.13 E+06$

$\begin{array}{llll}B R 83 & 3.07 E+02 & 2.00 E+02 & 3.28 E+03\end{array}$

$\begin{array}{llll}\text { KR83M } & 1.36 \mathrm{E}+02 & 1.10 \mathrm{E}+01 & 1.22 \mathrm{E}+01\end{array}$

$\begin{array}{llll}B R 84 & 6.23 E+04 & 4.73 E+04 & 7.21 E+05\end{array}$

$\begin{array}{llll}\text { KR85M } & 5.77 \mathrm{E}+03 \quad 3.52 \mathrm{E}+03 \quad 4.54 \mathrm{E}+04\end{array}$

$\begin{array}{llll}K R 85 & 1.06 E+02 & 6.50 E+01 & 1.04 E+03\end{array}$

$\begin{array}{llll}\text { KR87 } 3.10 E+04 & 2.30 E+04 & 3.60 E+05\end{array}$

RB87 $3.64 \mathrm{E}+00 \quad 7.30 \mathrm{E}-01 \quad 5.03 \mathrm{E}+00$ 
TABLE D.4. External Dose Rate Factors for Individual Radionuclides (EXTERNAL.DF) (contd)

$\begin{array}{llll}\text { KR88 } & 6.90 E+04 & 5.25 E+04 & 7.85 E+05 \\ \text { RB88 } & 2.55 E+04 & 1.92 E+04 & 2.90 E+05 \\ \text { KR89 } & 6.77 E+04 & 5.06 E+04 & 7.66 E+05 \\ \text { RB89 } & 7.62 E+04 & 5.72 E+04 & 8.52 E+05 \\ \text { SR89 } & 1.43 E+02 & 7.09 E+01 & 8.83 E+02 \\ \text { SR87M } & 1.13 E+04 & 7.69 E+03 & 1.22 E+05 \\ \text { RB86 } & 3.58 E+03 & 2.61 E+03 & 3.85 E+04 \\ \text { SR85 } & 1.86 E+04 & 1.28 E+04 & 2.17 E+05 \\ \text { SR90 } & 1.61 E+01 & 4.87 E+00 & 4.47 E+01 \\ \text { Y 90 } & 3.47 E+02 & 1.90 E+02 & 2.50 E+03 \\ \text { SR91 } & 2.61 E+04 & 1.89 E+04 & 2.81 E+05 \\ \text { Y91M } & 2.49 E+04 & 1.75 E+04 & 2.65 E+05 \\ \text { Y91 } & 2.87 E+02 & 1.76 E+02 & 2.38 E+03 \\ \text { SR92 } & 5.37 E+04 & 4.04 E+04 & 5.81 E+05 \\ \text { Y92 } & 1.11 E+04 & 8.11 E+03 & 1.20 E+05 \\ \text { Y93 } & 3.90 E+03 & 2.77 E+03 & 4.05 E+04 \\ \text { M093 } & 3.84 E+02 & 3.07 E+01 & 3.18 E+01 \\ \text { ZR93 } & 7.09 E-02 & 6.31 E-03 & 1.68 E-02 \\ \text { NB93M } & 7.97 E+01 & 6.37 E+00 & 6.60 E+00 \\ \text { ZR95 } & 2.95 E+04 & 2.10 E+04 & 3.05 E+05 \\ \text { NB95M } & 2.62 E+03 & 1.54 E+03 & 2.07 E+04 \\ \text { NB95 } & 3.25 E+04 & 2.34 E+04 & 3.31 E+05 \\ \text { ZR97 } & 7.17 E+03 & 5.19 E+03 & 7.75 E+04 \\ \text { NB97M } & 2.56 E+04 & 1.80 E+04 & 2.73 E+05 \\ \text { NB97 } & 2.65 E+04 & 1.86 E+04 & 2.82 E+05 \\ \text { NB94 } & 5.87 E+04 & 4.19 E+04 & 6.10 E+05 \\ \text { M099 } & 5.72 E+03 & 3.94 E+03 & 5.74 E+04 \\ \text { TC99M } & 4.52 E+03 & 2.59 E+03 & 2.88 E+04 \\ \text { TC99 } & 4.19 E+00 & 8.86 E-01 & 6.33 E+00 \\ \text { TC101 } & 1.47 E+04 & 1.00 E+04 & 1.59 E+05 \\ \text { RU103 } & 1.82 E+04 & 1.27 E+04 & 2.13 E+05 \\ \text { PD103 } & 1.04 E+03 & 8.89 E+01 & 3.95 E+02 \\ \text { RH103M } & 1.36 E+02 & 1.14 E+01 & 4.18 E+01 \\ \text { RU105 } & 3.13 E+04 & 2.14 E+04 & 3.27 E+05 \\ \text { RH105 } & 3.37 E+03 & 2.29 E+03 & 3.64 E+04 \\ \text { RU106 } & 8.72 E+03 & 6.08 E+03 & 9.57 E+04 \\ \text { PD107 } & 5.45 E-03 & 4.37 E-04 & 5.21 E-04 \\ \text { PD109 } & 7.09 E+02 & 1.35 E+02 & 1.22 E+03 \\ \text { AG110M } & 1.08 E+05 & 7.83 E+04 & 1.15 E+06 \\ \text { AG111 } & 1.11 E+03 & 7.34 E+02 & 1.14 E+04 \\ \text { CD109 } & 9.81 E+02 & 8.16 E+01 & 3.30 E+02 \\ \text { CD113M } & 1.81 E+01 & 5.73 E+00 & 5.41 E+01 \\ \text { CD115M } & 1.02 E+03 & 7.17 E+02 & 1.04 E+04 \\ \text { CD115 } & 7.33 E+03 & 5.05 E+03 & 8.49 E+04 \\ \text { IN115M } & 6.90 E+03 & 4.41 E+03 & 6.97 E+04 \\ \text { IN111 } & 1.46 E+04 & 8.38 E+03 & 1.07 E+05 \\ \text { IN114M } & 5.30 E+03 & 3.23 E+03 & 4.63 E+04 \\ \text { SN113 } & 1.28 E+03 & 2.08 E+02 & 1.96 E+03 \\ \text { IN113M } & 9.23 E+03 & 6.09 E+03 & 9.66 E+04\end{array}$


TABLE 0.4. External Dose Rate Factors for Individual Radionuclides (EXTERNAL.DF) (contd)

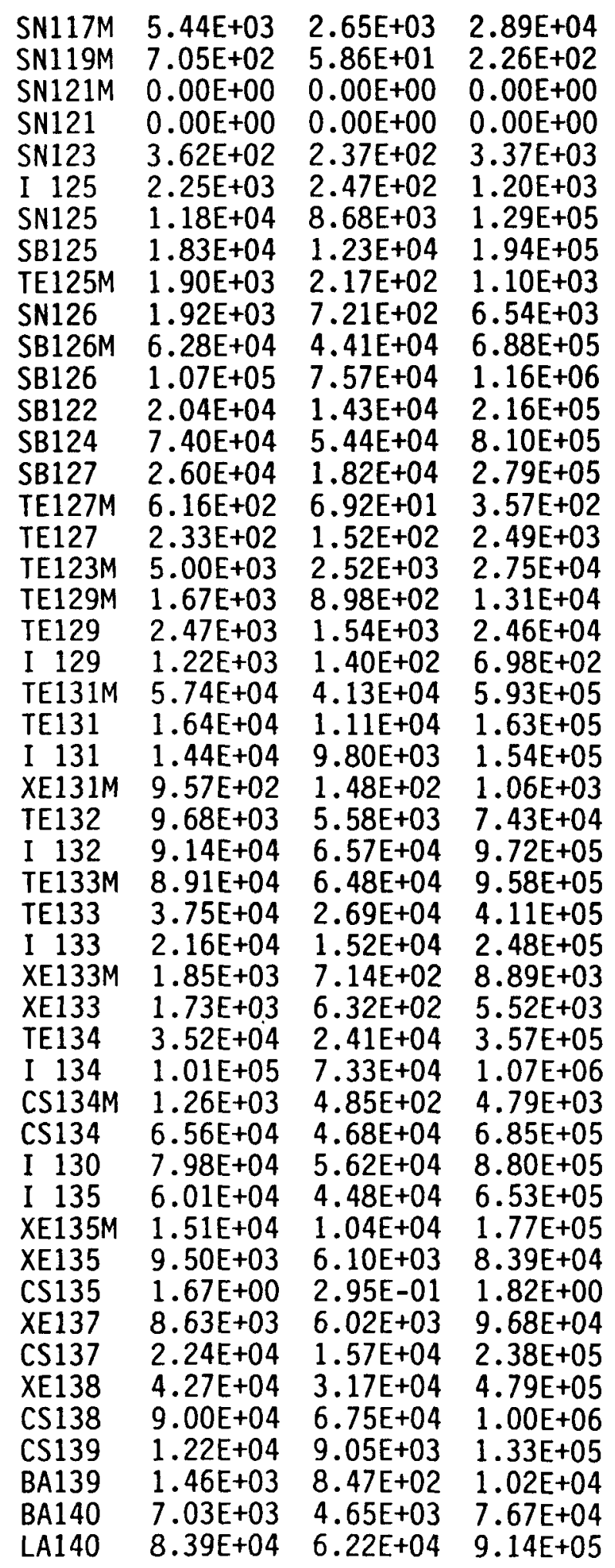


TABLE D.4. External Dose Rate Factors for Individual Radionuclides (EXTERNAL.DF) (contd)

$\begin{array}{llll}\text { CS136 } & 8.28 E+04 & 5.96 E+04 & 8.64 E+05 \\ \text { BA141 } & 3.37 E+04 & 2.36 E+04 & 3.49 E+05 \\ \text { LA141 } & 2.10 E+03 & 1.51 E+03 & 2.15 E+04 \\ \text { CE141 } & 2.74 E+03 & 1.47 E+03 & 1.60 E+04 \\ \text { BA142 } & 3.50 E+04 & 2.52 E+04 & 3.65 E+05 \\ \text { LA142 } & 9.75 E+04 & 7.42 E+04 & 1.14 E+06 \\ \text { CE143 } & 9.94 E+03 & 6.10 E+03 & 8.60 E+04 \\ \text { PR143 } & 4.80 E+01 & 1.93 E+01 & 2.11 E+02 \\ \text { CE144 } & 7.49 E+02 & 3.69 E+02 & 3.90 E+03 \\ \text { PR144M } & 5.71 E+02 & 1.14 E+02 & 6.70 E+02 \\ \text { PR144 } & 1.73 E+03 & 1.20 E+03 & 1.75 E+04 \\ \text { PR142 } & 2.33 E+03 & 1.70 E+03 & 2.42 E+04 \\ \text { ND147 } & 5.11 E+03 & 3.04 E+03 & 4.58 E+04 \\ \text { PM147 } & 2.38 E+00 & 4.83 E-01 & 3.44 E+00 \\ \text { SM147 } & 0.00 E+00 & 0.00 E+00 & 0.00 E+00 \\ \text { PM148M } & 8.39 E+04 & 5.93 E+04 & 9.02 E+05 \\ \text { PM148 } & 2.36 E+04 & 1.74 E+04 & 2.54 E+05 \\ \text { PM149 } & 4.69 E+02 & 2.93 E+02 & 4.02 E+03 \\ \text { PM151 } & 1.32 E+04 & 8.65 E+03 & 1.29 E+05 \\ \text { SM151 } & 1.19 E+00 & 9.88 E-02 & 2.44 E-01 \\ \text { SM153 } & 2.73 E+03 & 1.21 E+03 & 1.21 E+04 \\ \text { EU152M } & 1.26 E+04 & 8.88 E+03 & 1.27 E+05 \\ \text { EU152 } & 4.57 E+04 & 3.27 E+04 & 4.72 E+05 \\ \text { EU154 } & 4.73 E+04 & 3.41 E+04 & 4.91 E+05 \\ \text { EU155 } & 2.28 E+03 & 1.12 E+03 & 1.13 E+04 \\ \text { EU156 } & 5.09 E+04 & 3.79 E+04 & 5.60 E+05 \\ \text { GD153 } & 4.35 E+03 & 1.75 E+03 & 1.61 E+04 \\ \text { GD159 } & 1.57 E+03 & 9.38 E+02 & 1.40 E+04 \\ \text { TB160 } & 4.12 E+04 & 2.97 E+04 & 4.25 E+05 \\ \text { TB161 } & 9.49 E+02 & 2.55 E+02 & 1.87 E+03 \\ \text { DY165 } & 1.05 E+03 & 6.22 E+02 & 8.67 E+03 \\ \text { H0166M } & 6.07 E+04 & 4.22 E+04 & 6.10 E+05 \\ \text { H0166 } & 1.29 E+03 & 7.81 E+02 & 1.03 E+04 \\ \text { ER169 } & 6.69 E+00 & 1.44 E+00 & 1.07 E+01 \\ \text { ER171 } & 1.51 E+04 & 9.73 E+03 & 1.43 E+05 \\ \text { TA182 } & 5.07 E+04 & 3.67 E+04 & 5.18 E+05 \\ \text { W181 } & 1.34 E+03 & 4.75 E+02 & 3.47 E+03 \\ \text { W 185 } & 1.03 E+01 & 3.01 E+00 & 2.69 E+01 \\ \text { W 187 } & 1.86 E+04 & 1.28 E+04 & 1.95 E+05 \\ \text { RE187 } & 0.00 E+00 & 0.00 E+00 & 0.00 E+00 \\ \text { 0S185 } & 2.78 E+04 & 1.91 E+04 & 2.82 E+05 \\ \text { 0S191 } & 2.65 E+03 & 1.26 E+03 & 1.24 E+04 \\ \text { IR192 } & 3.34 E+04 & 2.29 E+04 & 3.63 E+05 \\ \text { HG203 } & 7.67 E+03 & 4.84 E+03 & 6.50 E+04 \\ \text { TH230 } & 5.98 E+01 & 9.06 E+00 & 5.15 E+01 \\ \text { RA226 } & 1.86 E+02 & 1.04 E+02 & 1.15 E+03 \\ \text { RN222 } & 6.85 E+04 & 5.00 E+04 & 7.38 E+05 \\ \text { PB210 } & 2.11 E+02 & 2.99 E+01 & 1.31 E+02 \\ \text { B1210 } & 7.08 E+01 & 3.09 E+01 & 3.58 E+02\end{array}$


TABLE D.4. External Dose Rate Factors for Individual Radionuclides (EXTERNAL.DF) (contd)

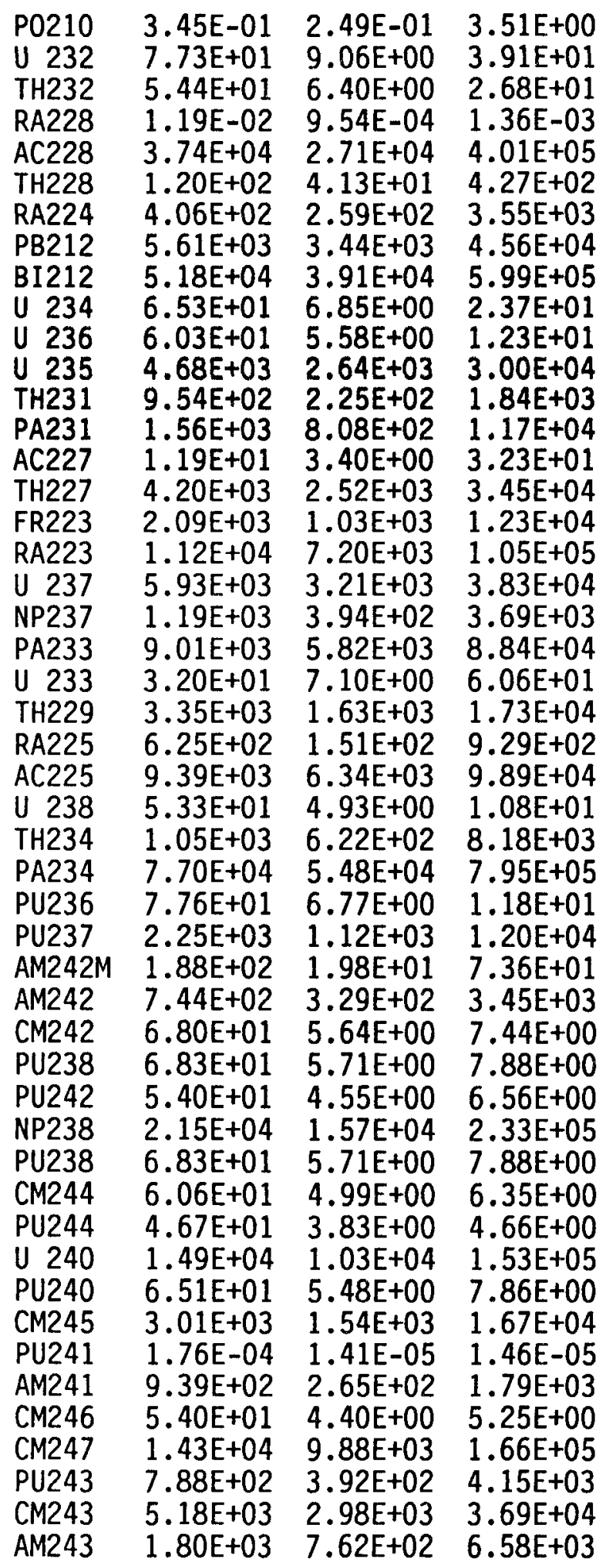


TABLE D.4. External Dose Rate Factors for Individual Radionuclides (EXTERNAL.DF) (contd)

$\begin{array}{llll}\text { NP239 } & 6.96 \mathrm{E}+03 & 4.05 \mathrm{E}+03 & 5.06 \mathrm{E}+04 \\ \text { PU239 } & 2.81 \mathrm{E}+01 & 3.44 \mathrm{E}+00 & 1.75 \mathrm{E}+01 \\ \text { CM248 } & 4.28 \mathrm{E}+01 & 3.56 \mathrm{E}+00 & 4.78 \mathrm{E}+00 \\ \text { CF252 } & 4.29 \mathrm{E}+01 & 3.62 \mathrm{E}+00 & 5.32 \mathrm{E}+00\end{array}$


TABLE D.5. External Dose Rate Factors for Radionuclide Chains in Equilibrium (EXTDAU.DF)

NRC Resid. Rad. External DFs w/daughters (rem/h per pCi/m2 or g) 27-Jan-90 RAP

Col 1: Infinite disk source, concrete

Col 2: $1 \mathrm{~cm}$ thick, $5 \mathrm{~m}$ disk source, concrete

Col 3: $15 \mathrm{~cm}$ thick infinite slab source, soil

H $3 \quad 3.4 \mathrm{E}-206.2 \mathrm{E}-16$ 6.4E-16

BE10 1.2E-14 8.6E-10 8.0E-09

C $14 \quad 7.2 \mathrm{E}-16 \quad 2.5 \mathrm{E}-11 \quad 1.4 \mathrm{E}-10$

F 18 2.3E-11 3.6E-06 6.2E-05

NA22 5.1E-11 $8.4 \mathrm{E}-06 \quad 1.3 \mathrm{E}-04$

NA24 9.5E-11 $1.7 \mathrm{E}-05 \quad 2.5 \mathrm{E}-04$

P 32 1.1E-13 1.4E-08 $1.8 \mathrm{E}-07$

P 33 2.1E-15 9.3E-11 $6.2 \mathrm{E}-10$

S 35 8.1E-16 2.9E-11 $1.6 \mathrm{E}-10$

CL36 1.9E-14 1.6E-09 $1.6 \mathrm{E}-08$

K $40 \quad 3.9 \mathrm{E}-12 \quad 6.6 \mathrm{E}-07$ 9.4E-06

CA41 4.6E-14 8.4E-10 8.7E-10

CA45 2.2E-15 9.8E-11 6.6E-10

SC46 5.1E-11 8.5E-06 $1.2 \mathrm{E}-04$

CR51 9.5E-13 1.4E-07 2.1E-06

MN54 2.1E-11 3.4E-06 4.8E-05

MN56 4.2E-11 7.1E-06 1.0E-04

FE55 1.1E-13 1.9E-09 2.0E-09

FE59 2.7E-11 4.6E-06 6.7E-05

C057 3.4E-12 4.2E-07 4.6E-06

C058 2.5E-11 4.0E-06 5.9E-05

C060 6.0E-11 1.0E-05 $1.4 \mathrm{E}-04$

NI59 1.3E-13 2.3E-09 2.4E-09

NI63 5.8E-17 1.2E-12 3.5E-12

NI65 1.4E-11 2.3E-06 3.4E-05

CU64 4.4E-12 7.0E-07 $1.2 \mathrm{E}-05$

ZN65 1.6E-11 2.6E-06 3.8E-05

ZN69M 1.1E-11 1.8E-06 3.0E-05

ZN69 3.1E-14 2.8E-09 3.1E-08

AS76 1.2E-11 2.0E-06 3.0E-05

SE75 9.4E-12 $1.3 \mathrm{E}-06 \quad 1.8 \mathrm{E}-05$

SE79 5.6E-16 $1.8 \mathrm{E}-11 \quad 9.8 \mathrm{E}-11$

BR82 6.9E-11 $1.1 \mathrm{E}-05 \quad 1.6 \mathrm{E}-04$

BR83 2.9E-13 3.1E-08 4.8E-07

BR84 4.0E-11 6.9E-06 $1.1 \mathrm{E}-04$

RB86 2.3E-12 3.8E-07 5.6E-06

RB88 1.6E-11 2.8E-06 4.2E-05

RB89 4.9E-11 8.4E-06 $1.2 \mathrm{E}-04$

SR85 1.2E-11 $1.9 \mathrm{E}-06 \quad 3.2 \mathrm{E}-05$

SR89 9.2E-14 $1.0 \mathrm{E}-08$ 1.3E-07

SR90 2.3E-13 2.8E-08 3.7E-07

SR91 2.6E-11 $4.3 \mathrm{E}-06 \quad 6.5 \mathrm{E}-05$

SR92 4.2E-11 7.1E-06 1.0E-04

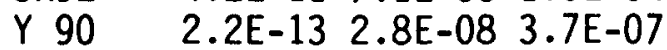

Y $91 \mathrm{M} \quad 1.6 \mathrm{E}-112.6 \mathrm{E}-06 \quad 3.9 \mathrm{E}-05$ 
TABLE D.5. External Dose Rate Factors for Radionuclide Chains in Equilibrium (EXTDAU.DF) (contd)

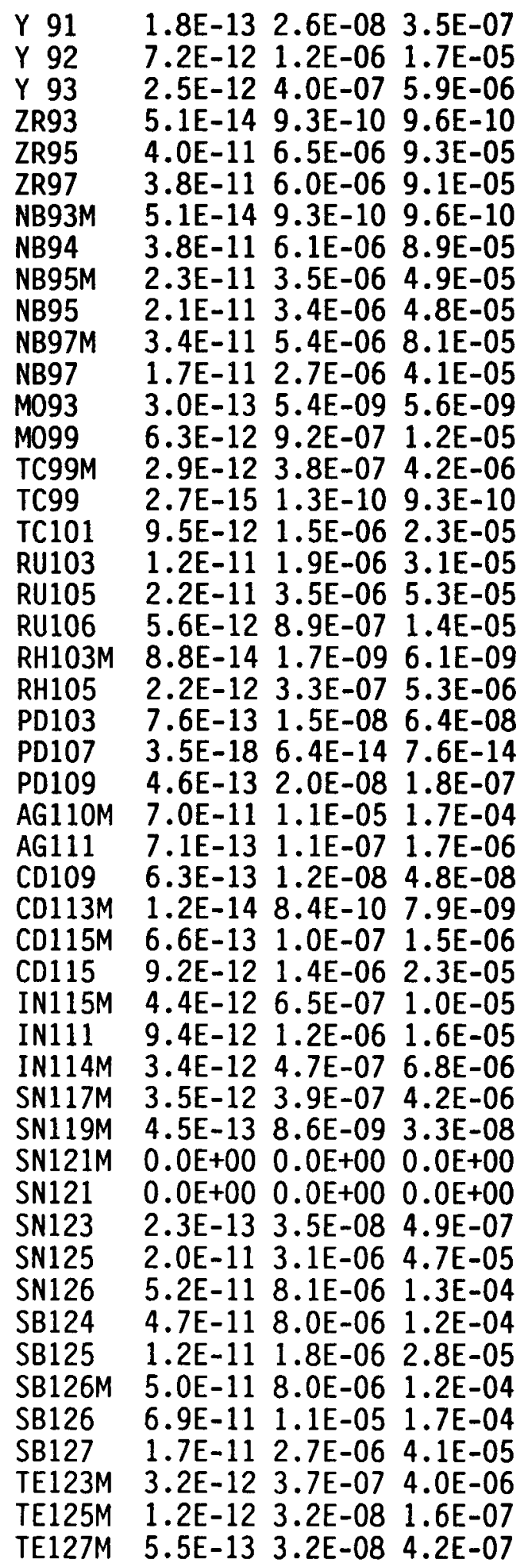


TABLE D.5. External Dose Rate Factors for Radionuclide Chains in Equilibrium (EXTDAU.DF) (contd)

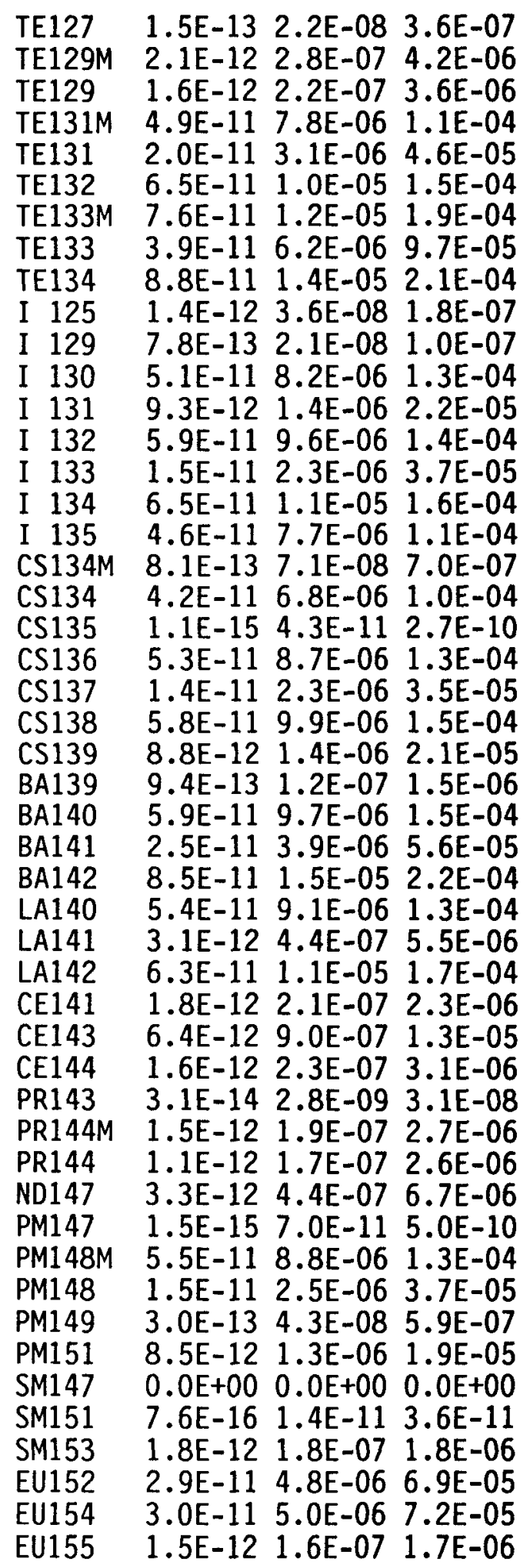


TABLE D.5. External Dose Rate Factors for Radionuclide Chains in Equilibrium (EXTDAU.DF) (contd)

\begin{tabular}{|c|c|c|}
\hline & $\begin{array}{ll}3.3 \mathrm{E}-11 & 5.5 \mathrm{E}-06 \\
2.8 \mathrm{E}-12 & 2.6 \mathrm{E}-07 \\
2.6 \mathrm{E}-11 & 4.3 \mathrm{E}-06 \\
3.9 \mathrm{E}-11 & 6.2 \mathrm{E}-06 \\
8.6 \mathrm{E}-13 & 6.9 \mathrm{E}-08 \\
6.6 \mathrm{E}-15 & 4.4 \mathrm{E}-10 \\
1.2 \mathrm{E}-11 & 1.9 \mathrm{E}-06 \\
0.0 \mathrm{E}+00 & 0.0 \mathrm{E}+00 \\
1.8 \mathrm{E}-11 & 2.8 \mathrm{E}-06 \\
1.7 \mathrm{E}-12 & 1.8 \mathrm{E}-07 \\
2.1 \mathrm{E}-11 & 3.3 \mathrm{E}-06 \\
4.9 \mathrm{E}-12 & 7.1 \mathrm{E}-07 \\
1.8 \mathrm{E}-13 & 8.9 \mathrm{E}-09 \\
3.7 \mathrm{E}-11 & 6.2 \mathrm{E}-06 \\
4.6 \mathrm{E}-14 & 4.6 \mathrm{E}-09 \\
3.3 \mathrm{E}-11 & 5.7 \mathrm{E}-06 \\
2.2 \mathrm{E}-16 & 3.6 \mathrm{E}-11 \\
7.2 \mathrm{E}-12 & 1.1 \mathrm{E}-06 \\
3.7 \mathrm{E}-11 & 6.3 \mathrm{E}-06 \\
6.4 \mathrm{E}-12 & 9.5 \mathrm{E}-07 \\
4.4 \mathrm{E}-11 & 7.4 \mathrm{E}-06 \\
6.1 \mathrm{E}-11 & 1.0 \mathrm{E}-05 \\
6.0 \mathrm{E}-12 & 9.2 \mathrm{E}-07 \\
9.9 \mathrm{E}-12 & 1.4 \mathrm{E}-06 \\
6.1 \mathrm{E}-11 & 1.0 \mathrm{E}-05 \\
9.9 \mathrm{E}-12 & 1.4 \mathrm{E}-06 \\
3.7 \mathrm{E}-11 & 6.3 \mathrm{E}-06 \\
8.6 \mathrm{E}-12 & 1.2 \mathrm{E}-06 \\
4.4 \mathrm{E}-11 & 7.4 \mathrm{E}-06 \\
6.1 \mathrm{E}-13 & 3.3 \mathrm{E}-08 \\
6.1 \mathrm{E}-11 & 1.0 \mathrm{E}-05 \\
7.6 \mathrm{E}-13 & 1.0 \mathrm{E}-07 \\
1.1 \mathrm{E}-11 & 1.5 \mathrm{E}-06 \\
5.8 \mathrm{E}-12 & 8.5 \mathrm{E}-07 \\
5.0 \mathrm{E}-11 & 8.0 \mathrm{E}-06 \\
5.0 \mathrm{E}-14 & 1.3 \mathrm{E}-09 \\
8.6 \mathrm{E}-12 & 1.2 \mathrm{E}-06 \\
4.2 \mathrm{E}-14 & 1.0 \mathrm{E}-09 \\
3.6 \mathrm{E}-12 & 4.2 \mathrm{E}-07 \\
3.9 \mathrm{E}-14 & 8.1 \mathrm{E}-10 \\
3.8 \mathrm{E}-12 & 4.7 \mathrm{E}-07 \\
7.9 \mathrm{E}-13 & 1.0 \mathrm{E}-07 \\
9.6 \mathrm{E}-12 & 1.5 \mathrm{E}-06 \\
6.6 \mathrm{E}-12 & 9.1 \mathrm{E}-07 \\
1.4 \mathrm{E}-11 & 2.3 \mathrm{E}-06 \\
4.5 \mathrm{E}-12 & 5.9 \mathrm{E}-07 \\
5.0 \mathrm{E}-14 & 9.9 \mathrm{E}-10 \\
1.4 \mathrm{E}-12 & 1.6 \mathrm{E}-07 \\
4.4 \mathrm{E}-14 & 8.3 \mathrm{E}-10\end{array}$ & $\begin{array}{l}-06 \\
-05 \\
-05 \\
-07 \\
-09 \\
-05 \\
+00 \\
-05 \\
-06 \\
-05 \\
-06 \\
-08 \\
-05 \\
-08 \\
-05 \\
-10 \\
-05 \\
-05 \\
-05 \\
-04 \\
-04 \\
-05 \\
-05 \\
-04 \\
-05 \\
-05 \\
-05 \\
-04 \\
-05 \\
-04 \\
-06 \\
-05 \\
-05 \\
-04 \\
-05 \\
-05 \\
-05 \\
-09 \\
-06 \\
-09 \\
-06\end{array}$ \\
\hline
\end{tabular}


TABLE D.5. External Dose Rate Factors for Radionuclide Chains in Equilibrium (EXTDAU.DF) (contd)

$\begin{array}{llll}\text { PU239 } & 1.8 \mathrm{E}-14 & 5.0 \mathrm{E}-10 & 2.6 \mathrm{E}-09 \\ \text { PU240 } & 4.2 \mathrm{E}-14 & 8.0 \mathrm{E}-10 & 1.1 \mathrm{E}-09 \\ \text { PU241 } & 6.1 \mathrm{E}-13 & 3.9 \mathrm{E}-08 & 2.6 \mathrm{E}-07 \\ \text { PU242 } & 3.5 \mathrm{E}-14 & 6.7 \mathrm{E}-10 & 9.6 \mathrm{E}-10 \\ \text { PU243 } & 3.3 \mathrm{E}-12 & 4.3 \mathrm{E}-07 & 5.4 \mathrm{E}-06 \\ \text { PU244 } & 9.6 \mathrm{E}-12 & 1.5 \mathrm{E}-06 & 2.2 \mathrm{E}-05 \\ \text { AM241 } & 6.1 \mathrm{E}-13 & 3.9 \mathrm{E}-08 & 2.6 \mathrm{E}-07 \\ \text { AM242M } & 6.7 \mathrm{E}-13 & 5.2 \mathrm{E}-08 & 5.2 \mathrm{E}-07 \\ \text { AM242 } & 5.5 \mathrm{E}-13 & 4.9 \mathrm{E}-08 & 5.1 \mathrm{E}-07 \\ \text { AM243 } & 5.6 \mathrm{E}-12 & 7.0 \mathrm{E}-07 & 8.4 \mathrm{E}-06 \\ \text { CM242 } & 8.8 \mathrm{E}-14 & 1.7 \mathrm{E}-09 & 2.2 \mathrm{E}-09 \\ \text { CM243 } & 5.4 \mathrm{E}-13 & 6.0 \mathrm{E}-08 & 6.3 \mathrm{E}-07 \\ \text { CM244 } & 3.9 \mathrm{E}-14 & 7.3 \mathrm{E}-10 & 9.3 \mathrm{E}-10 \\ \text { CM245 } & 2.5 \mathrm{E}-12 & 2.6 \mathrm{E}-07 & 2.7 \mathrm{E}-06 \\ \text { CM246 } & 3.5 \mathrm{E}-14 & 6.4 \mathrm{E}-10 & 7.6 \mathrm{E}-10 \\ \text { CM247 } & 1.3 \mathrm{E}-11 & 1.9 \mathrm{E}-06 & 3.0 \mathrm{E}-05 \\ \text { CM248 } & 2.7 \mathrm{E}-14 & 5.2 \mathrm{E}-10 & 7.0 \mathrm{E}-10 \\ \text { CF252 } & 2.8 \mathrm{E}-14 & 5.3 \mathrm{E}-10 & 7.8 \mathrm{E}-10\end{array}$


TABLE D.6. Internal Dose Rate Factors for Radionuclide Chains in Equilibrium (INTERNAL.DF)

Internal DF's for NRC Residual Radioactivity (10-Jan-90 RAP)

Inh 2nd Ing Air Ing Soi Ing Drink Water Maximum Organ Dose

H $3 \quad 8.2 \mathrm{E}-11$ 5.7E-11 2.3E-06 0.0E+00 6.7E-11 SI Wall

BE10 3.4E-07 4.6E-09 5.7E-05 3.7E-07 4.8E-08 LLI Wal1

C 14 1.9E-09 1.9E-09 7.2E-04 0.0E+00 2.3E-09 LLI Wall

F $18 \quad 7.7 \mathrm{E}-11$ 1.1E-10 7.8E-09 8.9E-08 1.1E-09 Stomach Wall

NA22 8.6E-09 1.4E-08 1.7E-03 5.7E-04 2.3E-08 Bone Surface

NA24 1.1E-09 1.4E-09 1.6E-06 1.7E-05 4.4E-09 Stomach Wal1

P $32 \quad 5.5 \mathrm{E}-09$ 8.5E-09 $\cdot 3.5 \mathrm{E}-04$ 4.3E-03 3.1E-08 Red Marrow

P $33 \quad 5.6 \mathrm{E}-10$ 8.7E-10 4.6E-05 4.6E-04 4.8E-09 Bone Surface

S 35 2.4E-09 4.0E-10 2.6E-05 1.0E-04 2.0E-09 LLI Wall

CL36 1.8E-09 2.8E-09 2.1E-04 1.1E-02 3.2E-09 SI Wall

K 40 9.9E-09 1.7E-08 5.9E-04 3.2E-03 2.0E-08 SI Wal1

CA41 1.2E-09 1.2E-09 6.1E-05 2.3E-05 1.5E-08 Bone Surface

CA45 6.4E-09 3.1E-09 1.4E-04 5.0E-05 1.9E-08 Bone Surface

SC46 2.8E-08 6.2E-09 6.9E-05 2.5E-06 3.7E-08 LLI Wall

CR51 3.1E-10 1.4E-10 1.8E-06 7.6E-09 9.2E-10 LLI Wall

MN54 6.1E-09 2.8E-09 3.5E-05 1.6E-05 8.5E-09 LLI Wall

MN56 3.3E-10 1.0E-09 9.7E-08 5.5E-06 5.2E-09 ULI Wall

FE55 1.2E-09 5.8E-10 1.3E-05 1.4E-07 2.1E-09 Spleen

FE59 1.2E-08 6.5E-09 7.3E-05 9.5E-07 3.1E-08 LLI Wal1

C057 8.3E-09 7.5E-10 1.2E-05 1.7E-06 4.8E-09 LLI Wall

C058 1.0E-08 2.9E-09 3.9E-05 5.9E-06 1.4E-08 LLI Wal1

C060 1.9E-07 1.0E-08 1.8E-04 2.4E-05 4.3E-08 LLI Wal1

NI59 7.9E-10 1.9E-10 8.1E-06 1.9E-06 9.7E-10 LLI Wal1

NI63 1.9E-09 5.1E-10 2.2E-05 5.1E-06 3.2E-09 LLI Wall

NI65 2.3E-10 6.5E-10 6.2E-08 2.3E-06 3.6E-09 ULI Wall

CU64 1.9E-10 4.8E-10 2.8E-07 1.2E-05 2.9E-09 LLI Wal1

ZN65 1.7E-08 $1.3 \mathrm{E}-08$ 1.4E-03 3.8E-03 $1.7 \mathrm{E}-08$ LLI Wal1

ZN69M 8.2E-10 1.4E-09 1.3E-06 1.3E-04 8.9E-09 LLI Wall

ZN69 3.8E-11 8.9E-11 3.2E-09 6.6E-06 7.8E-10 Stomach Wall

AS76 3.8E-09 5.3E-09 5.0E-06 2.1E-06 4.4E-08 LLI Wall

SE75 7.6E-09 8.9E-09 1.7E-03 4.6E-04 2.6E-08 Kidneys

SE79 8.8E-09 8.0E-09 2.7E-03 5.7E-04 4.8E-08 Kidneys

BR82 1.1E-09 1.6E-09 5.9E-06 6.0E-05 3.0E-09 Stomach Wal1

BR83 8.9E-11 9.4E-11 8.5E-09 2.8E-06 1.1E-09 Stomach Wall

BR84 9.4E-11 $1.8 \mathrm{E}-10$ 3.6E-09 5.2E-06 2.6E-09 Stomach Wal1

RB86 5.8E-09 9.1E-09 5.0E-04 6.2E-04 2.7E-08 Bone Surface

RB88 8.3E-11 $1.8 \mathrm{E}-10$ 2.0E-09 4.3E-06 2.8E-09 Stomach Wall

RB89 5.3E-09 8.8E-09 1.3E-04 5.5E-04 7.8E-08 LLI Wall

SR85 1.4E-09 1.7E-09 2.7E-05 1.1E-04 5.2E-09 LLI Wall

SR89 5.3E-09 8.7E-09 1.3E-04 5.5E-04 7.8E-08 LLI Wall

SR90 2.1E-07 1.3E-07 2.4E-03 7.7E-03 1.4E-06 Bone Surface

SR91 4.8E-08 1.2E-08 9.9E-05 1.4E-04 1.3E-07 LLI Wall

SR92 1.4E-09 3.6E-09 6.3E-07 8.9E-05 2.4E-08 ULI Wall

Y 90 8.5E-09 1.1E-08 2.2E-05 5.0E-06 1.2E-07 LLI Wall

Y 91M 4.7E-08 9.5E-09 9.8E-05 4.5E-06 1.1E-07 LLI Wal1

$Y$ 91 4.7E-08 9.4E-09 9.8E-05 4.5E-06 1.1E-07 LLI Wa11

Y 92 8.0E-10 2.0E-09 2.6E-07 9.2E-07 1.3E-08 ULI Wall 
TABLE D.6. Internal Dose Rate Factors for Radionuclide Chains in Equilibrium (INTERNAL.DF) (contd)

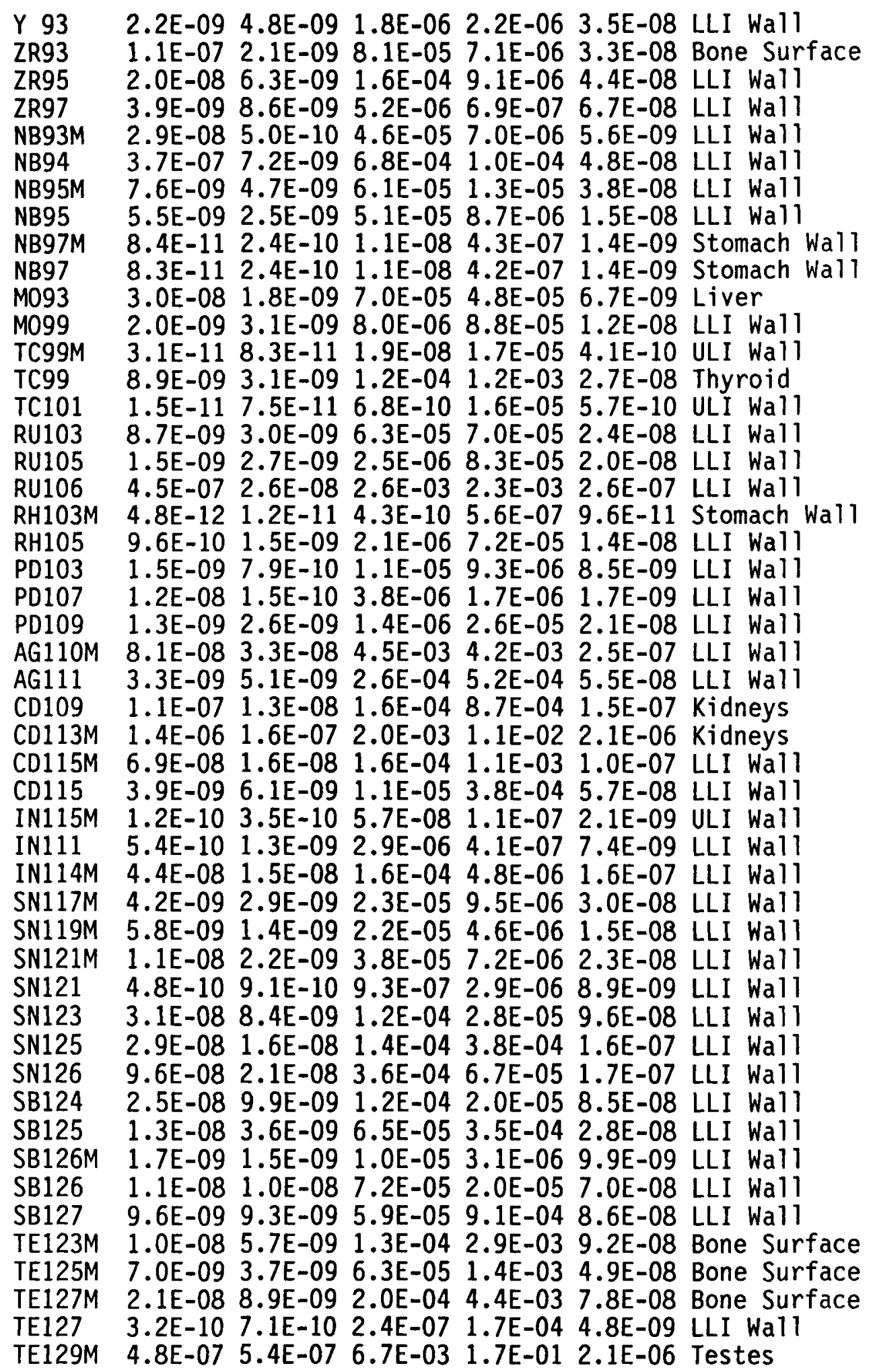


TABLE D.6. Internal Dose Rate Factors for Radionuclide Chains in Equilibrium (INTERNAL.DF) (contd)

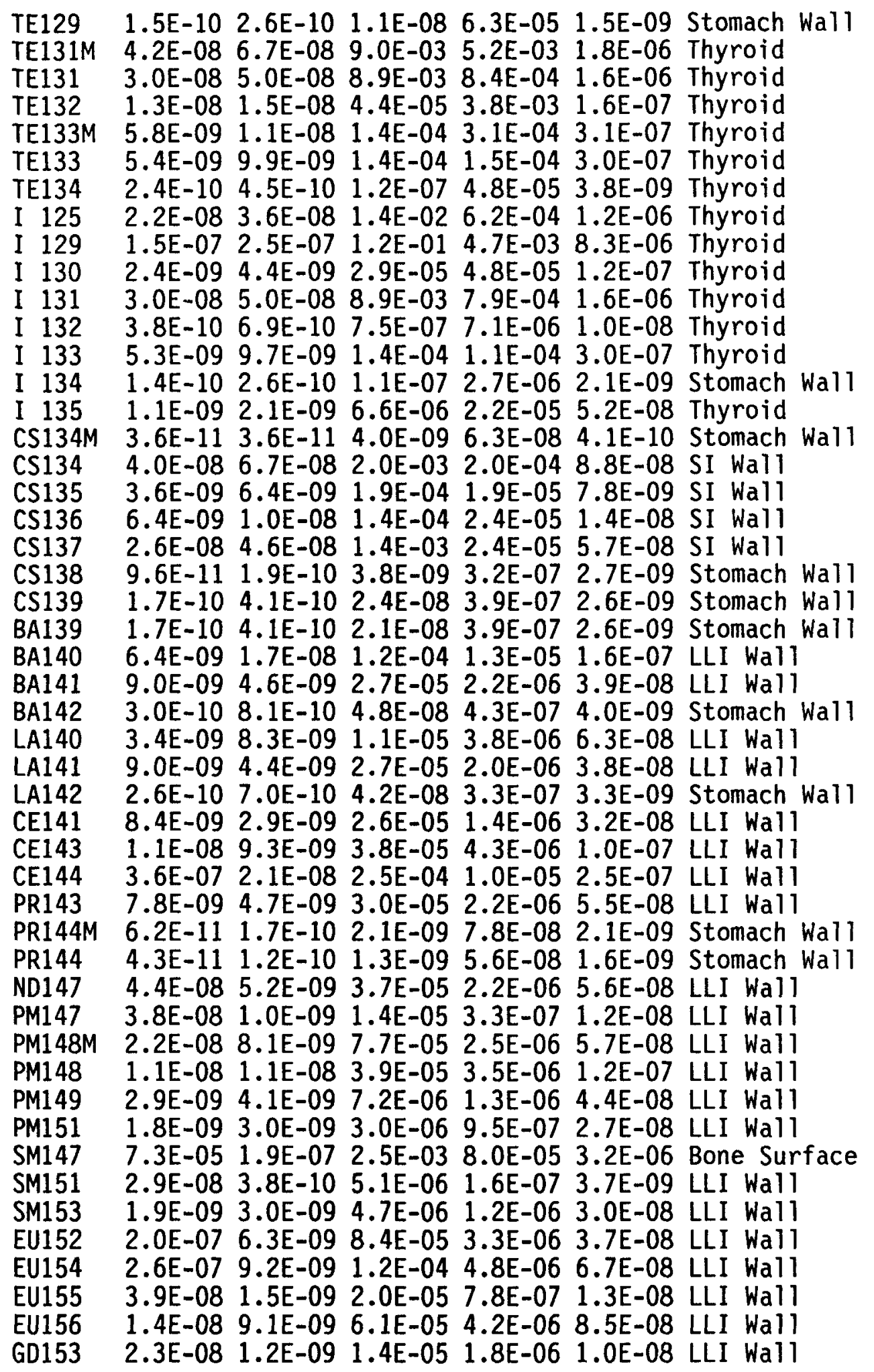


TABLE D.6. Internal Dose Rate Factors for Radionuclide Chains in Equilibrium (INTERNAL.DF) (contd)

TB160 2.4E-08 6.6E-09 7.1E-05 6.9E-07 5.5E-08 LLI Wa11 H0166M 7.2E-07 7.7E-09 1.0E-04 4.4E-06 4.1E-08 LLI Wa11 W $181 \quad 1.5 \mathrm{E}-102.9 \mathrm{E}-10 \quad 3.6 \mathrm{E}-06 \quad 1.0 \mathrm{E}-06 \quad 1.9 \mathrm{E}-09$ LLI Wa11 W $1857.5 \mathrm{E}-10 \quad 1.6 \mathrm{E}-09$ 1.9E-05 5.6E-06 1.7E-08 LLI Wa11 W $187 \quad 6.5 \mathrm{E}-10$ 2.1E-09 $1.8 \mathrm{E}-06 \quad 7.2 \mathrm{E}-06 \quad 1.7 \mathrm{E}-08$ LLI Wa11 RE187 5.6E-11 $1.4 \mathrm{E}-112.1 \mathrm{E}-07 \quad 5.8 \mathrm{E}-10$ 9.0E-11 ULI Wa11 0S185 9.2E-09 2.2E-09 $0.0 \mathrm{E}+00 \quad 0.0 \mathrm{E}+00$ 1.0E-08 LLI Wa11 0S191 2.2E-09 2.3E-09 $0.0 \mathrm{E}+00 \quad 0.0 \mathrm{E}+00$ 2.4E-08 LLI Wal1 IR192 2.6E-08 5.7E-09 6.1E-05 1.8E-05 4.8E-08 LLI Wal1 HG203 6.8E-09 1.1E-08 1.7E-04 3.8E-04 7.0E-08 Kidneys PB210 2.2E-05 7.3E-06 9.3E-02 5.0E-03 8.0E-05 Bone Surface PB212 1.8E-07 5.0E-08 1.9E-05 6.3E-05 6.7E-07 Bone Surface BI210 8.6E-06 1.9E-06 2.4E-02 9.2E-04 1.6E-05 Spleen

BI212 1.8E-08 1.1E-09 4.2E-08 3.0E-05 5.9E-09 Stomach wall P0210 8.4E-06 1.9E-06 2.3E-02 6.1E-04 1.6E-05 Spleen

RA223 7.5E-06 2.7E-07 1.7E-03 7.3E-04 4.1E-06 Bone Surface RA224 2.9E-06 1.8E-07 5.3E-04 4.2E-04 1.9E-06 Bone Surface RA225 1.5E-05 3.3E-07 2.7E-03 6.4E-04 5.9E-06 Bone Surface RA226 2.9E-05 8.2E-06 1.1E-01 7.7E-03 1.0E-04 Bone Surface RA228 3.2E-04 1.4E-06 1.9E-02 3.1E-03 3.0E-05 Bone Surface AC225 7.9E-06 1.1E-07 6.1E-04 2.8E-05 1.0E-06 LLI Wal1 AC227 1.3E-03 1.4E-05 1.7E-01 4.3E-03 2.5E-04 Bone Surface AC228 3.2E-04 5.8E-07 7.1E-03 7.3E-04 1.1E-05 Bone Surface TH227 2.3E-05 3.1E-07 3.2E-03 7.6E-04 4.3E-06 Bone Surface TH228 3.2E-04 5.7E-07 7.1E-03 7.3E-04 1.1E-05 Bone Surface TH229 1.7E-03 3.8E-06 4.7E-02 3.4E-03 9.4E-05 Bone Surface TH230 2.8E-04 8.8E-06 1.1E-01 8.1E-03 1.2E-04 Bone Surface TH231

TH232

TH234

PA231

PA233 8.1E-10 1.3E-09 1.2E-06 1.0E-06 1.3E-08 LLI Wa11 1.4E-03 4.2E-06 5.2E-02 5.2E-03 9.9E-05 Bone Surface 3.4E-08 1.3E-08 1.1E-04 1.1E-05 1.6E-07 LLI Wall

2.1E-03 2.5E-05 3.2E-01 2.3E-02 5.2E-04 Bone Surface .2E-09 3.6E-09 3.0E-05 6.0E-06 3.7E-08 LLI Wa11

U 232

U 233

U 234

U 235

U 236

U 237

U 238

U 240

NP237

NP238

NP239

8.2E-10 2.2E-09 5.5E-07 3.7E-06 1.2E-08 ULI Wall 6.0E-04 6.8E-08 9.3E-04 3.3E-05 9.7E-07 Bone Surface 1.8E-03 3.9E-06 4.7E-02 3.4E-03 9.4E-05 Bone Surface 1.3E-04 2.6E-08 3.5E-04 1.3E-05 1.9E-07 LLI Wa11 $1.2 \mathrm{E}-042.8 \mathrm{E}-08$ 3.8E-04 1.4E-05 2.1E-07 LLI Wa11

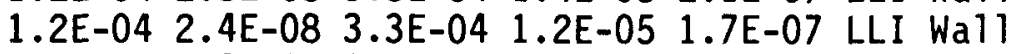
3.5E-09 3.2E-09 1.5E-05 1.5E-06 3.3E-08 LLI Wa11 1.1E-04 3.7E-08 4.9E-04 2.2E-05 3.3E-07 LLI Wa11 2.2E-09 4.7E-09 2.4E-06 2.2E-06 3.7E-08 LLI Wa11 6.1E-04 5.2E-06 6.4E-02 2.4E-03 1.2E-04 Bone Surface 2.8E-04 3.4E-07 4.1E-03 3.7E-05 5.9E-06 Bone Surface 2.4E-09 3.3E-09 6.1E-06 1.5E-06 3.3E-08 LLI Wa11 PU236 7.2E-05 6.3E-08 7.6E-04 6.6E-06 7.8E-07 Bone Surface PU237 1.8E-09 4.3E-10 4.1E-06 4.4E-08 3.7E-09 LLI Wa11 PU238 2.8E-04 3.4E-07 4.1E-03 3.5E-05 5.9E-06 Bone Surface PU239 2.9E-04 3.7E-07 4.5E-03 3.9E-05 6.7E-06 Bone Surface PU240 2.9E-04 3.7E-07 4.5E-03 3.8E-05 6.7E-06 Bone Surface 
TABLE D.6. Internal Dose Rate Factors for Radionuclide Chains in Equilibrium (INTERNAL.DF) (contd)

$\begin{array}{lllllll}\text { PU241 } & 4.3 \mathrm{E}-04 & 3.6 \mathrm{E}-06 & 4.7 \mathrm{E}-02 & 4.1 \mathrm{E}-03 & 6.8 \mathrm{E}-05 & \text { Bone Surface } \\ \text { PU242 } & 2.7 \mathrm{E}-04 & 3.4 \mathrm{E}-07 & 4.2 \mathrm{E}-03 & 3.6 \mathrm{E}-05 & 6.1 \mathrm{E}-06 & \text { Bone Surface } \\ \text { PU243 } & 1.4 \mathrm{E}-10 & 3.2 \mathrm{E}-10 & 5.6 \mathrm{E}-08 & 3.3 \mathrm{E}-08 & 2.1 \mathrm{E}-09 & \text { ULI Wal1 } \\ \text { PU244 } & 2.6 \mathrm{E}-04 & 3.6 \mathrm{E}-07 & 4.4 \mathrm{E}-03 & 3.9 \mathrm{E}-05 & 6.1 \mathrm{E}-06 & \text { Bone Surface } \\ \text { AM241 } & 4.3 \mathrm{E}-04 & 3.6 \mathrm{E}-06 & 4.7 \mathrm{E}-02 & 4.1 \mathrm{E}-03 & 6.7 \mathrm{E}-05 & \text { Bone Surface } \\ \text { AM242M } & 6.3 \mathrm{E}-04 & 3.8 \mathrm{E}-06 & 5.0 \mathrm{E}-02 & 4.0 \mathrm{E}-03 & 7.1 \mathrm{E}-05 & \text { Bone Surface } \\ \text { AM242 } & 2.4 \mathrm{E}-04 & 3.7 \mathrm{E}-07 & 4.5 \mathrm{E}-03 & 7.6 \mathrm{E}-05 & 6.3 \mathrm{E}-06 & \text { Bone Surface } \\ \text { AM243 } & 4.1 \mathrm{E}-04 & 3.6 \mathrm{E}-06 & 4.7 \mathrm{E}-02 & 4.1 \mathrm{E}-03 & 6.7 \mathrm{E}-05 & \text { Bone Surface } \\ \text { CM242 } & 2.9 \mathrm{E}-04 & 4.5 \mathrm{E}-07 & 5.4 \mathrm{E}-03 & 8.6 \mathrm{E}-05 & 7.5 \mathrm{E}-06 & \text { Bone Surface } \\ \text { CM243 } & 5.8 \mathrm{E}-04 & 2.9 \mathrm{E}-06 & 3.5 \mathrm{E}-02 & 1.2 \mathrm{E}-03 & 5.2 \mathrm{E}-05 & \text { Bone Surface } \\ \text { CM244 } & 2.3 \mathrm{E}-04 & 2.0 \mathrm{E}-06 & 2.4 \mathrm{E}-02 & 9.3 \mathrm{E}-04 & 3.6 \mathrm{E}-05 & \text { Bone Surface } \\ \text { CM245 } & 8.5 \mathrm{E}-04 & 7.3 \mathrm{E}-06 & 9.2 \mathrm{E}-02 & 5.8 \mathrm{E}-03 & 1.4 \mathrm{E}-04 & \text { Bone Surface } \\ \text { CM246 } & 4.2 \mathrm{E}-04 & 3.7 \mathrm{E}-06 & 4.5 \mathrm{E}-02 & 1.7 \mathrm{E}-03 & 7.0 \mathrm{E}-05 & \text { Bone Surface } \\ \text { CM247 } & 3.9 \mathrm{E}-04 & 3.4 \mathrm{E}-06 & 4.2 \mathrm{E}-02 & 1.6 \mathrm{E}-03 & 6.4 \mathrm{E}-05 & \text { Bone Surface } \\ \text { CM248 } & 1.5 \mathrm{E}-03 & 1.3 \mathrm{E}-05 & 1.6 \mathrm{E}-01 & 6.2 \mathrm{E}-03 & 2.5 \mathrm{E}-04 & \text { Bone Surface } \\ \text { CF252 } & 1.3 \mathrm{E}-04 & 1.1 \mathrm{E}-06 & 1.4 \mathrm{E}-02 & 1.1 \mathrm{E}-04 & 2.2 \mathrm{E}-05 & \text { Bone Surface }\end{array}$


TABLE D.7. Internal Dose Rate Factors for Individual Radionuclides (INTNODAU.DF)

Internal DF's for NRC Residual Radioactivity - No Daughters (10-Jan-90 RAP)

Inh 2nd Ing Air Ing Soi Ing Drink Water Maximum Organ Dose

H 3

BE10

C 14

F 18

NA22

NA24

P 32

P 33

S 35

CL36

K 40

CA41

CA45

SC46

CR51

MN54

MN56

FE55

FE59

C057

C058

C060

NI59

NI63

NI 65

CU64

ZN65

ZN69M

ZN69

AS76

SE75

SE79

BR82

BR83

BR84

RB86

RB88

RB89

SR85

SR89

SR90

SR91

SR92

Y 90

Y $91 \mathrm{M}$

$Y 91$

Y 92
8.2E-11 5.7E-11 2.3E-06 $0.0 \mathrm{E}+00$ 6.7E-11 SI Wall

3.4E-07 4.6E-09 5.7E-05 3.7E-07 4.8E-08 LLI Wall

1.9E-09 1.9E-09 7.2E-04 0.0E+00 2.3E-09 LLI Wal 1

7.7E-11 1.1E-10 7.8E-09 8.9E-08 1.1E-09 Stomach Wal1

8.6E-09 1.4E-08 1.7E-03 5.7E-04 2.3E-08 Bone Surface

1.1E-09 1.4E-09 1.6E-06 1.7E-05 4.4E-09 Stomach Wall

5.5E-09 8.5E-09 3.5E-04 4.3E-03 3.1E-08 Red Marrow

5.6E-10 8.7E-10 4.6E-05 4.6E-04 4.8E-09 Bone Surface

2.4E-09 4.0E-10 2.6E-05 1.0E-04 2.0E-09 LLI Wal1

1.8E-09 2.8E-09 2.1E-04 1.1E-02 3.2E-09 SI Wall

9.9E-09 1.7E-08 5.9E-04 3.2E-03 2.0E-08 SI Wall

1.2E-09 1.2E-09 6.1E-05 2.3E-05 1.5E-08 Bone Surface

6.4E-09 3.1E-09 1.4E-04 5.0E-05 1.9E-08 Bone Surface

2.8E-08 6.2E-09 6.9E-05 2.5E-06 3.7E-08 LLI Wal1

3.1E-10 1.4E-10 1.8E-06 7.6E-09 9.2E-10 LLI Wal1

$6.1 \mathrm{E}-092.8 \mathrm{E}-09$ 3.5E-05 1.6E-05 8.5E-09 LLI Wall

3.3E-10 1.0E-09 9.7E-08 5.5E-06 5.2E-09 ULI Wall

1.2E-09 5.8E-10 1.3E-05 1.4E-07 2.1E-09 Spleen

1.2E-08 6.5E-09 7.3E-05 9.5E-07 3.1E-08 LLI Wal1

8.3E-09 7.5E-10 1.2E-05 1.7E-06 4.8E-09 LLI Wal1

1.0E-08 2.9E-09 3.9E-05 5.9E-06 1.4E-08 LLI Wall

1.9E-07 1.0E-08 1.8E-04 2.4E-05 4.3E-08 LLI Wall

7.9E-10 1.9E-10 8.1E-06 1.9E-06 9.7E-10 LLI Wall

1.9E-09 5.1E-10 2.2E-05 5.1E-06 3.2E-09 LLI Wal1

2.3E-10 6.5E-10 6.2E-08 2.3E-06 3.6E-09 ULI Wall

1.9E-10 4.8E-10 2.8E-07 1.2E-05 2.9E-09 LLI Wall

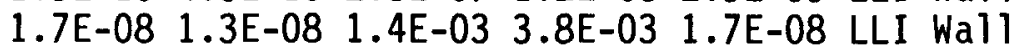

7.8E-10 1.3E-09 1.2E-06 1.2E-04 8.9E-09 LLI Wall

3.8E-11 8.9E-11 3.2E-09 6.6E-06 7.8E-10 Stomach Wal1

3.8E-09 5.3E-09 5.0E-06 2.1E-06 4.4E-08 LLI Wall

7.6E-09 8.9E-09 1.7E-03 4.6E-04 2.6E-08 Kidneys

8.8E-09 8.0E-09 2.7E-03 5.7E-04 4.8E-08 Kidneys

1.1E-09 1.6E-09 5.9E-06 6.0E-05 3.0E-09 Stomach Wall

8.9E-11 9.4E-11 8.5E-09 2.8E-06 1.1E-09 Stomach Wall

9.4E-11 1.8E-10 3.6E-09 5.2E-06 2.6E-09 Stomach Wall

5.8E-09 9.1E-09 5.0E-04 6.2E-04 2.7E-08 Bone Surface

8.3E-11 1.8E-10 2.0E-09 4.3E-06 2.8E-09 Stomach Wall

4.2E-11 9.5E-11 9.3E-10 2.3E-06 1.4E-09 Stomach Wall

1.4E-09 1.7E-09 2.7E-05 1.1E-04 5.2E-09 LLI Wall

5.3E-09 8.7E-09 1.3E-04 5.5E-04 7.8E-08 LLI Wall

2.0E-07 1.2E-07 2.2E-03 7.7E-03 1.4E-06 Bone Surface

8.6E-10 2.4E-09 8.9E-07 1.3E-04 1.4E-08 LLI Wal1

5.9E-10 1.6E-09 1.6E-07 8.8E-05 1.1E-08 ULI Wal1

8.5E-09 1.1E-08 2.2E-05 5.0E-06 1.2E-07 LLI Wal1

3.6E-11 4.1E-11 1.3E-09 1.9E-08 1.9E-10 Stomach wall

4.7E-08 9.4E-09 9.8E-05 4.5E-06 1.1E-07 LLI Wall

8.0E-10 2.0E-09 2.6E-07 9.2E-07 1.3E-08 ULI Wal1 
TABLE D.7. Internal Dose Rate Factors for Individual Radionuclides (INTNODAU.DF) (contd)

\begin{tabular}{|c|c|c|c|c|c|}
\hline $\begin{array}{l}\text { Y 93 } \\
\text { ZR93 } \\
\text { ZR95 } \\
\text { ZR97 } \\
\text { NB93M } \\
\text { NB94 } \\
\text { NB95M } \\
\text { NB95 } \\
\text { NB97M } \\
\text { NB97 } \\
\text { M093 } \\
\text { M099 } \\
\text { TC99M } \\
\text { TC99 } \\
\text { TC101 } \\
\text { RU103 } \\
\text { RU105 } \\
\text { RU106 } \\
\text { RH103M } \\
\text { RH105 } \\
\text { PD103 } \\
\text { PD107 } \\
\text { PD109 } \\
\text { AG110M } \\
\text { AG111 } \\
\text { CD109 } \\
\text { CD113M } \\
\text { CD115M } \\
\text { CD115 } \\
\text { IN115M } \\
\text { IN111 } \\
\text { IN114M } \\
\text { SN117M } \\
\text { SN119M } \\
\text { SN121M } \\
\text { SN121 } \\
\text { SN123 } \\
\text { SN125 } \\
\text { SN126 } \\
\text { SB124 } \\
\text { SB125 } \\
\text { SB126M } \\
\text { SB126 } \\
\text { SB127 } \\
\text { TE123M } \\
\text { TE125M } \\
\text { TE127M }\end{array}$ & $\begin{array}{l}7.9 \\
1.5 \\
3.8 \\
2.9 \\
3.7 \\
2.4 \\
5.5 \\
1.2 \\
8.3 \\
9.5 \\
1.9 \\
3.1 \\
8.9 \\
1.5 \\
8.7 \\
5.2 \\
4.5 \\
4.8 \\
9.6 \\
1.5 \\
1.2 \\
1.3 \\
8.1 \\
3.3 \\
1.1 \\
1.4 \\
6.9 \\
3.8 \\
1.2 \\
5.4 \\
4.4 \\
4.2 \\
5.8 \\
1.1 \\
4.8 \\
3.1 \\
1.5 \\
9.4 \\
2.5 \\
1.2 \\
2.9 \\
1.1 \\
5.8 \\
1\end{array}$ & $\begin{array}{l}4.8 \\
1.6 \\
3.7 \\
8.4 \\
5.0 \\
7.2 \\
2.3 \\
2.5 \\
3.9 \\
2.4 \\
1.4 \\
3.1 \\
8.3 \\
3.1 \\
7.5 \\
3.0 \\
1.3 \\
2.6 \\
1.2 \\
1.5 \\
7.8 \\
1.5 \\
2.6 \\
3.3 \\
5.1 \\
1.3 \\
1.6 \\
1.6 \\
5.7 \\
3.5 \\
1.3 \\
1.5 \\
2.9 \\
1.4 \\
1.5 \\
9.1 \\
8.4 \\
1.2 \\
1.9\end{array}$ & $\begin{array}{l}3.5 \\
4.6 \\
5.0 \\
4.6 \\
6.8 \\
8.7 \\
5.1 \\
2.5 \\
1.1 \\
2.4 \\
7.8 \\
1.9 \\
1.2 \\
6.8 \\
6.3 \\
2.1 \\
2.6 \\
4.3 \\
2.1 \\
1.1 \\
3.8 \\
1.4 \\
4.5 \\
2.6 \\
1.6 \\
2.0 \\
1.6\end{array}$ & $\begin{array}{ll}06 & 3.5 \mathrm{E} \\
08 & 3.3 \mathrm{E} \\
07 & 2.9 \mathrm{E} \\
07 & 6.7 \mathrm{E} \\
06 & 5.6 \mathrm{E} \\
04 & 4.8 \mathrm{E} \\
06 & 2.4 \mathrm{E} \\
06 & 1.5 \mathrm{E} \\
09 & 2.7 \mathrm{E} \\
07 & 1.4 \mathrm{E} \\
05 & 6.7 \mathrm{E} \\
05 & 1.1 \mathrm{E} \\
05 & 4.1 \mathrm{E} \\
03 & 2.7 \mathrm{E} \\
05 & 5.7 \mathrm{E} \\
05 & 2.4 \mathrm{E} \\
05 & 7.0 \mathrm{E} \\
03 & 2.6 \mathrm{E} \\
07 & 9.6 \mathrm{E} \\
05 & 1.4 \mathrm{E} \\
06 & 8.5 \mathrm{E} \\
06 & 1.7 \mathrm{E} \\
05 & 2.1 \mathrm{E} \\
03 & 2.5 \mathrm{E} \\
04 & 5.5 \mathrm{E} \\
04 & 1.5 \mathrm{E} \\
02 & 2.1 \mathrm{E} \\
03 & 1.0 \mathrm{E} \\
04 & 5.5 \mathrm{E} \\
07 & 2.1 \mathrm{E} \\
07 & 7.4 \mathrm{E} \\
06 & 1.6 \mathrm{E} \\
06 & 3.0 \mathrm{E} \\
06 & 1.5 \mathrm{E} \\
06 & 1.6 \mathrm{E} \\
06 & 8.9 \mathrm{E} \\
05 & 9.6 \mathrm{E} \\
05 & 1.3 \mathrm{E} \\
05 & 1.6 \mathrm{E} \\
05 & 8.5 \mathrm{E} \\
06 & 2.4 \mathrm{E} \\
07 & 9.6 \mathrm{E} \\
05 & 7.0 \mathrm{E} \\
05 & 7.4 \mathrm{E} \\
03 & 9.2 \mathrm{E} \\
03 & 4.9 \mathrm{E} \\
03 & 7.8 \mathrm{E} \\
04 & 4.8 \mathrm{E} \\
01 & . .1 \mathrm{E}\end{array}$ & 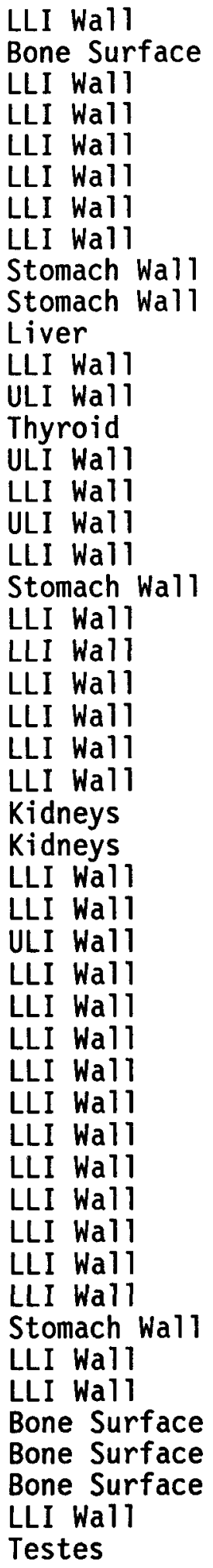 \\
\hline
\end{tabular}


TABLE D.7. Internal Dose Rate Factors for Individual

Radionuclides (INTNODAU.DF) (contd)

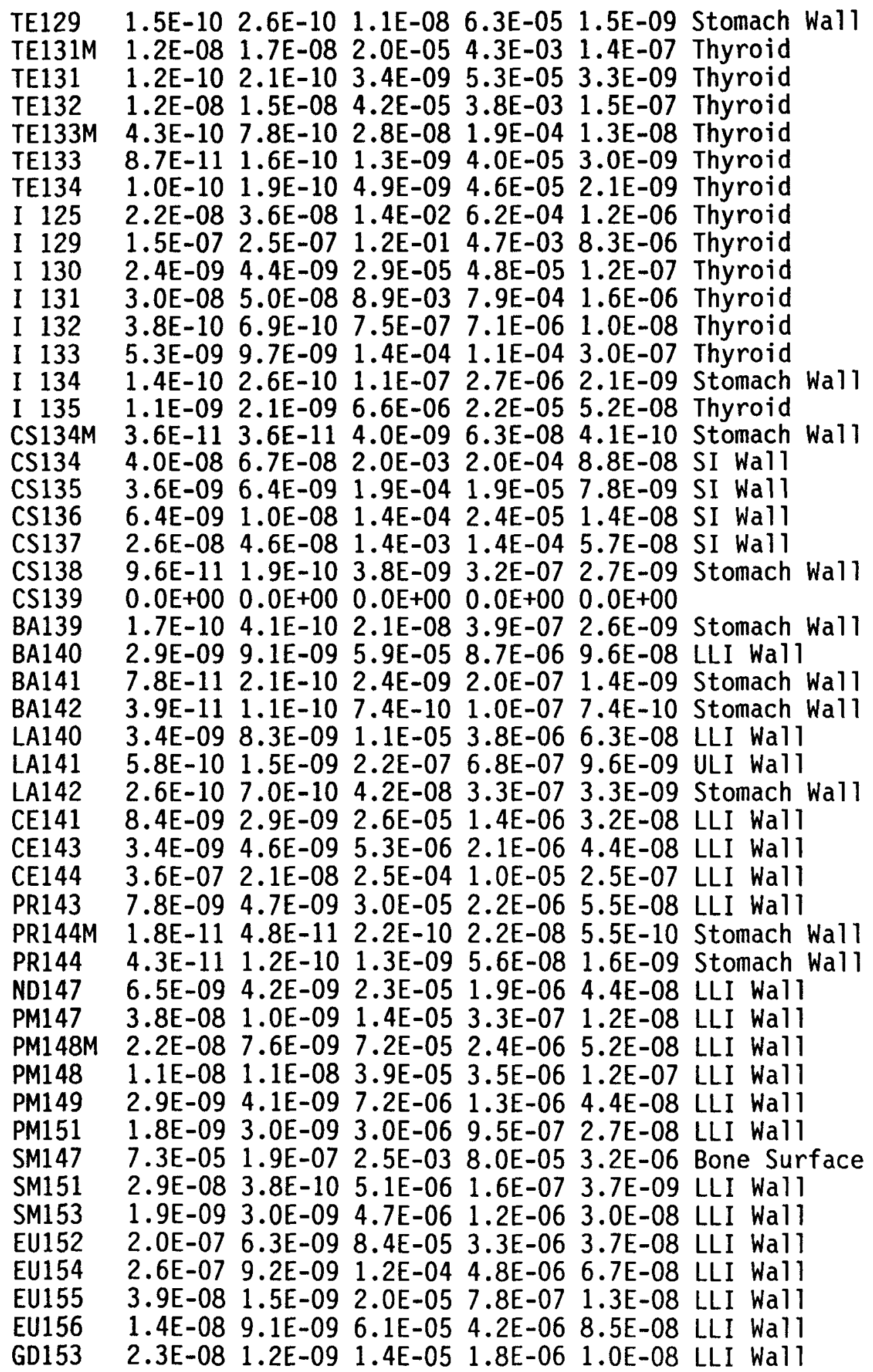




\section{TABLE D.7. Internal Dose Rate Factors for Individual} Radionuclides (INTNODAU.DF) (contd)
TB160
2.4E-08 6.6E-09 7.1E-05 6.9E-07 5.5E-08 LLI Wall
H0166M
7.2E-07 7.7E-09
1.0E-04 4.4E-06 4.1E-08 LLI Wall
W 181
$1.5 \mathrm{E}-102.9 \mathrm{E}-10$
3.6E-06 1.0E-06 1.9E-09 LLI Wa11
W 185
7.5E-10 1.6E-09 1.9E-05 5.6E-06 1.7E-08 LLI Wall
W 187
6.5E-10 2.1E-09 1.8E-06 7.2E-06 1.7E-08 LLI Wal1
RE187
5.6E-11 1.4E-11 2.1E-07 5.8E-10 9.0E-11 ULI Wall
OS185
9.2E-09 2.2E-09 $0.0 \mathrm{E}+00 \quad 0.0 \mathrm{E}+00 \quad 1.0 \mathrm{E}-08$ LLI Wal1
OS191
2.2E-09 2.3E-09 0.0E+00 0.0E+00 2.4E-08 LLI Wall
IR192
2.6E-08 5.7E-09 6.1E-05 1.8E-05 4.8E-08 LLI Wal1
HG203
6.8E-09 1.1E-08 1.7E-04 3.8E-04 7.0E-08 Kidneys
PB210
PB212
$1.3 \mathrm{E}-05$ 5.4E-06
6.7E-02 4.1E-03
8.0E-05 Bone Surface
BI210
$1.6 \mathrm{E}-07 \quad 4.9 \mathrm{E}-08$
$1.9 \mathrm{E}-05$
$3.6 \mathrm{E}-05$
Bone Surface
B1212
$1.9 \mathrm{E}-07 \quad 6.3 \mathrm{E}-09$
2.3E-05 1.8E-04 5.5E-08 LLI Wal1
P0210
$1.8 \mathrm{E}-08$ 1.1E-09
4.2E-08 3.0E-05
8.4E-06 1.9E-06
2.3E-02 6.1E-04
.9E-09 Stomach Wall
RA223
RA224
7.5E-06 2.7E-07 1.7E-03 7.3E-04
4.1E-06 Bone Surface
RA225
2.7E-06 1.3E-07 3.8E-04
$3.6 \mathrm{E}-04$
1.2E-06 Bone Surface
RA226
$7.1 \mathrm{E}-06$
2.2E-07
$1.6 \mathrm{E}-03$
6.1E-04
$5.6 \mathrm{E}-06$
Bone Surface
RA228
$7.9 \mathrm{E}-06$
9.5E-07 1.3E-02
2.7E-03
2.3E-05 Bone Surface
AC225
3.7E-06 8.4E-07 1.1E-02 2.3E-03
2.0E-05 Bone Surface
$7.9 \mathrm{E}-06$
1.1E-07
6.1E-04 2.8E-05
1.0E-06 LLI Wal1
AC227
1.3E-03 1.4E-05 1.7E-01 3.6E-03
2.5E-04 Bone Surface
AC228
TH227
1.1E-07 2.1E-09 4.9E-07 5.4E-07 1.1E-08 Bone Surface 1.6E-05 3.8E-08 2.8E-04 3.0E-05 3.4E-07 LLI Wal1
TH228
3.1E-04 4.0E-07 4.8E-03 3.1E-04 8.9E-06 Bone Surface
TH229
1.7E-03 3.5E-06 4.3E-02 2.8E-03 8.8E-05 Bone Surface
TH230
2.5E-04 5.4E-07 6.5E-03 4.2E-04 1.3E-05 Bone Surface
TH231
8.1E-10 1.3E-09 1.2E-06 1.0E-06 1.3E-08 LLI Wal1
TH232
1.1E-03 2.8E-06 3.3E-02 2.2E-03 6.9E-05 Bone Surface
TH234
3.4E-08 1.3E-08 1.1E-04 1.1E-05 1.6E-07 LLI Wal1
PA231
8.4E-04 1.1E-05 1.4E-01 1.9E-02 2.7E-04 Bone Surface
PA233
PA234
U 232
U 233
U 234
U 235
U 236
U 237
U 238
U 240
NP237
NP238
NP239
PU236
PU237
PU238
9.2E-09 3.6E-09 3.0E-05 6.0E-06 3.7E-08 LLI Wal1
8.2E-10 2.2E-09 5.5E-07 3.7E-06 1.2E-08 ULI Wal1
6.0E-04 6.8E-08 9.3E-04 3.3E-05 9.7E-07 Bone Surface
1.3E-04 2.6E-08 3.6E-04 1.3E-05 1.9E-07 LLI Wal1
1.3E-04 2.6E-08 3.5E-04 1.3E-05 1.9E-07 LLI Wal1
1.2E-04 2.6E-08 3.6E-04 1.3E-05 2.0E-07 LLI Wall
1.2E-04 2.4E-08 3.3E-04 1.2E-05 1.7E-07 LLI Wall
3.5E-09 3.2E-09 1.5E-05 1.5E-06 3.3E-08 LLI Wall
1.1E-04 2.4E-08 3.2E-04 1.1E-05 1.7E-07 LLI Wall
2.2E-09 4.7E-09 2.4E-06 2.2E-06 3.7E-08 LLI Wall
6.1E-04 5.2E-06 6.4E-02 2.4E-03 1.2E-04 Bone Surface
4.0E-08 4.0E-09 6.8E-06 1.9E-06 3.4E-08 LLI Wall
2.4E-09 3.3E-09 6.1E-06 1.5E-06 3.3E-08 LLI Wa11
7.2E-05 6.3E-08 7.6E-04 6.6E-06 7.8E-07 Bone Surface
1.8E-09 4.3E-10 4.1E-06 4.4E-08 3.7E-09 LLI Wal1
PU239
2.8E-04 3.4E-07 4.1E-03 3.5E-05 5.9E-06 Bone Surface
2.9E-04 3.7E-07 4.5E-03 3.9E-05 6.7E-06 Bone Surface
PU240
2.9E-04 3.7E-07 4.5E-03 3.8E-05 6.7E-06 Bone Surface 
TABLE D.7. Internal Dose Rate Factors for Individual Radionuclides (INTNODAU.DF) (contd)

$\begin{array}{lllllll}\text { PU241 } & 4.7 \mathrm{E}-06 & 6.8 \mathrm{E}-09 & 8.3 \mathrm{E}-05 & 7.1 \mathrm{E}-07 & 1.3 \mathrm{E}-07 & \text { Bone Surface } \\ \text { PU242 } & 2.7 \mathrm{E}-04 & 3.4 \mathrm{E}-07 & 4.2 \mathrm{E}-03 & 3.6 \mathrm{E}-05 & 6.1 \mathrm{E}-06 & \text { Bone Surface } \\ \text { PU243 } & 1.4 \mathrm{E}-10 & 3.2 \mathrm{E}-10 & 5.6 \mathrm{E}-08 & 3.3 \mathrm{E}-08 & 2.1 \mathrm{E}-09 & \text { ULI Wall } \\ \text { PU244 } & 2.6 \mathrm{E}-04 & 3.5 \mathrm{E}-07 & 4.3 \mathrm{E}-03 & 3.7 \mathrm{E}-05 & 6.1 \mathrm{E}-06 & \text { Bone Surface } \\ \text { AM241 } & 4.3 \mathrm{E}-04 & 3.6 \mathrm{E}-06 & 4.7 \mathrm{E}-02 & 4.1 \mathrm{E}-03 & 6.7 \mathrm{E}-05 & \text { Bone Surface } \\ \text { AM242M } & 3.9 \mathrm{E}-04 & 3.5 \mathrm{E}-06 & 4.5 \mathrm{E}-02 & 3.9 \mathrm{E}-03 & 6.4 \mathrm{E}-05 & \text { Bone Surface } \\ \text { AM242 } & 5.2 \mathrm{E}-08 & 1.4 \mathrm{E}-09 & 8.2 \mathrm{E}-07 & 1.4 \mathrm{E}-06 & 9.6 \mathrm{E}-09 & \text { LLI Wall } \\ \text { AM243 } & 4.1 \mathrm{E}-04 & 3.6 \mathrm{E}-06 & 4.7 \mathrm{E}-02 & 4.1 \mathrm{E}-03 & 6.7 \mathrm{E}-05 & \text { Bone Surface } \\ \text { CM242 } & 1.5 \mathrm{E}-05 & 1.1 \mathrm{E}-07 & 1.3 \mathrm{E}-03 & 5.1 \mathrm{E}-05 & 1.6 \mathrm{E}-06 & \text { Bone Surface } \\ \text { CM243 } & 2.9 \mathrm{E}-04 & 2.5 \mathrm{E}-06 & 3.0 \mathrm{E}-02 & 1.2 \mathrm{E}-03 & 4.5 \mathrm{E}-05 & \text { Bone Surface } \\ \text { CM244 } & 2.3 \mathrm{E}-04 & 2.0 \mathrm{E}-06 & 2.4 \mathrm{E}-02 & 9.3 \mathrm{E}-04 & 3.6 \mathrm{E}-05 & \text { Bone Surface } \\ \text { CM245 } & 4.2 \mathrm{E}-04 & 3.7 \mathrm{E}-06 & 4.5 \mathrm{E}-02 & 1.7 \mathrm{E}-03 & 6.8 \mathrm{E}-05 & \text { Bone Surface } \\ \text { CM246 } & 4.2 \mathrm{E}-04 & 3.7 \mathrm{E}-06 & 4.5 \mathrm{E}-02 & 1.7 \mathrm{E}-03 & 7.0 \mathrm{E}-05 & \text { Bone Surface } \\ \text { CM247 } & 3.9 \mathrm{E}-04 & 3.4 \mathrm{E}-06 & 4.2 \mathrm{E}-02 & 1.6 \mathrm{E}-03 & 6.4 \mathrm{E}-05 & \text { Bone Surface } \\ \text { CM248 } & 1.5 \mathrm{E}-03 & 1.3 \mathrm{E}-05 & 1.6 \mathrm{E}-01 & 6.2 \mathrm{E}-03 & 2.5 \mathrm{E}-04 & \text { Bone Surface } \\ \text { CF252 } & 1.3 \mathrm{E}-04 & 1.1 \mathrm{E}-06 & 1.4 \mathrm{E}-02 & 1.1 \mathrm{E}-04 & 2.2 \mathrm{E}-05 & \text { Bone Surface }\end{array}$


TABLE D.8. EXTDF Output for Surface Source Configuration

\section{EXTDF Dose Calculation Program}

(Version 1.429 19-Dec-89)

Case title: 5m Surface - Residual Radioactivity

Executed on: $12 / 21 / 89$ at $08: 26: 09$

Page

Shield composition $(\mathrm{gm} / \mathrm{cc})$ :

$\begin{array}{lccccc}\text { AIR } & 1 & 2 & 3 & 4 & 5 \\ & 1.3 \mathrm{E}-03 & 0.0 \mathrm{E}+00 & 0.0 \mathrm{E}+00 & 0.0 \mathrm{E}+00 & 0.0 \mathrm{E}+00\end{array}$

Shield thickness $(\mathrm{cm})$ :

$$
0.0 \mathrm{E}+00
$$

Geometry index:

DISC source

SLAB shield

Distance to detector:

Disc radius:

Taylor buildup data for shield:

with effective atomic number:

$1.00 \mathrm{E}+02 \mathrm{~cm}$

$5.00 \mathrm{E}+02 \mathrm{~cm}$ 1

7.0

Units are rem/hr per $\mathrm{Ci} / \mathrm{cm} 2$ or $\mathrm{cm} 3$

$\begin{array}{ll}\text { H } 3 & 5.29 E-05 \\ \text { BE10 } & 1.89 E+01 \\ \text { C 14 } & 1.12 E+00 \\ \text { N 13 } & 3.68 E+04 \\ \text { F 18 } & 3.56 \mathrm{E}+04 \\ \text { NA22 } & 7.98 \mathrm{E}+04 \\ \text { NA24 } & 1.48 \mathrm{E}+05 \\ \text { SI31 } & 1.63 \mathrm{E}+02 \\ \text { P } 32 & 1.70 \mathrm{E}+02 \\ \text { P 33 } & 3.33 \mathrm{E}+00 \\ \text { S 35 } & 1.26 \mathrm{E}+00 \\ \text { CL36 } & 2.91 \mathrm{E}+01 \\ \text { K 40 } & 6.01 \mathrm{E}+03 \\ \text { AR39 } & 1.88 \mathrm{E}+01 \\ \text { AR41 } & 4.64 \mathrm{E}+04 \\ \text { CA41 } & 7.19 \mathrm{E}+01 \\ \text { CA45 } & 3.45 \mathrm{E}+00 \\ \text { SC46 } & 7.92 \mathrm{E}+04 \\ \text { CR51 } & 1.48 \mathrm{E}+03 \\ \text { MN54 } & 3.27 \mathrm{E}+04 \\ \text { MN56 } & 6.49 \mathrm{E}+04 \\ \text { FE55 } & 1.64 \mathrm{E}+02 \\ \text { FE59 } & 4.23 \mathrm{E}+04 \\ \text { C057 } & 5.25 \mathrm{E}+03 \\ \text { C058 } & 3.86 \mathrm{E}+04\end{array}$


TABLE D.8. EXTDF Output for Surface Source Configuration (contd)

$\begin{array}{ll}\text { C060 } & 9.34 \mathrm{E}+04 \\ \text { NI59 } & 2.00 \mathrm{E}+02 \\ \text { NI63 } & 8.94 \mathrm{E}-02 \\ \text { NI65 } & 2.15 \mathrm{E}+04 \\ \text { CU64 } & 6.92 \mathrm{E}+03 \\ \text { ZN65 } & 2.44 \mathrm{E}+04 \\ \text { ZN69M } & 1.75 \mathrm{E}+04 \\ \text { ZN69 } & 4.82 \mathrm{E}+01 \\ \text { GA72 } & 1.02 \mathrm{E}+05 \\ \text { AS76 } & 1.91 \mathrm{E}+04 \\ \text { SE75 } & 1.46 \mathrm{E}+04 \\ \text { SE79 } & 8.66 \mathrm{E}-01 \\ \text { BR82 } & 1.07 \mathrm{E}+05 \\ \text { BR83 } & 3.07 \mathrm{E}+02 \\ \text { KR83M } & 1.36 \mathrm{E}+02 \\ \text { BR84 } & 6.23 \mathrm{E}+04 \\ \text { KR85M } & 5.77 \mathrm{E}+03 \\ \text { KR85 } & 1.06 \mathrm{E}+02 \\ \text { KR87 } & 3.10 \mathrm{E}+04 \\ \text { RB87 } & 3.64 \mathrm{E}+00 \\ \text { KR88 } & 6.90 \mathrm{E}+04 \\ \text { RB88 } & 2.55 \mathrm{E}+04 \\ \text { KR89 } & 6.77 \mathrm{E}+04 \\ \text { RB89 } & 7.62 \mathrm{E}+04 \\ \text { SR89 } & 1.43 \mathrm{E}+02 \\ \text { SR87M } & 1.13 \mathrm{E}+04 \\ \text { RB86 } & 3.58 \mathrm{E}+03 \\ \text { SR85 } & 1.86 \mathrm{E}+04 \\ \text { SR90 } & 1.61 \mathrm{E}+01 \\ \text { Y 90 } & 3.47 \mathrm{E}+02 \\ \text { SR91 } & 2.61 \mathrm{E}+04 \\ \text { Y 91M } & 2.49 \mathrm{E}+04 \\ \text { Y 91 } & 2.87 \mathrm{E}+02 \\ \text { SR92 } & 5.37 \mathrm{E}+04 \\ \text { Y 92 } & 1.11 \mathrm{E}+04 \\ \text { Y 93 } & 3.90 \mathrm{E}+03 \\ \text { M093 } & 3.84 \mathrm{E}+02 \\ \text { ZR93 } & 7.09 \mathrm{E}-02 \\ \text { NB93M } & 7.97 \mathrm{E}+01 \\ \text { ZR95 } & 2.95 \mathrm{E}+04 \\ \text { NB95M } & 2.62 \mathrm{E}+03 \\ \text { NB95 } & 3.25 \mathrm{E}+04 \\ \text { ZR97 } & 7.17 \mathrm{E}+03 \\ \text { NB97M } & 2.56 \mathrm{E}+04 \\ \text { NB97 } & 2.65 \mathrm{E}+04 \\ \text { NB94 } & 5.87 \mathrm{E}+04 \\ \text { M099 } & 5.72 \mathrm{E}+03 \\ \text { TC99M } & 4.52 \mathrm{E}+03 \\ \text { TC99 } & 4.19 \mathrm{E}+00 \\ \text { TC101 } & 1.47 \mathrm{E}+04 \\ & \\ \text { Th }\end{array}$


TABLE D.8. EXTDF Output for Surface Source Configuration (contd)

$\begin{array}{ll}\text { RU103 } & 1.82 E+04 \\ \text { PD103 } & 1.04 E+03 \\ \text { RH103M } & 1.36 E+02 \\ \text { RU105 } & 3.13 E+04 \\ \text { RH105 } & 3.37 E+03 \\ \text { RU106 } & 8.72 E+03 \\ \text { PD107 } & 5.45 E-03 \\ \text { PD109 } & 7.09 E+02 \\ \text { AG110M } & 1.08 E+05 \\ \text { AG111 } & 1.11 E+03 \\ \text { CD109 } & 9.81 E+02 \\ \text { CD113M } & 1.81 E+01 \\ \text { CD115M } & 1.02 E+03 \\ \text { CD115 } & 7.33 E+03 \\ \text { IN115M } & 6.90 E+03 \\ \text { IN111 } & 1.46 E+04 \\ \text { IN114M } & 5.30 E+03 \\ \text { SN113 } & 1.28 E+03 \\ \text { IN113M } & 9.23 E+03 \\ \text { SN117M } & 5.44 E+03 \\ \text { SN119M } & 7.05 E+02 \\ \text { SN121M } & 0.00 E+00 \\ \text { SN121 } & 0.00 E+00 \\ \text { SN123 } & 3.62 E+02 \\ \text { I 125 } & 2.25 E+03 \\ \text { SN125 } & 1.18 E+04 \\ \text { SB125 } & 1.83 E+04 \\ \text { TE125M } & 1.90 E+03 \\ \text { SN126 } & 1.92 E+03 \\ \text { SB126M } & 6.28 E+04 \\ \text { SB126 } & 1.07 E+05 \\ \text { SB122 } & 2.04 E+04 \\ \text { SB124 } & 7.40 E+04 \\ \text { SB127 } & 2.60 E+04 \\ \text { TE127M } & 6.16 E+02 \\ \text { TE127 } & 2.33 E+02 \\ \text { TE123M } & 5.00 E+03 \\ \text { TE129M } & 1.67 E+03 \\ \text { TE129 } & 2.47 E+03 \\ \text { I 129 } & 1.22 E+03 \\ \text { TE131M } & 5.74 E+04 \\ \text { TE131 } & 1.64 E+04 \\ \text { I1131 } & 1.44 E+04 \\ \text { XE131M } & 9.57 E+02 \\ \text { TE132 } & 9.68 E+03 \\ \text { I132 } 132 & 9.14 E+04 \\ \text { TE133M } & 8.91 E+04 \\ \text { TE133 } & 3.75 E+04 \\ \text { I133 } 133 & 2.16 E+04 \\ \text { XE133M } & 1.85 E+03 \\ & \end{array}$


TABLE D.8. EXTDF Output for Surface Source Configuration (contd)

$\begin{array}{ll}\text { XE133 } & 1.73 E+03 \\ \text { TE134 } & 3.52 E+04 \\ \text { I 134 } & 1.01 E+05 \\ \text { CS134M } & 1.26 E+03 \\ \text { CS134 } & 6.56 E+04 \\ \text { I 130 } & 7.98 E+04 \\ \text { I 135 } & 6.01 E+04 \\ \text { XE135M } & 1.51 E+04 \\ \text { XE135 } & 9.50 E+03 \\ \text { CS135 } & 1.67 E+00 \\ \text { XE137 } & 8.63 E+03 \\ \text { CS137 } & 2.24 E+04 \\ \text { XE138 } & 4.27 E+04 \\ \text { CS138 } & 9.00 E+04 \\ \text { CS139 } & 1.22 E+04 \\ \text { BA139 } & 1.46 E+03 \\ \text { BA140 } & 7.03 E+03 \\ \text { LA140 } & 8.39 E+04 \\ \text { CS136 } & 8.28 E+04 \\ \text { BA141 } & 3.37 E+04 \\ \text { LA141 } & 2.10 E+03 \\ \text { CE141 } & 2.74 E+03 \\ \text { BA142 } & 3.50 E+04 \\ \text { LA142 } & 9.75 E+04 \\ \text { CE143 } & 9.94 E+03 \\ \text { PR143 } & 4.80 E+01 \\ \text { CE144 } & 7.49 E+02 \\ \text { PR144M } & 5.71 E+02 \\ \text { PR144 } & 1.73 E+03 \\ \text { PR142 } & 2.33 E+03 \\ \text { ND147 } & 5.11 E+03 \\ \text { PM147 } & 2.38 E+00 \\ \text { SM147 } & 0.00 E+00 \\ \text { PM148M } & 8.39 E+04 \\ \text { PM148 } & 2.36 E+04 \\ \text { PM149 } & 4.69 E+02 \\ \text { PM151 } & 1.32 E+04 \\ \text { SM151 } & 1.19 E+00 \\ \text { SM153 } & 2.73 E+03 \\ \text { EU152M } & 1.26 E+04 \\ \text { EU152 } & 4.57 E+04 \\ \text { EU154 } & 4.73 E+04 \\ \text { EU155 } & 2.28 E+03 \\ \text { EU156 } & 5.09 E+04 \\ \text { GD153 } & 4.35 E+03 \\ \text { GD159 } & 1.57 E+03 \\ \text { TB160 } & 4.12 E+04 \\ \text { TB161 } & 9.49 E+02 \\ \text { DY165 } & 1.05 E+03 \\ \text { H0166M } & 6.07 E+04 \\ & \end{array}$


TABLE D.8. EXTDF Output for Surface Source Configuration (contd)

$\begin{array}{ll}\text { HO166 } & 1.29 E+03 \\ \text { ER169 } & 6.69 E+00 \\ \text { ER171 } & 1.51 E+04 \\ \text { TA182 } & 5.07 E+04 \\ \text { W } 181 & 1.34 E+03 \\ \text { W } 185 & 1.03 E+01 \\ \text { W } 187 & 1.86 E+04 \\ \text { RE187 } & 0.00 E+00 \\ \text { OS185 } & 2.78 E+04 \\ \text { OS191 } & 2.65 E+03 \\ \text { IR192 } & 3.34 E+04 \\ \text { HG203 } & 7.67 E+03 \\ \text { TH230 } & 5.98 E+01 \\ \text { RA226 } & 1.86 E+02 \\ \text { RN222 } & 6.85 E+04 \\ \text { PB210 } & 2.11 E+02 \\ \text { BI210 } & 7.08 E+01 \\ \text { P0210 } & 3.45 E-01 \\ \text { U 232 } & 7.73 E+01 \\ \text { TH232 } & 5.44 E+01 \\ \text { RA228 } & 1.19 E-02 \\ \text { AC228 } & 3.74 E+04 \\ \text { TH228 } & 1.20 E+02 \\ \text { RA224 } & 4.06 E+02 \\ \text { PB212 } & 5.61 E+03 \\ \text { BI212 } & 5.18 E+04 \\ U 234 & 6.53 E+01 \\ \text { U 236 } & 6.03 E+01 \\ \text { U 235 } & 4.68 E+03 \\ \text { TH231 } & 9.54 E+02 \\ \text { PA231 } & 1.56 E+03 \\ \text { AC227 } & 1.19 E+01 \\ \text { TH227 } & 4.20 E+03 \\ \text { FR223 } & 2.09 E+03 \\ \text { RA223 } & 1.12 E+04 \\ \text { U 237 } & 5.93 E+03 \\ \text { NP237 } & 1.19 E+03 \\ \text { PA233 } & 9.01 E+03 \\ \text { U 2333 } & 3.20 E+01 \\ \text { TH2229 } & 3.35 E+03 \\ \text { RA225 } & 6.25 E+02 \\ \text { AC225 } & 9.39 E+03 \\ \text { U 2338 } & 5.33 E+01 \\ \text { TH234 } & 1.05 E+03 \\ \text { PA234 } & 7.70 E+04 \\ \text { PU236 } & 7.76 E+01 \\ \text { PU237 } & 2.25 E+03 \\ \text { AM242M } & 1.88 E+02 \\ \text { AM2422 } & 7.444 E+02 \\ \text { CM242 } & 6.80 E+01 \\ & \end{array}$


TABLE D.8. EXTDF Output for Surface Source Configuration (contd)

$\begin{array}{ll}\text { PU238 } & 6.83 \mathrm{E}+01 \\ \text { PU242 } & 5.40 \mathrm{E}+01 \\ \text { NP238 } & 2.15 \mathrm{E}+04 \\ \text { PU238 } & 6.83 \mathrm{E}+01 \\ \text { CM244 } & 6.06 \mathrm{E}+01 \\ \text { PU244 } & 4.67 \mathrm{E}+01 \\ \text { U240 } & 1.49 \mathrm{E}+04 \\ \text { PU240 } & 6.51 \mathrm{E}+01 \\ \text { CM245 } & 3.01 \mathrm{E}+03 \\ \text { PU241 } & 1.76 \mathrm{E}-04 \\ \text { AM241 } & 9.39 \mathrm{E}+02 \\ \text { CM246 } & 5.40 \mathrm{E}+01 \\ \text { CM247 } & 1.43 \mathrm{E}+04 \\ \text { PU243 } & 7.88 \mathrm{E}+02 \\ \text { CM243 } & 5.18 \mathrm{E}+03 \\ \text { AM243 } & 1.80 \mathrm{E}+03 \\ \text { NP239 } & 6.96 \mathrm{E}+03 \\ \text { PU239 } & 2.81 \mathrm{E}+01 \\ \text { CM248 } & 4.28 \mathrm{E}+01 \\ \text { CF252 } & 4.29 \mathrm{E}+01\end{array}$


TABLE D.9. EXTDF Output for Volume Source Configuration

EXTDF Dose Calculation Program

(Version 1.429 19-Dec-89)

Case title: $5 \mathrm{~m}$ Volume, $1 \mathrm{~cm}$ thick - Residual Radioactivity

Executed on: $12 / 21 / 89$ at $08: 28: 32$

Shield composition (gm/cc):

$\begin{array}{lccccc} & 1 & 2 & 3 & 4 & 5 \\ \text { ORDCONC } & 2.4 E+00 & 0.0 E+00 & 0.0 E+00 & 0.0 E+00 & 0.0 E+00 \\ \text { AIR } & 0.0 E+00 & 1.3 E-03 & 0.0 E+00 & 0.0 E+00 & 0.0 E+00\end{array}$

Shield thickness $(\mathrm{cm})$ :

$$
1.0 E+00 \quad 0.0 E+00
$$

Geometry index:

END OF CYLINDER source

SLAB shields

Distance to detector:

Source length:

Source radius:

End of source to detector:

Source volume:

Integration specs:

NTHETA:

NPSI :

DELR:

NTHETA $\times$ NPSI $\times$ T(1) / DELR:

Taylor buildup data for shield:

with effective atomic number:

Units are rem/hr per $\mathrm{Ci} / \mathrm{cm} 2$ or $\mathrm{cm} 3$

$\begin{array}{ll}\text { H } 3 & 4.23 \mathrm{E}-06 \\ \text { BE10 } & 5.87 \mathrm{E}+00 \\ \text { C 14 } & 1.71 \mathrm{E}-01 \\ \text { N } 13 & 2.57 \mathrm{E}+04 \\ \text { F 18 } & 2.49 \mathrm{E}+04 \\ \text { NA22 } & 5.78 \mathrm{E}+04 \\ \text { NA24 } & 1.14 \mathrm{E}+05 \\ \text { SI31 } & 9.38 \mathrm{E}+01 \\ \text { P } 32 & 9.46 \mathrm{E}+01 \\ \text { P 33 } & 6.39 \mathrm{E}-01 \\ \text { S 35 } & 1.99 \mathrm{E}-01 \\ \text { CL36 } & 1.06 \mathrm{E}+01 \\ \text { K 40 } & 4.50 \mathrm{E}+03 \\ \text { AR39 } & 5.89 \mathrm{E}+00 \\ \text { AR41 } & 3.45 \mathrm{E}+04 \\ \text { CA41 } & 5.75 \mathrm{E}+00\end{array}$


TABLE D.9. EXTDF Output for Volume Source Configuration (contd)

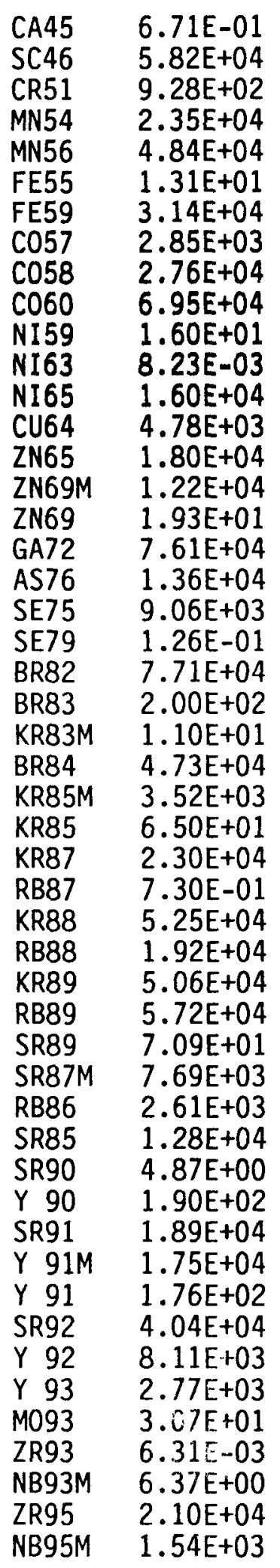


TABLE D.9. EXTDF Output for Volume Source Configuration (contd)

$\begin{array}{ll}\text { NB95 } & 2.34 E+04 \\ \text { ZR97 } & 5.19 E+03 \\ \text { NB97M } & 1.80 E+04 \\ \text { NB97 } & 1.86 E+04 \\ \text { NB94 } & 4.19 E+04 \\ \text { M099 } & 3.94 E+03 \\ \text { TC99M } & 2.59 E+03 \\ \text { TC99 } & 8.86 E-01 \\ \text { TC101 } & 1.00 E+04 \\ \text { RU103 } & 1.27 E+04 \\ \text { PD103 } & 8.89 E+01 \\ \text { RH103M } & 1.14 E+01 \\ \text { RU105 } & 2.14 E+04 \\ \text { RH105 } & 2.29 E+03 \\ \text { RU106 } & 6.08 E+03 \\ \text { PD107 } & 4.37 E-04 \\ \text { PD109 } & 1.35 E+02 \\ \text { AG110M } & 7.83 E+04 \\ \text { AG111 } & 7.34 E+02 \\ \text { CD109 } & 8.16 E+01 \\ \text { CD113M } & 5.73 E+00 \\ \text { CD115M } & 7.17 E+02 \\ \text { CD115 } & 5.05 E+03 \\ \text { IN115M } & 4.41 E+03 \\ \text { IN111 } & 8.38 E+03 \\ \text { IN114M } & 3.23 E+03 \\ \text { SN113 } & 2.08 E+02 \\ \text { IN113M } & 6.09 E+03 \\ \text { SN117M } & 2.65 E+03 \\ \text { SN119M } & 5.86 E+01 \\ \text { SN121M } & 0.00 E+00 \\ \text { SN121 } & 0.00 E+00 \\ \text { SN123 } & 2.37 E+02 \\ \text { I 125 } & 2.47 E+02 \\ \text { SN125 } & 8.68 E+03 \\ \text { SB125 } & 1.23 E+04 \\ \text { TE125M } & 2.17 E+02 \\ \text { SN126 } & 7.21 E+02 \\ \text { SB126M } & 4.41 E+04 \\ \text { SB126 } & 7.57 E+04 \\ \text { SB122 } & 1.43 E+04 \\ \text { SB124 } & 5.44 E+04 \\ \text { SB127 } & 1.82 E+04 \\ \text { TE127M } & 6.92 E+01 \\ \text { TE127 } & 1.52 E+02 \\ \text { TE123M } & 2.52 E+03 \\ \text { TE129M } & 8.98 E+02 \\ \text { TE129 } & 1.54 E+03 \\ \text { I129 } & 1.40 E+02 \\ \text { TE131M } & 4.13 E+04 \\ & \end{array}$


TABLE D.9. EXTDF Output for Volume Source Configuration (contd)

$\begin{array}{ll}\text { TE131 } & 1.11 E+04 \\ \text { I131 } & 9.80 E+03 \\ \text { XE131M } & 1.48 E+02 \\ \text { TE132 } & 5.58 E+03 \\ \text { I132 } & 6.57 E+04 \\ \text { TE133M } & 6.48 E+04 \\ \text { TE133 } & 2.69 E+04 \\ \text { I133 } & 1.52 E+04 \\ \text { XE133M } & 7.14 E+02 \\ \text { XE133 } & 6.32 E+02 \\ \text { TE134 } & 2.41 E+04 \\ \text { I 134 } & 7.33 E+04 \\ \text { CS134M } & 4.85 E+02 \\ \text { CS134 } & 4.68 E+04 \\ \text { I130 } & 5.62 E+04 \\ \text { I135 } & 4.48 E+04 \\ \text { XE135M } & 1.04 E+04 \\ \text { XE135 } & 6.10 E+03 \\ \text { CS135 } & 2.95 E-01 \\ \text { XE137 } & 6.02 E+03 \\ \text { CS137 } & 1.57 E+04 \\ \text { XE138 } & 3.17 E+04 \\ \text { CS138 } & 6.75 E+04 \\ \text { CS139 } & 9.05 E+03 \\ \text { BA139 } & 8.47 E+02 \\ \text { BA140 } & 4.65 E+03 \\ \text { LA140 } & 6.22 E+04 \\ \text { CS136 } & 5.96 E+04 \\ \text { BA141 } & 2.36 E+04 \\ \text { LA141 } & 1.51 E+03 \\ \text { CE141 } & 1.47 E+03 \\ \text { BA142 } & 2.52 E+04 \\ \text { LA142 } & 7.42 E+04 \\ \text { CE143 } & 6.10 E+03 \\ \text { PR143 } & 1.93 E+01 \\ \text { CE144 } & 3.69 E+02 \\ \text { PR144M } & 1.14 E+02 \\ \text { PR144 } & 1.20 E+03 \\ \text { PR142 } & 1.70 E+03 \\ \text { ND147 } & 3.04 E+03 \\ \text { PM147 } & 4.83 E-01 \\ \text { SM147 } & 0.00 E+00 \\ \text { PM148M } & 5.93 E+04 \\ \text { PM148 } & 1.74 E+04 \\ \text { PM149 } & 2.93 E+02 \\ \text { PM151 } & 8.65 E+03 \\ \text { SM151 } & 9.88 E-02 \\ \text { SM153 } & 1.21 E+03 \\ \text { EU152M } & 8.88 E+03 \\ \text { EU152 } & 3.27 E+04 \\ & \end{array}$


TABLE D.9. EXTDF Output for Volume Source Configuration (contd)

$\begin{array}{ll}\text { EU154 } & 3.41 E+04 \\ \text { EU155 } & 1.12 \mathrm{E}+03 \\ \text { EU156 } & 3.79 \mathrm{E}+04 \\ \text { GD153 } & 1.75 \mathrm{E}+03 \\ \text { GD159 } & 9.38 \mathrm{E}+02 \\ \text { TB160 } & 2.97 \mathrm{E}+04 \\ \text { TB161 } & 2.55 \mathrm{E}+02 \\ \text { DY165 } & 6.22 \mathrm{E}+02 \\ \text { H0166M } & 4.22 \mathrm{E}+04 \\ \text { H0166 } & 7.81 \mathrm{E}+02 \\ \text { ER169 } & 1.44 \mathrm{E}+00 \\ \text { ER171 } & 9.73 \mathrm{E}+03 \\ \text { TA182 } & 3.67 \mathrm{E}+04 \\ \text { W 181 } & 4.75 \mathrm{E}+02 \\ \text { W 185 } & 3.01 \mathrm{E}+00 \\ \text { W 187 } & 1.28 \mathrm{E}+04 \\ \text { RE187 } & 0.00 \mathrm{E}+00 \\ \text { OS185 } & 1.91 \mathrm{E}+04 \\ \text { OS191 } & 1.26 \mathrm{E}+03 \\ \text { IR192 } & 2.29 \mathrm{E}+04 \\ \text { HG203 } & 4.84 \mathrm{E}+03 \\ \text { TH230 } & 9.06 \mathrm{E}+00 \\ \text { RA226 } & 1.04 \mathrm{E}+02 \\ \text { RN222 } & 5.00 \mathrm{E}+04 \\ \text { PB210 } & 2.99 \mathrm{E}+01 \\ \text { B1210 } & 3.09 \mathrm{E}+01 \\ \text { P0210 } & 2.49 \mathrm{E}-01 \\ \text { U 232 } & 9.06 \mathrm{E}+00 \\ \text { TH232 } & 6.40 \mathrm{E}+00 \\ \text { RA228 } & 9.54 \mathrm{E}-04 \\ \text { AC228 } & 2.71 \mathrm{E}+04 \\ \text { TH228 } & 4.13 \mathrm{E}+01 \\ \text { RA224 } & 2.59 \mathrm{E}+02 \\ \text { PB212 } & 3.44 \mathrm{E}+03 \\ \text { B1212 } & 3.91 \mathrm{E}+04 \\ \text { U 234 } & 6.85 \mathrm{E}+00 \\ \text { U 236 } & 5.58 \mathrm{E}+00 \\ \text { U 235 } & 2.64 \mathrm{E}+03 \\ \text { TH231 } & 2.25 \mathrm{E}+02 \\ \text { PA231 } & 8.08 \mathrm{E}+02 \\ \text { AC227 } & 3.40 \mathrm{E}+00 \\ \text { TH227 } & 2.52 \mathrm{E}+03 \\ \text { FR223 } & 1.03 \mathrm{E}+03 \\ \text { RA223 } & 7.20 \mathrm{E}+03 \\ \text { U 237 } & 3.21 \mathrm{E}+03 \\ \text { NP237 } & 3.94 \mathrm{E}+02 \\ \text { PA233 } & 5.82 \mathrm{E}+03 \\ \text { U 233 } & 7.10 \mathrm{E}+00 \\ \text { TH229 } & 1.63 \mathrm{E}+03 \\ \text { RA225 } & 1.51 \mathrm{E}+02 \\ & \end{array}$


TABLE D.9. EXTDF Output for Volume Source Configuration (contd)

$\begin{array}{ll}\text { AC225 } & 6.34 \mathrm{E}+03 \\ U \text { 238 } & 4.93 \mathrm{E}+00 \\ \text { TH234 } & 6.22 \mathrm{E}+02 \\ \text { PA234 } & 5.48 \mathrm{E}+04 \\ \text { PU236 } & 6.77 \mathrm{E}+00 \\ \text { PU237 } & 1.12 \mathrm{E}+03 \\ \text { AM242M } & 1.98 \mathrm{E}+01 \\ \text { AM242 } & 3.29 \mathrm{E}+02 \\ \text { CM242 } & 5.64 \mathrm{E}+00 \\ \text { PU238 } & 5.71 \mathrm{E}+00 \\ \text { PU242 } & 4.55 \mathrm{E}+00 \\ \text { NP238 } & 1.57 \mathrm{E}+04 \\ \text { PU238 } & 5.71 \mathrm{E}+00 \\ \text { CM244 } & 4.99 \mathrm{E}+00 \\ \text { PU244 } & 3.83 \mathrm{E}+00 \\ \text { U 240 } & 1.03 \mathrm{E}+04 \\ \text { PU240 } & 5.48 \mathrm{E}+00 \\ \text { CM245 } & 1.54 \mathrm{E}+03 \\ \text { PU241 } & 1.41 \mathrm{E}-05 \\ \text { AM241 } & 2.65 \mathrm{E}+02 \\ \text { CM246 } & 4.40 \mathrm{E}+00 \\ \text { CM247 } & 9.88 \mathrm{E}+03 \\ \text { PU243 } & 3.92 \mathrm{E}+02 \\ \text { CM243 } & 2.98 \mathrm{E}+03 \\ \text { AM243 } & 7.62 \mathrm{E}+02 \\ \text { NP239 } & 4.05 \mathrm{E}+03 \\ \text { PU239 } & 3.44 \mathrm{E}+00 \\ \text { CM248 } & 3.56 \mathrm{E}+00 \\ \text { CF252 } & 3.62 \mathrm{E}+00\end{array}$


TABLE D.10. EXTDF Output for Soil Source Configuration

EXTDF Dose Calculation Program

(Version 1.429 19-Dec-89)

Case title: $15 \mathrm{~cm}$ Infinite Slab Source, soil for Residual Radioactivity Executed on: $12 / 21 / 89$ at $08: 50: 36$

Shield composition $(\mathrm{gm} / \mathrm{cc})$ :

$\begin{array}{lccccc}\text { ORDCONC } & 1.8 \mathrm{E}+00 & 0.0 \mathrm{E}+00 & 0.0 \mathrm{E}+00 & 0.0 \mathrm{E}+00 & 0.0 \mathrm{E}+00\end{array}$

$\begin{array}{llllll}A I R & 0.0 \mathrm{E}+00 & 1.3 \mathrm{E}-03 \quad 0.0 \mathrm{E}+00 & 0.0 \mathrm{E}+00 & 0.0 \mathrm{E}+00\end{array}$

Shield thickness $(\mathrm{cm})$ :

$$
1.5 \mathrm{E}+01 \quad 1.0 \mathrm{E}+02
$$

Geometry index:

INFINITE SLAB source

SLAB shield

Distance to detector:

Angle:

Source thickness:

Taylor buildup data for shield:

with effective atomic number:

$1.150 \mathrm{E}+02 \mathrm{~cm}$

0.0 degrees $15.00 \mathrm{~cm}$ 1

10.0

Units are $r e m / h r$ per $\mathrm{Ci} / \mathrm{cm} 2$ or $\mathrm{cm} 3$

$\begin{array}{ll}\text { H 3 } & 4.38 \mathrm{E}-06 \\ \text { BE10 } & 5.48 \mathrm{E}+01 \\ \text { C 14 } & 9.39 \mathrm{E}-01 \\ \text { N 13 } & 4.36 \mathrm{E}+05 \\ \text { F 18 } & 4.22 \mathrm{E}+05 \\ \text { NA22 } & 8.89 \mathrm{E}+05 \\ \text { NA24 } & 1.72 \mathrm{E}+06 \\ \text { SI31 } & 1.22 \mathrm{E}+03 \\ \text { P 32 } & 1.22 \mathrm{E}+03 \\ \text { P 33 } & 4.25 \mathrm{E}+00 \\ \text { S 35 } & 1.13 \mathrm{E}+00 \\ \text { CL36 } & 1.09 \mathrm{E}+02 \\ \text { K 40 } & 6.46 \mathrm{E}+04 \\ \text { AR39 } & 5.52 \mathrm{E}+01 \\ \text { AR41 } & 4.93 \mathrm{E}+05 \\ \text { CA41 } & 5.95 \mathrm{E}+00 \\ \text { CA45 } & 4.51 \mathrm{E}+00 \\ \text { SC46 } & 8.28 \mathrm{E}+05 \\ \text { CR51 } & 1.46 \mathrm{E}+04 \\ \text { MN54 } & 3.31 \mathrm{E}+05 \\ \text { MN56 } & 7.14 \mathrm{E}+05 \\ \text { FE55 } & 1.36 \mathrm{E}+01 \\ \text { FE59 } & 4.57 \mathrm{E}+05\end{array}$


TABLE D.10. EXTDF Output for Soil Source Configuration (contd)

$\begin{array}{ll}\text { C057 } & 3.15 E+04 \\ \text { C058 } & 4.01 E+05 \\ \text { C060 } & 9.93 E+05 \\ \text { NI59 } & 1.65 E+01 \\ \text { NI63 } & 2.39 E-02 \\ \text { NI65 } & 2.31 E+05 \\ \text { CU64 } & 8.05 E+04 \\ \text { ZN65 } & 2.58 E+05 \\ \text { ZN69M } & 2.07 E+05 \\ \text { ZN69 } & 2.10 E+02 \\ \text { GA72 } & 1.13 E+06 \\ \text { AS76 } & 2.04 E+05 \\ \text { SE75 } & 1.22 E+05 \\ \text { SE79 } & 6.70 E-01 \\ \text { BR82 } & 1.13 E+06 \\ \text { BR83 } & 3.28 E+03 \\ \text { KR83M } & 1.22 E+01 \\ \text { BR84 } & 7.21 E+05 \\ \text { KR85M } & 4.54 E+04 \\ \text { KR85 } & 1.04 E+03 \\ \text { KR87 } & 3.60 E+05 \\ \text { RB87 } & 5.03 E+00 \\ \text { KR88 } & 7.85 E+05 \\ \text { RB88 } & 2.90 E+05 \\ \text { KR89 } & 7.66 E+05 \\ \text { RB89 } & 8.52 E+05 \\ \text { SR89 } & 8.83 E+02 \\ \text { SR87M } & 1.22 E+05 \\ \text { RB86 } & 3.85 E+04 \\ \text { SR85 } & 2.17 E+05 \\ \text { SR90 } & 4.47 E+01 \\ \text { Y90 } & \cdot 2.50 E+03 \\ \text { SR91 } & 2.81 E+05 \\ \text { Y91M } & 2.65 E+05 \\ \text { Y 91 } & 2.38 E+03 \\ \text { SR92 } & 5.81 E+05 \\ \text { Y92 } & 1.20 E+05 \\ \text { Y93 } & 4.05 E+04 \\ \text { M093 } & 3.18 E+01 \\ \text { ZR93 } & 1.68 E-02 \\ \text { NB93M } & 6.60 E+00 \\ \text { ZR95 } & 3.05 E+05 \\ \text { NB95M } & 2.07 E+04 \\ \text { NB95 } & 3.31 E+05 \\ \text { ZR97 } & 7.75 E+04 \\ \text { NB97M } & 2.73 E+05 \\ \text { NB97 } & 2.82 E+05 \\ \text { NB94 } & 6.10 E+05 \\ \text { M099 } & 5.74 E+04 \\ \text { TC99M } & 2.88 E+04 \\ \text { To } & \end{array}$


TABLE D.10. EXTDF Output for Soil Source Configuration (contd)

$\begin{array}{ll}\text { TC99 } & 6.33 E+00 \\ \text { TC101 } & 1.59 E+05 \\ \text { RU103 } & 2.13 E+05 \\ \text { PD103 } & 3.95 E+02 \\ \text { RH103M } & 4.18 E+01 \\ \text { RU105 } & 3.27 E+05 \\ \text { RH105 } & 3.64 E+04 \\ \text { RU106 } & 9.57 E+04 \\ \text { PD107 } & 5.21 E-04 \\ \text { PD109 } & 1.22 E+03 \\ \text { AG110M } & 1.15 E+06 \\ \text { AG111 } & 1.14 E+04 \\ \text { CD109 } & 3.30 E+02 \\ \text { CD113M } & 5.41 E+01 \\ \text { CD115M } & 1.04 E+04 \\ \text { CD115 } & 8.49 E+04 \\ \text { IN115M } & 6.97 E+04 \\ \text { IN111 } & 1.07 E+05 \\ \text { IN114M } & 4.63 E+04 \\ \text { SN113 } & 1.96 E+03 \\ \text { IN113M } & 9.66 E+04 \\ \text { SN117M } & 2.89 E+04 \\ \text { SN119M } & 2.26 E+02 \\ \text { SN121M } & 0.00 E+00 \\ \text { SN121 } & 0.00 E+00 \\ \text { SN123 } & 3.37 E+03 \\ \text { I 125 } & 1.20 E+03 \\ \text { SN125 } & 1.29 E+05 \\ \text { SB125 } & 1.94 E+05 \\ \text { TE125M } & 1.10 E+03 \\ \text { SN126 } & 6.54 E+03 \\ \text { SB126M } & 6.88 E+05 \\ \text { SB126 } & 1.16 E+06 \\ \text { SB122 } & 2.16 E+05 \\ \text { SB124 } & 8.10 E+05 \\ \text { SB127 } & 2.79 E+05 \\ \text { TE127M } & 3.57 E+02 \\ \text { TE127 } & 2.49 E+03 \\ \text { TE123M } & 2.75 E+04 \\ \text { TE129M } & 1.31 E+04 \\ \text { TE129 } & 2.46 E+04 \\ \text { I 129 } & 6.98 E+02 \\ \text { TE131M } & 5.93 E+05 \\ \text { TE131 } & 1.63 E+05 \\ \text { I 131 } & 1.54 E+05 \\ \text { XE131M } & 1.06 E+03 \\ \text { TE132 } & 7.43 E+04 \\ \text { I 132 } & 9.72 E+05 \\ \text { TE133M } & 9.58 E+05 \\ \text { TE133 } & 4.11 E+05 \\ & \end{array}$


TABLE D.10. EXTDF Output for Soil Source Configuration (contd)

$\begin{array}{ll}\text { I 133 } & 2.48 E+05 \\ \text { XE133M } & 8.89 E+03 \\ \text { XE133 } & 5.52 E+03 \\ \text { TE134 } & 3.57 E+05 \\ \text { I 134 } & 1.07 E+06 \\ \text { CS134M } & 4.79 E+03 \\ \text { CS134 } & 6.85 E+05 \\ \text { I 130 } & 8.80 E+05 \\ \text { I 135 } & 6.53 E+05 \\ \text { XE135M } & 1.77 E+05 \\ \text { XE135 } & 8.39 E+04 \\ \text { CS135 } & 1.82 E+00 \\ \text { XE137 } & 9.68 E+04 \\ \text { CS137 } & 2.38 E+05 \\ \text { XE138 } & 4.79 E+05 \\ \text { CS138 } & 1.00 E+06 \\ \text { CS139 } & 1.33 E+05 \\ \text { BA139 } & 1.02 E+04 \\ \text { BA140 } & 7.67 E+04 \\ \text { LA140 } & 9.14 E+05 \\ \text { CS136 } & 8.64 E+05 \\ \text { BA141 } & 3.49 E+05 \\ \text { LA141 } & 2.15 E+04 \\ \text { CE141 } & 1.60 E+04 \\ \text { BA142 } & 3.65 E+05 \\ \text { LA142 } & 1.14 E+06 \\ \text { CE143 } & 8.60 E+04 \\ \text { PR143 } & 2.11 E+02 \\ \text { CE144 } & 3.90 E+03 \\ \text { PR144M } & 6.70 E+02 \\ \text { PR144 } & 1.75 E+04 \\ \text { PR142 } & 2.42 E+04 \\ \text { ND147 } & 4.58 E+04 \\ \text { PM147 } & 3.44 E+00 \\ \text { SM147 } & 0.00 E+00 \\ \text { PM148M } & 9.02 E+05 \\ \text { PM148 } & 2.54 E+05 \\ \text { PM149 } & 4.02 E+03 \\ \text { PM151 } & 1.29 E+05 \\ \text { SM151 } & 2.44 E-01 \\ \text { SM153 } & 1.21 E+04 \\ \text { EU152M } & 1.27 E+05 \\ \text { EU152 } & 4.72 E+05 \\ \text { EU154 } & 4.91 E+05 \\ \text { EU155 } & 1.13 E+04 \\ \text { EU156 } & 5.60 E+05 \\ \text { GD153 } & 1.61 E+04 \\ \text { GD159 } & 1.40 E+04 \\ \text { TB160 } & 4.25 E+05 \\ \text { TB161 } & 1.87 E+03 \\ & \end{array}$


TABLE D.10. EXTDF Output for Soil Source Configuration (contd)

$\begin{array}{ll}\text { DY165 } & 8.67 \mathrm{E}+03 \\ \text { H0166M } & 6.10 \mathrm{E}+05 \\ \text { H0166 } & 1.03 \mathrm{E}+04 \\ \text { ER169 } & 1.07 \mathrm{E}+01 \\ \text { ER171 } & 1.43 \mathrm{E}+05 \\ \text { TA182 } & 5.18 \mathrm{E}+05 \\ \text { W } 181 & 3.47 \mathrm{E}+03 \\ \text { W } 185 & 2.69 \mathrm{E}+01 \\ \text { W } 187 & 1.95 \mathrm{E}+05 \\ \text { RE187 } & 0.00 \mathrm{E}+00 \\ \text { OS185 } & 2.82 \mathrm{E}+05 \\ \text { OS191 } & 1.24 \mathrm{E}+04 \\ \text { IR192 } & 3.63 \mathrm{E}+05 \\ \text { HG203 } & 6.50 \mathrm{E}+04 \\ \text { TH230 } & 5.15 \mathrm{E}+01 \\ \text { RA226 } & 1.15 \mathrm{E}+03 \\ \text { RN222 } & 7.38 \mathrm{E}+05 \\ \text { PB210 } & 1.31 \mathrm{E}+02 \\ \text { B1210 } & 3.58 \mathrm{E}+02 \\ \text { P0210 } & 3.51 \mathrm{E}+00 \\ \text { U 232 } & 3.91 \mathrm{E}+01 \\ \text { TH232 } & 2.68 \mathrm{E}+01 \\ \text { RA228 } & 1.36 \mathrm{E}-03 \\ \text { AC228 } & 4.01 \mathrm{E}+05 \\ \text { TH228 } & 4.27 \mathrm{E}+02 \\ \text { RA224 } & 3.55 \mathrm{E}+03 \\ \text { PB212 } & 4.56 \mathrm{E}+04 \\ \text { B1212 } & 5.99 \mathrm{E}+05 \\ \text { U 234 } & 2.37 \mathrm{E}+01 \\ \text { U 236 } & 1.23 \mathrm{E}+01 \\ \text { U 235 } & 3.00 \mathrm{E}+04 \\ \text { TH231 } & 1.84 \mathrm{E}+03 \\ \text { PA231 } & 1.17 \mathrm{E}+04 \\ \text { AC227 } & 3.23 \mathrm{E}+01 \\ \text { TH227 } & 3.45 \mathrm{E}+04 \\ \text { FR223 } & 1.23 \mathrm{E}+04 \\ \text { RA223 } & 1.05 \mathrm{E}+05 \\ \text { U 237 } & 3.83 \mathrm{E}+04 \\ \text { NP237 } & 3.69 \mathrm{E}+03 \\ \text { PA233 } & 8.84 \mathrm{E}+04 \\ \text { U 233 } & 6.06 \mathrm{E}+01 \\ \text { TH229 } & 1.73 \mathrm{E}+04 \\ \text { RA225 } & 9.29 \mathrm{E}+02 \\ \text { AC225 } & 9.89 \mathrm{E}+04 \\ \text { U 238 } & 1.08 \mathrm{E}+01 \\ \text { TH234 } & 8.18 \mathrm{E}+03 \\ \text { PA234 } & 7.95 \mathrm{E}+05 \\ \text { PU236 } & 1.18 \mathrm{E}+01 \\ \text { PU237 } & 1.20 \mathrm{E}+04 \\ \text { AM2423M } & 7.36 \mathrm{E}+01 \\ & \end{array}$


TABLE D.10. EXTDF Output for Soil Source Configuration (contd)

$\begin{array}{ll}\text { AM242 } & 3.45 E+03 \\ \text { CM242 } & 7.44 E+00 \\ \text { PU238 } & 7.88 E+00 \\ \text { PU242 } & 6.56 E+00 \\ \text { NP238 } & 2.33 E+05 \\ \text { PU238 } & 7.88 E+00 \\ \text { CM244 } & 6.35 E+00 \\ \text { PU244 } & 4.66 E+00 \\ U 240 & 1.53 E+05 \\ \text { PU240 } & 7.86 E+00 \\ \text { CM245 } & 1.67 E+04 \\ \text { PU241 } & 1.46 E-05 \\ \text { AM241 } & 1.79 E+03 \\ \text { CM246 } & 5.25 E+00 \\ \text { CM247 } & 1.66 E+05 \\ \text { PU243 } & 4.15 E+03 \\ \text { CM243 } & 3.69 E+04 \\ \text { AM243 } & 6.58 E+03 \\ \text { NP239 } & 5.06 E+04 \\ \text { PU239 } & 1.75 E+01 \\ \text { CM248 } & 4.78 E+00 \\ \text { CF252 } & 5.32 E+00\end{array}$


TABLE D.11. GENEXT1.BAS Computer Program Listing

\section{GENEXT1}

GENII input file generator and batch file controller.

Surface external dose factors for NRC Residual Radioactivity

Utility program for the Hanford Environmental Dosimetry Software System

Last Modification: 27-Jan-90 RAP

DEFINT $\mathbf{i}-n$

CALL copyfile ("\resid \filext.sur","\resid \filename.dat", errs)

GENII input file--

fin\$ = "GENII.IN" : fing $\$=$ "\GENII \GENII.IN"

Check if DOSEQA file exists, then delete--

filO\$ = "\GENII \DOSEQA.OUT"

CALL exist (fi 10\$, ifound)

IF ifound THEN SHELL "erase "+filo\$

OPEN "I", \#1, "RMDLIBDA.SHT"

LINE INPUT \#1, dum\$

Process each record in batch control file-WHILE NOT EOF(1)

Read radionuclide record--

LINE INPUT \#1, $1 \$$

$\operatorname{rad} \$=\operatorname{LEFT} \$(1 \$, 6)$

Open new input file and write header--

OPEN "O", \#2, fin\$

PRINT \#2, "NRC Residual Rad. Surface External Dose Factors"

PRINT \#2, "Title: "+rad\$ + "Sur Ext ( $\mathrm{pCi} / \mathrm{m} 2)$ "

Open, read, and write options file--

OPEN "I", \#3, "EXTOPT.TPL"

WHILE NOT' EOF (3)

LINE INPUT \#3, $1 \$$

PRINT \#2, $1 \$$

WEND

CLOSE 3 
TABLE D.11. GENEXT1.BAS Computer Program Listing (contd)

Write inventory lines--

PRINT \#2," ";rad\$; SPACE\$(35); " 1.0"

Open, read, and write parameters file--

OPEN "I", \#3, "EXTPAR.TPL"

WHILE NOT EOF (3)

LINE INPUT \#3, $1 \$$

PRINT \#2, $1 \$$

WEND

CLOSE 3

Close input file and copy to GENII subdirectory--

CLOSE 2

CALL copyfile (fin\$, fing\$, ecode)

\section{Execute GENI I--}

IF NOT ecode THEN

SHELL "\genii\envin"

SHELL "Igeni i \env"

SHELL "\gen $i i \backslash$ dose"

ENDIF

WEND

CLOSE 1

CALL copyfile (fi10\$, "ext1.df", errs)

CALL copyfile ("Igenii Igenii.in", "ext1.gin", errs)

CALL copyfile ("Igeniilgenii.out", "ext1.out", errs)

CALL copyfile ("Igenii|dose.out", "ext1-2.out", errs) 
TABLE D.12. EXTOPT.TPL GENII Input File Template

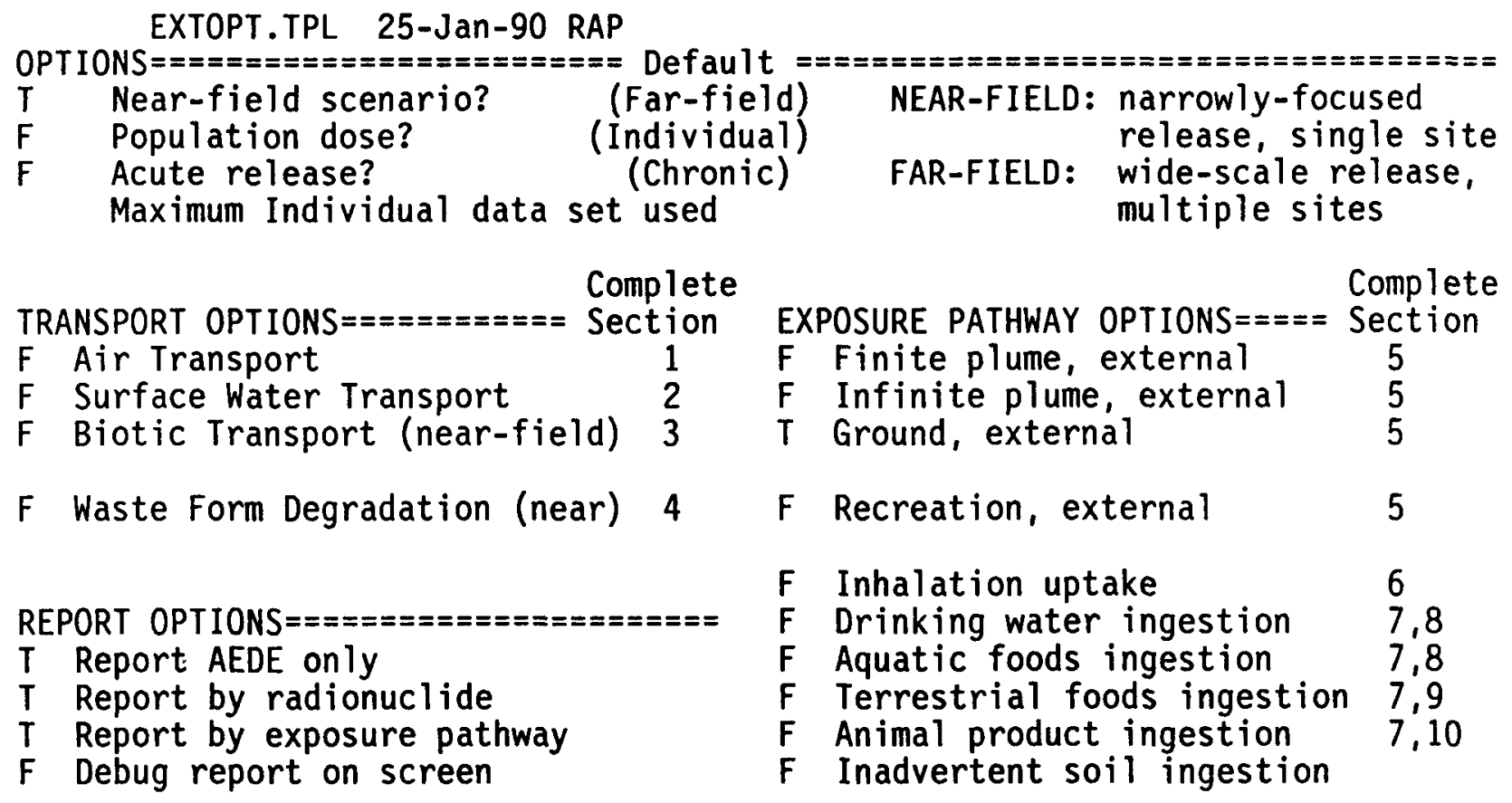

\section{INVENTORY \#\#\#\#\#\#\#\#\#\#\#\#\#\#\#\#\#\#\#\#\#\#\#\#\#\#\#\#\#\#\#\#\#\#\#\#\#\#\#\#\#\#\#\#\#\#\#\#\#\#\#\#\#\#\#\#\#\#\#\#\#\#\#\#\#\#\#}

1 Inventory input activity units: (1-pCi $2-\mathrm{uCi} 3-\mathrm{mCi} \quad 4-\mathrm{Ci}$ 5-Bq)

1 Surface soil source units $(1-\mathrm{m} 22-\mathrm{m} 33-\mathrm{kg})$

$\mathrm{T}$ Equilibrium question goes here

\begin{tabular}{l|r|r|}
\hline Use when & transport selected & near-field scenario, optionally \\
\hline Release & Surface Buried & Surface Deep
\end{tabular}

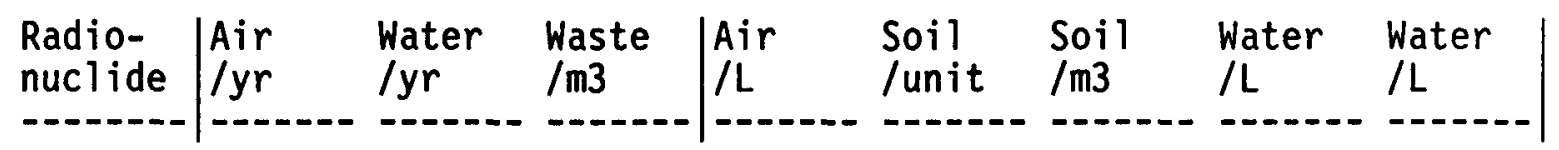


TABLE D.13. EXTPAR.TPL GENII Input File Template

\begin{tabular}{|c|c|c|c|c|}
\hline Use when & $\begin{array}{r}--- \text { Der } \\
\text { meas }\end{array}$ & $\begin{array}{l}\text { ived Con } \\
\text { ured val }\end{array}$ & es ar & known \\
\hline $\begin{array}{l}\text { idio- } \\
\text { iclide }\end{array}$ & $\begin{array}{l}\text { Terres. } \\
\text { Plant } \\
/ \mathrm{kg}\end{array}$ & $\begin{array}{l}\text { Animal } \\
\text { Product } \\
/ \mathrm{kg}\end{array}$ & $\begin{array}{l}\text { Drink } \\
\text { Water } \\
\text { /L }\end{array}$ & $\begin{array}{l}\text { Aquatic } \\
\text { Food } \\
/ \mathrm{kg}\end{array}$ \\
\hline
\end{tabular}

TIME $\# \# \# \# \# \# \# \# \# \# \# \# \# \# \# \# \# \# \# \# \# \# \# \# \# \# \# \# \# \# \# \# \# \# \# \# \# \# \# \# \# \# \# \# \# \# \# \# \# \# \# \# \# \# \# \# \# \# \# \# \# \# \# \# \# \# \# \# \# \# \# \# \# \#$

$1 \quad$ Intake ends after $(y r)$

50 Dose calc. ends after (yr)

0 Release ends after (yr)

0 No. of years of air deposition prior to the intake period

0 No. of years of irrigation water deposition prior to the intake period

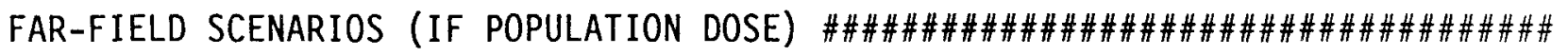

0 Definition option: 1-Use population grid in file POP.IN

0

2-Use total entered on this line

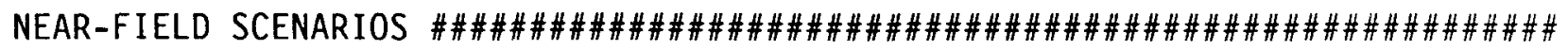

Prior to the beginning of the intake period: ( $y r)$

0

0

1.0

0.0

When was the inventory disposed? (Package degradation starts)

When was LOIC? (Biotic transport starts)

Fraction of roots in deep soil

0.0 Manual redistribution: deep soil/surface soil dilution factor

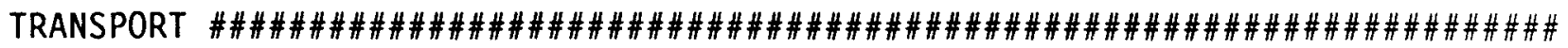

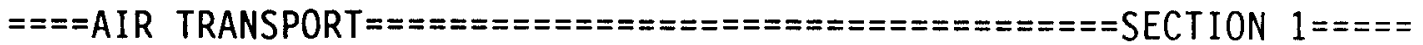
0 -Calculate PM

Option: 1-Use chi/Q or PM value F 2-Select MI dist \& dir 0

Stack release (T/F)

3-Specify MI dist \& dir

0

0

0

Chi/Q or PM value

MI sector index $(1=S)$

MI distance from release point (m)

Stack height (m)

Stack flow $(\mathrm{m} 3 / \mathrm{sec}$

Stack radius (m)

Effluent temp. (C)

Use joint frequency data, otherwise chi/Q grid

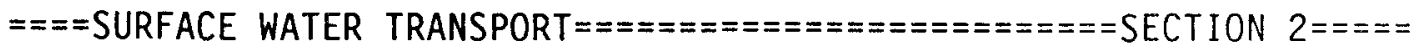

Mixing ratio model: 0-use value, 1-river, 2-lake, 3-river flow

Mixing ratio, dimensionless

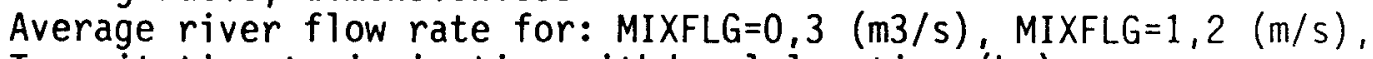

Transit time to irrigation withdrawl location (hr)

If mixing ratio model $>0$ :

Rate of effluent discharge to receiving water body $(\mathrm{m} 3 / \mathrm{s})$

Longshore distance from release point to usage location (m)

offshore distance to the water intake (m) 
TABLE D.13. EXTPAR.TPL GENII Input File Template (contd)

\begin{tabular}{|c|c|}
\hline & $\begin{array}{l}\text { Average water depth in surface water body }(\mathrm{m}) \\
\text { Average river width (m), MIXFLG=1 only } \\
\text { Depth of effluent discharge point to surface water }(\mathrm{m}) \text {, lake only }\end{array}$ \\
\hline $\begin{array}{l}.85 \\
.15\end{array}$ & $\begin{array}{l}====\text { WASTE FORM AVAILABILITY }=========================\text { SECTION } 3==== \\
\text { Waste form/package half life, }(y r) \\
\text { Waste thickness, }(\mathrm{m}) \\
\text { Depth of soil overburden, } m\end{array}$ \\
\hline
\end{tabular}

$T$
$T$

$====$ BIOTIC TRANSPORT OF BURIED SOURCE $==============$ SECTION $4====$ Consider during inventory decay/buildup period ( $T / F)$ ?

Consider during intake period (T/F)? 1-Arid non agricultural

Pre-Intake site condition............. 2-Humid non agricultural

3-Agricultural

EXPOSURE $\# \# \# \# \# \# \# \# \# \# \# \# \# \# \# \# \# \# \# \# \# \# \# \# \# \# \# \# \# \# \# \# \# \# \# \# \# \# \# \# \# \# \# \# \# \# \# \# \# \# \# \# \# \# \# \# \# \# \# \# \# \# \# \# \# \# \# \# \#$

0

1.0

0

0

0

0

0

0

1.0

2

1.0

0

0

0

0

$\mathrm{F}$

$\mathrm{F}$

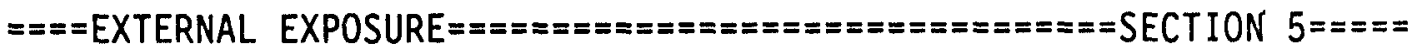 Exposure time:
Plume ( $h r$ )
Soil contamination ( $h r)$
Swimming $(\mathrm{hr})$
Boating (hr)
Shoreline activities (hr) 0
Residential irrigation:
Consider: $(T / F)$
Source: 1-ground water
2-surface water
Application rate (in/yr)
Duration (mo/yr)
Shoreline type: (1-river, 2-lake, 3-ocean, 4-tidal bas in)
Transit time for release to reach aquatic recreation $(\mathrm{hr})$
Average fraction of time submersed in acute cloud ( $\mathrm{hr} /$ person $\mathrm{hr}$ )

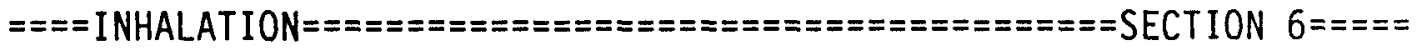
Hours of exposure to contamination per year

0 -No resus- 1-Use Mass Loading pension

Mass loading factor $(\mathrm{g} / \mathrm{m} 3)$

2-Use Anspaugh model

Top soil available $(\mathrm{cm})$

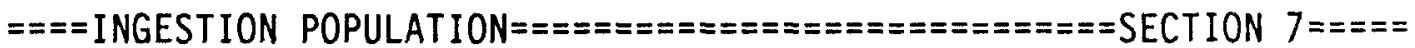
Atmospheric production definition (select option):

0 -Use food-weighted chi/Q, (food-sec/m3), enter value on this line

1-Use population-weighted chi/Q

2-Use uniform production

3-Use chi/Q and production grids (PRODUCTION will be overridden) Population ingesting aquatic foods, 0 defaults to total (person) Population ingesting drinking water, 0 defaults to total (person) Consider dose from food exported out of region (default $=F$ )

Note below: $S^{*}$ or Source: 0-none, 1-ground water, 2-surface water 3-Derived concentration entered above $====$ AQUATIC FOODS / DRINKING WATER INGESTION $========$ SECTION $8====$ Salt water? (default is fresh) 
TABLE D.13. EXTPAR.TPL GENII Input File Template (contd)

\begin{tabular}{|c|c|c|c|c|c|c|c|}
\hline $\begin{array}{l}\text { USE } \\
\stackrel{?}{\text { T/F }}\end{array}$ & TYOD & $\begin{array}{l}\text { TRAN- } \\
\text { SIT } \\
\text { hr }\end{array}$ & $\begin{array}{l}\text { PROD- } \\
\text { UCTION } \\
\mathrm{kg} / \mathrm{yr}\end{array}$ & $\begin{array}{l}\text {-CONSUMP } \\
\text { HOLDUP } \\
\mathrm{da}\end{array}$ & $\begin{array}{l}\text { PTION- } \\
\text { RATE } \\
\mathrm{kg} / \mathrm{yr}\end{array}$ & & DRINKING WATER \\
\hline & FISH & 0.00 & $0.0 \mathrm{E}+00$ & 0.00 & 0.0 & 0 & Source (see above) \\
\hline & MOLLUS & 0.00 & $0.0 \mathrm{E}+00$ & 0.00 & 0.0 & I T & Treatment? T/F \\
\hline & CRUSTA & 0.00 & $0.0 E+00$ & 0.00 & 0 . & 0 & Holdup/ \\
\hline & LANTS & 0.00 & $.0 E+00$ & 0.00 & 0.0 & 0 & Consumption (L/yr) \\
\hline
\end{tabular}

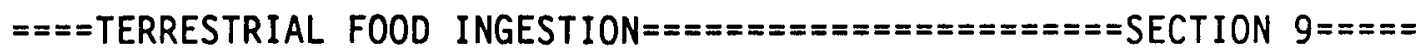

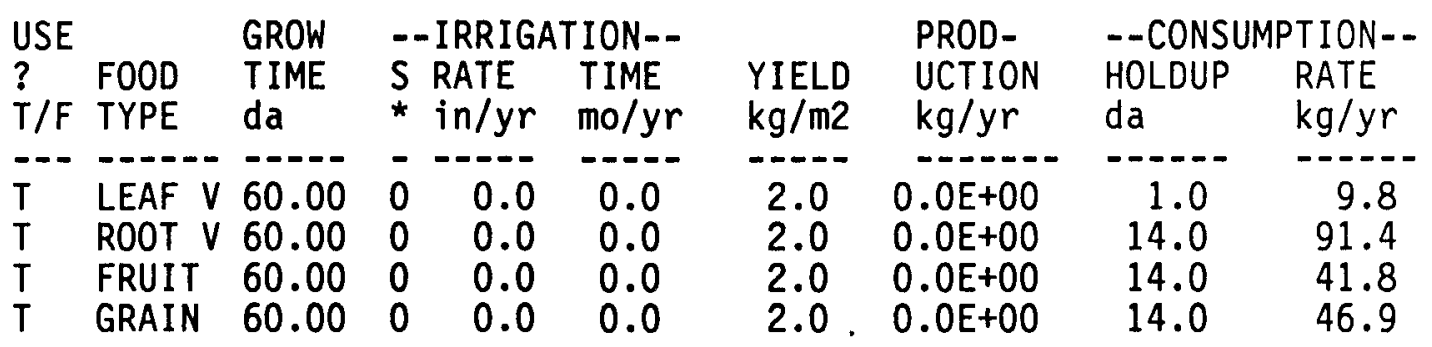

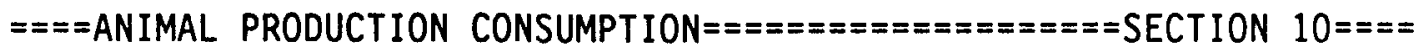

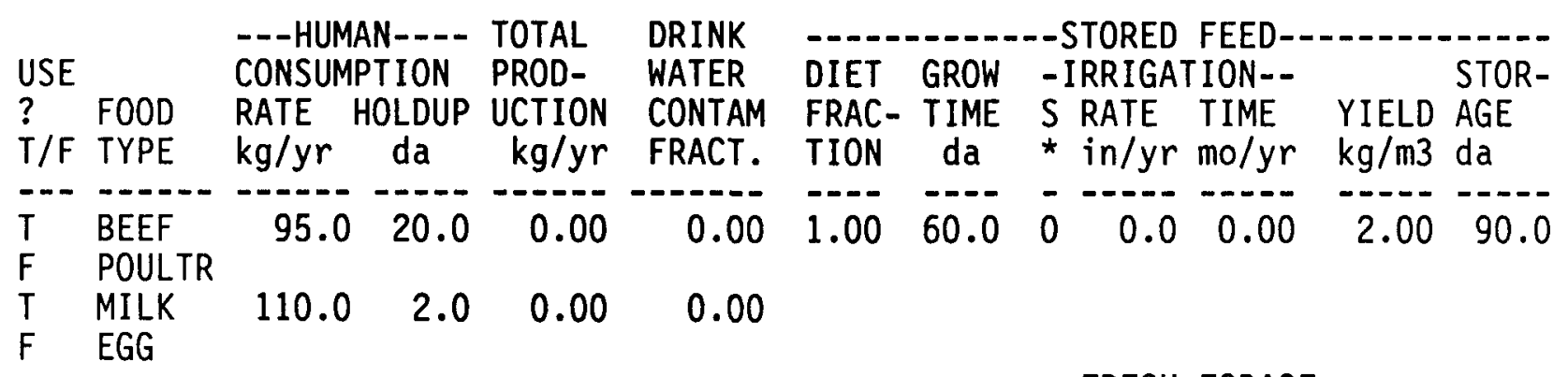

BEEF

MILK

$\begin{array}{lllllll}1.00 & 30.0 & 0 & 0.0 & 0.00 & 0.70 & 0.0\end{array}$

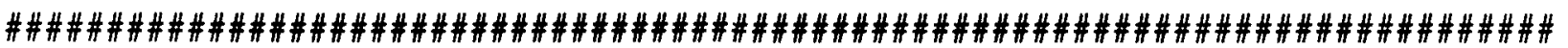


TABLE D.14 Control Program Usage for Generating Internal Dose

Factors Contained in Files INTERNAL.DF and INTNODAU.DF

\begin{tabular}{|c|c|c|c|c|}
\hline $\begin{array}{l}\text { Control } \\
\text { Program }\end{array}$ & $\begin{array}{c}\text { GENII } \\
\text { Input File } \\
\text { Templates } \\
\end{array}$ & $\begin{array}{l}\text { GENII } \\
\text { DOSEQA.OUT } \\
\text { Output File }\end{array}$ & Used In & Pathways Considered \\
\hline $\begin{array}{l}\text { GENALL.BAS } \\
\text { (Table D.11) }\end{array}$ & $\begin{array}{l}\text { SOILOPT.TPL } \\
\text { SOILPAR.TPL }\end{array}$ & SOILS.DF & INTERNAL.DF & $\begin{array}{l}\text { Soil contribution from } \\
\text { ingestion of food and } \\
\text { food products }\end{array}$ \\
\hline $\begin{array}{l}\text { GENALL2.BAS } \\
\text { (Table D.12) }\end{array}$ & $\begin{array}{l}\text { DRINKOPT.TPL } \\
\text { DRINKPAR.TPL }\end{array}$ & INHSI.DF & INTERNAL.DF & $\begin{array}{l}\text { Inhalation and } \\
\text { secondary ingestion }\end{array}$ \\
\hline $\begin{array}{l}\text { GENALL3.BAS } \\
\text { (Table D.13) }\end{array}$ & $\begin{array}{l}\text { SOILOPT.TPL } \\
\text { SOILPAR.TPL }\end{array}$ & AIRS.DF & INTERNAL.DF & $\begin{array}{l}\text { Air contribution from } \\
\text { ingestion of food and } \\
\text { food products }\end{array}$ \\
\hline $\begin{array}{l}\text { GENALL4.BAS } \\
\text { (Table D.14) }\end{array}$ & $\begin{array}{l}\text { DRINKOPT.TPL } \\
\text { DRINKPAR.TPL }\end{array}$ & DW.DF & INTERNAL.DF & $\begin{array}{l}\text { Drinking water } \\
\text { critical organ and } \\
\text { drinking water critical } \\
\text { organ dose }\end{array}$ \\
\hline
\end{tabular}

$\begin{array}{lll}\text { GENALLN.BAS } & \text { SOILOPT.TPL } & \text { SOILSNO.DF } \\ & \text { SOILPAR.TPL } & \end{array}$

INTNODAU.DF

Soil contribution from ingestion of food and food products

GENALL2N.BAS DRINKOPT.TPL INHSINO.DF DRINKPAR.TPL

INTNODAU.DF

Inhalation and secondary ingestion

GENALL3N.BAS SOILOPT.TPL AIRSNO.DF

INTNODAU.DF

Air contribution from ingestion of food and food products

GENALL4N.BAS DRINKOPT.TPL DWNO.DF DRINKPAR.TPL

INTNODAU.DF Drinking water critical organ and drinking water critical organ dose 
TABLE D.15. GENALL.BAS Computer Program Listing

\section{GENALL}

GENII input file generator and batch file controller.

Ingestion (soil contribution) dose factors for

NRC Residual Radioactivity

Utility program for the Hanford Environmental Dosimetry Software System

Last Modification: $4-0 \mathrm{ct}-89$ RAP

\section{DEFINT $i-n$}

CALL copyfile ("\resid\filename.sol","\genii\filename.dat", errs)

GENII input file--

fin\$ = "GENII.IN" : fing $\$=$ "\GENII \GENII.IN"

Check if DOSEQA file exists, then delete--

filO\$ = "\GENII \DOSEQA.OUT"

CALL exist (fi10\$, ifound)

IF ifound THEN SHELL "erase "+filo\$

OPEN "I", \#1, "RMDLIBDA.SHT"

LINE INPUT \#1, dum\$

Process each record in batch control file--

WHILE NOT EOF (1)

Read radionuclide record--

LINE INPUT \#1, 1\$

$\operatorname{rad} \$=\operatorname{LEFT} \$(1 \$, 6)$

Open new input file and write header--

OPEN "0", \#2, fin\$

PRINT \#2, "NRC Residual Radioactivity Ingestion Dose Factors (Soi1)"

PRINT \#2, "Title: "+rad\$ + "Soil (pCi/g)"

Open, read, and write options file--

OPEN "I", \#3, "SOILOPT.TPL"

WHILE NOT EOF (3)

LINE INPUT \#3, $1 \$$

PRINT \#2, $1 \$$

WEND

CLOSE 3 
TABLE D.15. GENALL. BAS Computer Program Listing (contd)

' Write inventory lines--

PRINT \#2," "; rad\$; SPACE\$(35); "1.0E+03"

Open, read, and write parameters file--

OPEN "I", \#3, "SOILPAR.TPL"

WHILE NOT EOF (3)

LINE INPUT \#3, $1 \$$

PRINT \#2, $1 \$$

WEND

CLOSE 3

$1 \quad$ Close input file and copy to GENII subdirectory--

CLOSE 2

CALL copyfile (fin\$, fing\$, ecode)

$1 \quad$ Execute GENII--

IF NOT ecode THEN

SHELL "\gen $i$ i\envin"

SHELL "Igen $i$ i env"

SHELL "\gen $i$ i $\backslash$ dose"

ENDIF

WEND

CLOSE 1

CALL copyfile (filo\$, "soils.df", errs)

CALL copyfile ("Igenii Igenii.in","soils.in", errs)

CALL copyfile ("geniilgenii.out", "soils.out", errs)

CALL copyfile ("geniildose.out", "soils2.out", errs) 
TABLE D.16. GENALL2.BAS Computer Program Listing

\section{GENALL2}

GENII input file generator and batch file controller for inhalation and secondary ingestion (NRC Residual Radioactivity)

Utility program for the Hanford Environmental Dosimetry Software System

Last Modification: 4-0ct-89 RAP

DEFINT $i-n$

CALL copyfile ("\resid \filename.sol", "\genii\filename.dat", errs)

GENII input file--

fin $\$=$ "GENII.IN" : fing\$ = "\GENII\GENII.IN"

Check if DOSEQA file exists, then delete--

fi $10 \$$ = "\GENII \DOSEQA.OUT"

CALL exist (filos, ifound)

IF ifound THEN SHELL "erase "+filo\$

OPEN "I", \#1, "RMDLIBDA.SHT"

LINE INPUT \#1, dum\$

Process each record in batch control file-WHILE NOT EOF (1)

Read radionuclide record--

LINE INPUT \#1, 1\$

$\operatorname{rad} \$=\operatorname{LEFT} \$(1 \$, 6)$

Open new input file and write header--

OPEN "0", \#2, fin\$

PRINT \#2, "GENII Input File for Residual Radioactivity Facility"

PRINT \#2, "Title: "rad\$ + " Inh/SI ( $\mathrm{pCi} / \mathrm{m3}$ or $\mathrm{pCi}) "$

Open, read, and write options file--

OPEN "I", \#3, "FACILOPT.TPL"

WHILE NOT EOF (3)

LINE INPUT \#3, $1 \$$

PRINT \#2, $1 \$$

WEND

CLOSE 3 
TABLE D.16. GENALL2.BAS Computer Program Listing (contd)

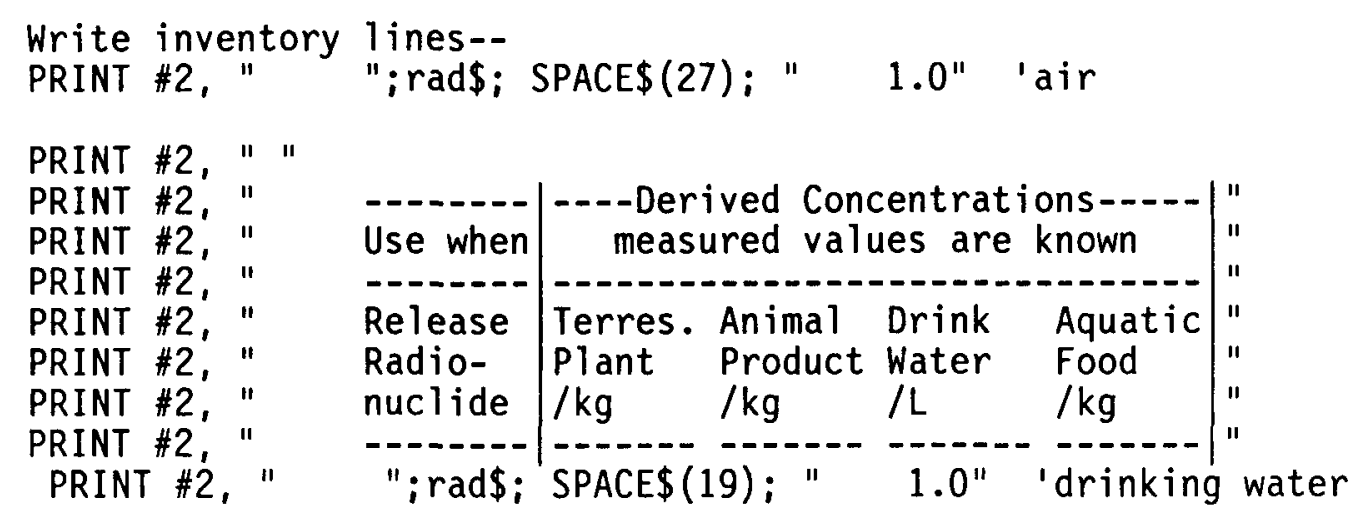

Write inventory lines--
PRINT \#2,"

Open, read, and write parameters file--

OPEN "I", \#3, "FACILPAR.TPL"

WHILE NOT EOF (3)

LINE INPUT \#3, $1 \$$

\section{WEND}

PRINT \#2, $1 \$$

CLOSE 3

Close input file and copy to GENII subdirectory--

CLOSE 2

CALL copyfile (fin\$, fing\$, ecode)

Execute GENII--

IF NOT ecode THEN

SHELL "\gen ii\envin"

SHELL "Igenii

SHELL "Igen $i$ idose"

\section{ENDIF}

\section{WEND}

CLOSE 1

CALL copyfile (filos, "inhsi.df", errs)

CALL copyfile ("Igenii Igenii.out", "inhsi.out", errs)

CALL copyfile ("Igenii\dose.out", "inhsi2.out", errs) 
TABLE D.17. GENALL3.BAS Computer Program Listing

\section{GENALL3}

GENII input file generator and batch file controller. Ingestion (air contribution) dose factors for NRC Residual Radioactivity

Utility program for the Hanford Environmental Dosimetry Software System

Last Modification: 5-0ct-89 RAP

DEFINT $i-n$

CALL copyfile ("\resid \filename.air","\genii\filename.dat", errs)

GENII input file--

fin $\$=$ "GENII.IN" : fing $\$=$ "\GENII\GENII.IN"

Check if DOSEQA file exists, then delete--

fi $10 \$$ = "\GENII $\backslash D O S E Q A . O U T "$

CALL exist (filo\$, ifound)

IF ifound THEN SHELL "erase "+filo\$

OPEN "I"，\#1, "RMDLIBDA.SHT"

LINE INPUT \#1, dum\$

Process each record in batch control file-WHILE NOT EOF(1)

Read radionuclide record--

LINE INPUT \#1, $1 \$$

$\operatorname{rad} \$=\operatorname{LEFT\$ }(1 \$, 6)$

Open new input file and write header--

OPEN "0", \#2, fin\$

PRINT \#2, "NRC Residual Radioactivity Ingestion Dose Factors (Air)"

PRINT \#2, "Title: "+rad\$ + " Air (pCi/m3)"

Open, read, and write options file (same as soil)--

OPEN "I", \#3, "SOILOPT.TPL"

WHILE NOT EOF(3)

LINE INPUT \#3, $1 \$$

WEND

PRINT \#2, $1 \$$

CLOSE 3 
TABLE D.17. GENALL3.BAS Computer Program Listing (contd)

Write inventory lines--

PRINT \#2," "; rad\$; SPACE\$(27); " 1.0"

Open, read, and write parameters file (same as soil)

OPEN "I" \#3, "SOILPAR.TPL"

WHILE NOT EOF(3)

LINE INPUT \#3, $1 \$$

PRINT \#2, $1 \$$

WEND

CLOSE 3

Close input file and copy to GENII subdirectory--

CLOSE 2

CALL copyfile (fin\$, fing\$, ecode)

Execute GENII--

IF NOT ecode THEN

SHELL "\genii\envin"

SHELL "Igeni i lenv"

SHELL "Igeni i dose"

ENDIF

WEND

CLOSE 1

CALL copyfile (filo\$, "airs.df", errs)

CALL copyfile ("Igeniilgenii.in", "airs.in", errs)

CALL copyfile ("Igeniilgenii.out", "airs.out", errs)

CALL copyfile ("Igeniildose.out", "airs2.out", errs) 
TABLE D.18. GENALL4.BAS Computer Program Listing

GENALL4

GENII input file generator and batch file controller for drinking water maximum organ (NRC Residual Radioactivity)

Utility program for the Hanford Environmental Dosimetry Software System

Last Modification: 10-0ct-89 RAP

DEFINT i-n

CALL copyfile ("\resid \filename.sol", "\geni i\filename.dat", errs)

GENII input file--

fin\$ = "GENII.IN" : fing\$ = "\GENII\GENII.IN"

Check if DOSEQA file exists, then delete--

fi10\$ = "\GENII \DOSEQA.OUT"

CALL exist (fi10\$, ifound)

IF ifound THEN SHELL "erase "+filo\$

OPEN "I", \#1, "RMDL IBDA.SHT"

LINE INPUT \#1, dum\$

Process each record in batch control file-WHILE NOT EOF (1)

Read radionuclide record--

LINE INPUT \#1, $1 \$$

$\operatorname{rad} \$=\operatorname{LEFT\$ }(1 \$, 6)$

Open new input file and write header--

OPEN "0", \#2, fin\$

PRINT \#2, "GENII Input File for Residual Radioactivity Facility" PRINT \#2, "Title: "+rad\$ + "DW (pCi/L)"

Open, read, and write options file-OPEN "I", \#3, "FACILOPT.TPL"

WHILE NOT EOF (3)

LINE INPUT \#3, $1 \$$

PRINT \#2, $1 \$$

WEND

CLOSE 3 
TABLE D.18. GENALL4.BAS Computer Program Listing (contd)

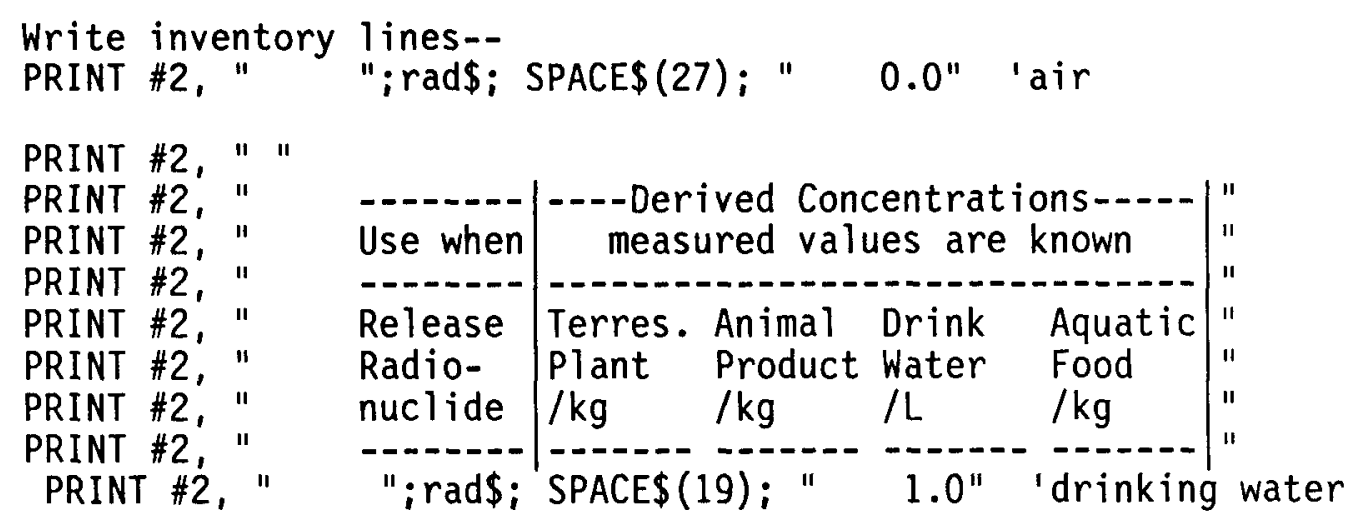

Open, read, and write parameters file--

OPEN "I", \#3, "FACILPAR.TPL"

WHILE NOT EOF (3)

LINE INPUT \#3, $1 \$$

PRINT \#2, $1 \$$

WEND

CLOSE 3

Close input file and copy to GENII subdirectory--

CLOSE 2

CALL copyfile (fin\$, fing\$, ecode)

$1 \quad$ Execute GENII--

IF NOT ecode THEN

SHELL "\geni i \envin"

SHELL "Igen i $i$ env"

SHELL "\geni $i \backslash$ dose"

\section{ENDIF}

\section{WEND}

CLOSE 1

CALL copyfile (filo\$, "dw.df", errs)

CALL copyfile ("Igenii \genii.out", "dw.out", errs)

CALL copyfile ("Igenii dose.out", "dw2.out", errs) 
TABLE D.19. SOILOPT.TPL GENII Input File Template

SOILOPT.TPL 2-0ct-89 RAP

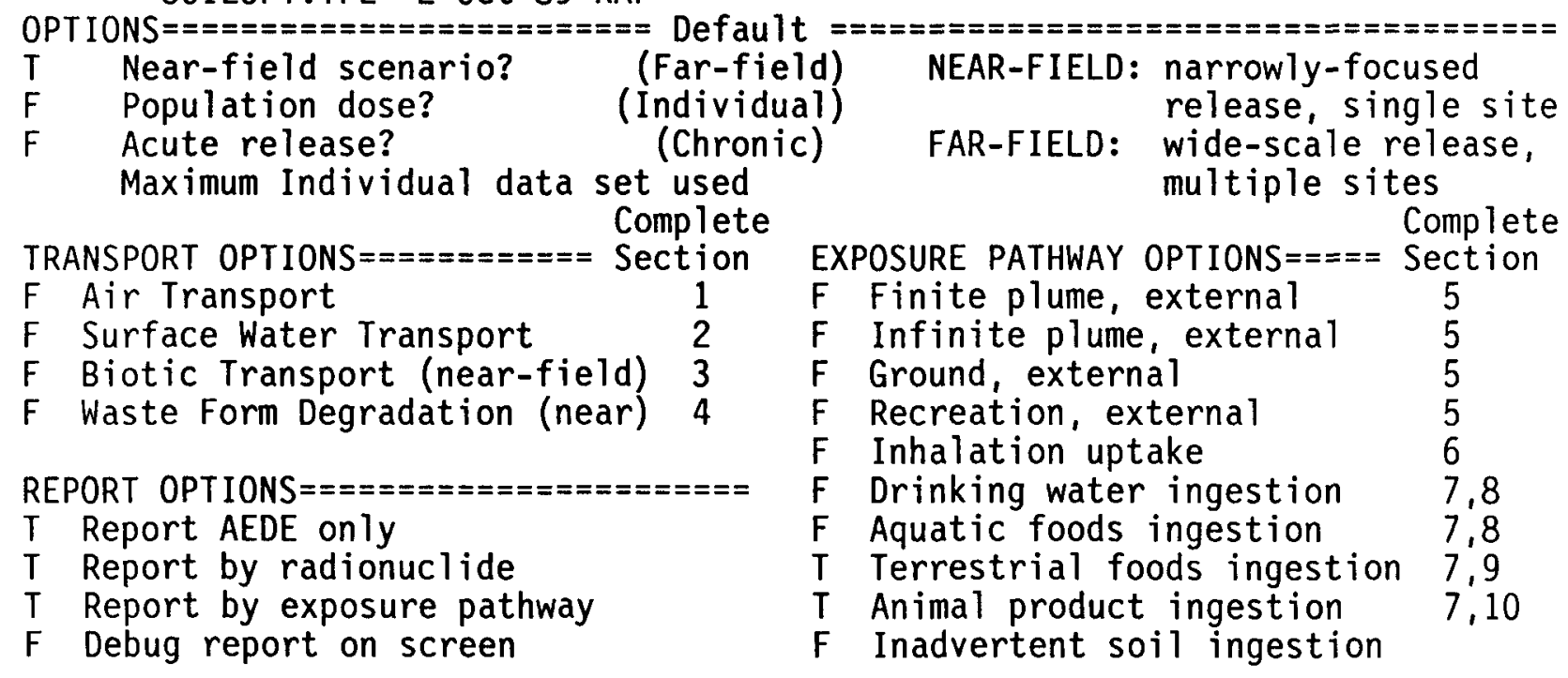

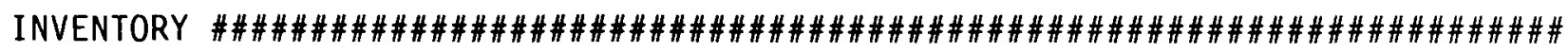

1 Inventory input activity units: (1-pCi $2-\mathrm{uCi} \quad 3-\mathrm{mCi} \quad 4-\mathrm{Ci} \quad 5-\mathrm{Bq})$

3 Surface soil source units (1- $\mathrm{m} 2 \quad 2-\mathrm{m} 3 \quad 3-\mathrm{kg})$

$T$ Equilibrium question goes here

\begin{tabular}{|c|c|c|c|c|c|c|c|c|}
\hline Use when & \multicolumn{3}{|c|}{$\mid \begin{array}{c}--- \text { Release } \\
\text { transporms }------ \\
\text { selected }\end{array}$} & \multicolumn{5}{|c|}{$=-B a$} \\
\hline$d 1$ & $\begin{array}{l}\text { Air } \\
\text { /yr }\end{array}$ & $\begin{array}{l}\text { Surface } \\
\text { Water } \\
\text { /yr }\end{array}$ & $\begin{array}{l}\text { Buried } \\
\text { Waste } \\
/ \mathrm{m} 3\end{array}$ & $\begin{array}{l}\text { Air } \\
/ L\end{array}$ & $\begin{array}{l}\text { Surface } \\
\text { Soil } \\
\text { /unit }\end{array}$ & $\begin{array}{l}\text { Deep } \\
\text { Soi } 1 \\
/ \mathrm{m} 3\end{array}$ & $\begin{array}{l}\text { Ground } \\
\text { Water } \\
/ L\end{array}$ & $\begin{array}{l}\text { Surface } \\
\text { Water } \\
/ L\end{array}$ \\
\hline
\end{tabular}


TABLE D.20. SOILPAR.TPL GENII Input File Template

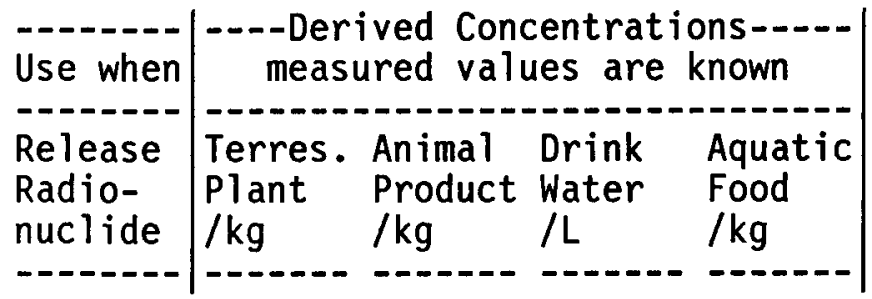

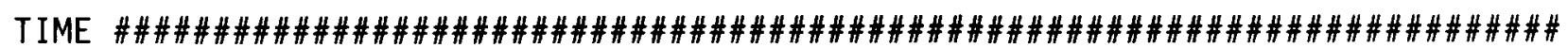

1 Intake ends after (yr)

50 Dose calc. ends after (yr)

0 Release ends after (yr)

0 No. of years of air deposition prior to the intake period

0 No. of years of irrigation water deposition prior to the intake period

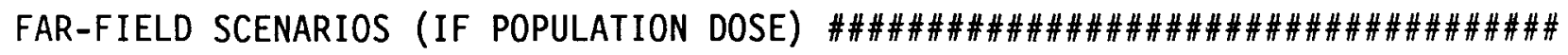

$0 \quad$ Definition option: 1-Use population grid in file POP.IN

0

2-Use total entered on this line

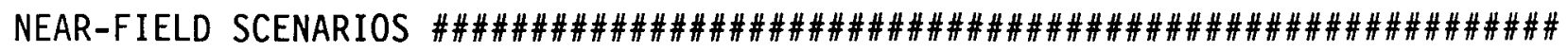

0

0

1.0

0.0

0.0

Prior to the beginning of the intake period: ( $y r)$ When was the inventory disposed? (Package degradation starts) When was LOIC? (Biotic transport starts)

Fraction of roots in upper soil (top $15 \mathrm{~cm}$ )

Fraction of roots in deep soil

Manual redistribution: deep soil/surface soil dilution factor

TRANSPORT

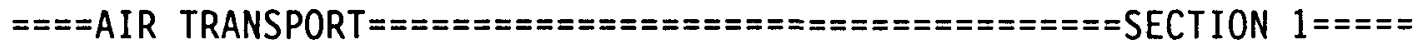

$$
0 \text {-Calculate PM }
$$

Option: 1-Use chi/Q or PM value F

2-Select MI dist \& dir 0

3-Specify MI dist \& dir 0

0

0

0

$\mathrm{T}$

Chi/Q or PM value

MI sector index $(1=S)$

MI distance from release point (m)

Stack release (T/F)

Stack height (m)

Stack flow (m $3 / \mathrm{sec}$

Stack radius (m)

Effluent temp. (C)

Use joint frequency data, otherwise chi/Q grid

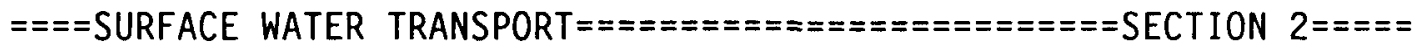
Mixing ratio model: 0-use value, 1-river, 2-lake, 3-river flow Mixing ratio, dimensionless

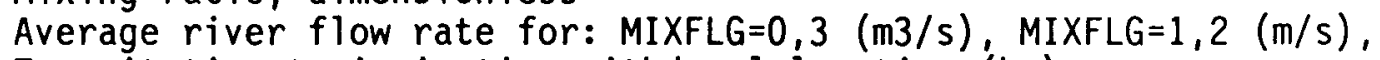
Transit time to irrigation withdrawl location (hr)

If mixing ratio model $>0$ :

Rate of effluent discharge to receiving water body $(\mathrm{m} 3 / \mathrm{s})$

Longshore distance from release point to usage location (m)

offshore distance to the water intake $(\mathrm{m})$ 
TABLE D.20. SOILPAR.TPL GENII Input File Template (contd)

0

0.85

0.15

T

Average water depth in surface water body $(m)$ Average river width (m), MIXFLG $=1$ only

Depth of effluent discharge point to surface water (m), lake only

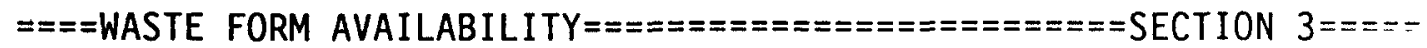
Waste form/package half life, (yr)

Waste thickness, (m)

Depth of soil overburden, $m$

$====$ BIOTIC TRANSPORT OF BURIED SOURCE $===============$ SECTION $4=====$ Consider during inventory decay/buildup period (T/F)?

Consider during intake period (T/F)? |1-Arid non agricultural

Pre-Intake site condition............ 2-Humid non agricultural

3-Agricultural

EXPOSURE $\# \# \# \# \# \# \# \# \# \# \# \# \# \# \# \# \# \# \# \# \# \# \# \# \# \# \# \# \# \# \# \# \# \# \# \# \# \# \# \# \# \# \# \# \# \# \# \# \# \# \# \# \# \# \# \# \# \# \# \# \# \# \# \# \# \# \# \# \#$

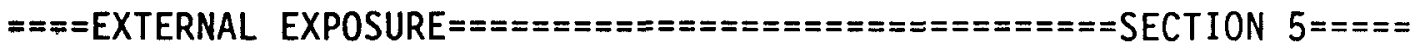
Exposure time: Plume (hr)

1.0

0

Soil contamination ( $h r)$

Swimming ( $h r$ )

Boating (hr)

Shoreline activities ( $h r) \quad 0$

Residential irrigation:

Consider: $(T / F)$

Source: 1-ground water

2-surface water

0

0

Shoreline type: (1-river, 2-lake, 3-ocean, 4-tidal basin)

Transit time for release to reach aquatic recreation $(\mathrm{hr})$

Average fraction of time submersed in acute cloud ( $\mathrm{hr} /$ person $\mathrm{hr}$ )

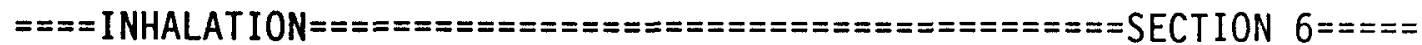

1.0 Hours of exposure to contamination per year

2

1.0

0-No resus- 1-Use Mass Loading

2-Use Anspaugh mode 1

pension Mass loading factor $(\mathrm{g} / \mathrm{m} 3)$ Top soil available $(\mathrm{cm})$

$====$ INGESTION POPULATION=m=s=s=s=s=s Atmospheric production definition (select option):

0 -Use food-weighted chi/Q, (food-sec/m3), enter value on this line 1-Use population-weighted chi/Q

2-Use uniform production

3-Use chi/Q and production grids (PRODUCTION will be overridden)

Population ingesting aquatic foods, 0 defaults to total (person) Population ingesting drinking water, 0 defaults to total (person) Consider dose from food exported out of region (defaul $t=F$ )

Note below: $S^{*}$ or Source: 0-none, 1-ground water, 2-surface water 3-Derived concentration entered above $====$ AQUATIC FOODS / DRINKING WATER INGESTION $========$ SECTION $8====$ 
TABLE D.20. SOILPAR.TPL GENII Input File Template (contd)

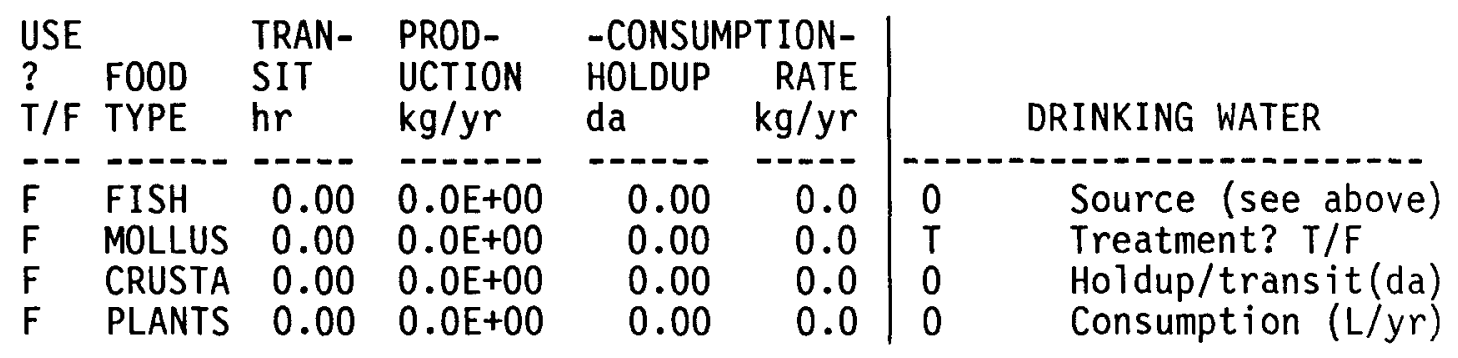

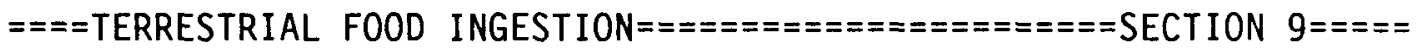

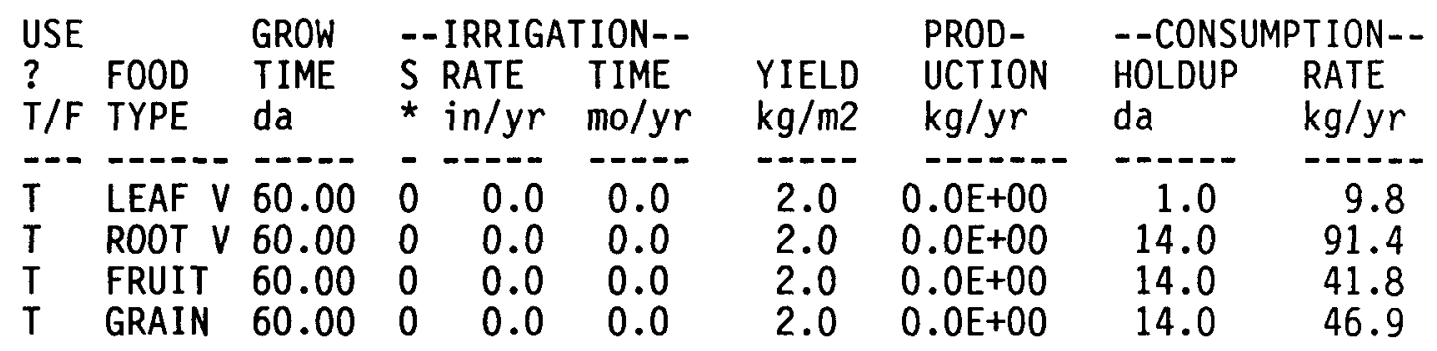

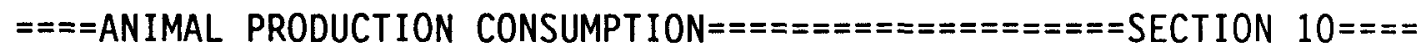

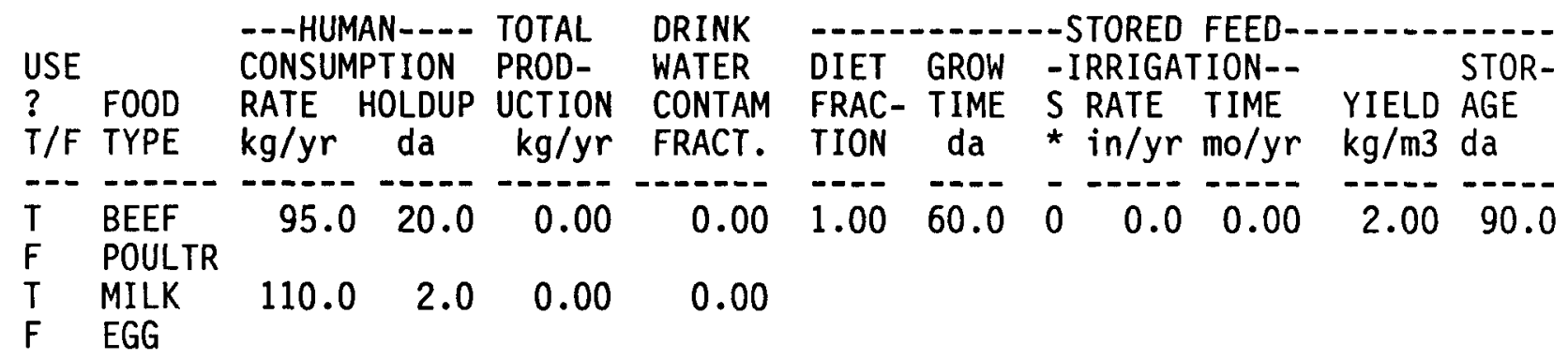

BEEF

MILK

$\begin{array}{lllllll}1.00 & 30.0 & 0 & 0.0 & 0.00 & 0.70 & 0.0\end{array}$ 
TABLE D.21. FACILOPT.TPL GENII Input File Template

FACILOPT.TPL 4-0ct-89 RAP

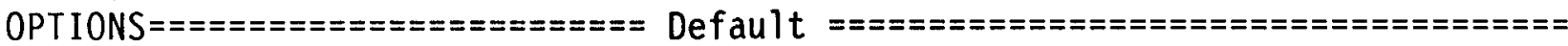

T Near-field scenario?

F Population dose?

$F \quad$ Acute release?

Maximum Individual data set used

(Far-field) NEAR-FIELD: narrowly-focused

(Individual)

release, single site

(Chronic)

Complete

TRANSPORT OPTIONS==========="= Section

FAR-FIELD: wide-scale release, multiple sites

Complete

F Air Transport

$F$ Surface Water Transport

EXPOSURE PATHWAY OPTIONS $=====$ Section

F Biotic Transport (near-field) 3

$F$ Waste Form Degradation (near) 4

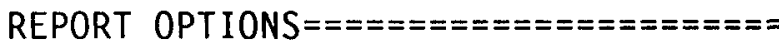

$T$ Report AEDE only

$F$ Report by radionuclide

$F$ Report by exposure pathway

$F$ Debug report on screen

$F$ Finite plume, external 5

$F$ Infinite plume, external 5

$F$ Ground, external

F Recreation, external

5

T Inhalation uptake 6

T Drinking water ingestion 7,8

$F$ Aquatic foods ingestion 7,8

F Terrestrial foods ingestion 7,9

F Animal product ingestion 7,10

$F$ Inadvertent soil ingestion

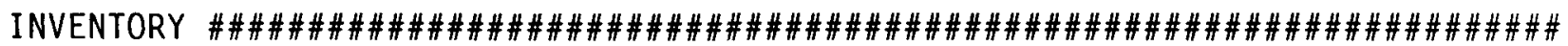

1 Inventory input activity units: ( $1-\mathrm{pCi} \quad 2-\mathrm{uCi} \quad 3-\mathrm{mCi} \quad 4-\mathrm{Ci} \quad 5-\mathrm{Bq})$

3 Surface soil source units (1- m2 2- $33 \quad 3-\mathrm{kg}$ )

$\mathrm{T}$ Equilibrium question goes here

\begin{tabular}{|c|c|c|c|c|c|c|c|c|}
\hline Use when & tr & $\begin{array}{l}\text { ease Ter } \\
\text { port sel }\end{array}$ & ected & & $\begin{array}{l}- \text {-Basic } \\
\text { ield sc }\end{array}$ & nari & opt & \\
\hline $\begin{array}{l}\text { lease } \\
\text { adio- } \\
\text { aclide }\end{array}$ & $\begin{array}{l}\text { Air } \\
\text { /yr }\end{array}$ & $\begin{array}{l}\text { Surface } \\
\text { Water } \\
\text { /yr }\end{array}$ & $\begin{array}{l}\text { Buried } \\
\text { Waste } \\
\text { /m3 }\end{array}$ & $\begin{array}{l}A i r \\
/ L\end{array}$ & $\begin{array}{l}\text { Surface } \\
\text { Soil } \\
\text { /unit }\end{array}$ & $\begin{array}{l}\text { Deep } \\
\text { Soi1 } \\
\text { /m3 }\end{array}$ & $\begin{array}{l}\text { Ground } \\
\text { Water } \\
/ L\end{array}$ & $\begin{array}{l}\text { Surface } \\
\text { Water } \\
/ L\end{array}$ \\
\hline
\end{tabular}


TABLE D.22. FACILPAR.TPL GENI I Input File Template

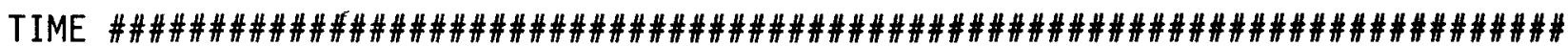

1 Intake ends after ( $y r)$

50 Dose calc. ends after (yr)

$0 \quad$ Release ends after (yr)

0 No. of years of air deposition prior to the intake period

0 No. of years of irrigation water deposition prior to the intake period

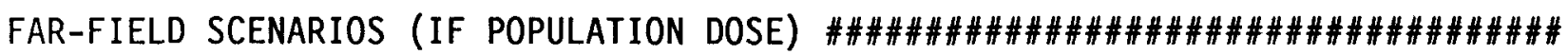

0 Definition option: 1-Use population grid in file POP.IN

0

2-Use total entered on this line

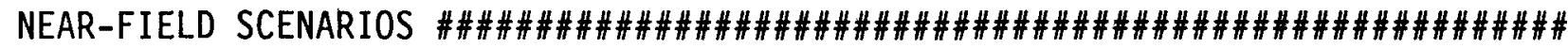

0

0

1.0

0.0

0.0

Prior to the beginning of the intake period: (yr) When was the inventory disposed? (Package degradation starts) When was LOIC? (Biotic transport starts)

Fraction of roots in upper soil (top $15 \mathrm{~cm}$ )

Fraction of roots in deep soil

Manual redistribution: deep soil/surface soil dilution factor

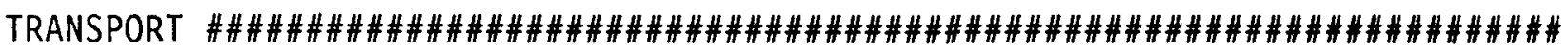

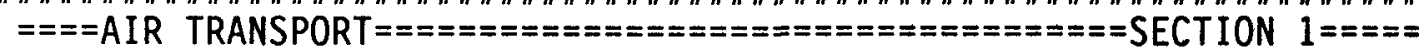
0-Calculate PM

Stack release (T/F)

Stack height (m) Stack flow (m3/sec Stack radius (m) Effluent temp. (C)

MI sector index $(1=S)$

MI distance from release point (m)

Use joint frequency data, otherwise chi/Q grid

$====$ SURFACE WATER TRANSPORT $============x=x=========$ SECTION $2=====$ Mixing ratio model: 0 -use value, 1-river, 2-lake, 3-river flow Mixing ratio, dimensionless

Average river flow rate for: MIXFLG $=0,3(\mathrm{~m} 3 / \mathrm{s}), \operatorname{MIXFLG}=1,2(\mathrm{~m} / \mathrm{s})$, Transit time to irrigation withdrawl location ( $h r$ ) If mixing ratio model $>0$ :

Rate of effluent discharge to receiving water body $(\mathrm{m} 3 / \mathrm{s})$ Longshore distance from release point to usage location (m) Offshore distance to the water intake (m) Average water depth in surface water body (m) Average river width (m), MIXFLG=1 only Depth of effluent discharge point to surface water (m), lake only $===$ WASTE FORM AVAILABILITY=s=s=s=s=s=s Waste form/package half life, (yr) Waste thickness, (m) Depth of soil overburden, $m$ 
TABLE D.22. FACILPAR.TPL GENII Input File Template (contd)

$===$ BIOTIC TRANSPORT OF BURIED SOURCE $==============$ SECTION $4=====$ Consider during inventory decay/buildup period (T/F)?

Consider during intake period (T/F)? |1-Arid non agricultural

Pre-Intake site condition.............. 2-Humid non agricultural

3-Agricultural

EXPOSURE \#\#\#\#\#\#\#\#\#\#\#\#\#\#\#\#\#\#\#\#\#\#\#\#\#\#\#\#\#\#\#\#\#\#\#\#\#\#\#\#\#\#\#\#\#\#\#\#\#\#\#\#\#\#\#\#\#\#\#

0

1.0

0

0

0

0

0

0

1.0

1

$1.0 \mathrm{E}-4$

0

0

0

0

$\mathrm{F}$

F

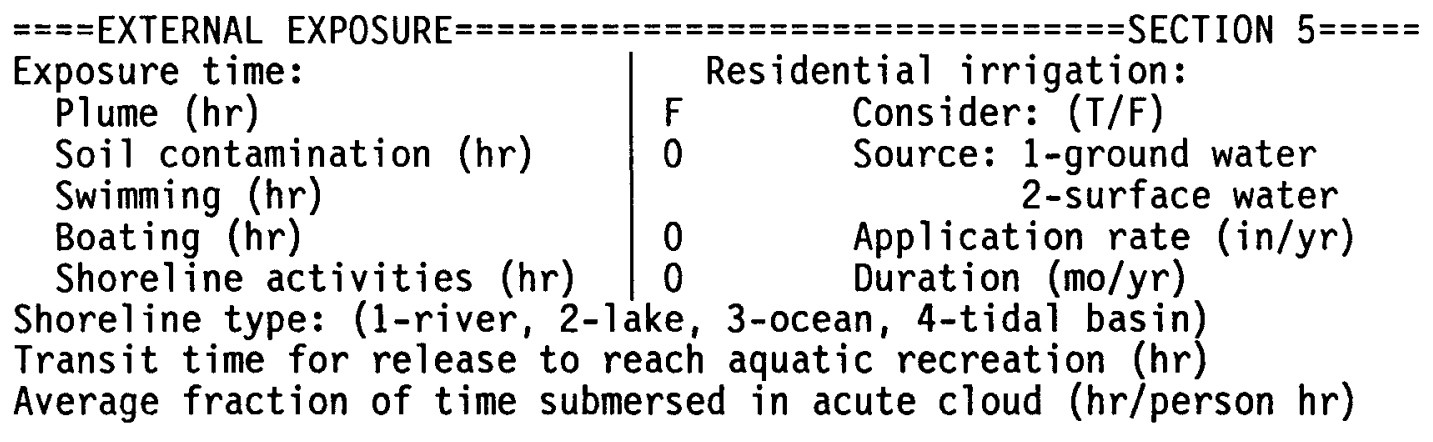

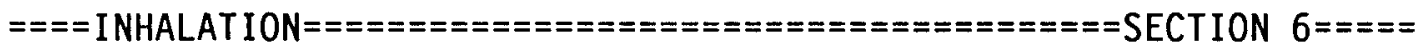
Hours of exposure to contamination per year

0 -No resus- 1-Use Mass Loading pension $\quad$ Mass loading factor $(\mathrm{g} / \mathrm{m} 3)$ Top soil available $(\mathrm{cm})$

$====$ INGESTION POPULATION $==========================$ SECTION $7=====$ Atmospheric production definition (select option):

0 -Use food-weighted chilQ, (food-sec/m3), enter value on this line 1-Use population-weighted chi/Q

2-Use uniform production

3-Use chi/Q and production grids (PRODUCTION will be overridden) Population ingesting aquatic foods, 0 defaults to total (person) Population ingesting drinking water, 0 defaults to total (person) Consider dose from food exported out of region (default=F)

Note below: $S^{*}$ or Source: 0-none, 1-ground water, 2-surface water 3-Derived concentration entered above $====$ AQUATIC FOODS / DRINKING WATER INGESTION $========$ SECTION $8====$

Salt water? (default is fresh)

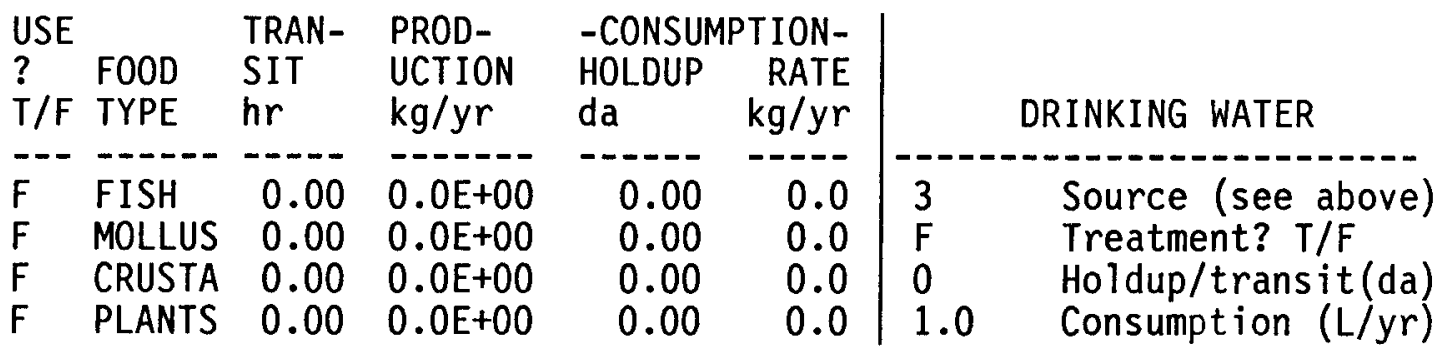


TABLE D.22. FACILPAR.TPL GENII Input File Template (contd)

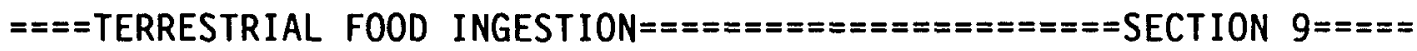

\begin{tabular}{|c|c|c|c|c|c|c|c|c|}
\hline USE & & GROW & - -IRRIG & TION-- & & PROD- & $--\operatorname{CONS}$ & PTION. \\
\hline$\stackrel{?}{\mathrm{~T} / \mathrm{F}}$ & $\begin{array}{l}\text { FOOD } \\
\text { TYPE }\end{array}$ & $\begin{array}{l}\text { TIME } \\
\text { da }\end{array}$ & $\begin{array}{l}\text { S RATE } \\
\text { * in/yr }\end{array}$ & $\begin{array}{l}\text { TIME } \\
\text { mo/yr }\end{array}$ & $\begin{array}{l}\text { YIELD } \\
\mathrm{kg} / \mathrm{m} 2\end{array}$ & $\begin{array}{l}\text { UCTION } \\
\mathrm{kg} / \mathrm{yr}\end{array}$ & $\begin{array}{l}\text { HOLDUP } \\
\mathrm{da}\end{array}$ & $\begin{array}{l}\text { RATE } \\
\mathrm{kg} / \mathrm{yr}\end{array}$ \\
\hline & -י- & $=--$ & $=-$ & ---- & ---- & $--\cdots--$ & ----- & \\
\hline$T$ & LEAF $V$ & 90.00 & 0.0 & 0.0 & 1.5 & $0.0 \mathrm{E}+00$ & 1.0 & 2.4 \\
\hline$T$ & ROOT V & 90.00 & 0.0 & 0.0 & 4.0 & $0.0 \mathrm{E}+00$ & . & 21.4 \\
\hline$T$ & FRUIT & 90.00 & 0.0 & 0.0 & 2.0 & $0.0 \mathrm{E}+00$ & 5.0 & 10.5 \\
\hline- & GRAIN & 90 & 0.0 & 0.0 & 0.8 & $0.0 \mathrm{E}+00$ & 180.0 & 12.8 \\
\hline
\end{tabular}

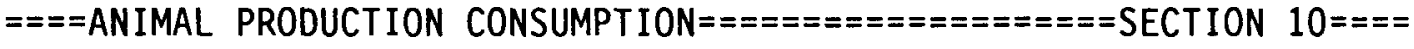

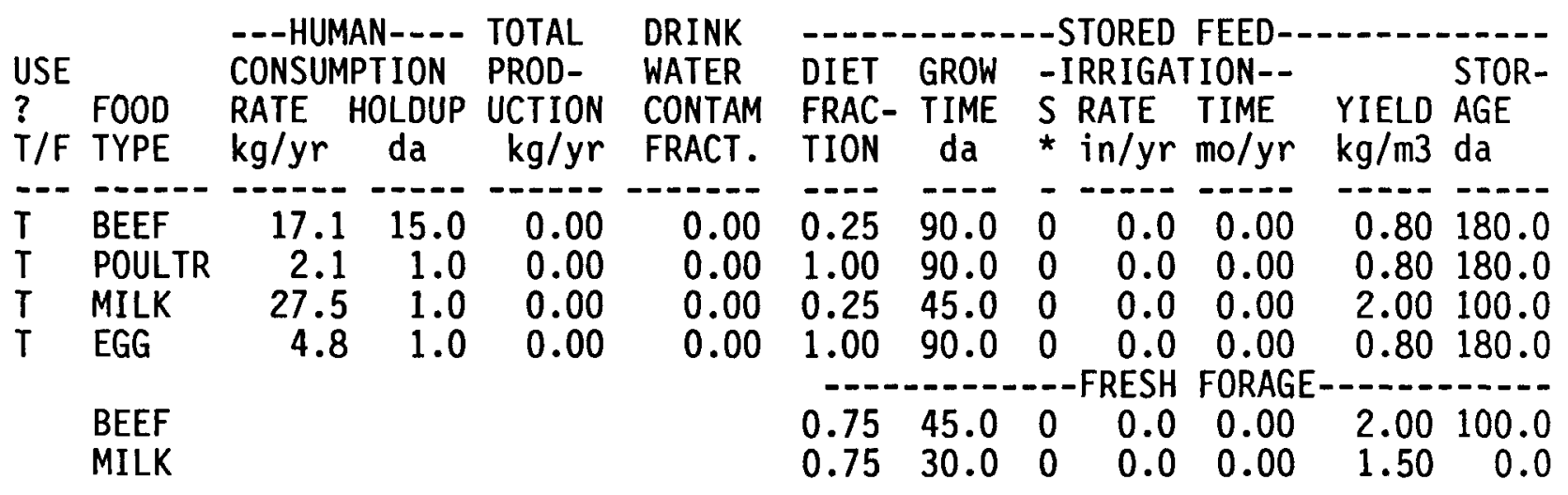


TABLE D.23. Radionuclide Master Library - Radionuclide Chains in Equilibrium (RMDLIB.DAT)

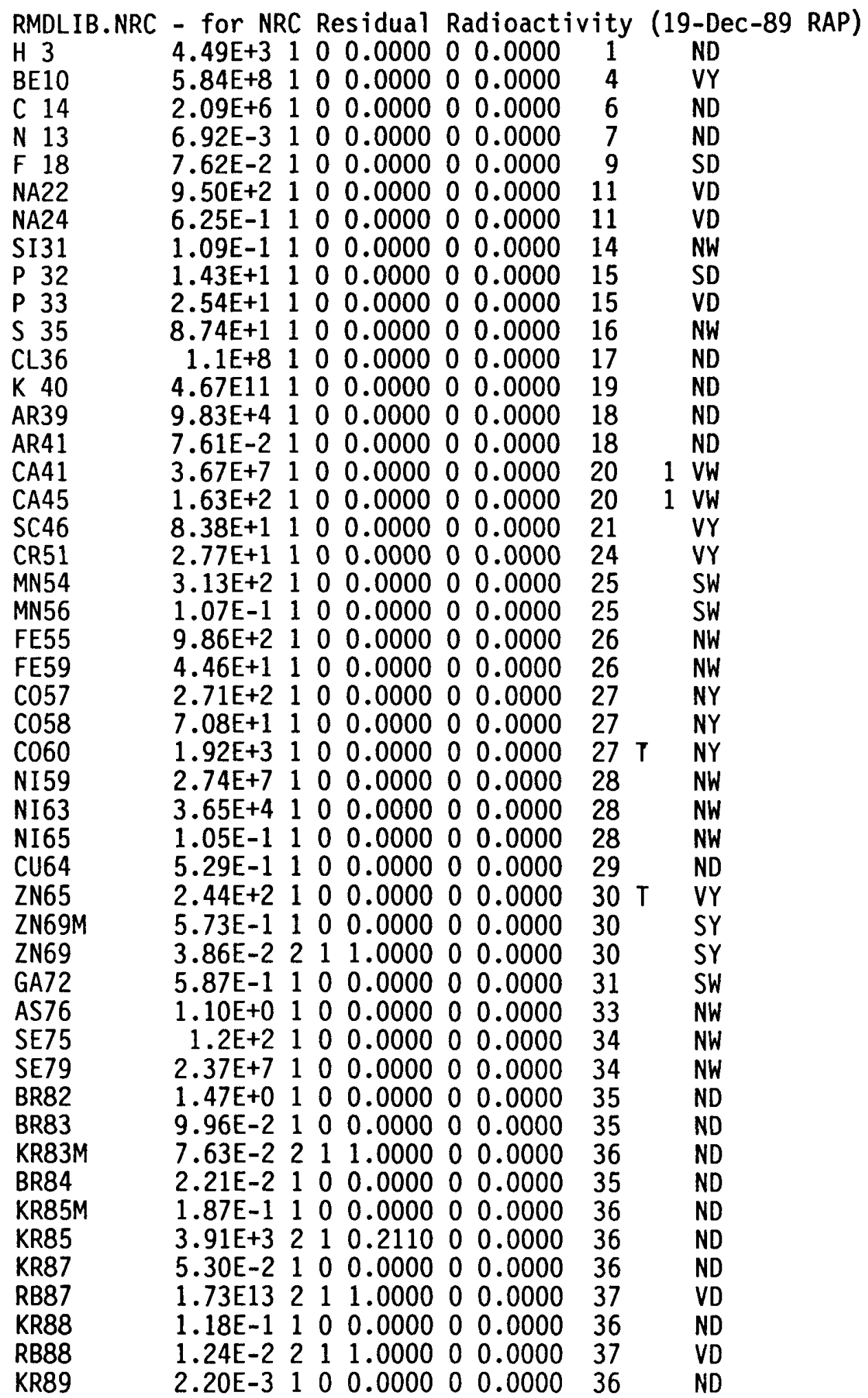


TABLE D.23. Radionuclide Master Library - Radionuclide Chains in Equilibrium (RMDLIB.DAT) (contd)

\begin{tabular}{|c|c|c|c|c|c|c|c|}
\hline $\begin{array}{l}\text { RB89 } \\
\text { SR899 } \\
\text { SR877M } \\
\text { RB86 } \\
\text { SR85 } \\
\text { SR90 } \\
\text { Y 90 } \\
\text { SR91 } \\
\text { Y 91M } \\
\text { Y } 91 \\
\text { SR92 } \\
\text { Y 92 } \\
\text { Y 93 } \\
\text { M093 } \\
\text { ZR93 } \\
\text { NB93M } \\
\text { ZR95 } \\
\text { NB95M } \\
\text { NB95 } \\
\text { ZR97 } \\
\text { NB97M } \\
\text { NB97 } \\
\text { NB94 } \\
\text { M099 } \\
\text { TC99M } \\
\text { TC99 } \\
\text { TC101 } \\
\text { RU103 } \\
\text { PD103 } \\
\text { RH103M } \\
\text { RU105 } \\
\text { RH105 } \\
\text { RU106 } \\
\text { PD107 } \\
\text { PD109 } \\
\text { AG110M } \\
\text { AG111 } \\
\text { CD109 } \\
\text { CD113M } \\
\text { CD115M } \\
\text { CD115 } \\
\text { IN115M } \\
\text { IN111 } \\
\text { IN114M } \\
\text { SN113 } \\
\text { IN113M } \\
\text { SN117M }\end{array}$ & $\begin{array}{ccc}1.07 \mathrm{E}-2 & 2 & 1 \\
5.06 \mathrm{E}+1 & 3 & 2 \\
1.17 \mathrm{E}-1 & 1 & 0 \\
1.87 \mathrm{E}+1 & 1 & 0 \\
6.5 \mathrm{E}+1 & 1 & 0 \\
1.04 \mathrm{E}+4 & 1 & 0 \\
2.67 \mathrm{E}+0 & 2 & 1 \\
3.96 \mathrm{E}-1 & 1 & 0 \\
3.45 \mathrm{E}-2 & 2 & 1 \\
5.85 \mathrm{E}+1 & 3 & 2 \\
1.13 \mathrm{E}-1 & 1 & 0 \\
1.48 \mathrm{E}-1 & 2 & 1 \\
4.21 \mathrm{E}-1 & 1 & 0 \\
1.28 \mathrm{E}+6 & 1 & 0 \\
5.58 \mathrm{E}+8 & 2 & 0 \\
5.33 \mathrm{E}+3 & 3 & 2 \\
6.40 \mathrm{E}+1 & 1 & 0 \\
3.61 \mathrm{E}+0 & 2 & 1 \\
3.51 \mathrm{E}+1 & 3 & 2 \\
7.04 \mathrm{E}-1 & 1 & 0 \\
6.94 \mathrm{E}-4 & 2 & 1 \\
5.01 \mathrm{E}-2 & 3 & 2 \\
7.41 \mathrm{E}+6 & 1 & 0 \\
2.75 \mathrm{E}+0 & 1 & 0 \\
2.51 \mathrm{E}-1 & 2 & 1 \\
7.77 \mathrm{E}+7 & 1 & 0 \\
9.86 \mathrm{E}-3 & 1 & 0 \\
3.94 \mathrm{E}+1 & 1 & 0 \\
1.70 \mathrm{E}+1 & 2 & 0 \\
3.90 \mathrm{E}-2 & 3 & 1 \\
1.85 \mathrm{E}-1 & 1 & 0 \\
1.47 \mathrm{E}+0 & 2 & 1 \\
3.68 \mathrm{E}+2 & 1 & 0 \\
2.37 \mathrm{E}+9 & 1 & 0 \\
5.61 \mathrm{E}-1 & 1 & 0 \\
2.50 \mathrm{E}+2 & 1 & 0 \\
7.46 \mathrm{E}+0 & 1 & 0 \\
4.64 \mathrm{E}+2 & 1 & 0 \\
5.00 \mathrm{E}+3 & 1 & 0 \\
4.46 \mathrm{E}+1 & 1 & 0 \\
2.23 \mathrm{E}+0 & 2 & 0 \\
1.82 \mathrm{E}-1 & 3 & 2 \\
2.8 \mathrm{E}+0 & 1 & 0 \\
5.00 \mathrm{E}+1 & 1 & 0 \\
1.15 \mathrm{E}+2 & 1 & 0 \\
6.91 \mathrm{E}-2 & 2 & 1 \\
1.40 \mathrm{E}+1 & 1 & 0 \\
2.93 \mathrm{E}+2 & 1 & 0 \\
2.01 \mathrm{E}+4 & 1 & 0\end{array}$ & $\begin{array}{l}1.0000 \\
1.0000 \\
0.0000 \\
0.0000 \\
0.0000 \\
0.0000 \\
1.0000 \\
0.0000 \\
0.5740 \\
1.0000 \\
0.0000 \\
1.0000 \\
0.0000 \\
0.0000 \\
0.0000 \\
1.0000 \\
0.0000 \\
0.0080 \\
0.9450 \\
0.0000 \\
0.9470 \\
1.0000 \\
0.0000 \\
0.0000 \\
0.8860 \\
0.0000 \\
0.0000 \\
0.0000 \\
0.0000 \\
0.9974 \\
0.0000 \\
1.0000 \\
0.0000 \\
0.0000 \\
0.0000 \\
0.0000 \\
0.0000 \\
0.0000 \\
0.0000 \\
0.0000 \\
0.0000 \\
1.0000 \\
0.0000 \\
0.0000 \\
0.0000 \\
1.0000 \\
0.0000 \\
0.0000 \\
0.0000\end{array}$ & $\begin{array}{ll}0 & 0.0000 \\
0 & 0.0000 \\
0 & 0.0000 \\
0 & 0.0000 \\
0 & 0.0000 \\
0 & 0.0000 \\
0 & 0.0000 \\
0 & 0.0000 \\
0 & 0.0000 \\
1 & 0.4260 \\
0 & 0.0000 \\
0 & 0.0000 \\
0 & 0.0000 \\
0 & 0.0000 \\
0 & 0.0000 \\
1 & 1.00000 \\
0 & 0.0000 \\
0 & 0.0000 \\
1 & 0.9922 \\
0 & 0.0000 \\
0 & 0.0000 \\
1 & 0.0530 \\
0 & 0.0000 \\
0 & 0.0000 \\
0 & 0.0000 \\
0 & 0.0000 \\
0 & 0.0000 \\
0 & 0.0000 \\
0 & 0.0000 \\
2 & 0.9997 \\
0 & 0.0000 \\
0 & 0.0000 \\
0 & 0.0000 \\
0 & 0.0000 \\
0 & 0.0000 \\
0 & 0.0000 \\
0 & 0.0000 \\
0 & 0.0000 \\
0 & 0.0000 \\
0 & 0.0000 \\
0 & 0.0000 \\
0 & 0.0000 \\
0 & 0.0000 \\
0 & 0.0000 \\
0 & 0.0000 \\
0 & 0.0000 \\
0 & 0.0000 \\
0 & 0.0000 \\
0 & 0.0000 \\
\end{array}$ & $\begin{array}{l}37 \\
38 \\
38 \\
37 \\
38 \\
38 T \\
39 \mathrm{~T} \\
38 \\
39 \\
39 \\
38 \\
39 \\
39 \\
42 \\
40 \\
41 \\
40 \\
41 \\
41 \\
40 \\
41 \\
41 \\
41 \\
42 \\
43 \\
43 \\
43 \\
44 \\
46 \\
45 \\
44 \\
45 \\
44 \mathrm{~T} \\
46 \\
46 \\
47 \\
47 \\
48 \\
48 \\
48 \\
48 \\
49 \\
49 \\
49 \\
50 \\
49 \\
50 \\
50 \\
50\end{array}$ & 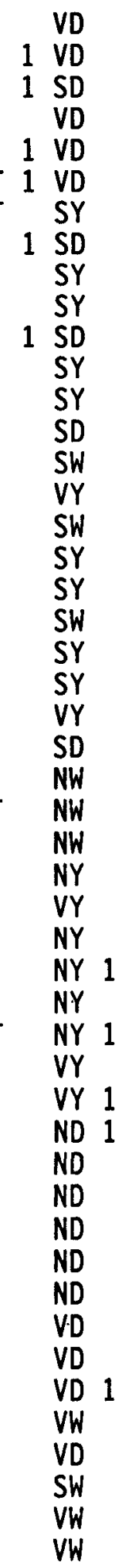 & $\begin{array}{l}\text { RH105M } \\
\text { RH106 } \\
\text { AG109M } \\
\text { AG110 } \\
\text { IN114 }\end{array}$ & $\begin{array}{l}1.000 \\
1.000 \\
1.000 \\
0.985\end{array}$ \\
\hline
\end{tabular}


TABLE D.23. Radionuclide Master Library - Radionuclide Chains in Equilibrium (RMDLIB.DAT) (contd)

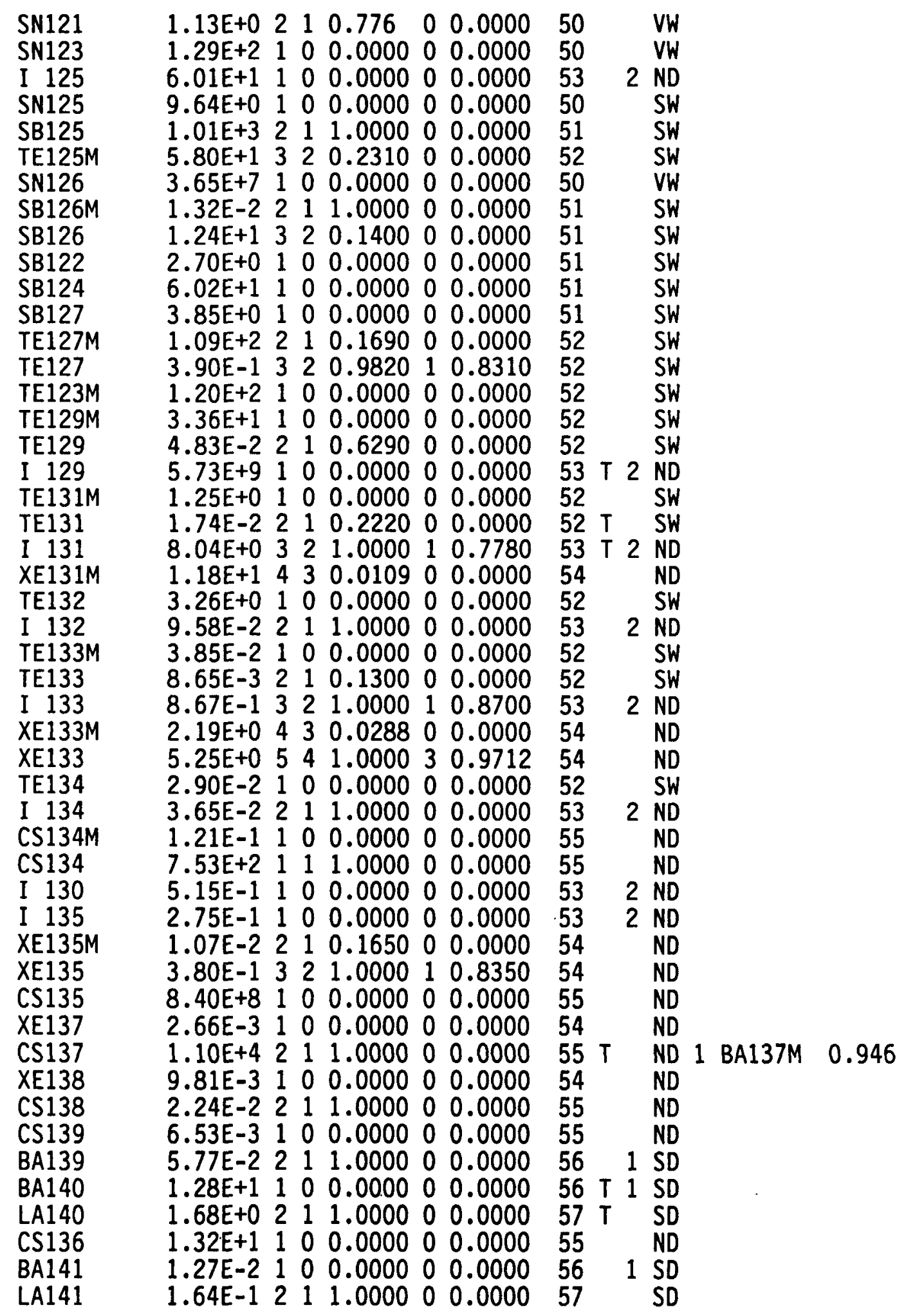


TABLE D.23. Radionuclide Master Library - Radionuclide Chains in Equilibrium (RMDLIB.DAT) (contd)

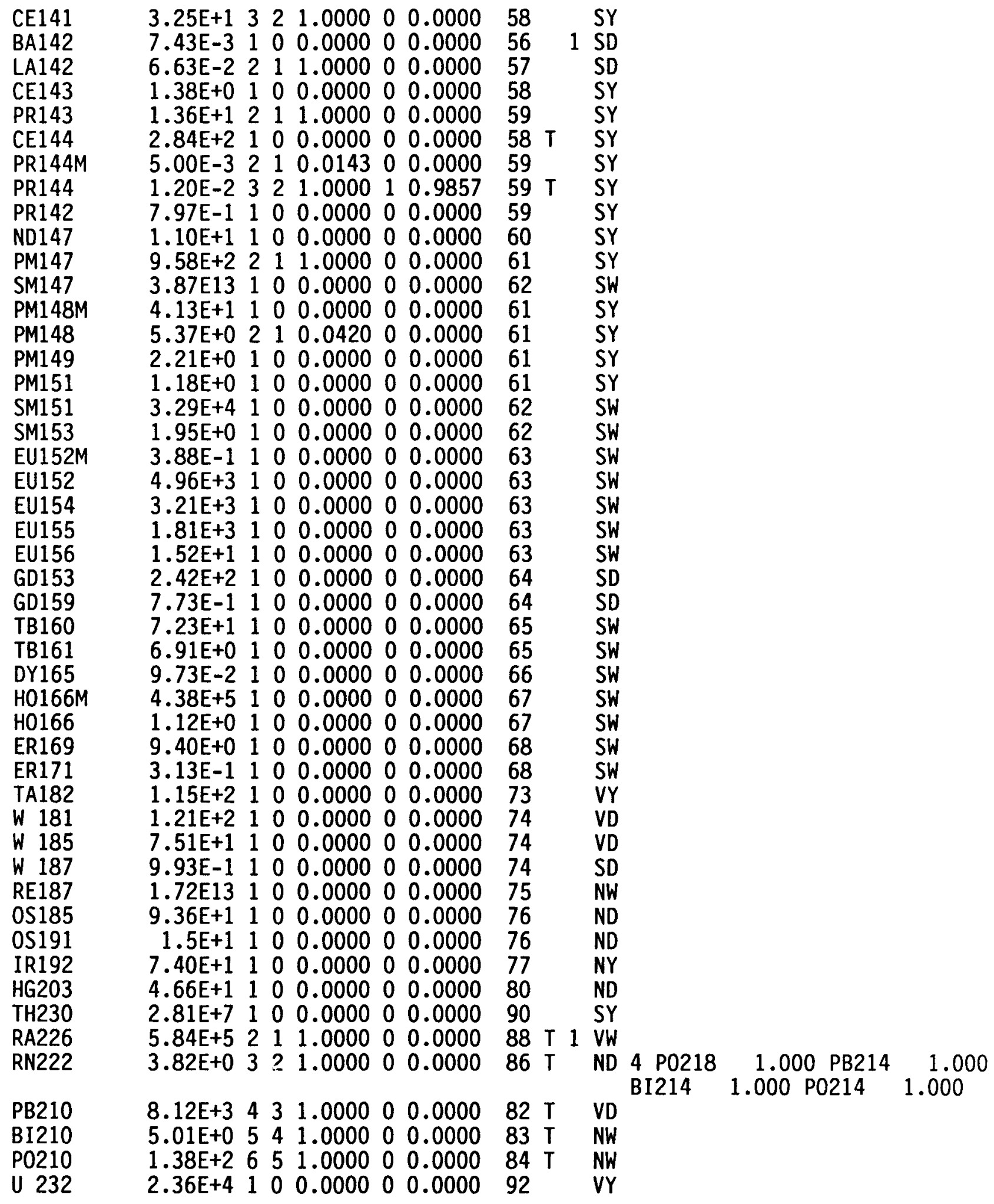


TABLE D.23. Radionuclide Master Library - Radionuclide Chains in Equilibrium (RMDLIB.DAT) (contd)

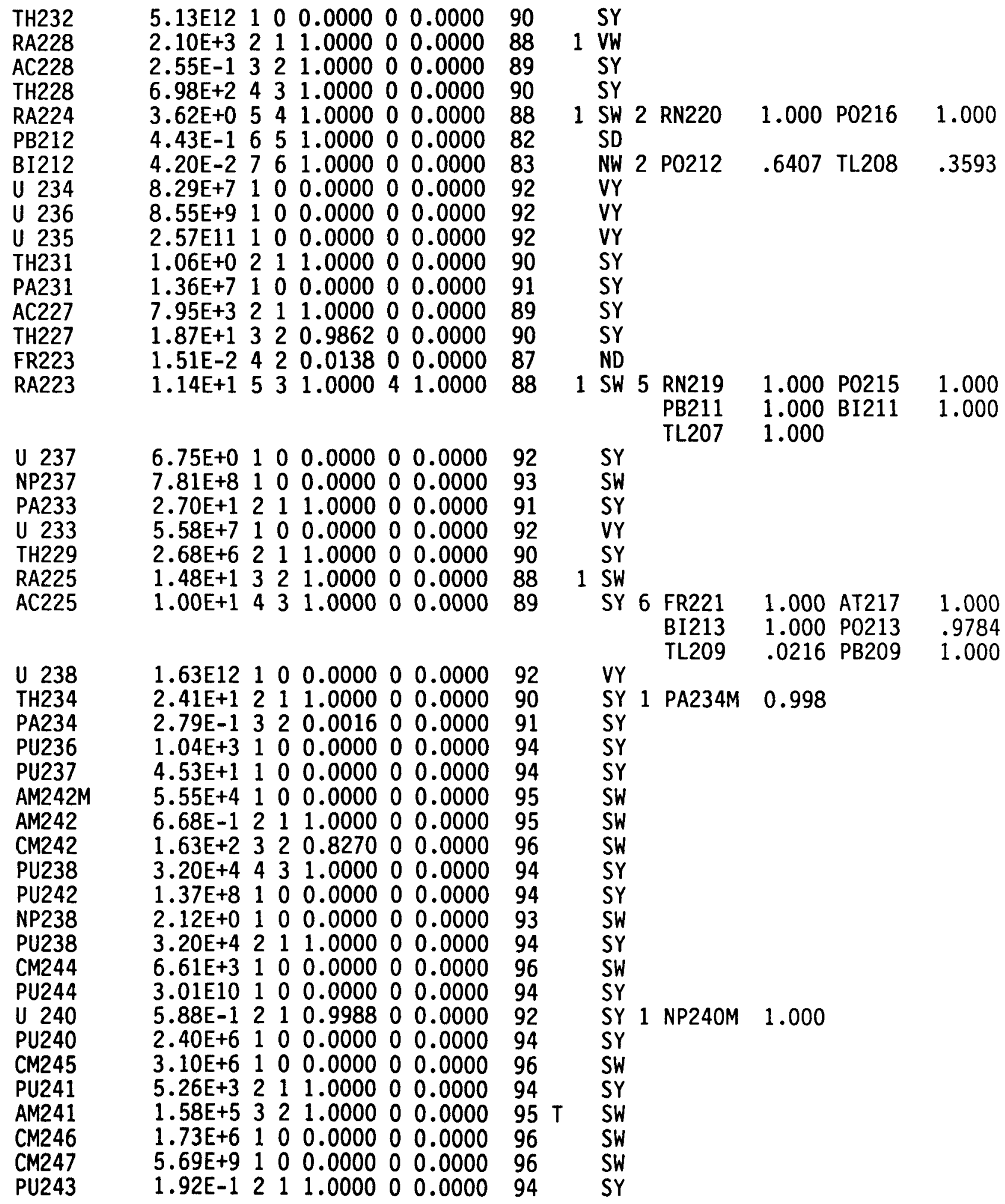


TABLE D.23. Radionuclide Master Library - Radionuclide Chains in Equilibrium (RMDLIB.DAT) (contd)

$\begin{array}{lllllllllll}\text { CM243 } & 1.04 E+4 & 1 & 0 & 0.0000 & 0 & 0.0000 & 96 & \text { SW } \\ \text { AM243 } & 2.69 E+6 & 2 & 1 & 0.0024 & 0 & 0.0000 & 95 & \text { SW } \\ \text { NP239 } & 2.36 E+0 & 3 & 2 & 1.0000 & 0 & 0.0000 & 93 & \text { SW } \\ \text { PU239 } & 8.81 E+6 & 4 & 1 & 0.9976 & 0 & 0.0000 & 94 & \text { SY } \\ \text { CM248 } & 1.24 E+8 & 1 & 0 & 0.0000 & 0 & 0.0000 & 96 & \text { SW } \\ \text { CF252 } & 9.63 E+2 & 1 & 0 & 0.0000 & 0 & 0.0000 & 98 & \text { SW } \\ & & & 0 & 0 & 0.0000 & 0 & 0.0000 & & \end{array}$


TABLE D.24. Radionuclide Master Library - Radionuclides without Explicit Daughters

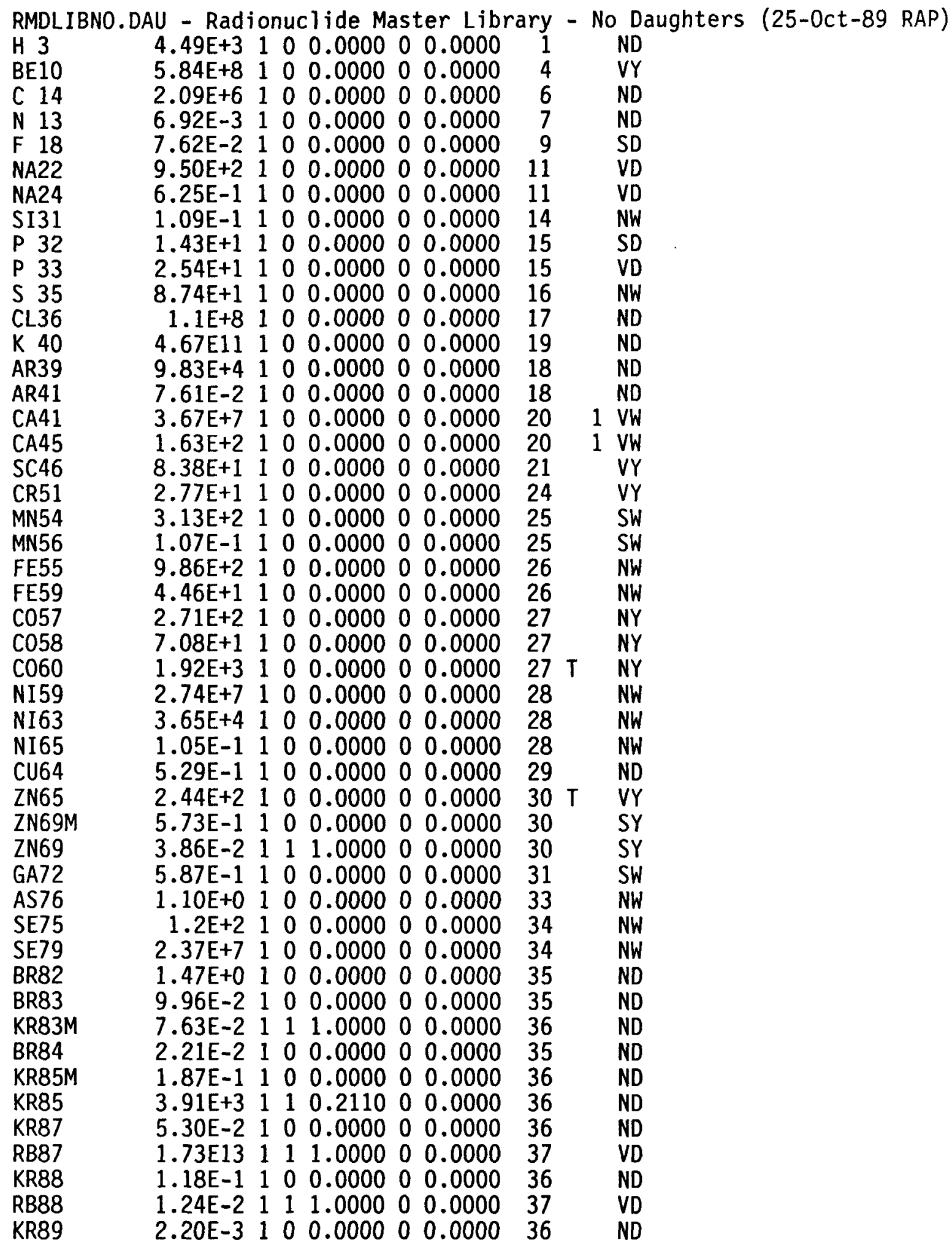


TABLE D.24. Radionuclide Master Library - Radionuclides without Explicit Daughters (contd)

\begin{tabular}{|c|c|c|c|c|c|c|c|}
\hline $\begin{array}{l}\text { RB89 } \\
\text { SR89 } \\
\text { SR87M } \\
\text { RB86 } \\
\text { SR85 } \\
\text { SR90 } \\
\text { Y 90 } \\
\text { SR91 } \\
\text { Y 91M } \\
\text { Y 91 } \\
\text { SR92 } \\
\text { Y 92 } \\
\text { Y 93 } \\
\text { M093 } \\
\text { ZR93 } \\
\text { NB93M } \\
\text { ZR95 } \\
\text { NB95M } \\
\text { NB95 } \\
\text { ZR97 } \\
\text { NB97M } \\
\text { NB97 } \\
\text { NB94 } \\
\text { M099 } \\
\text { TC99M } \\
\text { TC99 } \\
\text { TC101 } \\
\text { RU103 } \\
\text { PD103 } \\
\text { RH103M } \\
\text { RU105 } \\
\text { RH105 } \\
\text { RU106 } \\
\text { PD107 } \\
\text { PD109 } \\
\text { AG110M } \\
\text { AG111 } \\
\text { CD109 } \\
\text { CD113M } \\
\text { CD115M } \\
\text { CD115 } \\
\text { IN115M } \\
\text { IN111 } \\
\text { IN114M } \\
\text { SN113 } \\
\text { IN113M } \\
\text { SN117M } \\
\text { SN119M } \\
\text { SN121M }\end{array}$ & $\begin{array}{r}1.07 \mathrm{E}-2 \\
5.06 \mathrm{E}+1 \\
1.17 \mathrm{E}-1 \\
1.87 \mathrm{E}+1 \\
6.5 \mathrm{E}+1 \\
1.04 \mathrm{E}+4 \\
2.67 \mathrm{E}+0 \\
3.96 \mathrm{E}-1 \\
3.45 \mathrm{E}-2 \\
5.85 \mathrm{E}+1 \\
1.13 \mathrm{E}-1 \\
1.48 \mathrm{E}-1 \\
4.21 \mathrm{E}-1 \\
1.28 \mathrm{E}+6 \\
5.58 \mathrm{E}+8 \\
5.33 \mathrm{E}+3 \\
6.40 \mathrm{E}+1 \\
3.61 \mathrm{E}+0 \\
3.51 \mathrm{E}+1 \\
7.04 \mathrm{E}-1 \\
6.94 \mathrm{E}-4 \\
5.01 \mathrm{E}-2 \\
7.41 \mathrm{E}+6 \\
2.75 \mathrm{E}+0 \\
2.51 \mathrm{E}-1 \\
7.77 \mathrm{E}+7 \\
9.86 \mathrm{E}-3 \\
3.94 \mathrm{E}+1 \\
1.70 \mathrm{E}+1 \\
3.90 \mathrm{E}-2 \\
1.85 \mathrm{E}-1 \\
1.47 \mathrm{E}+0 \\
3.68 \mathrm{E}+2 \\
2.37 \mathrm{E}+9 \\
5.61 \mathrm{E}-1 \\
2.50 \mathrm{E}+2 \\
7.46 \mathrm{E}+0 \\
4.64 \mathrm{E}+2 \\
5.00 \mathrm{E}+3 \\
4.46 \mathrm{E}+1 \\
2.23 \mathrm{E}+0 \\
1.82 \mathrm{E}-1 \\
2.8 \mathrm{E}+0 \\
5.00 \mathrm{E}+1 \\
1.15 \mathrm{E}+2 \\
6.91 \mathrm{E}-2 \\
1.40 \mathrm{E}+1 \\
2.93 \mathrm{E}+2 \\
2.01 \mathrm{E}+4\end{array}$ & $\begin{array}{lll}1 & 1 & 1.0000 \\
1 & 2 & 1.0000 \\
1 & 0 & 0.0000 \\
1 & 0 & 0.0000 \\
1 & 0 & 0.0000 \\
1 & 0 & 0.0000 \\
1 & 1 & 1.0000 \\
1 & 0 & 0.0000 \\
1 & 1 & 0.5740 \\
1 & 2 & 1.0000 \\
1 & 0 & 0.0000 \\
1 & 1 & 1.0000 \\
1 & 0 & 0.0000 \\
1 & 0 & 0.0000 \\
1 & 1 & 1.0000 \\
1 & 3 & 1.0000 \\
1 & 0 & 0.0000 \\
1 & 1 & 0.0080 \\
1 & 2 & 0.9450 \\
1 & 0 & 0.0000 \\
1 & 1 & 0.9470 \\
1 & 2 & 1.0000 \\
1 & 0 & 0.0000 \\
1 & 0 & 0.0000 \\
1 & 1 & 0.8860 \\
1 & 2 & 1.0000 \\
1 & 0 & 0.0000 \\
1 & 0 & 0.0000 \\
1 & 0 & 0.0000 \\
1 & 1 & 0.9974 \\
1 & 0 & 0.0000 \\
1 & 1 & 1.0000 \\
1 & 0 & 0.0000 \\
1 & 0 & 0.0000 \\
1 & 0 & 0.0000 \\
1 & 0 & 0.0000 \\
1 & 0 & 0.0000 \\
1 & 0 & 0.0000 \\
1 & 0 & 0.0000 \\
1 & 0 & 0.0000 \\
1 & 0 & 0.0000 \\
1 & 2 & 1.0000 \\
1 & 0 & 0.0000 \\
1 & 0 & 0.0000 \\
1 & 0 & 0.0000 \\
1 & 1 & 1.0000 \\
1 & 0 & 0.0000 \\
1 & 0 & 0.0000 \\
1 & 0 & 0.0000 \\
0\end{array}$ & $\begin{array}{ll}0 & 0.0000 \\
0 & 0.0000 \\
0 & 0.0000 \\
0 & 0.0000 \\
0 & 0.0000 \\
0 & 0.0000 \\
0 & 0.0000 \\
0 & 0.0000 \\
0 & 0.0000 \\
1 & 0.4260 \\
0 & 0.0000 \\
0 & 0.0000 \\
0 & 0.0000 \\
0 & 0.0000 \\
0 & 0.0000 \\
2 & 1.0000 \\
0 & 0.0000 \\
0 & 0.0000 \\
1 & 0.9922 \\
0 & 0.0000 \\
0 & 0.0000 \\
1 & 0.0530 \\
0 & 0.0000 \\
0 & 0.0000 \\
0 & 0.0000 \\
1 & 0.1140 \\
0 & 0.0000 \\
0 & 0.0000 \\
0 & 0.0000 \\
2 & 0.9997 \\
0 & 0.0000 \\
0 & 0.0000 \\
0 & 0.0000 \\
0 & 0.0000 \\
0 & 0.0000 \\
0 & 0.0000 \\
0 & 0.0000 \\
0 & 0.0000 \\
0 & 0.0000 \\
0 & 0.0000 \\
0 & 0.0000 \\
0 & 0.0000 \\
0 & 0.0000 \\
0 & 0.0000 \\
0 & 0.0000 \\
0 & 0.0000 \\
0 & 0.0000 \\
0 & 0.0000 \\
0 & 0.0000\end{array}$ & $\begin{array}{l}37 \\
38 \\
38 \\
37 \\
38 \\
38 \\
39 \\
38 \\
38 \\
39 \\
39 \\
38 \\
39 \\
39 \\
42 \\
40 \\
41 \\
40 \\
41 \\
41 \\
40 \\
41 \\
41 \\
41 \\
42 \\
43 \\
43 \\
43 \\
44 \\
46 \\
45 \\
44 \\
45 \\
44 \\
46 \\
46 \\
47 \\
47 \\
48 \\
48 \\
48 \\
48 \\
49 \\
49 \\
49 \\
50 \\
49 \\
50 \\
50 \\
50\end{array}$ & 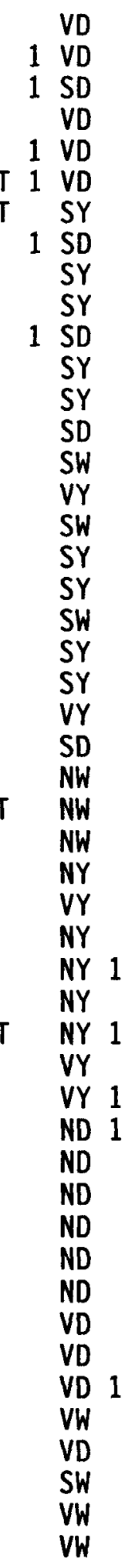 & $\begin{array}{l}\text { RH105M } \\
\text { RH106 } \\
\text { AG109M } \\
\text { AG110 }\end{array}$ & $\begin{array}{l}1.000 \\
1.000 \\
1.000 \\
0.985\end{array}$ \\
\hline
\end{tabular}


TABLE D.24. Radionuclide Master Library - Radionuclides without Explicit Daughters (contd)

\begin{tabular}{|c|c|c|c|c|c|c|c|}
\hline N12 & $\begin{array}{ll}1.13 E+0 & 1 \\
1.29 E+2 & 1 \\
6.01 E+1 & 1 \\
9.64 E+0 & 1 \\
1.01 E+3 & 1 \\
5.80 E+1 & 1 \\
3.65 E+7 & 1 \\
1.32 E-2 & 1 \\
1.24 E+1 & 1 \\
2.70 E+0 & 1 \\
6.02 E+1 & 1 \\
3.85 E+0 & 1 \\
1.09 E+2 & 1 \\
3.90 E-1 & 1 \\
1.20 E+2 & 1 \\
3.36 E+1 & 1 \\
4.83 E-2 & 1 \\
5.73 E+9 & 1 \\
1.25 E+0 & 1 \\
1.74 E-2 & 1 \\
8.04 E+0 & 1 \\
1.18 E+1 & 1 \\
3.26 E+0 & 1 \\
9.58 E-2 & 1 \\
3.85 E-2 & 1 \\
8.65 E-3 & 1 \\
8.67 E-1 & 1 \\
2.19 E+0 & 1 \\
5.25 E+0 & 1 \\
2.90 E-2 & 1 \\
3.65 E-2 & 1 \\
1.21 E-1 & 1 \\
7.53 E+2 & 1 \\
5.15 E-1 & 1 \\
2.75 E-1 & 1 \\
1.07 E-2 & 1 \\
3.80 E-1 & 1 \\
8.40 E+8 & 1 \\
2.66 E-3 & 1 \\
1.10 E+4 & 1 \\
9.81 E-3 & 1 \\
2.24 E-2 & 1 \\
6.53 E-3 & 1 \\
5.77 E-2 & 1 \\
1.28 E+1 & 1 \\
1.68 E+0 & 1 \\
1.32 E+1 & 1 \\
1.27 E-2 & 1 \\
1.64 E-1 & 1 \\
1.04\end{array}$ & $\begin{array}{llll}1 & 1 & 0.776 & 0 \\
1 & 0 & 0.0000 & 0 \\
1 & 0 & 0.0000 & 0 \\
1 & 0 & 0.0000 & 0 \\
1 & 1 & 1.0000 & 0 \\
1 & 2 & 0.2310 & 0 \\
1 & 0 & 0.0000 & 0 \\
1 & 1 & 1.0000 & 0 \\
1 & 2 & 0.1400 & 0 \\
1 & 0 & 0.0000 & 0 \\
1 & 0 & 0.0000 & 0 \\
1 & 0 & 0.0000 & 0 \\
1 & 1 & 0.1690 & 0 \\
1 & 2 & 0.9820 & 1 \\
1 & 0 & 0.0000 & 0 \\
1 & 0 & 0.0000 & 0 \\
1 & 1 & 0.6290 & 0 \\
1 & 2 & 1.0000 & 1 \\
1 & 0 & 0.0000 & 0 \\
1 & 1 & 0.2220 & 0 \\
1 & 2 & 1.0000 & 1 \\
1 & 3 & 0.0109 & 0 \\
1 & 0 & 0.0000 & 0 \\
1 & 1 & 1.0000 & 0 \\
1 & 0 & 0.0000 & 0 \\
1 & 1 & 0.1300 & 0 \\
1 & 2 & 1.0000 & 1 \\
1 & 3 & 0.0288 & 0 \\
1 & 4 & 1.0000 & 3 \\
1 & 0 & 0.0000 & 0 \\
1 & 1 & 1.0000 & 0 \\
1 & 0 & 0.0000 & 0 \\
1 & 1 & 1.0000 & 0 \\
1 & 0 & 0.0000 & 0 \\
1 & 0 & 0.0000 & 0 \\
1 & 1 & 0.1650 & 0 \\
1 & 2 & 1.0000 & 1 \\
1 & 3 & 1.0000 & 0 \\
1 & 0 & 0.0000 & 0 \\
1 & 1 & 1.0000 & 0 \\
1 & 0 & 0.0000 & 0 \\
1 & 1 & 1.0000 & 0 \\
1 & 0 & 0.0000 & 0 \\
1 & 1 & 1.0000 & 0 \\
1 & 0 & 0.0000 & 0 \\
1 & 1 & 1.0000 & 0 \\
1 & 0 & 0.0000 & 0 \\
1 & 0 & 0.0000 & 0 \\
1 & 1 & 1.0000 & 0\end{array}$ & $\begin{array}{l}0.0000 \\
0.0000 \\
0.0000 \\
0.0000 \\
0.0000 \\
0.0000 \\
0.0000 \\
0.0000 \\
0.0000 \\
0.0000 \\
0.0000 \\
0.0000 \\
0.0000 \\
0.8310 \\
0.0000 \\
0.0000 \\
0.0000 \\
0.3710 \\
0.0000 \\
0.0000 \\
0.7780 \\
0.0000 \\
0.0000 \\
0.0000 \\
0.0000 \\
0.0000 \\
0.8700 \\
0.0000 \\
0.9712 \\
0.0000 \\
0.0000 \\
0.0000 \\
0.0000 \\
0.0000 \\
0.0000 \\
0.0000 \\
0.8350 \\
0.0000 \\
0.0000 \\
0.0000 \\
0.0000 \\
0.0000 \\
0.0000 \\
0.0000 \\
0.0000 \\
0.0000 \\
0.0000 \\
0.0000 \\
0.0000\end{array}$ & $\begin{array}{l}50 \\
50 \\
53 \\
50 \\
51 \\
52 \\
50 \\
50 \\
51 \\
51 \\
51 \\
51 \\
51 \\
51 \\
52 \\
52 \\
52 \\
52 \\
52 \\
52 \\
53\end{array}$ & \multicolumn{2}{|c|}{$\begin{array}{l}\text { VW } \\
\text { VW } \\
2 \text { ND } \\
\text { SW } \\
\text { SW } \\
\text { SW } \\
\text { VW } \\
\text { SW } \\
\text { SW } \\
\text { SW } \\
\text { SW } \\
\text { SW } \\
\text { SW } \\
\text { SW } \\
\text { SW } \\
\text { SW } \\
\text { SW } \\
2 \text { ND } \\
\text { SW } \\
\text { SW } \\
2 \text { ND } \\
\text { ND } \\
\text { SW } \\
2 \text { ND } \\
\text { SW } \\
\text { SW } \\
2 \text { ND } \\
\text { ND } \\
\text { ND } \\
\text { SW } \\
2 \text { ND } \\
\text { ND } \\
\text { ND } \\
2 \text { ND } \\
2 \text { ND } \\
\text { ND } \\
\text { ND } \\
\text { ND } \\
\text { ND } \\
\text { ND } \\
\text { ND } \\
\text { ND } \\
\text { ND } \\
1 \text { SD } \\
1 \text { SD } \\
\text { SD } \\
\text { SD }\end{array}$} & 0.946 \\
\hline
\end{tabular}


TABLE D.24. Radionuclide Master Library - Radionuclides without Explicit Daughters (contd)

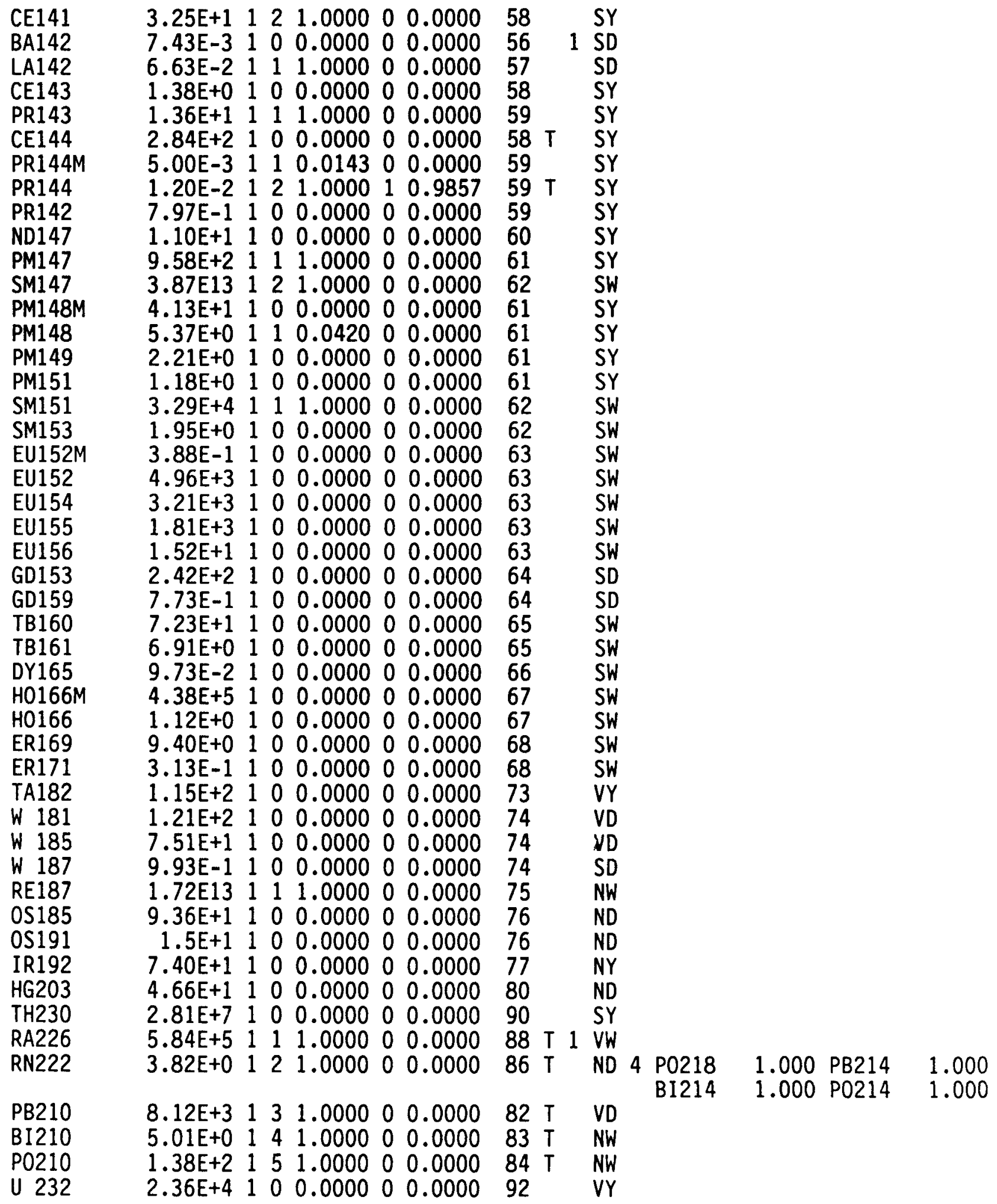


TABLE D.24. Radionuclide Master Library - Radionuclides without Explicit Daughters (contd)

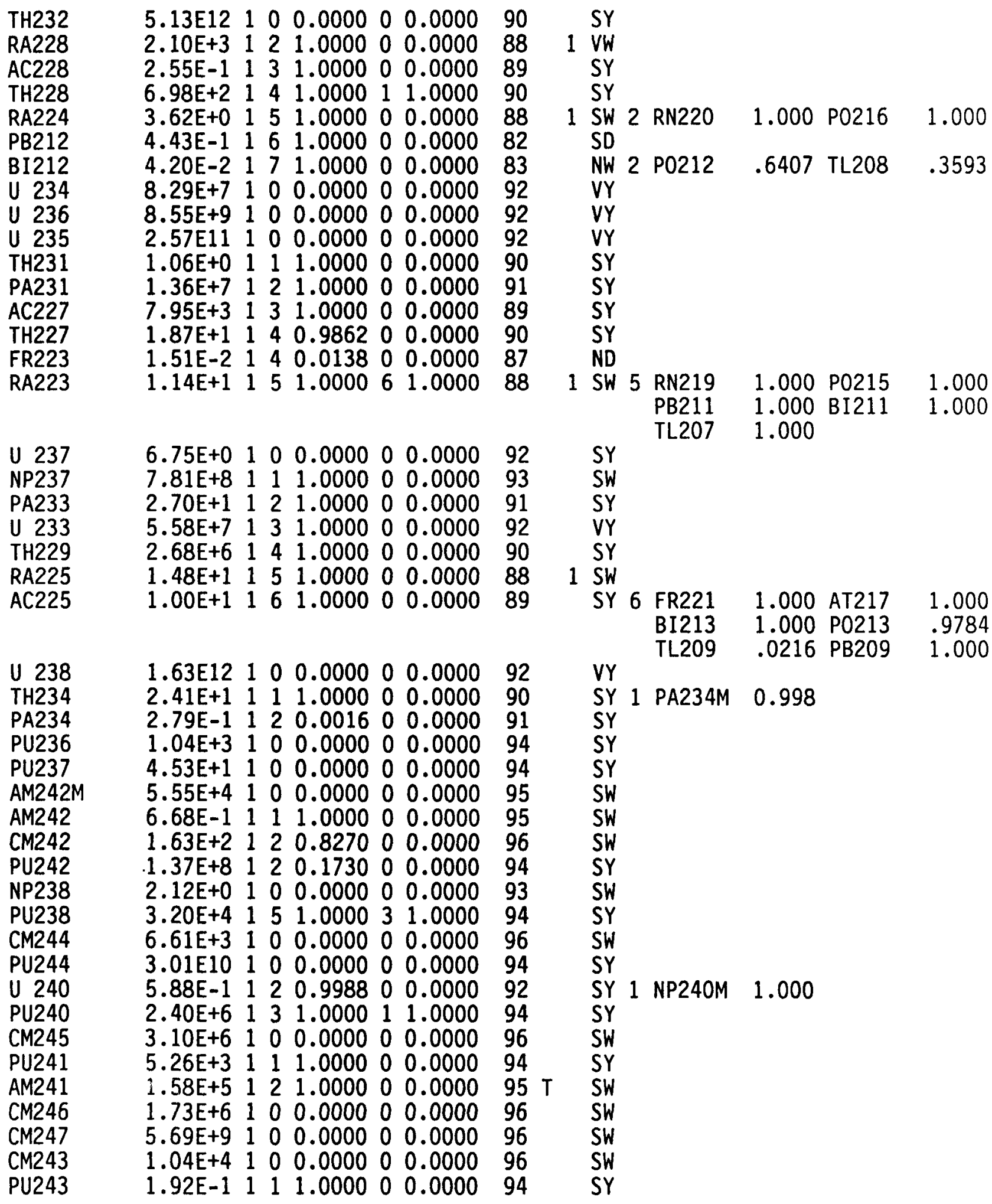


TABLE D.24. Radionuclide Master Library - Radionuclides without Explicit Daughters (contd)

$\begin{array}{llllllllll}\text { AM243 } & 2.69 \mathrm{E}+6 & 1 & 3 & 1.0000 & 2 & 0.0024 & 95 & & \text { SW } \\ \text { NP239 } & 2.36 \mathrm{E}+0 & 1 & 4 & 1.0000 & 0 & 0.0000 & 93 & & \text { SW } \\ \text { PU239 } & 8.81 \mathrm{E}+6 & 1 & 5 & 1.0000 & 2 & 0.9976 & 94 & \mathrm{~T} & \text { SY } \\ \text { CM248 } & 1.24 \mathrm{E}+8 & 1 & 0 & 0.0000 & 0 & 0.0000 & 96 & \text { SW } \\ \text { CF252 } & 9.63 \mathrm{E}+2 & 1 & 0 & 0.0000 & 0 & 0.0000 & 98 & & \text { SW } \\ & & & 0 & 0.0000 & 0 & 0.0000 & & & \end{array}$

D.116 


\section{TABLE D.25. Food Transfer Coefficient Data for Soil}

Contribution to Food Product Ingestion

10 CFR 61 Transfer Factors when Available, then Reg Guide 1/109 (5-0ct-89 RAP)

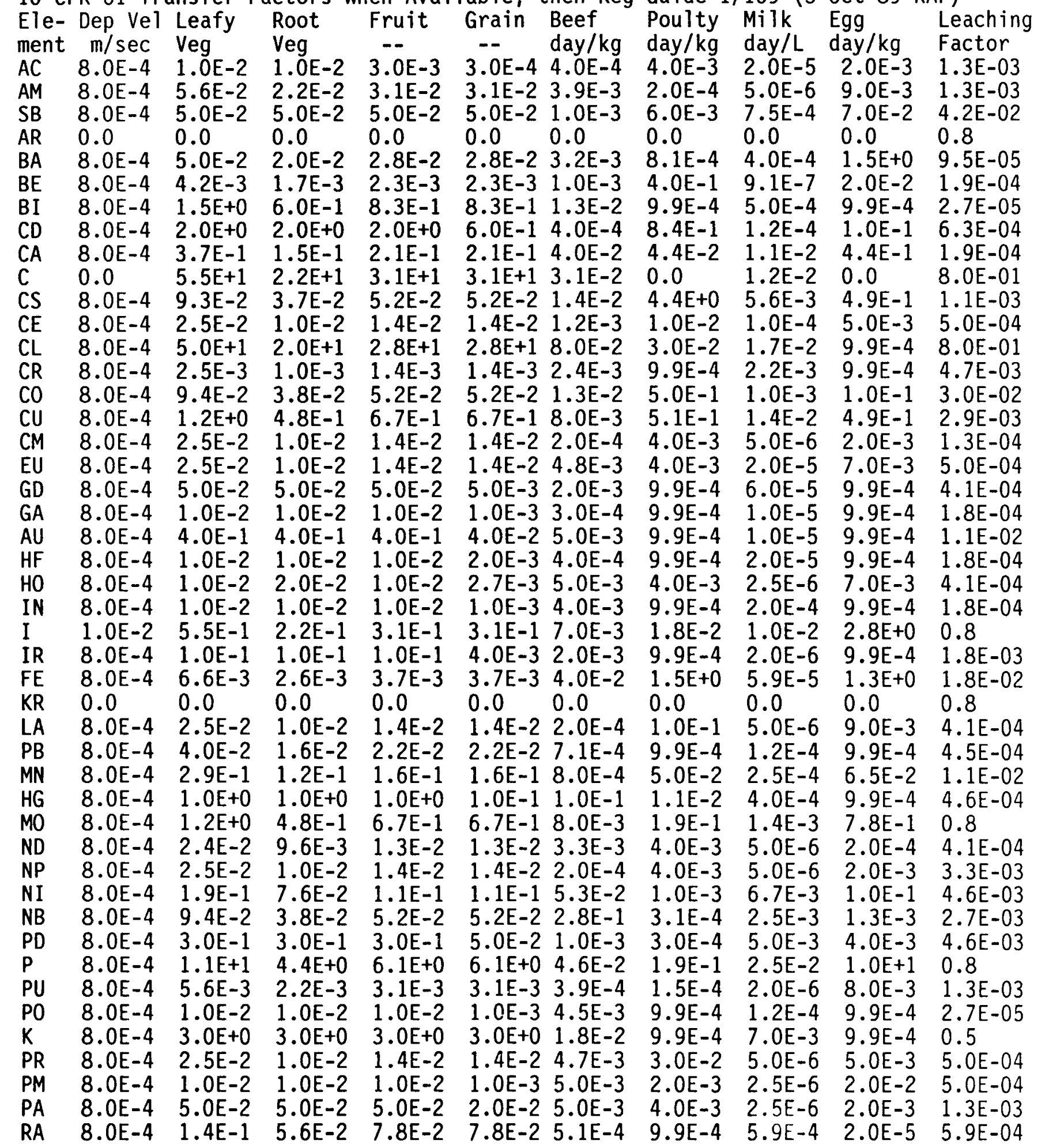


TABLE D.25. Food Transfer Coefficient Data for Soil

Contribtution to Food Product Ingestion (contd)

\begin{tabular}{|c|c|c|c|c|c|c|c|c|c|}
\hline $\begin{array}{l}.0 \mathrm{E}-4 \\
.0 \mathrm{E}-4 \\
.0 \mathrm{E}-4 \\
.0 \mathrm{E}-4 \\
.0 \mathrm{E}-4 \\
.0 \mathrm{E}-4 \\
.0 \mathrm{E}-4 \\
.0 \mathrm{E}-4 \\
.0 \mathrm{E}-4\end{array}$ & $\begin{array}{l}1.3 \mathrm{E}+ \\
5.0 \mathrm{E}- \\
1.0 \mathrm{E}- \\
1.0 \mathrm{E}- \\
1.5 \mathrm{E}+ \\
5.0 \mathrm{E}- \\
5.2 \mathrm{E}- \\
2.9 \mathrm{E}+ \\
2.0 \mathrm{E}+ \\
1.1 \mathrm{E}+ \\
1.3 \mathrm{E}+ \\
4.2 \mathrm{E}- \\
1.0 \mathrm{E}- \\
1.8 \mathrm{E}- \\
2.5 \mathrm{E}- \\
0.0 \\
2.6 \mathrm{E}- \\
4.0 \mathrm{E}+ \\
1.7 \mathrm{E}- \\
4.8 \mathrm{E}+ \\
7.5 \mathrm{E}+ \\
2.0 \mathrm{E}- \\
1.0 \mathrm{E}- \\
7.6 \mathrm{E}- \\
2.6 \mathrm{E}- \\
0.0 \\
2.5 \mathrm{E}- \\
9.9 \mathrm{E}- \\
9.9 \mathrm{E}- \\
9.9 \mathrm{E}- \\
9.9 \mathrm{E}- \\
9.9 \mathrm{E}- \\
3.5 \mathrm{E}- \\
1.0 \mathrm{E}-\end{array}$ & $\begin{array}{l}5 . \\
2 . \\
1 . \\
1 . \\
6 . \\
5 . \\
2 . \\
1 . \\
2 . \\
5 . \\
1 . \\
1 . \\
7 .\end{array}$ & $\begin{array}{l}1.4 \\
7.2 \mathrm{E} \\
2.8 \mathrm{E} \\
1.0 \mathrm{E} \\
1.0 \mathrm{E} \\
8.3 \mathrm{E} \\
5.0 \mathrm{E} \\
2.9 \mathrm{E} \\
1.6 \mathrm{E} \\
2.0 \mathrm{E} \\
6.1 \mathrm{E} \\
7.2 \mathrm{E} \\
2.3 \mathrm{E} \\
1.0 \mathrm{E} \\
1.0 \mathrm{E} \\
1.4 \mathrm{E} \\
0.0 \\
1.4 \mathrm{E} \\
2.2 \mathrm{E} \\
9.4 \mathrm{E} \\
2.7 \mathrm{E} \\
7.5 \mathrm{E} \\
2.0 \mathrm{E} \\
1.0 \mathrm{E} \\
7.6 \mathrm{E} \\
2.6 \mathrm{E} \\
0.0 \\
2.5 \mathrm{E} \\
9.9 \mathrm{E} \\
9.9 \mathrm{E} \\
9.9 \mathrm{E} \\
9.9 \mathrm{E} \\
9.9 \mathrm{E} \\
3.5 \mathrm{E} \\
1.0 \mathrm{E}\end{array}$ & $\begin{array}{l}7 . \\
2 . \\
1 . \\
1 . \\
1 . \\
0 . \\
1 .\end{array}$ & $\begin{array}{l}3.0 \\
5.0 \\
6.0 \\
1.0 \\
1.0 \\
3.0 \\
3.0 \\
2.0 \\
8.0 \\
7.7 \\
2.0 \\
1.0 \\
1.3 \\
3.4 \\
0.0 \\
4.6\end{array}$ & $\begin{array}{l}2 . \\
7 . \\
4 . \\
4 . \\
9 . \\
8 . \\
1 . \\
3 . \\
9 . \\
3 . \\
8 . \\
4 . \\
9 . \\
9 . \\
1 . \\
0 . \\
1 . \\
6 . \\
6 . \\
0 . \\
9 . \\
9 . \\
8 . \\
4 . \\
4 . \\
0 . \\
4 . \\
9 . \\
9 . \\
9 .\end{array}$ & $\begin{array}{l}2 \\
6 \\
5 \\
6 \\
2 \\
2 \\
2 \\
3 \\
2 \\
3 \\
3 \\
6 \\
3 \\
4 \\
4\end{array}$ & $\begin{array}{l}2 \\
2 \\
1 \\
0 \\
9 \\
9 \\
9 \\
1 \\
7 \\
0 \\
2 \\
9 \\
1 \\
9 \\
9 \\
9 \\
0\end{array}$ & $\begin{array}{l}1 \\
0 \\
2 \\
3 \\
0 \\
0 \\
5 \\
2 \\
1 \\
1 \\
0 \\
5 \\
2 \\
5 \\
8 . \\
0 \\
0 \\
1 . \\
1 . \\
4 . \\
0 \\
4 \\
4 \\
1 \\
3 \\
4 \\
0 \\
8 \\
4\end{array}$ \\
\hline
\end{tabular}


TABLE D.26. Food Transfer Coefficient Data for Air Contribution to Food Product Ingestion

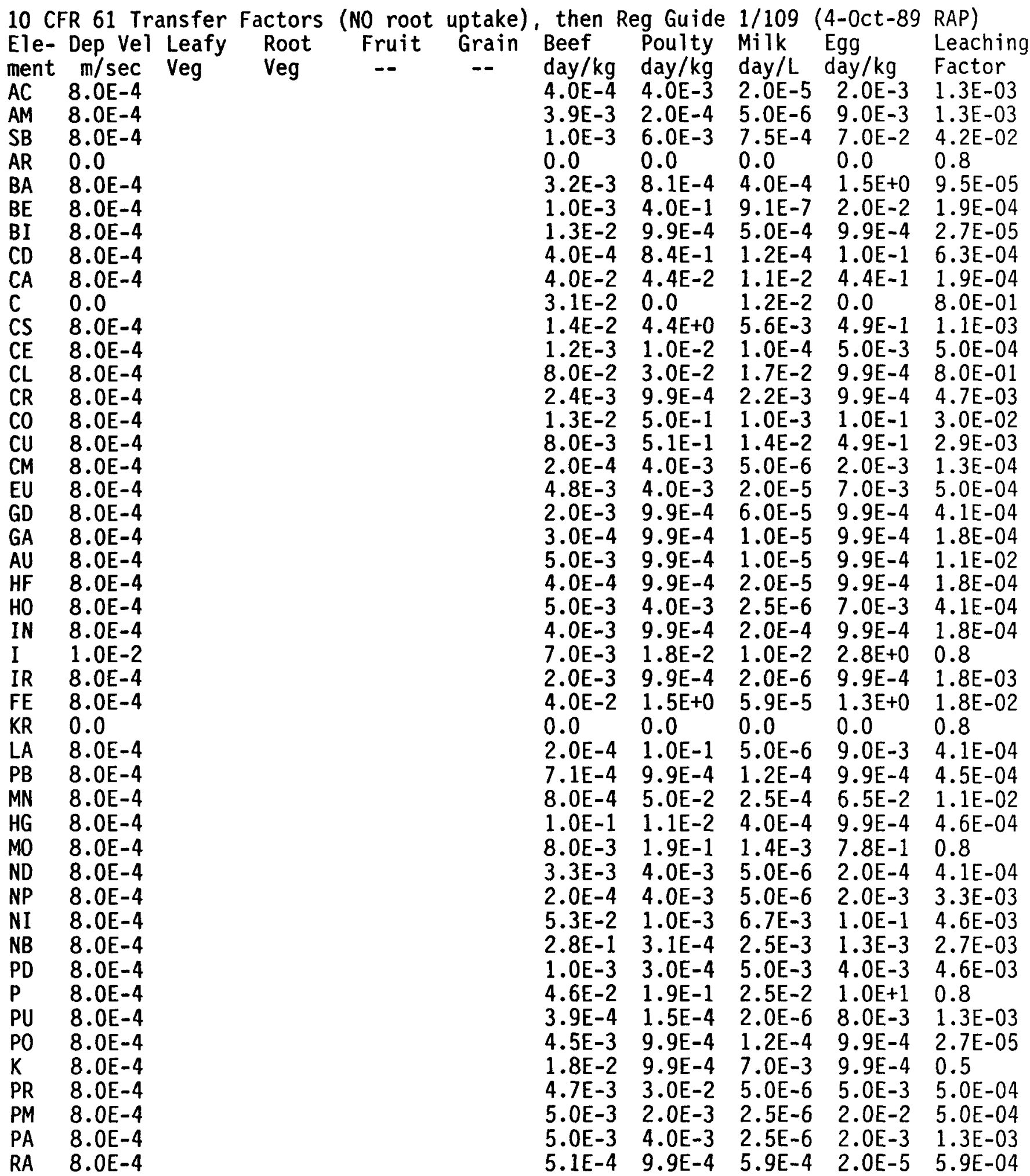


TABLE D.22. Food Transfer Coefficient Data for Air Contribution to Food Product Ingestion (contd)

$\begin{array}{ll}\text { RH } & 8.0 \mathrm{E}-4 \\ \text { RB } & 8.0 \mathrm{E}-4 \\ \text { RU } & 8.0 \mathrm{E}-4 \\ \text { SM } & 8.0 \mathrm{E}-4 \\ \text { SC } & 8.0 \mathrm{E}-4 \\ \text { AG } & 8.0 \mathrm{E}-4 \\ \text { SE } & 8.0 \mathrm{E}-4 \\ \text { NA } & 8.0 \mathrm{E}-4 \\ \text { SR } & 8.0 \mathrm{E}-4 \\ \text { S } & 8.0 \mathrm{E}-4 \\ \text { TC } & 8.0 \mathrm{E}-4 \\ \text { TE } & 8.0 \mathrm{E}-4 \\ \text { TH } & 8.0 \mathrm{E}-4 \\ \text { SN } & 8.0 \mathrm{E}-4 \\ \text { W } & 8.0 \mathrm{E}-4 \\ \text { U } & 8.0 \mathrm{E}-4 \\ \text { XE } & 0.0 \\ \text { Y } & 8.0 \mathrm{E}-4 \\ \text { ZN } & 8.0 \mathrm{E}-4 \\ \text { ZR } & 8.0 \mathrm{E}-4 \\ \text { H } & 0.0 \\ \text { N } & 8.0 \mathrm{E}-4 \\ \text { F } & 8.0 \mathrm{E}-4 \\ \text { AS } & 8.0 \mathrm{E}-4 \\ \text { BR } & 8.0 \mathrm{E}-4 \\ \text { TB } & 8.0 \mathrm{E}-4 \\ \text { RN } & 0.0 \\ \text { CF } & 8.0 \mathrm{E}-4 \\ \text { DY } & 8.0 \mathrm{E}-4 \\ \text { MG } & 8.0 \mathrm{E}-4 \\ \text { RE } & 8.0 \mathrm{E}-4 \\ \text { TA } & 8.0 \mathrm{E}-4 \\ \text { TL } & 8.0 \mathrm{E}-4 \\ \text { SI } & 8.0 \mathrm{E}-4 \\ \text { ER } & 8.0 \mathrm{E}-4 \\ & \\ & \end{array}$

$\begin{array}{lllll}8.0 \mathrm{E}-3 & 3.0 \mathrm{E}-4 & 1.3 \mathrm{E}-3 & 4.0 \mathrm{E}-3 & 4.4 \mathrm{E}-0 \\ 3.1 \mathrm{E}-2 & 2.0 \mathrm{E}+0 & 3.0 \mathrm{E}-2 & 3.0 \mathrm{E}+0 & 4.4 \mathrm{E}-0 \\ 4.0 \mathrm{E}-1 & 7.0 \mathrm{E}-3 & 1.0 \mathrm{E}-6 & 6.0 \mathrm{E}-3 & 7.6 \mathrm{E}-0 \\ 5.0 \mathrm{E}-3 & 4.0 \mathrm{E}-3 & 2.0 \mathrm{E}-5 & 7.0 \mathrm{E}-3 & 4.1 \mathrm{E}-0 \\ 6.0 \mathrm{E}-3 & 4.0 \mathrm{E}-3 & 2.5 \mathrm{E}-6 & 9.9 \mathrm{E}-4 & 2.7 \mathrm{E}-0 \\ 1.7 \mathrm{E}-2 & 9.9 \mathrm{E}-4 & 5.0 \mathrm{E}-2 & 9.9 \mathrm{E}-4 & 2.7 \mathrm{E}-0 \\ 1.0 \mathrm{E}+0 & 8.5 \mathrm{E}+0 & 2.3 \mathrm{E}-2 & 9.3 \mathrm{E}+0 & 1.7 \mathrm{E}-0 \\ 3.0 \mathrm{E}-2 & 1.0 \mathrm{E}-2 & 4.0 \mathrm{E}-2 & 2.0 \mathrm{E}-1 & 0.5 \\ 3.0 \mathrm{E}-4 & 3.5 \mathrm{E}-2 & 2.4 \mathrm{E}-3 & 3.0 \mathrm{E}-1 & 2.7 \mathrm{E}-0 \\ 2.0 \mathrm{E}-1 & 9.9 \mathrm{E}-4 & 1.5 \mathrm{E}-2 & 9.9 \mathrm{E}-4 & 3.4 \mathrm{E}-0 \\ 8.7 \mathrm{E}-3 & 3.0 \mathrm{E}-2 & 9.9 \mathrm{E}-3 & 3.0 \mathrm{E}+0 & 0.8 \\ 7.7 \mathrm{E}-2 & 8.5 \mathrm{E}-2 & 1.0 \mathrm{E}-3 & 5.2 \mathrm{E}+0 & 0.8 \\ 2.0 \mathrm{E}-4 & 4.0 \mathrm{E}-3 & 5.0 \mathrm{E}-6 & 2.0 \mathrm{E}-3 & 5.3 \mathrm{E}-0 \\ 1.0 \mathrm{E}-2 & 9.9 \mathrm{E}-4 & 1.0 \mathrm{E}-3 & 9.9 \mathrm{E}-4 & 2.7 \mathrm{E}-0 \\ 1.3 \mathrm{E}-3 & 9.9 \mathrm{E}-4 & 5.0 \mathrm{E}-4 & 9.9 \mathrm{E}-4 & 1.8 \mathrm{E}-0 \\ 3.4 \mathrm{E}-4 & 1.2 \mathrm{E}+0 & 6.1 \mathrm{E}-4 & 9.9 \mathrm{E}-1 & 1.3 \mathrm{E}-0 \\ 0.0 & 0.0 & 0.0 & 0.0 & 0.8 \\ 4.6 \mathrm{E}-3 & 1.0 \mathrm{E}-2 & 1.0 \mathrm{E}-5 & 2.0 \mathrm{E}-3 & 5.0 \mathrm{E}-0 \\ 3.0 \mathrm{E}-2 & 6.5 \mathrm{E}+0 & 3.9 \mathrm{E}-2 & 2.6 \mathrm{E}+0 & 2.8 \mathrm{E}-0 \\ 3.4 \mathrm{E}-2 & 6.4 \mathrm{E}-5 & 5.0 \mathrm{E}-6 & 1.9 \mathrm{E}-4 & 5.3 \mathrm{E}-0 \\ 1.2 \mathrm{E}-2 & 0.0 & 1.0 \mathrm{E}-2 & 0.0 & 8.0 \mathrm{E}-0 \\ 9.9 \mathrm{E}-4 & 9.9 \mathrm{E}-4 & 1.1 \mathrm{E}-2 & 9.9 \mathrm{E}-4 & 0.8 \\ 2.0 \mathrm{E}-2 & 9.9 \mathrm{E}-4 & 7.0 \mathrm{E}-3 & 9.9 \mathrm{EE}-4 & 0.8 \\ 1.5 \mathrm{E}-3 & 8.3 \mathrm{E}-1 & 8.0 \mathrm{E}-5 & 9.9 \mathrm{EE}-4 & 1.3 \mathrm{E}-0 \\ 2.0 \mathrm{E}-2 & 4.0 \mathrm{E}-3 & 2.0 \mathrm{E}-2 & 1.6 \mathrm{EE}+0 & 1.1 \mathrm{E}-01 \\ 5.0 \mathrm{E}-3 & 4.0 \mathrm{E}-3 & 2.5 \mathrm{E}-6 & 7.0 \mathrm{E}-3 & 4.1 \mathrm{E}-0< \\ 0.0 & 0.0 & 0.0 & 0.0 & 0.8 \\ 5.0 \mathrm{E}-3 & 4.0 \mathrm{E}-3 & 7.0 \mathrm{E}-7 & 2.0 \mathrm{E}-3 & 4.1 \mathrm{EE}-04 \\ 5.3 \mathrm{EE}-3 & 9.9 \mathrm{E}-4 & 5.0 \mathrm{E}-6 & 9.9 \mathrm{E}-4 & 4.1 \mathrm{E}-04 \\ 1.8 \mathrm{E}-2 & 9.9 \mathrm{E}-4 & 9.9 \mathrm{E}-4 & 1.6 \mathrm{E}+0 & 1.9 \mathrm{E}-04 \\ 9.9 \mathrm{E}-4 & 9.9 \mathrm{E}-4 & 1.0 \mathrm{E}-3 & 9.9 \mathrm{EE}-4 & 3.3 \mathrm{E}-02 \\ 9.9 \mathrm{E}-4 & 9.9 \mathrm{E}-4 & 3.0 \mathrm{E}-6 & 9.9 \mathrm{E}-4 & 4.1 \mathrm{EE}-04 \\ 9.9 \mathrm{E}-4 & 9.9 \mathrm{E}-4 & 2.0 \mathrm{E}-3 & 9.9 \mathrm{E}-4 & 0.5 \\ 4.0 \mathrm{E}-5 & 9.9 \mathrm{E}-4 & 2.0 \mathrm{E}-5 & 9.9 \mathrm{EE}-4 & 8.8 \mathrm{E}-03 \\ 4.0 \mathrm{E}-3 & 9.9 \mathrm{E}-4 & 2.0 \mathrm{E}-5 & 9.9 \mathrm{E}-4 & 4.1 \mathrm{E}-04 \\ & & & & \\ & & & & \end{array}$


APPENDIX E

GLOSSARY 


$$
\text { , }
$$




\section{APPENDIX E}

\section{GLOSSARY}

"Absorbed dose" means the energy imparted by ionizing radiation per unit mass of irradiated material. The units of absorbed dose are the rad and the gray (Gy).

"Activity" is the rate of disintegration (transformation), or decay of radioactive material. The units of activity are the curie ( $\mathrm{C} i$ ) and the becquerel $(\mathrm{Bq})$.

"Airborne radioactive material" means radioactive material dispersed in the air in the form of dusts, fumes, particulates, mists, vapors, or gases.

"Annual Dose" means an accounting for external exposure during a year plus the effective committed dose equivalent from intake by inhalation and ingestion during a year. The duration of exposure for each pathway is determined by the scenario considered and need not be $8760 \mathrm{~h} / \mathrm{yr}$. For example, an individual may reside or work at a contaminated site for a fraction of the year.

"Class" (or "lung class" or "inhalation class") means a classification scheme for inhaled material according to its rate of clearance from the pulmonary region of the lung. Materials are classified as $D, W$, or $Y$ which applies to a range of clearance half-times for $D$ (Days) of less than 10 days, for $W$ (Weeks) from 10 to 100 days, and for $Y$ (Years) of greater than 100 days.

"Collective dose" is the sum of the individual doses received in a given period of time by a specified population from exposure to a specified source of radiation.

"Commission" means the Nuclear Regulatory Commission (NRC) or its duly authorized representatives.

"Committed dose equivalent" ( $H T, 50)$ means the dose equivalent to organs or tissues of reference $(T)$ that will be received from an intake of radioactive material by an individual during the 50 -year period following the intake.

"Committed effective dose equivalent" $\left(\mathrm{HE}_{E}, 50\right)$ is the sum of the products of the weighting factors applicable to each of the body organs or tissues which are irradiated and the committed dose equivalent to these organs or tissues $\left(H_{E, 50}=\Sigma T W T H T, 50\right)$.

"Conservative" means the application of a cautious approach to a dose analysis that is likely to produce an overestimate of the expected result. A conservative analysis involves the deliberate selection of parameter values that maximize the expected result.

"Deep dose equivalent" $\left(H_{d}\right)$, which applies to external whole body exposure, is the dose equivalent at a tissue depth of $1 \mathrm{~cm}\left(1000 \mathrm{mg} / \mathrm{cm}^{2}\right)$. 
"Dose" or "radiation dose" is a generic term which means absorbed dose, dose equivalent, effective dose equivalent, committed dose equivalent, committed effective dose equivalent, or total effective dose equivalent, as defined in other paragraphs of this section.

"Dose equivalent" ( $\left.H_{T}\right)$ means the product of the absorbed dose in tissue, quality factor, and a 11 other necessary modifying factors at the location of interest. The units of dose equivalent are the rem and sievert (Sv).

"Effective dose equivalent" ( $H_{E}$ ) is the sum of the products of the dose equivalent to the organ or tissue (HT) and the weight factors (WT) applicable to each of the body organs or tissues which are irradiated $(H E=\Sigma T W T H T)$.

"Exposure" means being exposed to ionizing radiation or to radioactive material.

"External dose" means that portion of the dose equivalent received from radiation sources outside of the body.

"Gray" (Gy) is the SI unit of absorbed dose. One gray is equal to an absorbed dose of 1 joule/kilogram (100 rad).

"Individual" means any human being.

"Internal dose" means that portion of the dose equivalent received from radioactive material taken into the body.

"License" means a license issued under the regulations in Parts 30 through $35,39,40,50,60,61,70$, or 72 of this chapter.

"Licensee" means the holder of a license.

"Licensed material" means source material, special nuclear material, or byproduct material received, possessed, used, or transferred under a general or specific license issued by the Commission.

"Member of the public" means an individual in a controlled or unrestricted area. However, an individual is not a member of the public during any period in which the individual receives an occupational dose.

"NRC" means the Nuclear Regulatory Commission or its duly authorized representatives.

"Pathway" means the potential routes through which people may be exposed to radiation or radioactive materials. Typical radiation exposure pathways include external exposure to penetrating radiation, inhalation of airborne materials, and ingestion of materials contained in surface contamination, food products, or drinking water. 
"Public dose" means the dose received by a member of the public from exposure to radiation and to radioactive material released by a licensee, or to another source of radiation either within a licensee's controlled area or in unrestricted areas. It does not include occupational dose, or dose received from natural background, as a patient from medical practices, or from voluntary participation in medical research programs.

"Rad" is the special unit of absorbed dose. One rad is equal to an absorbed dose of $100 \mathrm{ergs} / \mathrm{gram}$ or 0.01 joule/kilogram ( 0.01 gray).

"Radiation" (ionizing radiation) means alpha particles, beta particles, gamma rays, x-rays, neutrons, high-speed electrons, high-speed protons, and other particles capable of producing ions. Radiation, as used in this part, does not include nonionizing radiation, such as sound, radio, or microwaves, or visible, infrared, or ultraviolet light.

"Reference man" means a hypothetical aggregation of human physical and physiological characteristics arrived at by international consensus. These characteristics may be used by researchers and public health workers to standardize results of experiments and to relate biological insult to a common base.

"Rem" is the special unit of dose equivalent. The dose equivalent in rem is equal to the absorbed dose in rad multiplied by the quality factor ( 1 rem = 0.01 sieverts).

"Scenario" means a combination of radiation exposure pathways in an effort to conceptually model the potential conditions, events, and processes that result in radiation exposure to individuals or groups of people.

"Sievert" is the SI unit of dose equivalent. The dose equivalent is sieverts is equal to the absorbed dose in grays multiplied by the quality factor (1 Sv $=100$ rem).

"Total Effective Dose Equivalent" means the sum of the deep dose equivalent (for external exposures) and the committed effective dose equivalent (for internal exposures).

"Uranium fuel cycle" means the operations of milling of uranium ore, chemical conversion of uranium, isotopic enrichment of uranium, fabrication of uranium fuel, generation of electricity by a light-water-cooled nuclear power plant using uranium fuel, and reprocessing of spent uranium fuel, to the extent that these activities directly support the production of electrical power for public use. Uranium fuel cycle does not include mining operations, operations at waste disposal sites, transportation of radioactive material in support of these operations, and the reuse of recovered non-uranium special nuclear and byproduct materials from the cycle. 
"Weight factor," WT, for an organ or tissue $(T)$ is the proportion of the risk of stochastic effects resulting from irradiation of that organ or tissue to the total risk of stochastic effects when the whole body is irradiated uniformly. For calculating the effective dose equivalent, the values of $w_{T}$ are:

\begin{tabular}{ll}
\hline Organ or Tissue & WT \\
\hline Gonads & 0.25 \\
Breast & 0.15 \\
Red bone marrow & 0.12 \\
Lung & 0.12 \\
Thyroid & 0.03 \\
Bone surfaces & 0.03 \\
Remainder & $0.30(\mathrm{a})$ \\
\hline Whole body & \\
\hline
\end{tabular}

(a) 0.30 results from 0.06 for each of 5 "remainder organs" (excluding the skin and the lens of the eye) that receive the highest doses).

(b) For the purpose of weighting the external whole body dose (for adding it to the internal dose), a single weight factor, $w_{T}=1.0$, has been specified. The use of other weighting factors for external exposure will be approved on a case-by-case basis until such time as specific guidance is issued.

"Whole body" means, for purposes of external exposure, head, trunk (including male gonads), arms above the elbow, or legs above the knee. 